
Research Laboratories of Anthropology

University of North Carolina

Monograph Series No. 1

\section{THE SIOUAN PROJECT: SEASONS I AND II}

Edited by

Roy S. Dickens, Jr.

H. Trawick Ward

R. P. Stephen Davis, Jr.

Contributors :

Linda F. Carnes

I. Randolph Daniel, Jr.

R. P. Stephen Davis, Jr.

Roy S. Dickens, Jr.

Kristen Johnson Gremillion

Julia E. Hammett

Mary Ann Holm

James H. Merrell

Gary L. Petherick

Bryan P. Sorohan

V. Ann Tippitt

H. Trawick Ward

Homes Hogue Wilson

Chape1 Hill, North Carolina 1987 


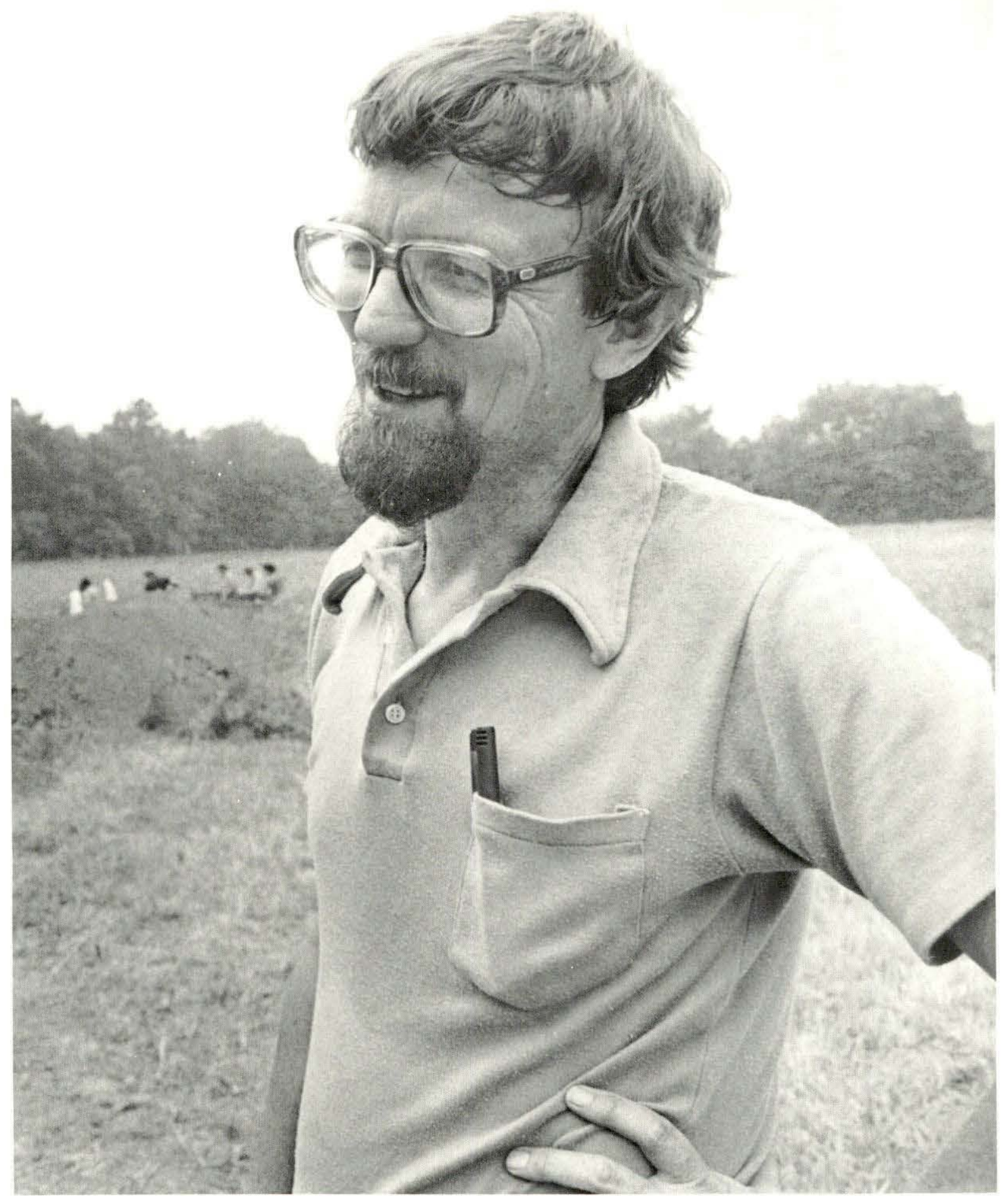

Roy Selman Dickens, Jr.

1938-1986 


\section{Table of Contents}

Page

LIST OF TABLES . . . . . . . . . . . . . . . . . . viii

LIST OF FIGURES. • • • . • . . . . . . . . . . . . . xi

ACKNOWLEDGMENTS. • • • . . . . . . . . . . . . . . xvii

PREFACE. . . . . . . . . . . . . . . . . . . . xviii

CHAPTER 1. INTRODUCTION, by Roy S. Dickens, Jr., H. Trawick Ward, and R. P. Stephen Davis, Jr .............. 1

Historical Background. . . . . . . . . . . . 1

Archaeological Background. . . . . . . . . . 3

Siouan Project . . . . . . . . ...... 5

1983 Fieldwork . . . . . . . . . . . . 6

1984 Fieldwork ................. 7

1985-86 Fieldwork at the Fredricks Site. . . . . 13

Report Overview. . . . . . . . . . . 14

CHAPTER 2. "THIS WESTERN WORLD": THE EVOLUTION OF THE PIEDMONT,

1525-1725, by James H. Merrell. . . . . . . . . . 19

CHAPTER 3. ARCHITECTURE AND FEATURES AT THE FREDRICKS, WALL, AND

MITCHUM SITES, by Gary L. Petherick . . . . . . . . . 29

Introduction ............... 29

Wall Site. . . . . . . . . . . . . 30

Mitchum Site................. 49

Fredricks Site................. 58

Comparisons and Conclusions. . . . . . . . . 77

CHAPTER 4. MORTUARY PATTERNS AT THE FREDRICKS, WALL, AND MITCHUM

SITES, by H. Trawick Ward . . . . . . . . . . 81

Introduction . . . . . . . . . . . . 81

Approaches to Mortuary Analysis. . . . ..... 81

Ethnohistoric Background . . . . . . . . . . 82

Archaeological Background. . . . . . . . . 85

The Susquehannock Connection .......... . 89

Fredricks Site Burials . . . . . . . . . . 90

Discussion of Fredricks Site Burials . . . . . . . 101

Wal1 Site Burials $(1938,1940-41) . . . . . . . . .105$

Wa11 Site Burials (1983) . . . . . . . . . 106

Discussion of Wall Site Burials. . . . . . . . 107

The Mitchum Site Burial. . . . ........ 107

Summary of Mortuary Data . . . . . . . . . 108

Socio-Political Implications of the Fredricks Site

Burials . . . . . . . . . . . 108

CHAPTER 5. HUMAN SKELETAL REMAINS FROM THE WALL AND FREDRICKS

SITES, by Homes Hogue Wilson. . . . . . . . . . . . . 111

Introduction . . . . . . . . . . ... 111

Demographic Analysis . . . . . . . . . . . 111

Pathologies................. 121 
Diet, Health, and Trace Element Analysis . . . . . 127 Biological Distance and Diversity. . . . . . . 130 Conclusions. . . . . . . . . . . . . . 138

CHAPTER 6. EUROAMERICAN ARTIFACTS FROM THE FREDRICKS, WALL, AND MITCHUM SITES, by Linda F. Carnes............ . 141

Introduction . . . . . . . . . . . . . 141 Methods. . . . . . . . . . . ... 141 Euroamerican Artifacts by Functional Group . . . . . 142 Interpretations. . . . . . . . . . . . 158 Conclusions. . . . . . . . . . . . . 164

CHAPTER 7. SHELL ARTIFACTS FROM THE CAROLINA PIEDMONT, by Julia E. Hammett. . . . . . . . . . . . . . . . 167

Introduction . . . . . . . . . . . . 167 Beadmaking Technology. . . . . . . . . . . 167 Ornaments from the Siouan Project. . . . . . . 169 A Regional Perspective.............. . 182 Serrated Shells................. . 183 Conclusions. . . . . . . . . . . . . 183

CHAPTER 8. POTTERY FROM THE FREDRICKS, WALL, AND MITCHUM SITES,

by R. P. Stephen Davis, Jr. . . . . . . . . . . . 185

Introduction . . . . . . . . . . . . 185

Analytic Methods ................ 186

Fredricks Site................. 189

Wall Site. . . . . . . . . . . . . 198

Mitchum Site. . . . . . . . . . . . . 204

Intersite Comparisons. . . . . . . . . . . 209

Summary and Conclusions. . . . . . . . . . 214

CHAPTER 9. LITHIC ARTIFACTS FROM THE FREDRICKS, WALL, AND MITCHUM

SITES, by V. Ann Tippitt and I. Randolph Daniel, Jr . . . . 217

Introduction . . . . . . . . . . . . 217

Analytic Methods ............... 217

Artifact Types ................. . . 217

Raw Materials. . . . . . . . . . . . 219

Assemblage Descriptions. . . . . . . . . . 220

Comparisons. ................. 230

Summary and Conclusions. . . . . . . . . 236

CHAPTER 10. FAUNAL REMAINS FROM THE WALL AND FREDRICKS SITES, by

Mary Ann Holm . . . . . . . . . . . . . . . 237

Research Questions . . . . . . . . . . 237 Ethnohistoric References to the Use of Faunal Resources. 239 Excavation and Recovery Techniques Affecting Faunal

Remains . . . . . . . . . . . . . 242

Sampling and Analytic Techniques . . . . . . . . 242

Results of Analysis: Wall Site. . . . . . . . . 244

Results of Analysis: Fredricks Site . . . . . . 247

Comparison of the Two Assemblages. . . . . . . . 251

Habitat Preferences and Seasonality. . . . . . . 255

Diversity. . . . . . . . . . . . . 256

Conclusions. . . . . . . . . . . . . 257 
CHAPTER 11. PLANT REMAINS FROM THE FREDRICKS, WALL, AND MITCHUM

SITES, by Kristen Johnson Gremillion. . . . . . . . . 259

Introduction . . . . . . . . . . . . 259

Methodological Issues. . . . . . . . . . . 259

Comparison of Plant Remains Assemblages. . . . . . 263

A Cost-Benefit Approach to Change and Contact. . . . 272

Reconstruction of Plant Use Patterns at the Fredricks

Site................. 273

Summary and Conclusions. . . . . . . . . . 276

REFERENCES CITED . . . . . . . . . . . . . . . . 279

APPENDIX A. SUMMARY REPORT OF 1985 FREDRICKS SITE EXCAVATIONS, by

Roy S. Dickens, Jr., H. Trawick Ward, and R. P. Stephen

Davis, Jr.. . . . . . . . . . . . . . . . 295

Introduction . . . . . . . . . . . . 295

Results. . . . . . . . . . . . . . 295

Conclusions. . . . . . . . . . . . . 299

APPENDIX B. SUMMARY REPORT OF 1986 FREDRICKS SITE EXCAVATIONS, by

H. Trawick Ward and R. P. Stephen Davis, Jr.. . . . . . 303

Introduction ............... 303

Results. . . . . . . . . . . . . . 303

Conclusions. . . . . . . . . . . . . . 306 


\section{List of Tables}

Table

1.1. Summary of archaeological features at the Fredricks site, 1983-1986 . . .

3.1. Summary of feature attributes for pits and basins at the Wall site. .

3.2. Nonbotanical remains, wood charcoal, and plant food remains from the 19831984 excavations at the Wall site . 39

3.3. Summary of structure attribute measurements at the Wall site . . 42

3.4. Selected data related to structure maintenance and placement at the Wall site.......... 47

3.5. Summary of feature attributes at the Mitchum site. . . . . . 54

3.6. Amounts and percentages of nonbotanical remains, wood charcoal, and plant food remains from feature fill at the Mitchum site. . . . 56

3.7. Summary of feature attributes at the Fredricks site. . . . . . . 6

3.8. Nonbotanical remains, wood charcoal, and plant food remains from the 19831984 excavations at the Fredricks site. . . . . . . . . 64

3.9. Summary of structure attribute measurements at the Fredricks site. . 68

4.1. Pit dimensions of the Fredricks site burials...... . . . . 93

4.2. Acculturation indices for Fredricks site burial groupings . . . . . 104

4.3. Distribution of artifacts associated with the Fredricks site burials by age categories. ......... 104

5.1. Age of subadults from the Wall and Fredricks sites ......... 112

5.2. Age of adults from the Wall and Fredricks sites . . . . . . . 112

5.3. $2 \times 2$ contingency table to test the significance of male and female mortality at the prehistoric Shannon site........... 116

5.4. $2 \times 3$ contingency table to test the significance of male and female mortality at the prehistoric Shannon site. ......... 116
Table

Page

5.5. $2 \times 2$ contingency table to test the significance of male and female mortality at Upper Saratown . . . 116

5.6. $2 \times 3$ contingency table to test the significance of male and female mortality at Upper Saratown . . . 117

5.7. $2 \times 2$ contingency table to test the significance of male mortality at the Shannon and Upper Saratown sites ........... 117

5.8. $2 \times 3$ contingency table to test the significance of male mortality at the Shannon and Upper Saratown sites. . 117

5.9. $2 \times 2$ contingency table to test the significance of female mortality at the Shannon and Upper Saratown sites ........... 117

5.10. $2 \times 3$ contingency table to test the significance of female mortality at the Shannon and Upper Saratown

sites........... 117

5.11. Life table for the Shannon site . . 118

5.12. Life table for the Wall site. . . . 118

5.13. Life table for the Upper Saratown site........... 119

5.14. Life table for the Fredricks site . 119

5.15. Estimated population size of the Fredricks site. . ....... 120

5.16. ICP of trace elements in bones from the Wall site. . . . . . . 129

5.17. ICP of trace elements in bones from the Fredricks site. . . . . 129

5.18. Mean cranial indices. . . . . . 132

5.19. Post-cranial indices. . . . . . 133

6.1. Euroamerican trade items from trade lists for the Fredricks, Mitchum, and Upper Saratown sites. . . . . 142

6.2. Frequency of historic artifacts by site, context, and functional classification. . . . . . . . 143

6.3. Kaolin pipe stem fragments by context and bore diameter . . . . 154 
6.4. Frequency of Euroamerican artifacts as burial associations and in pitfill-plowzone contexts . . . . 162

6.5. Frequency of Euroamerican artifacts in burial pitfill and non-burial pitfill . . . . . . . . 164

7.1. Preliminary inventory of shel1 artifacts from selected Southeastern sites. . . . . . 173

7.2 Summary of margine1la ornaments from the Wall site . . . . . . . 178

7.3 Inventory of shell ornaments from the Wall site . . . . . . . 179

7.4 Summary of wampum from the Fredricks site. . . . . . . 180

7.5 Inventory of she11 ornaments from the Fredricks site........ 181

8.1 Frequency of Fredricks site sherds and vessels by size....... 189

8.2 Frequency of Fredricks site sherds by temper and exterior surface treatment ......... 190

8.3 Frequency of Fredricks site sherds from feature and burial contexts. . 191

8.4 Summary of coarse sand-tempered sherds from the Fredricks site. . . 192

8.5. Summary of medium sand-tempered sherds from the Fredricks site. . . 192

8.6. Summary of fine sand-tempered sherds from the Fredricks site . . . . 192

8.7. Summary of coarse crushed quartztempered sherds from the Fredricks site. ........... 192

8.8. Summary of medium crushed quartztempered sherds from the Fredricks site. . . . . . . . . 192

8.9. Summary of fine crushed quartztempered sherds from the Fredricks site. . . . . . . . . 193

8.10. Summary of coarse crushed feldspartempered sherds from the Fredricks site. . . . . . . . . 193

8.11. Summary of fine crushed feldspartempered sherds from the Fredricks site........... 193

8.12. Evaluation of differences in spatial distribution of selected sherd categories at the Fredricks site. . 196
8.13. Definition of ceramic groups, based on attribute similarities, at the Fredricks site. . ....... 196

8.14. Frequency of Wall site sherds and vessels by size . . . . . . . 198

8.15. Frequency of Wall site sherds by temper and exterior surface treatment . . . . . . . . 199

8.16. Summary of Wall site sherds by exterior surface treatment. . . . 200

8.17. Comparison of selected attributes for plowzone and midden contexts at the Wall site. . . . . . . 204

8.18. Frequency of Mitchum site sherds by size . . . . . . . . 205

8.19. Frequency of Mitchum site sherds by temper and exterior surface treatment . . . . . . . . 205

8.20. Summary of selected sherd categories from the Mitchum site... . . 207

8.21. Summary of assemblages used in intersite ceramic analysis. . . . 211

8.22. Surface treatment percentages for analyzed ceramic assemblages. . . 211

8.23. Correlation matrix for data used in the intersite ceramic analysis. . . 212

8.24. Factor loading matrix . . . . . 213

9.1. Distribution of debitage by site. . 220

9.2. Distribution of tool types by site. . 221

9.3. Distribution of utilized/retouched flakes by site. . . . . . . 222

9.4. Fredricks site lithic artifact distribution by context . . . . 224

9.5. Comparison of Fredricks and Forbush Creek 1ithic assemblages by inferred functional group. . . . . . . 231

9.6. Mean projectile point length, width, and thickness by site... . . 232

9.7. Distribution of triangular projectile point forms by site. . . 235

9.8. Percent of raw material types represented by debitage from the Wal1, Fredricks, and Mitchum sites. . 236

9.9. Percent of flakes and chipped-stone tools of local raw materials. . . 236 
10.1. Animal remains from the Wall site . 245

10.2. Animal remains from the Fredricks

site. .......... 248

10.3. Expected and actual representation of deer skeletal elements . . . . 252

10.4. Estimated meat yield in pounds. . . 254

10.5. Summary of species diversity measures. ......... 256

11.1. Summary of plant remains from the Wa11, Fredricks, and Mitchum sites. . 261

11.2. Percent of plant food remains from the Wa11, Fredricks, and Mitchum sites . . . . . . . . 262
11.3. Ubiquity of plant remains, as percent of flotation samples, at the Wall, Fredricks, and Mitchum sites. . . 264

11.4. Seed counts from the Fredricks site.......... 265

11.5. Seed counts from the Wall and Mitchum sites...... 266

11.6. Percent of nutshell from the Wall, Fredricks, and Mitchum sites. . . 267

11.7. Comparison of plant remains from burials, features, and structures at the Fredricks site . . . . 268

11.8. Distribution of seeds from the Wall, Mitchum, and Fredricks sites. . . 270 


\section{List of Figures}

Figure

1.1. Hillsborough locality showing the Wall and Fredricks sites. . . . . 2

1.2. Edward Moseley's 1733 map locating "Acconeechy" on the Eno River... . 3

1.3. Archaeological sites in the Siouan Project area.......... 4

1.4. 1938 excavations at the Wall site. . 5

1.5. Areal view of 1983 test excavation at the Fredricks site showing rectangular burial pits....... 7

1.6. Hillsborough archaeological district. . . . . . . . . 9

1.7. Aerial view of 1984 excavations at the Wall site and Fredricks site. . 10

1.8. Removing plowzone at the Fredricks site. . . . . . . 10

1.9. Trowelling the top of subsoil to expose archaeological features at the Fredricks site . . . . . . 11

1.10. Overview of the 1984 excavation at the Fredricks site showing palisade and village cemetery......... 11

1.11. Plan of 1983-84 excavations at the Fredricks site. . . . . . . 12

1.12. Area covered by 1983-86 excavations at the Fredricks site . . . . . 13

1.13. Fredricks site plan showing the results of $1983,1984,1985$, and 1986 excavations. . . . . . . 15

3.1. 1938, 1940-41, and 1983-84 excavation areas at the Wall site. . . . . . 31

3.2. Excavation plan at the Wall site, 1938-1984..............32

3.3. Plan of architecture and features at the Wall site. . . . . . . . 33

3.4. Areal extent of the midden at the Wall site......... 34

3.5. Site stratigraphy at the Wall site, N-S sections.......... 35

3.6. Sq. $370 \mathrm{R} 530$ at the Wall site showing midden preserved beneath plowzone.

\section{Figure}

Page

3.7. Plan and profile of Feature 32, a small shallow basin, at the Wall site.......... 38

3.8. Plan and profile of Feature 54, a large shallow basin, at the Wall site. . . . . . . . . 38

3.9. Plan and profile of Feature 45, a large, amorphous shallow basin located within Structure $J$, at the Wall site.......... . 38

3.10. Plan and profiles of Feature 23 and 3-84, small deep pits, at the Wall

site. . . . . . . . . 38

3.11 Plan and profile of Feature 4-84, an irregular trench-like feature associated with Structure $G$, at the Wall site............ . 39

3.12 Structure $G$ at the Wall site after excavation of postholes and pits. . 44

3.13 SYMAP of daub in the vicinity of Structures $\mathrm{G}$ and $\mathrm{H}$ at the Wall site............ 45

3.14 Excavation plan of the Mitchum site, 1983........... 50

3.15 Feature 1, a deep basin-shaped pit, at the Mitchum site...... 51

3.16 Plan and profile of Feature 1 at the Mitchum site......... 51

3.17 Feature 6, a deep pit, at the Mitchum site......... 51

3.18 Plan and profile of Feature 6 at the Mitchum site. . . . . . 51

3.19 Feature 8, a large shallow basin, at the Mitchum site. . . . . . 52

3.20. Plan and profile of Feature 8 at the Mitchum site. . . . . . . 52

3.21. Feature 14 , a small shallow basin, at the Mitchum site... . . . 53

3.22. Plan and profile of Feature 14 at the Mitchum site. . . . . . . 53

3.23. Structure 1 at the Mitchum site after excavation of postholes . . . . 57 
3.24. Excavation plan of the Fredricks site, 1983-1984....... . 59

3.25. SYMAP of historic aboriginal ceramics in the plowzone at the Fredricks

site.......... 60

3.26. Overview of the 1984 excavations at the Fredricks site, showing portions of the palisade and cemetery. . . .

3.27. Feature 9 at the Fredricks site, prior to excavation . . . . . 62

3.28. Feature 9 at the Fredricks site, after excavation. . . . . . 62

3.29. Plan and profile of Feature 9 at the Fredricks site. . . . . . 62

3.30. Feature 9 at the Fredricks site, showing stratigraphy of pit fill. . 63

3.31. Bottom of Feature 9 at the Fredricks site, showing rock clusters and charred plant remains on pit floor. .

3.32. Feature 10 at the Fredricks site, after excavation. . . . . . . 64

3.33. Plan and profile of Feature 10 at the Fredricks site . . . . .

3.34. Feature 11 at the Fredricks site, after excavation. . . . . . 65

3.35. Plan and profile of Feature 11 at the Fredricks site.......

3.36. Feature 12 at the Fredricks site, after excavation. . . . . . 66

3.37. Plan and profile of Feature 12 at the Fredricks site . . . . 66

3.38. Feature 13 at the Fredricks site, after excavation. . . . . . 67

3.39. Plan and profile of Feature 13 at the Fredricks site . . . . . 67

3.40. Plan of architecture and features at the Fredricks site . . . . .

3.41. Structure 1 and Feature 9 at the Fredricks site, prior to excavation. . . . . . . . 70

3.42. Structure 1 and Feature 9 at the Fredricks site, after excavation. . . 70

3.43. SYMAP of daub in the plowzone at the Fredricks site.......

3.44. Map showing the results of soil auger testing at the Fredricks site . . .
3.45. SYMAP of features at the Fredricks site, based on the results of auger testing . . . . . . . . 74

3.46. Excavation plan of the Fredricks site showing activity areas . . . 75

3.47. Relative frequency histogram of feature classes at the Wall, Mitchum, and Fredricks sites. . . 77

3.48. Histogram showing the floor area of structures at the Wall, Mitchum, and Fredricks sites, and their associated storage pit volumes ...... 79

4.1. Results of soil auger testing at the Fredricks site, showing the cemetery. . . . . . . . . 91

4.2. Burial 1 at the Fredricks site. . . 92

4.3. Plan and profile of Burial 1 at the Fredricks site. . . . . . . 92

4.4. Artifact cluster from Burial at the Fredricks site . . . . . 92

4.5. Burial 2 at the Fredricks site. . . 93

4.6. Plan and profile of Burial 2 at the Fredricks site...... 93

4.7. Artifact cluster from Burial 2 at the Fredricks site.. . . . 94

4.8. Small check-stamped pottery vesse1 from Burial 2 at the Fredricks site. . . . . . . . 94

4.9. Burial 3 at the Fredricks site. . . 95

4.10. Plan and profile of Burial 3 at the Fredricks site .. . . . 95

4.11. Artifact cluster from Burial 3 at the Fredricks site . . . . . 95

4.12. Smoking kit from Burial 3 at the Fredricks site . . . . 96

4.13. Burial 4 at the Fredricks site. . . 96

4.14. Plan and profile of Burial 4 at the Fredricks site... . . 96

4.15. Burial 5 at the Fredricks site. . . 97

4.16. Plan and profile of Burial 5 at the Fredricks site . . . . . 97

4.17. Artifact cluster from Burial 5 at the Fredricks site... . . 98

4.18. Burial 6 at the Fredricks site. . . 99 
4.19. Plan and profile of Burial 6 at the Fredricks site . . . . . 99

4.20. Bells associated with Burial 7 at the Fredricks site . . . . . 100

4.21. Burial 8 at the Fredricks site. . 100

4.22. Plan and profile of Burial 8 at the Fredricks site . . . . . 100

4.23. Artifact cluster from Burial 8 at the Fredricks site . . . . 101

4.24. Pot with artifact cluster from Burial 8 at the Fredricks site. . . 101

4.25. Burial 9 at the Fredricks site. . 102

4.26. Plan and profile of Burial 9 at the Fredricks site . . . . . 102

4.27. Fil1 profiles of Burial Groups 1 and 2 at the Fredricks site . . . 103

4.28. Fill profiles of Burial Groups 3 and 4 at the Fredricks site . . . 103

4.29. Burial 1-83 at the Wall site. . . 106

4.30. Burial 1 at the Mitchum site. . . 108

4.31. Schematic plan of village-burial spatial relationships . . . . 109

5.1. Mortality curves for the Fredricks, Wal1, Upper Saratown, and Shannon skeletal samples. . . . . . . 114

5.2. Mortality curves by sex for the Shannon and Upper Saratown skeletal samples......... 116

5.3. Left humerus compared with right humerus of Burial 1 at the

Wall site......... 122

5.4. Right parietal fragment of Burial 4 from the Fredricks site, showing the cut mark or traumatic injury. . . . 123

5.5. Vertebral lipping of Burials 1 and 3 from the Wall site. . . . . . 123

5.6. Patella and calcaneus of Burial 3 from the Wall site, showing possible tendonitis . . . . . 124

5.7. Thoracic vertebrae of Burials 4 and 9 from the Fredricks site, with herniated disc or Schmorl's nodes . 125

5.8. Closeup of Burial 4 skull from the Wall site, with orbits showing cribra orbitalia. . . . . . 126
5.9. Top view of cranium of Burial 4 from the Wall site, showing frontal and parietal pitting related to spongy hyperostosis . . . . . 126

5.10. Front view of crania from Fredricks site Burial 5 and Wall site Burial 1. . . . . . . . . 134

5.11. Top view of crania from Wall site Burial 1 and Fredricks site Burial 5. ........... 134

5.12. Side view of crania from Wall site Burial 1 and Fredricks site Burial 5. . . . . . . . 135

5.13. Front view of crania from Upper Saratown Burial 73 and Fredricks site Burial 5. . . . . . . 135

5.14. Top view of crania from Fredricks site Burial 5 and Upper Saratown Burial 73 . . . . . . . 136

5.15. Side view of crania from Upper Saratown Burial 73 and Fredricks site Burial 5. . . . . . . 136

5.16. Front view of crania from Upper Saratown Burial 73 and Wall site Burial 1.......... 137

5.17. Top view of crania from Wall site Burial 1 and Upper Saratown Burial 73 . . . . . . . . 137

5.18. Side view of crania from Upper Saratown Burial 73 and Wall site Burial $1 . . . . . . . .138$

6.1. Sample of spherical lead ammunition from the Fredricks site... . 144

6.2 Gunflints from the Fredricks site . 144

6.3. Dog-lock hammers from Upper Saratown that are similar in style to the gun from Burial 6 at the Fredricks site............ 146

6.4. Close-up of dog-1ock firing mechanism from Burial 6 at the Fredricks site........... 146

6.5. Sample of clothing fasteners from the Fredricks site. . . . . . 147

6.6. Scissors from burials at the Fredricks site. . . . . . . 147

6.7. Wine (rum) bottle from Burial 3 at the Fredricks site... . . 148

6.8. Wine (rum) bottle from Burial 4 at the Fredricks site . . . . 148 
6.9. Profile of whole wine bottle from Burial 4 at the Fredricks site. . . 149

6.10. Latten spoon from Burial 8 at the Fredricks site. . . . . . . 150

6.11. Close-up of touch mark on Burial 8 spoon bowl. . . . . . . . 150

6.12. Sample of glass and ivory beads from the Fredricks site . . . . 151

6.13. Sample of brass bells from Burial 7 and Feature 13 at the Fredricks site........... 152

6.14. C-shaped wire bracelets from Burial 6 at the Fredricks site . . . . . 153

6.15. Sample of aboriginally modified metal artifacts from the Fredricks site . 153

6.16. Sample of pipes from the Fredricks site. . . . . . . . . 154

6.17. Cast pewter tulip bowl style pipe from Burial 3 at the Fredricks site. . . . . . . . . 155

6.18. Hand-carved pipe stem from Burial 6 and pewter pipe bowl liner from Feature 13 at the Fredricks site. . 155

6.19. Artist's reconstruction of pewter pipestem with wooden bowl and pewter bowl liner . . . . . . . 155

6.20. Iron ember tongs from Burial 3 at the Fredricks site . . . . . 155

6.21. Cleaned and treated iron axe head from Burial 5 at the Fredricks site.......... 156

6.22. Broad-bladed iron hoe from Burial 6 at the Fredricks site . . . . . 157

6.23. Narrow-bladed iron hoe from Burial 9 at the Fredricks site...... 157

6.24. Bone-handled, steel-bladed case knives from Burial 1 at the Fredricks site. . . . . . . 157

6.25. Spatial distribution of iron nails at the Fredricks site . . . . . 159

6.26. Spatial distribution of brick fragments at the Fredricks site . . . 159

6.27. Spatial distribution of glass artifacts at the Fredricks site . . . 159

6.28. Spatial distribution of European ceramics at the Fredricks site. . . 159
6.29. Spatial distribution of metal artifacts at the Fredricks site . . . 160

6.30. Spatial distribution of gunflints at the Fredricks site . . . . 160

6.31. Spatial distribution of miscellaneous historic artifacts at the Fredricks site........... 160

6.32. Spatial distribution of European trade pipes at the Fredricks site. . 160

6.33. Spatial distribution of aboriginal pipes at the Fredricks site . . . 161

7.1. Pendants and gorgets....... 170

7.2. Close-up of pendants from Burial 1 at the Fredricks site . . . . . 170

7.3. Comparison of designs on large pendant from Burial 1 and on spoon from Burial 8 at the Fredricks site. . . 171

7.4. Evidence of beadmaking. . . . . 171

7.5. Tube beads from the Fredricks site, Vir196, and Upper Saratown; tube blank from Upper Saratown . . . . 174

7.6. Bead types from the Wall site... 174

7.7. Bead types from the Fredricks site. 175

7.8. Shell disk beads from the Wall site and Fredricks site . . . . 176

7.9. Frequency distribution of she11 disk beads by screen size . . . . 177

7.10. Serrated shells from the Wall site showing similarity of edge to scrape marks on inside of potsherd . . . 183

8.1. Definition of vessel portion attribute states. . . . . . . 186

8.2. Definition of rim and lip form attribute states. . . . . . 186

8.3. Relative frequency distributions and statistical evaluation of selected attributes for medium sand-tempered plain, check-stamped, and netimpressed sherds from the Fredricks site........... 195

8.4. Plain, brushed, and cord-marked sherds from the Fredricks site. . . 197

8.5. Check-stamped sherds from the Fredricks site. . . . . . . . 
8.6. Net-impressed, brushed, simplestamped, check-stamped, and cobimpressed sherds from the Fredricks site. . .......... 197

8.7. Whole vessels from Burial 2, Burial 8 , and Burial 6 at the Fredricks site. ........ 19

8.8. Profiles of vessels and selected vessel fragments from the Fredricks site. . . . . . . . . 198

8.9. Simple-stamped sherds from the Wall site . . . . . . . . 202

8.10. Check-stamped and plain sherds from the Wall site . . . . . . . 202

8.11. Undecorated and decorated plain vessel sections from the Wall site. . 202

8.12. Whole vessels from Burial 3-83 and Burial 1-83 at the Wall site. . . . 202

8.13. Relative frequency distributions and statistical evaluation of selected attributes for plain, simple-stamped, and check-stamped sherds from the Wall site . . . . . . . . 203

8.14. Profiles of vessels and selected vessel fragments from the

Wall site . . . . . . . . 204

8.15. Plain, simple-stamped, brushed, and cob-impressed sherds from the Mitchum site. . . . . . . 206

8.16. Net-impressed sherds from the Mitchum site. . . . . . . 206

8.17. Relative frequency distributions and statistical evaluation of selected attributes for plain, brushed, simple-stamped, and net-impressed sherds from the Mitchum site. . . 208

8.18. Map locating sites used in the comparative ceramic analysis. . . 210

8.19. Dendrogram showing results of ceramic cluster analysis. . . . . 213

9.1. Flake tools from the Fredricks site . 222

9.2. Choppers and chipped-stone disks from the Fredricks site. . . . . 222

9.3. Triangular projectile points from the Fredricks site. . . . . . 223

9.4. Archaic and Woodland projectile points from the Fredricks site. . . 223
9.5. Ground-stone artifacts from the Fredricks site. . . . . . . 223

9.6. Triangular projectile points from the Wall site........ 227

9.7. Flake tools from the Wall site. . . 227

9.8. Paleoindian and Archaic projectile points from the Wall site . . . 228

9.9. Triangular projectile points from the Mitchum site. . . . . . 228

9.10. Flake tools from the Mitchum site . 228

9.11. Triangular projectile points from Early Upper Saratown. . . . . . 229

9.12. Flake tools from Early Upper Saratown. . . . . . . . 229

9.13. Triangular projectile points from Upper Saratown. . . . . . . 230

9.14. Flake tools from Upper Saratown . . 230

9.15. Cumulative frequency distribution of projectile point length. . . . 233

9.16. Cumulative frequency distribution of projectile point width . . . . 234

9.17. Bivariate plot of mean projectile point length versus mean width. . . 235

10.1. Bone beamer and awls from the Wall site. . . . . . . . . 247

10.2. Bivariate plots of rank values for 12 animal species at the Wall site and Fredricks site. . . . . . 254

A. 1. Burial 10, excavated. . . . . 296

A.2. Burial 11, excavated....... 296

A. 3. Burial 13, excavated. . . . . 297

A.4. Feature 19, excavated . . . . . 297

A.5. Feature 28, excavated . . . . 297

A.6. Feature 18, excavated . . . . 297

A.7. Structure 5 and Structure 6 at top of subsoil........... 298

A.8. Clay pots recovered from Burial 10 and Burial 11 . . . . . . . . 299

A.9. Iron hoe and axe fragments. . . . 300

A. 10. Selected small artifacts recovered during 1985 excavations . . . . 300 
B.1. Burial 12, excavated. . . . . 304

B.2. Burial 14, excavated. . . . . 304

B.3. Feature 46, excavated ...... 305

B.4. Feature 56, excavated . . . . 305

B.5. Feature 53, fill at top of Zone 2. 305

B.6. Reconstructed check-stamped pots. . 307
B.7. Reconstructed plain and cord-marked pots............ 307

B. 8. Small Euroamerican artifacts. . . 308

B.9. Aboriginal artifacts. . . . . 308

B. 10. Settlement plan of the Occaneechi village . . . . . . . 308 


\section{Acknowledgments}

The Siouan Project marks a major undertaking in the annals of archaeological studies conducted by the Research Laboratories of Anthropology at the University of North Carolina. Although only the initial phases of the project are reported in the chapters that follow, they, nonetheless, represent a major effort requiring the labor, encouragement, and support of a large number of dedicated individuals. In particular, the contributors worked hard and long in a concerted and unselfish effort to lend their expertise to a wide range of complex archaeological issues. Most were full-time graduate students during the time the chapters were prepared and often were called upon to stretch already tight academic schedules to meet project demands. During the field seasons, they also rose early and labored long digging plowzone, excavating features and burials, and keeping the myriad record files current.

Thank you Linda Carnes, Randy Daniel, Kristen Gremillion, Julia Hammett, Mary Ann Holm, Gary Petherick, Beverly Sizemore, Ann Tippitt, and Homes Wilson. Although not a contributor to this volume, Dan Simpkins deserves a special thanks for his dedicated and herculean efforts in the field. He also generously shared his indepth knowledge of Siouan settlement patterns, and his insights were particularly helpful in weaving a web of continuity between the various chapters.

The Siouan Project is not only a graduate student project; it has also provided many undergraduates with their first taste of field archaeology. Most proved to be excellent students and are too numerous to mention individually. However, we cannot overstate the importance of their eagerness to learn and willingness to work in making the field seasons productive as well as enjoyable. Two of these students, Jane McManus and Bryan Sorohan, developed particularly keen excavation skills and an unwhetted appetite for fieldwork. These uncommon qualities made them invaluable field assistants during subsequent summers, and their capable services are most appreciated.

We also wish to acknowledge the strong support and sage advise of Stephen Birdsall, Associate Dean of Arts and Sciences; Donald Brockington, acting Director of the Research Labs; George Holcomb, Chairman of the Anthro- pology Department; and Richard Yarne11, Professor of Anthropology.

Jack Wilson, Jr. of the Historic Sites Section of the N. C. Division of Archives and History has been a friend of the project since its inception, and his indepth knowledge of the Piedmont Siouans has made him a most valued colleague. Bruce Jones of the N.C. Commission of Indian Affairs has not only provided encouragement but also enhanced our awareness of the sensitivities of Native Americans and the need for a common bond between all of us who are dedicated to understanding the past.

We also wish to thank the volunteers who have participated in the project at one time or another. Margo Price and Steve Wood were particularly helpful along with many members of the Archaeological Society of North Carolina who devoted their Saturdays to our cause.

It is a gross understatement to say that we owe a deep debt of gratitude to the landowners. Without their cooperation, the Siouan Project would have remained an idea whose time had yet to come. Frank Fredricks, Cyrus Hogue III, and Dr. Kenneth Mitchum freely gave us permission to excavate on their property. They also made us welcome in their communities and provided innumerable services above and beyond the call of duty. We are especially appreciative of Richard Jenrette, a UNC alumnus whose generosity both facilitated the 1986 field season at the Fredricks site and insured the future of archaeological research at several other sites, including the Wall site, in the immediate vicinity.

Last, but not least, we wish to thank Estella Stansbury for her patient assistance in the preparation of this book and for keeping the lab running smoothly while we indulged ourselves in more pleasurable pursuits.

Funding for the Fredricks site excavations was generously provided by the National Geographic Society. Additional support for Siouan Project research has been provided by the University of North Carolina, the National Park Service (administered by the N. C. Division of Archives and History), and the National Science Foundation. 


\section{Preface}

With one exception, the chapters in this monograph were written during the spring of 1985 by students and staff of the Research Laboratories of Anthropology. The one exception is Chapter 2 which was written by James H. Merre11, an historian at Vassar College. Jim's contribution is a summary of extensive documentary research on the Piedmont Indians during the Contact period. The remaining chapters present detailed analyses of all major data categories retrieved during 1983-84 archaeological excavations at the Fredricks, Wall, and Mitchum sites.

Excavations at the Fredricks site after the spring of 1985 resulted in new information which, in some instances, altered interpretations based on earlier work. In an effort to make this publication as current as possible, results of the 1985 and 1986 field seasons at the Fredricks site are summarized briefly in the Introduction, and where these data gainsay interpretations reached in 1985, brief comments describe these differences. More detailed accounts of the 1985 and 1986 excavations are presented in Appendixes A and B.

The spring of 1985 was a particularly creative and productive period in the Research Labs' long history of archaeological research in North Carolina. The impetus behind this strong surge of research activity was Roy S. Dickens, $\mathrm{Jr}$. who had become the Director of the Research Labs in the summer of 1982. The Siouan Project was born shortly thereafter. Excavations at the Wall, Fredricks, and Mitchum sites commenced the following summer, and work at the Fredricks site was expanded considerably during the 1984 field season.

Roy conducted a seminar on the Siouan Project during the spring of 1985 in which the contributors to this monograph participated. This seminar provided a fertile medium for ideas to germinate and grow. Discussions were open and wide-ranging; debates were intense and sometimes heated, but always constructive. When the seminar was concluded, it was agreed that the results of the Siouan Project research, up to that point, should be disseminated. Roy felt strongly that this should be accomplished by revising and publishing the seminar papers as the first volume in a new Research Labs' monograph series.

Roy's untimely death in May 1986 delayed temporarily the publication of this volume, but his energy, exuberance, dedication, and love of archaeology did not die. They inspired the momentum which led to the completion of this monograph and launched a long-term program of Siouan research that continues today. These uncommon qualities live in his work and in all of us who knew him. This monograph is only a small reflection of the total contribution he made to North Carolina archaeology during his short tenure as Director of the Research Laboratories of Anthroplogy.

H. Trawick Ward

R. P. Stephen Davis, Jr. 


\title{
Introduction
}

\author{
by \\ Roy S. Dickens, Jr. \\ H. Trawick Ward \\ R. P. Stephen Davis, Jr.
}

This book focuses on archaeological research conducted by the Research Laboratories of Anthropology of the University of North Carolina at Chapel Hill on three Indian village sites in the Piedmont region of North Carolina. The Fredricks site (Figure 1.1), which was occupied between about 1680 and 1710 , represents the last major village of the Occaneechi tribe; the Wall site is a protohistoric (ca. 1545) village of an unknown group; and the Mitchum site is a village, probably of the Saxapahaw tribe, that was occupied between about 1660 and 1680 .

Data for the Fredricks site were obtained from excavations conducted in 1983 and 1984; the 1984 season was sponsored by a grant from the Committee for Research and Explorations of the National Geographic Society. Information obtained during 1983 and 1984 from the Wall site was supplemented in some categories by data from work done in 1938, 1940, and 1941. Information on the Mitchum site was collected in 1983. Work continued at the Fredricks site during the summers of 1985 and 1986 and additional excavations were conducted at the Mitchum site during the fall of 1986. With few exceptions, the results of these later excavations have not been incorporated into the following chapters. However, in subsequent sections of this chapter, summary results of the 1985 and 1986 excavations are described. If patterns or interpretations based on the research through 1984 were significantly altered by the addition of more recent data, these changes are discussed. Descriptive reports of the 1985-86 fieldwork are also included in Appendixes $\mathrm{A}$ and $\mathrm{B}$.

Investigations at these three sites are part of a larger project--the Siouan Project-which has as its goal the elucidation of culture change among Indian groups of the North Carolina-southern Virginia Piedmont during the Historic period. The Fredricks, Wall, and Mitchum sites are important links in this research effort. The former two sites are located in the same bend of the Eno River in Orange County, North Carolina. Their spatial proximity and the fact that they are separated in time by about 150-200 years, make them excellent candidates for comparison within the culture-change theme. The Mitchum site, which lies on the Haw River in Chatham County only a short distance from the pievious two sites and which represents a point in time intermediate to the other two, also fits well into this research theme.

When information from these three closelyspaced sites is complemented by information from other excavated sites in the larger research area, a basis is obtained for examining specific questions about changes in technology, settlement patterns, social organization, mortuary practices, subsistence activities, and physical conditions on the Piedmont during a time that spans initial European contact to the disintegration of many of the Indian societies. As the reader will see, however, only preliminary interpretations can be offered by most of the researchers. Nevertheless, some productive results have been obtained, and there is good reason to believe that additional data from these and other sites will contribute to secure answers in the near future.

Research, funded by the National Science Foundation, is currently underway in the Haw River drainage and will commence in the Dan River basin of the northern Piedmont in 1988 . The final phase of this project will see a resumption of investigations along the Eno and its sister streams in the central Piedmont. These additional investigations should not only fill in any gaps in the current work but also broaden the geographic base and facilitate inter-regional comparisons of Siouan culture change.

\section{HISTORICAL BACKGROUND}

When European explorers first entered the Virginia and North Carolina Piedmont, they found it occupied by several small Indian tribes who shared a common culture and a similar language. These Siouan tribes also shared a mixed subsistence of hunting, gathering, and agriculture, and a social system regulated by ties of kinship and reciprocity.

As the colonial frontier was pushed into the Piedmont and as Indian and European interaction was intensified, the Occaneechi tribe became prominent among the Siouan groups. The Occaneechi controlled much of the deerskin 


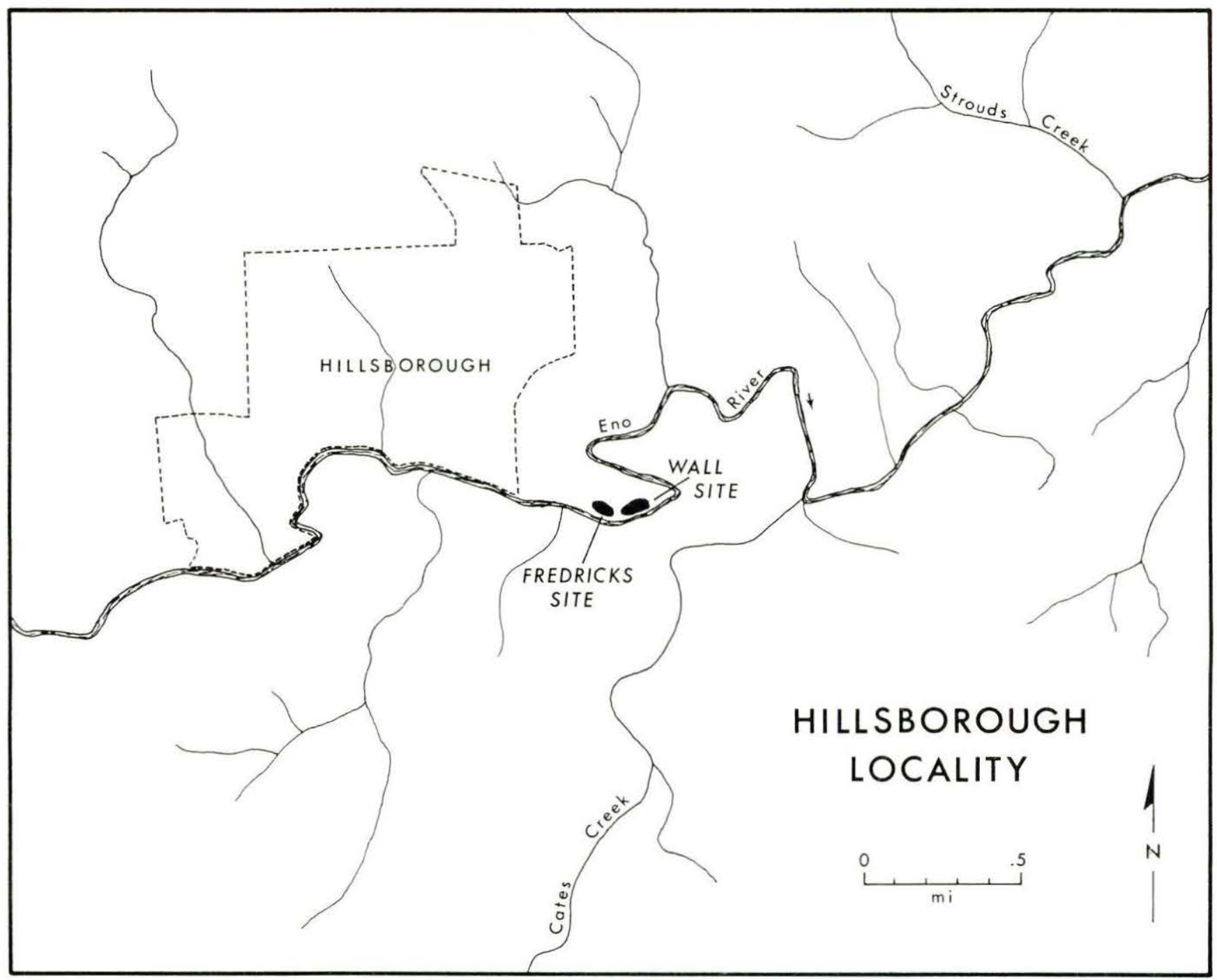

Figure 1.1

Hillsborough locality showing the Wall and Fredricks sites.

trade, and their language became the lingua franca of the Piedmont. Their pivotal role in the fur trade came about partly because one of their villages, on an island in the Roanoke River, was astride the Great Trading Path from Virginia to Georgia.

The island village of the Occaneechi was visited by John Lederer in 1670 (Cumming 1958). After the Occaneechis "barbarously murthered" six Cherokees who were attempting to establish trade relations with the Virginia colonists, Lederer, fearing for his life, cut short his visit. James Needham and Gabriel Arthur, who traveled through the same territory in 1673, observed that the Occaneechis controlled the colonial trade, which endowed them with an importance that far exceeded their numbers (Alvord and Bidgood 1912). They seem to have maintained and reinforced their role in the trade network through warfare and intimidation. Thus, the Occaneechi tribe earned a fierce and pugnacious reputation, which eventually led to an eruption of armed hostilities with Nathaniel Bacon's militia in 1676 .

After pursuing a group of Susquehannock Indians into Occaneechi territory, Bacon convinced some "Manakins" and "Annalectins," who had also joined the Occaneechi, to aid his forces in defeating the Susquehannocks. After that victory was accomplished, Bacon then attacked the Occaneechis (Billings 1975:267269).

After the battle with Bacon, the Occaneechis were so reduced in numbers that they could no longer defend their island stronghold on the Roanoke. The survivors abandoned their home territory, retreated southward, and reestablished a village on the Eno River, near present Hillsborough, North Carolina (Figure 1.2). In 1701, English surveyor John Lawson visited the relocated Occaneechi Town where he 


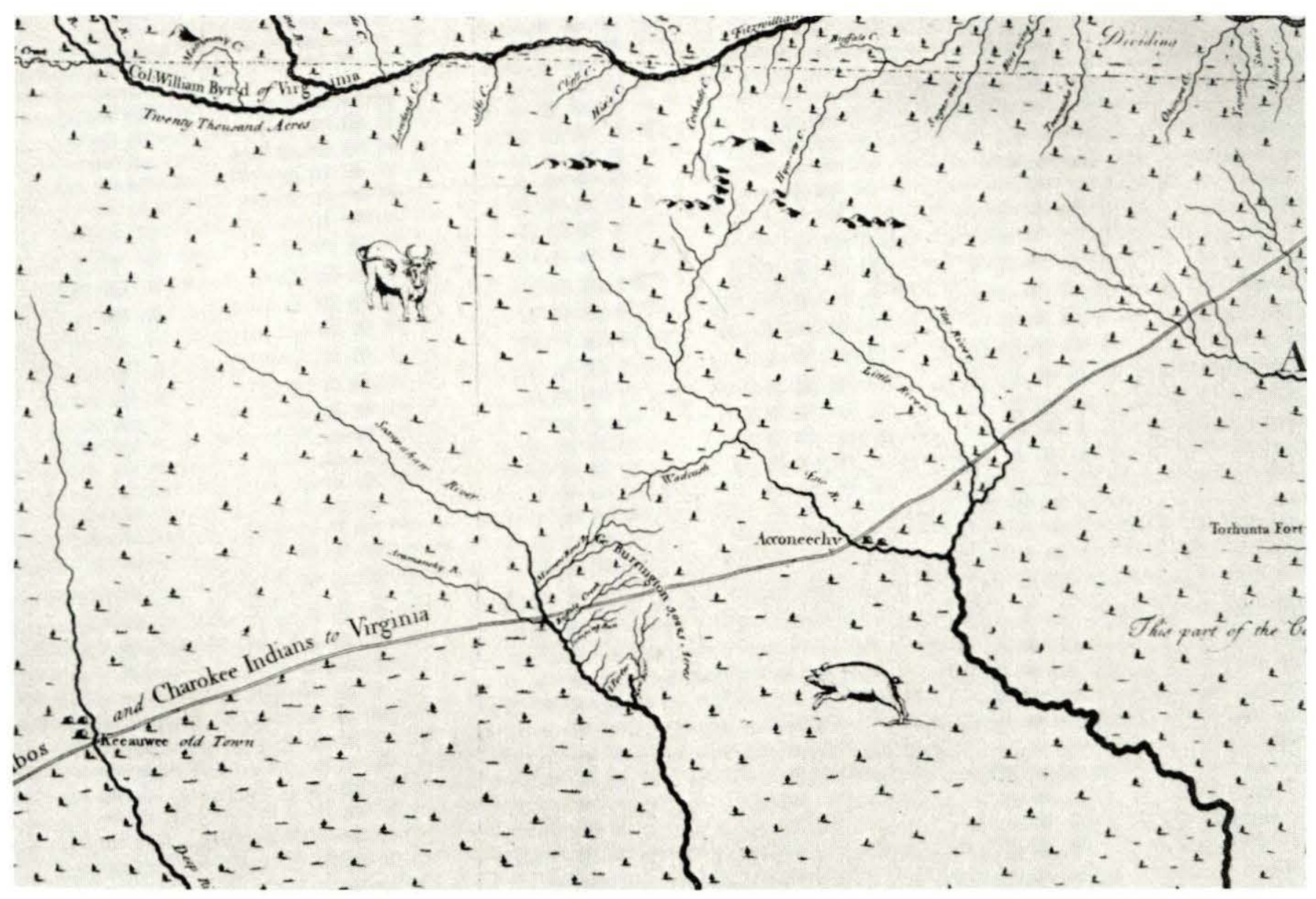

Figure 1.2

Edward Moseley's 1733 map locating "Acconeechy" on the Eno River.

observed that there were "no Indians having greater Plenty of Provisions than these" (Lefler 1967:61).

After Lawson's visit, conditions worsened for the Occaneechi, as well as for the other Siouan tribes, and by 1722, disease, warfare, and rum had virtually destroyed Indian societies in the Piedmont. Remnants of once autonomous groups either huddled together around Fort Christanna in Virginia or moved to join their cousins, the Catawba, in South Carolina. By 1730 , except for a few isolated Indian families, the North Carolina Piedmont lay mostly vacant, awaiting the arrival of hordes of colonists from Virginia, Maryland, and Pennsylvania.

\section{ARCHAEOLOGICAL BACKGROUND}

Archaeologists first became interested in studying the remains of the Piedmont Siouans in the 1930s, when village sites thought to be associated with the Keyauwee, Sara, Saponi, and Occaneechi were subjected to excavations of varying intensity (Coe 1937; Lewis 1951). Though broad in scope, these early efforts were not focused by a structured research design. At most sites, only small areas were tested, and collections were gathered primarily with an eye toward identifying pottery types of the different tribes. As part of this early research, extensive excavations were carried out between 1938 and 1941 at the Wall site on the Eno River near Hillsborough (Figures 1.3 and 1.4). This site was thought to represent the Occaneechi village visited by Lawson in 1701 .

The next archaeological research in the Siouan area was undertaken in the 1940s on the Roanoke River, prior to the inundation of Kerr Reservoir in North Carolina and Virginia (Miller 1962). Under the auspices of the Smithsonian Institution's River Basin Salvage Program, extensive excavations were conducted in the reservoir area at the Clarksville site on the east bank of the river opposite "Occaneechi Island," and on the island itself at the Tollifero site (Figure 1.3). These two sites contained information on the prehistoric Siouan inhabitants of the area, but no evidence was found of the 1670 Occaneechi village visited by Lederer, Needham and Arthur, and Bacon.

In 1972, the Research Laboratories of Anthropology at the University of North Carolina began excavations at the Upper Saratown site on the Dan River in Stokes County, North Carolina (Figure 1.3). These investigations, which 


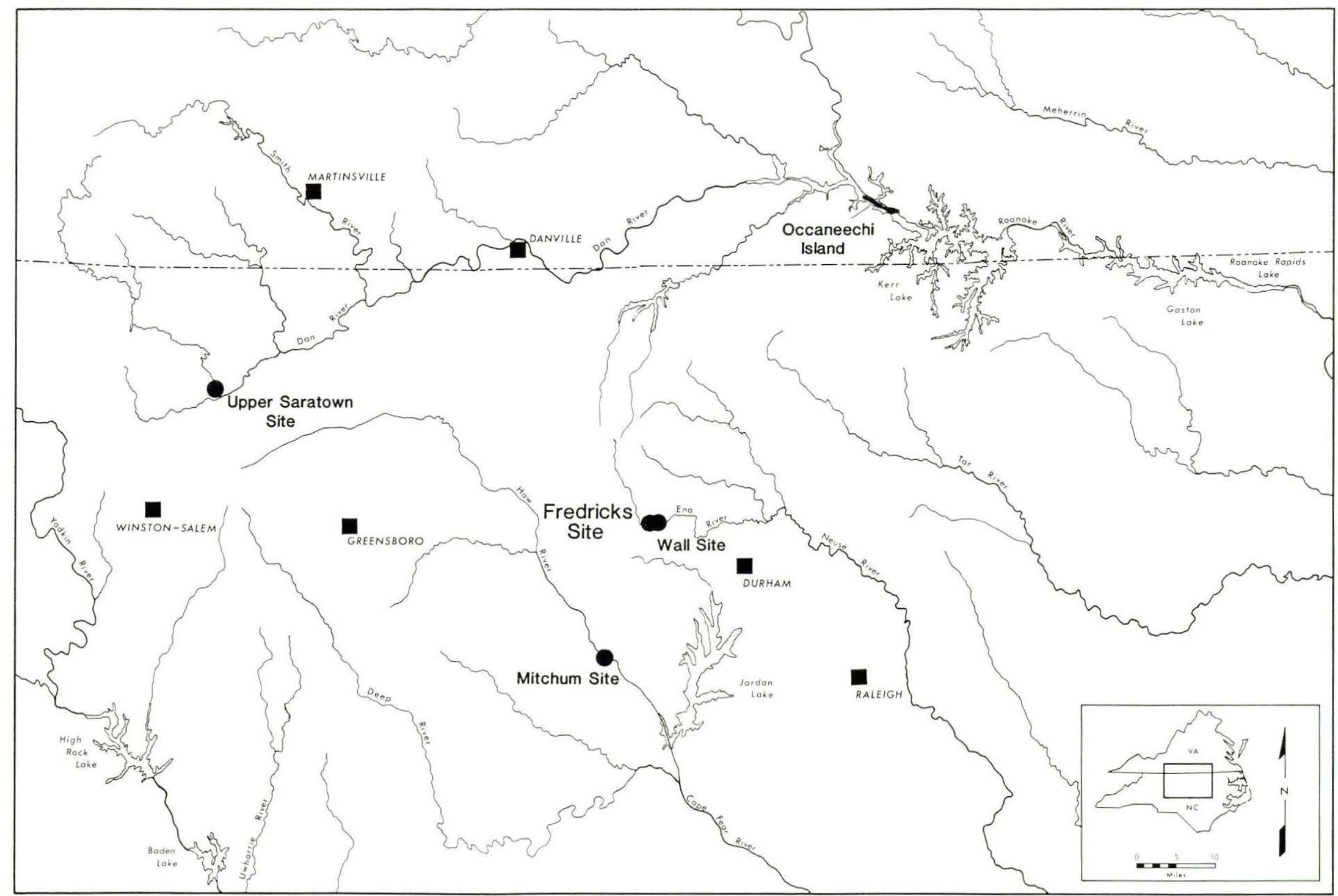

Figure 1.3

Archaeological sites in the Siouan Project area. 


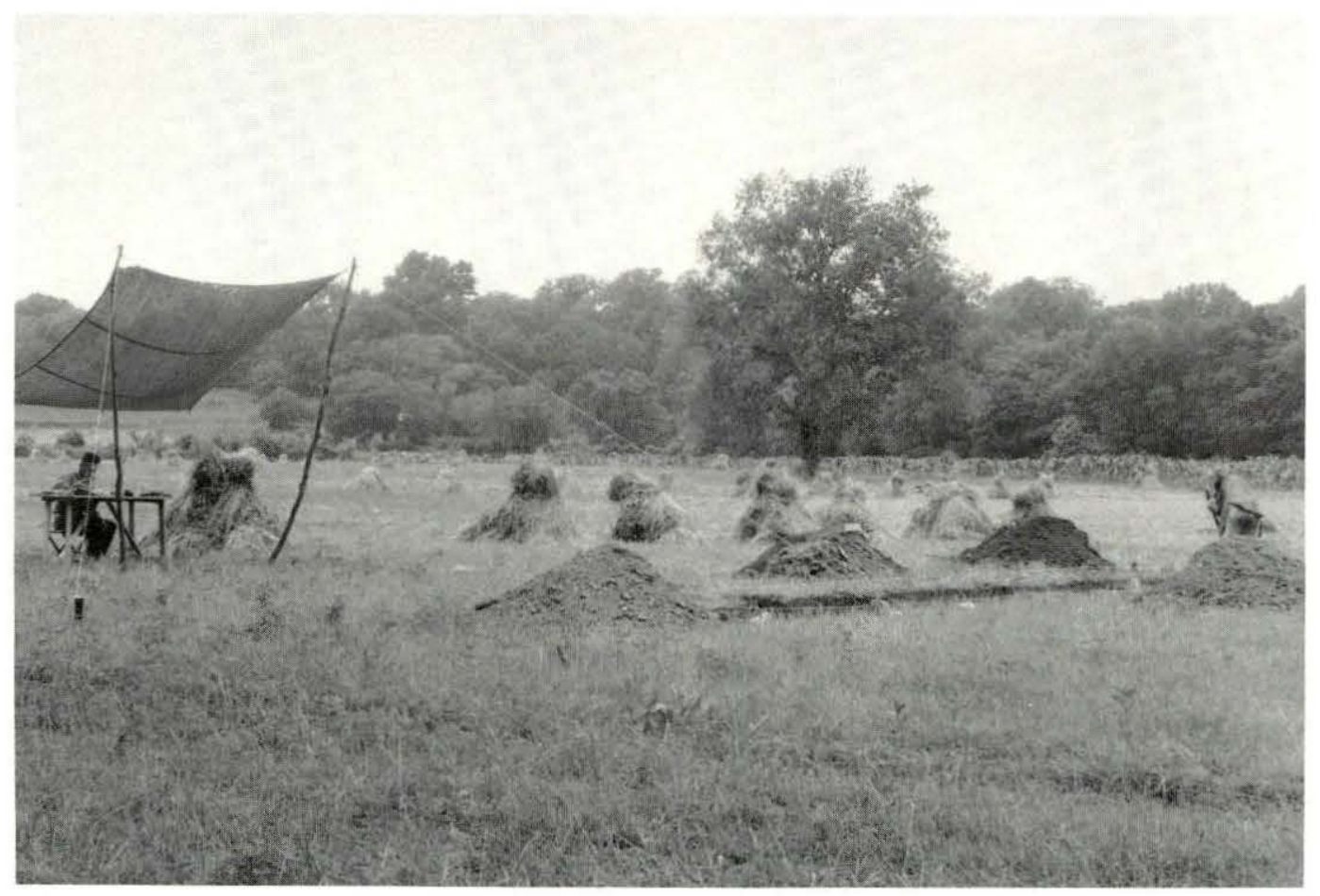

Figure 1.4

1938 excavations at the Wall site.

lasted for ten consecutive field seasons, exposed a group of circular houses with associated storage pits and burials, as well as a sequence of village palisades (Ward 1980; J. Wilson 1983). Most of the burials were accompanied by nonutilitarian (ornamental) European trade items. Ethnohistoric records and the recovered trade artifacts suggested that this site was occupied during the late 1600 s by the Sara, one of the Piedmont Siouan tribes and neighbors of the Occaneechi.

When combined, these initial efforts to investigate the "Siouan problem" seem substantial. Each project, however, was developed as an end in itself and was not guided by an overall set of research objectives. Consequent1y, archaeological coverage of the Siouan area was uneven. For example, the upper Dan River valley was extensively investigated, whereas the Haw and Eno drainages to the southeast received relatively little attention. Surveys were opportunistic rather than systematic, and a few larger sites were tested and excavated at the virtual exclusion of many small ones. Despite all of their shortcomings, these previous investigations do provide a foundation for more systematic studies of Piedmont Siouan culture.

\section{SIOUAN PROJECT}

Although the need to approach Siouan archaeology with a set of specific goals, opera- tionalized by an overall research strategy, has been obvious, such a course of study was not formulated until the winter and spring of 1983. At that time, staff of the Research Laboratories of Anthropology developed a research design which included a set of questions focused on Siouan culture change and the archaeological correlates of that change.

Initial archaeological investigations of the Siouan Project have focused on the Dan, Eno, and Haw River drainages, heartland of the Piedmont Siouans during the Historic period. Extant ethnohistoric and archaeological information suggest that there was considerable cultural diversity among the groups in these three river systems, reflecting possible differences in ethnicity, microenvironmental adaptation, and intensity of interaction with the English. Although the Siouan tribes seem to have commonly shifted their villages and to have even changed their territories, by 1675 the locations of their settlements were more or less stabilized within the confines of these three drainages. The Sara, Tutelo, and Saponi occupied the territory drained by the Dan and its tributaries; the Eno basin was the homeland of the Eno and Shakori, and (after 1680) the Occaneechi; and the Haw River area was occupied by the Sissipahaw and possibly others.

Since the Siouan Project is concerned with studying changes in aboriginal culture brought about by contact and interaction with English colonists, a primary goal has been to locate and identify towns occupied by the various Indian 
tribes at specific temporal intervals from the Late Prehistoric period through the Contact period. These intervals are: Late Prehistoric (A.D.1300-1525), Protohistoric (A.D.1526-1625), Early Contact (A.D.1626-1675), Middle Contact (A.D.1676-1710), Late Contact (A.D.1711-1740), and Euroamerican (A.D.1741-Present). Once sites representing all (or most) intervals have been located in each drainage area, it will be possible to address more specific questions concerning how the different Piedmont groups adapted within local environments to increasing exposure to European materials, ideas, and institutions.

Some initial questions being considered are: What were the Siouan cultures like prior to European contact? After initial European contact, what aspects of culture changed first, and with what relative intensity? As contact became protracted, did the Indians move more toward the adoption of European ways, or more toward making adjustments in their existing cultural patterns to cope with the European presence? What were the short-term and long-term effects of European epidemic diseases? What effects did the deerskin trade have on the native economy, technology, and social organization? How did man-land interactions change through time?

\section{FIELDWORK}

Although the ethnohistoric record contains little precise information on the locations of Siouan towns, a description in Lawson's journal (Lefler 1967) and the survival of "Occaneechi" as a placename provide a strong case for locating the 1701 town of Occaneechi immediately southeast of present-day Hillsborough (Rights 1957; Lefler 1967). Although the location at the Wall site is also approximately correct according to the above information, a cursory re-examination of the 1938-1941 excavation data (Coe 1952) immediately called to question the identification of that site as Lawson's Occaneechi. Euroamerican artifacts from the Wall site either dated too late or had been found in disturbed contexts. Additional field investigations were needed to clarify the temporal placement of the Wall site and to critically evaluate its identification as Occaneechi Town.

\section{Wa11 Site}

Therefore, in the summer of 1983, after a hiatus of 42 years, excavations were resumed at the Wall site. Initially, sections of the old excavations were isolated and a site grid re-established. A portion of the rich midden surrounding the village was excavated and subjected to fine-scale recovery techniques. In addition, three burials were removed and portions of two circular house patterns exposed. The few European artifacts recovered were from disturbed plowzone, and most of them dated to the latter half of the eighteenth century or early nineteenth century. Three radiocarbon samples from undisturbed contexts yielded an average corrected date of A.D. $1545+80$ years. These data, in conjunction with a review of the earlier investigations, led to the irrefutable conclusion that the Wall site was too old to be historic Occaneechi Town.

\section{Fredricks Site}

During 1983, other village sites were found in the vicinity of the Wall site. At one of these, the Fredricks site, numerous European artifacts, along with aboriginal remains, were found on the surface. Test excavations were undertaken at this site late in the field season, while work was still in progress at the Wall site. These initial tests revealed five sharp-cornered rectangular pits and a line of small postholes (Figure 1.5). Both the posthole pattern and the pits were neatly arranged in a NW-SE direction. Four of the pits were excavated, and three contained human skeletal remains accompanied by grave goods of both European and Indian manufacture. The fourth pit, identical in shape to the other three, appeared to represent a burial but contained no bones or grave goods. Two of the burials were the remains of children between three and eight years old at death. Included with these burials were European trade items such as knives, scissors, and a variety of glass beads. Aboriginal artifacts included shell gorgets, shell beads, and a ceramic vessel. One adult male burial contained a wealth of European artifacts, including an intact rum bottle, scissors, knives, a pewter pipe and buttons, a pair of ember tongs, and an iron ax head.

Most of the trade artifacts from the Fredricks site dated to the late 1600 s or very early 1700s, the appropriate period for Occaneechi Town. The site seemed to be well preserved, with no evidence of disturbance other than shallow plowing. From these observations, it became obvious that more intensive work was needed at the Fredricks site. Hence, plans were immediately begun for a project in 1984 that would combine a major excavation and testing program at the Fredricks site, along with continued work at the neighboring Wall site (Figures 1.6 and 1.7).

\section{Mitchum Site}

During 1983, three weeks of fieldwork were also conducted at the Mitchum site, an historic village site on the Haw River about twenty miles southwest of the Wall and Fredricks sites. Excavations were resumed at the site in September, 1986 and continued through the middle of october. The Mitchum site is believed to contain remains of a seventeenth-century Sissipahaw village. Excavations have revealed a complete wall pattern of a house, 36 pit features, two burials, and a segment of a palisade line. Trade artifacts from this site (primarily 


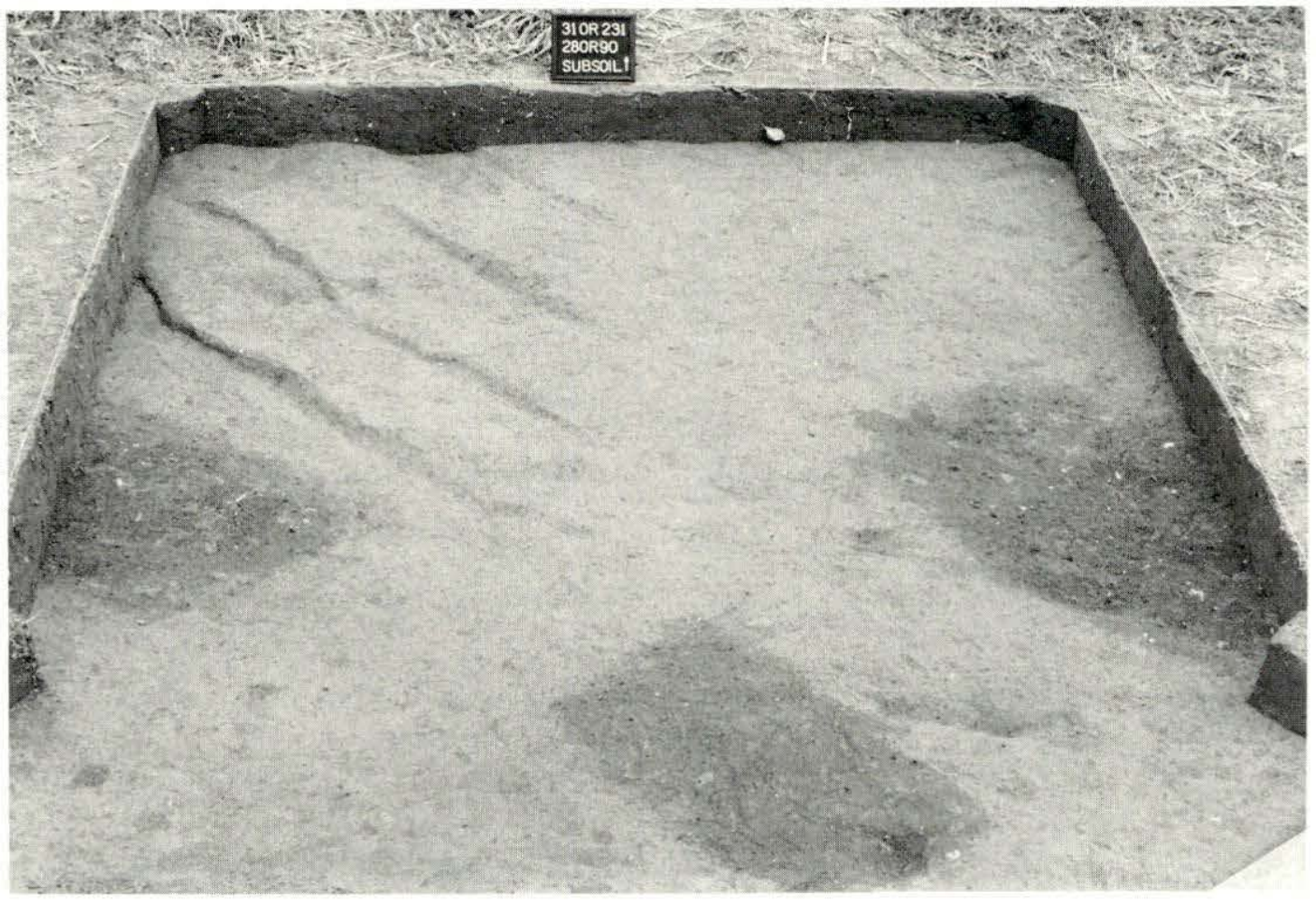

Figure 1.5

Areal view of 1983 test excavation at the Fredricks site showing rectangular burial pits.

glass beads and brass ornaments) have been preliminarily dated to the third quarter of the 1600 s.

\section{FIELDWORK}

\section{Fredricks Site}

The 1984 archaeological investigations at the Fredricks site, sponsored by a grant from the National Geographic Society, were undertaken in two phases. The first phase, which consisted of the excavation of $2700 \mathrm{ft}^{2}$ adjacent to the 1983 excavation block, was begun on May 22 and completed on July 14. The purpose of these excavations was to obtain additional data on mortuary behavior from the previously discovered cemetery area of the site and to begin sampling adjacent domestic areas. The second phase, which consisted of systematic subsurface testing (augering) of unexcavated portions of the site, was undertaken between February 24 and October 17 , as weather and time permitted. This latter phase of fieldwork provided data for delimiting probable settlement boundaries, and for making a preliminary assessment of internal site structure.

Site preparation prior to excavation consisted of bushhogging the work area (ca. 100x150 ft), establishing a site grid and a reference point for elevations, and constructing a storage shed and sluices for waterscreening. All plow- zone was excavated in $10 \times 10-f t$ units, with soil being dry screened through 1/2-inch wire mesh using hand sifters (Figure 1.8). A 20liter soil sample from the plowzone of each unit was waterscreened through $1 / 16-i n c h$ mesh to assess small artifact content.

Following the removal of plowzone, the bottom of each excavation unit was carefully trowelled in order to identify and record pits and postholes (Figure 1.9). The trowelled surface was documented by black-and-white and color photographs and by maps at a scale of 1 in=2 $\mathrm{ft}$. The drawings of each excavation unit subsequently were combined to produce an overall plot of the excavations. Photographs were also made of all procedures and of the general progress of work. Horizontal and vertical control was maintained through reference to the site grid and by using a transit and rod to determine elevations. In addition to these excavations, six 10x10-ft units excavated in 1983 were re-exposed. Once accomplished, the entire excavation area was cleaned and photographed (Figure 1.10).

The 1983 and 1984 excavations at the Fredricks site exposed numerous archaeological features, including 10 human burials, 5 pits, a sweat house, an alignment of postholes forming a 90-ft segment of the village palisade, and approximately 800 additional postholes representing all or portions of at least five houses (Figure 1.11, Table 1.1). All postholes were systematically recorded but most were not 
Table 1.1

Summary of archaeological features at the Fredricks site, 1983-1986.

\begin{tabular}{|c|c|c|c|c|c|c|}
\hline \multirow{2}{*}{$\begin{array}{l}\text { Feature/Burial } \\
\text { Number }\end{array}$} & \multirow{2}{*}{$\begin{array}{l}\text { Excav. } \\
\text { Season }\end{array}$} & \multirow[b]{2}{*}{ Feature Type } & \multirow{2}{*}{$\begin{array}{l}\text { Center } \\
\text { Location }\end{array}$} & \multicolumn{2}{|c|}{ Dimensions } & \multirow{2}{*}{$\begin{array}{l}(\mathrm{ft}) \\
\mathrm{D}\end{array}$} \\
\hline & & & & $\mathrm{L}$ & W & \\
\hline $\mathrm{Bu} \cdot 1$ & 1983 & Burial & $276.8 \mathrm{R} 90.3$ & 3.6 & 2.6 & 2.4 \\
\hline Bu. 2 & 1983 & Burial & $279.3 \mathrm{R} 85.8$ & 3.1 & 2.6 & 2.1 \\
\hline Bu. 3 & 1983 & Burial & $282.7 \mathrm{R} 89.1$ & 4.4 & 3.2 & 3.0 \\
\hline Fea. 1 & 1983 & Probable Burial & $282.7 R 80.7$ & 3.9 & 2.9 & 2.8 \\
\hline Fea. $2 / \mathrm{Bu}, 4$ & 1984 & Burial & $293.5 R 76.5$ & 3.2 & 2.2 & 2.1 \\
\hline Fea. 3/Bu. 5 & 1984 & Burial & $299.2 R 69.5$ & 5.0 & 2.8 & 2.1 \\
\hline Fea. 4/Bu. 6 & 1984 & Burial & $300.6 R 75.7$ & 5.6 & 4.0 & 2.3 \\
\hline Fea. 5/Bu. 7 & 1984 & Burial & $290.0 \mathrm{R} 80.4$ & 3.4 & 2.3 & 1.4 \\
\hline Fea. 6/Bu. 8 & 1984 & Burial & $306.5 R 61.7$ & 4.0 & 2.5 & 2.5 \\
\hline Fea. 7/Bu. 9 & 1984 & Burial & $308.7 R 68.2$ & 5.1 & 3.5 & 2.3 \\
\hline Fea. 8 & 1984 & Tree Stump & $290.0 R 58.0$ & 2.4 & 2.2 & 2.3 \\
\hline Fea. 9 & 1984 & Fire Pit & $247.4 \mathrm{R} 56.6$ & 5.0 & 4.7 & 2.9 \\
\hline Fea. 10 & 1984 & Storage Pit & $251.6 \mathrm{R} 70.0$ & 2.6 & 2.3 & 3.1 \\
\hline Fea. 11 & 1984 & Pit & $249.5 R 77.4$ & 3.0 & 2.4 & 1.5 \\
\hline Fea. 12 & 1984 & Pit & $264.0 \mathrm{R} 85.5$ & 3.4 & 3.2 & 1.1 \\
\hline Fea, 13 & 1984 & Pit & $254.0 \mathrm{R} 85.7$ & 2.8 & 2.4 & 1.5 \\
\hline Fea. 14/Bu. 11 & 1985 & Burial & $315.2 R 66.2$ & 4.9 & 3.1 & 3.1 \\
\hline Fea. 15 & 1985 & Tree Stump & $318.8 \mathrm{R} 69.3$ & 2.6 & 1.5 & 1.4 \\
\hline Fea. 16 & 1985 & Shallow Basin & $253.0 \mathrm{R} 96.6$ & 1.3 & 1.1 & 0.2 \\
\hline Fea. 17 & 1985 & Storage Pit & $233.5 R 77.5$ & 2.7 & 2.4 & 2.1 \\
\hline Fea. 18 & 1985 & Pit & $236.3 \mathrm{R} 70.3$ & 3.3 & 3.3 & 0.9 \\
\hline Fea. 19 & 1985 & Storage Pit & $234.5 R 87.6$ & 2.7 & 2.6 & 2.4 \\
\hline Fea. 20 & 1985 & Pit & $224.0 R 71.5$ & 3.0 & 2.8 & 1.5 \\
\hline Fea. 21 & 1985 & Sha1low Depression & $248.9 \mathrm{R} 91.1$ & 1.2 & 1.1 & 0.1 \\
\hline Fea. 22 & 1985 & Shallow Depression & 251.1893 .7 & 0.8 & 0.7 & 0.2 \\
\hline Fea. 23 & 1985 & Pit & $291.1 R 20.0$ & 2.2 & 1.9 & 1.5 \\
\hline Fea. 24 & 1985 & Shallow Basin & $286.0 \mathrm{R} 28.5$ & 4.3 & 2.2 & 0.5 \\
\hline Fea. 25 & 1985 & Shallow Basin & $252.2 R 48.5$ & 2.3 & 2.3 & 0.6 \\
\hline Eea. 26/Bu. 13 & 1985 & Burial & $312.0 R 58.0$ & 4.6 & 3.2 & 2.3 \\
\hline Fea. $27 / \mathrm{Bu} \cdot 10$ & 1985 & Burial & $316.5 \mathrm{R} 53.2$ & 3.5 & 2.8 & 2.9 \\
\hline Fea. 28 & 1985 & Storage Pit & $318.0 \mathrm{R} 42.5$ & 3.2 & 3.2 & 3.0 \\
\hline Fea. 29 & 1985 & Storage Pit & $324.7 R 40.7$ & 3.0 & 2.8 & 3.4 \\
\hline Fea. 30 & 1985 & Storage Pit & $271.5 R 21.5$ & 2.9 & 2.8 & 2.2 \\
\hline Fea. 31 & 1986 & Probable Burial & $267.5 \mathrm{R} 16.0$ & 3.1 & 2.2 & 2.0 \\
\hline Fea. 32 & 1985 & Rodent Disturbance? & $266.0 \mathrm{R} 23.0$ & - Not & Excava & ated- \\
\hline Fea. 33 & 1985 & Pit & $281.5 R 25.9$ & 3.0 & 2.6 & 1.7 \\
\hline Fea. 34 & 1985 & Hearth & $286.0 R 22.3$ & 3.2 & 3.0 & - \\
\hline Fea. 35 & 1985 & Cob-Filled Pit & $307.8 \mathrm{R} 36.6$ & 0.9 & 0.8 & 0.6 \\
\hline Fea. 36 & 1985 & Cob-Filled Pit & $300.4 \mathrm{R} 22.3$ & 1.8 & 0.9 & 0.3 \\
\hline Fea. 37 & 1985 & Shallow Basin & $292.6 \mathrm{R} 07.0$ & 1.8 & 1.0 & 0.5 \\
\hline Fea. 38 & 1985 & Shallow Basin & $305.5 R 11.5$ & 2.5 & 1.3 & 0.3 \\
\hline Fea. 39 & 1985 & Shallow Basin & $308.2 \mathrm{R} 39.8$ & 2.1 & 1.6 & 0.7 \\
\hline Fea. 40 & 1985 & Shallow Basin & $318.5 \mathrm{R} 33.5$ & 1.3 & 1.0 & 0.2 \\
\hline Fea. 41 & 1985 & Storage Pit & $288.5 R 05.0$ & 3.5 & 3.2 & 1.9 \\
\hline Fea. 42 & 1986 & Pit & $198.0 \mathrm{R} 73.0$ & 3.0 & 3.0 & 1.8 \\
\hline Fea. 43 & 1986 & Probable Hearth & $209.5 R 80.5$ & 2.0 & 1.5 & - \\
\hline Fea. 44 & 1986 & Storage Pit & $201.2 R 59.3$ & 2.8 & 2.0 & 2.5 \\
\hline Fea. 45 & 1986 & Pit & $207.5 R 58.8$ & 2.7 & 2.6 & 1.5 \\
\hline Fea. 46 & 1986 & Storage Pit & $211.5 R 34.5$ & 2.6 & 2.4 & 2.0 \\
\hline Fea. 47 & 1986 & Pit & $203.8 R 62.5$ & 2.7 & 2.6 & 1.6 \\
\hline Fea. 48 & 1986 & Irregular Trench & - & - & - & - \\
\hline Fea. 49 & 1986 & Probable Burial & $212.5 R 49.2$ & 2.5 & 1.5 & 1.2 \\
\hline Fea. $50 / \mathrm{Bu} \cdot 12$ & 1986 & Burial & $212.9 \mathrm{R} 11.3$ & 2.4 & 2.0 & 1.1 \\
\hline Fea. 51 & 1986 & Storage Pit & $224.2 R 05.2$ & 2.4 & 2.4 & 2.0 \\
\hline Fea. 52 & 1986 & Shallow Basin & $251.4 \mathrm{~L} 04.3$ & $-\mathrm{Not}$ & Excava & ated- \\
\hline Fea. 53 & 1986 & Storage Pit & $216.0 \mathrm{R} 19.0$ & 2.9 & 2.7 & 2.1 \\
\hline Fea. $54 / \mathrm{Bu} .14$ & 1986 & Burial & $183.4 \mathrm{R} 36.5$ & 3.5 & 2.5 & 1.3 \\
\hline Fea. 55 & 1986 & Pit & $190.5 \mathrm{R} 36.7$ & 2.9 & 2.6 & 0.6 \\
\hline Fea. 56 & 1986 & Storage Pit & $252.5 \mathrm{~L} 09.0$ & 2.9 & 2.8 & 3.3 \\
\hline Fea. 57 & 1986 & Pit & $215.9 \mathrm{R} 26.3$ & 2.4 & 2.3 & 1.3 \\
\hline Fea. 58 & 1986 & Pit & $195.3 \mathrm{R} 36.3$ & 2.6 & 2.2 & 0.8 \\
\hline Fea. 59 & 1986 & Pit & $235.0 R 00.6$ & 3.6 & 2.5 & 1.7 \\
\hline Fea. 60 & 1986 & Probable Pit & $211.5 \mathrm{R} 00.0$ & - Not & Excava & ated- \\
\hline Fea. 61 & 1986 & Probable Pit & $223.8 \mathrm{~L} 03.8$ & 3.7 & 3.2 & 2.1 \\
\hline
\end{tabular}




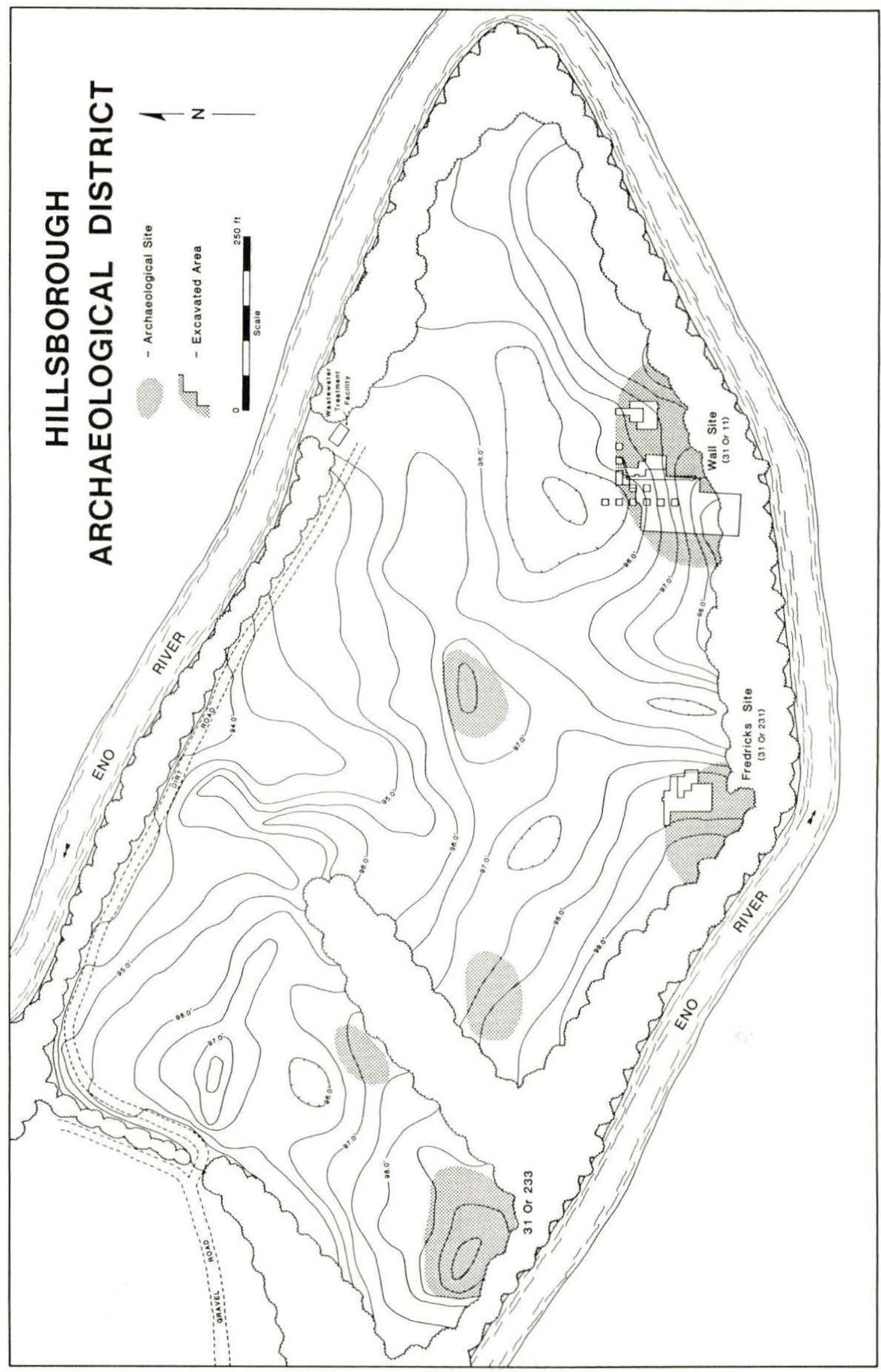

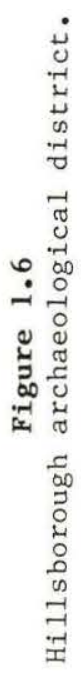




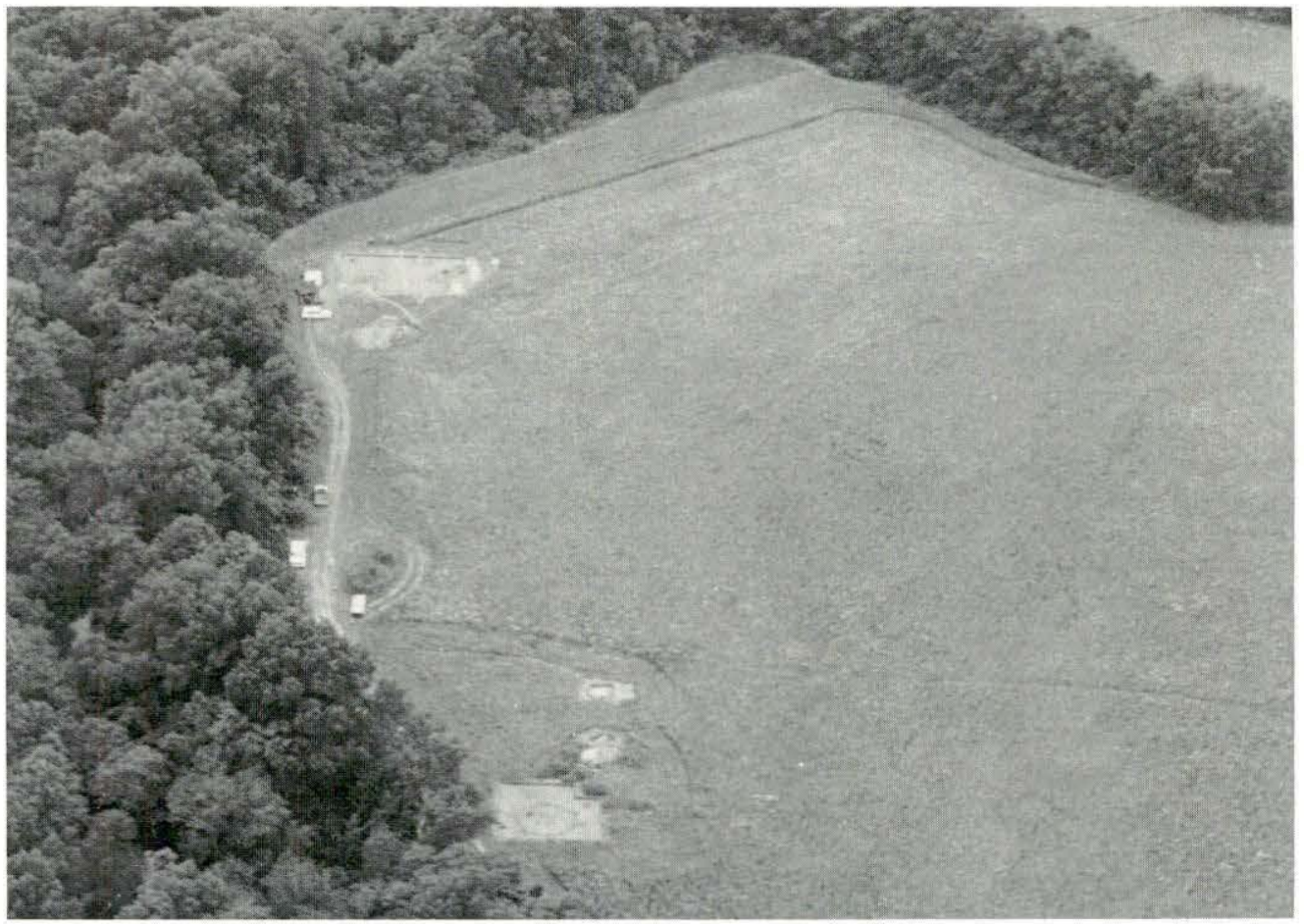

Figure 1.7

Aerial view of 1984 excavations at the Wall site (foreground) and Fredricks site (background).

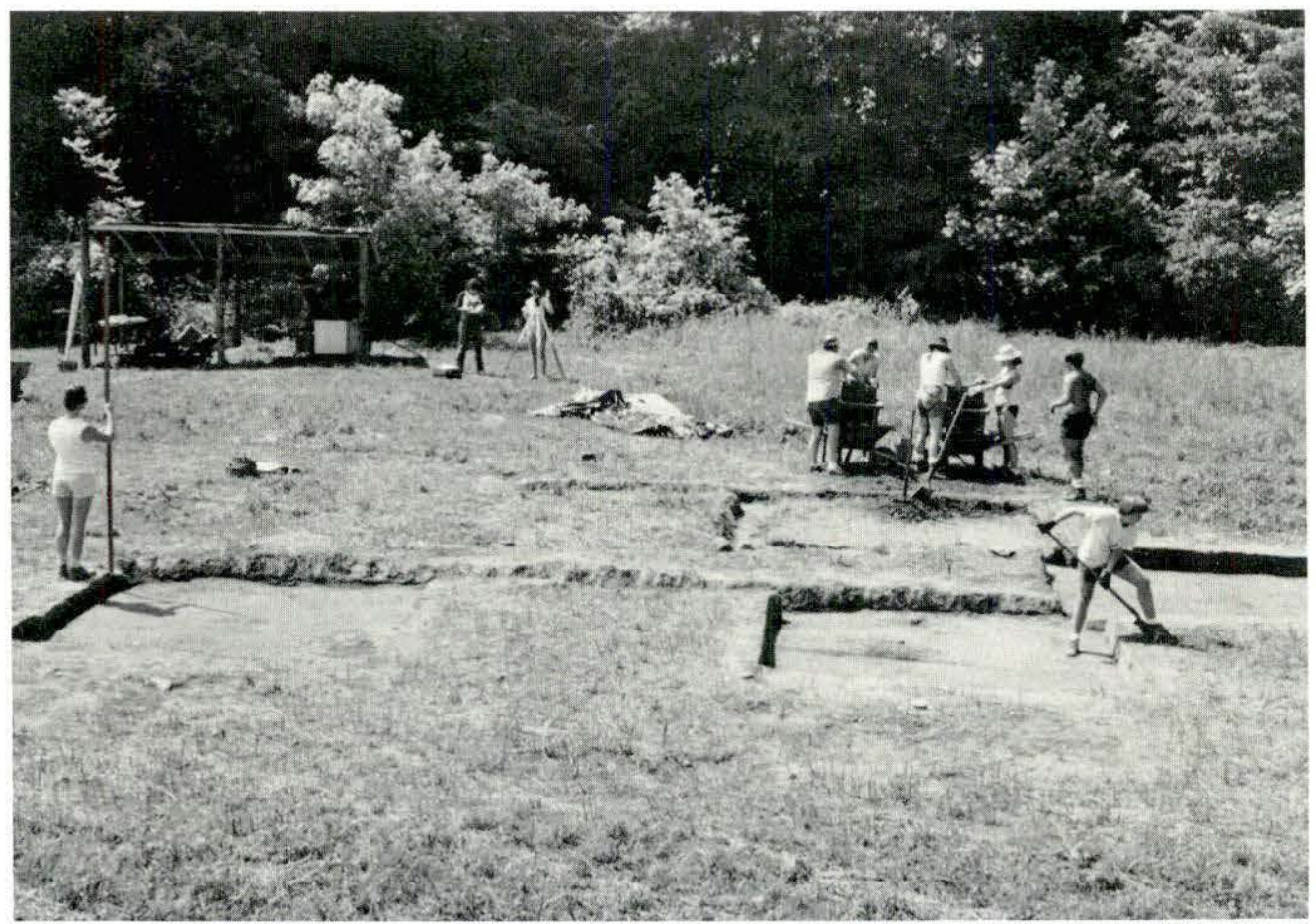

Figure 1.8

Removing plowzone at the Fredricks site. 


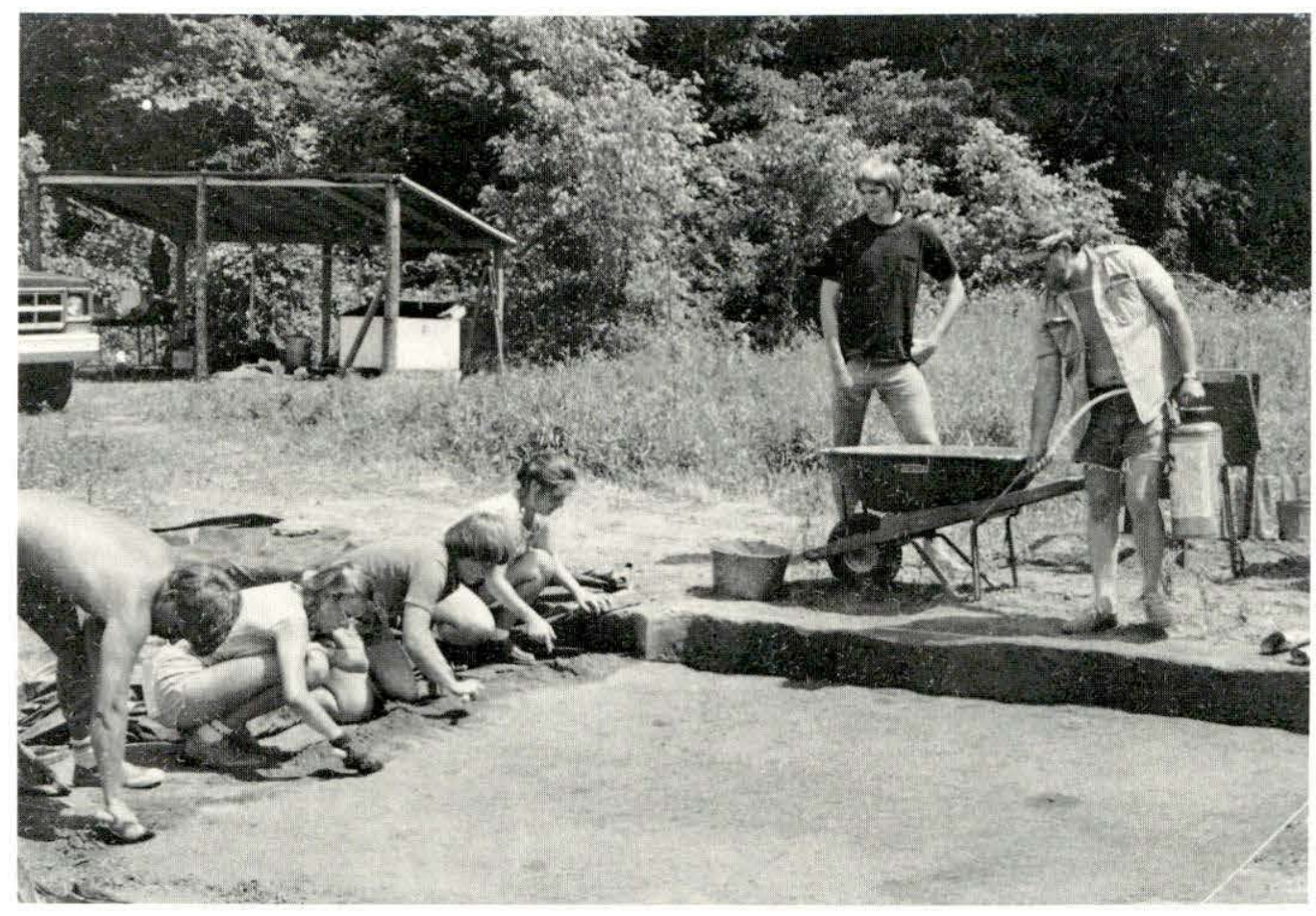

Figure 1.9

Trowelling the top of subsoil to expose archaeological features at the Fredricks site.

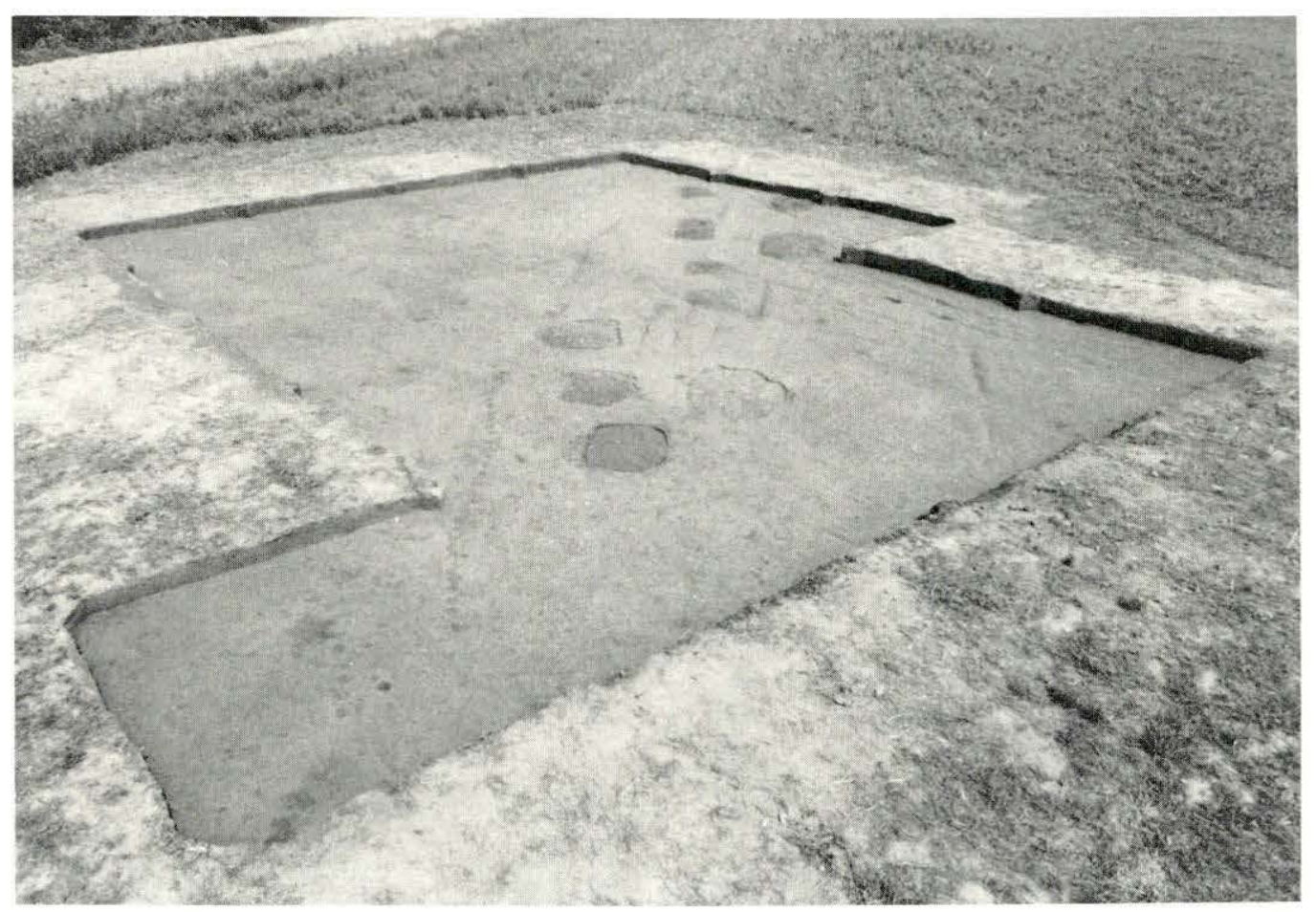

Figure 1.10

Overview of the 1984 excavation at the Fredricks site showing palisade and village cemetery. 


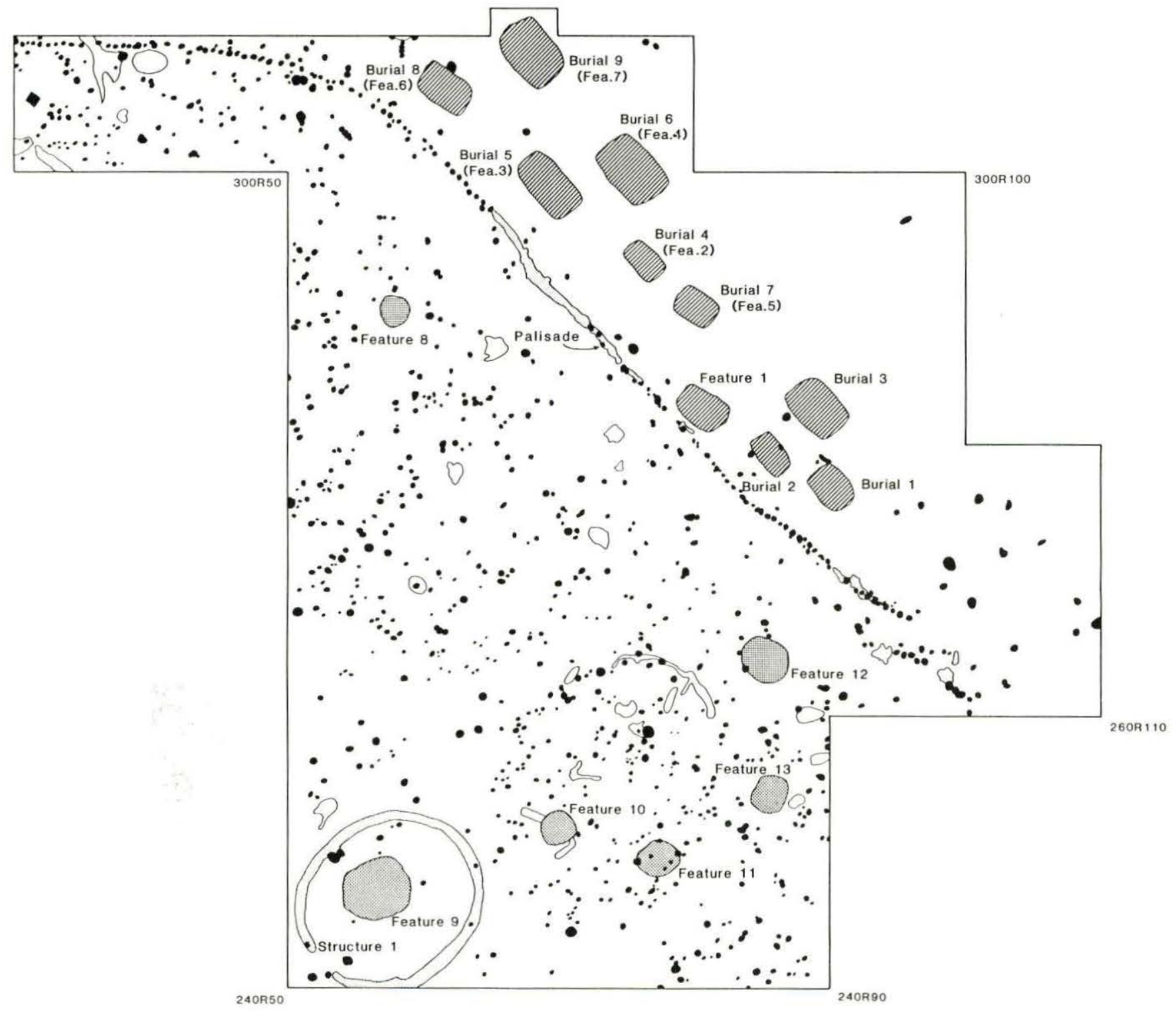

FREDRICKS SITE

Plan of 1983-84 Excavations

$\mathrm{N}$

Figure 1.11

Plan of 1983-84 excavations at the Fredricks site. 
excavated. Excavation of features, burials, and the sweat house, was accomplished using trowels, grapefruit knives, brushes, and other small tools. Sunscreens, constructed of wooden frames and bedsheets, were erected over features during excavation to minimize the damage to feature contents by the summer sun. Feature fill was removed in natural zones, when evident, and all fill was waterscreened through sluice boxes having a sequence of 1/2-inch, 1/4-inch, and 1/16-inch wire mesh. This technique permitted the recovery of minute artifacts, including shell and glass beads, lead shot, small animal bones, and carbonized plant remains. Standard 10-liter soil samples from each zone of each feature were simultaneously processed by flotation to retrieve very small, extremely fragile carbonized seeds that might otherwise be lost in the waterscreening. Elevations were taken following the removal of each soil zone of a feature in order to establish precise provenience for zone contents and to permit the calculation of soil volume.

Upon completion, a11 features and burials were extensively documented by black-and-white and color photography, and by drawings in profile and plan at a scale of 1 in=1 ft. Also, extensive notes were kept by all excavators in both field journals and on standardized feature and burial data forms.

Special care was taken with human burials to ensure that all bones and associated artifacts were kept in place during excavation. The primary objective during burial excavation was to preserve the integrity of contextual relationships among human remains and burial furniture while removing the fill dirt. In instances where burial remains (e.g., bones, bead clusters, and corroded metal artifacts) were too fragile or complex to permit thorough cleaning and full documentation in situ, the surrounding soil was cut away to form a pedestal beneath the remains. This pedestal was then wrapped with a band of fine fiberglass screen and covered with a layer of Bondo to prevent disintegration when removed from the ground. The pedestal was then removed to the conservation laboratory where the remains could be carefully cleaned, documented, and preserved.

\section{Wall Site}

During the 1984 field season, in addition to the National Geographic Society-sponsored work at the Fredricks site, $1200 \mathrm{ft}^{2}$ were excavated at the nearby Wall site. Funds for this work were provided by the University of North Carolina. Of the total excavation, 1100 $\mathrm{ft}^{2}$ comprised a contiguous set of $10 \times 10-\mathrm{ft}$ units immediately south of the main 1983 excavation, on what was probably the northeast edge of the village. Also, one $10 \times 10-\mathrm{ft}$ unit was opened in an area of dense midden in the north-central part of the site near a point where the 1938, 1940-41, and 1983 excavations converged.
The same procedures of preparation, excavation, recovery, and recording described for the Fredricks site were employed at the Wall site. As a result of the 1984 investigations, the overlapping posthole patterns for two structures, which had been partly defined in 1983, were fully exposed. Also, in this same area were found two small pit features and an irregular disturbance associated with the southern margin of one of the structures.

\section{5-86 FIELDWORK AT THE FREDRICKS SITE}

Fieldwork continued at the Fredricks site during the summers of 1985 and 1986, sponsored by grants from the National Geographic Society. The excavation procedures outlined above were again employed, insuring data comparability.

The 1985 excavation opened up nearly twice as much area as had been exposed during the two previous field seasons and permitted an accurate estimate of the size of the village. At this point, approximately $60 \%$ of the village area within the palisade had been excavated (Figure 1.12). Three additional burials were recovered from the cemetery area, and considerable information was obtained concerning domestic architecture and the overall village plan.

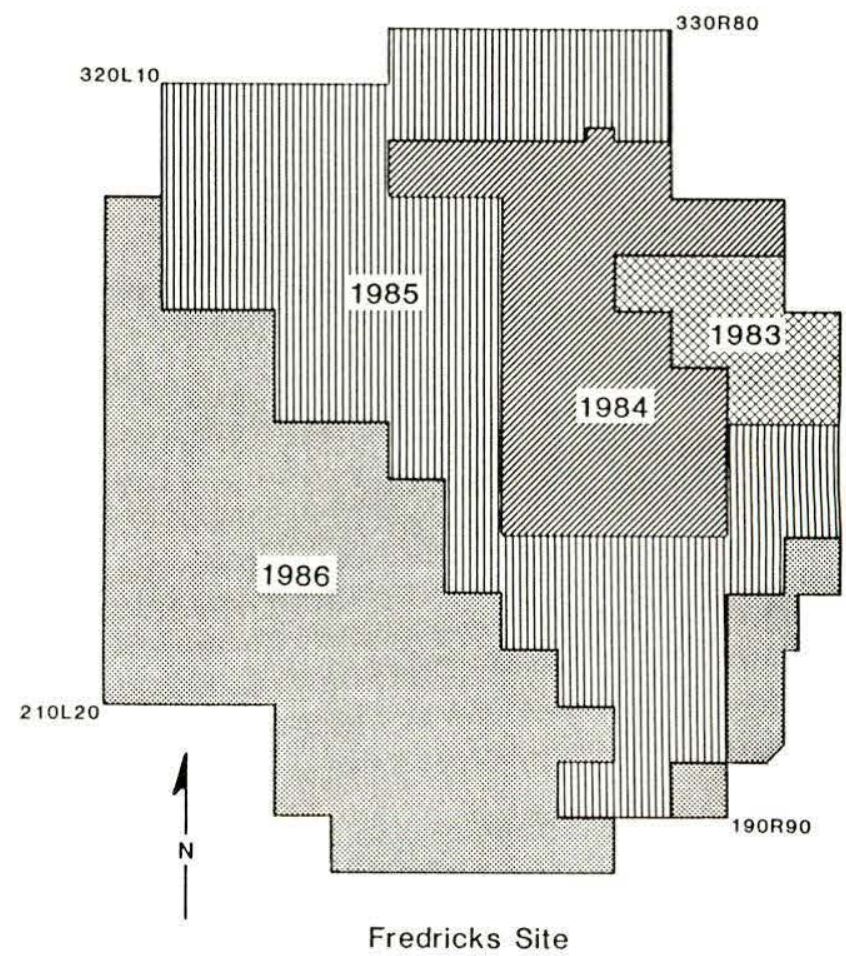

Summary of Excavations (1983-1986)

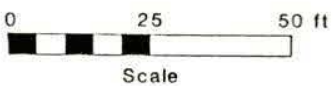

Figure 1.12

Area covered by 1983-86 excavations at the Fredricks site. 
In addition to identifying six more structures (including another possible sweat house), the expanded excavation in 1985 also permitted a more secure interpretation of the domestic structures exposed in 1984. These new data indicated that houses were of wall-trench as well as single-post construction, were of variable shape ranging from sub-rectangular to circular, and provided approximately 175-250 $\mathrm{ft}^{2}$ of interior floor space. Hearths were centrally located and some houses had interior, subterranean storage facilities. The houses encircled a common area, or plaza, which contained few architectural features. The central feature within this plaza, as well as within the larger village, was the oval sweat lodge (Structure 1) identified in 1984.

The 1986 excavation at the Fredricks site uncovered the remainder of the village area enclosed within the palisade except for a small section in the southwest corner where large trees prevented soil removal (Figure 1.13). Although still small, the village was more irregular in shape than predicted after the 1985 field season. The irregular outline resulted from the fact that the palisade "bowed-out" or expanded to the southwest, thus creating a D-shaped rather than oval configuration. Although much of the structural evidence continued to consist of somewhat vague posthole clusters, two additional wall-trench structures were defined, and the pit features associated with the structures were extremely rich.

Of particular interest were two burials excavated in 1986. Neither was associated with the cemetery, and both were contained in pits that were very different from those of the cemetery burials. Although human skeletal remains were absent, two other pit features were excavated that resembled burials in form and fill characteristics. Both were in close proximity to the above burials.

\section{REPORT OVERVIEW}

The remainder of this book covers detailed data analyses and interpretations from the first two years of research, 1983-84, and with the exception of the historical overview in Chapter 2, is organized by data category. Any significant changes or modifications in interpretations resulting from the research conducted during $1985-86$ are discussed below as the individual chapters are previewed.

The various authors did not conduct their work in isolation; rather, there was ongoing interaction and information exchange throughout the planning, fieldwork, laboratory processing, analysis, and writing stages of the research. And, there were two semester-long seminars in which all project participants--director, staff archaeologists, and students--discussed and developed together the course of the investi- gations. Such an integrative process was essential given the culture-change theme of the project, a subject which requires discovery of interrelationships between the cultural and environmental components of the systems involved.

James H. Merrell, an historian, provides in Chapter 2 an historical background on the period spanned by the occupations of the Wall, Mitchum, and Fredricks sites. Using the documentary, rather than material record, he reconstructs several stages of an evolutionary process of increasingly more intensified interaction between European and Indian. His discussion serves well to set the stage for interpreting the evidence of change manifested in the archaeological record.

In Chapter 3, Gary L. Petherick describes in detail the stratigraphy, features, and architecture of the three primary sites. Even though less than $40 \%$ of the Wall site and probably less than $10 \%$ of the Fredricks site had been excavated at the time of his analysis, he is able to define patterns and relationships within these settlements and to explain these observations in social terms. He notes that the two historic sites contain more storage-type pits and less substantial architecture than the protohistoric site. According to Petherick, these differences are indicative of decreasing permanence and increasing mobility of Piedmont groups during the Historic period. This interpretation is consistent with the ethnohistoric record and with other categories of archaeological data. Petherick's reconstruction of the intrasite structure at the Fredricks site proved to be remarkably accurate in light of the completed village excavations in 1986.

In his section on mortuary practices (Chapter 4), H. Trawick Ward provides evidence for major differences in patterns of burial and attendant ritual between the Occaneechi people at the Fredricks site and the earlier Wall site occupants. The Mitchum and Upper Saratown burials retain mostly pre-contact characteristics, except that both have European grave goods and the Upper Saratown burials are more numerous than one would expect for a village of its size and term of occupation. The Occaneechi buried in cemeteries, similar to the Susquehannocks and other Northeastern groups; they lavished large numbers of both ornamental and utilitarian items on their dead; and they included in the fill of their burials large amounts of food and other refuse, which Ward considers to be evidence of ritual "feasting with the dead."

The two interments excavated in 1986 were both subadults deposited in shaft-and-chamber pits and accompanied by ornamental artifacts of European and native origin. The European artifact associations suggest contemporaneity with the cenetery burials. These individuals may be members of an ethnic group distinct from that represented by the cemetery. In fact, the pit configurations are much more similar to the 


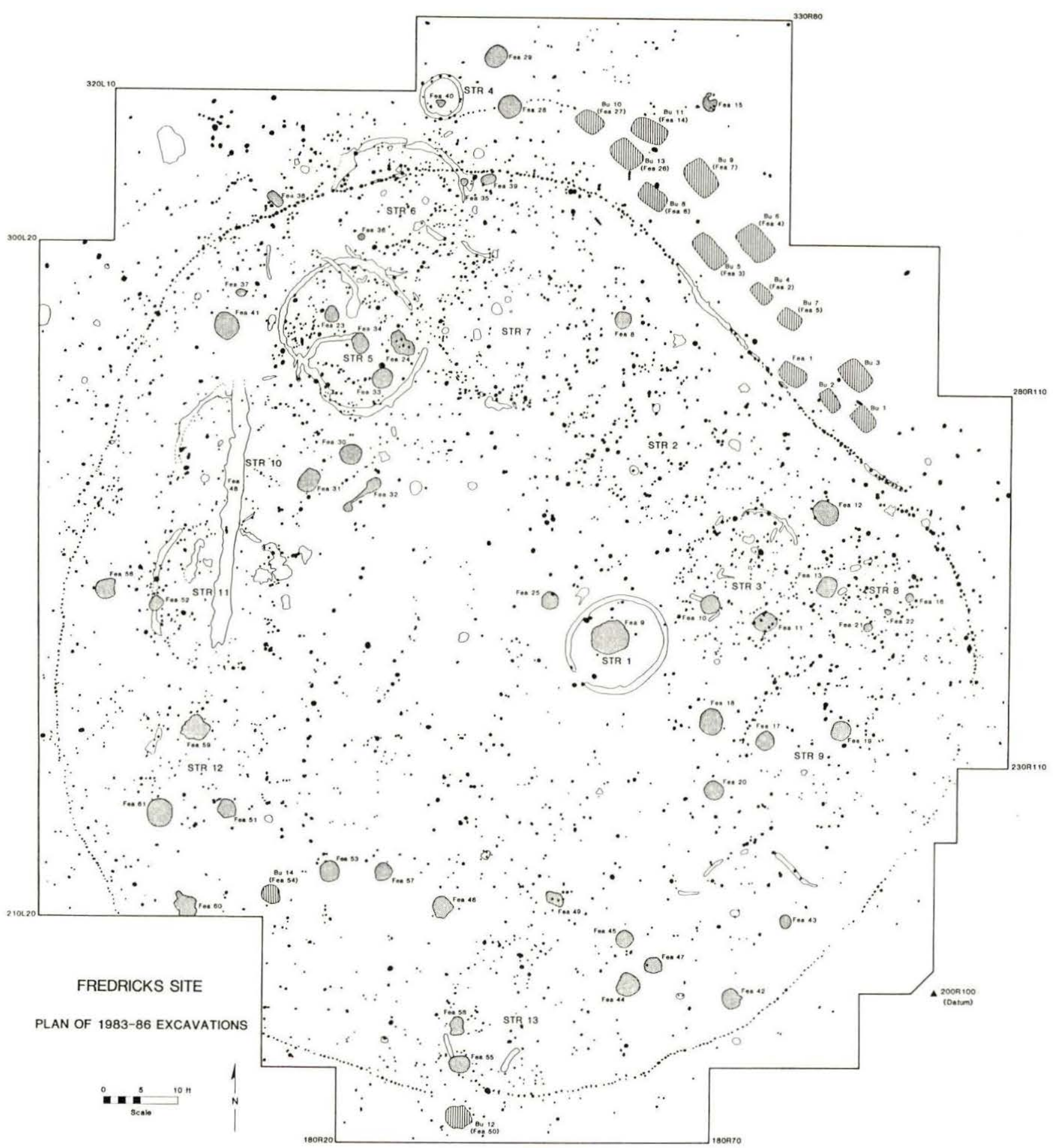

Figure 1.13

Fredricks site plan showing the results of 1983, 1984, 1985, and 1986 excavations. 
"typical" Siouan pattern than those of the cemetery.

An analysis of the human skeletal remains by Homes Hogue Wilson (Chapter 5) offers important preliminary information on differences in diet, nutrition, and health between late prehistoric/protohistoric populations and historic populations. All the cemetery burials from the Fredricks site, including those excavated in 1985, are included in the analysis. These and the Wall site burial sample are supplemented with data from the previously investigated Shannon site (Late Prehistoric) in Virginia and Upper Saratown site (Historic) in North Carolina. Wilson concludes that an increase in physical stress from warfare, long-distance hunting, food deprivations, and European diseases are reflected in increases in pathologies and disease conditions in the historic skeletal samples. The results of her efforts to determine relative biological distance among the populations, although suggestive, are inconclusive because of the small size of the primary samples.

Since the most intense involvement of the Piedmont Indians with Europeans was through the deerskin trade, careful analysis of the European artifacts from the study sites is an important aspect of the present research. In Chapter 6, Linda F. Carnes gives detailed descriptions of the Euroamerican artifacts from the three primary sites and then groups these artifacts into functional categories. She then evaluates the intrasite distributions of each category by context. At the Fredricks site, although she finds that there are no significant differences between categories from various fill contexts (e.g., feature fill vs. burial pitfill), she does encounter significant differences in categories used as burial associations and those in fill and plowzone contexts. Items selected by the Indians from their trade assemblage to place in burials included both ornamental and utilitarian items, and she notes that there is a significant increase in utilitarian items in the Fredricks site burials over those at the slightly earlier and more remote Upper Saratown site. The kinds of reworked and recycled Euroamerican items, together with contextual relationships and the nature of the overall assemblage, suggest that Euroamerican ornaments, tools, and utensils were integrated into an existing technology, to be used alongside their aboriginal counterparts, rather than producing changes in the direction of European functions or technological innovation.

Julia E. Hammett's analysis of the shell artifacts from the study sites (Chapter 7) focuses on shell ornaments, which occur mostly in burial contexts. Hammett begins by establishing a classification for these ornaments, a task that has been neglected by Southeastern archaeologists. She then attempts to define changes in styles, contexts, and sources through time. She concludes that, during the Historic period, a pre-existing network for trade in she11 was maintained and probably extended. Not only was trade carried out over longer distances, but there was an important shift from southern to northern material sources. Some traditional forms, especially of beads, were replaced with new shell forms or with European glass forms. Replacement forms seem to have been used in contexts similar to their most comparable older forms.

For the three primary sites, pottery sherds are one of the few kinds of artifacts that occur in numbers amenable to quantitative analysis. In Chapter 8, R. P. Stephen Davis, Jr. examines these ceramic artifacts on both intrasite and intersite levels using a computer-aided analysis format that includes variables of context, morphology, technology, and style. These data are then statistically manipulated to determine if the pottery assemblage from each site is homogeneous and to ascertain if there are relationships between the sites.

Davis concludes that the variability in ceramics from the Fredricks and Mitchum sites, where net-impressed and carved paddle-stamped pottery co-occurs, is probably the result of multiple, chronologically separate occupations. Conversely, the Wall site pottery is attributed to a single, rather short-term occupation. Sherd assemblages from these and other sites, when analyzed collectively, reflect a discontinuity in the spatial distribution of ceramic styles, centered upon the Haw River basin, that began in Late Prehistoric times and persisted through the Contact period. Relative continuity involving a carved paddle-stamped tradition is recognized from the Wall to Fredricks assemblages, and there is additional ceramic evidence for ethnic relationships through time between the occupants of the upper Eno drainage and those of the middle Roanoke drainage to the northeast.

V. Ann Tippitt's and I. Randolph Daniel's study of the lithic artifacts (Chapter 9) represents one of only a few attempts to systematically analyze stone tools from late contexts in the Piedmont Southeast. They use a computer-aided analysis format to document the lithic tools and debitage by stage of manufacture, raw material, tool category, spatial distribution, and overall morphology. All of the lithic data recovered from the Fredricks site through 1985 are included in the analysis.

The results indicate no major differences between the lithic artifact assemblages from the Wal1, Mitchum, and Fredricks sites, which suggests that the introduction of European metal tools did not appreciably affect the composition of the native assemblage. Such an interpretation must take into consideration, however, that variation could be masked by a lack of information on tools from sites away from the main habitations. There does appear to be a possible trend toward slightly larger triangular 
points, or at least greater variability in point size, during the Historic period. Also, a significantly larger amount of Ridge-and-Valley chert occurs at the Mitchum site, evidence for the same westerly connections indicated in Davis' ceramic analysis.

The 1986 excavations at the Fredricks site recovered a significantly larger number of ground-stone tools than had been previously found. Many of these may have been used to hone the metal cutting tools introduced by the colonial traders.

Mary Ann Holm's analysis in Chapter 10 of the faunal remains from the Wall and Fredricks sites (almost no faunal remains were found in the 1983 test excavations at the Mitchum site) suggests that there was very little change in animal exploitation from protohistoric to historic times. In fact, similarities in the two assemblages are far more striking than the differences. Although there is similar reliance on deer as expected, the comparable secondary use of catfish is somewhat unexpected. When one considers, however, that the large bend in the Eno where both of these sites are found, probably provided an unusually favorable habitat for sluggish-water species, such a common reliance is not surprising. The only evidence for domesticated animals at the Fredricks site is one bone each of horse and pig, which suggests that European-introduced animals were not an important food source in this area, even as late as 1700. One of Holm's most striking discoveries is a lack of differences in deer remains, and hence in hunting/butchering between the two sites. If hunting and butchering practices changed to accommodate the deerskin trade, as one would expect for a group like the Occaneechi, there should be evidence for this change in the animal bone assemblages. Since there are no such differences in the assemblages under consideration, Holm concludes that the Occaneechi either were not hunting deer for skins close to their village (i.e., most hunting for skins was conducted far enough away from the village that only the skins were brought back), that the contexts excavated thus far are not a true reflection of the total faunal assemblage at the site, or that they were not hunting at all to procure skins for the trade (i.e., they functioned mostly as "middlemen" in the trade).

Most of the faunal remains from the Fredricks site included in Holm's analysis were recovered from burial contexts and led to Ward's suggestion that feasting was part of the mortuary ritual. The faunal data recovered during 1985-86 came primarily from domestic contexts (i.e., re-filled storage pits). A comparison of the two samples reveals no significant differences in terms of the overall inventory of species or their relative frequencies.

Finally, Kristen Johnson Gremillion examines the paleoethnobotanical remains from the Wall, Mitchum, and Fredricks sites (Chapter 11). Gremillion was responsible for both field recovery and laboratory analysis of these remains. The botanical assemblages, like the faunal assemblages, exhibit far more similarities than differences. Corn, of major importance at all three sites, may show an increase through time. The 1983-84 data indicated that hickory nut increased in importance relative to acorn and walnut. However, subsequent analysis of the 1985 botanical remains revealed this contrast to be rather weak. The only European-introduced plant, present at both Mitchum and Fredricks, is peach. Except for the presence of watermellon within 1986 botanical samples, additional data collected from the Fredricks site during the last two field seasons do not alter significantly the patterns of plant food utilization based on the 1983-84 excavations. 


\title{
Chapter 2
}

\section{"This Western World": The Evolution of the Piedmont, 1525-1725}

by

\author{
James H. Merrell
}

On January 26, 1701, an adventurous Englishman named John Lawson left an Indian village along the Catawba River and swung northeast, completing an arc through the Carolina interior that had begun in Charleston a month before and would end on the lower Pamlico River a month hence. At the halfway mark of his journey, Lawson was a seasoned traveler, having already survived cold weather, poor food, wild animals, and an occasional angry native. Yet the thrill of discovery remained as he traversed the North Carolina Piedmont, "Every Step presenting some new Object, which still adds Invitation to the Traveller in these Parts" (Lefler 1967:54). Rich soil, tall trees, abundant wildlife, pleasant streams--Lawson was so taken by the scenery in what he called "this Western World" that he considered settling in the area (Lefler 1967:52).

Fascinated as he was by the landscape, Lawson thought that the native inhabitants he encountered were more interesting still. Between the Catawba River and the Coastal Plain were the Saponi, Tutelo, Keyauwee, Eno, Shakori, and Occaneechi, and the explorer surveyed them as carefully as he did the soil or streams. The Saponi headman had lost an eye while measuring gunpowder; the Tutelo blew a special powder into their eyes to improve their sight; the Keyauwee painted their faces with a lead ore; inside their houses the Occaneechi hung bear meat and dried venison; the Eno loved to play a game they called "Chenco" (Lefler 1967:52, 54, 61, 62). At each village, Lawson found something new and different to remark upon. His observations offer a window onto a lost world.

The English traveler was so struck by the diversity of these Indians that he failed to appreciate that they were probably related. Like the Catawba he had just left, the Monacan inhabiting central Virginia, and the Sara then living on the upper Roanoke River, the Occaneechi and their neighbors in the North Carolina upcountry were descended from Siouanspeaking migrants who had come over the mountains several centuries before Columbus arrived in America. As the newcomers fanned out along the rivers slicing through the region, their cultural uniformity slowly dissolved. A "people" became one or a cluster of villages, with its own dialect, its own customs, its own identity. Still, the differences were mere variations on a common theme. All spoke different forms of Siouan, and may have used Occaneechi as "a sort of general Language" to converse across group boundaries (Beverley 1947:191). ${ }^{2}$ A11 dwelt in the lands between the Coastal Plain and the Mountains, what the Europeans labeled "the Upper Country," the "hilly Parts," or "Hil1Country" (Lefler 1967:xxxi, 56, 89; see also Cumming 1958:9-10). All built villages of circular bark houses along the rivers and creeks. All followed a seasonal subsistence routine that balanced farming the bottomlands along the river, fishing the nearby waterways, hunting in the hills or canebrakes, and gathering wild plants at selected sites. Despite the barriers imposed by time, distance, and dialect, a fundamental unity underlay Piedmont life, a unity grounded in a shared cultural heritage and a common physical environment.

These Piedmont peoples also shared a common destiny once Europeans landed on America's shores. Between the 1520s, when explorers first touched the Carolina coast, and the 1740s, when most Indians had left the region, inhabitants of the upcountry went through four different stages of development. The first era, covering roughly the years from 1525 to 1625, was characterized primarily by indirect contacts with the visitors from the 0ld World. Material goods and lethal bacteria must have been carried into the interior by coastal Indians who had visited Spanish outposts to the south or later English settlements at Roanoke and Jamestown. Any face-toface encounters that did occur probably were fleeting. During the mid-sixteenth century, Spanish armies commanded by Hernando De Soto and Juan Pardo marched up the Catawba River valley before swinging west toward the mountains (Hudson et al. 1984:72-74, Figure 1; Depratter et al. 1983). From the east came tentative English probes up the Roanoke and the James (Quinn 1977:332-333, 451-452; Barbour 1964:222$225,237-239)$. Some Piedmont Indians may have headed in the opposite direction, drawn to the lowcountry by a desire to see the strange new beings for themselves (Barbour 1969:300-301).

Direct contact became much more frequent in the second stage of historical development, which began in the 1620 s with the defeat of the Powhatan Confederacy and a concomitant increase 
in Virginia's interest in lands beyond the falls of the James. A series of English explorers-some famous, most obscure--ventured inland to search for valuable mines or a westward passage (Cumming 1958:15-41; Alvord and Bidgood 1912: 183-205, 209-226; Morrison 1921:217-236). Close on their heels came other men eager to trade with the Indians. While natives welcomed the traders, this expanded contact was not without risk. In the 1650 s and again in the 1670 s there were bitter clashes between Piedmont warriors and colonial forces, with the Indians generally the losers. Nonetheless, by the time the Virginia rebel Nathaniel Bacon and his followers destroyed the Occaneechis' Roanoke River trading center in 1676, Virginians had penetrated to the far corners of the Southern Piedmont, and natives there had at least occasional encounters with an alien culture (Cumming 1958:16, 22; Washburn 1957:42-46; Wright 1981:87-90; Miche1 1916:30).

The destruction of the Occaneechi stronghold ushered in a new age on the Piedmont. With the Occaneechi no longer blocking the principal route into the upcountry, intercultural exchange flourished. The Indians Laws on saw in 1701 were accustomed to regular visits by Virginia traders, who often stayed for months at a time before heading home. Thus colonists had at last become a familiar sight in the upcountry, and the marvelous goods they brought had become a part of everyday life.

This period ended in the early years of the eighteenth century. Soon after Lawson passed through the area, incessant raids by powerful native foes combined with the lure of English trade goods to pull peoples from the interior toward the coast. By 1711, when Carolina began a decade of intercultural conflict with the Tuscarora and Yamasee, the Saponi had joined the Tutelo, Occaneechi, and Monacan under Virginia's protective umbrella. To the south, the Sara, Eno, and Keyauwee had drifted into South Carolina's orbit, later to merge with peoples in the Catawba valley to form the polyglot Catawba Nation. North of that isolated native island-an island that remains to this day--the Piedmont was mostly empty of human settlements until the first European colonists moved into the area during the middle decades of the eighteenth century. Before these permanent intruders arrived, the silence was broken only by an occasional hunting party, a band of Iroquois warriors after a Catawba scalp, a colonial packhorse train bound for Cherokee villages, or an isolated Indian farmstead.

A world that vanished in the space of two centuries is easier to mourn than to study. Few American Indian groups have left as little trace of themselves in the historical record as the peoples of the Carolina-Virginia Piedmont. Distant from initial European settlements, overshadowed by more prominent neighbors like the Powhatan and Cherokee, the upcountry Indian attracted little attention from observers willing and able to put their impressions down on paper for the benefit of posterity. With no chronicler like John Smith or James Adair to tell their tale, these peoples lived and died in obscurity, an obscurity that, for the most part, has continued to this day. Nonetheless, it is possible to shed some light on this shadowy world beyond the skeletal chronology of its demise offered above. Careful digging in the documents, when combined with equally careful digging in the Piedmont soil currently being carried out by the Research Laboratories of Anthropology at the University of North Carolina, enables us to piece together a fragmentary sketch of these peoples in historic times. The story that emerges is one of societies that adapted to dramatic change while remaining securely anchored to past ways.

By far the most drastic upheavals were caused by invisible invaders, the foreign bacteria introduced from the 01d World. Native populations cut off from Europe, Africa, and Asia for millenia were utterly without immunity to smallpox, measles, and other ailments the newcomers unwittingly brought across the seas. These maladies may have reached the Piedmont well before the first European. Spanish explorers who marched through what is now central South Carolina in 1540 came upon villages abandoned during an epidemic that had struck the area two years before (Bourne 1904:66). Did natives living farther north suffer similar devastation? Did illnesses that swept through the aboriginal population near the English settlement on Roanoke Island in the 1580s find their way upriver (Hariot 1972:27-28)? The answers require further archaeological research. But it seems unlikely, even impossible, that inhabitants of the uplands managed to escape all of the epidemics raging through eastern North America during these years. Certainly by Lawson's day Piedmont Indians were all too familiar with the lethal consequences of contact. The Sewee along the South Carolina coast were "now very much decreas'd...," he reported, "and all other Nations of Indians are observ'd to partake of the same Fate, where the Europeans come, the Indians being a People very apt to catch any Distemper they are afflicted witha1..." (Lefler 1967:17).

Precisely how "apt" upcountry natives were to succumb to alien infections remains unclear, but by any standard of measurement the devastation was severe. Lawson talked of entire communities being wiped out by smallpox and estimated that since 1660 only one Indian in six living within two hundred miles of English settlements had survived (Lefler 1967:232). Archaeological evidence from a Sara settlement on the Dan River offers grim confirmation of Lawson's claim. The site, occupied for a single generation in the latter half of the seventeenth century, contained so many bodies in such a short span of time that few could have escaped the terrible scourge (Ward 1980:182; Navey 1982:83). 
Those who did manage to survive had to pick up the pieces of their lives. It was not an easy task amidst the despair that followed in the wake of demographic disaster. One village along the Pee Dee River yielded mute testimony of the psychological havoc natives may have suffered. The area contained a markedly higher proportion of infant burials, suggesting a sharp increase in infant mortality due to disease. Moreover, the strikingly more elaborate ornamentation interred with each body hints at a heightened sense of loss among the survivors (Lewis 1951:328-329).

There was little opportunity to dwe1l on the past, however. Survival demanded that people cope with the present and think of the future. A village that had been reduced from 600 to 100 , or 300 to fifty, could no longer carry on alone. Thus the tattered remnants of Piedmont peoples began to merge with others to create new communities. Most of these unions went unseen or unrecorded by Europeans, and their documentation therefore must await further archaeological inquiry. But the first step was probably to join kinfolk from a nearby town or hamlet. In this manner a "people" that had once comprised a whole series of settlements scattered through a particular region or river valley now collapsed into a single town (Simpkins 1984). That "people" would still be set off from the world outside by language, customs, appearance, a kinship network--in short, by all of the characteristics that had always combined to create a distinct identify. But it would now occupy one site rather than many. The Monacan living along the James River in 1700, for example, were probably descendants of the entire cluster of villages John Smith had recorded for the lands above the falls almost a century before (Mooney 1894:18-22; McCary 1957; Bushne11 1920; Miche1 1916:29-30, 122-123). Similarly, it seems likely that the Occaneechi, Saponi, Keyauwee, Tutelo, and others Lawson visited were remnants, mere shadows, of more populous and more complex societies.

Lawson himself arrived on the scene in the midst of the second stage of the native response to depopulation. Continued visitations by alien diseases (the most recent smallpox epidemic had occurred only three years before) eventually reduced native numbers so drastically that survivors were compelled to look further afield for others with whom to unite. This process may have been underway in some areas by 1600 (Benthall 1969:45-48). By the time Lawson penetrated the Carolina interior, the Eno, Shakori, and Adshusheer had already come together to form a single community, and the Saponi, Tutelo, and Keyauwee were about to do the same (Lefler $1967: 61,53)$. None of these particular combinations endured. In 1708, the Saponi--alone-moved to the Virginia frontier, and during the next few years the Tutelo, Occaneechi (themselves a collection of groups as early as the 1670s [Alvord and Bidgood 1912:225]), and the Monacan remnants followed them during the next few years (Merrel1 1982a:107, 113; Mooney 1894: 18-19, 21). Meanwhile, the Shakori applied to South Carolina for protection, and the Keyauwee, Eno, and Sara combined forces and headed in the same southerly direction (Merre11 1982a:107-108, 113).

It is virtually impossible to retrace the steps of each native group, to reconstruct the shuffling and reshuffling that became almost routine as Indians sought to adjust to a new disease environment. It is even more difficult to gauge the impact of these stresses on the people involved. We can surmise, however, that the effect was profound. All of the available evidence suggests that inhabitants of the Piedmont, although culturally related, were intensely localistic, focusing their existence within a tightly circumscribed cultural and geographical range. This localism found expression in a number of ways. Lawson was astonished to find "a strange Difference in the Proportion and Beauty of these Heathens. Altho' their Tribes or Nations border one upon another, yet you may discern as great an Alteration in their Features and Dispositions, as you can in their Speech, which generally proves quite different from each other, though their Nations be not above 10 or 20 Miles in Distance" (Lefler 1967:35). Thus the Keyauwee had whiskers, the Tutelo were "tall, likely Men," while the Eno were of "mean stature" (Lefler 1967:58, 54; Cumming 1958:27). Cultural differences were equally clear. In 1670 the explorer John Lederer learned that Occaneechis were ruled by two headmen, Eno society was "Democratick," while the Saponi were governed by "an absolute Monarch" (Cumming 1958:25, 27, 24). Lederer also learned to distinguish among neighboring groups by their signs: the Occaneechi marked themselves with a serpent, the Saponi with three arrows, symbolic embodiments of their separate identities (Cumming 1958:13; Beverley 1947:161).

Natural boundaries--rivers, swamps, upland ridges, areas of poor soil--had long served to reinforce localism. Buffer zones, or "deserts" in English parlance, clearly divided Piedmont from Coastal Plain, and within the upcountry itself less obvious "deserts" set peoples off from another (Arber and Bradley 1910:59, 70; Baker 1975:25-36; Turner 1978:42-46; MacCord 1983:9-10; Simpkins 1984). Tales of the evils awaiting those who dared venture beyond local boundaries strengthened environmental barriers. A common story about a fierce creature inhabiting the headwaters of Neuse River frightened hunters away and may have been designed to keep people out of a border region (Lefler 1967:130). Explorers who traveled through uninhabited areas and had to live off the land rather than off the natives were unwittingly charting the ancient physical and cultural boundaries dividing peoples (Varner and Varner 1951:283-284, 331; Bourne 1904:59-62).

In the aftermath of an epidemic, Indians had to cross these boundaries as never before. 
Strangers became friends, the tall and the short lived side by side, necessity invented a common language and a common council of leaders from different groups. Those involved made the difficult process of adjustment less painful by keeping their old identity as much as possible. The Saponi, Tutelo, Occaneechi, and Stuckanock (Monacan), who collected in a single village at Fort Christanna on the Meherrin River, were considered "one Nation" by Virginia authorities (Great Britain, Public Record Office, Colonial Office, Series 5, 1714:1316:622). The Indians thought differently. Even after merging, each group continued to choose its own headmen and to adhere to its own customs (Brock 1885:88; Wright 1966:315-316). The Sara in the Catawba Nation did the same, not only heeding their own chiefs more than a generation after joining the Catawba but also living in their own village (Merrell 1982a:311, 400-401).

Archaeological evidence indicates that segregation extended from this world to the next. In aboriginal times Piedmont folk buried their dead at random in the village, in effect making the entire community a graveyard and suggesting that everyone in the settlement possessed a common identity. During the Historic period, on the other hand, interments were made in clusters, creating a handful of discrete cemeteries. Did several hitherto independent groups now occupy one town and, still acutely conscious of their own identities, express that consciousness by creating distinct burial grounds (Ward 1984)? At this point the pattern unearthed is suggestive rather than conclusive. But it fits well with the other habits recorded by European observers. Piedmont peoples did not shed their ancient identities overnight or even over a generation. The Saponi, Occaneechi, and Tutelo had a common destiny but separate identities until the era of the American Revolution, and as late as the mid-nineteenth century the Sara among the Catawba still spoke their own language at home (Hale 1883:10; Pearson 1842:5). Eroded by disease and by time, ethnic feelings nonetheless endured for generations.

Piedmont peoples' attachment to the past extended well beyond maintaining ancient loyalties to embrace many aspects of traditional life. The result of demographic disaster could have been profound despair and cultural ruin, stemming from a feeling that the supernatural forces protecting a society had failed and should be abandoned. Evidence turned up in the archives and in the earth suggests otherwise. Even the burials uncovered along the Dan and the Pee Dee, which speak so poignantly of the devastation wrought by an epidemic, also testify to a faith unshaken by recent events. Orientation of the body, positioning of the remains, inclusion of burial goods to accompany the soul into the afterlife--all suggest careful attention to time-honored customs designed to meet the demands of the supernatural. Recently discovered hints of feasts that took place at the burial site after interment only strengthen the idea that the old ways still endured in a Piedmont world periodically wracked by inexplicable disaster (Navey 1982; Dickens et a1. $1984: 30-37,52$ ).

If these burial feasts did persist, they were only part of a regular round of ceremonies that stretched across the generations into Lawson's time. Lawson himself ran across many more examples of rituals with roots in the remote past. "All the Indians hereabouts," he wrote while among the Keyauwee, "carefully preserve the Bones of the Flesh they eat, and burn them, as being of Opinion, that if they omitted that Custom, the Game would leave their Country, and they should not be able to maintain themselves by their Hunting" (Lefler 1967:58). Native travelers were no less cautious about propitiating the gods by adding a stone to a pile at a sacred site or placing some tobacco in the hollow of a large rock beside the trail (Lefler 1967:50, 63). Nor could time erase the fear of breaking customary rules. While exploring the interior in 1728, William Byrd II discovered that his Indian companion, a Saponi named Ned Bearskin, vehemently objected when the Englishmen in the party tried to cook venison and turkey in the same pot. No amount of cajoling or ridicule could make Bearskin drop the subject. He knew--as Southeastern Indians had always known (Hudson 1976:148, 165, 302)-that mixing inhabitants of the earth and the sky would bring disaster. Byrd considered it the silliest superstition; to the Saponi it was very real and very frightening indeed (Wright 1966: $116-117,243-244$ ).

Bearskin believed because he, like other Piedmont Indians, had seen sufficient proof that the old magic still worked. The priests were powerless to stop the new diseases sweeping through their villages, but experience was teaching them how not to respond when smallpox struck. "Now they are become a little wiser" about the best means of treating it, Lawson reported (Lefler 1967:232). Moreover, in other cures their wisdom was still unsurpassed. Using local herbs and ancient skills, natives astonished Lawson again and again with their abilities (Lefler 1967:17, 27, 48, 49). At the Saponi town, an Indian "Doctor" ushered the English traveler into his dwelling and proudly "shew'd me a great Quantity of medicinal Drugs, the Produce of those Parts; Relating their Qualities..., and what great Maladies he had heal'd by them" (Lefler 1967:54). Near the village stood several stone sweat lodges which "they make such Use of" as a cure for a wide variety of symptoms, and archaeological research reveals that the Occaneechi also retired to sweat lodges when they felt ill (Lefler 1967:55; Ewan and Ewan 1971:379; Alexander 1972:97; Dickens et al. 1984:3, 39, 42). Nor were priestly powers confined to healing. When a fierce wind struck the Saponi settlement the day after Lawson had admired the "Doctor's" pharmacy, the frightened Englishman rushed from his bed to find the headman in the center of the 
community busy with "his Necromantick Practice." Lawson's initial skepticism faded rapidly, for "in two Minutes, the Wind was ceas'd, and it became as great a Calm, as ever I knew in my Life" (Lefler 1967:55). If Lawson were converted, for others this merely confirmed what they already knew: the gods lived.

Given the continuing power of the ancient system of belief and behavior, it is not surprising that traditional authority figures continued to exert enormous influence. The elder1y, customarily the repositories of secret skills and ancient lore, had been depleted by disease; but their authority remained. "01d Age [is] held in as great Veneration amongst these Heathens, as amongst any People you shall meet withal in any Part of the World," Lawson wrote (Lefler 1967:43). Piedmont society set off old from young by different titles, secret languages, and special access to temples or other sacred places. They took precedence in welcoming visitors, speaking in council, and making decisions (Lefler 1967:43, 177-178, 210, 219, 231; Lederer 1958:14, 27, 41). Were the aged also differentiated by special burial customs, more elaborate burial goods, or some other distinguishing features? The answer awaits further archaeological inquiry (Navey 1982:191-194).

Thus the upheavals caused by sickness had not divorced Piedmont societies from their past. Life could never be the same again for those lucky enough to come through an epidemic alive. Still, they managed to rearrange their lives under the most difficult conditions without forgetting the lessons handed down from their ancestors.

A similar blend of persistence amidst change marked the upcountry Indians' response to European technology, the second important innovation introduced from abroad after 1525 . The manner in which these unfamiliar wares found their way into the hands of upcountry Indians remains unclear. The first trickle of material goods must have appeared in the sixteenth century. European explorers routinely passed out gifts--a knife here, a metal cross there, some beads further on--to ease their passage through Indian territory, and some of these prized possessions probably ended up among more distant communities (Lewis and Loomie 1953:111). Some natives were not content to wait. In 1609 , for example, Indians living along the Santee River had already made their way north to Jamestown and returned home bearing hatchets, knives, and biscuits made of wheat flour (Barbour 1969:312). Future archaeological research may reveal whether other curious natives from the Carolina interior made a similar trek and were similarly rewarded.

As trade with Virginia developed during the mid-seventeenth century, the pattern of exchange became clear. Each Piedmont settlement went through two distinct stages in its growing acquaintance with new technology, stages that even a novice like John Lederer recognized and that have been tentatively confirmed by recent archaeological work. The first step, which Lederer reported among the "remoter Indians" and archaeologists have found at Upper Saratown, was marked by a certain native naivete about the range of merchandise available from AngloAmerica. These people were happy to barter for "trinkets" such as mirrors and pictures, glass beads and bracelets, knives and scissors, "and all manner of gaudy toys and knacks for children..." (Cumming 1958:42). The archaeological portrait of Upper Saratown matches Lederer's description closely. This Sara village, filled with beads and "trinkets," was virtually devoid of European weapons or other metal goods (Wilson 1984).

A potsherd found at Upper Saratown with the outline of a musket etched into it by some unknown native artisan hints at a growing awareness of the material cornucopia available from Europeans and may signal the beginning of the second phase of a people's relationship with an alien material culture. Lederer found that "neighbour-Indians," more experienced in the art of intercultural exchange would not be satisfied with "trinkets." They demanded not only knives and scissors but also arms and ammunition, not only beads and bracelets but cloth, axes, hoes, "and all sorts of edg'd tools" (Cumming 1958: 41). By the end of the century, Indians throughout the Piedmont were behaving like "neighbour-Indians." At the Fredricks site, the probable location of the Occaneechi town Lawson visited in 1701, archaeologists have uncovered an inventory of goods quite unlike that at Upper Saratown, occupied a generation earlier. Inhabitants of the Fredricks site possessed muskets and pistols, glass bottles and metal pipes, iron axes and pewter porringers--in short, they were more thoroughly integrated into the colonial trade system (Dickens et al. 1984:27-39; Wilson 1984).

It is easy to exaggerate the changes wrought by this deepening involvement in intercultural exchange. In fact, whereas European goods and colonial intruders were certainly novelties, traders and trade were not, and Piedmont natives fitted the new men and the new merchandise into established patterns of exchange and existence. Archaeological evidence demonstrates that Indians in the interior had long traded with their coastal neighbors for a variety of items (Merrel1 1982a:22). Some of that trade remained wholly untouched by the growing colonial presence. In Lawson's day, for example, towns along the coast still gathered yaupon plants (from which Indians brewed the ceremonial "black drink") and sea shells, carried them inland, and swapped them with "remote Indians" for a root that grew near the mountains and was used to make red paint (Lefler $1967: 98,218,174)$.

With the structure of exchange so well established, Indian traders living near the 
English could easily begin to add new products to the supply of merchandise they hauled into the upcountry. In 1670, John Lederer met some Sara traders at a village along the Catawba River (Cumming 1958:31). Since the Sara were in touch with Virginians around that time (Wright 1966:400), it seems likely that some of the goods they carried were of European manufacture. By the time Lawson passed through, this trend was clear: coastal Indian traders were peddling everything from stolen horses to jugs of liquor in Piedmont villages (Lefler 1967:44, 54, 232).

Colonial traders gradually supplemented, then supplanted, the native middleman, but they still had to fulfill Indian expectations by conforming to local codes of conduct (Merrell 1982b:5-7) and satisfying their hosts' taste in trade goods. Despite the new and wonderful products a Virginia trader dangled before their eyes, natives insisted that he also bring goods traditionally carried past the Fall Line from the lowcountry. "This yeere [1682] the Indyans will have Roanoake," complained Cadwallader Jones, a colonist heavily involved in the Piedmont trade, "not with standing all other com[m]odities be $p[\mathrm{re}]$ sented.... I having at this time a considerable parcell of other goods amongst them unsold" (Great Britain, Public Record Office, Colonial Office, Series 1 1682:48:115-116). In 1691, William Byrd I, another businessman active in the uplands, also complained that the Indians wanted shell beads more than anything else (Tinling 1977:163). The natives' insistence on acquiring shells may explain a curious shift in the types of shells found at Piedmont villages in the Historic period (Sizemore 1984). The Fredricks site contained shells from northern shores, and it seems likely that these items arrived there on the backs of colonial packhorses, for colonial traders often looked northward for the supplies Indians demanded. In 1671 John Lederer received a commission from Maryland's Lord Baltimore to trade with natives in the Southern Piedmont (Cumming 1958:99-100). A decade later Cadwallader Jones also looked to Lord Baltimore, begging Maryland's Lord Proprietor to grant him permission to collect shells along the colony's Eastern Shore (Great Britain, Public Record Office, Colonial Office, Series 1 1682:48:115116). And a decade after that, William Byrd I went even further afield, writing the governor of New York to ask that he send some shell beads to be passed along to Carolina natives (Tinling 1977:163). The source of supply had changed; native tastes had not.

Piedmont inhabitants were no less selective in the European wares they did purchase. To his dismay, the colonial trader learned that Indians would not accept every item he happened to have on hand. Dark blue cloth sold best, as did larger hoes and smaller glass beads. But not just any beads: villages west of Virginia wanted blue and red ones, those to the south, black and white (Tinling 1977:30, 41, 57, 64; Ewan and Ewan 1971:385). Why? The colonist either did not know or did not say. He knew only that such idiosyncrasies could spell the difference between profit and loss.

Those items natives did accept were grafted onto existing ways. Some were simply substitutions of European for aboriginal manufactures. Indians happily donned cloth instead of deerskins, painted their faces with vermillion rather than cinnabar, became expert marksmen with a musket as well as a bow, dug graves with iron rather than stone tools (Ward 1984), and adorned themselves and their dead with glass beads as well as shells. At other times they reshaped an item to suit themselves. Lawson reported, and archaeologists have since confirmed, that Piedmont men fashioned arrowheads from broken glass bottles (Lefler 1967:63; Charles 1983:31). Meanwhile, the women might take a copper kettle and cut it up to make into ornaments, or an Indian fortunate enough to obtain a horse used the animal in ways that made an Englishmen cringe. The Saponi headman proud1y showed Lawson "2 of his Horses, that were as fat, as if they had belong'd to the Dutch Troopers." Natives never rode these creatures, and scarcely ever used them as beasts of burden, preferring instead to keep them as status symbols and stuff them with corn like some pampered pet (Lefler 1967:54, 44).

The merchandise Indians gave in return for a11 of these European goods further strengthened attachments to past ways, for each was firmly rooted in aboriginal skills. The cane baskets William Byrd's men brought back from Piedmont towns were products of a long craft tradition among women there, a tradition flourishing in de Soto's time (Ewan and Ewan 1971:384; Varner and Varner 1951:313,315-316). Similarly, the deerskins that made up the bulk of a colonial trader's return cargo entailed no radical departure from previous modes of existence. Deer were already a vital part of everyday life, and Indians were adept at stalking, killing, and processing the animals. Even the occasional Indian slave that Virginians brought out of the interior required no revolutionary reversal of customary ways. Piedmont warriors had habitually captured enemy Indians for adoption, torture, or servitude. This reservoir of outsiders could now be tapped to supply colonial demands, and replenished by new forays against traditional foes (Merre11 1982a:78-80).

In short, neither the wares Piedmont folk acquired nor the articles they handed over in exchange revolutionized their lives. It is therefore not surprising to find traditional patterns of belief and behavior intact despite heavy engagement in intercultural trade. Historic sources and archaeological evidence agree that routine subsistence practices did not break down despite the time Indians devoted to the trade. Natives had added peaches to their repertoire of foodstuffs, but otherwise a Piedmont Indian in 1700 ate much the same dishes as his ancestors a century or two earlier (Lefler 
1967:24, 35, 115-116; Ewan and Ewan 1971:376; Wilson 1977:83, 115-116; Ward 1980:196, 198; Johnson 1984). Young men still helped with planting, women still tended the crops and gathered wild plants, hunters still went out for food as well as deerskins, bringing back turkey as wel1 as venison (Lefler 1967:17, 31, 34-35, $59,177)$. Nor had the demands of the trade wrenched people free from the ancient system of values. A hunter stalking deer to sell the hides to colonists was no less eager to propitiate the gods than his ancestor (Lefler 1967: 58). And when that hunter died, his possessions--even the coveted musket--went with him into the earth and the afterlife rather than being passed on to his kinfolk as people with European notions of property would have done (Dickens et al. 1984:35, 49).

Thus the threads binding upcountry Indians to the past remained unbroken by the steady expansion of trade with colonists. Nonetheless, the evidence of cultural persistence cannot altogether obscure signs of profound changes set in motion by the trade. The most obvious of these changes was alcohol, one European product Indians could not easily incorporate. Lawson ranked it with smallpox as a killer (Lefler $1967: 232)$. While exaggerated, his assessment does point to the havoc created by a keg of rum. Indians would sell all they possessed to acquire it, would not stop drinking until completely intoxicated, and then, freed of customary restraints, proceeded to maim or kill themselves and their townspeople (Lefler 1967:18, 184, 211, 240). According to Lawson, inhabitants of the interior had only recently become acquainted with liquor (Lefler 1967:232). If so, they quickly learned how destructive it could be and took steps to combat it. By 1712, the Saponi were petitioning Virginia authorities to prohibit the sale of liquor to Indians, an appeal repeated many times with no real effect (McIlwaine 1928:312-313). Officials in Williamsburg proved as helpless to stop colonists from selling it as native headmen were to prevent their people from purchasing it (Wright $1966: 315$ ).

Alongside the obvious addiction to alcohol was a more subtle, more pervasive, and ultimately more destructive addiction to European technology in general. The erosion of ancient craft skills, virtually undetectable at the time, is clear in the archaeological record, as arrowheads and clay pots became cruder in design and clumsier in execution with the passage of time (Lewis 1951:310; Coe 1964:49-50; Trinkley and Hogue 1979:11). This ominous development meant that Indians were steadily becoming more dependent upon fresh supplies of European merchandise, a dependence that left them at the mercy of distant markets and unknown forces.

The shifting Piedmont settlement pattern also leaves subtle clues of the growing importance of trade. With the exception of the Tutelo and Sara, by 1701 all of the remaining
Piedmont groups had chosen to settle astride the principal trail from Virginia to the populous Catawba River towns, the better to waylay itinerant colonial traders headed to those lucrative markets. Between the time Lawson passed through and the publication of his book eight years later, all of these peoples had taken another important step down the road to dependence, leaving the upcountry to bypass middlemen like the Tuscarora and get closer to their colonial suppliers. The Sara and others moved south into unfamiliar terrain along the Pee Dee River to establish a trade connection with Charleston (Anonymous 1715). The Saponi, Occaneechi, and Tutelo chose to forget their old quarrels with Virginia, and they settled along trading paths on the colony's southwestern frontier where they had easy access to the colonial trading community (Cumming 1958:16, 22; McIlwaine 1928:188, $196,296,566)$.

While disease had compelled independent peoples to consolidate and trade had pulled these remnant groups toward English settlements, the catalyst in these developments--the force driving survivors together and then pushing them out of the Piedmont--was Iroquois warfare, the third and final major change Piedmont groups endured in the Historic period. The beginnings of this bitter conflict date from the last half, perhaps even from the last quarter, of the seventeenth century. Before that time the Five Nations of Iroquois were preoccupied with native peoples elsewhere. Only after 1660 did northern visitors begin to penetrate the Southern Piedmont, and even then intruders were rare-colonial explorers like Lederer made no mention of them. The real threat to the uplands came in the late 1670s, when the Susquehannock--driven from Maryland by colonial militia in 1675, attacked the next year upcountry warriors in Virginia, the year following incorporated with the Five Nations--returned south with their new friends to settle old scores. Before long, northern war parties regularly "infested" the southern upcountry, attacking villages and carrying off prisoners (Merrel1 1984a:3).

Warfare was hardly new to the Piedmont. Archaeological research at earlier village sites has turned up unmistakable signs of palisades, clear evidence that inhabitants of the precontact world feared attacks from someone (Benthall 1969:20; Holland 1970:115; Egloff 1980:130). Nonetheless, these Iroquois incursions were probably unprecedented in their frequency and their ferocity. The peoples Lawson met between the Catawba and the Tuscarora huddled in their fortified villages in daily fear of another raid. Lest they forget the dangers, piles of stones marking the graves of earlier victims or the occasional escapee from Iroquois captivity served as painful reminders of the harsh reality (Lefler 1967:50, 59). The recent discovery of two burials at Occaneechi Town--one showing signs of scalping, the other with a musket ball lodged in its leg--further attests to the precarious existence of Piedmont 
Indians in this period (Dickens et al. 1984:32, $37,48)$. They banded together, acquired firearms, captured prisoners, even ventured north to gain revenge--but nothing they did could halt enemy incursions (Lefler 1967:53; Hazard 1851: 138). Ultimately it was the search for shelter from this Iroquois storm that drove the Sara and their confederates into South Carolina's embrace while the Occaneechi and others sought refuge in Virginia (Wright 1966:398; Lefler 1967:242).

Peace proved elusive, however. For a time the Sara and their Keyauwee and Eno compatriots along the Pee Dee River enjoyed the best of both worlds: they kept their ties to Virginia while making new friends with South Carolina. When men from Charleston stopped at the Sara village in 1712 on their way to fight the Tuscaroras then raiding North Carolina, forty-two Sara warriors were heading in the opposite direction to join the Yamasee and others in an attack on South Carolina, and they carried on the fight with grim determination long after most Indians had made peace. As if South Carolina's enmity were not enough, the Sara also learned that they could run from the Iroquois invaders, but they could not hide. In 1716 and again in 1723, war parties from the Five Nations wreaked havoc along the Pee Dee River. By the end of the $1730 \mathrm{~s}$, most of the inhabitants had abandoned their new homes to take refuge among the Catawba. They soon discovered that the Catawba Nation was more target than refuge, and during the 1740s Sara leaders were again talking of moving someplace "where they might have fewer Enemies." Colonists and Catawbas convinced them to stay, and they agreed, perhaps in large part because past experience had taught them the futility of escaping their implacable northern foes (Merrel1 1982a:223, 234, 250, 303, 309, $363,390)$.

The Occaneechi, Tutelo, and Saponi followed a path in some ways different but also quite similar. In 1714, they signed a treaty with Virginia's Lieutenant Governor Alexander Spotswood that formalized their relationship with the province. The Indians would live beside the Meherrin River in the shadow of Fort Christanna, an outpost to be built by a trading company and manned by colonial rangers. In return for their promises to help defend the frontier and pay tribute to Williamsburg, the natives were to receive protection, trade, a reservation, and--Spotswood's pet project--instruction in "civilization" and Christianity. Spotswood was optimistic, the Indians pronounced themselves satisfied, and a visitor to the settlement in 1716 found native children sitting attentively in a classroom under the watchful eye of an English tutor (Beaudry 1981:2-13; Alexander 1972:90-99).

For all the high hopes, the experiment was short-lived. Competing trade interests managed to get the trading company abolished before the end of the decade, and in the $1720 \mathrm{~s}$ the "Christanna Indians" found themselves harassed by nearby settlers, attacked by northern war parties, and abandoned by colonial officials. In 1728 , they marched back into the Piedmont along the old trading path to join other refugees heading toward the Catawbas. Their stay in the Nation turned out to be as brief as the Christanna experiment, however. By 1732, they were back in Virginia, from whence they scattered in several directions. Some went to live with the Tuscarora still in North Carolina; others drifted into the backwaters of Virginia society; still others found a home with a North Carolina planter engaged in trade with the Catawba; most eventually drifted north to join their old enemies, the Six Nations, and became one of the "props" in the Iroquois Longhouse (Merrel1 1982a:305-3089; McIlwaine 1930:239; Saunders 1968:537-538, 5:321, 6:616; Holland 1982:42; Grinnan 1895-96:189-191; White 1981:6569; Hale 1883:5-10).

The different fates of the Sara and Occaneechi constellations remains puzzling. Why did Piedmont peoples sort themselves out into this particular configuration and not some other?. Why did the Sara-Eno-Keyauwee groups eventually establish permanent residence with the Catawbas, while the Saponi and their associates did not? There was no overt antagonism between the two: the Sara and Eno had considered joining the Christanna experiment, and when Christanna Indians returned from the Catawba Nation in 1732 some Saras came along while some Saponis remained behind (McIlwaine 1930:269; Merre11 1982a:113, n.197). The Siouan Project currently being conducted by the Research Laboratories of Anthropology may yield clues to these and the many other riddles Piedmont peoples bequeathed to future generations. The answers lie buried beneath the surface of the land these Indians once called home, at Upper Saratown, the Fredricks site, or other places yet uncharted. Rediscovering that lost world will take time. But the marriage of history and archaeology has already begun to piece together its outlines, to show that, however different their ultimate fate, the inhabitants of the uplands shared a common history during the first two centuries of European contact, a history marked by creative if painful adaptation to the changes the intruders brought to "this Western World."

\section{NOTES}

1. Until further archaeological research is carried out, the claims put forth in this paragraph remain speculative. It should be noted that the linguistic and cultural patterns in the Piedmont are matters of considerable dispute. Earlier scholars placed a large number of groups under the Siouan umbrella; more recently, many have demanded firmer evidence. While agreeing that the first students of the problem may have been too eager to label a people 
"Siouan" and that nonlinguistic considerations cannot prove linguistic relationships, I am persuaded by William Sturtevant's argument (Sturdevant 1958:741) that where we lack linguistic evidence, we must make do with whatever sources are available. Here geographical proximity, cultural parallels, aboriginal or historic political relationships, and contemporary statements by colonists about linguistic similarities all suggest that most if not all of the Indians in the Piedmont at the time of English contact spoke some form of Siouan. Included among these are Catawbas, Saras, Saponis, Tutelos, Occaneechis, Monacans, Mannahoacs for certain, and Waterees, Enos, Keyauwees, Sugarees, Esaws, Shuterees, and Shakoris most probably. The linguistic debate may be followed in Mooney (1894), Siebert (1945), Miller (1957), Sturdevant (1958), Binford (1959), and Hudson (1970:5-9, 27-28). For the archaeological work done (other than the Siouan Project currently underway) see Griffin (1945), Coe (1952), Wilson (1977:12), and Mouer 1983:21-24).

2. It is possible that Occaneechi developed as a trade language only in historic times.

3. The following analysis of native response to disease and trade has been adapted from work I have already published on the Catawba Nation and its neighbors. See Merre11 1984b:542-555. 


\title{
Architecture and Features at the Fredricks, Wall, and Mitchum Sites
}

\author{
by
}

\author{
Gary L. Petherick
}

\section{INTRODUCTION}

This section describes and compares the internal site structure of the Wall, Mitchum, and Fredricks sites from data available through the 1984 field season. This research is part of a larger project to investigate the processes of culture change among Indian groups of the Carolina Piedmont during the period of European exploration, trade, and colonization. Information on the structure of these three sites, which represent settlements from the Protohistoric, Early Contact, and Middle Contact periods, allow some preliminary assessments of the cultural diversity, continuity, and change as reflected in the organization of the archaeological record at intrasite and intersite levels.

Specific research questions about site structure are listed below:

1. How were the villages of the Protohistoric period structured in terms of their spatial organization and architecture?

2 . Do these villages reflect increasing populations, or were they relatively stable in terms of size?

3. Were the villages seasonally abandoned or were they occupied all year?

4. Does spatial structure at these sites reflect differences in status between the members of these societies?

5. Do differences between the sites reflect differences in native cultural traditions or European-induced culture change?

6. Did participation in the deerskin trade bring about an increase in group mobility?

7. Did the introduction of metal tools or Euroamerican ideas concerning architecture and construction methods cause changes in the traditional ways of house building?

8. With depopulation, did people become consolidated in small inter-ethnic composite villages?
9. Did settlements become more compact and nucleated as a response to increased warfare?

Although the data are insufficient to approach these questions with certainty, some preliminary observations can be made by comparing aspects of the structure of the three sites under study. Complete answers to these questions will have to be derived from the integration of findings from a larger number of sites representing a greater range of time. It is hoped, however, that this discussion will play some small part in leading to a more complete understanding of the cultural systems of the Native Americans who once thrived in this area.

An eclectic, and largely descriptive approach, is taken in this section. This approach is justified by a belief that accurate and complete descriptive data are necessary in order to reach correct interpretations. Limitations of the data have led in many instances to the development of preliminary interpretations that may seem to border on speculation. Such statements, however, are offered in an effort to provide a datum from which future research can be developed.

The basic assumption used in this research is that the spatial organization of a site, as it is observed archaeologically, reflects either directly or indirectly the spatial organization of the cultural system that produced the site. More specifically, the spatial arrangement of structures, facilities, and open areas reflect the cultural and physical processes that created them. Their patterned interrelationships reflect patterned behavior associated with them. These patterns also reflect ideas shared by members of a community as to how space should be organized (Ha11 1969; Rapoport 1969; Clarke 1977; Hodder 1978, 1982).

The method followed within this analysis is a search for patterns: patterns of postholes, patterns of structure location, patterns of features both in form and context, patterns in materials making up fill within features, patterns in the distribution of cultural residues. The interrelationships between these kinds of patterns may themselves form patterns. It is 
these larger patterns that allow for interpretations that are more secure and meaningful in terms of past human behavior. This analysis is confined to what Clarke (1977) has classified as the micro and semi-micro scales of cultural systems. That is, the analysis is confined to patterns and processes visible at the site level and at levels within the site. It is acknowledged that such an analysis would be incomplete without considering the larger settlement system of which villages are only a part. Nevertheless, it is believed that for an analysis of the larger system, detailed information concerning the parts are required. An attempt is made to interpret the observed patterns in behavioral, social, and cultural terms where possible and to offer some preliminary hypotheses when the meaning of the pattern is unclear. With these basic ideas in mind, each of the sites are discussed and comparisons and preliminary interpretations offered. The latter are directed at the research questions outlined above.

The size of the area investigated at each site is different, and this presents some problems in comparing the sites at the scale necessary for defining site patterns. It is difficult, if not impossible, to compare the village structure as it appears from over 14,000 $\mathrm{ft}^{2}$ of excavation, with the structure as it appears from $850 \mathrm{ft}^{2}$. Similarly, it is difficult to compare features and feature fill zones from two sites when one set of features was excavated before archaeologists became aware of the need to use fine-scale data recovery techniques. Such are some of the problems with the data from these sites affecting comparability.

\section{WALL SITE}

The Wall site is a relatively small, palisaded village which dates to the mid-sixteenth century. Three radiocarbon dates from wood charcoal recovered from feature excavation yield an average date of A.D. 1545+80 (GX9718, GX9719, GX9834). The village itse $\overline{1} f$ covers approximately one and one-quarter acres and lies within a horseshoe bend of the Eno River in Orange County, North Carolina.

Investigations at the Wall site were conducted in 1938, 1940-41, and 1983-84 (Figure 3.1). In 1938, a 100-ft exploratory trench revealed the stratigraphy of the site and a portion of a circular structure. The 1940-41 excavations were more extensive and exposed a large portion of the western half of the village. At the end $g^{f}$ the 1941 field season approximately $12,000 \mathrm{ft}^{2}$ of the village had been explored. In 1983, after a hiatus of 42 years, investigations were resumed at the Wall site. The 1983 work was designed to determine the exact location and extent of the earlier investigations. Additionally, a $600 \mathrm{ft}^{2}$ area to the east of the earlier excavation was exposed, revealing a group of three burials, portions of two circular structures, and a section of one of the village palisades. The focus of the 1984 work, which totaled $1100 \mathrm{ft}^{2}$, was to expose and excavate the circular structures identified the previous year.

As of 1984 , nearly $26 \%$ of the village had been excavated $\left(14,300 \mathrm{ft}^{2}\right)$. These excavations have revealed a fairly detailed picture of the internal organization of the village (Figure $3.2)$.

Ten complete structures have been identified from the investigations so far (Figure 3.3). In addition, two structures are partly defined but extend beyond the boundaries of the excavations. Midden deposits were well preserved along the northern third of the excavations. The densest deposits have been observed in the north-central part of the site in the vicinity of entrances through the various palisades (Figure 3.4). The features observed and investigated consist mostly of shallow basins of different sizes and shapes. Three small storage pits also have been identified, two of which were discovered during the 1984 excavations in the eastern part of the site. A total of eight burials have been excavated. These were located both within and near the structures at the site (see Ward, Wilson, this report).

\section{Site Stratigraphy}

At the Wall site, cultural remains are contained within four soil layers that are differentially preserved across the site (Figure 3.5). The uppermost layer (Zone I) is a plow-disturbed light brown clay loam that ranges from 0.55 to $0.95 \mathrm{ft}$ thick. Artifacts recovered from this zone consist of fragments of aboriginal and Euroamerican ceramics, chippedstone tools and debris, fire-cracked rock, daub, brick fragments, metal fragments, glass, and small amounts of charcoal and animal bone. These materials derive from both aboriginal and Euroamerican occupation of the site vicinity. The vast majority of these remains are from the mid-sixteenth-century village occupation, being derived from plowing of the upper portions of postmolds, pits, house floors, and midden. (For a description of the pre-village remains see Tippitt and Daniel, this report; and for a description of the historic remains see Carnes, this report). Thus, Zone I was created by plowing through the tops of the features and mixing their contents with debris from both earlier and later occupations of the area.

The plowzone is underlain by either Zone II, III, or IV depending on the portion of the site examined. Zone II consists of an intact midden deposit which accumulated on the original occupation surface. This midden is extensive and well preserved along the northern third of the site, where the original land surface sloped 


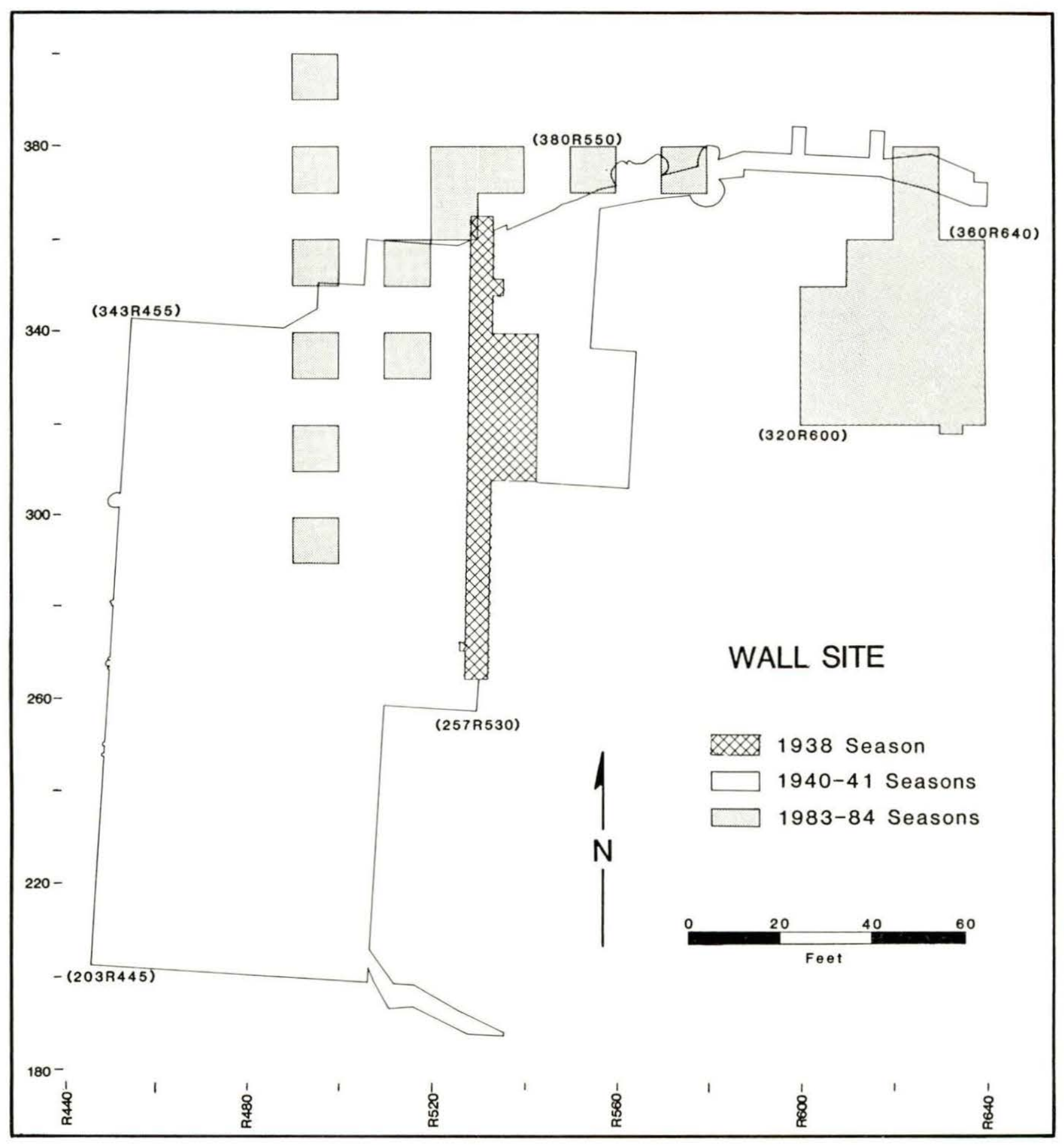

Figure 3.1

1938, 1940-41, and 1983-84 excavation areas at the Wall site. 


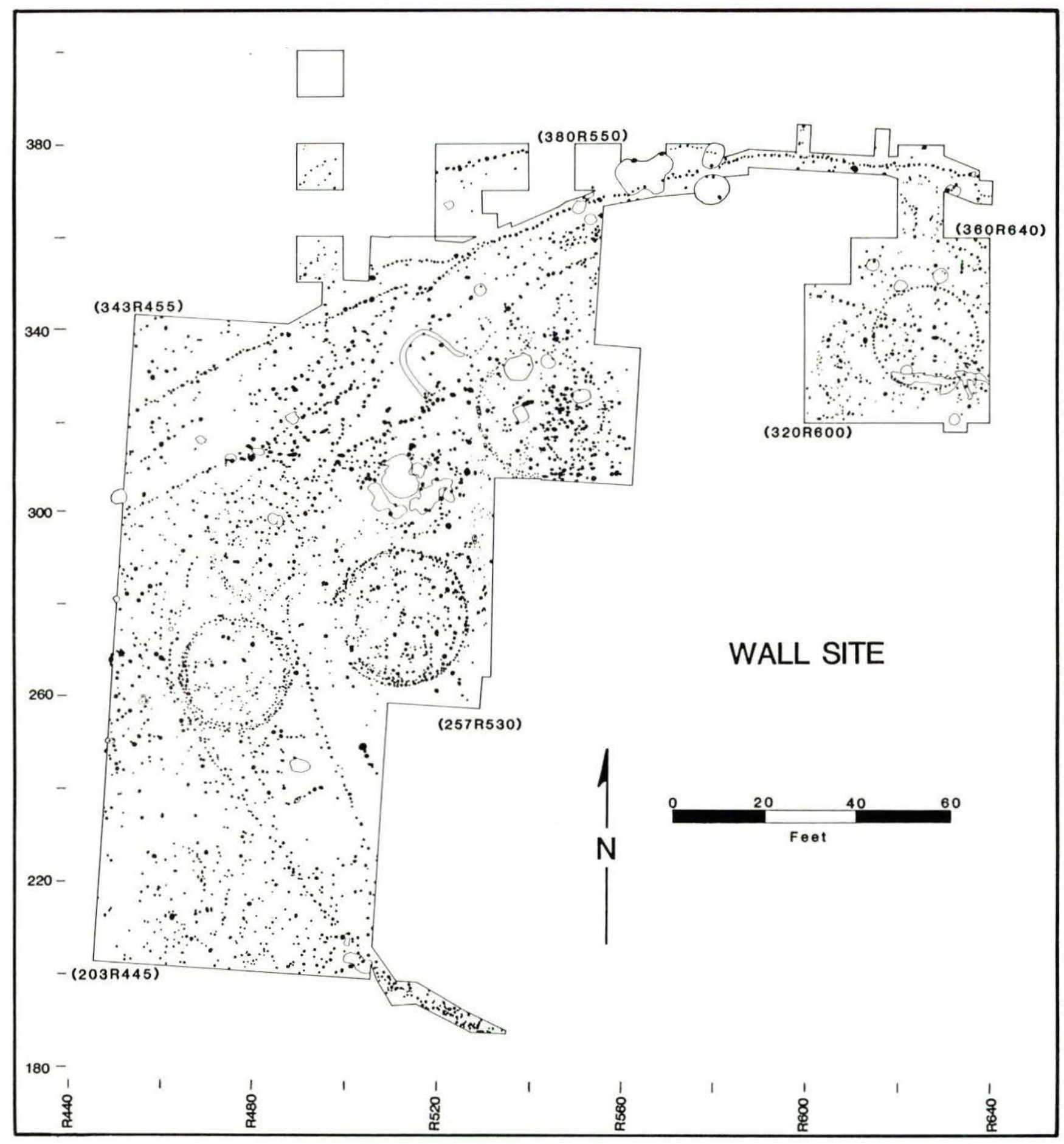

Figure 3.2

Excavation plan at the Wall site, 1938-1984. 


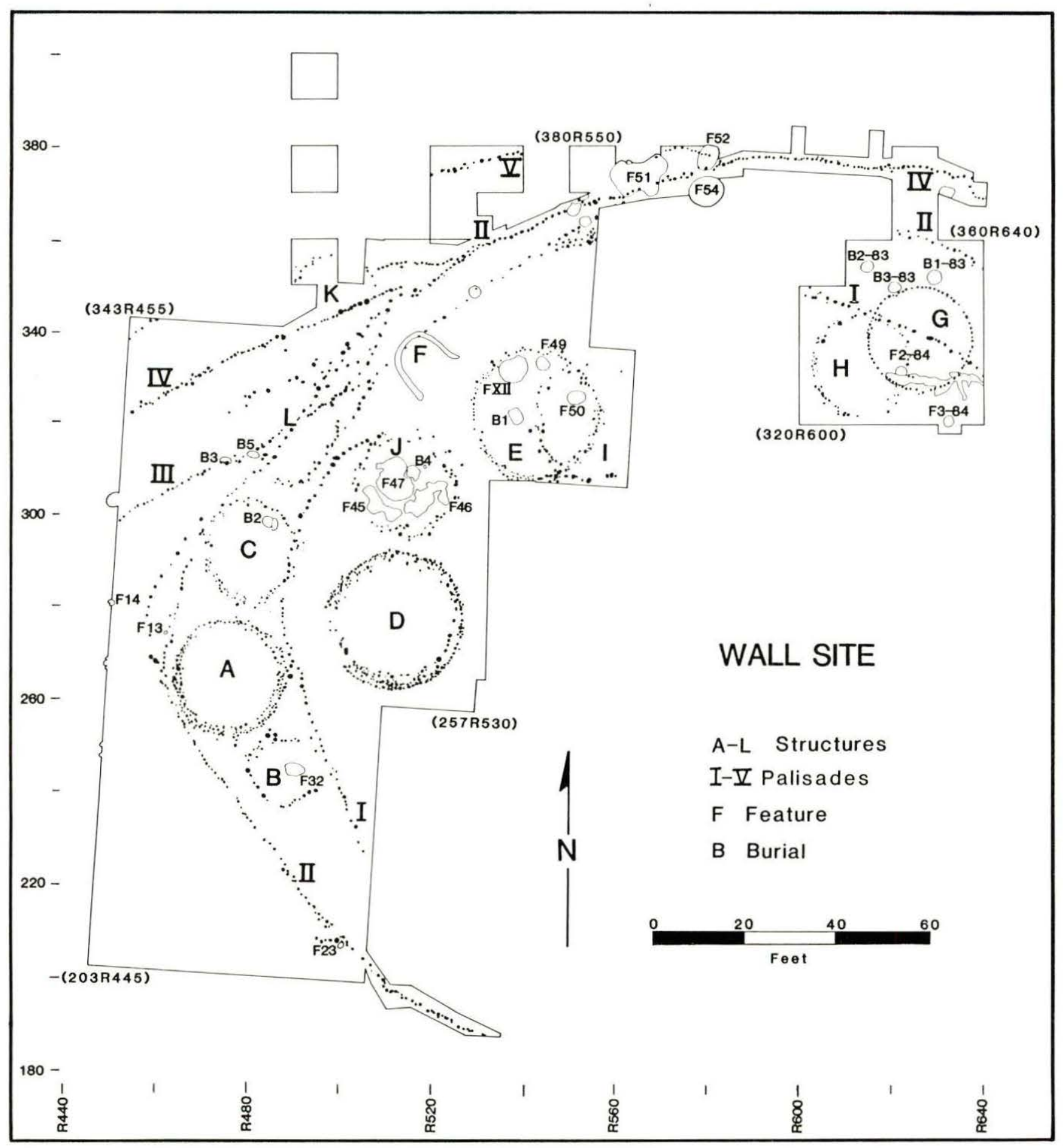

Figure 3.3

Plan of architecture and features at the Wall site. 


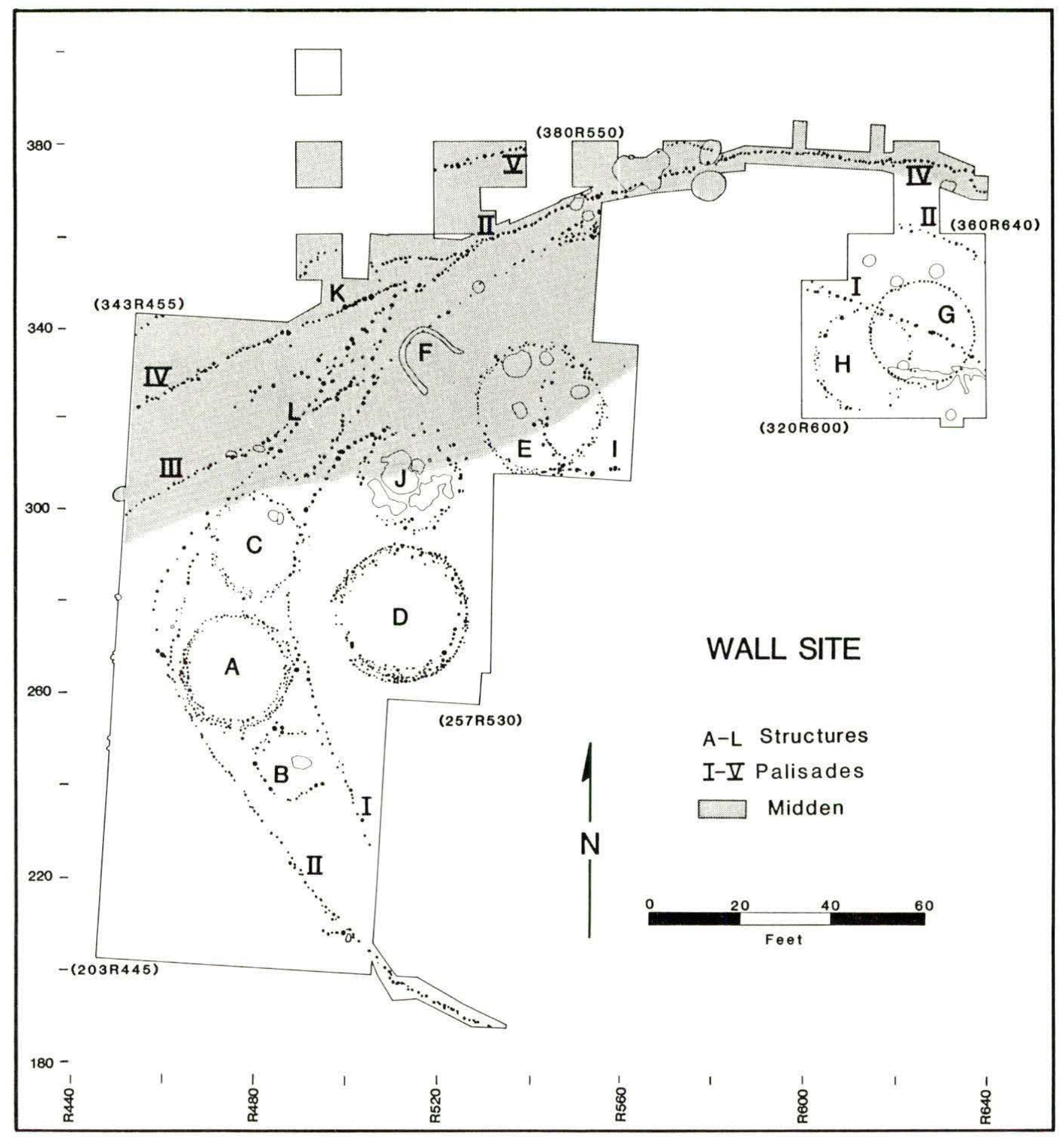

Figure 3.4

Areal extent of the midden at the Wall site. 
PROFILE A

s

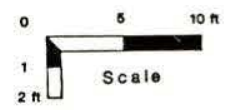

PROFILE B

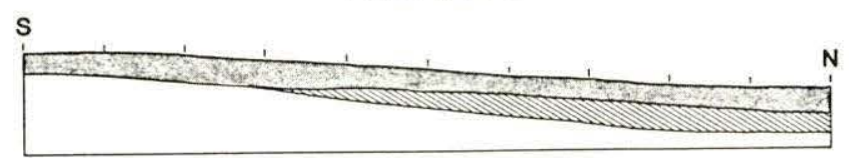

E. Plowzone

Midden

- Humus

- Subsoil

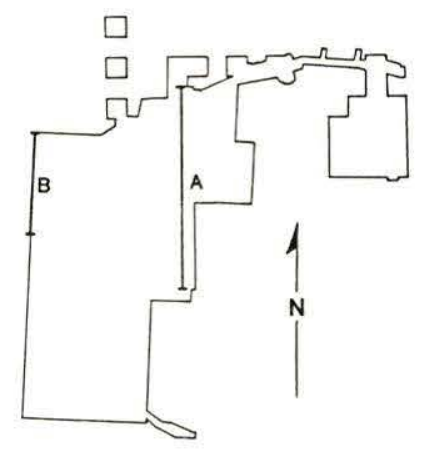

Figure 3.5

Site stratigraphy at the Wall site, N-S sections.

downward and away from the village. Material accumulated in these low areas to a thickness that allowed for their preservation beneath the deepest penetration of the plow. Zone II ranges from $0.0 \mathrm{ft}$ to $1.25 \mathrm{ft}$ thick and is a dark brown loam rich in cultural and organic material. This midden contains numerous potsherds, chipped- and ground-stone tools and debris, large quantities of animal bone and charred plant remains, daub, fire-cracked rock, shell, and wood charcoal. The artifacts usually are in larger pieces and are less weathered than those of Zone I. This rich cultural deposit provides a diverse assemblage of materials that has been analyzed for information concerning faunal exploitation (Holm, this report), plant exploitation (Gremillion, this report), shell utilization (Hammett, this report), ceramic tradition and technology (Davis, this report), and lithic technology (Tippitt and Daniel, this report). Zone II was excavated in two levels. The uppermost level (Level 1) was darker and appeared to have a higher organic content than Level 2. The relatively large amounts of cultural debris recovered from Level 2 suggest that this level, although much lighter in color, is not the original (pre-village) surface but is a deposit overlying and grading into the original surface.

Zone III is a light tan sandy clay loam which grades from the overlying midden zone to the underlying subsoil. This soil layer is interpreted to be a relict of the original humus on which the midden accumulated. The upper portion of this humus probably was disturbed aboriginally and is contained in the lower portion of Zone II. One feature (Feature 5-84) was observed within Zone III and has been interpreted as hearth materials redeposited adjacent to the village prior to the accumulation of midden in that area.

Underlying Zone III, and Zone I where the midden is not present, is Zone IV. This is a yellowish-brown sandy clay subsoil of undetermined depth. Cultural remains from within this zone constitute various intrusive features excavated by the aboriginal inhabitants. These features consist of postholes, burial pits, and shallow and deep pits used in food preparation, heating, clay procurement, and storage or caching. These features are evident at the top of Zone IV as stains that contrast with the subsoil in both color and texture.

\section{Midden}

The midden at the Wall site is preserved as a continuous deposit rich in organic material and artifacts in the vicinity of the various palisades (Figures 3.4 and 3.6). It is present inside Palisade I and extends well beyond Palisades II and IV. Its presence inside the earliest palisade suggests that the extent of the midden may have been much greater before recent plowing truncated it. The thickest part of the preserved midden is in the vicinity of Palisade IV. The deposit becomes much thinner at distances greater than $20 \mathrm{ft}$ from this palisade. Profile drawings (Figure 3.5) show that the original land surface was much lower than it is today in the area where the midden is preserved. Thus, the original boundaries of the village may have conformed to the higher area between the river and this depressed area. Refuse was deposited along the outer edge of the village and eventually built up this low area. 


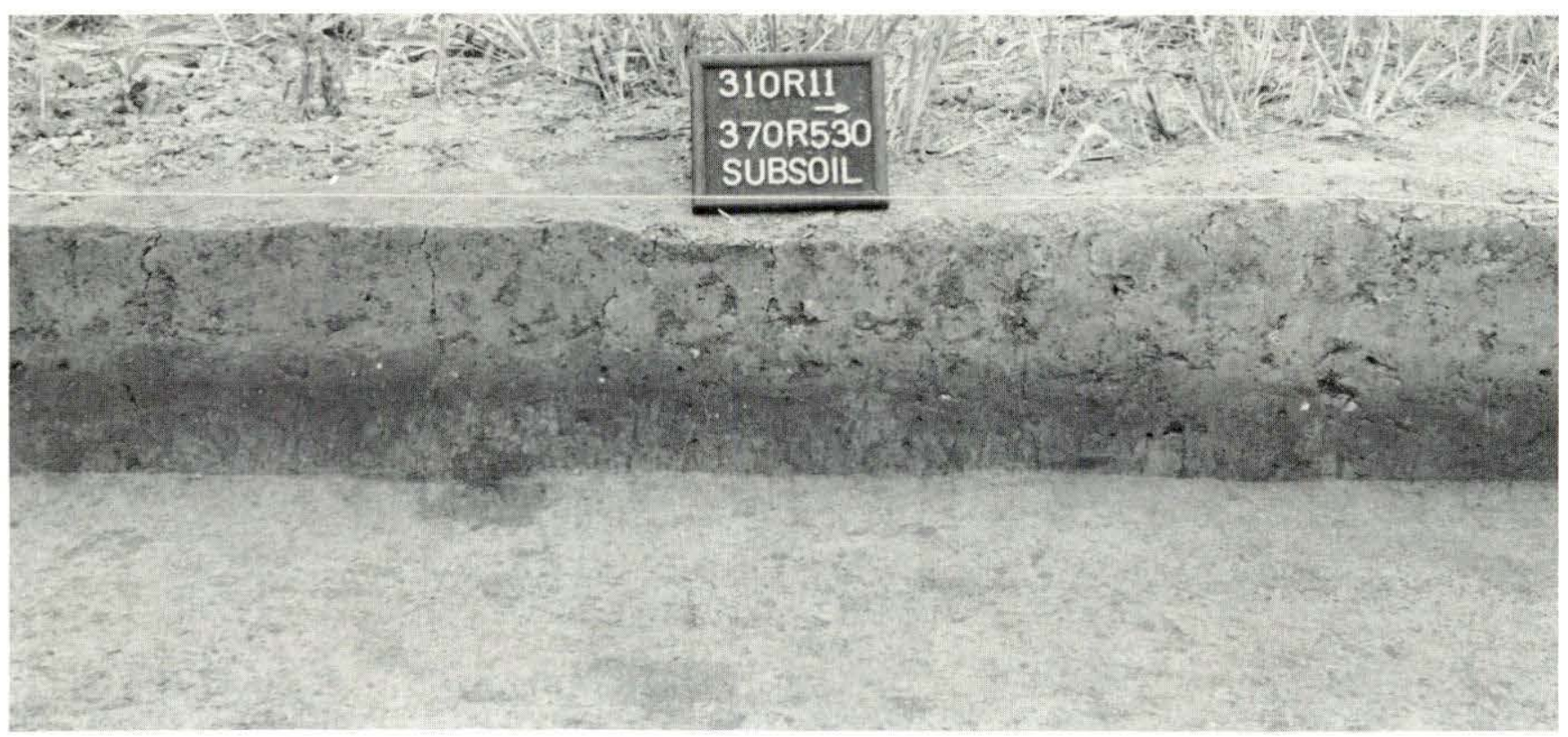

Figure 3.6

Sq. 370 R530 at the Wall site showing midden preserved beneath plowzone.

This process created additional area onto which the village expanded.

The full extent to which the midden originally accumulated is not known. Postoccupational processes of plowing and erosion have disturbed the upper portions of this deposit and obliterated it in areas where it was only a thin sheet. A second problem in defining the extent of the midden results from the data recovery and record-keeping methods of the 1940-41 investigations. The materials recovered from these excavations were recorded either as coming from "plowed soil" or from "undisturbed soil." The distribution of units with material recovered from undisturbed soil suggests that most of this is midden along the palisades. Several excavation units in the central part of the site in the vicinity of Structure $J$, however, also had undisturbed soil below the plowzone. If this undisturbed soil is village midden, it must have accumulated after the abandonment of Structure $\mathrm{J}$ because these deposits overlay three features and a burial that are associated with the structure.

\section{Features}

Thus far, 73 features have been found at the Wall site. (Features identified in 1938 were assigned numbers I-XIII, and those excavated in 1940-41 were assigned numbers 1-54. When work was resumed in 1983, features were assigned consecutive numbers beginning with 1-84. Because these feature designations are embedded in the excavation records of this site, original numbers are used in this discussion.) Thirteen of these were identified during the 1938 excavations. Of these 13, al1 but two appear to be postholes. Features XII and XIII have their locations and plan form known only and will not be discussed further.

Fifty-four features were identified during the 1940-41 excavations. Of these, al1 but 13 probably represent large postholes. One is a small wall trench structure (Structure F).

The 1983-84 excavations revealed five additional features, bringing the total number of features (excluding postholes and structures) to 17. Locations of these features are shown in Figure 3.2, and size attributes of pits and basins are summarized in Table 3.1 .

Features 32, 49, 50, and 52 were small and medium-sized shallow basins (Figure 3.7). These features were oval to circular in plan and ranged from $2.5 \mathrm{ft}$ to $5.0 \mathrm{ft}$ in diameter. Three were located within structures. Feature 32 was located within Structure B and Feature 49 was located within Structure E. Feature 50 was located within the area where Structures E and I overlap, and was probably associated with Structure I. As only a sketch was made of the profile of Feature 50, its exact depth is not known, but it does appear to have been a shallow basin. Feature 52 was located in the northeastern part of the site and was intruded by Palisade IV. This was the largest of the four features in this group.

Although it is possible that these features represent soil recovery facilities (borrow pits), their context suggests that most were basins used in tasks which took place inside of houses, perhaps related to food processing. Contents were removed in single units and no fine-screening or flotation was used to recover small materials such as carbonized plant 


\begin{tabular}{|c|c|c|c|c|c|c|c|}
\hline Feature & $\begin{array}{l}\text { Length } \\
(\mathrm{ft})\end{array}$ & $\begin{array}{l}\text { Width } \\
\text { (ft) }\end{array}$ & $\begin{array}{l}\text { Depth } \\
(\mathrm{ft})\end{array}$ & $\begin{array}{l}\text { Estimated } \\
\text { Original } \\
\text { Depth ( } f t)\end{array}$ & $\begin{array}{c}\text { Depth/ } \\
\text { Diameter }\end{array}$ & $\begin{array}{c}\text { Estimated } \\
\text { Volume } \\
\left(\mathrm{ft}^{3}\right)\end{array}$ & Feature Form \\
\hline 32 & 3.70 & 2.50 & 0.40 & 1.15 & 0.28 & 13 & Shallow Basin \\
\hline 49 & 3.80 & 3.15 & 0.35 & 0.45 & 0.13 & 4 & Shallow Basin \\
\hline 50 & 4.25 & 3.10 & - & - & - & - & Shallow Basin \\
\hline 52 & 5.00 & 4.25 & 0.60 & 0.60 & 0.13 & 8 & Shallow Basin \\
\hline 51 & 13.00 & 7.90 & 1.00 & 1.00 & 0.10 & 47 & Large Shallow Basin \\
\hline 54 & 7.30 & $6 \cdot 30$ & 0.80 & 0.80 & 0.12 & 24 & Large Shallow Basin \\
\hline 47 & 9.30 & 8.20 & 1.10 & 1.10 & 0.13 & 44 & Large Shallow Basin \\
\hline 23 & 2.00 & 1.80 & 2.30 & 3.05 & 1.52 & 10 & Deep Pit \\
\hline $2-84$ & 2.60 & 1.75 & 1.45 & 2.20 & 0.98 & 7 & Deep Pit \\
\hline $3-84$ & 2.95 & 2.63 & 1.45 & 2.20 & 0.88 & 9 & Deep Pit \\
\hline 45 & 9.70 & 3.20 & 0.60 & 0.60 & - & 10 & Amorphous Basin/Depression \\
\hline 46 & 11.10 & 3.80 & 0.85 & 0.85 & - & 11 & Amorphous Basin/Depression \\
\hline 13 & 1.00 & 0.80 & - & - & - & - & Cob-Filled Pit/Basin \\
\hline 14 & 1.80 & 0.75 & 0.35 & 1.10 & - & - & Cob-Filled Pit/Basin \\
\hline
\end{tabular}

remains. The field records do not describe the fill qualities, and it is not possible to ascertain whether the fill in these features represents in situ deposits from food preparation, or secondary refuse. Their relatively small size and their contexts within domestic structures suggest that they functioned primarily in household activities.

Features 47,51 , and 54 were large shallow basins (Figure 3.8). They were oval to circular in plan and ranged in size from about six feet in diameter for the circular basins to about thirteen by eight feet for the oval forms. Depths of these features ranged from $0.80 \mathrm{ft}$ to $1.10 \mathrm{ft}$ below subsoil.

Two of these features were located adjacent to one of the outermost palisade lines in the northeastern area of the site. Feature 47 was located in the central area of Structure J. The contents recovered from these features consisted of a wide variety of cultural materials, including several items that were poorly represented in the midden. Bone tools, such as awls and beamers, were present. Larger quanti- ties of she11 tools, such as scrapers, and unaltered fresh water she11, probably representing food remains, also were present. Whether the contents of the features represent in situ deposits or secondary deposits is not known given the limitations of the data. However, differences in contents of these features and the midden do suggest that they resulted from different behavior. The largest of the two features was rather irregular in form, and it is probable that this one was used originally as a borrow pit for clay and subsequently as a refuse container. An alternative interpretation is that its irregular form resulted from the overlap of several more regularly shaped basins that were constructed in the same location at different times.

Two large amorphous basins, Features 45 and 46, were located within Structure $J$ and conformed to its inside perimeter. They were irregular in both plan and profile (Figure 3.9) and could represent either soil recovery facilities or depressed and erosional surfaces associated with activity inside the structure. The absence of such worn surfaces inside other 


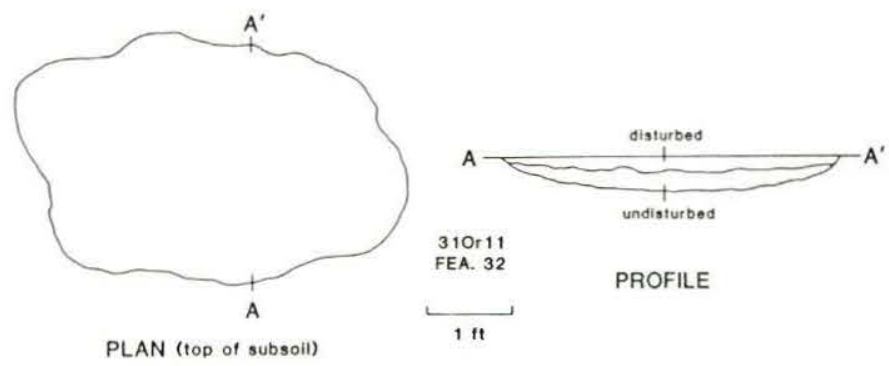

Figure 3.7

Plan and profile of Feature 32, a small shallow basin, at the Wall site.

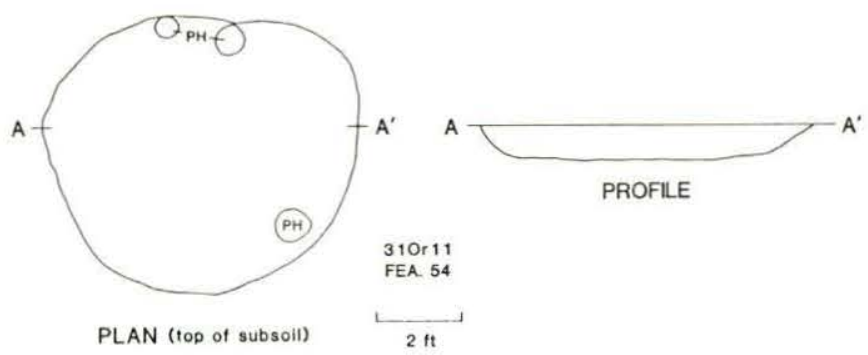

Figure 3.8

Plan and profile of Feature 54, a large shallow basin, at the Wall site.

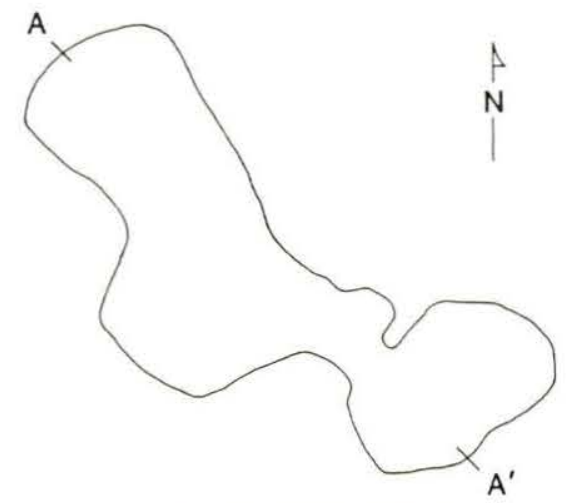

PLAN (top of subsoil)

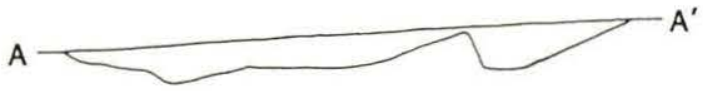

310 r 11

PROFILE

FEA. 45

$1 \mathrm{ft}$

Figure 3.9

Plan and profile of Feature 45, a large, amorphous shallow basin located within Structure J, at the Wall site. structures at the Wall site argues against the latter interpretation. The features, therefore, can best be interpreted as areas where clay was obtained to be used in the maintenance of the structures in this vicinity.

Two small pit/basins filled with charred corncobs were excavated during the 1940-41 seasons. Both of these features were located near Structures A and C. Feature 13 was within the area enclosed by Palisade II, and Feature 14 was located between Palisade II and Structure C. The fill characteristics of these features indicate that they were small facilities in which corncobs were used as fuel. Their size and shape, as well as their contents and context, suggest that they were used as hide smoking facilities (Binford 1967).

Feature 23 (Figure 3.10) was a small deep be11-shaped pit located adjacent to a possible southwestern entrance for Palisade II. Very few cultural materials were recovered from this pit, and it does not appear to be associated with any structures.

The five features discovered during the 1983-84 excavations were entirely different from the previously described features, and each warrants individual description.

Feature 1-84 was a large shallow amorphous depression that overlay the southern portions of Structures $G$ and $H$. It measured $20.3 \mathrm{ft}$ in length and $3.75 \mathrm{ft}$ in width and had a maximum

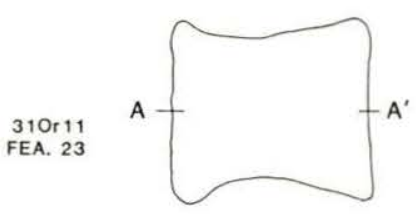

PLAN (top of subsoil)

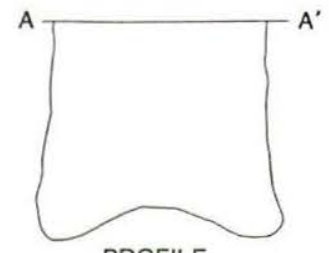

PROFILE

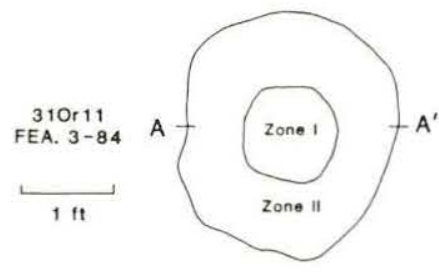

PLAN (top of subsoil)

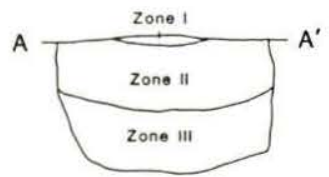

PROFILE

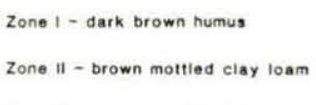

Zone III - orange mottled clay

Figure 3.10

Plan and profiles of Features 23 and 3-84, small deep pits, at the Wall site. 
depth of $0.15 \mathrm{ft}$ below subsoil. This feature consisted of a laminated and lensed matrix of gray sandy soils. Although forty-six sma11 sherds were recovered from this fill, all but two were too small to analyze. No other artifacts were recovered, and only a very small amount of charred corn was recovered through flotation. The feature overlay Structures G and $\mathrm{H}$, as well as a portion of Feature 4-84. The amorphous form, shallow depth, and its orientation across two overlapping structures suggest that it is not associated with either of the structures. The low artifact density and their small size suggest that post-occupation processes were responsible for its formation. Numerous 18th- and 19th-century Euroamerican artifacts were recovered from the plowzone in this part of the site, an indication of intense Euroamerican activity in the vicinity.

Feature 2-84 was a semicircular pit located in the area where Structures $G$ and H overlap. The pit was $2.60 \mathrm{ft}$ long, $1.75 \mathrm{ft}$ wide, and 1.45 ft deep below subsoil. The fill of Feature 2-84 consisted of two distinct zones. Zone I was a thin layer of mottled brown and orange clay loam that contained small amounts of hickory nutshe11 and acorn shell (recovered from 101 flotation sample). Zone I overlay Zone III (in situ subsoil along the southern third of the feature was mistakenly identified as Zone II) and was an orange-brown clay loam with brown mottling. This fill was very similar to relatively sterile zones of burial fill; no artifacts were recovered. The fill texture and contents suggest that this pit was only open for a brief time and refilled rapidly with mostly sterile clay subsoil.

Feature 3-84, an oval pit, was located south of Structure $G$. The pit was $2.60 \mathrm{ft}$ long, $2.63 \mathrm{ft}$ wide, $1.50 \mathrm{ft}$ deep below subsoil, and had a profile similar to Feature 2-84 (Figure $3.10)$. There were three distinct zones of fill in this pit. Zone $I$, a thin layer of dark brown humus and charcoal, was confined to the top center of the pit. Cultural remains from this zone consisted of one chipped-stone projectile point, one flake, and a few fragments of animal bone. Zone II, a brown mottled clay loam, underlay Zone I and was approximately $0.75 \mathrm{ft}$ in thickness. Cultural remains from this zone included animal bone, charcoal, a chipped-stone projectile point, and one potsherd. Zone III was an orange mottled clay loam, $0.75 \mathrm{ft}$ thick and sterile. The concave bottom of the pit, the shape of Zone II, and the fact that Zone I was confined to the extreme central area of the pit, imply that Zone $I$ is a slump of midden that probably was characteristic of the village surface in this area. Zone II seems to represent secondary deposits of household debris associated with food preparation and consumption. Both wood charcoal and charred plant remains were abundant (Table 3.2), and animal bone was present.

Feature 4-84 (Figure 3.11) consisted of two
Table 3.2

Nonbotanical remains, wood charcoal, and plant food remains from the 1983-1984 excavations at the Wall site (grams per 10 11ter flotation sample).

\begin{tabular}{lcccr}
\hline & $\begin{array}{c}\text { Nonbotanical } \\
\text { Remains }\end{array}$ & $\begin{array}{c}\text { Wood } \\
\text { Charcoal }\end{array}$ & $\begin{array}{c}\text { Plant Food } \\
\text { Remains }\end{array}$ & Total \\
\hline Fea. 1-84, Zone I & 31.81 & 0.03 & 0.08 & 31.92 \\
Fea. 2-84, Zone I & 18.94 & 0.10 & 0.06 & 19.10 \\
Fea. 3-84, Zone II & 46.92 & 15.08 & 0.45 & 62.45 \\
Fea. 4-84, Trench 1, Zone I & 77.46 & 1.98 & 0.03 & 79.47 \\
Fea. 4-84, Trench 2, Zone I & 36.99 & 0.71 & 0.11 & 37.81 \\
Fea. 4-84, Trench 2, Zone II & 120.22 & 0.77 & 0.01 & 121.00 \\
Fea. 5-84, Zone I & 121.18 & 0.95 & 0.28 & 122.41 \\
\hline
\end{tabular}

elongated and irregularly-shaped trenches 1ocated immediately southeast of Structure $G$ and south of Palisade I. The shape, orientation, and location of these trenches indicate that they may have been part of an entrance to Structure G. The feature was overlain by gray sandy deposits of Feature 1-84 along the northern part of both sections. The western trench was intruded by three postholes. Both

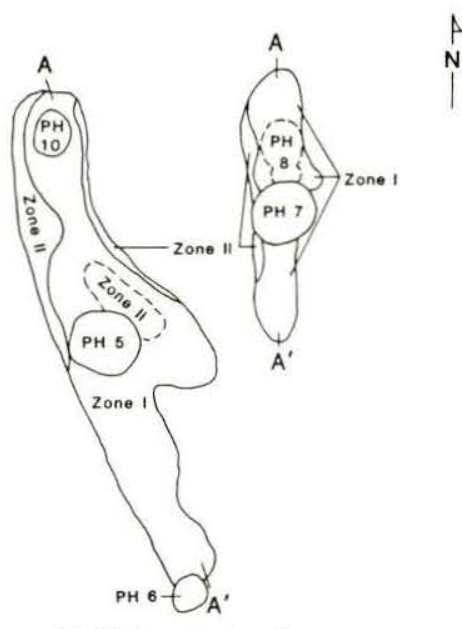

PLAN (top of subsoil)

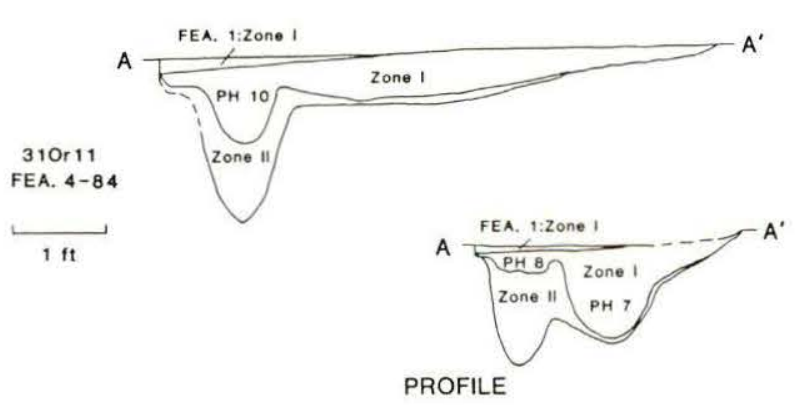

Zone 1 - medium brown loam

Zone II - mottied yellow sand

Figure 3.11

Plan and profile of Feature 4-84, an irregular trench-like feature associated with

Structure G, at the Wall site. 
trenches are comprised of two zones of fill. Zone I was a medium brown loam, both hickory nutshell and acorn remains were recovered in small quantities from this zone. Zone II underlay Zone I in all but the southernmost part of the trenches. This zone was thickest in areas where the posts appeared to intrude the feature. The shape of Zone II and the trench floor conform to the shape of the posts and are not intruded by them. This suggests that the posts and trench are associated.

Feature $5-84$ is a secondary deposit of hearth contents identified near the bottom of the midden in Excavation Unit 360R530, outside of Palisade IV. The deposit was $1.7 \mathrm{ft}$ in diameter and was $0.35 \mathrm{ft}$ thick. It did not extend into the subsoil and was confined to the lower portion of the midden (Zone II) and the old humus (Zone III). This feature consisted of fired clay, ash, and charcoal. No artifacts or fire-cracked rock were associated with it.

Aside from postholes, pits and basins are the most common features represented at the Wa11 site. The absence of hearths, which certainly were present when the site was occupied, is assumed to be a consequence of post-occupation processes of plowing.

Subsurface features may have their form changed as the facilities are transferred from one systemic context to another by processes of recycling. They need not necessarily change their function for their form to change, as this may be altered by processes of maintenance or rejuvenation (Schroed1 1980:17). Additionally, it is important to keep in mind that a subsurface facility may be abandoned at any point in its use-life. It is at this point that a facility may once again be transferred from one systemic context to another (e.g., a storage pit may become a container for refuse). It is only after a facility is ultimately abandoned that it enters the archaeological context (Schiffer 1972, 1976).

The production of a subsurface facility creates two end-products. First, there is the soil removed during its creation, and secondly, there is the space created (i.e., the facility itself). Either or both of these products may be the desired goal of the excavation of a pit or basin. If soil recovery is the sole desired goal, then the empty space created by its removal is an incidental by-product. If the desired goal is the space created, then the excavated soil is the incidental by-product. It is likely that these goals are often simultaneous, so that both the soil and the space are the desired end-products.

Because processes of recycling, maintenance, and rejuvenation intervene between the time that a facility is initially created and the time that it enters the archaeological record, it is not possible to trace these processes for individual features. However, because a facility may enter the archaeological record at any point in its use-life, the variation in feature form, size, and context observed archaeologically should reflect at least some of the various stages that a facility goes through before being abandoned (Schroedl 1980:20). A feature's size and form at abandonment may provide some insight into how it functioned prior to abandonment.

The attributes of feature fill sometimes contain important information about how a feature functioned, but only if the deposits resulted directly from behavior related to the feature. Even if the deposits are secondary refuse, the fill characteristics can be useful in making inferences about the behavior that created the fill prior to its secondary deposition in pits and basins (Wilson 1977, 1985). The fill also can provide important information about the season in which a pit was abandoned, which may be useful in making inferences about the function of the facility (Dickens 1985) and about aspects of subsistence behavior (see Gremillion, this report).

Interpretations of fill classes from the features at the Wall site are limited to Features 2-84 and 3-84, which seem to have been subsurface storage facilities. Interpretation of Features 1-84, 4-84, and 5-84 has been offered earlier.

Zone I of both Features 2-84 and 3-84 seems to be midden that slumped into these pits when they settled. Both zones contain a variety of cultural remains, including stone flakes, animal bones, charcoal, and other debris which accumulated on the village surface. Very little plant food remains or wood charcoal were present in these zones of fill. Zone III of Feature 2-84 was almost sterile of cultural material and seems to represent an episode of very rapid filling with subsoil, perhaps even with the soil that was originally excavated from this pit. This suggests that if Feature 2-84 functioned as a storage facility, it may have been a very temporary one that was quickly filled in.

Zone II of Feature 3-84 appears to have been a secondary deposit of refuse. The large amount of wood charcoal, plant food remains, and nonbotanical debris observed in the flotation sample (Table 3.2), along with a few small lithic tools, imply that this fill resulted from house and hearth cleaning of one of the nearby structures.

\section{Primary Functions of the Wall Site Features}

Schroedl (1980) has suggested that features whose primary function was storage and those whose primary function was soil recovery should be distinguishable by attributes of form and size. Specifically, storage facilities should be deep relative to their diameter, and large if they were to store large amounts of materials. Soil recovery facilities, on the other hand, 
should be shallow relative to their diameter. The variability in volume of these shallow facilities should be a measure of the extent to which they were used. This is not to say that functions of features can be determined solely by analyzing their size and shape. Context also must be taken into consideration in order to arrive at meaningful interpretations (cf. Dickens 1985; Ward 1985; Wilson 1985).

Table 3.1 summarizes the size attributes of the features excavated at the Wall site. The depth/diameter ratios and volumes include estimates of the portions of the features that were truncated by plowing. Where features were present beneath intact midden, the observed dimensions of the features are used for determining volumes and depth/diameter ratios. Volumes are rounded off to the nearest cubic foot. The depth/diameter ratios for the pit and basin-shaped features at the Wall site range frgm 0.10 to 1.52. Volumes range from 4 to 47 $\mathrm{ft}^{3}$. These features can be divided into four classes.

Feature Class $1 \quad(n=4)$ is represented by Features 32, 49, 50, and 52. These are small-to-medium oval-to-circular shallow basins (Figure 3.7). This feature class has a depth/ diameter range of 0.13 to 0.28 and volumes between 4 and $13 \mathrm{ft}^{3}$.

Feature Class $2 \quad(n=3)$ is represented by Features 51, 54, and 47. These features have a depth/diameter range of 0.10 to 0.13 and volumes between 24 and $47 \mathrm{ft}^{3}$. They are large shallow basins, with oval or circular plans (Figure $3.8)$.

Feature Class $3 \quad(n=3)$ is represented by Features 23, 2-84, and 3-84. They have depth/ diameter ratios between 0,88 and 1.52 and volumes between 7 and $10 \mathrm{ft}^{3}$. They are sma11 deep pits of two forms: two have straight to slightly insloping sides, and one has slightly undercut walls (Figure 3.10).

Feature Class $4 \quad(n=2)$ is represented by Features 45 and 46, large amorphous basin/ depressions (Figure 3.9). The irregular shapes of these features make depth/diameter ratios difficult to determine. If [max. length $+\max$. width]/2 is used as an estimate of diameter, these two features would have depth/diameter ratios of 0.09 and 0,11 , respectively, and volumes of 10 and $11 \mathrm{ft}^{3}$. Their depth/diameter ratio falls within the range of Feature Class 2 and their volumes fall within the range of Feature Class 1.

Three of the four features in Feature Class 1 are located inside structures and the fourth is located adjacent to Palisade IV. Although these features may represent small soil recovery facilities, perhaps related to individual structure maintenance, their relationships with the insides of structures suggest that their primary function was probably related to food processing or other domestic tasks.

Two of the Class 2 features, excavated in 1940-41, are located along the palisade in the northern part of the site. The third is associated with Structure J. The primary function of these features may have been as large scale soil recovery facilities, an assumption which is based on the fact that their fill represents secondary refuse. Feature 51 probably represents a soil recovery facility related to maintenance of the palisades in that vicinity. Such large, shallow basins associated with palisade maintenance have been observed at the Warren Wilson site in western North Carolina (Dickens 1985; Ward 1985). Features 47 and 52 are much more regular in shape and may represent large-scale food processing facilities, such as the large "earth ovens" at the site of Upper Saratown (Wilson 1977, 1985).

Two of the three features in Class 3 were excavated during the 1983-84 investigations and are located within and adjacent to structures. These two features are interpreted as small storage facilities. The volume of these features is small, and it is unlikely that they were used for bulk storage of food. Instead, they may represent caching facilities used to conceal items of value during short trips away from the village (Ward 1985). The third feature of this class is a small bell-shaped pit located near a possible entrance to Palisade II in the southwestern part of the site.

In some ways the presence of just a few storage facilities is enigmatic. The general absence of such facilities may indicate that most of the food storage was carried out above ground in facilities such as granaries or cribs. Such structures are known for the area from descriptions by early European travelers (e.g., Cumming 1958; Lefler 1967).

\section{Structures}

Structures built at the Wall site are defined by patterns of postholes that can be observed at the top of subsoil (in areas of midden, the patterns are less clear). Figure 3. 3 shows the distribution of the recognized houses and the several palisade constructions. Although some patterns are better defined than others, it appears that there were 12 structures in this area. Two of them (Structures $B$ and $F$ ) are small open-ended buildings that probably were not habitations. The remaining 10 structures show considerable variability in size and amounts of rebuilding and/or maintenance. Descriptions of each structure are presented below and are followed by Table 3.3 which summarizes the attributes of the structures as a group.

Structure A was defined by a three-post wide, circular pattern of postholes with an entrance on the south side. The structure was $25.5 \mathrm{ft}$ in diameter on the outside and $21.5 \mathrm{ft}$ in diameter on the inside. The mean exterior 
Table 3.3

Summary of structure attribute measurements at the wall site.

\begin{tabular}{|c|c|c|c|c|c|c|c|c|c|}
\hline Structure & $\begin{array}{l}\text { Outside } \\
\text { Diameter } \\
\text { (ft) }\end{array}$ & $\begin{array}{c}\text { Inside } \\
\text { Diameter } \\
(\mathrm{ft})\end{array}$ & $\begin{array}{c}\text { Exteri } \\
\overline{\mathrm{X}}\end{array}$ & $\begin{array}{l}\text { or Post } \\
\text { (ft) } \\
\text { sd }\end{array}$ & $\begin{array}{c}\text { Size } \\
\mathrm{n}\end{array}$ & $\begin{array}{l}\text { Inter } \\
\overline{\mathrm{x}}\end{array}$ & $\begin{array}{l}\text { or Post } \\
(f t) \\
\text { sd }\end{array}$ & $\begin{array}{l}\text { Size } \\
n\end{array}$ & $\begin{array}{c}\text { Floor Area } \\
\left(\mathrm{ft}^{2}\right)\end{array}$ \\
\hline A & 22.5 & 21.5 & 0.54 & 0.12 & 187 & 0.57 & 0.17 & 60 & 363 \\
\hline B & $13.5 \times 16.0$ & $12.5 \times 13.0$ & - & - & - & - & - & - & 163 \\
\hline c & 22.0 & 18.5 & 0.53 & 0.12 & 87 & 0.57 & 0.12 & 58 & 269 \\
\hline D & 31.0 & 23.5 & 0.56 & 0.13 & 240 & 0.58 & 0.14 & 118 & 434 \\
\hline E & 28.5 & 25.0 & 0.49 & 0.16 & 142 & 0.46 & 0.13 & 51 & 491 \\
\hline F & $11.5 \times 12.0$ & $9.5 \times 10.5$ & - & - & - & - & - & - & 100 \\
\hline G & 23.0 & 22.0 & 0.52 & 0.07 & 79 & 0.56 & 0.13 & 46 & 380 \\
\hline $\mathrm{H}$ & 22.0 & 21.0 & 0.52 & 0.15 & 58 & 0.56 & 0.17 & 45 & 346 \\
\hline I & 26.0 & 23.0 & 0.59 & 0.18 & 38 & 0.65 & 0.16 & 20 & 415 \\
\hline $\mathrm{J}$ & 24.5 & 20.0 & 0.60 & 0.13 & 57 & 0.69 & 0.18 & 27 & 314 \\
\hline K & 24.5 & 22.0 & - & - & - & - & - & - & 380 \\
\hline L & 22.5 & 19.0 & - & - & - & - & - & - & 284 \\
\hline
\end{tabular}

post size was $0.54 \mathrm{ft}(n=187, s d=0.12)$. There were numerous interior postholes distributed around the inside perimeter of the structure. The interior postholes show more variation in size than the exterior postholes, probably because of the different functions of the interior posts. Postholes larger than the exterior postholes probably represent support posts for the roof. The smaller postholes probably represent built-in furnishings, such as the sleeping platforms so often described in early historic accounts of the Eastern North American Indians (e.g., Tyler 1907; Lefler 1967; Alexander 1972; Sturtevant 1975). Structure A was located adjacent to and inside of Palisade II in the western part of the site. It was also close to Structures $B$ and $C$.

Structure B was defined by an irregular oval-to-square pattern of postholes inside of Palisade II and immediately southeast of Structure A. This structure was approximately $16.0 \mathrm{ft}$ by $13.5 \mathrm{ft}$ outside and $13.0 \mathrm{ft}$ by 12.5 $\mathrm{ft}$ inside. Feature 32 was located in the approximate center of this structure.

Structure C was defined by a two-post wide, circular pattern of postholes to the north of and close to Structure A. Burial 2 was located within this structure. Structure $C$ had an outside diameter of $22.0 \mathrm{ft}$ and an inside diameter of $18.5 \mathrm{ft}$. The mean exterior posthole size was $0.53 \mathrm{ft} \quad(n=87, s d=0.12)$. The mean interior posthole size was $0.57 \mathrm{ft} \quad(\mathrm{n}=58, \mathrm{sd}=$ 0.12). The structure appeared to abut Palisade
II on its northwest side and to overlap part of Palisade I with one of its exterior postholes intruding into a posthole of this palisade.

Structure $D$ was defined by a five-post wide, circular posthole pattern located to the east of Structure $A$ and $C$ and within the area enclosed by Palisade I. The mean posthole diameter was $0.56 \mathrm{ft}(n=240, s d=0.13)$. The interior postholes were mostly clustered around the inside perimeter of the structure with another cluster of postholes near the center of the structure. The mean interior posthole size was $0.58 \mathrm{ft}$ in diameter $(n=118, \quad \mathrm{sd}=0.14)$. Structure $J$ was located immediately to the north.

Structures E and I were overlapping structures located in the north-central part of the site. Structure E was defined by a two-post wide, circular pattern of postholes with an outside diameter of $28.5 \mathrm{ft}$ and an inside diameter of $25.0 \mathrm{ft}$. Structure I was only partly exposed by the excavations. It appears to have been a two-post wide circular structure with an estimated diameter of $24.5 \mathrm{ft}$ outside and $23.0 \mathrm{ft}$ inside. Burial 1, Feature XII, and Feature 49 were located within Structure E. These structures overlap and it was not possible to determine, without some question, which of the structures was constructed earlier. Feature 50 was located within the area where the structures overlap and the relative absence of postholes intrusive into this feature suggests that it was associated with the later of the two 
structures. The presence of a similar type of feature (Feature 49) clearly inside Structure E may suggest that Feature 50 was associated with Structure I. This, in turn, may suggest that Structure E predated Structure I. The mean exterior posthole diameter for Structure $\mathrm{E}$ was $0.49 \mathrm{ft}(\mathrm{n}=142, \mathrm{sd}=0.16)$. The mean interior posthole diameter (not including those in the area of structure overlap) was $0.46 \mathrm{ft} \quad(n=51$, $s d=0.13$ ). The western portion of this structure was excavated in 1938 and the eastern portion excavated during 1940-41. All of the interior postholes in the area where Structures $E$ and I do not overlap were excavated in 1938. In general, the postholes observed in 1940-41 were larger than those observed and recorded in 1938 . In using data from 1938 and 1940-41 to determine exterior post size, and using only data from the 1938 excavations to determine interior post size, problems of comparability have been introduced. The mean exterior post size is effected by the combination of two seasons of observation, while the interior post size is not. This resulted in the mistaken conclusion that the exterior posts were larger than the interior posts. If only the interior posts within the area excavated in $1940-41$ are considered, the mean interior post diameter is $0.69 \quad(n=49$, $s d=0.21)$. It appears, therefore, that the exterior posts were smaller than the interior posts, as with the other structures at the Wall site.

Structure $\mathrm{F}$ was defined by a U-shaped wall trench located to the northwest of Structure $E$. The structure was $12.0 \mathrm{ft}$ by $11.5 \mathrm{ft}$ outside and $10.5 \mathrm{ft}$ by $9.5 \mathrm{ft}$ inside, with the open portion on the southeast side. It was the only structure defined by a wall trench at the site. In one portion the wall trench intrudes into a posthole of Palisade I, suggesting that the structure postdated the earliest palisade. The function of this structure is not known. It is similar in size and form to Structure B and may represent an enclosed activity area associated with one of the structures in this vicinity. Alternatively, its proximity to an entrance in Palisade IV may mean that it was part of the defensive fortification of the village. Scaffold-like towers were described by John Smith for some of the palisades at Indian villages in tidewater Virginia during the early 17 th century (Tyler 1906:148-149).

Structures G and H (Figure 3.12), overlapping structures, were located in the eastern part of the site. These were the only two structures excavated during the 1983-84 investigations. Structure $G$ was defined by a one post-wide, circular pattern of postholes with an entrance to the south. This structure had an outside diameter of $23.0 \mathrm{ft}$ and an inside diameter of $21.0 \mathrm{ft}$. The mean interior post diameter was $0.52 \mathrm{ft} \quad(n=79, s d=0.07)$, and the mean interior post diameter was $0.56 \mathrm{ft} \quad(n=46$, $s d=0.13)$. The densest area of interior postholes was in the area where the two structures overlap. The arrangement of interior postholes suggests that there were interior roof supports, to which were attached other constructions requiring smaller posts.

Structure $\mathrm{H}$, located to the southwest of Structure G, was defined by a two post-wide, circular posthole pattern with an outside diameter of $22.0 \mathrm{ft}$ and an inside diameter of $21.0 \mathrm{ft}$. The northeastern third of this structure was intruded by Structure G. Structure H abutted Palisade I on its northeast side. The mean exterior posthole diameter was $0.52 \mathrm{ft}$ $(n=45, s d=0.17)$. Feature $2-84$ was located within the area where the structures overlap. The absence of intrusive postholes and the probability that Structure $G$ postdates Structure $H$ suggests that Feature $2-84$ is associated with Structure G.

Structure $J$ was defined by a discontinuous circular pattern of postholes north of and adjacent to Structure D. This structure was within the area enclosed by Palisade I. Features 45,46 , and 47 were located within the structure and conformed roughly to its interior perimeter and central area. Burial 4 was in the center of the structure and intruded into the northeastern part of Feature 47. The structure was $24.5 \mathrm{ft}$ in outside diameter and $20.0 \mathrm{ft}$ inside. The mean exterior posthole diameter was $0.60 \mathrm{ft} \quad(n=57, s d=0.13)$, and the mean interior posthole diameter was $0.69 \mathrm{ft} \quad(n=27, s d=0.18)$. The conformity of Features 45, 46, and 47 to structure walls and interior area suggests that these features were created while Structure J was still standing.

Structure $\mathrm{K}$ was defined by a discontinuous circular posthole pattern to the northwest of Structure $F$ and near the entrance to Palisade II/IV. This structure was only partly exposed. It is represented by two arcs of postholes, which, if joined, would produce a circular structure approximately $24.5 \mathrm{ft}$ in outside diameter and $22.0 \mathrm{ft}$ in inside diameter. This structure appeared to intrude across the entrance to Palisade II/IV but to fall within the area enclosed by suspected Palisade V.

Structure L was represented by a poorly defined pattern of postholes between Structures $C$ and $K$ and to the northwest of Structure $J$. Burial 5 was located within this structure, and Burial 3 was outside and to the southwest. This structure intrudes upon Palisades II and III in an area of the site where there are thick deposits of midden. The structure's poor definition may have come about because exterior posts did not extend into the subsoil below the midden. The pattern was better defined on its southern side where the midden deposits were thinner. This structure appears to have been $22.5 \mathrm{ft}$ in outside diameter and $19.0 \mathrm{ft}$ in inside diameter.

The structures at the Wall site, with the exception of Structure F, were all of singlepost construction. The exterior postholes for 


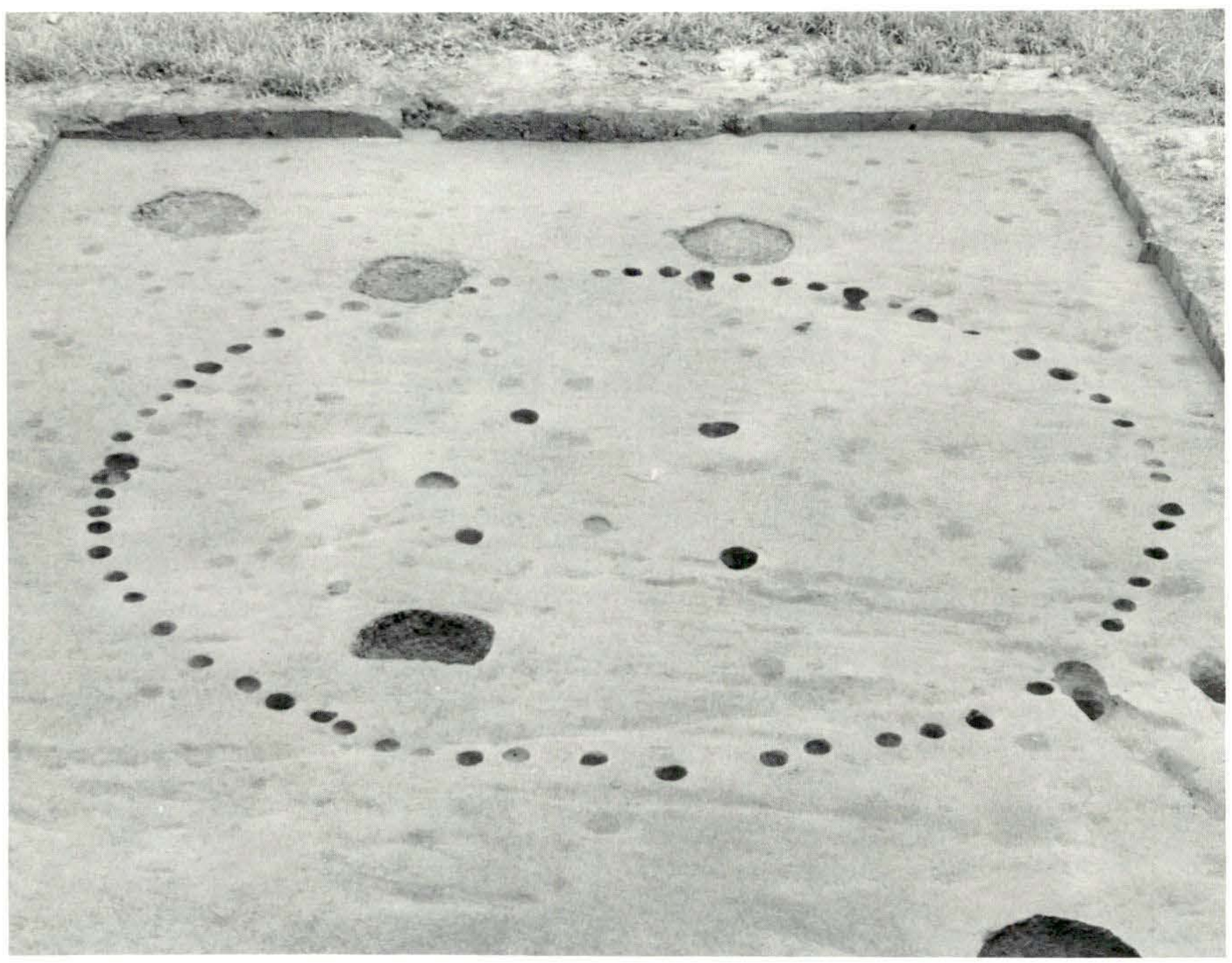

Figure 3.12

Structure G at the Wall site after excavation of postholes and pits.

all of these structures had a mean outside posthole diameter of $0.54 \mathrm{ft}(\mathrm{sd}=0.13 \mathrm{ft})$, and a mean interior posthole diameter of $0.58 \mathrm{ft}$ $(\mathrm{sd}=0.15 \mathrm{ft})$. The majority of the interior postholes were in the 0.4 to $0.6 \mathrm{ft}$ range, however, each structure also had a few interior postholes in the 0.8 to $1.0 \mathrm{ft}$ range. The large interior posts were spaced around the central area of the structures, while the small postholes were clustered between the larger postholes and the exterior walls. This suggests that the posts were of two different types: support posts for the roof, and posts used in the construction of built-in furnishings such as sleeping platforms. The centers of the structures were fairly devoid of postholes of both types, suggesting that a hearth was located in the center of each.

The use of large roof support posts implies that these structures were probably not of the domed "wigwam" form, which is so frequently described for Indians of this area during the Historic period (e.g., Wright 1947; Cumming 1958; Lefler 1967). Domed structures would not require the use of large central support posts because the roof is essentially self supporting. In a domed structure each exterior post or pole would be pulled over towards the center and lashed to posts or poles pulled over from the opposite side of the structure. The tension created by the opposing poles would be of an upward and outward direction. This would probably be sufficient to support a lightweight covering of hides or bark.

The fairly large exterior postholes $(>0.55$ $\mathrm{ft}$ ) suggest that the roofs and exterior walls of the Wall site structures were separate architectural features. The posts were probably set in the ground vertically and separate rafter elements erected between the outer wall and beams supported by the interior posts. This type of roof construction would not necessarily be self-supporting. The direction of force of the roof would be downward and outward. The roofs at the Wall site, therefore, probably were conical, with the overall structure being similar to the winter houses of the Cherokee (cf. Schroedl 1983). 
The type of material used as covering for the roofs at the Wall site is difficult to determine from the archaeological evidence. The roof covering may have been either grass thatch, hides, or bark. The historical evidence suggests that houses were usually covered with bark or hides (Hariot 1590; Tyler 1907; Wright 1947; Lefler 1967). However, these structures were usually described as having domed or arched roof constructions. The wall covering may have been bark or daub, although evidence for either is meager. Some daub was recovered from the plowzone during 1983-84 in the vicinity of Structures $G$ and $H$. The average amount of this material recovered from each excavation unit $\left(100 \mathrm{ft}^{3}\right)$ was only $15.30 \mathrm{~g}$ (range=0.00-39.90; $\mathrm{sd}=13.70)$. Although such small amounts of daub seem to deny the use of this material for construction, factors governing its preservation must be considered.

Daub is a mixture of clay and plant materials, the latter being added as a binder. After the mixture is applied and sun dries, it is subject to weathering by the natural processes of rain, freezing and thawing, and drying out. As a result of these processes, daub probably continually enters the archaeological context, but usually as small particles. Should a structure burn, daub is greatly hardened and its durability is increased, favoring its preservation. Post-depositional processes also would effect the preservation of daub. Trampling and cleaning up after a fire would transfer some of the material from its original depositional context in the vicinity of a structure to a secondary context elsewhere. Once it entered an archaeological context, natural processes would effect the daub, breaking it down into its constituent elements of clay and fiber. Cultural post-depositional processes, especially plowing would reduce the size of the particles even further. This would create greater surface area upon which both natural and cultural processes could act to further reduce the particles (Schiffer 1976:36).

Except in cases where daub entered the archaeological record in especially large quantities or was burned, it seems unlikely that much of it will be preserved for the archaeologist to recover. Nevertheless, small amounts of this material will probably be preserved, and its distribution, rather than its quantity, may be evidence for its use.

The distribution of daub in the plowzone of the eastern area excavations in the vicinity of Structures $\mathrm{G}$ and $\mathrm{H}$ is shown in Figure 3.13 . Highest relative density is in the southwestern and northeastern areas of the excavations. The southwestern concentration of daub corresponds to the location of Structures $G$ and $H$. The area showing the greatest density is near the western side of Structure $H$. Numerous postholes in this area contained charcoal. This may indicate that part of Structure $\mathrm{H}$ burned and modified the daub in a manner that contributed to its preserva-

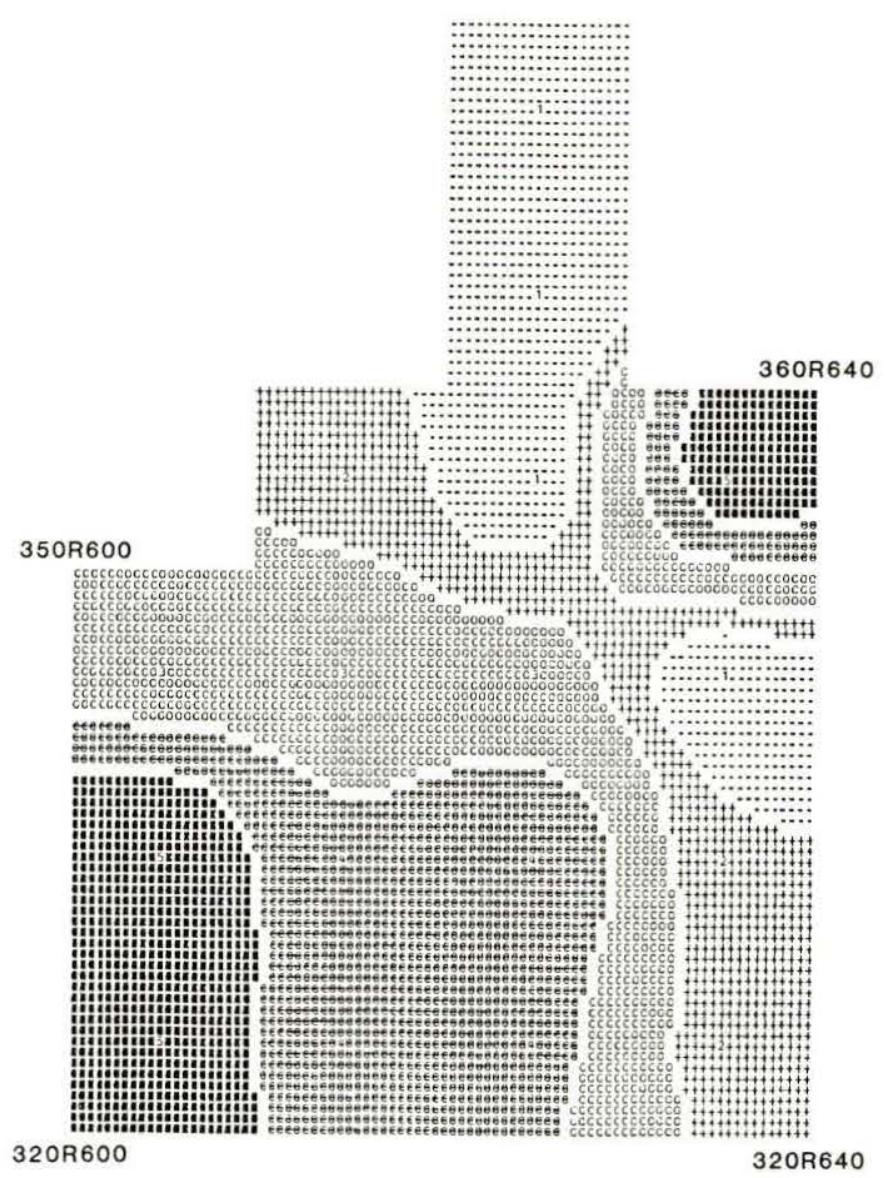

Figure 3.13

SYMAP of daub in the vicinity of Structures $\mathrm{G}$ and $\mathrm{H}$ at the Wall site.

tion. A smaller concentration of daub extending northeast toward the center of Structure G may be the remains of fire-hardened clay associated with a hearth in the center of that structure. Although no visible remains of a hearth were observed beneath the plowzone, the location of a daub concentration in this area is suggestive. The third daub concentration is in the northwestern portion of the excavations. This concentration seems to be associated with several postholes (containing charcoal) of Palisade II.

Although the amount of daub recovered from the Wall site excavations is rather small, its concentration in areas of architectural remains that show some evidence of having been burned, suggests that it may have been used as a covering for house walls and the palisades.

\section{Palisades}

Five separate palisades have been identified at the Wall site (Figure 3.3). These features are characterized by long continuous posthole patterns that enclose all or some of the identified structures. The palisade postholes are larger than those associated with structures and range from 0.60 to $1.25 \mathrm{ft}$ in 
diameter. They are fairly uniformly spaced, usually between 0.5 and $1.5 \mathrm{ft}$ apart.

Palisade $I$ was the innermost of the identified palisades. It enclosed the area occupied by Structures D, E, H, I, and J. Structure $\mathrm{F}$ also may have been just within this palisade. Ninety-two postholes associated with this palisade were observed in the western area of the excavation and an additional 28 postholes were observed in the eastern area excavated during 1983-84. The portion in the eastern area was intruded by Structure G, and a possible entrance through this palisade was observed in the vicinity of Structure J (Figure 3.3).

Palisade II was roughly parallel to and outside of Palisade I. It has been identified by 194 postholes, 180 of which are in the western area of the excavations, and 14 which are in the eastern area excavated in 1983-84. Palisade II was intruded by Structures C, K, and L.

Palisade III was defined by a linear pattern of postholes in the northwestern area of the excavations. This palisade appeared to cut through Palisade II and join with Palisade I in the vicinity of Structure F. Structure L intruded upon this palisade.

Palisade IV was parallel to and outside of Palisade III. This palisade was defined by a linear pattern of 67 closely-spaced postholes with a well-defined entrance where Palisade IV intersected and joined with Palisade II. Structure $\mathrm{K}$ intruded upon this palisade.

Palisade $V$ was located outside of Palisade IV. It was defined by a discontinuous linear posthole pattern observed in the extreme northwest corner of the 1940-41 excavations and in several of the 1983-1984 excavation units in the northern part of the site. This palisade appeared to join with Palisade II/IV in the vicinity of Features 50, 51, and 54 .

The five described palisades and their spatial arrangement suggest that the village represented at the wall site went through at least two expansions following the construction of the first two palisades. It is not possible to determine if the village was unpalisaded in its earliest form.

Although it is possible that each palisade represents an episode of village expansion, it is more likely that a double palisade type of fortification was used by the inhabitants of the Wall site and that Palisades I and II were constructed first and used simultaneously. The archaeological evidence also suggests that the expansion phases may not have been symmetrical. This is indicated by the fact that various palisades are differentially exhibited in the eastern and western areas of the site. Also, the later palisades attached to earlier ones, which means that the expansions were more lateral than concentric.

Palisades I and II were constructed first and established the general limits of the village along its axis perpendicular to the Eno River. Subsequent palisade building episodes expanded the village limits laterally, paralle 1 to the river. The first village expansion was accomplished by the erection of Palisades III and IV. These palisades joined with central sections of the pre-existing palisades in the vicinity of Structures $F$ and $K$. Palisade IV extended east from where it intersected with Palisade II. Palisade III apparently only extended to the west, as it was not observed in the eastern area of the excavations.

As Figures 3.1-3.2 show, the eastern and western areas of the excavation were joined by a trench excavated during the 1940-1941 investigations. This trench was excavated to trace the extent of Palisade IV to the east of the main excavation area. The 1983-84 excavation area coincidentally tied into the easternmost extension of the 1940-41 trench. The interpretation of the directions of Palisades I and II through the unexplored portion of the site is preliminary and subject to modification with additional data. Nevertheless, it now appears that Palisade III does not extend into the eastern part of the site. This poses a problem in interpreting the construction phases of the village. If, as suggested, the palisades were paired, then at least portions of Palisade II must have been standing when Palisade IV was constructed.

A general interpretation of the sequence of structure and palisade building is offered as follows:

Phase 1: Palisades I and II were constructed, enclosing the area occupied by Structures D, E, J, and possibly Structure F.

Phase 2: Structures A, B, C, and $\mathrm{H}$ were constructed. Structure I also may have been constructed at this time, to replace Structure E. This expansion phase was accomplished without the construction of additional palisades by using the space between Palisades I and II. Palisade I may have been taken down at this time, or sections of it may have been left standing if they did not interfere with activity areas or house construction. Structure H, which is the only structure to abut Palisade I, may have been constructed early in this phase and later replaced by Structure G.

Phase 3: Palisades III and IV were constructed. This resulted in lateral expansion of the village, at least to the west and southwest. Although no structures associated with this phase of construction were observed in the excavated area, there 
are several areas of high posthole density in the extreme southwestern area of the excavations.

Phase 4: Palisade V was constructed and portions of Palisades III and IV were dismantled. Structures $\mathrm{K}$ and $\mathrm{L}$ were subsequently constructed.

The variability in the number of concentric patterns of postholes defining the structures in the western area of the site is evidence for the proposition that the village was expanding. These data suggest that while the village was expanding, houses were being maintained and the population was probably growing. Abundant evidence of structure maintenance and the small number of overlapping structures suggest that all of the non-overlapping structures were standing and occupied during the maximum period of expansion reflected in the excavations.

The number of concentric posthole patterns that define the exterior walls of structures range from one (Structures $G$ and $L$ ) to five (Structure D). The ten structures were ranked according to the number of the concentric patterns they exhibited. The structures were also ranked according to their distance from Palisade IV. It is assumed here that the number of concentric patterns of postholes is a function of the amount of maintenance invested in the structure during the course of its use. It is further assumed that these features are related to the length of time that a structure was occupied or otherwise used. Distance to Palisade IV relates each structure to a common architectural feature that is observable across the entire site. If the village expanded through time then there should be a strong positive correlation between the distance of a structure to Palisade IV and the amount of maintenance exhibited for each structure. Table 3.4 presents the data on these variables. Spearman's rank-order correlation coefficient (Blalock 1960:434-436) was computed using these data to test the strength of the correlation. A value of $r=0.6788$ was obtained showing a strong positive correlation between the variables ( $p<.03$, one-tail test). The small sample size and the frequency of ties in the maintenance ranking suggest that these results should be accepted cautiously. However, the results tentatively support an interpretation of an expanding village and growing population.

\section{Spatial Organization of the Structures}

The relatively small sample of structures places limits on the extent to which the spatial organization of these structures might be understood in terms of the social life of the inhabitants of the Wall site. The social order and cultural development of all communities are probably expressed at least partially in spatial terms (Hall 1969; Clarke 1977; Hodder 1978). The problem comes in making valid inferences about sociocultural factors from the static
Table 3.4

Selected data related to structure malntenance and placement at the Wall site.

\begin{tabular}{|c|c|c|c|c|}
\hline Structure & $\begin{array}{l}\text { No. of Rebuilding } \\
\text { Episodes }\end{array}$ & $\begin{array}{l}\text { Ma1ntenance } \\
\text { Rank }\end{array}$ & $\begin{array}{c}\text { Distance to } \\
\text { Pallsade D }(\mathrm{ft})\end{array}$ & $\begin{array}{l}\text { D1stance } \\
\text { Rank }\end{array}$ \\
\hline A & 3 & 2 & 47 & 2 \\
\hline c & 2 & 4.5 & 27 & 5.5 \\
\hline D & $3-5$ & 1 & 51 & 1 \\
\hline E & $2-3$ & 3 & 22 & 8 \\
\hline G & 1 & 9.5 & 24 & 7 \\
\hline H & $1-2$ & 7 & 31 & 3.5 \\
\hline I & 2 & 4.5 & 31 & 3.5 \\
\hline $\mathrm{J}$ & $1-2$ & 7 & 27 & 5.5 \\
\hline$k$ & $1-2$ & 7 & -13 & 10 \\
\hline 1. & 1 & 9.5 & 8 & 9 \\
\hline
\end{tabular}

patterns of structures, palisades, burials, and other archaeological features.

It is assumed for the purpose of this discussion that social distance is reflected partly in the spatial organization of the archaeological record. The small size of the Wall site village, even at its point of maximum expansion, suggests that its inhabitants regularly and consistently associated with one another. Kin-based institutions such as clans and moieties probably provided a framework through which every individual understood his relationship to every other individual belonging to the village, and to the wider settlement system of which the Wall site village was only a part. One aspect of social organization that is visible in the archaeological record at the Wall site is the spatial configuration of the domestic structures.

The distance between structures in the western area of the excavations was examined. The limited excavations in the eastern area do not provide enough information to examine the spatial relationships of Structures $G$ and $H$ to other structures that certainly must be present in that area of the site. The minimum distance between adjacent circular structures is $2.0 \mathrm{ft}$ and the maximum distance is $8.5 \mathrm{ft}$. The spatial relationships between Structures A, B, C, D, and $J$ are examined in terms of their proximity to one another and their contemporaneity as defined in the proposed phases of village growth. The range of distance between adjacent structures in this group is $1.5 \mathrm{ft}$ to $13.5 \mathrm{ft}$. When structure size is considered, the distribution of the distances between adjacent structures reveals a distinct pattern. The closest spatial relationships are between Structures A and B, Structures $A$ and $C$, and between Structures D and $J$. The furthest distances are between Structures B and $D$, and between Structures C and J. Structures $A, B$, and $C$ are considered to be related because of their close proximity to one another and 
their spatial relationship to Palisade II. Structures D and $J$ are related through proximity to one another and to Palisade I.

Structures A, B, and C seem to represent a structure compound that was probably used by a single household. The central structure of these three, Structure A, is also the largest. The spatial relationship between Structures A and $\mathrm{C}$ is mirrored by the relationship between Structures D and $J$. It is probable that Structures A and C, and Structures D and J represent pairs, each pair being used either year-round by a separate family or as winter and summer houses by the same family. The differences in size of these structures (Table $3.3)$, coupled with their spatial relationships, gives somewhat greater weight to the latter interpretation.

Following this line of reasoning, Structures $\mathrm{L}$ and $\mathrm{K}$ may represent another structure pair. Structure $\mathrm{L}$ is the smaller of the two and is nearly equidistant from both Structures $C$ and J. Thus, while Structures A and C, D and J, and $\mathrm{K}$ and $\mathrm{L}$ seem to represent structure pairs, Structures C, L, and J form a group of three. These three structures create a somewhat symmetrical and approximately rectangular space between them. This space, bounded on its fourth side by Structure D (the largest and oldest structure of the six in this area), is interpreted as a small plaza/activity area shared by the inhabitants of these four structures.

Rapoport (1969) has proposed that houses are not simply shelters, but are a cultural phenomenon that reflects a particular social and cultural context. Furthermore, he suggests that houses cannot be viewed in isolation; they must be considered as parts of a larger whole. Houses, and the space created and defined by their interrelationships, undoubtedly facilitated the social interaction among their occupants.

With this in mind, it can be suggested that the structure pairs represent summer and winter houses. Paired structures like this are known ethnographically for much of the Southeast (Hudson 1976). Usually the winter houses were round and the summer houses square or rectangular; however, the Creek Indians had both rectangular summer and winter houses (Hudson 1976:216). Ethnohistoric accounts of the Carolina and Virginia Piedmont Indians (e.g., Cumming 1958; Lefler 1967) describe both winter houses and summer, arbor-like structures. Additionally, paired structures which probably represented winter and summer houses have been identified archaeologically at several sites in the Southeast (Faulkner 1977; Schroed1 1980, 1983; Ward 1984a). The posthole patterns of the smaller structures at the Wall site are not clearly defined and have numerous gaps in their exterior walls. This suggests that the smaller structures represent partially enclosed, roofed buildings with built-in interior benches. The larger structures have more clearly and completely defined exterior walls and generally exhibit much evidence of maintenance, which implies that they were used primarily during the cold and rainy seasons of winter and spring. Ward (1984a) reported a similar pattern of clearly defined and ephemerally defined structures at the Warren Wilson site in western North Carolina.

The fact that the smaller (summer) structures are in closer proximity to each other than are the larger (winter) houses suggests that the level of social interaction between household groups may have been greater during the summer months. A possible explanation for this seasonal difference lies in the subsistence strategies of the Wall site people (see Holm, Gremillion, this report). A mixture of hunting, gathering, and horticulture is reflected in the plant and animal remains recovered from the excavations. These activities were probably scheduled seasonally, with most of the hunting being in the fall and winter, and most of the gathering and horticulture in the spring, summer, and early fall. It is also probable that larger social groups cooperated in horticultural activities than in hunting or gathering, and that the horticultural activities were at their peak during the months that the smaller structures were occupied. Therefore, the spatial relationships between the structures at the Wall site, especially the arrangement of probable summer houses, seems to reflect seasonal variation in social interaction and cooperation between household groups.

\section{Estimates of Village and Household Populations}

In recent years, several studies (e.g., Narol1 1962; LeBlanc 1971; Kramer 1979) have suggested that there is a relationship between floor area and population size. Other studies have focused on total settlement area and correlated populations (Weisner 1974; Sumner 1979). The studies using floor area to arrive at population estimates suggest that about $10 \mathrm{~m}^{2}$ of roofed building space per person is a close approximation of space needs. Thus, household population may be estimated by dividing the amount of floor space by 10. None of the ethnographic examples used to determine this correlation, however, seem to use paired winter and summer houses.

In an attempt to arrive at a population estimate for the village at the Wall site, only the larger of the paired structures were used. It is also assumed that all of the nonoverlapping structures were contemporary during some point in the occupation of the site. With these assumptions, five structures representing distinct households are present in the portion of the Wall site excavated to date. These are Structures A, D, E, G, and K. The combined area for these structures is $2048 \mathrm{ft}^{2}$ or $190.27 \mathrm{~m}^{2}$. Using an estimate of $10 \mathrm{~m}^{2}$ per person, one arrives at a population of 19 persons for the 
excavated portion of the site, and 3.8 persons per structure. This estimate is believed to be very low and in error. Ethnohistoric literature indicates that Indian societies of the East and Southeast at the time of European contact a11 lived in extended family households (Tyler 1907; Lefler 1967; Hudson 1976). These households certainly consisted of more than three or four persons.

A more accurate method to estimate population for this site is to suggest an "average" household size based on the ethnohistoric literature and multiply this by the number of structures that can be assumed to have been occupied at the same time. Lawson (Lefler 1967:182) states that the households of the Carolina Indians consisted of several related families, and John Smith (Tyler 1907:101) relates that the Algonkian Indians of Virginia were living in multigenerational, extendedfamily households. Such a group would fall within the range of 6-20 persons.

In considering the households at the Wall site village, it is assumed that the lower end of this 6-20 range is appropriate, given the relatively small size of the domestic structures. Using an estimate of between eight and ten persons per household, the population for the portion of the site excavated would have been between 40 and 50 persons. Assuming that the portion of the site excavated thus far is representative of the entire village and that the entire village is about four times larger than the excavated portion, it can be estimated that between 150 and 200 persons lived in the village during the period of maximum village size. The density of population would have been about 120 to 160 persons per acre and the average floor space per person would have been between four and five square meters.

\section{Summary}

The analysis and interpretation of the spatial structure of the Wall site provides a baseline necessary for the study of the processes of culture change that occurred in this area as a result of European contact and interaction. The Wall site has been shown to have been a village that grew in size during its period of occupation. The investment in maintenance of domestic structures and palisades and absence of storage pits suggests that these people were relatively sedentary. If the Wall site can be considered typical of other villages of this region during the Protohistoric period, some generalizations can be offered. Sites are likely to be rather small, ranging in size from about $3 / 4$ acre to about $1-1 / 2$ acres. They should show evidence of fortification, implying that by this time warfare was a fact of life. They were comprised of clusters of extended households that may have cooperated with each other in certain labor-intensive activities such as agriculture and hunting. The spatial organization of these household clusters implies that there was greater social interaction at the village during the months when agricultural activities dominated the subsistence cycle. This suggests that at least some labor was organized on a level larger than the household.

The data and interpretations presented above will be compared with the Mitchum and Fredricks sites in order to begin to assess some of the effects that the European invasion had on the native societies of the Carolina Piedmont.

\section{MITCHUM SITE}

The Mitchum site has received only limited excavation to date. The site is located on the Haw River approximately 17 miles southwest of the Wall and Fredricks sites. Davis (personal communication) has suggested that the site dates to the third quarter of the 17 th century. Carnes (this report) places the site occupation between 1625 and 1665, based on her analysis of the glass trade beads. The excavation consisted of one area of $725 \mathrm{ft}^{2}$ and an additional excavation unit of $100 \mathrm{ft}^{2}$ to the east of the larger area.

The excavations revealed an oval structure, one burial, and 14 features (Figure 3.14). The small size of the excavations at the Mitchum site prevents determination of the relationship of the structure to the entire settlement. Also, the site limits are not known. The discussion below, therefore, will consist of a description of the oval structure, the features, and their relationships.

The stratigraphy observed at the Mitchum site consists of three distinct soil zones. Zone 1 is a medium brown, sandy loam that contains a mixture of both historic and prehistoric artifacts. This zone was created by periodic episodes of plowing during the Historic period. Plowing caused the displacement of cultural materials that aboriginally were deposited on the surface or within the upper portions of subsurface facilities. This thickness of the plowzone across the portion of the site investigated ranges from $0.60 \mathrm{ft}$ to a little over $1.0 \mathrm{ft}$.

Underlying the plowzone is a light brown sand, Zone II. Numerous postholes and subsurface features were observed to extend into this zone, which is interpreted as an organically enriched sand that was the lower portion of a humic topsoil that developed before the area was occupied during the Early Contact period. Zone II ranges in thickness from $0.14 \mathrm{ft}$ to $0.23 \mathrm{ft}$. Zone III is a yellow sandy soil. This soil is alluvial in origin and is culturally sterile except for numerous intrusive postholes, pits, and basins. The thickness of the Zone III soil is unknown.

It is likely, given the sedimentary origin 

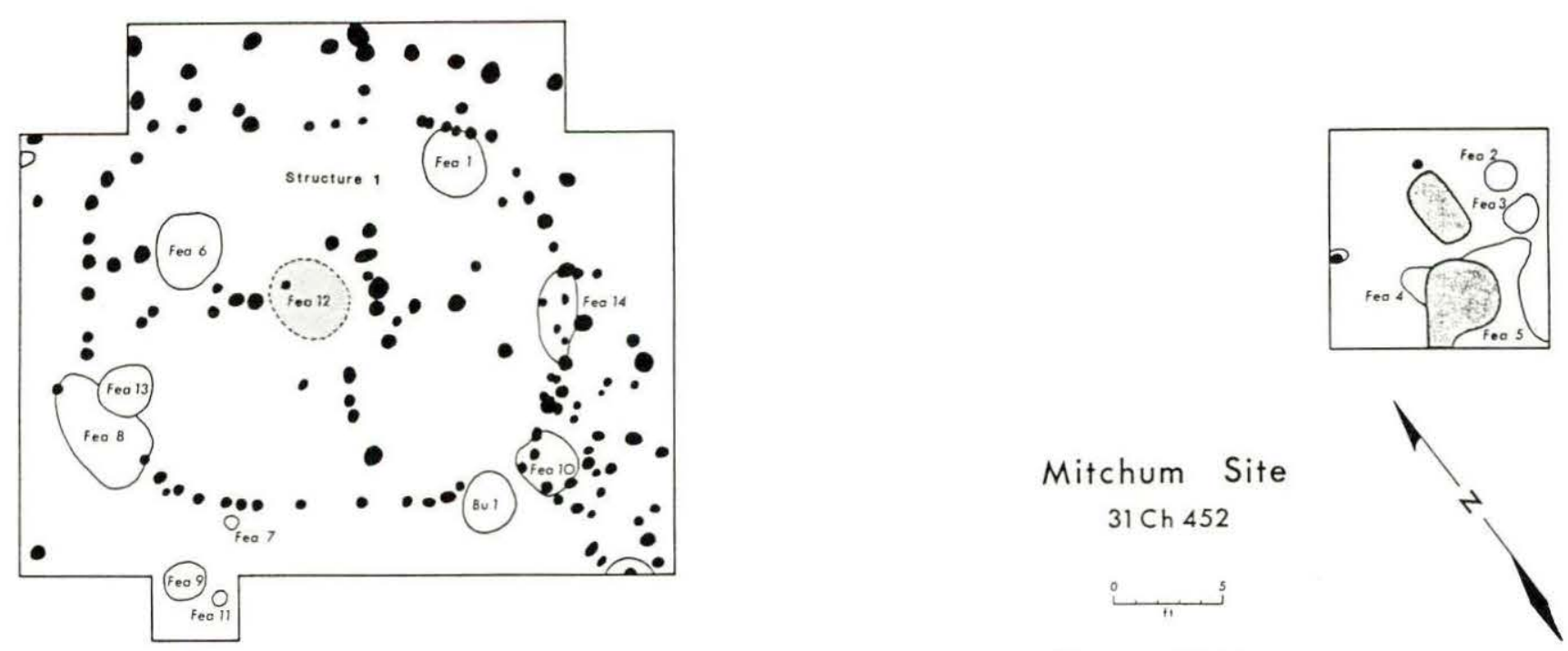

- Heorth i: Pothole

Figure 3.14

Excavation plan of the Mitchum site, 1983.

and unconsolidated nature of the sandy soils at the Mitchum site, that additional distinct soil horizons are present. It is also possible that earlier, sealed cultural components are present within these buried strata.

\section{Features}

Other than postholes and the single burial, the largest class of features consisted of pits and basin-shaped facilities. Although a Late Woodland component may be present at the Mitchum site (see Davis, this report), the features with culturally diagnostic materials are related to an early historic occupation (Davis, personal communication).

Feature 1 (Figures 3.15-3.16) was a deep basin. It was nearly circular in plan with a diameter of approximately $3.0 \mathrm{ft}$. The feature had a depth of $0.88 \mathrm{ft}$ below the top of the yellow sand zone (Zone III). The fill consisted of a single zone of dark brown sand with charcoal mixed throughout. Cultural material from this fill consisted of potsherds, stone artifacts, aboriginal clay pipe fragments, glass trade beads, mussel shells, carbonized plant remains, and a few fragments of animal bone. This feature was located within Structure 1, adjacent to the northeast portion of the exterior wall.

Feature 2 was a very small pit located about $45 \mathrm{ft}$ east of Structure 1, in the separate 10x10-ft excavation unit. It was approximately circular, about $1.0 \mathrm{ft}$ in diameter, and $0.76 \mathrm{ft}$ deep from the top of Zone III. The fill consisted of brown sandy soil containing one potsherd, a few stone flakes, a few fragments of animal bone and mussel shell, and a small amount of charred plant remains.
Feature 3 was a small circular pit, $1.6 \mathrm{ft}$ in diameter and $0.88 \mathrm{ft}$ in depth below the top of Zone III. The fill consisted of a single zone of brown sandy soil containing a few potsherds, a single glass trade bead, stone flakes, charred plant remains, and small amounts of animal bone. The plant remains consisted of small amounts of wood charcoal, hickory nutshe11, peach pits, and corn.

Features 4 and 5, basin-shaped facilities, were intruded by a large pit recently dug by pothunters. Feature 4 contained a single zone of dark brown, sandy soil. Very little cultural material was recovered from the remains of this feature. Feature 5 contained a single zone of light brown, sandy soil in which there were a few potsherds and lithic artifacts, a small amount of animal bone fragments, and a small but diverse assemblage of plant remains that included wood charcoal, hickory nutshell, peach pits, walnut shell, corn, and maygrass seeds.

Feature 6 (Figures 3.17-3.18) was a deep oval pit with undercut walls giving it a slight "bel1" shape when viewed in profile. It was 3.6 $x 3.1 \mathrm{ft}$ in plan and had a depth of $2.34 \mathrm{ft}$ below the top of Zone III. This feature, which lay within the northwest quadrant of Structure 1 , contained four zones of fill. The upper zone (Zone I), a basin-shaped deposit of gray ashy loam, was rich in artifacts and botanical remains. One hundred twenty-one potsherds were recovered. Stone artifacts, burnt clay, small amounts of animal bone and shell, and glass trade beads were also found in this zone of fill. Plant remains consisted of a large amount of wood charcoal, hickory nutshell, corn, cucurbit rind, and small amounts of acorn shell. Also, numerous charred seeds (mostly maygrass) were recovered. Zone I was surrounded on its sides by Zone Ia, a brown, sandy loam with 


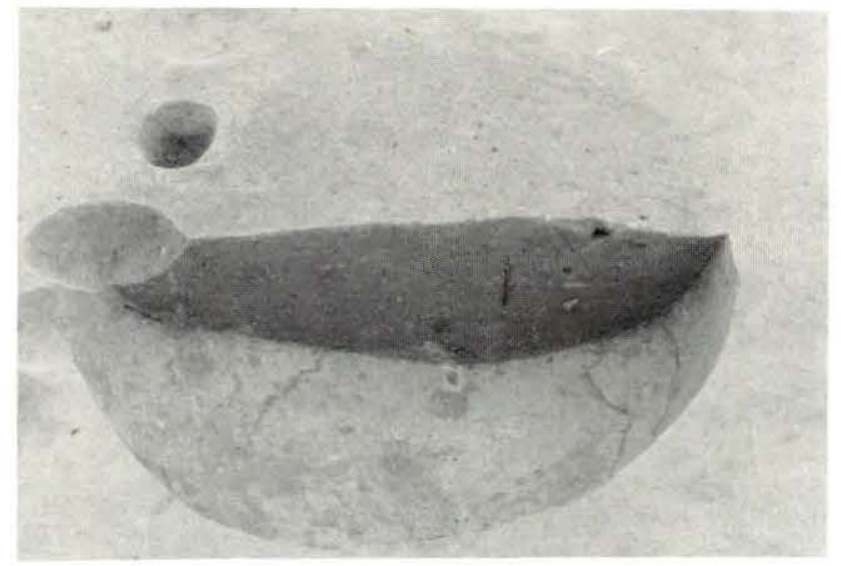

Figure 3.15

Feature 1, a deep basin-shaped pit (shown in profile), at the Mitchum site.

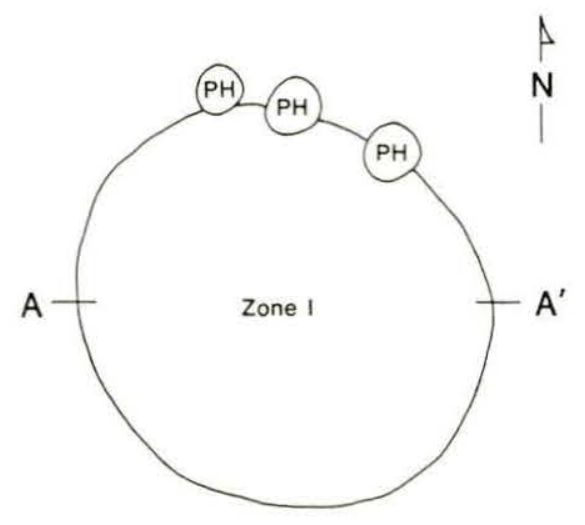

PLAN (top of subsoil)

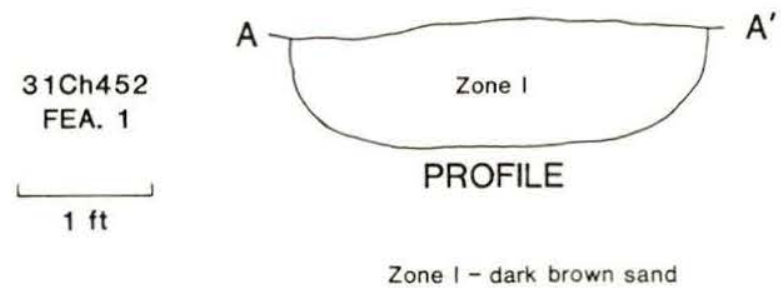

Figure 3.16

Plan and profile of Feature 1 at the Mitchum site.

charcoal and burnt clay. Although this zone contained a variety of cultural remains, it had a much smaller amount of charcoal than Zone I.

Zone II underlay Zones I and Ia. This was a tan sandy loam containing potsherds, stone flakes, animal bone fragments, she11, glass trade beads, and small amounts of wood charcoal and charred hickory nutshell and maygrass seeds.

Zone III, a reddish-tan sandy loam, underlay Zone II and filled the bottom $1.25 \mathrm{ft}$ of the feature. Sparse cultural materials were

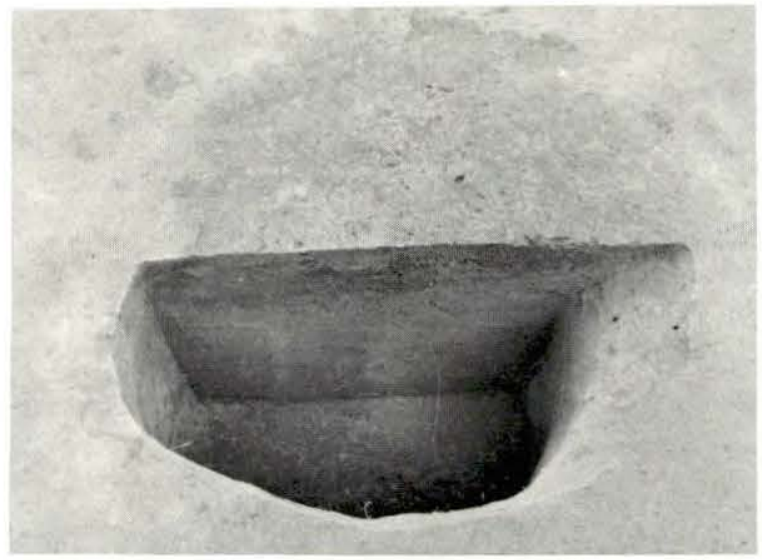

Figure 3.17

Feature 6, a deep pit (shown in profile), at the Mitchum site.

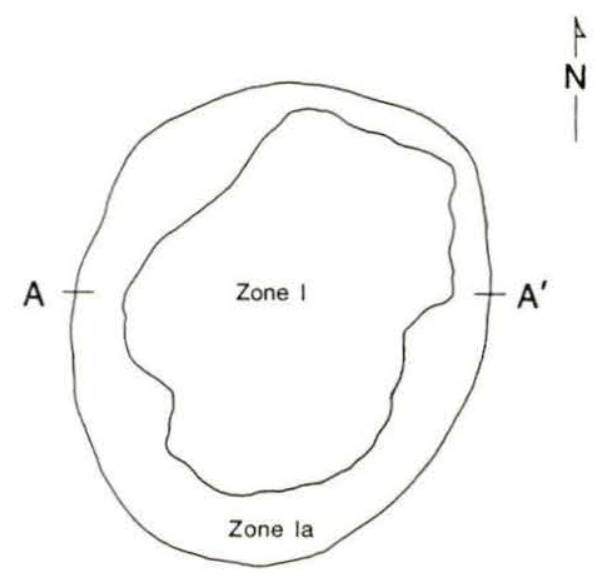

PLAN (top of subsoil)

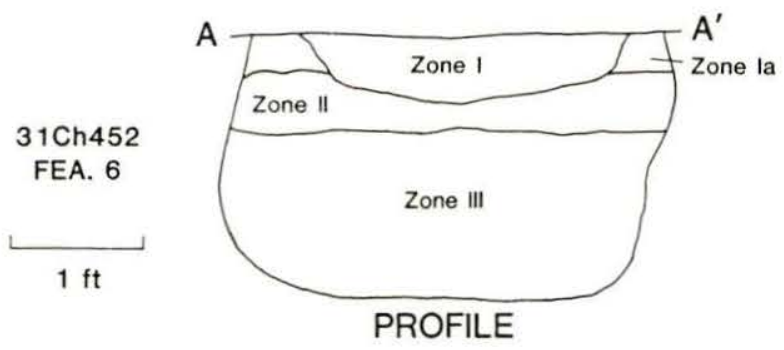

Zone I - gray ash

Zone la - brown sand

Zone II - tan sandy loam

Zone III - reddish tan sandy loam

Figure 3.18

Plan and profile of Feature 6 at the Mitchum site.

recovered from Zone III. These consisted of lithic artifacts, fragments of animal bone, and a small amount of charred plant remains. The plant remains consisted of hickory nutshell, walnut shell, and acorn shell. 
Features 7 and 11 were small, charred corncob-filled pits. Both were located outside and immediately to the south of Structure 1 . Feature 7 was $0.65 \mathrm{ft}$ in diameter and $0.25 \mathrm{ft}$ in depth below the top of Zone III. Feature 11 was oval ( $0.90 \mathrm{ft} E-W \times 0.85 \mathrm{ft} \mathrm{N}-\mathrm{S})$ and $0.35 \mathrm{ft}$ in depth from the top of Zone III. Both of these features contained a homogeneous fill of charred whole and partial corncobs.

Feature 8 (Figures 3.19-3.20) was a large, oblong basin which was intruded by Structure 1 and Feature 13. It was $5.6 \mathrm{ft}$ long and $2.9 \mathrm{ft}$ wide, and had a maximum depth of $0.60 \mathrm{ft}$ below the top of Zone III. The fill of this feature consisted of a single zone of dark brown sand containing a single potsherd, glass trade beads, stone artifacts, and charred plant remains. The plant remains consisted of mostly wood charcoal (several large pieces of charred wood were recovered) and a small amount of charred plant food remains consisting of hickory nutshell and a fragment of peach pit.

Feature 9 was a small oval basin located south of Structure 1 and near Features 7 and 11 . This feature was $2.0 \mathrm{ft} 10 \mathrm{ng}, 1.65 \mathrm{ft}$ wide, and $0.55 \mathrm{ft}$ deep (from the top of Zone III). It was filled with a single zone of reddish-brown sand mottled with charcoal and fired clay. Three potsherds and a small amount of lithic debris were recovered along with charred plant remains consisting of a small amount of hickory nutshe11 and corn.

Feature 10 was a circular pit, measuring $2.5 \mathrm{ft}$ across the top and having a depth of 1.22 $\mathrm{ft}$ below the top of Zone III. The fill consisted of a single zone of mottled, light brown sandy loam, with a few animal bone fragments, a small amount of charred plant food remains, a single aboriginal clay pipe fragment, and lithic debris. The plant remains consisted of abundant wood charcoal and small amounts of hickory nutshell, acorn, corn, and grape seeds.

Feature 12 was a thin and diffuse area of fired clay, probably a hearth, located in the approximate center of Structure 1 . No artifacts or food remains were associated with the feature. It measured $4.0 \mathrm{ft}$ by $3.8 \mathrm{ft}$.

Feature 13 was a deep circular pit, $2.8 \mathrm{ft}$ in diameter, which intruded Feature 8 and Structure 1. The pit was $2.8 \mathrm{ft}$ in depth below the top of Zone III. Three zones of fill were observed. Zone I, a light brown sand, contained glass trade beads, lithic debris, and small amounts of plant and animal remains. The plant remains consisted of wood charcoal, hickory nutshell, corn, persimmon seeds, and maygrass seeds. This zone represented approximately half of the pit fill.

Zone II, a dark reddish brown sand, underlay Zone I and was only $0.35 \mathrm{ft}$ thick. It contained eight potsherds, some lithic debris, a few animal bone fragments, glass trade beads,

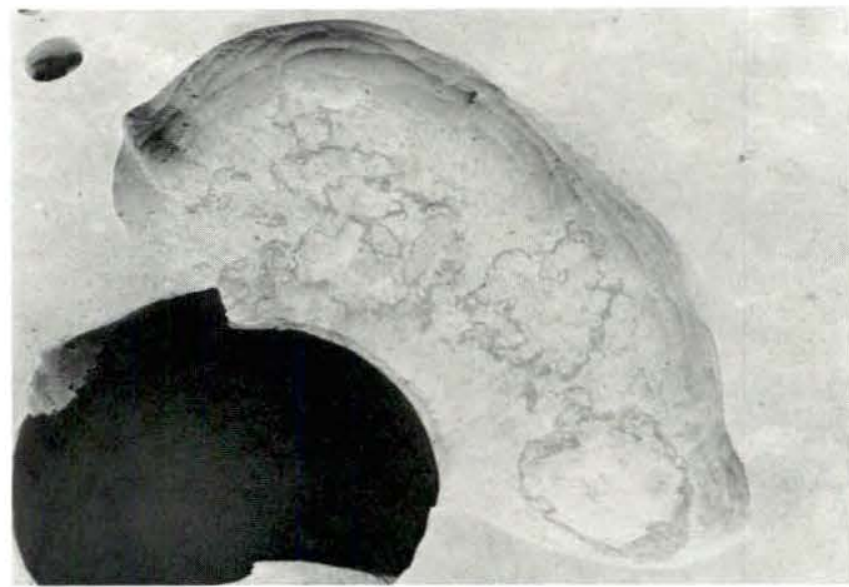

Figure 3.19

Feature 8, a large shallow basin (after excavation), at the Mitchum site.

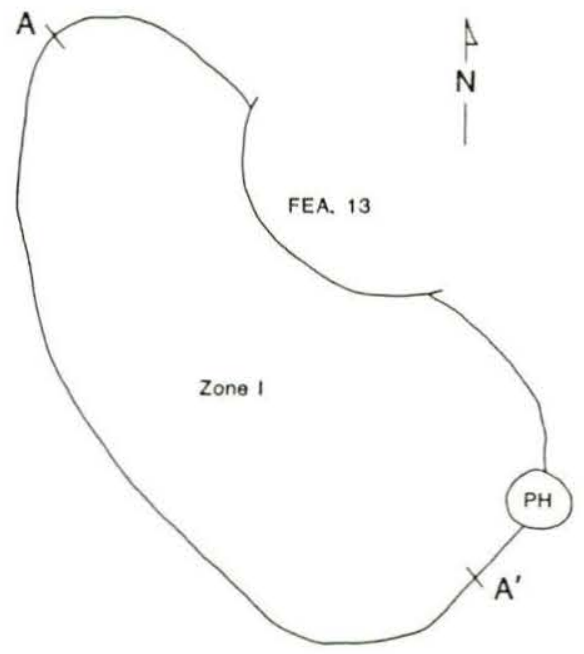

PLAN (top of subsoil)

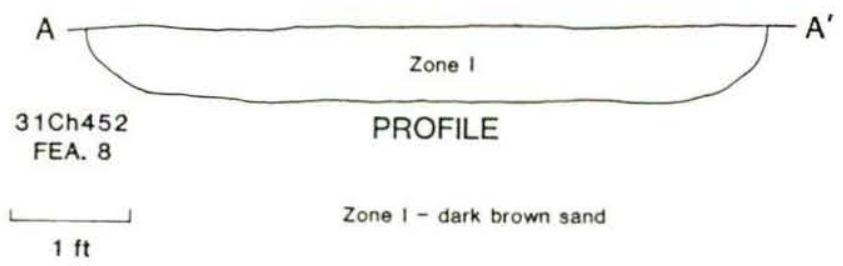

Figure 3.20

Plan and profile of Feature 8 at the Mitchum site.

and charred wood and other plant remains. The plant food remains consisted of hickory nutshell, acorn shell, peach pits, corn, maypop seeds, and persimmon seeds.

Underlying Zone II was Zone III, a brown sand containing a mixture of small amounts of pottery, animal bone, lithics, and wood char- 
coal. The plant food remains consisted of hickory nutshe11, acorn shel1, corn, maypop seeds, and persimmon seeds. This zone was 1.25 ft thick and extended to the bottom of the pit.

Feature 14 (Figures 3.21-3.22), an oblong basin, was intruded by the eastern side of Structure 1. The feature measured $4.0 \mathrm{ft}$ long, $1.7 \mathrm{ft}$ wide, and extended $0.35 \mathrm{ft}$ below the top of Zone III. The fill consisted of a single zone of dark brown sand containing small amounts of pottery, lithics, animal bone fragments, and charred plant remains. The plant remains consisted of hickory nutshe11, corn, maypop seeds, and butterfly pea seeds.

The dimensions of the features at the Mitchum site are summarized in Table 3.5. The estimated volumes and depth/diameter ratios are based on extrapolations that take into consideration the portions of these features that were truncated by plowing.

There is considerable variation in the kinds of features represented at the Mitchum site. Feature 12 is interpreted as the remnant of a hearth associated with Structure 1. Features 7 and 11 are small charred cob-filled pits which probably functioned as hide-smoking facilities. Feature 2 is probably a large posthole, perhaps associated with a suspected palisade that was partly defined north of Structure 1. The remainder of the features are pits and basins having fill that contains refuse and other secondary deposits. These features can be divided into four general classes: small shallow basins, large shallow basins, small deep basin/pits, and large deep pits.

The small shaliow basins are defined by a depth/diameter ratio of $<0.40$ and a volume of $<20 \mathrm{ft}^{3}$. Feature 14 is the only feature in this class (Figures 3.21-3.22).

The large shallow basins (Figures 3.193.20) are defined by a depth/diameter ratio of $<0.40$ and a volume of $>20 \mathrm{ft}^{3}$. Feature 8 is representative of this feature class. Feature 5 is also probably of this class, although since it had been disturbed by pothunters an accurate determination of its original form could not be made.

Features in Class III are small, deep basins and pits (Figures 3.15-3.16). They are characterized by a deptb/diameter ratio of $>0.40$ and a volume of $<20 \mathrm{ft}^{3}$. Features $1,3,9$, and 10 are Class III features. Feature 4, which was disturbed by recent pothunting, is also probably of this class.

Feature Class IV (Figures 3.17-3.18) consists of pits with depth/diameter ratios of $>0.40$ and volumes of $>20 \mathrm{ft}^{3}$. Features 6 and 13 are of this type. Both of these are cylindrical to bell-shaped pits having stratified zones of fill.

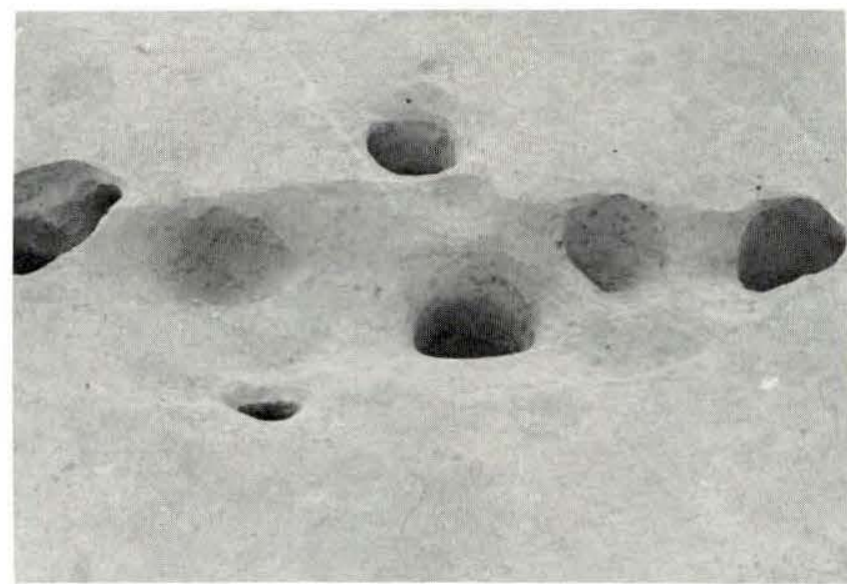

Figure 3.21

Feature 14, a small shallow basin (after excavation), at the Mitchum site.

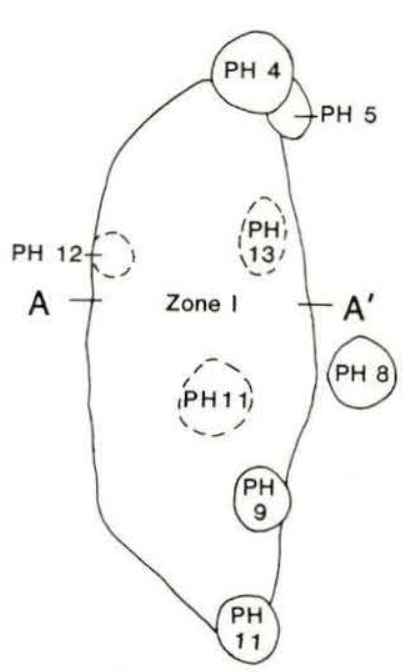

PLAN (top of subsoil)

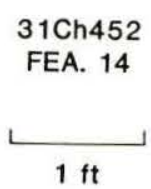

A

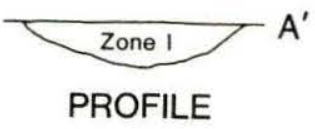

Zone I - dark brown sand

Figure 3.22

Plan and profile of Feature 14 at the Mitchum site.

Following Schroed1 (1980), it is assumed that features whose primary function was for storage should have a relatively high ratio of depth to diameter. The features in Classes III and IV are of this form. Although their usefulness as storage facilities in terms of volume varies from $6 \mathrm{ft}^{3}$ to $26 \mathrm{ft}^{3}$, they are all 


\begin{tabular}{|c|c|c|c|c|c|c|c|}
\hline Feature & $\begin{array}{l}\text { Length } \\
(\mathrm{ft})\end{array}$ & $\begin{array}{l}\text { Width } \\
\text { (ft) }\end{array}$ & $\begin{array}{l}\text { Depth } \\
\text { (ft) }\end{array}$ & $\begin{array}{l}\text { Estimated } \\
\text { Original } \\
\text { Depth }(\mathrm{ft})\end{array}$ & $\begin{array}{c}\text { Depth/ } \\
\text { Diameter }\end{array}$ & $\begin{array}{l}\text { Estimated } \\
\text { Vol ume } \\
\left(\mathrm{ft}^{3}\right)\end{array}$ & Feature Form \\
\hline 1 & 3.20 & 2.85 & 0.88 & 1.92 & 0.63 & 13 & Deep Basin/Pit \\
\hline 2 & 1.10 & 1.00 & 0.76 & 1.80 & 1.80 & 1.5 & Large Posthole? \\
\hline 3 & 1.60 & 1.60 & 0.88 & 1.75 & 1.09 & 3 & Small Pit \\
\hline $4 *$ & 1.70 & 1.10 & 0.23 & 1.27 & 0.91 & - & Shallow Basin \\
\hline $5 *$ & 4.70 & 4.50 & 0.20 & 1.24 & 0.27 & - & Large Basin \\
\hline 6 & 3.60 & 3.10 & 1.95 & 3.00 & 0.90 & 26 & Deep Pit \\
\hline 7 & 0.65 & 0.65 & 0.25 & 1.29 & - & - & Cob-Filled Pit/Basin \\
\hline 8 & 5.60 & 2.90 & 0.60 & 1.64 & 0.36 & 25 & Large Basin \\
\hline 9 & 2.00 & 1.65 & 0.55 & 1.59 & 0.66 & 6 & Deep Basin \\
\hline 10 & 2.50 & 2.50 & 1.22 & 2.26 & 0.87 & 10 & Deep Pit \\
\hline 11 & 0.90 & 0.85 & 0.35 & 1.39 & - & - & Cob-Filled Pit/Basin \\
\hline 12 & 4.00 & 3.80 & - & - & - & - & Hearth Remnant \\
\hline 13 & 2.90 & 2.70 & 2.80 & 3.85 & 1.51 & 19 & Deep Pit \\
\hline 14 & 4.00 & 1.70 & 0.35 & 1.39 & 0.35 & 12 & Shallow Basin \\
\hline
\end{tabular}

* Partially disturbed by pothunters. Size and form estimated from undisturbed portion.

relatively deep in comparison to their horizontal area.

Class I and II features probably served as food processing facilities. The sandy soil at the Mitchum site disputes their use as borrow pits for clay.

The various zones of fill in the Mitchum site features can be divided into three general groups based on fill color and texture. Fill Group I, gray ash with charcoal, contained abundant wood charcoal, charred plant food remains, and artifacts. Zone $I$ of Feature 6 is the only example of this type of fill. Feature fill Group II is a reddish-tan, brown sandy loam containing small amounts of wood charcoal. Zone I of Feature 9, Zone II of Features 13, and Zone III of Feature 6 are of this type. The majority of the feature fill zones are of Group III. This fill is a light to dark brown sand with various amounts of wood charcoal.

Besides color and texture, there are other differences in the composition of the zones of fill at the Mitchum site. The zones vary in terms of the amounts and proportions of nonbotanical remains, wood charcoal, and charred plant food remains (Table 3.6).

The gray ashy fill, Zone I, of Feature 6 contained a proportion of wood charcoal that was large in comparison to other types of refuse. The plant food remains were diverse and contained a high percentage of charred seeds. The non-botanical remains were rich in animal bone and shell. This fill zone contained only small amounts of artifacts. This fill probably represents a rather restricted set of behaviors involved in the preparation of food for either consumption or storage (Wilson 1985).

Fill Group II zones contained very small quantities of cultural materials. The largest proportions of refuse represented in this fill was wood charcoal. Artifacts are poorly represented in the flotation samples; only one of the three zones contained lithic debris and none had pottery. However, small amounts of pottery were recovered from the waterscreened fill, and glass 
trade beads were recovered from two of the three zones. Zone I from Feature 9 contained only a minute amount of plant food remains. The other two fill zones, although only represented by small weights of plant remains, contained among the largest amounts of plant food remains when considered as a proportion of the remains making up the fill. The reddish color of the soil suggests the fill originated from activities involving the preparation of plant foods in two cases and non-cooking fire-related activity in the case of Feature 9.

Feature fill Group III shows considerable variability in fill composition. The fill zones in this category fall into three classes. Class I, represented by Zones 1 of Feature 8 and Feature 13, contain large proportions of wood charcoal.

Material recovered from Feature 8 fill consisted of little other than wood charcoal and very small amounts of hickory nutshell and peach. Zone I fill of Feature 13 contained a more diverse assemblage of materials, including plant remains, lithic debris, animal bone, and glass trade beads. Since Feature 13 intrudes into Feature 8, it is difficult to be certain that there was no aboriginal mixing between the two. The variety of material in Zone I of Feature 13 suggests that this fill may have resulted from general house-cleaning. Feature 8 fill reflects a much more restricted set of behaviors that probably were not related to the preparation of food. Feature $10 \mathrm{fill}$ consisted of a mixture of non-botanical and botanical remains. The few artifacts found within this fill consisted of lithic debris and a single clay pipe fragment. No ceramics were recovered. Most of the non-botanical remains were nonartifact debris and small fragments of animal bone. This diverse assemblage of materials, with a large percentage of charcoal, suggests that this fill originated from domestic food preparation and consumption.

The remainder of the Group III zones at the Mitchum site have non-botanical materials as the major component of the fill. These remains are a mixture of debris, animal bone, glass beads, pottery, and lithics. It is suggested that the fill in these zones represents behavior that was not narrowly restricted temporally and was associated with ongoing village activities. The high proportion of debris implies that natural processes such as erosion and slumping, as well as cultural processes, operated to create them.

\section{Structure 1}

The remains of a single structure were uncovered at the Mitchum site (Figures 3.14 and 3.23). Structure 1 was defined by an oval pattern of single postholes. The exterior dimensions of this structure were $18.0 \times 24.0 \mathrm{ft}$ and the inside dimensions were $17.0 \times 22.0 \mathrm{ft}$. There was no evidence of post replacement or rebuilding of the structure's exterior. The exterior wall posts have a mean diameter of 0.49 ft $(n=58, s d=0.09)$. The use of small posts or poles and the oval shape suggest that this was a domed-roof structure similar to the wigwams so frequently described in the ethnohistoric literature (e.g., Wright 1947; Lefler 1967). Lawson describes the construction of this type of house as follows:

These Savages live in Wigwams, or Cabins built of Bark, which are made round like an Oven, to prevent any Damage by hard Gales of Wind. They make the Fire in the middle of the House, and have a Hole at the Top of the Roof right above the Fire, to let out the Smoke. These Dwellings are as hot as Stoves, where the Indians sleep and sweat all Night. The Floors thereof are never paved nor swept, so that they have always a loose Earth on them....

The Bark they make their Cabins withal, is generally Cyprus, or red or white Cedar; and sometimes, when they are a great way from any of these Woods, they make use of Pine-Bark, which is the worser sort. In building these Fabricks, they get very long Poles, of Pine, Cedar, Hiccory, or any Wood that will bend; these are the Thickness of the Small of a Man's Leg, at the thickest end, which they generally strip of the Bark, and warm them well in the Fire, which makes them tough and fit to bend; afterwards, they stick the thickest ends of them in the Ground, about two Yards asunder, in a Circular Form, the distance they design the Cabin to be, (which is not always round, but sometimes oval) then they bend the Tops and bring them together, and bind their ends with Bark of Trees, that is proper for that use, as Elm is, or sometimes the Moss that grows on the Trees, and is a Yard or two long, and never rots; then they brace them with other Poles, to make them strong; afterwards, cover them all over with Bark, so that they are very warm and tight, and will keep up firm against all the Weathers that blow (Lefler 1967:180-182).

The postholes observed inside Structure 1 are difficult to interpret. Their large size (diameter: $\overline{\mathrm{x}}=0.60 \mathrm{ft}, \mathrm{sd}=0.15$ ) and distribution along the long axis of the structure suggests that they may have been used to support the roof. This type of roof, created by pulling tops of the wall poles over the center, should not have required substantial support. It is possible, although unlikely, that these postholes predate the structure. It is more likely that most of these posts were used by the occupants of the structure both as supports for the roof, ceiling beams, partitions, and for sleeping platforms.

The covering material of this structure was probably hides or bark. Approximately $95 \mathrm{~g}$ of material cataloged as daub was recovered from some of the excavation units in and around the 
Table 3.6

Amounts and percentages of nonbotanical remains, wood charcoal, and plant food remains from feature fill at the Mitchum site (grams per 10 liter flotation sample).

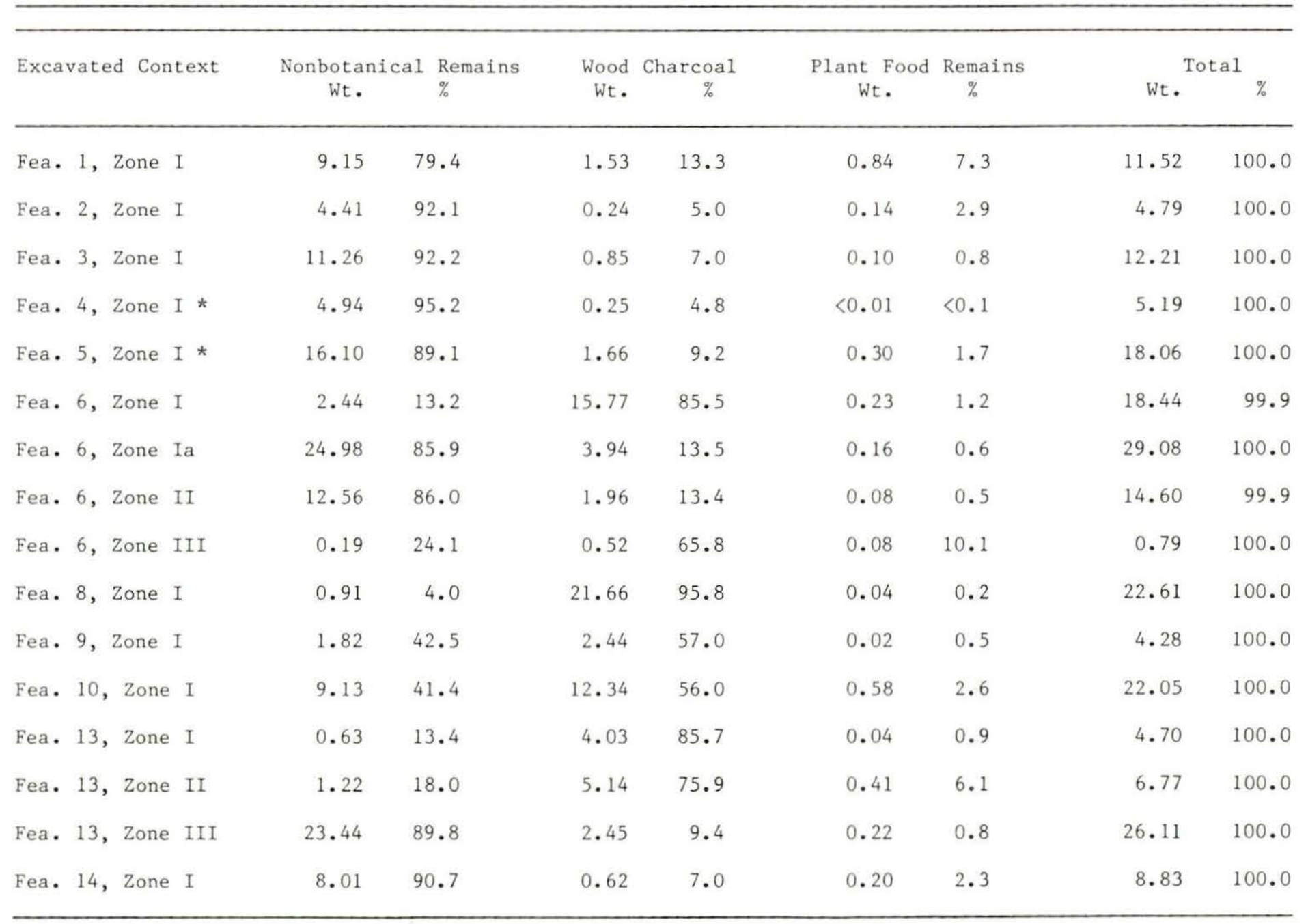

* Flotation samples taken from undisturbed fill only. 


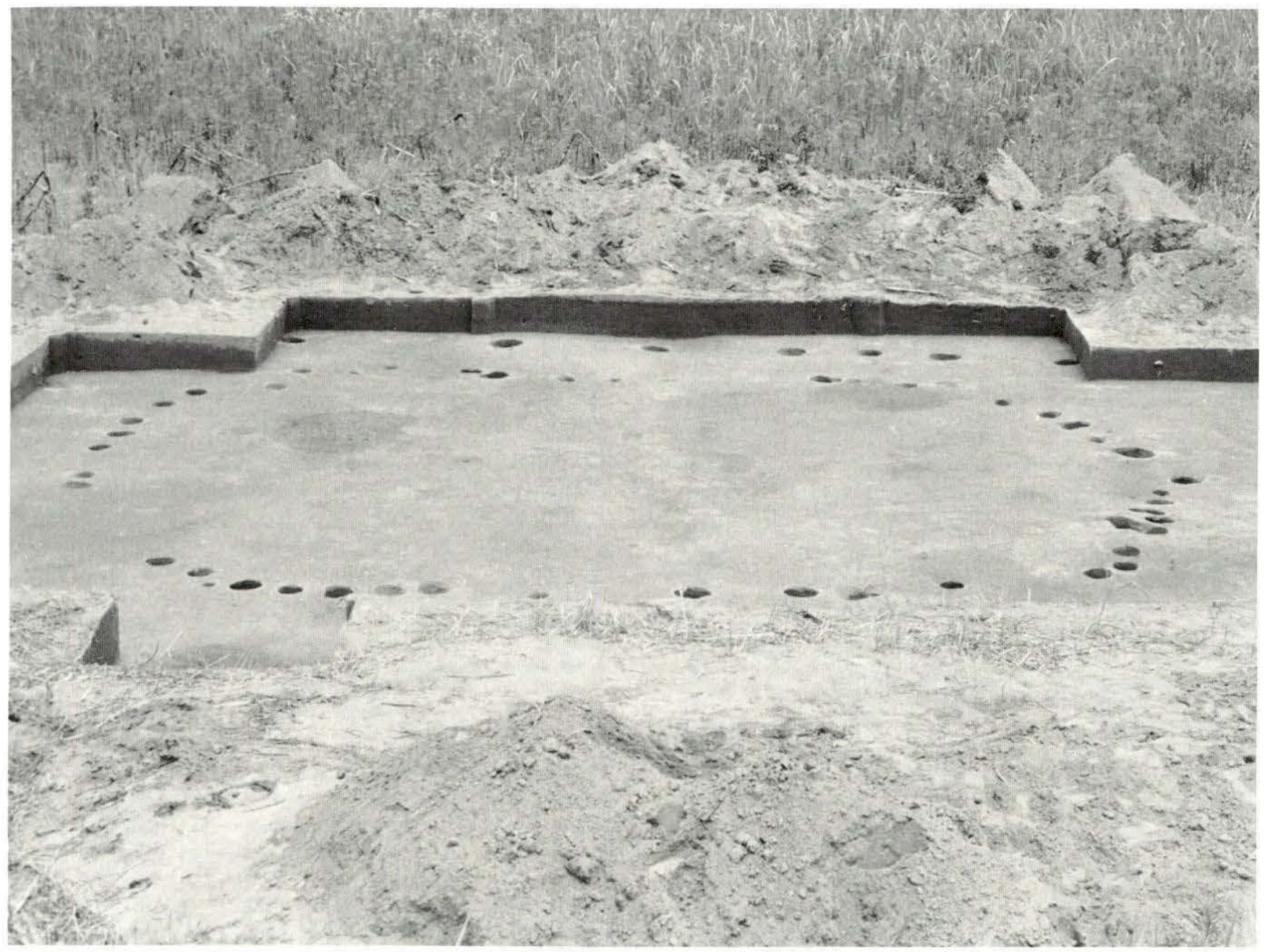

Figure 3.23

Structure 1 at the Mitchum site after excavation of postholes.

structure. The majority $(62 \%)$ of this daub was concentrated in the excavation unit near the center of the structure. Feature 12 is located in this unit and is a diffuse area of fired clay. Hence, the "daub" probably originated from a prepared-clay hearth in the center of Structure 1 .

A line of nine large postholes was observed approximately two feet north of Structure 1. These fairly widely spaced postholes, arranged parallel to the long axis of Structure 1, may represent a portion of a palisade. It is equally possible that these posts are part of another domestic structure. Additional excavations will be required to determine what this apparent posthole alignment represents.

of the features observed at the Mitchum site, only Features 1, 6, and 12 were definitely associated with Structure 1. It is possible that Features 7, 9, and 11, located close together and to the south of the structure, also resulted from activities of the domestic group that occupied Structure 1. If Features 7 and 11 (cob-filled pits) are associated with Structure
1, they probably were used in an activity that needed to be carried out outside of the structure.

Features 8,10 , and 14 predate the construction of Structure 1 , as they all are intruded by exterior wall posts of the structure. Burial 1 (see Ward, Wilson, this report) may postdate the occupation of Structure 1; Feature 13 may also postdate the occupation.

A spatial analysis of the materials recovered from the plowzone was not undertaken, since the area of the site exposed thus far is so sma11.

\section{Summary}

The excavations at the Mitchum site have yielded important but limited information concerning the native culture of this area during the Early Contact period. The excavations to date are only large enough to expose Structure 1 and a small area surrounding it. Relationships between this structure, outside activity areas, other structures in the vicinity, and the vil- 
lage as a whole cannot be attempted at this time.

The features observed and excavated in the $10 \times 10-f t$ excavation unit just to the east of Structure 1 were badly disturbed by pothunters. Additional excavation will be required to determine associations of these features.

Even with the limitations imposed by the small size of the excavations at the Mitchum site, the complete exposure of Structure 1 has provided insight into domestic architecture of the village, and the feature data have yielded information on food storage and processing strategies and technologies of the occupants. Such data will be essential for comparing the site structure of the three sites examined in this chapter.

One of the most obvious differences between the Wall site and the Mitchum site is in the number and kinds of subsurface facilities. The number of deep facilities with constricted openings, defined by a depth/diameter ratio of $>0.40$, is significantly greater at the Mitchum site. Six of the features observed at the Mitchum site could have been used for caching or storage facilities. Their meaning, in terms of human behavior and adaptive strategies, will be addressed following the discussion of the Fredricks site.

The single structure observed at the Mitchum site is of very different construction than those at the Wall site. The use of a bent-pole, bark- or hide-covered structure agrees with the ethnohistoric accounts of this period, and may reflect greater mobility of Piedmont Indian groups during the Early Contact period. There does seem to be a general continuity in the density of occupation within domestic structures, if it can be assumed that an extended family was living together in a single structure, Structure 1 has a floor area of about $290 \mathrm{ft}^{2}$. Assuming that between 8 and 10 persons comprised the social unit of an extended family, there was a density of approximately one person per 30-40 $\mathrm{ft}^{2}$ of floor space. Although this is somewhat smaller than the figures derived using the Wall site structure data, it is not impossible that small houses like Structure 1 were densely occupied. In fact, structures of similar size and form of the Western Niantic, a New England Indian tribe, were observed in the mid-18th century to have an even greater density of occupants than has been suggested for the structures at either the Wall or Mitchum sites (Sturdevant 1975). Whether such a density of house occupation would have occurred year-round or only seasonally is a difficult question to answer. The seasonal profile based on plant remains (see Gremillion, this report) of the upper fill zones of the two pit/basin features associated with Structure 1, indicates that these facilities did not function as storage facilities during summer. Therefore, the facilities probably functioned as storage pits during the winter. Thus, it is possible that Structure 1 was occupied primarily during the late fall and winter and was abandoned during the summer.

\section{FREDRICKS SITE}

Two seasons (1983-1984) of investigation at the Fredricks site have exposed $3500 \mathrm{ft}^{2}$ of the village (Figure 3.24) and have provided information for a preliminary interpretation of site structure. From the existing excavations and auger testing, it appears that the village covered no more than one acre. The Fredricks site undoubtedly represents a Middle Contact period (A.D. 1680-1710) village occupied by the Occaneechi who had moved to this area from the vicinity of the Staunton and Dan River confluence in the late 17th century (see Merre11, this report). Historic artifacts recovered from the excavations support the temporal placement of the site in the late 17 th and very early $18 \mathrm{th}$ century (see Carnes, this report).

\section{Site Stratigraphy}

The archaeological manifestations of the village occupation are contained within two soil zones at the Fredricks site. The upper zone, Zone $\mathrm{I}$, is a light brown clay loam consisting of a mixture of soil, artifacts, and organic matter that have accumulated over the past several thousand years. These materials have been intermixed by agricultural plowing, which has been extensive since Europeans settled in this area in the mid-18th century (see Tippitt and Daniel, Davis, this report, for a discussion of the prehistoric remains in the plowzone; and Carnes, this report, for a discussion of the European component that postdates the historic Indian occupation). The materials associated with the Occaneechi occupation at the Fredricks site and contained within this plowzone are derived from the upper portion of the various pits and postholes that have been truncated by plowing, and of materials that accumulated on the original surface of the village either as scatters or in middens. Although no intact midden has been observed during the excavations, relatively larger amounts of artifacts have been found along the palisade especially in the northwestern area of the site (Figure 3.25). The underlying subsoil in this area was also more organically stained than in areas to the east and southeast. The subsoil, Zone II, underlying the plowzone is a yellow-brown clay of undetermined depth. This soil contains numerous boulders of blue-gray phyllite at a depth between two and three feet below the base of the plowzone. Cultural remains within the subsoil consist of various intrusive pits and postholes along with their contents. These features were clearly evident at the top of the subsoil. Once the plowzone had been excavated, they stood out as brown-to-black stains contrasting with the yellow-brown clay subsoil 


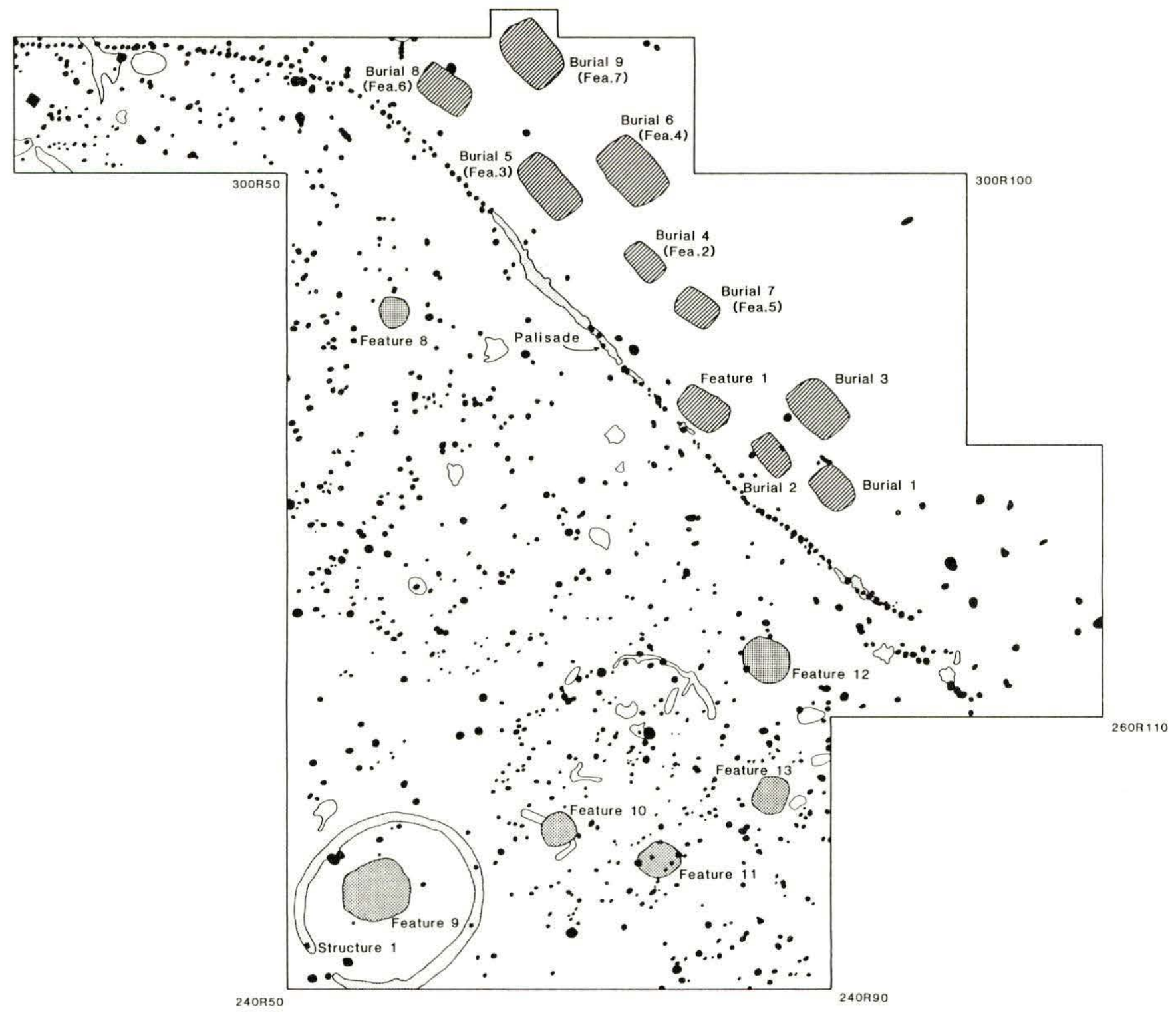

FREDRICKS SITE

Plan of 1983-84 Excavations

N

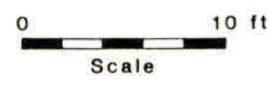

Figure 3.24

Excavation plan of the Fredricks site, 1983-1984. 


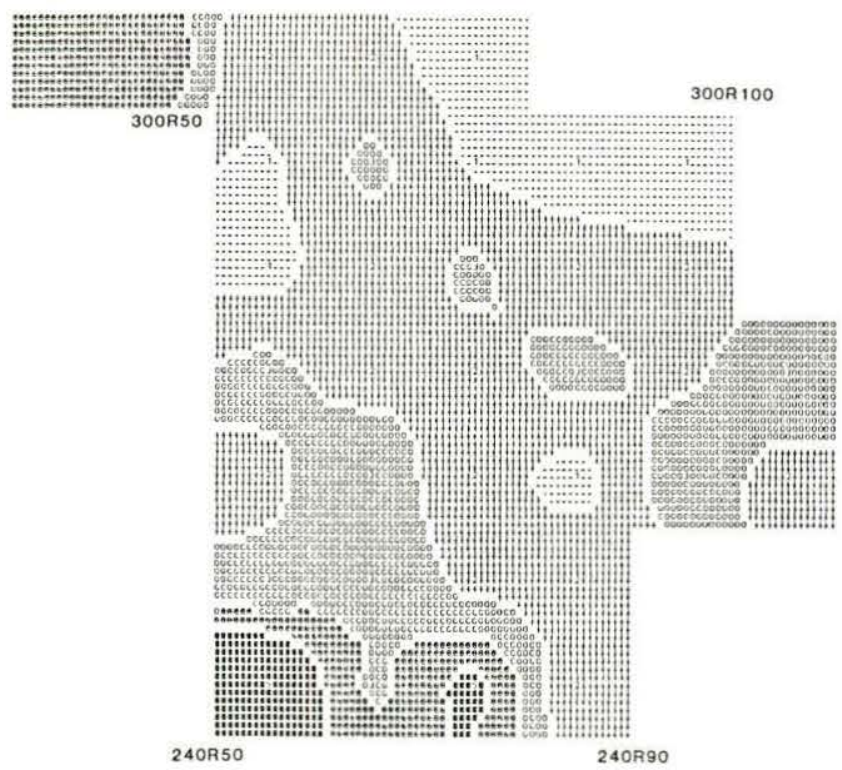

Figure 3.25

SYMAP of historic aboriginal ceramics in the plowzone at the Fredricks site.

\section{(Figure 3.26).}

The excavations exposed a varied set of archaeological features including a cemetery (see Ward, Wilson, this report), five subsurface pit facilities, the remains of several kinds of structures, and a portion of a small palisade that enclosed the village (Figures 3.24).

Immediately southwest of Palisade 1 was an area dense in postholes, with two complete structures and portions of additional structures that extended beyond the limits of the excavation. Several pit features were also located within this $30-\mathrm{ft}$ wide area parallel to the palisade. To the southwest of this area was Structure 1, an oval wall trench centered on a large cylindrical pit.

The discussion of site structure focuses on features, structures, and their interrelationships. Also discussed is the spatial distribution of some of the artifact classes recovered from the plowzone. Finally, the results of the systematic auger testing are presented and the information recovered from this testing is incorporated into the interpretations of site structure. The spatial organization of the cemetery and its associated burials is not addressed in this analysis. Although this area and the behavior it represents is pertinent to the interpretation of site structure, the spatial organization of the area is discussed elsewhere (see Ward, this report).

\section{Features}

At the Fredricks site, 13 subsurface intrusions were assigned feature numbers. Fea- tures 1 through 7 were burials and Feature 8 proved to be a tree disturbance upon excavation. Features 9 through 13 were aboriginally excavated pits of various sizes. The physical dimensions of these features are summarized in Table 3.7 .

All of these features could have been used effectively as storage facilities. The depth/ diameter ratios ranged from 0.51 to 1.55 and their volumes (estimates which include the portion of the pits ${ }_{3}$ runcated by plowing) ranged from 12 to $59 \mathrm{ft}^{3}$. Feature 10 had undercut walls, which indicated that it may have been cleaned periodically and maintained as a storage facility for a longer period than the other pits. Features 11 through 13 were shallower than Feature 10 and all had straight-toinsloping sides. These features might have functioned as storage facilities or as expediently produced pits to cache valuables. Each of these features is described in detail below.

Feature 9 (Figures 3.27-3.31) was a large deep cylindrical pit located within Structure 1. The pit was $5.0 \mathrm{ft} 1 \mathrm{ong}, 4.7 \mathrm{ft}$ wide, and 2.85 ft deep below subsoil. A massive rock that extended into the pit on the south side appeared to have been heated repeatedly. Its depth/ diameter ratio $(0.73)$ and large volume (59 $\mathrm{ft}^{3}$ ) are similar to large storage facilities observed archaeologically at Historic Cherokee sites in Tennessee (Schroedl 1980). It must be remembered, however, that pits used for different purposes may have similar forms and volumes. Additionally, pits like other artifacts may undergo substantial changes in function as needs change. A feature's form at an abandonment stage in its life-cycle is, in part, the result of all of the uses to which it was put. It may or may not contain evidence of each different use. It is for this reason that functional interpretations of features must consider attributes of form, fill, and especially context (Wilson 1985). Feature 9 is an excellent example of a pit facility that may have undergone several transformations in its function prior to its abandonment. The fill zones of this feature (Figures 3.29-3.30) reflect these potential transformations.

The bottom zone (Zone IIIb) consisted of masses of charred bark and clusters of carbonized corn kernels, all on the pit floor. The masses of corn had been contained in woven baskets, portions of which were recovered in the excavations. These baskets seem to have been intentionally placed on the pit floor around the inside perimeter adjacent to the walls. The pit floor beneath the bark lining, as well as the lower pit walls, was colored brick-red from having been fired. The only animal remains from this zone were 134 fragments of charred foot bones from an unidentified small mammal (Holm, this report). A few flakes and small glass trade beads completed the inventory from this zone. 


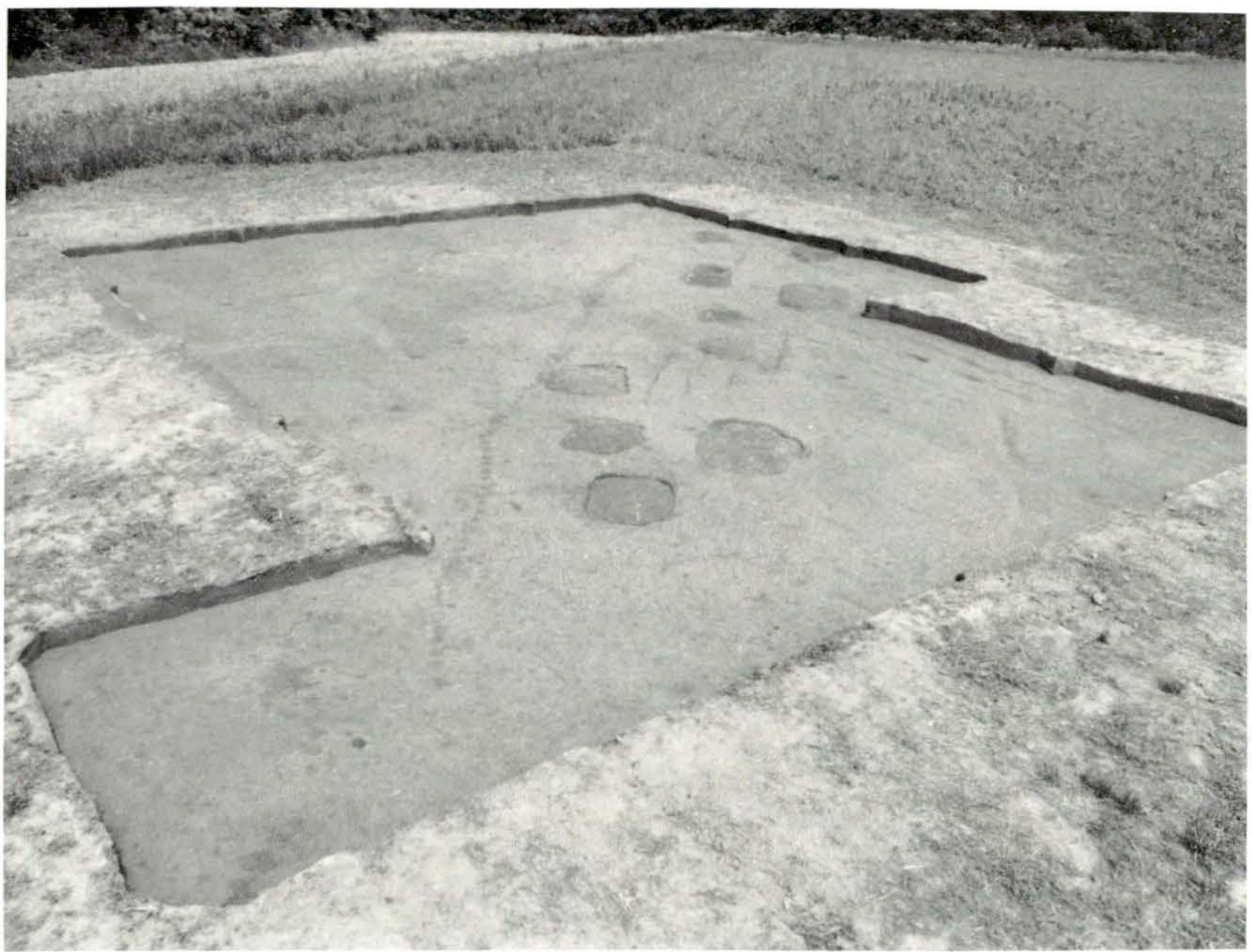

Figure 3.26

Overview of the 1984 excavation at the Fredricks site, showing portions of the palisade and cemetery.

Table 3.7

Summary of feature attributes at the Fredricks site.

\begin{tabular}{|c|c|c|c|c|c|c|c|}
\hline Feature & $\begin{array}{c}\text { Length } \\
(\mathrm{ft})\end{array}$ & $\begin{array}{l}\text { Width } \\
\text { (ft) }\end{array}$ & $\begin{array}{l}\text { Depth } \\
(\mathrm{ft})\end{array}$ & $\begin{array}{l}\text { Estimated } \\
\text { Original } \\
\text { Depth ( } f t)\end{array}$ & $\begin{array}{c}\text { Depth/ } \\
\text { Diameter }\end{array}$ & $\begin{array}{l}\text { Estimated } \\
\text { Volume } \\
\left(\mathrm{ft}^{3}\right)\end{array}$ & Feature Form \\
\hline 9 & 5.00 & 4.70 & 2.85 & 3.55 & 0.73 & 59 & Large Deep Pit \\
\hline 10 & 2.60 & 2.30 & 3.10 & 3.80 & 1.55 & 20 & Deep Pit \\
\hline 11 & 3.30 & 2.60 & 1.65 & 2.35 & 0.80 & 14 & Small Deep Pit \\
\hline 12 & 3.60 & 3.00 & 1.14 & 1.84 & 0.51 & 17 & Small Deep Pit \\
\hline 13 & 2.80 & 2.40 & 1.60 & 2.30 & 0.85 & 12 & Small Deep Pit \\
\hline
\end{tabular}




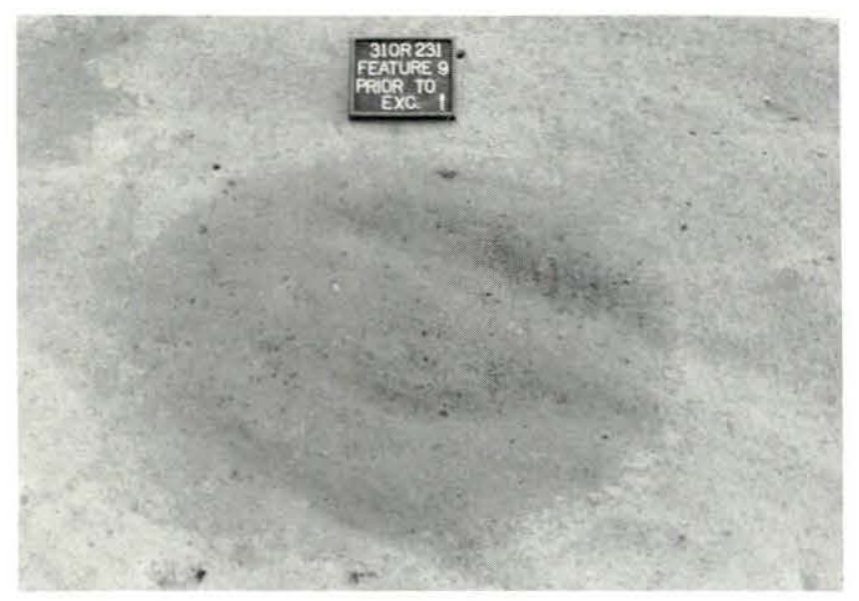

Feature 3.27

Feature 9 at the Fredricks site, prior to excavation.

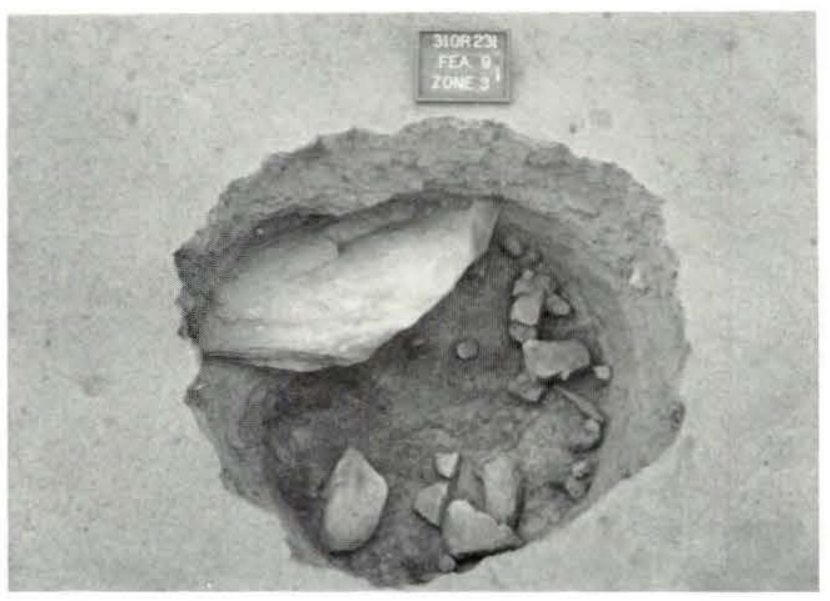

Feature 3.28

Feature 9 at the Fredricks site, after excavation.

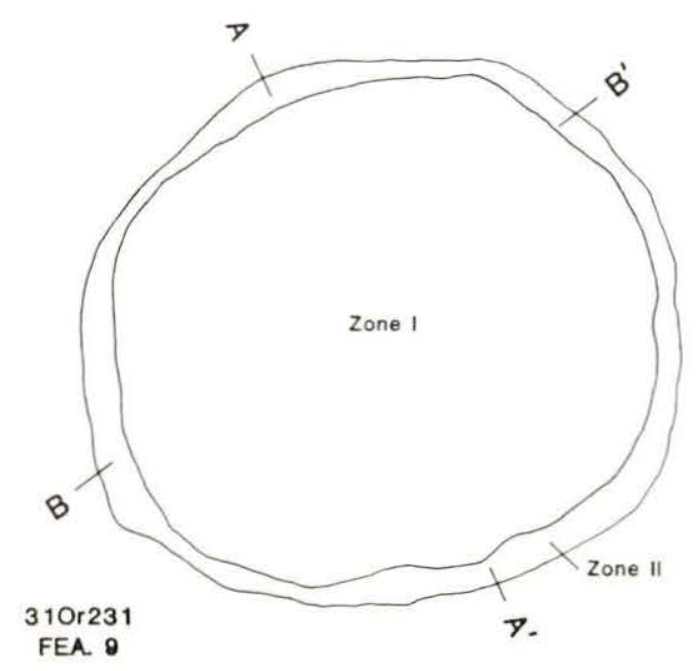

PLAN (top of subsoil)

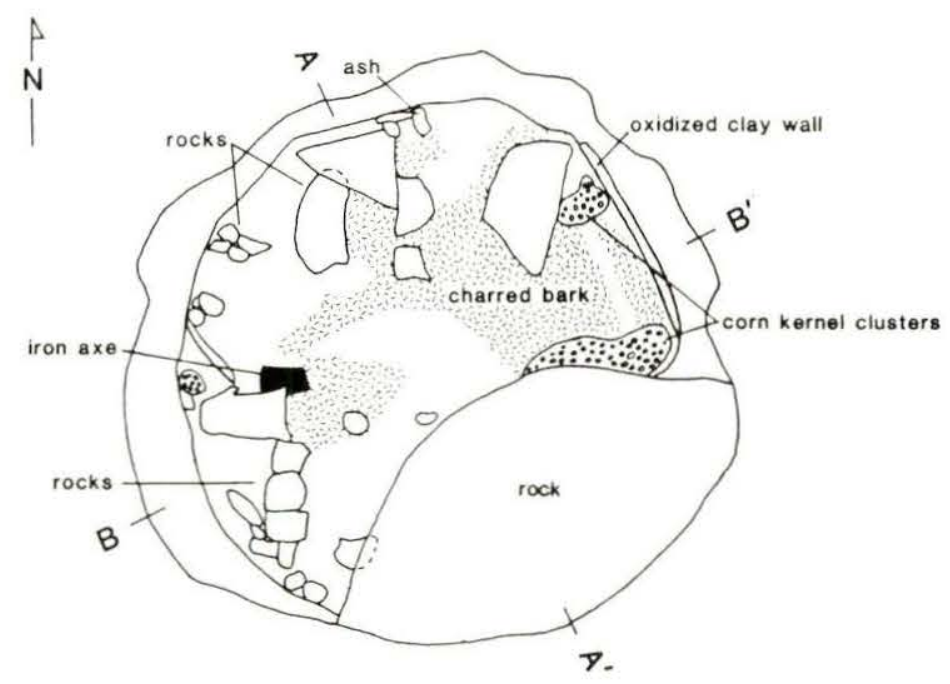

PLAN (excavated)

$1 \mathrm{ft}$

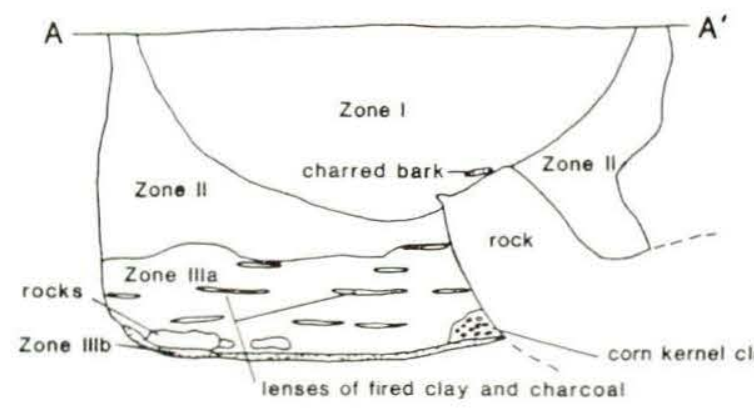

PROFILE

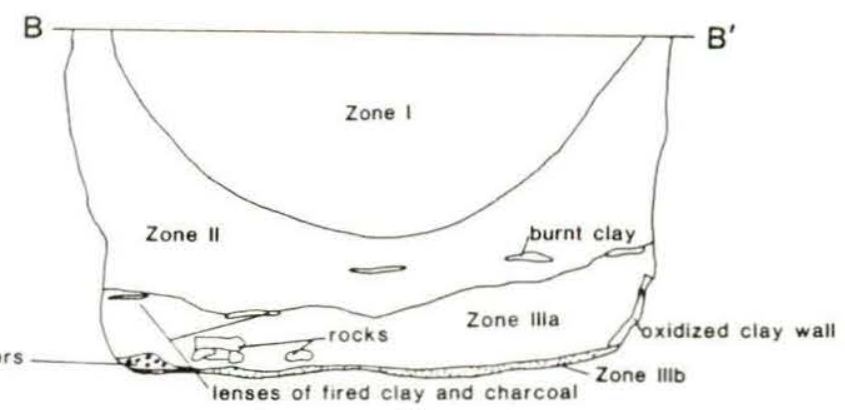

PROFILE 


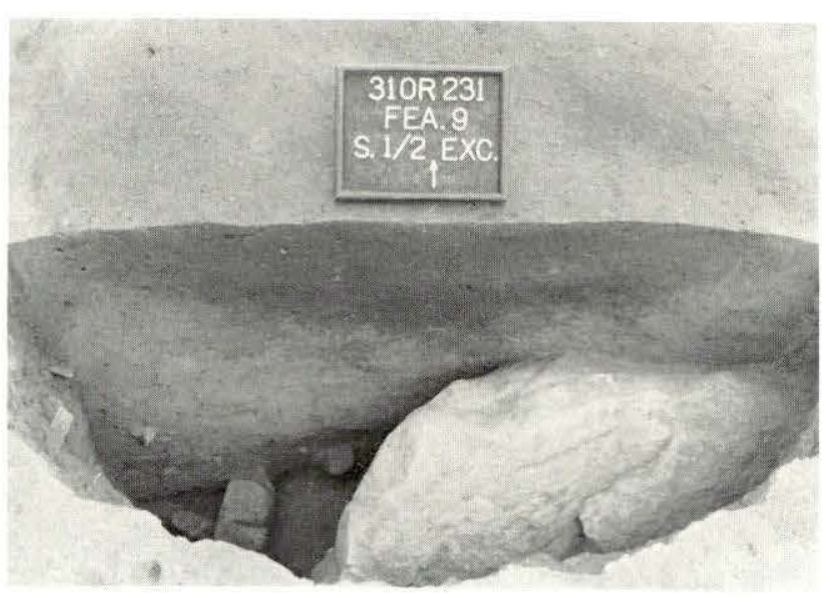

Figure 3.30

Feature 9 at the Fredricks site, showing stratigraphy of pit fill.

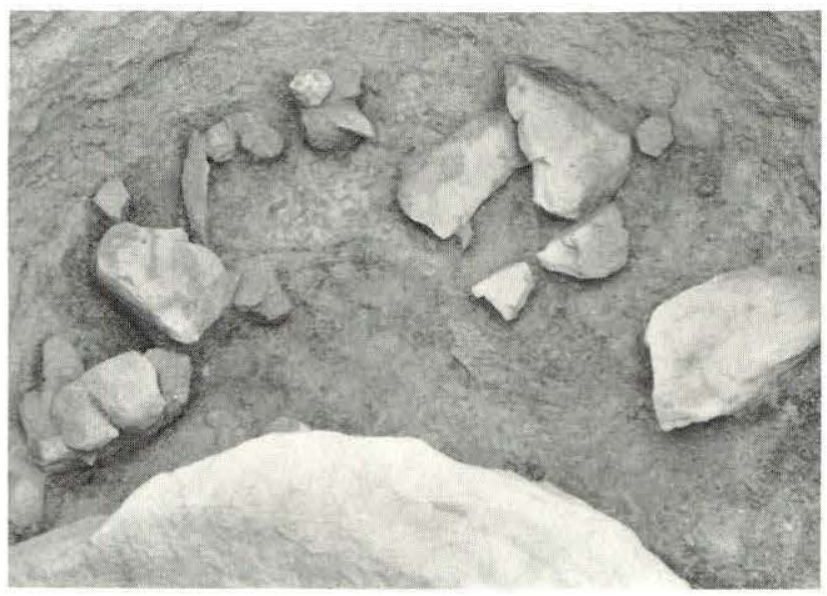

Figure 3.31

Bottom of Feature 9 at the Fredricks site, showing rock clusters and charred plant remains on pit floor.

If Feature 9 had functioned as a storage facility the bark lining and corn could represent materials that were left in place when the first observable transformation of the facility occurred. Although most corn was probably stored on the husk, Harrington (1908:589) observed that the Seneca stored roasted and dried green corn in bags.

An alternative interpretation of Zone IIIb is that it represents initial preparation of the feature for use as a fire pit (This does not rule out the possibility that the pit was originally used for storage). The containers of corn kernels may reflect ritual behavior associated with feasts of thanksgiving at the end of the corn harvest, such as those described by Lawson (Lefler 1967:67,177). Such harvest ceremonies were common throughout much of North
America (Hudson 1976).

Zone IIIa accumulated on top of Zone IIIb. This zone had evidence of repeated episodes of fire building. Numerous fire-cracked rocks and uncracked hearth stones were present in this zone (Figure 3.31). Associated with hearth stones was a damaged and burnt steel axe head (Carnes, this report), several lumps of sandtempered potter's clay, and a small hammerstone (Tippitt and Daniel, this report). One of the large rocks showed evidence of having been used as a grinding stone. These materials were contained within a rich organic, ashy-clay loam matrix. Numerous lenses of brick-red fired clay were present throughout this zone as were many fragments of charred wood and bark. The soil matrix, rocks, fired clay, and fire-reddened and hardened pit walls and floor strongly suggest that this zone of fill accumulated in-place as a result of repeated episodes of fires.

The plant food remains from this zone were the most abundant and diverse of all the fill zones in either the refuse-filled pits or the burials excavated at the site (Gremillion, this report). This seems to imply that plant food preparation on a large scale might have been an important behavioral component in the formation of this zone. However, it is very possible that much of the corn (which represents $68 \%$ of the plant food remains by weight) might have originated from the same activities that produced the corn kernel clusters in the underlying zone. Although there was a diverse assemblage of seeds present in the fill, most of these seeds were of weedy species and could have been introduced into the fill by natural processes. It fact, an open fire pit might be expected to "capture" an assortment of seeds from nearby plant communities. The other seeds represented in this zone were grape, maypops, and sumac, all of which could have been consumed while people were sitting around taking sweat baths.

Zone II, a mottled clay loam, contained a mixture of cultural materials, including potsherds, 1ithic artifacts, animal bone fragments, glass trade beads, wood charcoal, and charred plant food remains. The mottled composition of the fill suggests that it was a mixture of subsoil and humus excavated to fill the pit after it ceased to function as a fire pit. The ceramics were diverse and represent a mixture of prehistoric materials and historic aboriginal sherds. This zone was more organically rich where it was bounded by the underlying and overlying zones of rich organic material. All of the species of plant food remains from this fill were present in the overlying Zone I deposit and may have originated from the same activity that produced that zone. Zone II extended to the top of the feature (base of the plowzone). At that level it appeared as a 0.3 ft ring surrounding a central ashy deposit (Zone I). It is probable that Zone II had extended to the surface and that Zone $I$ was intrusive into it. 
The upper zone of fill (Zone I) was a basin-shaped deposit of dark, yellowish-brown sandy ash containing a mixture of diverse plant and animal remains, along with a variety of artifacts. The artifacts within this zone consisted of potsherds, lithic debris and tools, glass trade beads, kaolin pipe fragments, wood charcoal, fired clay, and daub. This zone was a very homogeneous and fine-grained deposit of ash. In composition, it contained the least amount of nonbotanical remains of any of the fill zones at the site (Table 3.8). There was a relatively high percentage of plant food remains, which included hickory nutshell, acorn shell, peach pits, walnut shells, corn, grape seeds, and maypop seeds. A variety of other carbonized seeds representing non-plant food remains were also present. Four species of animals (deer, raccoon, bear, and a single horse molar) were represented as bone fragments in the fill. The textural qualities of the fill and the relatively small amounts of nonbotanical remains represented in the flotation sample suggest that this zone accumulated in situ. The basin shape of the deposit probably resulted from partial re-excavation (cleaning out) of the pit aboriginally. The large and diverse assemblage of plant food remains imply that this facility may have been used for the large scale preparation of food, perhaps with feasting that occurred as part of the mortuary practices of the occupants of the village (see Ward, this report).

Feature 10 (Figures 3.32-3.33) was a deep cylindrical pit with undercut walls that gave it a bell-shaped profile. This feature was $2.6 \mathrm{ft}$ long, $2.3 \mathrm{ft}$ wide, and was $3.1 \mathrm{ft}$ deeper than the top of subsoil. The ${ }_{3}$ depth/diameter ratio (1.55) and volume (20 $\left.\mathrm{ft}^{3}\right)$ suggest that the pit was used for storage of food or other materials. It was located within a small oval structure

Table 3.8

Nonbotanical remains, wood charcoal, and plant food remains from the 1983-84 excavations at the Fredricks site (grams per 10 11ter flotation sample).

\begin{tabular}{lcccc}
\hline & $\begin{array}{c}\text { Nonbotanical } \\
\text { Remains }\end{array}$ & $\begin{array}{c}\text { Wood } \\
\text { Charcoal }\end{array}$ & $\begin{array}{c}\text { Plant Food } \\
\text { Remains }\end{array}$ & Total \\
\hline Fea. 9, Zone I & 62.06 & 1.10 & 3.08 & 66.24 \\
Fea. 9, Zone II & 89.31 & 1.20 & 2.02 & 92.53 \\
Fea. 9, Zone IIIa & 469.32 & 7.41 & 8.43 & 485.16 \\
Fea. 9, Zone IIIb & 401.20 & 37.48 & 8.15 & 446.83 \\
Fea. 10, Zone I & 141.35 & 8.40 & 0.98 & 150.73 \\
Fea. 10, Zone II & 130.79 & 5.91 & 0.19 & 136.89 \\
Fea. 11, Zone I & 135.98 & 2.15 & 0.01 & 138.14 \\
Fea. 12, Zone I & 145.62 & 1.19 & 0.94 & 147.75 \\
Fea. 12, Zone II & 112.22 & 1.02 & 0.99 & 114.23 \\
Fea. 13, Zone I & 105.08 & 12.52 & 0.58 & 118.18 \\
Fea. 13, Zone II & 134.24 & 0.95 & 0.78 & 135.97 \\
\hline
\end{tabular}

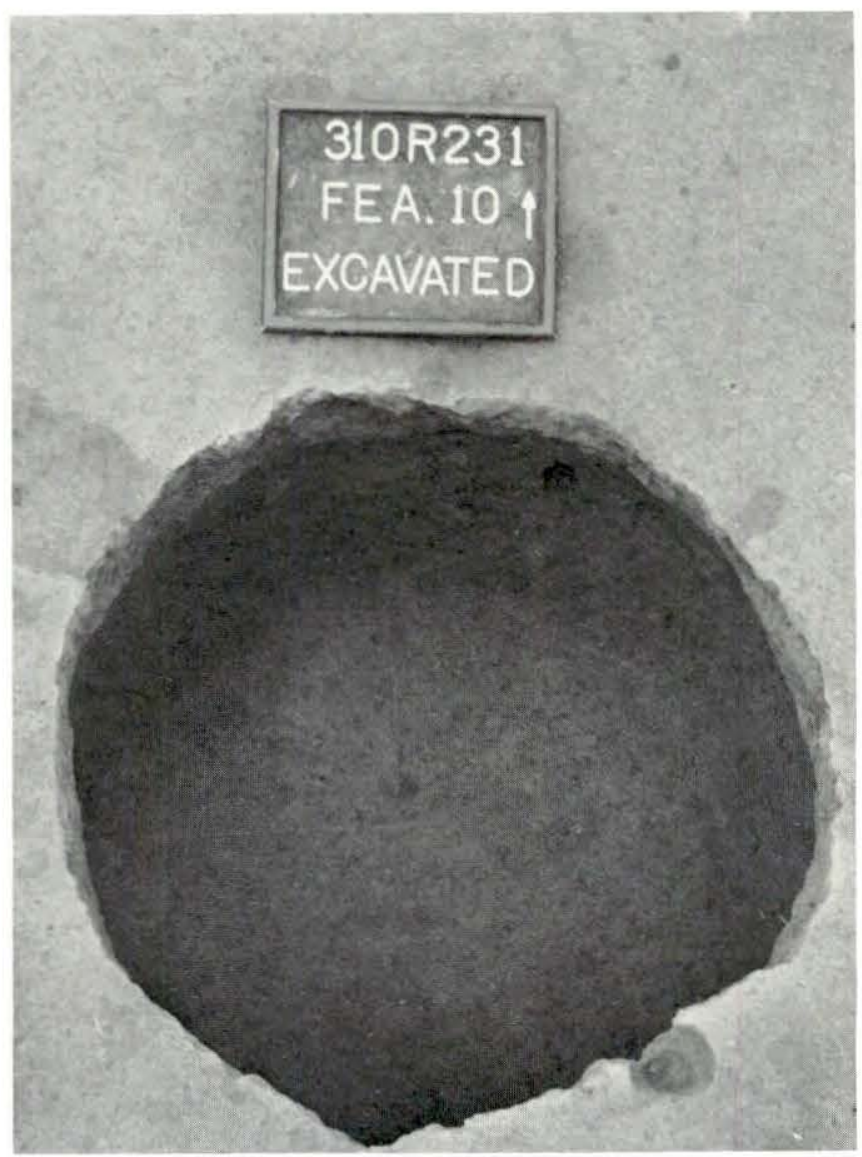

Figure 3.32

Feature 10 at the Fredricks site, after excavation.

(Structure 3) and probably served as the primary subterranean storage facility for the members of that household.

Feature 10 contained two zones of fill. The upper zone, Zone I, was a dark brown loam that contained a variety of cultural remains. These remains consisted of potsherds, lithic artifacts, kaolin pipe fragments, glass trade beads, animal bone, wood charcoal, and charred plant food remains. Deer was the only identifiable animal species represented. The plant remains recovered were hickory nut, acorn, peach, and corn; wood charcoal also was fairly abundant. The lower portion of this zone contained most of the above material, as well as a small concentration of fire-cracked rock, charcoal, and sandy ash. The fill probably represents a brief episode of disposal of household debris, including hearth materials. Zone II was a deposit of dark, brownish-orange, mottled clay loam that extended to the bottom of the pit. This zone was about $2.5 \mathrm{ft}$ thick and contained over $700 \mathrm{~g}$ of animal bone fragments representing deer, box turtle, squirrel, and turkey. A small amount of plant food remains were recovered, consisting of hickory nut and a trace of corn. Wood charcoal was well-preserved but in a smaller amount than in the overlying zone. Potsherds were more abundant than in Zone 


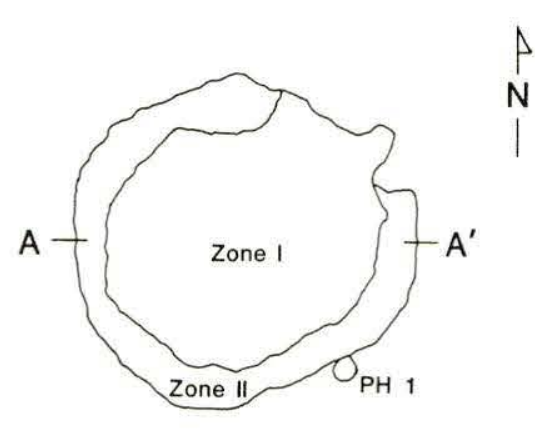

PLAN (top of subsoil)

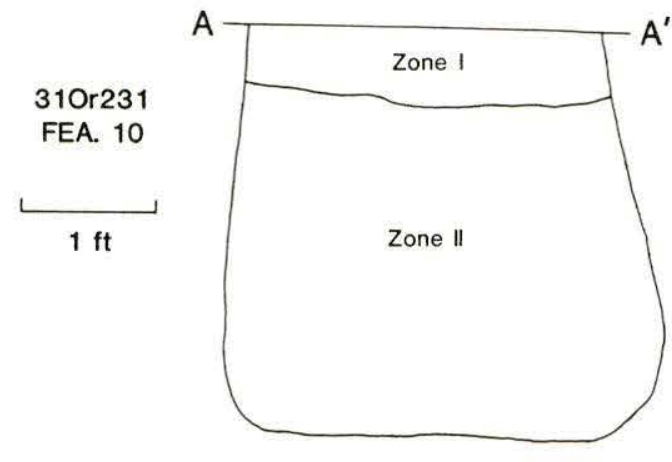

PROFILE

Zone I - dark brown mottled loam

Zone II - orange-brown mottled clay

Figure 3.33

Plan and profile of Feature 10

at the Fredricks site.

I. Zone II seems to represent the initial filling of this pit upon its abandonment as a storage facility. A lack of lensing in the fill suggests that it accumulated fairly rapidly. It is not possible to determine the origin of this fill, although its mottled color and variety of cultural debris may identify it as a redeposited mixture of humus soil, midden, and clay subsoil.

Features 11, 12, and 13 were small deep refuse-filled pits. Feature 11 (Figures 3.343.35) was an oval pit located within and adjacent to the southeastern wall of Structure 3. This feature was $3.0 \mathrm{ft}$ long, $2.4 \mathrm{ft}$ wide, and $1.53 \mathrm{ft}$ in depth below the top of subsoil. A large rock extended into the pit from the surrounding subsoil and the pit wall had been undercut around this rock, perhaps in an attempt to remove it. The depth/diameter ratio was 0.80 and it probably functioned as a small storage facility. The small volume (14 $\left.\mathrm{ft}^{3}\right)$ suggests that it was not used for bulk storage, but

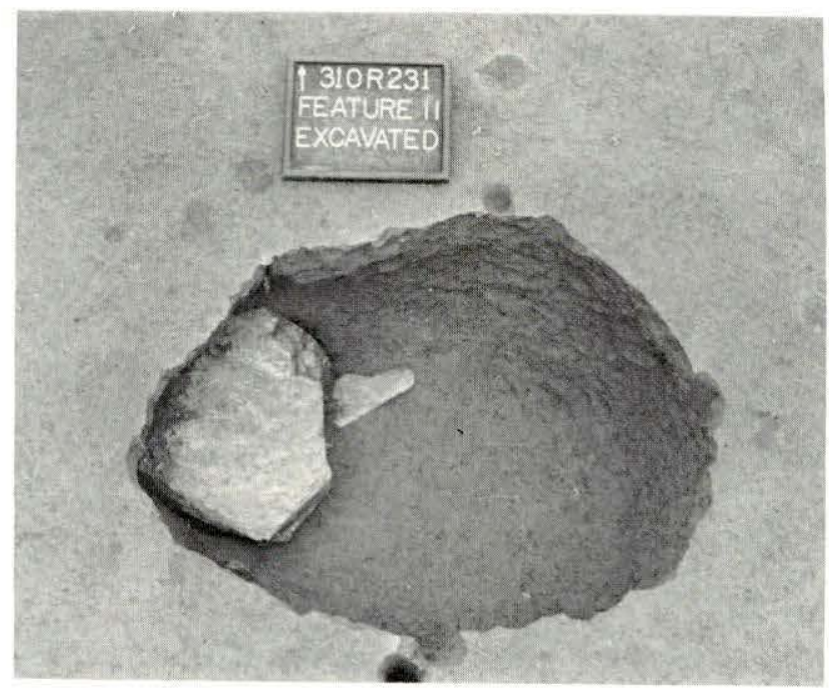

Figure 3.34

Feature 11 at the Fredricks site, after excavation.

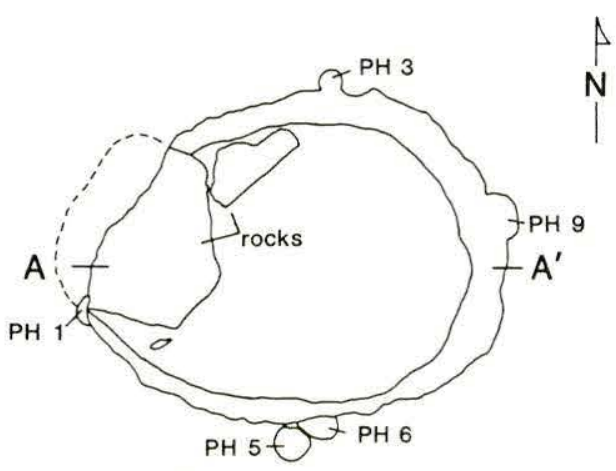

PLAN (excavated)

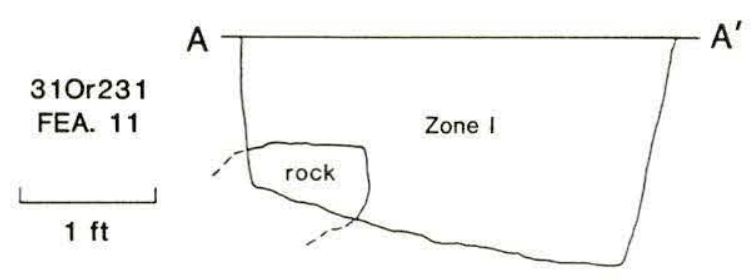

PROFILE

Zone I - medium dark brown sandy loam

\section{Figure 3.35}

Plan and profile of Feature 11 at the Fredricks site.

rather that it may have functioned as a cache for non-food items. 
Feature 11 contained a single zone of brown sandy clay loam with charcoal and fired clay. This fill contained only a small amount of plant remains $(0.01 \mathrm{~g}$ of hickory nutshell per 101 flotation sample). Animal remains were present but in very poor condition; only 13 of 94 fragments were identified, all of which were deer remains. Other cultural materials consisted of potsherds, stone flakes, rocks, kaolin pipe fragments, and glass trade beads. The fill in this feature contained the fewest cultural remains of all the features excavated at the Fredricks site, and the sloped bottom of the pit gave it a somewhat "unfinished" appearance. It may be that it was perceived as unsuitable once the rock was encountered and efforts to remove it failed.

Feature 12 (Figures 3.36-3.37) was a sma11 deep oval pit located to the northeast of Structure 3, between it and the opening in Palisade 1. It was $3.4 \mathrm{ft}$ long, $3.2 \mathrm{ft}$ wide, and $1.4 \mathrm{ft}$ deeper than the top of subsoil. The depth/diameter ratio was 0.51 , indicating that it could have functioned as a storage facility. The location of this pit is somewhat enigmatic because of its proximity to the palisade entrance, in what was probably an area of heavy traffic. It is possible that this feature represents a small soil recovery facility rather than a storage pit.

Feature 12 contained two zones of fill. The upper zone, Zone I, was a dark reddish-brown sandy loam containing a variety of historic trade items, aboriginal potsherds, charcoal, fired clay, charred plant food remains, and a large quantity of animal bone. The animal remains consisted of box turtle, deer, and bear. The plant food remains were mostly hickory nutshell, with a small amount of acorn shell, peach pit, and corn. Less wood charcoal was present than in the other features. Among the trade items were glass beads, copper wire, and the handle and blade of an iron knife. This zone was relatively thin and confined to the central area of the feature. The composition and texture of this fill suggests that it originated as household debris representing a variety of activities.

Zone II was a homogeneous deposit of dark brown sandy loam mottled with subsoil. Numerous animal bone fragments, charred plant remains, wood charcoal, historic trade items, lithic artifacts, potsherds, and plant remains were recovered in this zone. The animal bone consisted of deer, box turtle, turkey, squirrel, and bear. The plant food remains were mostly hickory nutshell with a small amount of corn and cucurbit. The homogeneous brown color of the fill, the small amount of wood charcoal, and the diversity of animal and plant species suggest that this fill was redeposited midden and humus.

Feature 13 (Figures 3.38-3.39) was an ova1 pit $2.8 \mathrm{ft}$ long, $2.4 \mathrm{ft}$ wide, and $1.47 \mathrm{ft}$ in depth below the top of subsoil. It was located

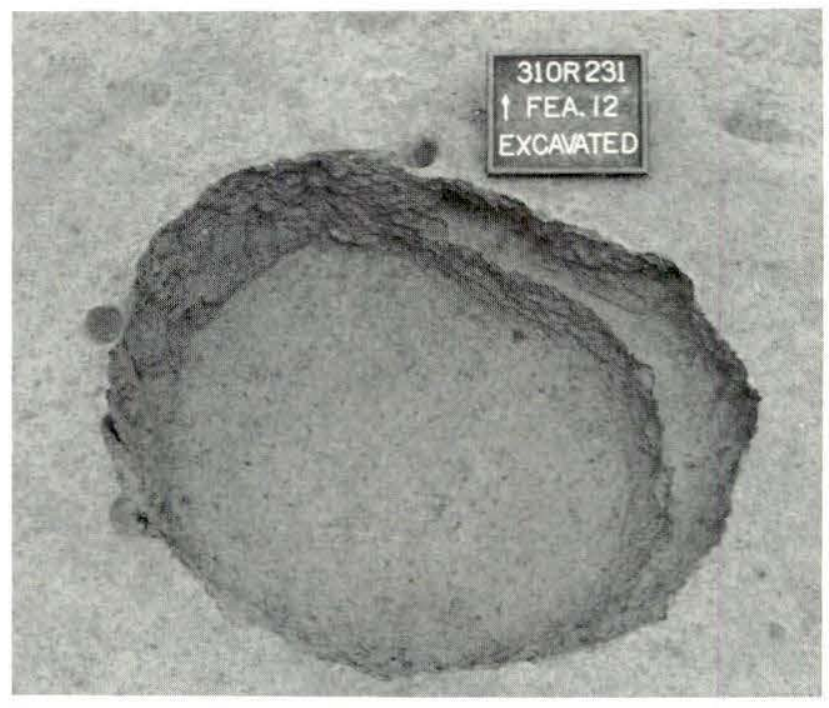

Figure 3.36

Feature 12 at the Fredricks site, after excavation.

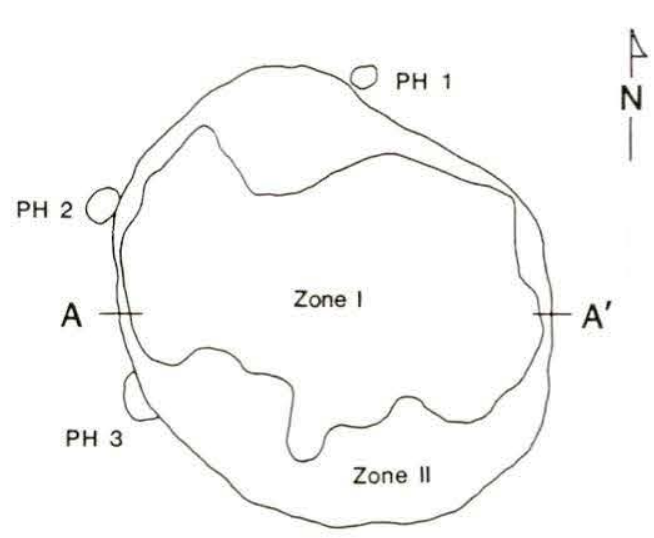

PLAN (top of subsoil)

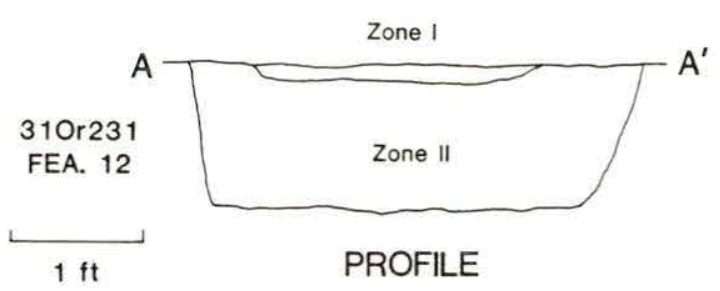

Zone I - dark reddish-brown mottled sandy loam

Zone II - dark brown mottled sandy loam

Figure 3.37

Plan and profile of Feature 12 at the Fredricks site.

adjacent to, and east of, Structure 3. This area has numerous postholes that may define a portion of a structure extending beyond the limits of the current excavations. Feature 13 
was the smallest of the features excavated at the Fredricks site. It had a depth/diameter ratio of 0.85 and an estimated original volume of $12 \mathrm{ft}^{3}$.

Two zones of fill were present in this feature. The upper zone (Zone II) was a thin deposit of mottled yellow clay which contained only a sparse amount of cultural material other than charcoal, animal bone, and plant remains. The animal species represented were deer and raccoon. The plant food remains consisted of hickory nutshell, peach pits, and a trace of corn. The lower zone of fill (Zone I) was a dark brown, highly organic, loam containing diverse animal and plant food remains, abundant wood charcoal, potsherds, lithic artifacts, and a variety of European trade items (see Carnes, this report). Many of these items, including a brass bell, metal fishhook, brass wire, and aboriginal as well as kaolin pipes, were more complete and less worn out than most of the trade artifacts, except those from burials. The brass bell appeared to have been associated with one of the two complete turtle carapaces recovered from this zone.

Feature 13 had a ledge about $0.2 \mathrm{ft}$ wide around the western half of the feature about $0.65 \mathrm{ft}$ above the pit floor. Six large rocks were found on the pit floor, two of which showed evidence of intentional modification (see Tippitt and Daniel, this report). One of these had been bifacially modified along the edges,

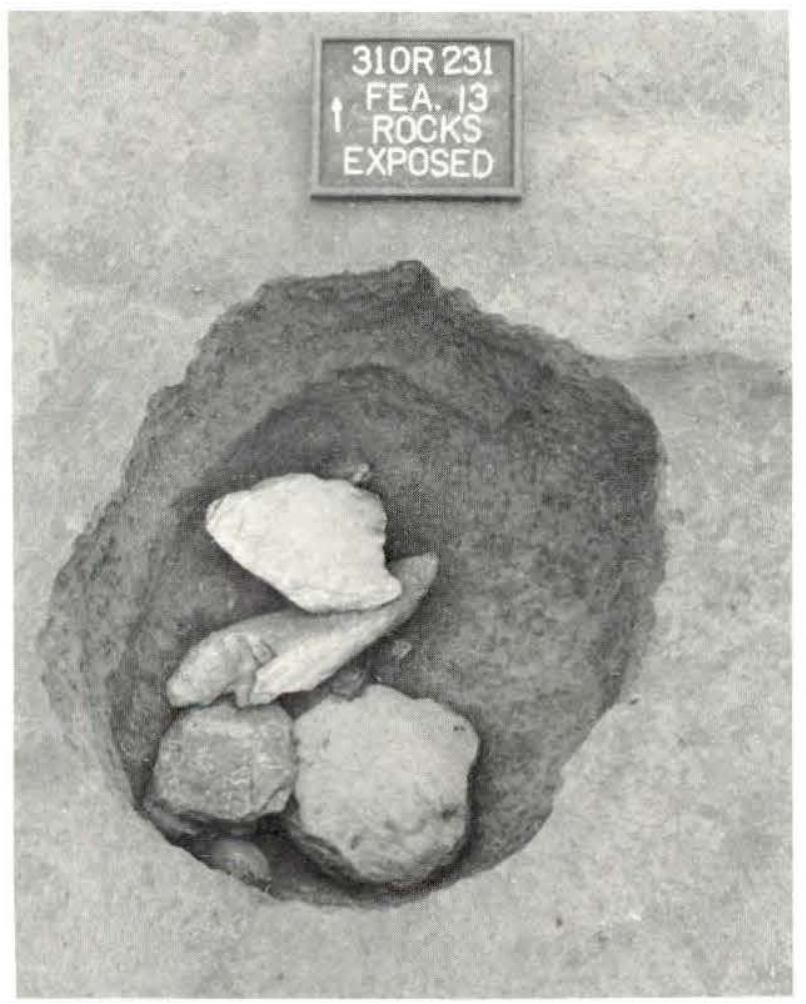

Figure 3.38

Feature 13 at the Fredricks site, after excavation. creating a shape that conformed to the pit wall. The six large rocks seemed to fit together and may have originally formed a cover (at about the level of the ledge) over items cached in the bottom of this pit.

The animal remains from Zone I represent the most diverse group of species found in any of the nonburial features present. These species consist of box turtle, deer, passenger pigeon, turkey, frog, gray squirrel, fox squirrel, raccoon, and bear. This assemblage of animal remains is very similar to that found in several of the zones of fill from the burials at the site. The closest similarity appears to be with the upper burial fill from Feature 3/Burial 5. Fourteen species are represented in the combination of Feature 13, Zone I and Burial 5, Zone II. Nine of these species are present in both zones. Thirteen species are represented in the combination of Feature 13, Zone I and Burial 5, Zone I. Eight of these are present in both zones. The plant food remains show the same association between zones of fill. In both of them, hickory, peach, corn, and grapes are the only plant foods represented.

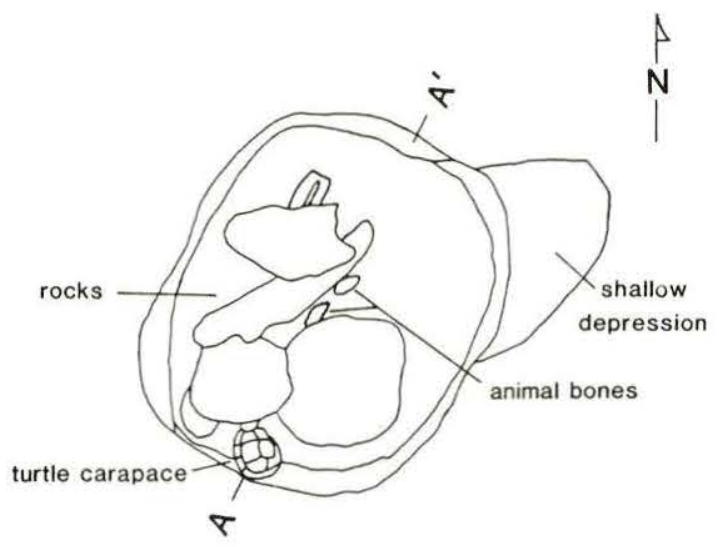

PLAN (excavated)

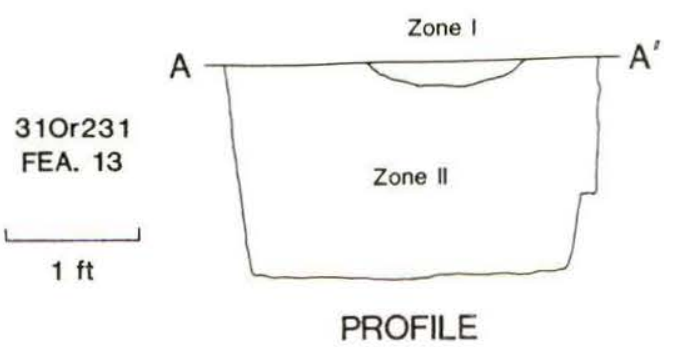

Zone 1 - medium dark brown loam

Zone II - mottled yellow clay

Figure 3.39

Plan and profile of Feature 13 at the Fredricks site. 
The food remains from Zone I of Feature 13 suggest that the feature was filled at the time of the ritual feasting associated with the burial of the adult male in Burial 5 .

\section{Structures}

Three structures were observed at the Fredricks site (Figure 3.40). Each of these structures was different from the others in form and with respect to associated features. Table 3.9 summarizes the formal attributes of these structures.

Structure 1 (Figures 3.41-3.42) was defined by an oval wall trench with an opening to the southwest. This structure was oriented NE-SW and had overall dimensions of $14 \times 12.5 \mathrm{ft}$. The wall trench varied in width from $0.60-1.00 \mathrm{ft}$, with an average of about $0.80 \mathrm{ft}$. The opening in the southwest end was just under $2.0 \mathrm{ft}$ wide. The fill of the wall trench, a mottled brown soil, contained glass trade beads, a fragment of possible mirror glass (see Carnes, this report), plant food remains, and a small amount of wood charcoal.

No postholes or postmolds were observed in the wall trench. The absence of remains of posts suggests that the structure was a domeshaped building constructed of small flexible poles which were pulled together at the top. The resulting tension would have held the poles against the outer wall of the trench.

The large pit (Feature 9) at the center of the floor of Structure 1 functioned as a fire pit. The small size of the structure, $110 \mathrm{ft}^{2}$ (including the area taken up by Feature 9), indicates that it was not a dwelling. It is interpreted to be the remains of a sweat lodge. Such structures are frequently mentioned in the early historic accounts for this region (e.g., Wright 1947:218-219; Jones 1956:55; Lefler 1967:48,55; Myers 1970:49). John Fontaine, a traveler who visited the "Saponi" town near Fort Christanna in 1716, when some of the Occaneechi were residing there, described the sweathouses as follows:
Between the town and the river side there are several little huts built with wattles in the form of an oven with a small door in one end of it. These wattles are plaistered without side with clay very close, and they are big enough to hold a man. They call those houses sweating houses, for when they have any sickness they get 10 or 12 pebble stones which they make very hot in a fire and when they are red hot they carry them in those little huts and the sick man or woman goes in naked, only a blanket with him and they shut the door upon them and there they sit and sweat until they are no more able to support it and then they go out naked and immediately jump into the water over head and ears. This is the remedy they have for a11 distempers (Alexander 1972:97).

Architecturally, Structure 1 conforms very well to this description. Daub and fired clay are very abundant in the vicinity of Structure 1 (Figure 3.43), indicating that Structure 1 was daub-covered.

A major discrepancy between the ethnohistoric description and the archaeological remains appears to be the way in which heat was introduced into the structure. The historic accounts describe a process by which hot stones, heated outside of the sweat lodge, were brought inside where water was poured over them to create steam. The fire pit inside of structure 1 is somewhat anomalous in this respect. It is suggested that Feature 9 was originally excavated not for use as a fire pit, but probably to be used as a storage facility. The large inclusive rock within the pit may have been perceived as potentially useful as a substitute for more traditional ways of heating a sweat lodge. The lower deposits of this feature show that repeated fires were built in the pit. Such fires would have heated the large rock and there would have been no need to build fires outside. It then would have been necessary only to bring in water to make steam.

Structure 2 (Figure 3.40) was a rectangular

Table 3.9

Summary of structure attribute measurements at the Fredricks site.

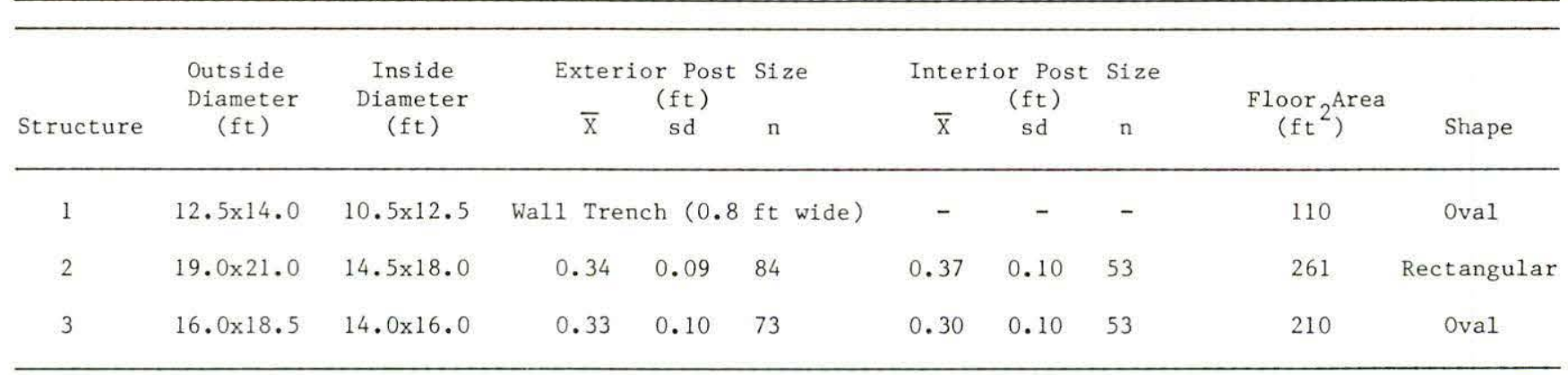




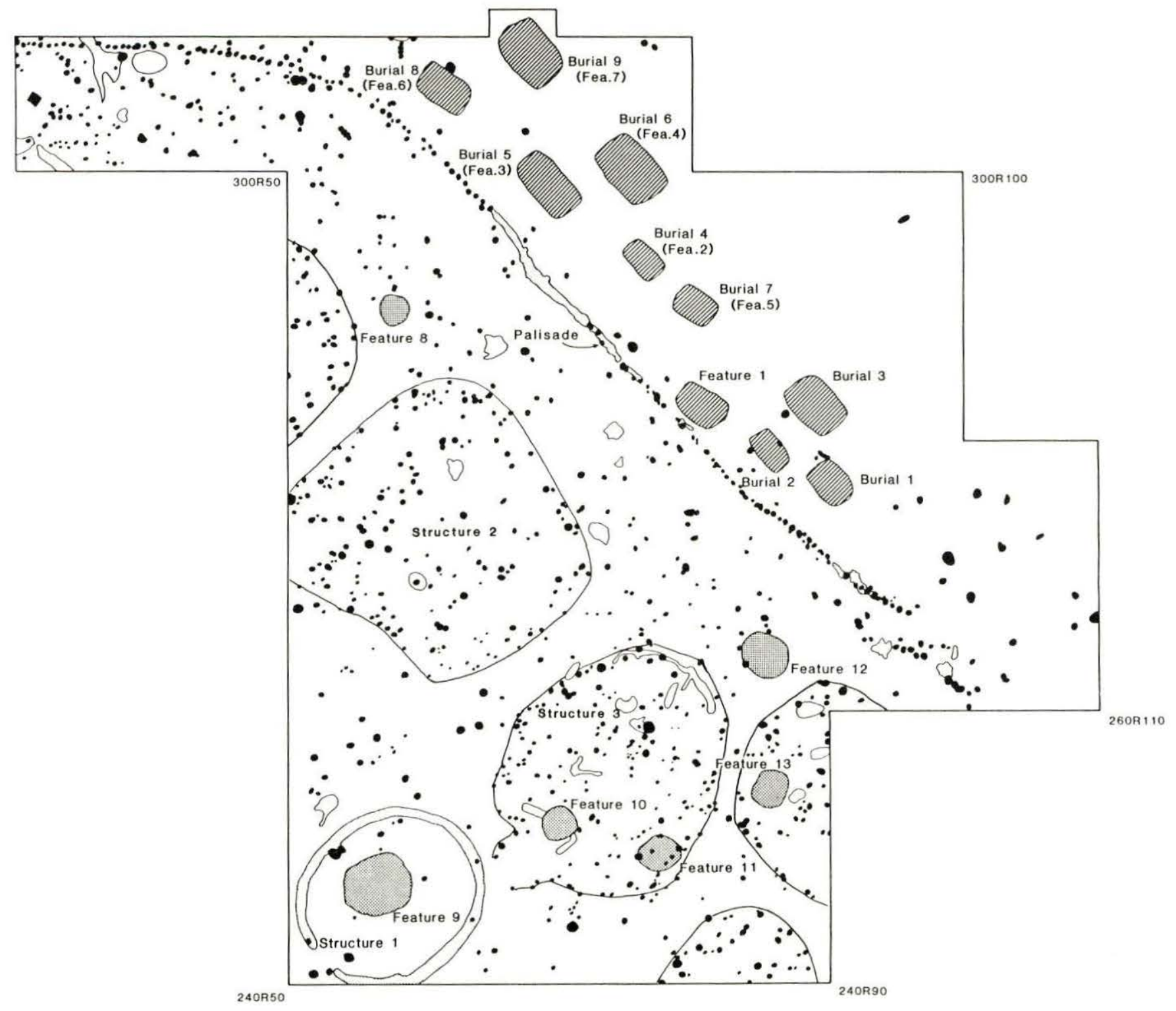

FREDRICKS SITE

Plan of 1983-84 Excavations

N

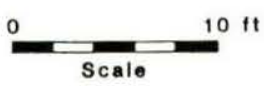

Figure 3.40

Plan of architecture and features at the Fredricks site. 


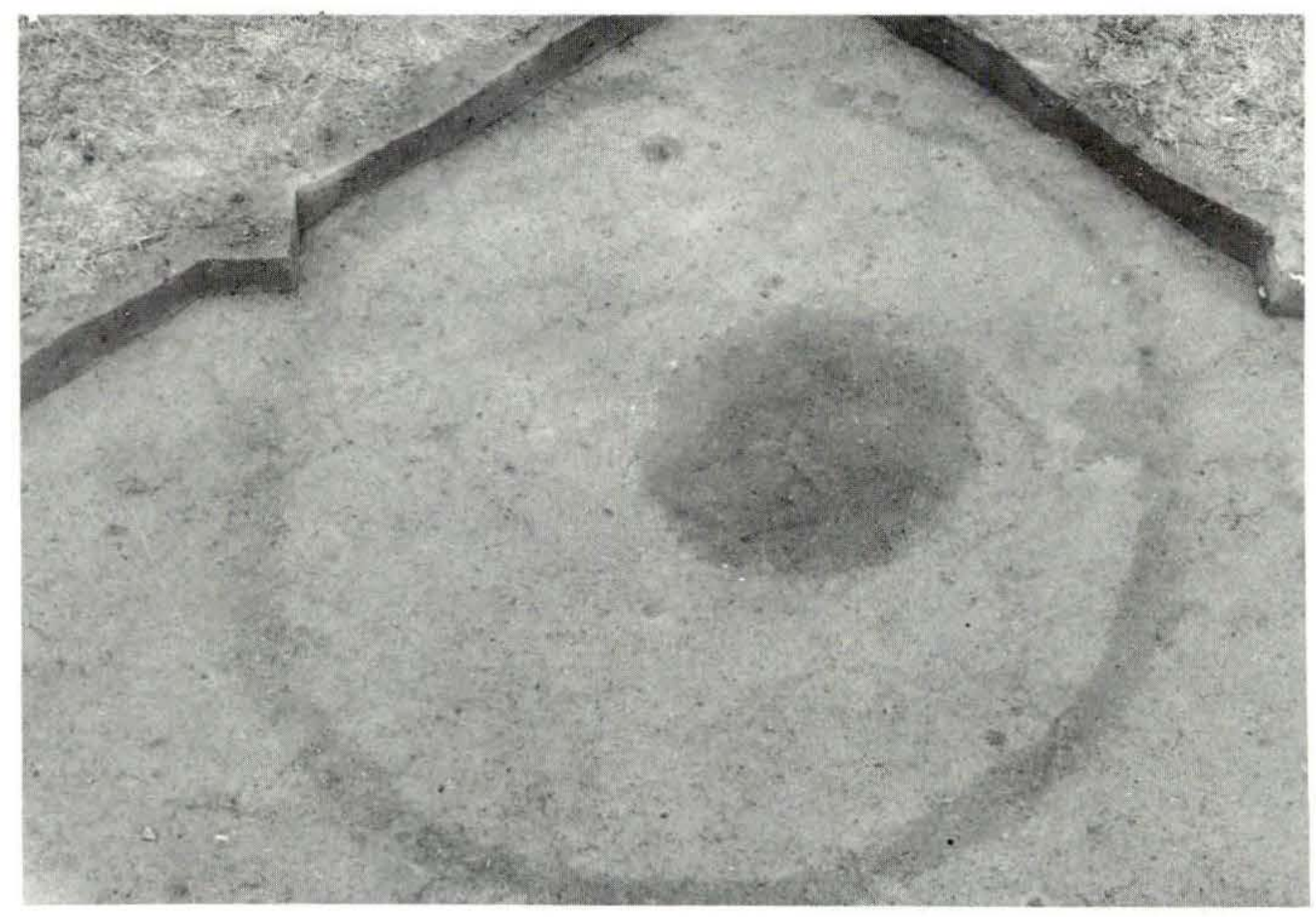

Figure 3.41

Structure 1 and Feature 9 at the Fredricks site, prior to excavation.

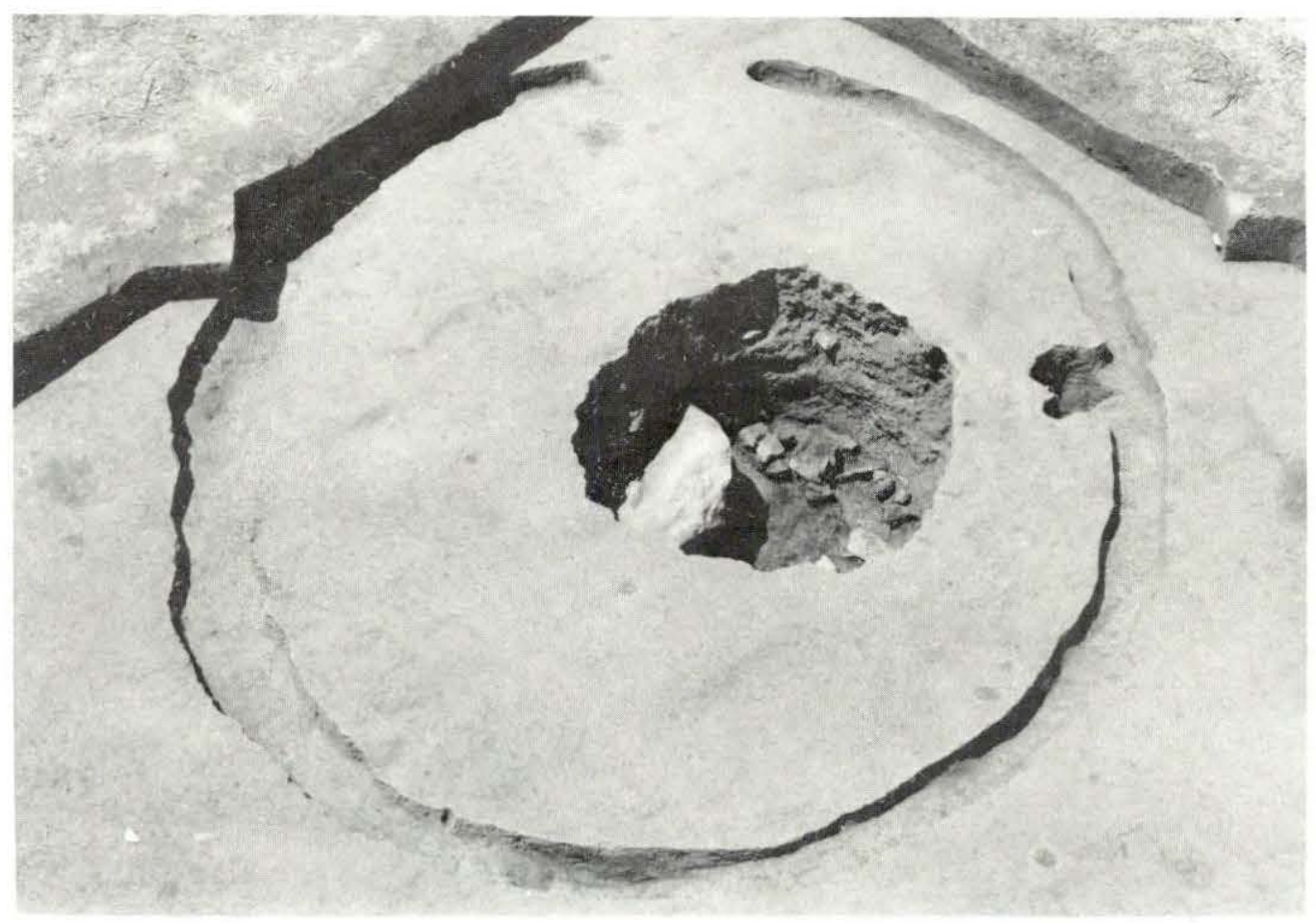

Figure 3.42

Structure 1 and Feature 9 at the Fredricks site, after excavation. 
structure measuring $19.0 \times 21.0 \mathrm{ft}$ and located about five feet north of Structure 1. It was of a single-post construction with no clearly defined entrance. The postholes defining its exterior walls were rather small with an average diameter of $0.34 \mathrm{ft}$. They were irregularly spaced about one-half foot apart. The exterior wall pattern ranged from two to three posts wide, suggesting that the structure had been maintained for some time, with replacement of some posts. The interior postholes were slightly larger than the exterior ones, averaging about $0.37 \mathrm{ft}$ in diameter. These postholes formed a rectangular pattern concentric with the outside wall pattern, and probably were roof supports and supports for benches and sleeping platforms around the inside perimeter of the structure. Although no hearth was observed within the structure, an area relatively clear of posts at the center of the floor may have once contained a hearth.

Structure 3 (Figure 3.40) was an oval structure located two feet to the southeast of Structure 2. This structure measured $18.5 \mathrm{ft}$ by $16.0 \mathrm{ft}$ on the outside with its long axis oriented parallel to Structure 1. An opening in the posthole pattern on the southwest end probably represents an entrance. The structure seems to have been of single-post construction, although a discontinuous band of dark mottled soil conforming to the northeast portion of the exterior wall may be a remnant of a wall trench that has been mostly plowed away. Feature 10, a deep storage pit, was located near the entrance of the structure. Although several of the exterior wall posts intruded into the southeastern side of Feature 11, there were also exterior wall posts outside the feature. The presence of these latter posts may indicate that the feature was associated with an early phase of the structure and was filled before the structure was abandoned.

The outside wall postholes of Structure 3 had an average diameter of $0.33 \mathrm{ft}$. The interior posts were generally smaller, averaging about $0.30 \mathrm{ft}$ in diameter. These postholes were concentrated around the inside perimeter of the structure and probably served as supports for sleeping platforms. The size and shape of this structure suggests that it was an oval, domed structure similar to the one observed at the Mitchum site, and to those described in the early historic accounts of the Piedmont Indians (e.g., Lefler 1967:180-182).

Material preliminarily classified as daub is relatively abundant at the Fredricks site, with a heavy concentration in the vicinity of Structure 1 (Figure 3.43). Aside from the concentration around the sweat lodge, "daub" is distributed uniformly across the portion of the site inside the palisade. While there are not separate concentrations conforming to the posthole patterns defining Structures 2 and 3, the presence of daub as a linear concentration inside the palisade and in the vicinity of the

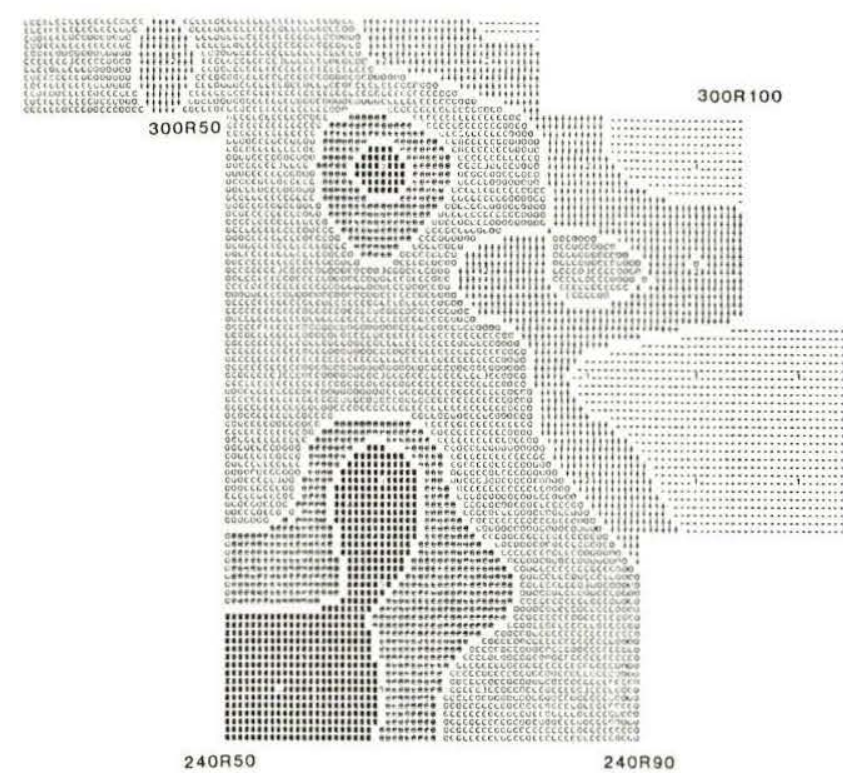

Figure 3.43

SYMAP of daub in the plowzone at the Fredricks site.

structures suggests that daub was used to cover these structures.

Parts of three additional structures seem to be present in the excavated portion of the site. One of these structures was located northwest of Structure 2 and was defined by a linear pattern of postholes parallel to and 1.5 ft away from Structure 2. This pattern appeared to turn to the northwest at about $10-12 \mathrm{ft}$ from the palisade and may represent the corner of another rectangular structure. The northeast corner of another possible structure was partially defined in the extreme southwest corner of the excavations where a linear cluster of postholes was observed about $2.5-3.0 \mathrm{ft}$ away from, and parallel to, Structure 3 .

A third area with high posthole density was observed to the east of Structure 3. These postholes may define part of a structure that extends beyond the excavation. Feature 13 was located within this area.

\section{Palisade 1}

Aside from the structures, the major architectural feature at the Fredricks site is a palisade, $90 \mathrm{ft}$ of which has been exposed in the north and northwestern part of the excavations (Figures 3.24-3.26). This linear feature separates the cemetery from the habitation area of the site.

The section of the palisade observed in the excavations consisted of 135 small postholes with a mean diameter of $0.35 \mathrm{ft}$. Some of these postholes were observed within a narrow wall trench. Only discontinuous portions of the wall trench were observed and it is uncertain whether 
the entire palisade originally consisted of posts erected within a wall trench, or if only portions of it were constructed in this manner. The wall trench was not observed in the extreme northwestern area of the excavations, even though it appeared that the village remains were better preserved in that area.

A single entrance, just southwest of the cemetery, was observed in Palisade 1. Associated with the entrance were a series of paired postholes that formed an arc extending outward from just south of the entrance and towards the first group of burials in the cemetery. This posthole pattern may represent a tower or similar feature, associated with the village entrance.

\section{Auger Testing}

In conjunction with the excavations undertaken at the Fredricks site, systematic auger testing was conducted to obtain subsurface data on unexcavated portions of the site.

A 1-in Oakfield soil-sampling tool was used to obtain soil cores at $2.5 \mathrm{ft}$ intervals on the site grid. Limited testing using this technique was undertaken prior to the 1984 excavations and was found to be $100 \%$ effective in locating features encountered later during excavation.

An area of $9200 \mathrm{ft}^{2}$ (beyond the area excavated in 1983-1984) was tested using the soil auger. This procedure produced 120 positive tests, 41 of which probably represent pit types of features, and 79 of which probably represent postholes, shallow subsurface disturbances, or remnants of midden. The distribution of the positive tests is shown in Figure 3.44 .

Figure 3.45 shows the relative density of the suspected pit features based on the results of the auger tests. (also included is the feature density in the excavated area as it would have appeared through auger tests placed in this area). It does not depict the density of numbers of individual features because positive tests adjacent to each other likely intruded into the same feature. The sampled area was divided into 5-ft square units for the purpose of computer mapping (SYMAP), and the number of positive tests encountering pit features for each unit was determined. Where positive tests were on boundaries between units the value of that test was divided between the units. The range of values for positive tests per unit was between 0.00 and 2.00 .

When feature density is mapped at this scale some large patterns emerge that relate to the internal structure of the Fredricks site. The cemetery stands out as a prominent feature as does the sweat lodge. Features 11-13 appear to be the northern most end of a band of features extending toward the southwest. Areas devoid of features are depicted on both sides of this arc and probably relate to the location of structures, and activity areas.

A different pattern of feature density and distribution is apparent to the west of the excavated area. The predicted density of features in this part of the site is much greater than in the area that has been excavated. This pattern suggests that the occupation of the site may have been denser, or is better preserved, to the west. The absence of features southeast of the present excavations suggests that the palisade is turning fairly sharply toward the south. Thus, the 1983-84 excavations appear to be on the eastern periphery of the village.

Several relatively open areas are also evident. They probably represent locations of additional structures, especially in areas west of the excavations in line with Structures 2 and 3. The open area to the southwest of Structure 1 may represent a plaza in the center of the village.

\section{Summary}

Investigations undertaken thus far suggest that the Fredricks site represents a village site of the Occaneechi Indians during the Middle Contact period. The village appears to have been surrounded by a palisade of small posts, and to have had a cemetery on the outside, and adjacent to, the palisade. Domestic structures, located about 10-12 ft inside of and parallel to the palisade, were rather small (210-260 $\left.\mathrm{ft}^{2}\right)$ and closely spaced. Two forms of domestic structures were present--rectangular and oval. The rectangular structure observed in the excavation did not have any features associated with it. Two features were located within the oval structure--a large, deep storage facility and a smaller pit. Additional pits were located outside and around the structure.

It is possible that Structures 2 and 3 represent summer and winter houses. It can probably be assumed that storage pits were designed for concealment of materials (DeBoer 1984; Ward 1985). Caching facilities would have been useful during periods when men were away on hunting or trading trips or when there was threat of a raid. They may not have been necessary during the spring and summer when agricultural activities dominated subsistence activities. Structure 3 may represent a winter house, and Structure 2 a summer shelter, possibly an open arbor such as described by Lawson in the early 18 th century.

They have other sorts of Cabins without Windows, which are for their Granaries, Skins, and Merchandizes; and others that are covered overhead; the rest left open for the Air. These have Reed-Hurdles, like Tables, to lie and sit on, in Summer, and serve for pleasant Banqueting-Houses in the Hot Season of the Year (Lefler 1967:182).

Storage facilities were used as trash 


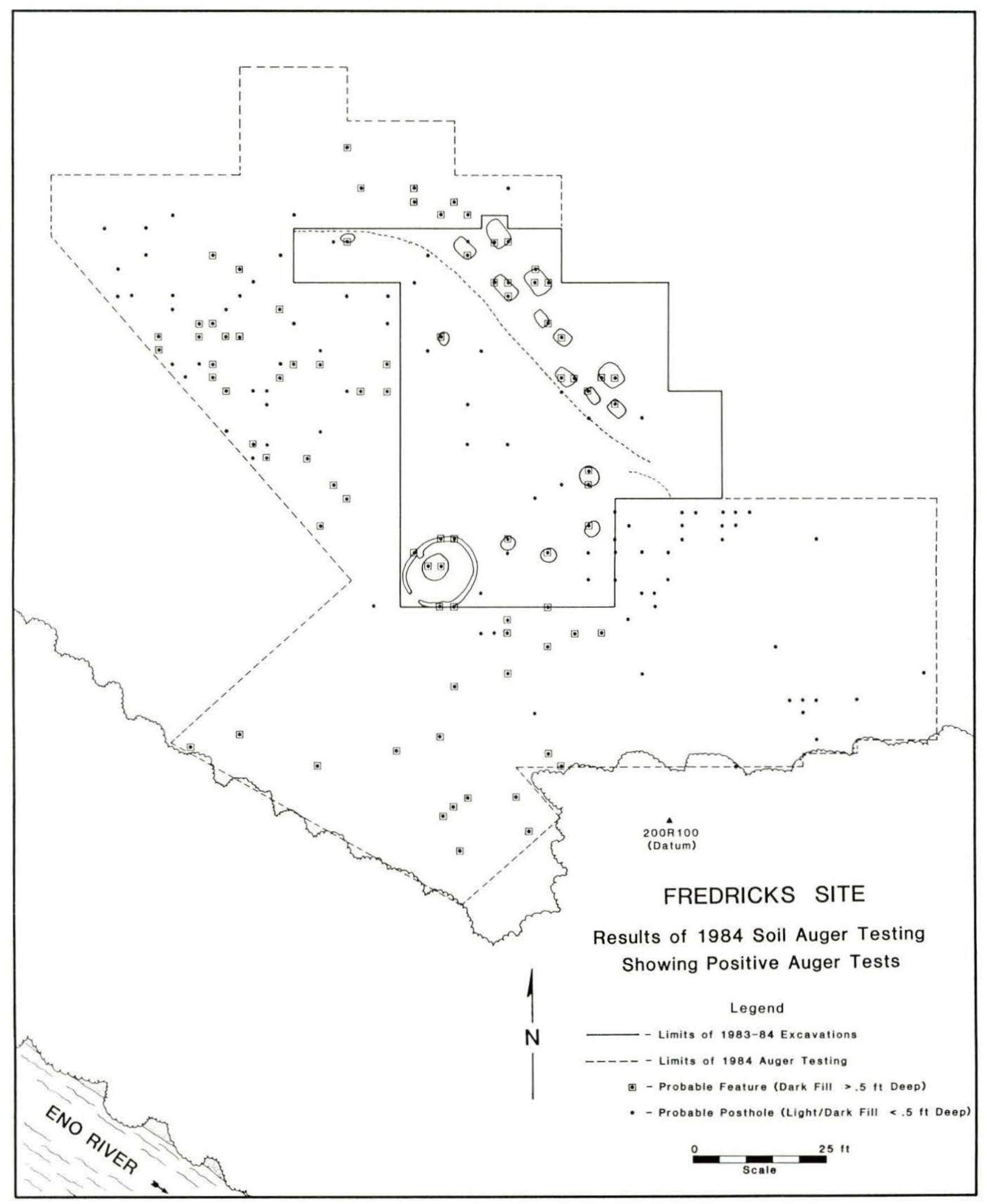

Figure 3.44

Map showing the results of soil auger testing at the Fredricks site. 


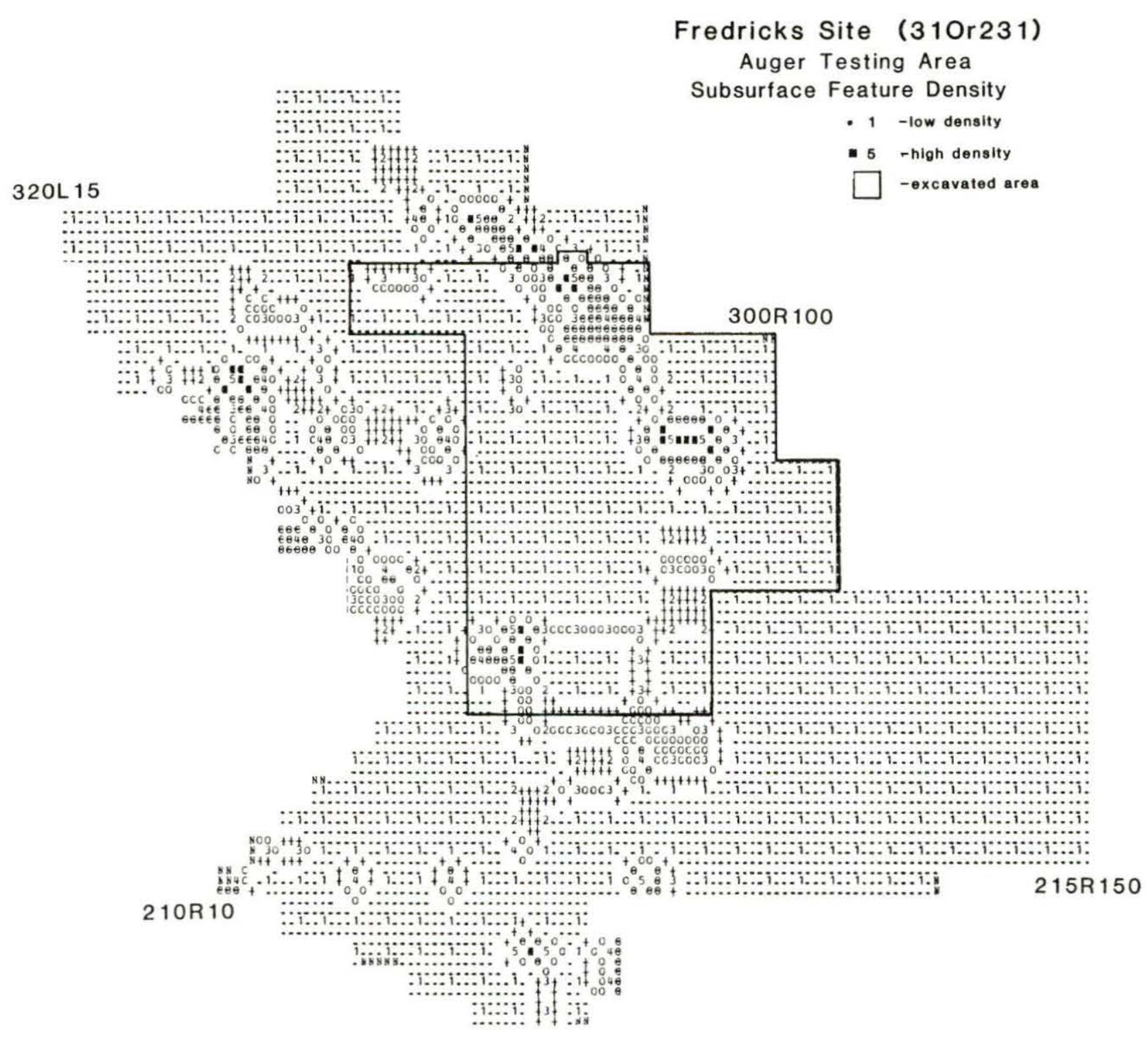

Figure 3.45

SYMAP of features at the Fredricks site, based on the results of auger testing.

receptacles when they were no longer needed. It is likely that other refuse was disposed of around the periphery of the village. The plowzone distribution of aboriginal ceramics from the historic occupation (see Davis, this report) indicate that household waste was discarded outside of houses, either in pits, against the palisade, and just outside the village entrance (Figure 3.25).

It is not yet entirely clear how the village was structured spatially. Four distinct activity areas are evident (Figure 3.46), but a larger area will have to be investigated in order the substantiate this interpretation.

The outer area consists of a long and narrow (approximately $12 \mathrm{ft}$ wide) special purpose area which was reserved for mortuary activities. This area is parallel to the palisade. Data from this context have provided valuable information on ritual mortuary behavior, technology, subsistence, and biology of these peoples (see Ward, Carnes, Holm, Gremillion, and Wilson, this report). Comparisons of characteristics of the pitfill from these burials and the features suggest that ritual feasting contributed to the formation of the burial fill.

The second activity area consists of a narrow zone between the palisade and the structures. The density of postholes observed in the extreme northwest of the excavation suggests this zone may be confined to the part of the site already excavated. If this is proven by future work, then it might be profitable to consider this area an activity area associated 


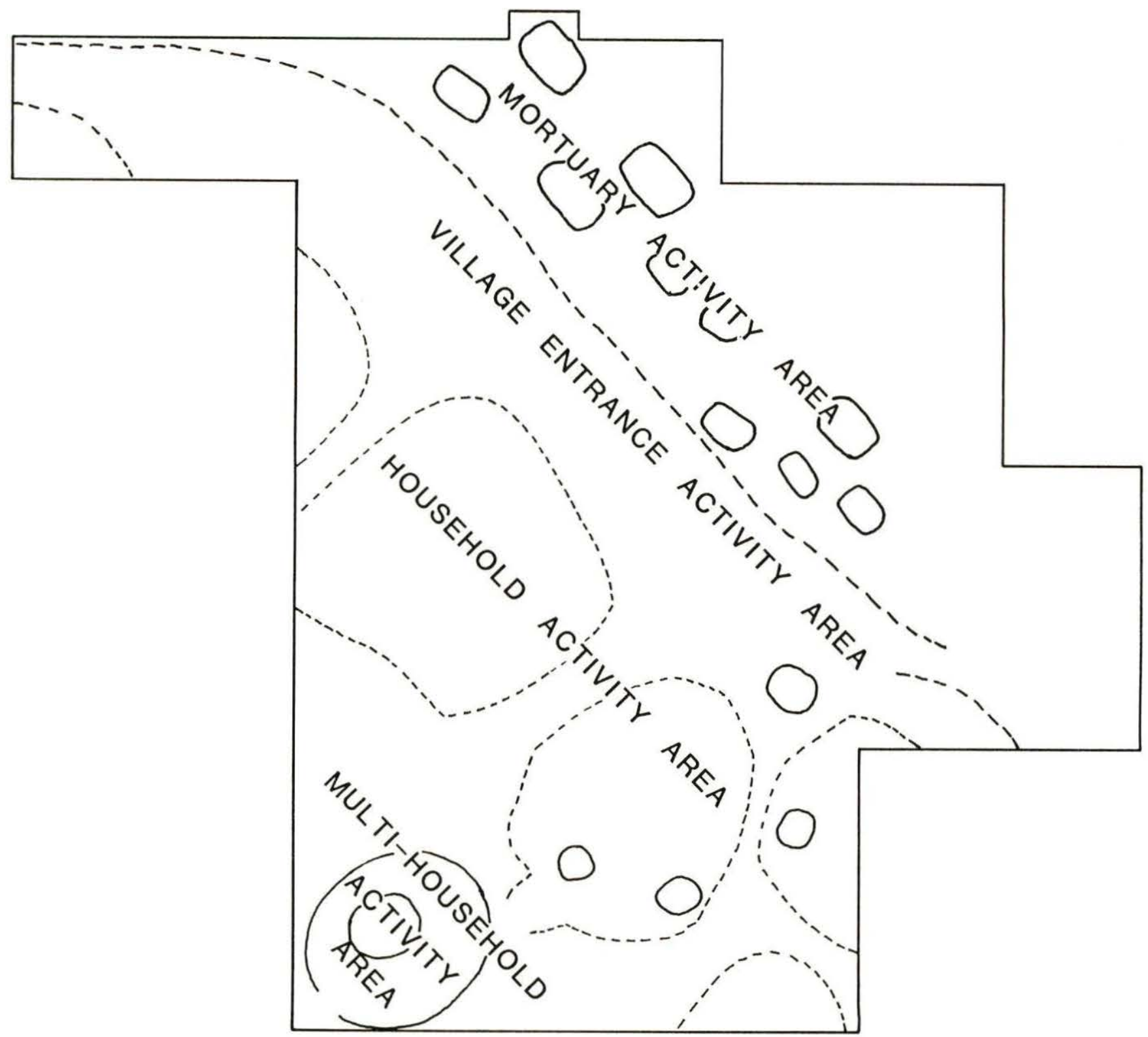

Figure 3.46

Excavation plan of the Fredricks site showing activity areas.

with the structures in this vicinity rather than as an area used as a passageway around the village. There is some evidence that refuse may have been disposed of in the part of this area nearest the palisade.

The third activity area is identified as a domestic use area and is defined by several domestic structures and storage facilities. It is about $22 \mathrm{ft}$ wide and appears to extend about $30 \mathrm{ft}$ into the village. The associated activities were probably related to a variety of day-to-day and seasonal tasks that took place within and around houses. Such behavior is reflected in the trash deposits recovered from abandoned storage facilities. The many small pits suggest that surplus was organized at a household level, and that caching of materials was a necessary precaution during times when hunting and warfare necessitated seasonal abandonment of the village by at least a portion of the population.

Because of the orientation of the present excavation, only a small area interior to the third activity area has been investigated. This fourth area is defined by the presence of a sweat lodge and represents a different set of activities than those in the domestic area. The area probably was reserved for activities involving members of several different households or the entire village and may be the edge of a plaza. According to historic accounts, sweat lodges functioned as therapeutic facilities. Beverly (Wright 1947:218-219) noted in 1705 that among the Virginia Algonquin Indians 
"sweating-houses" were maintained by a village "doctor." He described these facilities as follows :

in every Town they have a Sweating-House, and a Doctor is paid by the Publick to attend it. They commonly use this to refresh themselves, after they have been fatigu'd with Hunting, Travel, or the like, or else when they are troubl'd with Aches, or Pains in their Limbs. Their method is thus, the Doctor takes three or four large stones, which after having heated red hot, he places 'em in the middle of the stove, laying on them some of the inner Bark of Oak beaten in a Mortar, to keep them from burning. This being done, they creep in six or eight at a time, or as many as the place will hold, and then close up the mouth of the Stove, which is usually made like an Oven, in some Bank near the Water side. In the mean while, the Doctor, to raise a Steam, after they have been stewing a little while, pours cold water on the Stones, and now and then sprinkles the Men to keep them from fainting. After they have sweat as long as they can well endure it, they sally out, and (tho it be the depth of Winter) forthwith plunge themselves over Head and Ears in cold water, which instantly closes up the Pores, and preserves them from taking cold...they instantly recover their Strength, and find their Joynts as supple and vigorous as if they never had travel1'd, or been indispos'd.

They also probably functioned as social gathering places and as individual retreats (Alexander 1972; Myers 1970; Lefler 1967). The social context is interpreted to be at the level of multiple households. It is doubtful that every household maintained such a facility.

One can hypothesize that additional activity areas will be characteristic of this site. The historic descriptions of towns are filled with accounts of gaming fields (Cumming 1958: 26-27), and plaza areas (Alexander 1972:96-97; Morton 1956:55) used for activities involving larger portions of the village population.

In summary, the excavation of the Fredricks site has provided information allowing a preliminary interpretation of the spatial organization of the village. If the area of present excavation is representative of the entire site, the data suggest that this village was densely occupied regardless of its total size. It is only possible to estimate the village size within a range of area as it is not known as yet whether the palisade extended to the river edge or whether it enclosed a smaller area away from the river. Nevertheless, it appears that between $8 \%$ and $20 \%$ of the site has been excavated thus far. From this range, it can be estimated that between 10 and 25 houses were present, along with other burial areas and sweat houses, and possibly other kinds of special-function areas. Overall, the village spatial organization appears to be very similar by Fontaine in 1716. He described the town as follows :

(April 17, 1716) The fifth day. After breakfast I went down to the Saponey town, which is about a muskey shot from the fort. This town lieth in a plain by the river side. I walked round the town to view it. The houses join all the one to the other and altogether make a circle. The walls of their houses are large pieces of timber, which are squared and being sharpened at the lower end, they put above two feet in the ground and about seven feet above the ground. They laid them as close as they could the one to the other, and when these posts are all fixed after this manner then they make a sort of roof with rafters and cover the house with oak or hickory bark, which they strip off in great flakes, and lay it so closely that no rain can come in. Some of their houses are covered in a circular manner which they do by getting long saplings and stick each end in the ground and so cover them with bark, but there is none of the houses in this town so covered. There is three ways of coming into this town or circle of houses which are passages of about 6 feet wide between two of the houses. All the doors of the houses are on the inside of the ring and it is very level withinside which is in common with all the people to divert themselves. There is also in the centre of the inside circle a great stump of a tree. I asked the reason they left it stand, and they informed me that it was for one of their head men to stand on when he had any thing of consequence to relate to them, that being raised, he may better be heard.... Between the town and the river side there are several little huts.... They call those houses sweating-houses.... (Alexander 1972:96-97).

Identification of these activity areas within the Fredricks site is essential. Each of these areas was utilized for different activities by social groups of different size and composition. Features associated with each of these areas contained information relevant to different problems and questions. For example, ritual behavior represented in the first activity area is reflected in the burial remains. The diversity of animal and plant remains from the fill in these features may not reflect normal subsistence activities. Nevertheless, they probably do represent the breadth of the resources available at any one time. Questions regarding household activities can be addressed through artifacts, structures, and features from the domestic activity areas. Similarly, questions about non-mortuary activity involving groups larger than the household, or comprised of individuals from several households, may be 
addressed by data derived from the fourth activity area, perhaps characterized by special purpose facilities like the sweat lodge.

\section{COMPARISONS AND CONCLUSIONS}

Comparisons of data from the three sites used in this study are limited in some respects by differences in the scale of excavation at each site. The Mitchum site has received the least investigation so far, and the scale of excavation makes it impossible to even speculate on the overall configuration of the village. The Wall site has received the largest amount of excavation, yet a large majority of this work was undertaken when data recovery techniques and the questions being asked were different from what they are today. The Fredricks site received the most attention during the 1983-1984 field seasons, and the quality of the data is excellent. However, the excavations have exposed only a portion of the village. Nevertheless, some comparisons between the sites are possible. These comparisons are presented below along with interpretations of what the differences observed between the sites may mean in terms of the research questions posed at the onset of this study.

Differences in the kinds and amounts of features at the three sites undoubtedly reflect cultural changes throughout the period from A.D. 1500-1720. Figure 3.47 shows the percentages of features by class observed at the three sites. These data should be used cautiously, however, since the different scales of excavations at the sites may mean that additional feature classes will be found in parts of the Mitchum and Fredricks sites not yet investigated. The area of the Wall site investigated is sufficiently large that all of the feature types present at this site are represented in the sample.

Feature Class I consists of small shallow basins that make up $28.6 \%$ of the features at the Wa11 site, $8.3 \%$ at the Mitchum site, and none at the Fredricks site. These features probably represent food processing facilities, although clay recovery may also have been a function of those basins at the Wall site. It is not known what kinds of foods were processed using these facilities, but the decline in their use may be related to the decreased emphasis on acorn utilization (see Gremillion, this report). Acorns require a leaching process to be edible and small basins could have functioned in this manner (Driver 1969:91).

Feature Class II consists of large shallow basins and is represented by $21.4 \%$ of the features at the Wall site and $16.7 \%$ of the features at the Mitchum site. No Class II features were observed at the Fredricks site. These features probably represent two functional classes at the Wall site, large-scale soil-recovery facilities
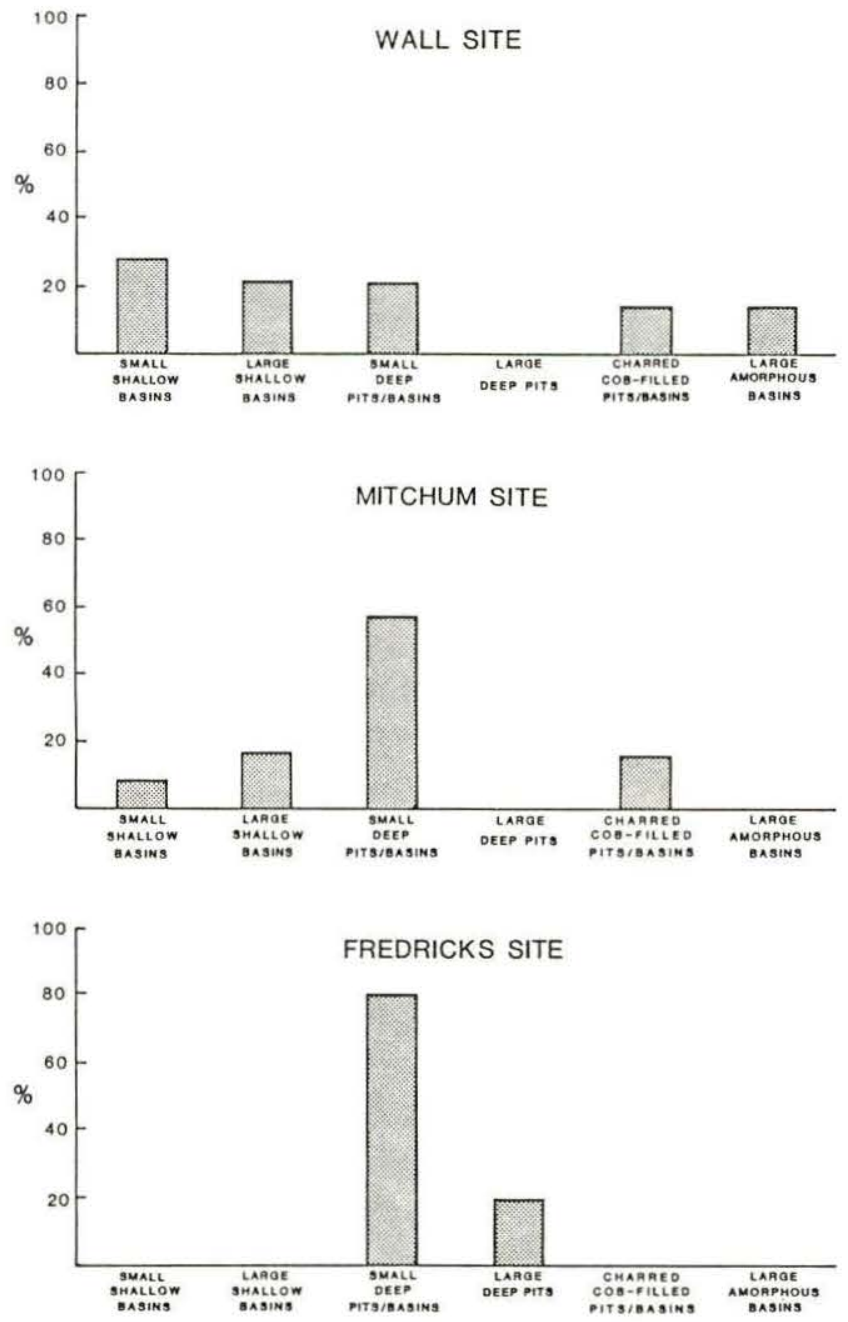

Figure 3.47

Relative frequency histogram of feature classes at the Wall, Mitchum, and Fredricks sites.

and large-scale food processing facilities. The large shallow basins at the Mitchum site were probably used as food processing facilities. Such facilities probably are also present in unexcavated areas of the Fredricks site. The excavated areas at that site are confined primarily to the domestic and mortuary activity areas. The basin-shaped deposit of Zone I in Feature 9 at the Fredricks site has been interpreted as in situ deposits representing largescale plant food processing, perhaps associated with ritual feasting. While this deposit has a depth/diameter ratio of greater than 0.40 , it probably functioned in a similar manner to the larger but shallower features of this class.

Class III features are small and mediumsized deep pits which include both deep, be11-shaped pits with constricted openings and smaller straight-sided and sloped-sided deep pits and basins. The relative proportion of features shows an increase from $21.4 \%$ at the Wall site, to $58.3 \%$ at the Mitchum site, and to $80 \%$ at the Fredricks site. All of the features 
in this group would have been useful for below-ground storage of small quantities of foodstuffs or caching of valuable items (Schroed1 1980; DeBoer 1984; Ward 1985). This pattern demonstrates a fundamental shift in storage behavior from the Protohistoric to the Historic period.

Only one Class IV feature was present at any of the three sites. This is the large pit at the Fredricks site which has been discussed in detail above. It is likely that this pit was originally excavated for storage purposes and was later transformed into a fire pit for the sweat lodge. This strengthens the argument for the shift to below-ground storage.

Cob-filled pits make up $14.3 \%$ of the feature inventory at the Wall site and $16.7 \%$ at the Mitchum site. No such pits were observed at the Fredricks site. Assuming that these facilities were used in the preparation of animal hides and skins, it is somewhat surprising that they are absent from the Fredricks site feature inventory. What this may imply, if sampling error is not responsible for the absence of these features, is that as the participation in the deerskin trade increased, there was a shift in hide-processing strategies. Since both dressed and undressed deerskins were traded (France 1985), time previously devoted to hide preparation was spent in skin procurement. In an examination of the trade inventories in the historic records, France (1985) noted that the bulk of trade items consisted of cloth products, which may reflect a shift from animal-skin clothing to clothes made from incoming European fabrics.

The structural evidence from the three sites is difficult to compare because of the great differences in areas excavated. Circular, oval, and rectangular structures are present. Palisades are present at both the Fredricks site and the Wall site and possibly at the Mitchum site. Figure 3.48 summarizes the structure data from the three sites. Included in this figure are the volumes of Class III features associated with each structure, and the ratio of pit volume to floor area $\left(\mathrm{ft}^{3} / \mathrm{ft}^{2}\right)$.

Al1 of the habitation structures at the Wall site are circular, and they have an average area of $367.60 \mathrm{ft}^{2}$ (range $=284-491$ ). As discussed earlier, these structures seem to be paired. The single structure identified at the Mitchum site is oval and has $290 \mathrm{ft}^{2}$ of floor space. The two habitation structures at the Fredricks site represent both oval and rectangular house forms. The absence of subsurface features in the rectangular structure and its proximity to the oval structure may suggest that these are paired winter/summer structures. If this is true, there is a continuity in function for domestic architecture, even though the house forms are different.

It appears that the structures at the Wall site were fairly substantial buildings that received considerable maintenance. They may have been daub-covered as we11. The structures at the Mitchum and Fredricks sites were built using much smaller posts and do not exhibit as much evidence of maintenance as those at the Wall site. This may be partly because of briefer periods of occupation at the Fredricks and Mitchum sites, possibly reflecting more frequent population movements during the Historic period. The structures at the Fredricks site also appear to be more closely spaced than those at the Wall site.

The differences in the palisades at the Wall and Fredricks sites is notable. The Wall site's palisade was built with large posts and probably served as a formidable defensive work. The Fredricks site's palisade was constructed of much smaller posts and could not have provided much of a barrier to intruders.

The most striking difference between the sites is in the dramatic increase in the number of subterranean storage pits at the Mitchum and Fredricks sites compared to the Wall site. This shift is reflected in the changing ratio of storage volume to floor area of associated structures, which DeBoer (1984:18) has proposed as a measure of subterranean "storage-percapita."

The Wall site had 10 structures, for which only one has associated storage pits. The volume/floor area ratio for the structure in 0.042 . The rest of the structures have ratios of 0.00 which yields an average storage/floor area ratio of 0.004 . The Mitchum site is represented by a single structure with two storage pits associated with it. The combined volume of these two facilities is $39 \mathrm{ft}^{3}$ yielding a volume/floor area ratio of 0.134 . The Fredricks site is represented by three structures. One of the structures has no associated storage pits, one has two, and the third structure is a sweat lodge with a large pit that may have originally been used for storage. Since the association of this pit with a domestic habitation structure is not clear, it is not used in the intersite comparison. The two habitation structures have volume/floor area ratios of 0.00 and 0.162 , yielding an average of 0.081 for the two structures. The difference between the Mitchum and Fredricks site ratios is probably a result of sampling error, especially since only one structure has been excavated at the Mitchum site.

Both DeBoer (1984) and Ward (1985) have argued, from ethnographic and archaeological data, that subterranean storage is a concealment strategy used when settlements are seasonally abandoned by all or a portion of the inhabitants, such as by hunting/raiding groups of males. The deerskin trade, displacement of populations, and population reduction created a condition which led to increased interaction between the Native American societies of the 


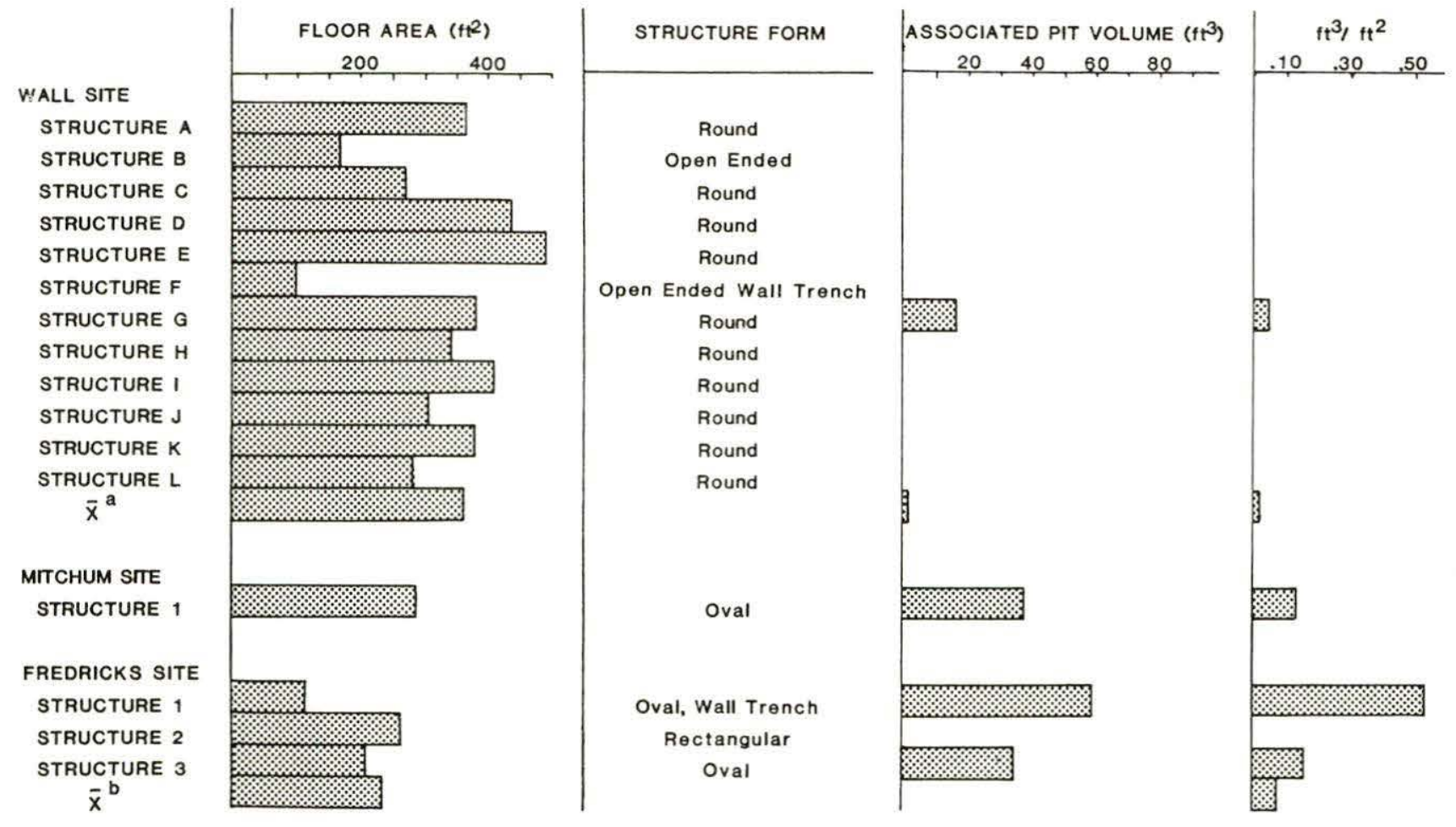

a does not include structures $B$ and $F$

b does not include strutcture 1

Figure 3.48

Histogram showing the floor area of structures at the Wa11, Mitchum, and Fredricks sites, and their associated storage pit volumes.

North Carolina-Virginia area (Ward 1984b; Dickens 1984). This interaction was complex and resulted in both increased intertribal warfare and consolidation of formerly distinct societal systems. Participation in the deerskin trade and warfare resulted in parties of men being away from the village for varying periods, especially during fall and winter after crops were harvested and stored. The absence of men from the villages during these expeditions would leave the settlements vulnerable to raids. It is precisely under these conditions that concealment of surplus would be adaptive. The risk of spoilage from burying items below ground would be outweighed by the risk of loss from raiding parties.

This shift from stable, long-term village occupation to less permanently occupied villages is dramatically illustrated in the respective archaeological remains of the Wall and Fredricks sites. At the Wall site intensive investments were made by the community in the construction and maintenance of a defensive fortification. Houses were lived in and maintained for long periods of time, and the village expanded in response to its growing population. Storage of surplus crops was accomplished with the use of above-ground storage facilities like corn cribs. This general pattern is in marked contrast to the pattern described above for the Fredricks site. It is not known whether the Wall site was abandoned because its population grew too large for the local environment to support, or its development was cut short by the disruptive effects of 16 th century Spanish intrusions into the interior of the Southeast. The data from the Fredricks site clearly show that by A.D. 1700, about 150 years after the abandonment of the Wall site, adaptation to the same local physical environment had changed greatly as a result of changes in the social and cultural environment.

It is now possible to offer some preliminary answers to the research questions raised at the beginning of this paper. One important question concerned the nature of village structure during the Protohistoric period. Assuming that the Wall site was representative of a portion of the larger settlement system of which it was a part, it appears that Protohistoric villages of this area were small palisaded communities consisting of several groups of extended families. The spatial arrangement of houses were in clusters of summer and winter houses arranged around small plazalike open areas. The groups occupying these houses probably cooperated in collective endeavors such as agriculture and village maintenance. Storage of surplus food was in aboveground facilities; subterranean storage was 
minimal. A double set of palisades was used for defense, and villages were occupied year-round. Domestic structures were circular, conicalroofed buildings that probably were plastered with daub. At least some of these villages underwent expansion in response to increased population growth during the Protohistoric period.

There is still little evidence on which to base an interpretation of socio-political organization during the Protohistoric period. No large communal structures were observed at the Wall site, nor was there evidence of social stratification in the burials. Nevertheless, given the scale of social investment by the inhabitants in the village fortification, the village expansion in area and population, and the evidence that it was occupied year-round, it is possible that the stage may have been set for the development of low-level chiefdoms in this area, such as those observed in the coastal regions of Carolina and Virginia in the late 16 th century (Hariot 1590).

Differences in house forms between the Wall site and the Mitchum and Fredricks sites are pronounced. These differences may have ethnic rather than temporal implications. The ceramics represented at these sites support the idea that at least two distinct ethnic groups occupied the different drainages in the study area (see Davis, this report).

Analysis of site structure of the Wall, Mitchum, and Fredricks sites shows that mobility increased during the Early and Middle Contact periods. This shift is probably a result of the complex interaction of factors relating to participation in the deerskin trade, population decimation from disease, increased tribal interaction, and increased warfare.

The introduction of metal tools during the Historic period does not seem to have greatly affected traditional architecture and construction techniques. A few square postmolds were observed at the Fredricks site but were not associated with any of the domestic structures. It is possible that the introduction of metal digging tools such as hoes, led to a change from construction of structures using posts set individually to the construction of structures using posts set in wall trenches, excavated with metal tools. It is likely that subterranean facilities were constructed using metal tools but these facilities retained traditional oval and circular forms. Burial pits, which were rectangular with sharp edges and corners, undoubtedly were excavated with metal tools.

Evidence for the overall configuration of the Fredricks site is insufficient at this point to address the question of whether or not different ethnic groups were consolidated and living together in the village. It is probable that population density at the Fredricks site was higher than at the Wall site. The structures, although smaller, appear to have been much more closely spaced. Additional excavations will be necessary to determine if ethnic differences are reflected in either architecture or spatial patterning of features and artifacts. If the village was a composite of formerly distinct groups, these groups may have demarcated themselves in distinctive ways as part of an effort to maintain their identity. Ward (this report) argues that the spatial patterning of the Fredricks site cemetery reflects possible ethnic differences. It would be surprising if efforts to maintain group identities were confined to mortuary behavior.

The analysis of the spatial structure of the Wa11, Mitchum, and Fredricks sites has provided a baseline of information on village and household structure and behavior. The interpretations of the spatial patterns at these three sites are not intended to be conclusive. Instead, these interpretations are meant to be working hypotheses to be tested with additional data from these and other sites within the region. 


\title{
Mortuary Patterns at the Fredricks, Wall, and Mitchum Sites
}

by

\author{
H. Trawick Ward
}

\section{INTRODUCTION}

In this section, an attempt will be made to describe the burials from the Fredricks site, and to discuss the mortuary complex as it reflects the organization of later 17 th-century Siouan Indian society and the processes of prehistoric-to-historic culture change. Comparative data will be presented from the archaeological and ethnohistoric records to reconstruct changing mortuary patterns of Siouan peoples as well as those of neighboring groups.

Mortuary practices at Siouan sites occupied during the Prehistoric and early Historic periods will be compared with the mortuary pattern at the late 17 th-century Fredricks site to elucidate temporal changes in spatial organization, burial pit morphology, and grave associations. Finally, questions concerning Indian-colonial interaction and acculturation will be addressed in the context of the Fredricks site mortuary complex.

The Occaneechis were very influential in the development of Siouan societies during the Historic period. They occupied a strategic position in a trade network that was comprised of Native American and colonial groups. It is probable that their prominence in this network was established early in the Historic period because of pre-existing ties and relationships with groups to the north. The Susquehannocks, for example, seem to have been at least partially responsible for the development of the Occaneechis as a major link in the VirginiaCarolina trading system. As the intensity of colonial influence increased during the latter part of the 17 th century, the Occaneechis, as with other interior tribes, had to cope with depopulation and other consequences of the colonial expansion. Social and cultural adjustments should be reflected in Siouan mortuary practices.

\section{APPROACHES TO MORTUARY ANALYSIS}

Over the past two decades, studies of mortuary behavior have dramatically increased. This trend is often seen as a direct consequence of the rise of anthropological archaeology and the acceptance of the analysis of social organization as a proper domain of archaeological inquiry. As a result, mortuary data are no longer used only to speculate about primitive belief systems but rather to provide the main focus for studying social differentiation, cultural complexity, culture change, and demography (Bartel 1982:52; O'Shea 1984:13). In fact, mortuary ritual probably contains more information concerning social processes and culture change than does any other data category available to the archaeologist (Goody 1962:142; Tainter 1978:110).

It should also be kept in mind that the primary archaeological manifestation of mortuary behavior, a burial, represents only one link in the behavioral chain surrounding the ceremonial treatment and disposal of the dead. Mortuary practices involve several distinct stages: death, body preparation, burial chamber preparation, interment, and post-interment activities (Bartel 1982:53). The burial itself, however, should inform on precedent and subsequent behaviors (i.e., what happened before and after the body was placed in a pit or other receptacle).

Most studies of mortuary practices by American archaeologists have been concerned with status differentiation, particularly within ranked societies, and have used grave associations as their primary source of information (e.g., Brown 1971). Tainter (1978:121), however, has found that social distinctions were symbolized by mortuary associations in less than five percent of a sample of 93 mortuary systems described in the ethnographic 1iterature. Because the way a culture disposes of its dead can mirror a complex web of economic and sociopolitical variables as well as ideological beliefs, Bartel (1982:52), Brown (1971), Binford (1971), Rothchild (1979), and others have pointed out that for studies of mortuary behavior to be productive, they must take into account the structure and organization of the total mortuary system, not simply the material content resulting from burial behavior.

After critically reviewing recent approaches to the analysis of mortuary behavior, O'Shea (1984:14) proposes that if mortuary remains are to be understood directly it is 
necessary to assume that only a single set of cultural directives governing mortuary treatment was in operation throughout the duration of a burial group. Following Binford (1971:13-18), 0 'Shea further states that there are regularities that link a society and how it disposes of its dead. The most important relationships are: 1) mortuary differentiation is patterned and integrated with other components of the cultural system; 2) mortuary differentiation accorded an individual is consistent with and reflects his social position (i.e., "social persona") in the living society; and 3) mortuary differentiation becomes more complex as societal complexity increases (0'Shea 1984:21).

In societies with little complexity, the dimensions of status differentiation are based on age, sex, and "differential capacities" for performing cultural tasks. In more complex societies, on the other hand, status differentiation is determined by culturally defined sociocentric statuses (Binford 1971:18; Service 1962:155). At the same time, there are no set rules concerning the degree of mortuary differentiation within any given society. Some may permit a lot of variation, whereas others permit only a little. And all social differences may not be recognized through differential mortuary treatment. In some societies, for example, the way a person dies may have primary influence in determining mortuary treatment ( $0^{\prime}$ Shea 1984:36).

The spatial dimensions of the structure and organization of mortuary systems can be a sensitive barometer of social variability (Saxe 1971). Peebles $(1971: 87)$ in his analysis of Moundville burials found that individuals of high status were spatially separated from lower status individuals. Persons were segregated within cemeteries, and cemeteries within the site were ranked relative to one another. Individuals buried in mounds were further segregated from those buried in cemeteries. A similar mound-cemetery segregation has been reported at Etowah (Larson 1971) and Spiro (Brown 1971). "Status space" is a characteristic and significant feature of the mortuary practices of complex, ranked societies, and the structure of cemetery burials is reflective of the hierarchical nature of their social organization.

Cemetery burial may also provide information on social variables other than status. Tainter (1978:123) suggests that the presence of cemeteries reflects the importance of individual corporate groups; Saxe (1971:51) interprets cemeteries among egalitarian societies as indicating strong lineal affiliation; and Bartel (1982:51-52) states that societies with social structures characterized by clan or lineage organization usually will have distinct geographical burial locations within cemeteries. Thus, cemeteries may be expected in unranked, as well as ranked, societies as long as strong unilineal kinship ties define corporate groups.
One of the primary reasons for the acceptance of mortuary analysis into the mainstream of archaeological thought is that it can be tied directly to ethnohistoric and ethnographic data ( 0 'Shea 1984:1). Although the use of these data does not suggest a one-to-one correlation between the acts of one culture and the material remains of another, descriptive accounts of mortuary practices can reveal behaviors that may be detected as patterns in the archaeological record. Obviously, the correlation between ethnographic or ethnohistoric observations and archaeological remains is considerably strengthened if there is an historic connection between the two, as there is with the Occaneechis. Nonetheless, ethnohistoric descriptions in particular must be closely scrutinized because they are usually filtered through the biased eyes of individuals from an alien culture. The ultimate test of whether such accounts are relevant to the interpretation of archaeological remains depends on how close the fit is between facts revealed by the archaeological record and the ethnohistoric or ethnographic model (see Ucko 1969:263).

\section{ETHNOHISTORIC BACKGROUND}

To follow are ethnohistoric descriptions of Siouan mortuary behavior as well as accounts of such behavior in other, neighboring cultures, particularly groups to the northeast where there is a rich reservoir of ethnohistoric data. There were strong cultural affiliations between some of the interior northern tribes and the Siouans, particularly the Occaneechi. This relationship is best supported by the fact that the Tutelo, Occaneechis, and Saponi all ultimately settled with the tribes of the Iroquois Confederacy in New York (Mooney 1894:55).

In searching the ethnohistoric record for descriptions of burial practices among Siouan groups and their neighbors, one quickly discovers a paucity of such observations by White visitors. The extant written records are by individuals who visited the tribes for relatively brief intervals. Given communities with populations of about 200, and a high mortality rate of 30 per 1000 , there would be an average of about six deaths per year (Gruber 1971:64-65). It is obvious, therefore, that the chance of a traveler such as John Lawson or John Lederer observing a burial ceremony first-hand would be very slight. Certainly the frequency of observations would not have been sufficient to allow detailed descriptions of patterns of mortuary behavior. Also, it should be recognized that, even if an outsider did arrive at a village at the time of a death, it is unlikely that he would be told about the death or allowed to observe the mortuary ritual. This point is made clear by Adair (1930:189), who observed among the Cherokee that

they will not associate with us, when we 
are burying any of our people, who die in their land: and they are unwilling we should join with them while they are performing this Kindred duty to theirs.

Thus, because of the relatively low frequency of death and burial, and the fact that most groups probably did not allow outsiders to observe or participate in burial ceremonies, most of those descriptions that are available probably are second-hand.

John Lawson, the most detailed chronicler of the Indians of the Carolina Piedmont, wrote concerning the South Carolina Indians that their tombs were located near the cabins, with the implication that these tombs were grouped. "Near to these Cabins are several Tombs made after the manner of these Indians; the largest and chiefest of them was the Sepulche of the late Indian King of the Santees..." (Lefler 1967:27). Lawson goes on to describe in detail the burial customs of the Santee.

The manner of their /interment is thus: A Mole or Pyramid of Earth is raised, the Mould thereof being worked very smooth and even, sometimes higher or lower, according to the Dignity of the Person whose Monument it is. On the Top thereof is an Umbrella made Ridgeways, like the Roof of an House, this is supported by nine stakes, or small posts, the Grave being about six or eight Foot in Length and four foot in Breadth; about it is hung Gourds, Feathers and other such Trophies, placed there by the dead Man's Relations, in respect to him in the Grave (Lefler 1967:28).

Lawson continues by describing pre-burial mortuary behavior and states that when someone dies, they are laid out in the sun and "seasoned" with a concoction made of bear fat and a red root. This ointment is also used by the living as a hair dressing. After two or three days, the body is covered with pine or cypress bark. The worldly possessions of the deceased are brought to the body, and a close male re1ative sings "a mournful Ditty" for three or four more days. Finally, when it decays to the extent that it can be stripped from the bone, the flesh is removed and burned. The bones are then thoroughly cleaned, oiled, and put into a wooden box to be kept by the deceased's closest relative (Lefler 1967:28).

Lawson also mentions a burial ceremony that took place while he was visiting a Tuscarora town and stated that it was "much the same as that of the Santees, who make a great feast at the interment of their Corpse" (Lefler 1967:66).

Lawson (Lefler 1967:185-189) also discusses mortuary rituals in his general description of the Indians of Carolina. He lists a sequence of the events that is very similar to his description of the Santee ritual. There is a great deal of mourning by the nearest relatives, and the corpse, after lying in an outbuilding constructed for that purpose for a day and a night, is wrapped in a blanket or match coat and two or three cane or rush mats. This bundle is then enclosed by a web of woven reeds or cane. Next, the body is taken outside the village ("into an orchard of Peach-Trees") where the individual's kinsmen and other members of his tribe as well as representatives from allied tribes listen to a shaman ("Doctor or Conjurer") give a detailed account of the highlights of the dead person's life. After this lengthy discourse, the corpse is carried to the burial pit which is six feet deep and eight feet long. A forked branch of pitch pine or light wood is driven down either side of the grave, and several layers of bark are placed on the bottom. The corpse is then laid down gently, and a pole is placed across the two forked sticks.

Having a great many Pieces of Pitch-Pine logs about two foot and a half long, they stick them in the sides of the Grave down each End and near the top thereof, where the other ends lie on the Ridge-Pole, so that they are declining like the Roof of a House. These being very thick placed, they cover them (Many times double) with Bark; then they throw the Earth thereon that came out of the Grave, and beat it down very firm; by this means the dead Body lies in a Vault, nothing touches him; so that when I saw this way of Burial, I was mightily pleased with it, esteeming it very decent and pretty, as having seen a great many Christians buried without the tenth part of that Ceremony and Decency (Lefler 1967:18).

After the flesh had rotted, the bones were taken out and dressed in deer skins and placed in a charnel house to accompany the remains of other "Kings" and "War-Captains." Lawson continues his description by saying that, although the burial ceremonies differ slightly among the various Indians, all had in common

the Mourning, which is, to appear every night at the Sepulchre, and howl and weep in a very dismal manner.... If the dead Person was a Grandee, to carry on the Funeral Ceremonies, they hire People to cry and lament over the dead Man (Lefler $1967: 189)$.

The first part of Lawson's descriptions seems appropriate and generally conforms to the archaeological record. However, there is no archaeological evidence that the Occaneechis or other Siouan burials were unearthed, defleshed, and placed in communal burial houses. It appears that Lawson combined some attributes of Siouan mortuary customs with the Algonquian practice of defleshing bodies and storing them in charnel houses until they were interred in a communal pit or ossuary. There is abundant archaeological evidence from Siouan sites, including Occaneechi Town, that bodies were wrapped prior to interment and that the graves 
contained chambers or vaults (Navey 1982).

The only other description of Siouan burial ritual comes from John Lederer, written in 1670 . Lederer's account is interesting because he clearly states that individuals were buried in cemeteries.

Their places of Burial they divide into four quarters, assigning to every Tribe one: for, to mingle their bodies, even when dead, they hold wicked and ominous. They commonly wrap up the corpse in beasts skins, and bury with it Provision and Household stuff for its use in the other world. When their great men die, they likewise slay prisoners of war to attend them (Cummings 1958:14).

As with Lawson, Lederer mentions that the bodies were wrapped before burial. He also states that grave goods, in particular utilitarian items, were placed with the dead. Unfortunately, it is not possible to ascertain what social divisions Lederer's "tribe" refers to. The implication is that these were clans of one village, since they shared a common cemetery that was divided into spatial units. There are no historical accounts or archaeological evidence to support his contention that prisoners of war were killed at the time of the death of their "great men." This statement, rather, seems to reflect Lederer's familiarity with de Acosta's account of the Mexican Indians rather than a first-hand observation of Siouan mortuary behavior (see Cummings 1958:13, footnote).

There are several ethnohistoric accounts of mortuary behavior of the Indians located just north of the Siouan area. These accounts are important because they seem to have some close counterparts in the archaeological record of the Occaneechis. A recurring theme in virtually all of these accounts is the emphasis placed on feasting in the mortuary ritual. Lawson, however, only refers to feasting indirectly when comparing the Santee burial customs with those of the Tuscarora. Another important aspect of mortuary behavior shared by most of the northern groups is the fact that gift-giving and redistribution also were part of the mortuary pattern. Among Algonquian groups in Maine, the Jesuit Pere Pierre Biard observed that the village prepared a feast which continued, day and night, from the time of death until all of the food was gone (Bushne11 1920:12). In describing the burial ritual Biard states that

They arch the graves over with sticks, so that the earth will not fall back into it, and thus they cover up the tomb... If it is some illustrious personage they build a Pyramid or monument of interlacing poles... If it is a man, they place there as a sign and emblem, his bow, arrows, and shield; if a woman, spoons, Matachias, or jewels, ornaments, etc...they bury with the dead man all that he owns, such as his bag, his arrows, his skins and all his other articles and baggage (Biard quoted in Bushne11 1920:13).

The description of the grave with sticks arching over it to prevent dirt from touching the body is very similar to Lawson's account. Also of interest is the mention of grave offerings being differentiated by sex and of the graves being marked on the surface by some of the belongings of the deceased. Apparently among these Algonquians, all of the personal property of the deceased was buried with him and not redistributed among the living.

A description of the burial of a Delaware chief's wife in 1762 clearly indicates the importance of feasting and the redistribution of goods, particularly European trade goods, in the burial ritual (Bushnel1 1920:22). As with the Algonquians, burial was in a cemetery located outside the village, and graves were marked with painted or decorated posts. The deceased was wrapped and covered to avoid contact with the dirt. At the end of the funeral procession from the village to the cemetery area, "two stout men [carried] loads of European manufactured goods upon their backs." After the grave was covered and surrounded by a palisade, food was prepared and passed out.

Then presents were distributed the many things which had been carried by the two men in the rear of the procession. Those who had rendered assistance were given the most valuable and highly prized pieces, but no one was omitted...At dusk after the burial, a kettle of food was placed upon the grave, and this was renewed every evening for three weeks (Heckewelder quoted in Bushne11 1920:22).

A couple of important inferences can be drawn from the above description. First, the re-distribution of European goods as part of the mortuary ritual may provide a clue as to how these goods were generally distributed within various social groups. Second, not only was there a feast for the living, but food was also placed on the grave so that the deceased might share in the feast. The fact that food is placed on the grave itself has archaeological implications that will be discussed later in this section.

The Delaware also put

tobacco pouch, knife, tinder box, tobacco and pipe, bow and arrow, gun powder and shot, skins and cloth for clothes, paint, a small bag of Indian corn or dried bilberries, sometimes the kettle, hatchet, and other furniture of the deceased, into the grave, supposing that the departed spirits would have the same wants and occupations in the land of souls (Loskiel quoted in Heye and Pepper 1915:77). 
There are also detailed descriptions of the mortuary behavior of the Shawnee who are thought to be related to the Seneca and Delaware. These accounts state that the graves were lined with wood or bark which also covered the body. Before burial, gifts were brought to the dead person's kinsmen and redistributed. After burial, a small house was constructed over the grave, and a large feast was served to the funeral guests. After the third day, an allnight vigil was held. A meal was prepared for the deceased and served to the dead and his blood kin prior to and during the vigil (Voegelin 1944:240-245).

Food and fire were placed on the grave for three nights during the journey to the other world. On the fourth morning, food for a feast was again set near the grave. At this time, the funeral leader spoke to the dead while burning tobacco in a small fire made near the grave. The Shawnee believed that the smoke created by the tobacco being thrown in the fire would take the leader's words upward to the dwelling place of the spirit of the dead (Voegelin 1944: 261-268).

Another interesting feature of Shawnee burial ritual is that all the dirt excavated from the burial pit had to be placed back on top of the grave. If this was not done, the Shawnee believed that another death would occur shortly in the same family (Voegelin 1944:390). Feasts were held and gifts were redistributed one year after an individual died. Accounts prior to 1687 indicate that these feasts were given annually for a period of four years (Voegelin 1944:297).

The most detailed description of Susquehannock culture comes from the 1666 writings of George Alsop, who published the following account of their mortuary practices:

When any among them depart this life, they give him no other intombment, then to set him upright upon his breech in a hole dug five feet long and three feet deep, covered with the Bark of Trees Arch-wise, with his face Du-West, only leaving a hole half a foot square open. They dress him in the same Equipage and Gallantry that he to be trim'd in when he was alive, and so bury him (if a Soldier) with his Bows, Arrows, and Target, together with all the rest of his implements and weapons of War, with a Kettle of Broth, and Corn standing before him, lest he should met with bad quarters in his way... They bury all within the wall of Palisade'd impalement of their City or Cannadogo as they call it (Alsop quoted in Kent 1984:41).

Kent (1984:41) finds that Alsop's description of the Susquehannock form of burial fits fairly well with the archaeological evidence. According to Kent, individuals originally placed in burial pits in a sitting position later would have fallen over from pressure of collapsing dirt, thus creating the flexed posture normally found in Susquehannock burials. Alsop's description of grave goods also matches the archaeological record.

Every detail of the ethnohistoric descriptions should not be accepted uncritically, but there are regularities and trends that suggest that the more general aspects of the descriptions are accurate. For example, the burials were always wrapped to avoid contact with the earth. In addition, they were usually covered and placed in a dirt-free chamber. Some had other coverings, in the form of small house-like structures, placed on the top of the grave. In some cases, the burials were protected by palisades. All the ethnohistoric accounts indicate that burials were grouped in cemeteries spatially distinct from the habitation areas. Feasts were prepared as part of the mortuary ritual, and in some instances, these feasts were renewed for several years on the anniversary of the death. Usually, although these feasts were prepared by the deceased's relatives, they were participated in by the village as a whole. Food was also prepared for the dead and placed on or near the grave. A redistribution of material goods, usually items of European manufacture, almost always accompanied the redistribution of food. In most cases, special attention was given leaders, with "grandees" and "kings" receiving greatest accord.

It is also important to note that food and personal property were placed with the burials. Among the Susquehannocks, these items included implements and weapons that the individual had owned during life. Algonquians buried with the dead all that he owned, and the Delaware placed food, weapons, and tools with the dead. A common theme seems to be that an individual was given what his kinsmen determined that he would need in the afterlife, and these needs were perceived as being similar to those the individual had while alive.

\section{ARCHAEOLOGICAL BACKGROUND}

Having presented ethnohistoric descriptions of the mortuary rituals of the Siouans and other groups along the Eastern Seaboard, it is now appropriate to turn to the archaeological record to isolate correlates to the ethnohistoric accounts. First, archaeological sites that are known to have been occupied by Siouan groups during the Late Prehistoric and early Historic periods will be discussed. Then archaeological data from groups known to have interacted with the Siouans during the Historic period will be presented. These latter data are presented because it is believed that there are closer similarities between the Occaneechi burials at the Fredricks site and those of groups such as the Susquehannock and Delaware than there are 
between the prehistoric and historic Siouan mortuary patterns.

The Wall site, located immediately east of the Fredricks site, dates to the Protohistoric period. Although roughly $16,000 \mathrm{ft}^{2}$ have been excavated, only eight burials have been identified. All the burials were inside or in the vicinity of houses. The individuals were flexed, and all but one were placed in ovoid shaft-and-chamber pits with their heads usually positioned to the southeast. Grave goods consisted entirely of aboriginal artifacts. Decorative items such as shell beads and gorgets were found along with aboriginal pots and smoking pipes. The latter were associated with adults, whereas the former were found with children. The burials from this site will be discussed in detail later in this section.

At the Clarksville site, located on the North Carolina-Virginia border and dating to the Late Prehistoric period, burials were randomly dispersed across the excavation area. Although these burials probably also were in the vicinity of houses, no such structures were identified by the excavators (Miller 1962). The overall inventory of grave goods at the Clarksville site was very similar to that from the Wall site.

During the summer of 1983, excavations at the Mitchum site, an historic village site, uncovered an oval house structure and a single shaft-and-chamber burial lying within the floor area of the house. Grave goods were represented by a necklace of small glass trade beads and two copper ear ornaments. A detailed description of this burial also will be presented later in this section.

At the Madison site, located in Rockingham County, North Carolina, 120 burials were removed from an area of approximately $14,000 \mathrm{ft}^{2}$. Unfortunately, most of these burials were taken out by pot-hunters. However, some records of the excavations were kept. This site probably dates to ca. 1650-1670 and was occupied at about the same time as Upper Saratown, discussed below. Twelve of the burials were arranged in a semicircle around a cluster of refuse pits, and the remaining burials radiated out from the primary group in a more or less random fashion. The burials were flexed and all but one was oriented in an eastward direction. European trade goods, found in $70 \%$ of the graves, consisted mostly of glass beads and brass ornaments (Gravely 1969:11). In general, this pattern is very similar to that described below at Upper Saratown. Postholes and house patterns among the burials probably were present but were not observed by the excavators.

At Upper Saratown, occupied during the middle 17th century, an area similar in size to that of the Wall and Madison sites has been excavated and 111 burials identified. All these interments were found either inside or in the vicinity of house structures. Navey (1982:152) notes that, "It can be stated with certainty only that the preferred burial locations were within the village and in the proximity of houses". Only $12 \%$ of the burials were in shaft-and-chamber pits. The most popular pit type was ovoid to rectangular in shape and usually had shelves for a burial covering near the top of the pit. The bodies were flexed and the heads generally oriented in an easterly direction (Navey 1982:158-168). The most common grave goods were glass beads and brass ornaments, followed by aboriginal shell ornaments (Navey 1982:170).

At the Wall, Madison, and Upper Saratown sites, areas almost equal in size have been excavated. A like number of burials were found at the latter two sites, 120 at Madison Cemetery and 111 at Upper Saratown, whereas only eight were found at the Wall site. A comparison of the Wall site and Upper Saratown shows that a similar number of houses was constructed at both sites, although there is more evidence for rebuilding and superimpositions at Upper Saratown. That Upper Saratown was occupied somewhat longer is also suggested by a denser concentration of artifacts, but there is no reason to suspect that it was a viable community for more than 50 years. In all likelihood, the Wall site was occupied for about 20 years, given the multiple palisade alignments and a rich midden deposit around the periphery of the village. The difference in numbers of burials between the two sites, therefore, is much too large to be dismissed as the result of sampling error or different durations of occupation. It is rather a clear indication of the devastating impact of European diseases on the aboriginal populations, and that this is one area where the accounts of the early traders and explorers were not exaggerated (e.g., Lefler 1967:232).

In summary, there seems to be a definite pattern in the dimensions of Siouan mortuary behavior during the Late Prehistoric and Historic periods. During the Late Prehistoric period there was a preference for ovoid shaftand-chamber burial pits. Although this type of pit was continued into the early Historic period, its popularity diminished. Grave goods consisted of aboriginal utilitarian and decorative artifacts with shell ornaments being most popular. During the middle 17 th century, the most popular grave was ovoid to rectangular in shape and usually had shelves to support a covering. Aboriginal decorative artifacts were replaced by glass beads and brass or copper ornaments. Aboriginal utilitarian artifacts, however, were still popular (Wilson 1984).

During the Late Prehistoric and early Historic periods, bodies were flexed and the heads usually pointed in an easterly direction. During both periods, most burials were randomly distributed in the villages, although some may have been associated with house structures. With the possible exception at the Madison site, graves were not spatially segregated into 
clusters or aligned with one another.

An early archaeological account that describes a cemetery complex is the Munsee Cemetery report (Heye and Pepper 1915). The Munsee site, located in southern New Jersey, consists of a circular palisaded village and cemetery occupied by members of the Delaware tribe during the middle 17th century. The cemetery was excavated during the summer of 1914, and there are some problems in interpreting the remains because of the relatively poor archaeological field methods at that time. It is clear, however, that the cemetery contained burials that were aligned with one another along a northwest-southeast axis. Several of the pits also contained upper zones of refuse, and in some instances deer bones and charcoal were intermingled with the human ske1etal material. Heye and Pepper (1915:22) interpreted the animal remains as representing refuse from a feast. The clearest evidence of feasting activity was found in a child's burial.

In association with this burial there were evidences of a feast, for over the body there was a broad discolored area in which were much charcoal and many cracked animal bones, mostly those of deer. Other burials showed evidences of accompanying feastpits, but none was so strongly marked as this (Heye and Pepper 1915:28).

Pewter pipes and artifact bundles were also reported associated with the burials. "Resting against the left shoulder was a deposit of objects consisting of two flints and fragments of a steel, two circular mirrors with metal backs, a clay pipe of European manufacture, and a pewter pipe" (Heye and Pepper 1915:53). Several other pewter pipes were also found, and these were determined to have been imports from Iroquois groups to the west and north (Heye and Pepper 1915:53). In addition to the pipes, brass kettles and bracelets, metal spoons, glass and shell beads, and fragments of European fabric were found in the graves. In summary, the inventory of grave offerings at the Munsee site, though larger than that of the Fredricks site, is quite similar in overall content, as will be seen in the following descriptions.

Several Susquehannock sites exhibit marked similarities, through time, to the Siouan sites. The Ibaugh site, located near Washington Boro in Lancaster County, Pennsylvania, is a Susquehannock cemetery and village that dates between 1600 and 1625, a time when the Susquehannock were first becoming intensively involved in the fur trade. The population of the Ibaugh site is estimated to have been around 1000 (Witthoft et al. 1959:119). Here, the grave depths averaged 31-in and appeared to have been dug with hoes. The pits were oval in outline with flat bottoms and sloping sides. In most cases, the pits were larger than necessary to accommodate the burial (Witthoft et al. 1959:105).
At the Ibaugh site, all the bodies except infants were flexed. Most were loosely flexed; only two were tightly flexed. The dominant orientation of the burials was west or southwest, with the heads oriented westward. In a single incident of secondary burial, two bundled individuals had been placed in the same pit with a flexed skeleton. Whitthoft et al. (1959:109) state that

it seems quite obvious that the secondary nature of this interment was not the result of any traditional burial practice, but that it represents the remains of two persons who had died away from home, perhaps in warfare or in a hunting accident. The bones had been found in the woods at a somewhat later date and had then been carried home and placed in the first available grave, an open one which had just received a fresh corpse.

Most of the burials contained both aboriginal artifacts and Euroamerican trade goods. Large quantities of glass beads were found in clusters as if sown on clothing. Small white and blue glass "seed" beads were most common, with only a few tubular shell "wampum" beads being present. The graves of children and infants contained more beads than those of adults. Iron artifacts included knives, axes, hoes, scissors, and a few nails. Also recovered were pipes, kettles, bracelets, and tubular beads made from glass. Artifacts sown on clothing were discoidal shell beads, conch columella beads, shale beads, perforated elk and bear tusks, brass cones and bells, and a sheet brass breast ornament. Most of the burials also contained aboriginal ceramic vessels that contained the remains of food offerings (Witthoft et al. 1959:110-115).

The Strickler site, also located near Washington Boro, is a large palisaded Susquehannock village with at least three associated cemeteries. It is estimated that the village was occupied between 1650 and 1675 (Futer 1959:147) or between 1645 and 1665 (Kent 1984:367). It may have contained as many as 3000 inhabitants (Kent 1984:363). Burials at the Strickler site averaged 25-in deep below the subsoil surface. As with those at the Ibaugh site, the pits were bath-tub shaped and had an average horizontal measurement of 65-in by 30-in. Most skeletons were flexed either on their right or left sides; $23 \%$ were extended (Kent 1984:365). By in large, the bodies were oriented northwest-southeast, with the heads to the northwest (Futer 1959: 136).

Most of the Strickler burials were accompanied by brass kettles, gun parts, metal or kaolin pipes, and glass beads, in addition to aboriginal clay pots and pipes. Four pewter pipes were recovered, and fragments of trade cloth and blankets were found preserved by contact with the metal artifacts. The inventory 
also included several flintlock and doglock muskets, axes, hoes, knives, swords, a single pistol, and over 200 musket balls. Other artifacts included hawkbells, Jews harps, and buckles (Futer 1959:137-140). In short, "Grave offerings at Strickler consist of virtually every kind of material item made by, or which came into the hands of, the Susquehannocks" (Kent 1984:366).

The final site to be occupied by the Susquehannock was Conestoga Town, located in Lancaster County, Pennsylvania. This village, established after the 1675 defeat of the Susquehannocks by the Iroquois, is believed to have been occupied between 1690 and 1730 (Kent 1984:386). Excavations in 1972 revealed striking differences between this site and earlier Susquehannock villages. Instead of long houses, the houses were more cabin-like, and the space between houses was greatly increased over earlier villages. The small settlement, ${ }_{2}$ confined to an area less than 90,000 $\mathrm{ft}^{2}$, is estimated to have been occupied by only 100 to 200 individuals (Kent 1984:282-283).

Conestoga Town contained five distinct clusters of burials, which seem to have been spatially related to different groups of houses. It is known that different ethnic groups occupied the site at the same time (Susquehannocks, Seneca and possibly others), and these distinctions may be reflected in the burial clusters. Most of the burials were extended (supine position) instead of being flexed, and each grave pit was dug just large enough for the body. A few apparently were coffin burials. Most of the skeletons had their heads oriented to the west-northwest; however, a few graves were oriented toward the southwest, southeast, east, and northeast, with greater variability than at earlier Susquehannock cemeteries (Kent 1984:387).

All the burials at Conestoga Town that had not been looted contained some form of grave goods, ranging from a few beads or a knife to thousands of glass beads accompanied by a variety of other trade artifacts. "Generally it was the adolescents who had the largest quantities of objects interred with them" (Kent $1984: 387)$. The most common grave associations were beads, iron knives, and brass kettles, the latter often serving as repositories for artifact caches. Wooden spoons and fragments of split-cane baskets were sometimes found with the kettles. The overwhelming majority of burial artifacts were of European origin, and the list includes almost every imaginable item from beads and buttons to guns and bullets (Kent 1984:389).

The various kinds of objects and their quantities found buried with the dead at Conestoga Town are indicative of the retention of certain old native beliefs, together with a cumbersome admixture of ideas borrowed from Christianity. In our opinion the kinds of quantities of objects do not reflect anything about individual status or economic conditions of the community (Kent 1984:390).

The earliest historic Siouan villages, such as Upper Saratown, do not compare with a Susquehannock town such as Ibaugh. The latter are at least 10 times as large and have spatially distinct cemetery areas, whereas the former are relatively small and have burials distributed throughout the village. However, in both cases the vast majority of the bodies are loosely flexed and oriented in specific directions; the Siouans usually to the east, and the Susquehannocks usually to the west. There are also similarities in the kinds of European artifacts used as burial furniture. Although nonutilitarian goods such as beads are predominate at Upper Saratown and the Ibaugh site, axes, hoes, and other utilitarian objects are also found with the burials at both sites. The Ibaugh site, however, appears to have produced a greater variety of iron and brass artifacts than Upper Saratown.

There are no known parallels in the Siouan area to the Strickler site. Most of the Siouans never coalesced into a single village or group of villages that match the Strickler site in size and complexity. It was, however, during the span of occupation of the Strickler site that most of the cultural interaction took place between the Susquehannocks and the Siouans. And the original home of the Occaneechis on Occaneechi Island may have been in the process of becoming like Strickler when it was raided by Bacon in 1676. Three "forts" were occupied on the island when Bacon attacked. Immediately before Bacon's attack, the Occaneechi King, Posseclay, started:

Massing his Indians and also the Hayhelocks, and Manakins and Annalectons, man all his forts and lined the other side of the river thick with men so that wee could neither well attack nor depart the Island (Billings 1975:268).

As the militia tried to stop the Indians from entering the fort, King Posseclay attempted to appease Bacon by blaming the Manakins and Annalectons who were "too numerous for him [the king] to control" (Billings 1975:268).

From this account, it would seem that the Occaneechis and their allies who occupied Occaneechi Island in 1676 were somewhat more numerous than the archaeological and ethnohistoric records indicate for other Siouan villages and in some ways more comparable to the Strickler site. Unfortunately, Occaneechi Island is now inundated, and archaeological research on the island prior to its flooding failed to locate the village and forts of the Occaneechis (Miller 1962).

There are numerous similarities and a few differences between the Fredricks site as it is 
currently known and Conestoga Town. As for the differences, most of the burials at Conestoga Town were extended and a few were in coffins, perhaps reflecting Christian influence. Also, burial orientation was variable. In contrast, the burials at the Fredricks site were all flexed and oriented in the same direction. The two sites are similar in the presence of a wide range of Euroamerican trade artifacts used as grave offerings and in the clustering of burials into small cemeteries. Although only one such cluster has, so far, been found at the Fredricks site--compared with five at Conestoga Town--it may be that additional cemeteries are present at Fredricks. The two sites are also similar in the fact that subadults received a great deal of attention at the time of burial. In terms of overall village size, the two sites seem to be very similar, and both villages included a mix of once distinct tribal groupings. It is believed that the mixed ethnic composition at both sites is the reason for the relatively small and distinct burial clusters.

\section{THE SUSQUEHANNOCK CONNECTION}

It was stated in the introduction of this section that cultures located north of the Occaneechi and the other Siouan tribes had a significant impact on the development of Siouan cultures during the Historic period. Ethnohistoric data indicate that the Susquehannocks were particularly influential in the southern Piedmont, and that they may have been responsible for setting up the Occaneechis as "middlemen" in the Carolina-Virginia deerskin trade.

From the beginning, trade with Europeans along the Atlantic Seaboard was controlled and managed by a relatively small number of Indian tribes. No doubt, in many cases, these strategic positions had been at least partly established during the Late Prehistoric period, and the large-scale trade with the colonists simply enhanced and entrenched previously established trade networks (Merrell 1982:72).

In the northeast, European trade was controlled by the tribes of the Iroquois Confederacy: the Mohawks, Onondagas, Cayugas, Oneida, and Seneca, the latter being the westernmost and the largest of these groups. The Seneca also appear to have been the most hostile in their relations with other tribes, particularly those to the south (Abler and Tooker 1978:505). Initially Seneca raids were aimed primarily at the Susquehannocks who were located in a strategic position in the lower Susquehanna valley. Many of these raids were prompted by Susquehannock attacks on the Seneca's shipments of furs to their eastern markets (Hunter 1959:15). After the defeat of the Susquehannocks in 1675, the Seneca continued raiding the southern frontier and into Siouan territory. In 1684, William Byrd I mentioned that he had spoken with 50 Seneca Indians who, "promised to behave themselves hereafter very peaceable towards the English" (Trinling 1977:16). In 1701, John Lawson was warned by the white trader Massey, "to strike down the Country for Ronoack, and not think of Virginia, because the Sinnagers, of whom they were afraid, though so well armed and numerous" (Lefler 1967:61).

As mentioned above, the Susquehannocks occupied a strategic position in the trade network and acted as intermediaries as early as 1608, when John Smith reported that the Tockwkogh living at the head of Chesapeake Bay had knives, hatchets, and pieces of iron and brass they had received from the Susquehannocks (Kent 1984:26). The geographic location of the Susquehannocks and their role as entrepreneurs placed them in a continuing state of hostilities with the Seneca. For a time they were allied with the Maryland colony against the Seneca. However, in 1674, Maryland made peace with the Seneca and declared war against the Susquehannocks, who were defeated by the Seneca in 1675. Weakened, the Susquehannocks were later pursued by the Maryland and Virginia militia and sought refuge with the Occaneechis who were, at that time, living on an island in the Roanoke River.

In contrast to the hostile relations the Susquehannocks had with the Seneca, they appeared to have lived in harmony with the neighboring Delaware Indians, as well as with groups to the south, especially the Occaneechis (Hunter 1959:15). Because of this relationship, the Susquehannocks appear to have established themselves as middlemen in the fur trade with the Siouans prior to 1670. And in establishing this position, they also made the Occaneechis, located astride the major north-south trading path, their primary trade agents. Up to this period, few white traders had yet ventured into the southern Piedmont. John Lederer, on his second voyage in 1670, hired a Susquehannock guide, Jackzetavon, to lead him through Siouan territory. This guide may have been familiar with the Carolina Piedmont from participating in earlier Susquehannock trading expeditions, an interpretation that is supported by Lederer on his approach to a Siouan town.

You must by your scouts inform your self whether they hold any correspondence with the Sasquesahanaughs: for to such you must fire notice of your approach by a gun; which amongst other Indians is to be avoided, because being ignorant of their use, it would affright and dispose them to some treacherous practice against you (Cummings 1958:41).

Lederer's comments suggest that not only did the Susquehannocks trade with the Siouans, but they traded in firearms, and probably other Euroamerican utilitarian goods, and not just in beads and trinkets.

Shortly after 1670, the Occaneechis had established their own reputation as trade middle- 
men. In 1673, Abraham Woods observed that the Occaneechis' store of arms and powder made them "the mart for all the Indians for at least 500 miles" (Woods, quoted in Merrell 1982:91). During this same period the Occaneechi had established ties with several other tribes, and their village was said to be:

Strongly fortified by nature and that makes them so insolent for they are but a handfull of people besides what Vagabonds repaire to they it being a receptakle for rogues (Alvord and Bidgood 1912:225).

The Susquehannock-0ccaneechi connection is clearly illustrated by the fact that after being defeated by the Iroquois in 1675, and being chased by the Virginia and Maryland militia, a band of Susquehannocks sought refuge among the Occaneechis who at the time lived on their island stronghold in the Roanoke River. In 1675 "Manakins" and "Annalectins" had also retreated to Occaneechi Island. Nathanial Bacon conspired with these two groups to betray the Susquehannocks, which they did along with capturing 30 individuals who were turned over to Bacon's forces and put to death (Billings 1975:267). It is important to note that the Occaneechis were not involved in the Susquehannocks' betrayal but rather continued to play their role as middlemen by trying to stall Bacon. Their strategy did not work, and the Occaneechis were attacked by Bacon and so devastated by the attack that they were forced to abandon the island and retreat southward to the vicinity of present day Hillsborough, North Carolina (Billings 1975:267-268).

It is hypothesized that ties of trade brought with them bonds of social responsibility, and it is likely that a strong trade relationship was sanctioned by an equally strong network of social ties. This relationship of mutual obligations is evident in the above accounts and in an earlier 1663 report of the Virginia General Assembly in which it was stated that some of the "ill-omened and murderous Doeg (Susquehannock) Indians" had taken up sanctuary with the Occaneechis (Cummings and Rights 1958: 119). Social bonds between the Siouans and Susquehannocks are also evidenced by the fact that neither Lederer nor his Susquehannock guide were threatened by any of the Siouans they visited. This is in sharp contrast to the reception given by the Occaneechis to five Cherokees who were visiting among them at the same time as Lederer. The Cherokees wanted to establish trade relations directly with the Virginia colonists, and this so angered the Occaneechis, that they murdered their visitors (Cummings 1985:261). A similar fate met James Needham in 1674, when he also attempted to establish trade relations independent of the Occaneechis (Alvord and Bidgood 1912:217).

Thus, the ethnohistoric and archaeological records argue for a strong connection between the development of Piedmont Siouan tribes and tribes of the Susquehanna valley. The northern groups first felt the invasion of the Europeans, and early in the 17 th century, they established extensive trade networks with them and other Indian groups. By the middle of the 17th century, trade competition from the Iroquois Confederacy to the north forced the Susquehannocks to look to the southern Piedmont for potential trading partners. It is believed that they found in the Occanneechis, both geographically and culturally, an ally that would allow them to monopolize the Siouan trade. The Occaneechis were in a sense "set up" by the Susquehannocks as middlemen with whom they could deal directly. And, as Susquehannock influence was on the wane after 1670, the Occaneechis were just coming into their own as a major trading influence when attacked by Nathaniel Bacon. This no doubt affected their unchallenged prominence in the Siouan fur trade. However, even after moving to Hillsborough in the late 17 th century, they were apparently still prosperous.

During the early 1700s, European diseases and slavery greatly reduced the tribes of the middle Atlantic region. Remnants of various groups coalesced to maintain social solidarity and to try to overcome the effects of depopulation. By 1700 , similar cultural patterns had developed in both the Susquehanna valley and in the hills of the Eno, and, as a consequence, Conestoga town and Occaneechi Town shared many characteristics that are still visible in the archaeological record.

\section{FREDRICKS SITE BURIALS}

In 1983, four burial pits were uncovered at the Fredricks site. These burials were situated just outside a palisade that surrounds at least part of the village. By the end of the 1984 field season, nine burials had been excavated and auger tests indicated that from three to five additional burials were present (Figure 4.1).

All the burials were rectangular in outline, regularly spaced, and consistently aligned in a northwest-southeast direction. These spatial dimensions clearly define a cemetery where individuals were buried in locations that required a knowledge of previous interments. Either the burials were dug more or less simultaneously, or their locations were designated by above-ground markers. Heretofore, cemeteries such as this had not been reported in the North Carolina Piedmont. Given the configuration of the village, it is suspected that other cemeteries may be present and will be uncovered by additional excavations.

Burial 1 (Figures 4.2-4.4)

Pit Morphology. The pit for Burial 1 was first observed as a rectangular patch of dark brown humus containing a generous amount of charcoal, animal bones, and other refuse. It 


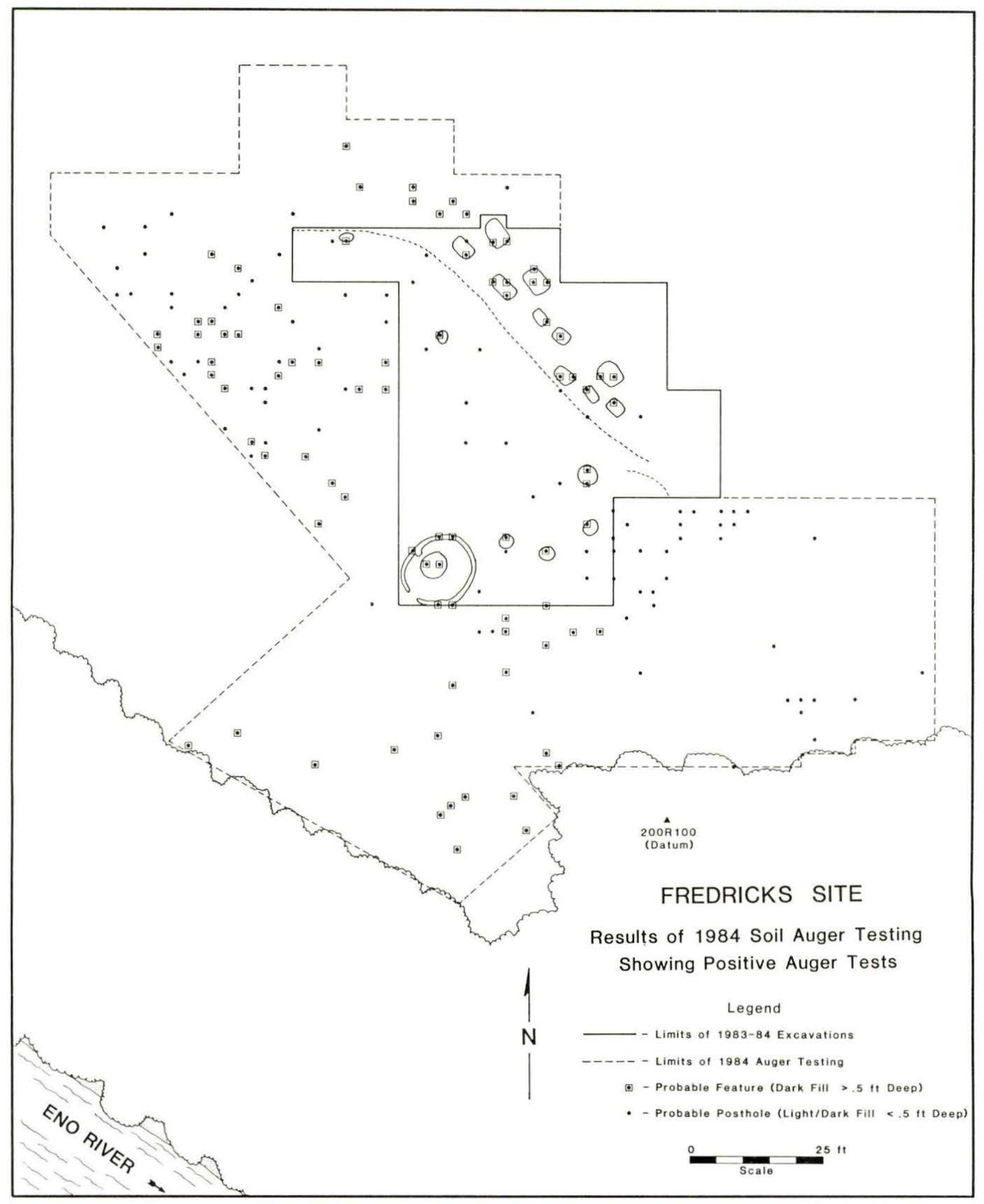

Figure 4.1

Results of soil auger testing at the Fredricks site, showing the cemetery. 


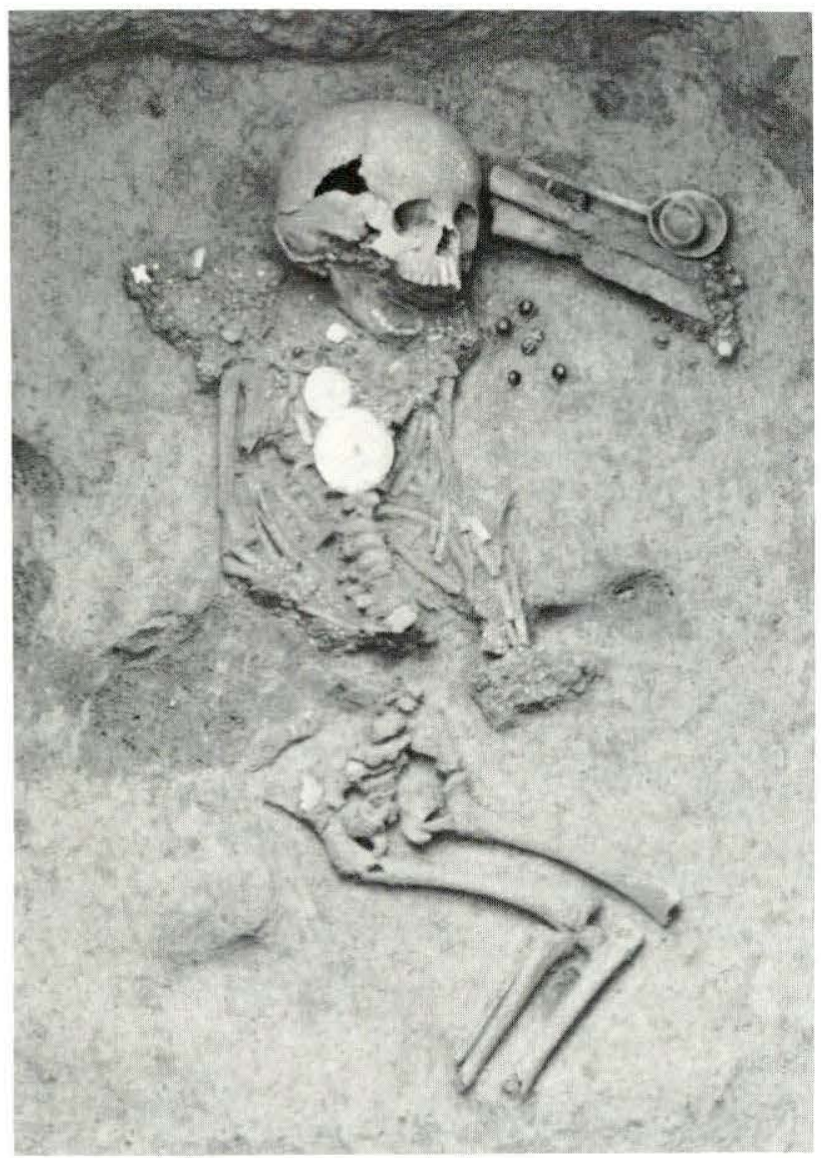

Figure 4.2

Burial 1 at the Fredricks site.

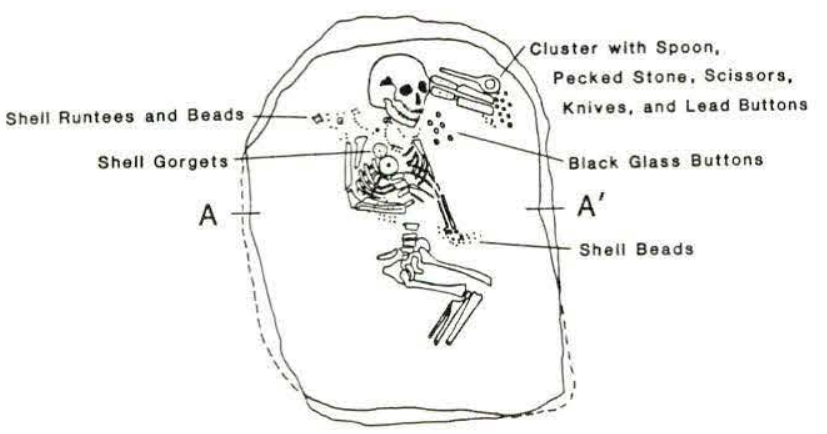

PLAN

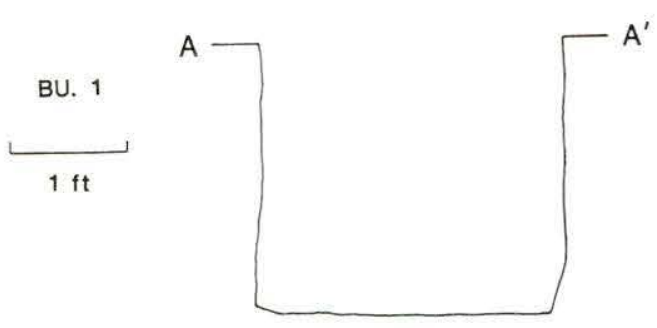

PROFILE

Figure 4.3

Plan and profile of Burial 1 at the Fredricks site.
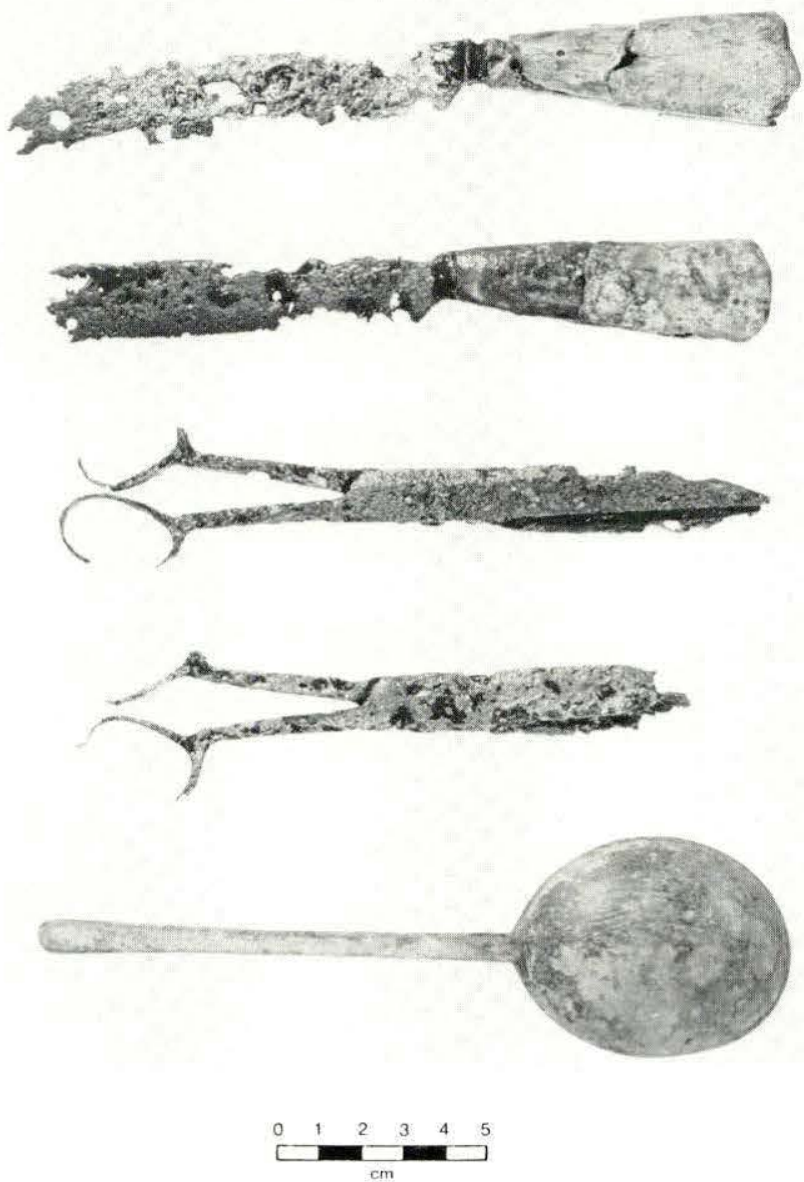

Figure 4.4

Artifact cluster from Burial 1 at the Fredricks site.

was oriented northwest-southeast, had a maximum length of $3.6 \mathrm{ft}$ and a maximum width of $2.6 \mathrm{ft}$, and was rectangular in plan. When excavated, the pit was found to have straight walls, a flat bottom, and to measure $2.4 \mathrm{ft}$ in depth (Table 4.1). The relatively flat walls and bottom showed evidence of having been dug with bladed (probably metal) digging implements. The fill was comprised of three distinct soil zones. Zone 1 consisted of a dark brown humus rich in refuse including charred food debris (animal bones and charred plant parts), potsherds, part of a corroded iron knife blade, and numerous glass beads. The northern half of Zone 1 was noticeably richer than the southern half. Zone 1 rested upon an irregular layer of mottled orange clay with ashy lens (Zone 2) that appeared to intrude Zone 1 in the northern section of the pit. It also contained lens of greyish black fill which were excavated separately from the mottled orange clay. Zone 2 probably represents a transitional face between Zone 1 and the bottom zone, Zone 3. The latter consisted solely of mottled orange clay (probably a portion of the soil excavated to create the pit) typical of burial fill at other 
Table 4.1

Pit dimensions of the Fredricks site burials.

\begin{tabular}{cccc}
\hline Burial & $\begin{array}{c}\text { Length } \\
(\mathrm{ft})\end{array}$ & $\begin{array}{c}\text { Width } \\
(\mathrm{ft})\end{array}$ & $\begin{array}{c}\text { Depth } \\
(\mathrm{ft})\end{array}$ \\
\hline $1 *$ & 3.6 & 2.6 & 2.4 \\
$2 \star$ & 3.1 & 2.6 & 2.1 \\
3 & 4.4 & 3.2 & 3.0 \\
4 & 3.2 & 2.2 & 2.1 \\
5 & 5.0 & 2.9 & 2.0 \\
6 & 5.6 & 4.0 & 2.3 \\
$7{ }^{*}$ & 3.4 & 2.3 & 1.4 \\
$8 *$ & 4.0 & 2.5 & 2.5 \\
9 & 5.1 & 3.5 & 2.3 \\
${ }_{\text {Fea. }} 1$ & 3.9 & 2.9 & 2.3 \\
Mean $_{\text {All }}$ & 4.1 & 2.0 & 2.3 \\
Mean $_{\text {Subadult }}$ & 3.5 & 2.5 & 2.1 \\
Mean $_{\text {Adult }}$ & 4.7 & 3.2 & 2.3 \\
\hline
\end{tabular}

* Subadult

Piedmont sites. The homogeneity of Zone 1 could have resulted only from the intentional filling of the upper portion of the burial pit with refuse-rich soil.

Burial Deposition. The burial was that of a child from four to five years old at death loosely flexed, lying on its left side. The skull was positioned to the southeast. The right arm lay across the chest, whereas the left arm lay straight along the left side.

Grave Goods. Around the wrist, waist, and shoulders were numerous shell beads that probably had been sewn to a blanket or garment. A bundle containing a latten spoon with a round pecked stone in the bowl, two bone handled iron knives, two pairs of scissors, seven lead buttons, and numerous glass beads were located between the face and the southwest corner of the pit (Figure 4.4). Over the sternum were two she11 gorgets with punctated designs.

Burial 2 (Figures $4.5-4.8$ )

Pit Morphology. The pit was visible at the base of the plowzone as a rectangular stain comprised of a medium-to-dark brown gritty sandy fill with a large amount of charcoal. A thin mottled orange collar was evident along the northwest and southwest edge of the stain. The pit was oriented northwest-southeast and measured $3.1 \mathrm{ft}$ long and $2.6 \mathrm{ft}$ wide and had a rectangular plan. It extended to a depth of 2.1 $\mathrm{ft}$ below the base of the plowzone (Table 4.1). Two postholes intruded the pit edge, one along the southwest edge and the other midway along the northeast edge. The sides were straight,

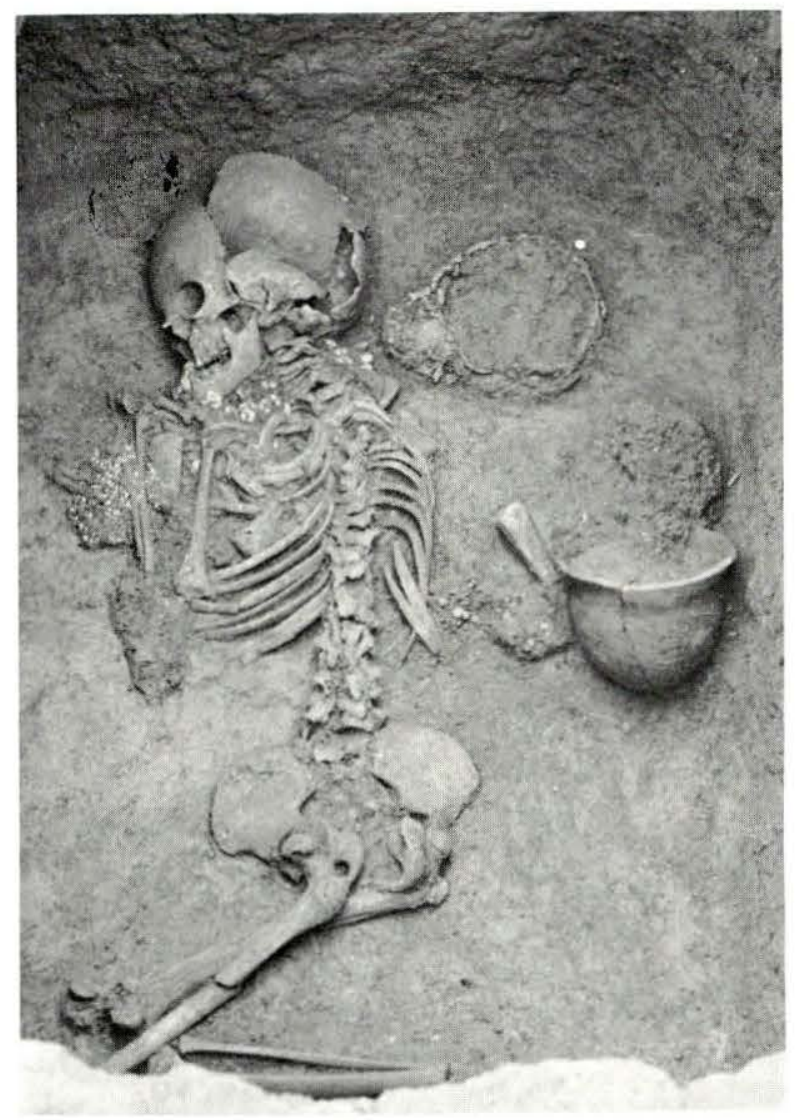

Figure 4.5

Burial 2 at the Fredricks site.

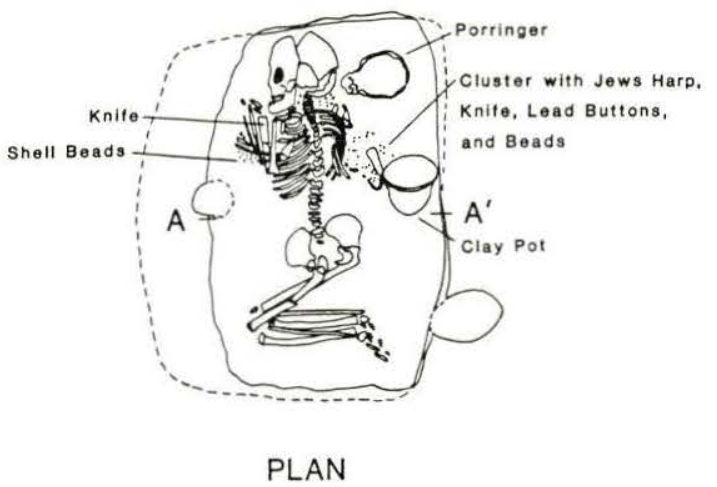

BU. 2

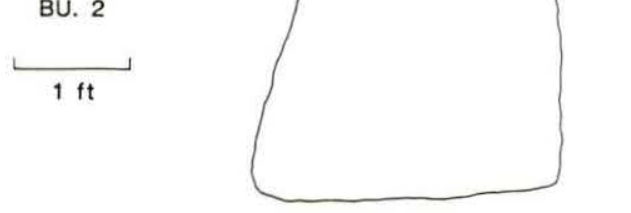

PROFILE

Figure 4.6

Plan and profile of Burial 2 at the Fredricks site. 


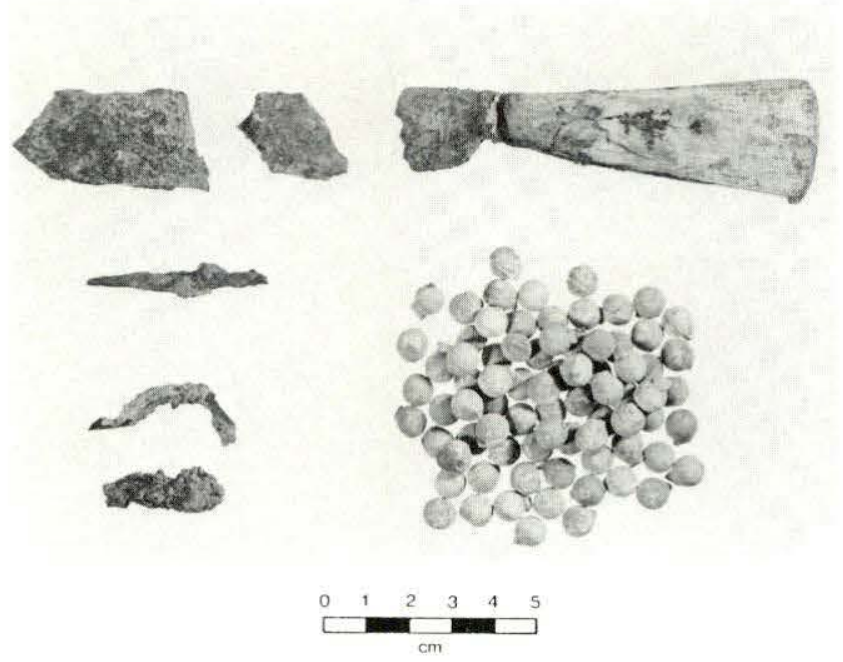

Figure 4.7

Artifact cluster from Burial 2 at the Fredricks site.

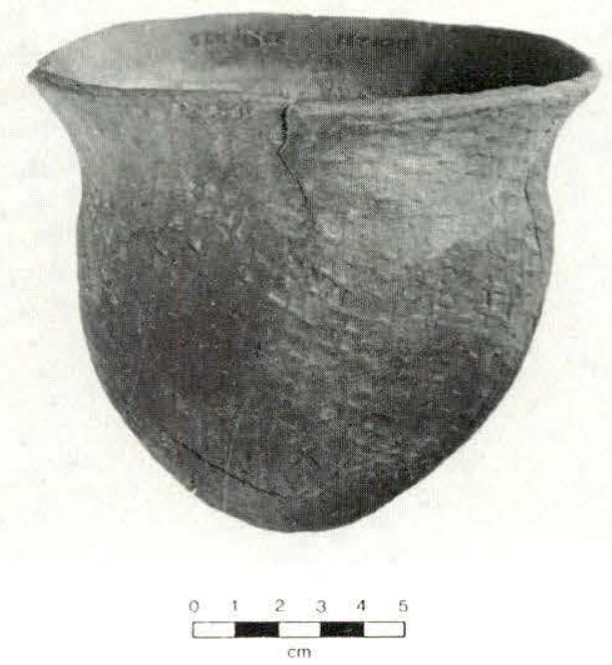

Figure 4.8

Small check-stamped pottery vessel from Burial 2 at the Fredricks site.

except for the northeast wall which was undercut to create a bell-shaped bulge at the bottom of the pit. The pit bottom was flat and smooth, which suggests that metal implements were used in its excavation.

The fill was excavated as two separate zones. The top layer (Zone 1) consisted of a brown gritty or sandy humic soil that was rich in refuse, including ceramics, some animal bone, and ethnobotanical remains. This zone was very similar to Zone 1 in Burial 1 except that it was not as rich in animal bone and was thicker (extended for approximately $1 \mathrm{ft}$ below the top of the subsoil). The bottom of this zone was mottled, containing lens of ash and charcoal. Zone 2 consisted of a mottled yellow clay with some charcoal that extended to the bottom of the pit. It was roughly $1.5 \mathrm{ft}$ thick.
Burial Deposition. The burial was that of a subadult between seven and eight years old at death. The skeleton was loosely flexed, lying on the right side with the skull oriented to the southeast. The arms were bent with the hands lying in front of the face.

Grave Goods. A bundle of artifacts believed to have been contained in a beaded bag was located on the left side of the chest (Figure 4.7). That bundle contained a Jew's harp, several lead shot, three lead buttons, and three unidentified fragments of iron. Several small white glass beads, thought to have been sewn on the bag, were located in the same area. A bone-handled knife may also have been part of the bundle. Between this cluster of artifacts and the pit wall was a small check-stamped pottery vessel (Figure 4.8). The pot was lying on its side and contained a dark stain around the orifice, perhaps the residue of food originally placed in the vessel. Adjacent to the back of the skull lay a pewter porringer. Another bone-handled knife and a cluster of small shell beads were observed adjacent to the wrists on the right side of the burial. Large columella beads were found in the neck area.

Burial 3 (Figures 4.9-4.12)

Pit Morphology. The pit for this burial was visible at the base of the plowzone as a rectangular area of brown humus that contained charcoal, animal bone, and other food refuse. The major axis of the pit was northwestsoutheast. It measured $4.4 \mathrm{ft}$ by $3.2 \mathrm{ft}$ and was $3.0 \mathrm{ft}$ deep making it the deepest pit in the cemetery (Table 4.1). Pit walls usually were vertical; however, the southwest wall of this pit sloped in slightly at the bottom and a narrow ledge was present along the bottom of the northeast wall. Several large rocks, natural inclusions in soil, protruded into the pit at various points along the bottom edge. The flat surfaces of the walls and bottom again indicate that it was excavated with Euroamerican metal tools. As with Burials 1 and 2, this pit contained two major zones of fill. The upper Zone 1 averaged a little over $1 \mathrm{ft}$ thick and consisted of a dark brown humic soil rich in animal bones, ethnobotanical remains, and ceramics. A layer of small pebbles extended through the middle of this zone. Zone 2 was comprised of a mottled orange clay, which in some areas extended upward around the rim of the pit, thus encircling Zone 1. In places, Zone 2 was nearly $2 \mathrm{ft}$ thick.

Burial Deposition. The skeleton, that of an adult male between 20-25 years at death, was tightly flexed, lying on its right side, with its skull to the southeast. The skeleton occupied only the southwestern half of the pit and was tucked against the southwest wall. The arms were bent at an acute angle with the hands in front of the face.

Grave Goods. A bundle of artifacts located 


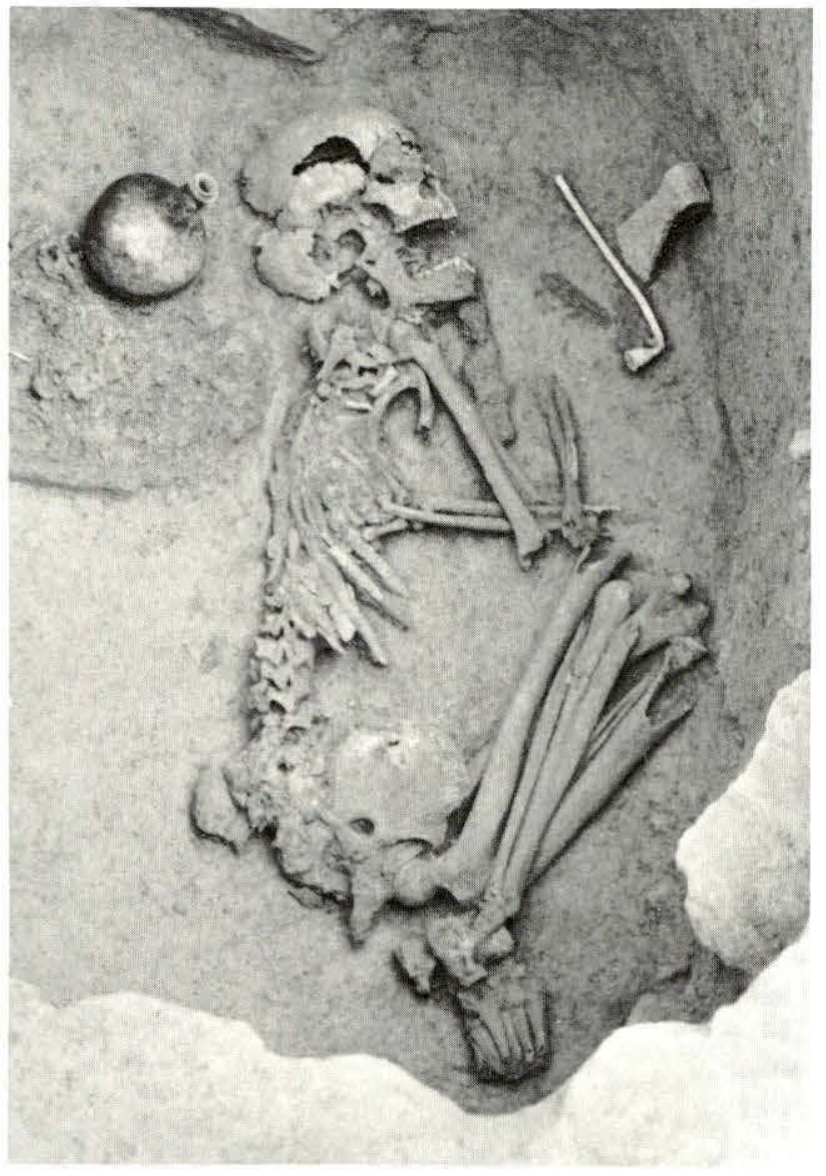

Figure 4.9

Burial 3 at the Fredricks site.
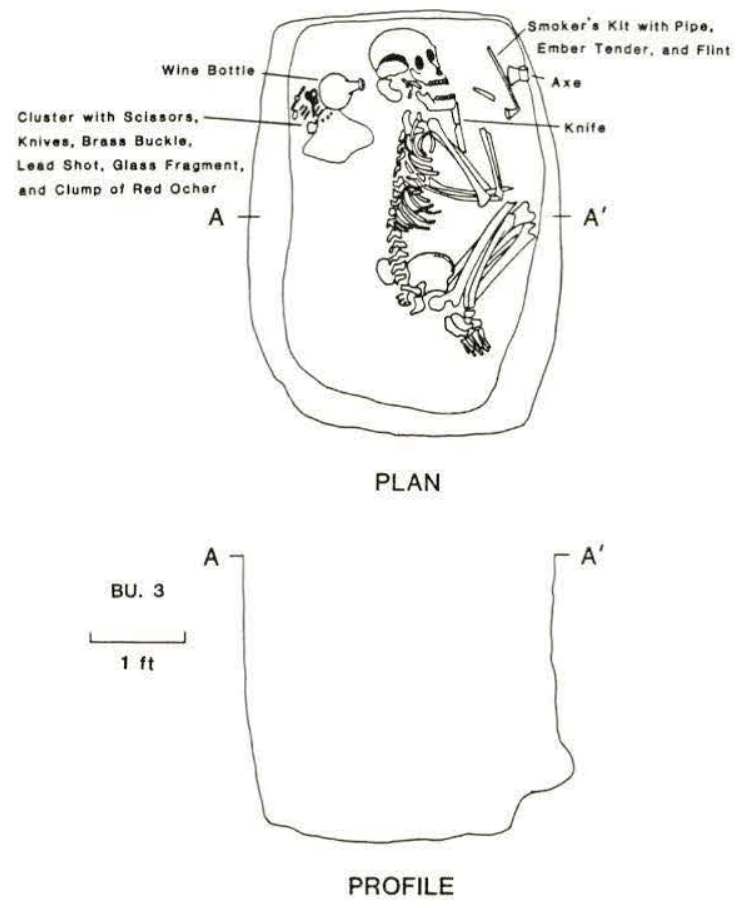

Figure 4.10

Plan and profile of Burial 3 at the Fredricks site. between the back of the neck and the northeast pit wall (Figure 4.11) was comprised of two pairs of scissors, two bone-handled knives, three pewter buttons, a fragment of dark-faceted glass, a metal tack, a brass buckle with part of a leather strap preserved, lead shot, a dog lock musket spring, several unidentified fragments of iron, and a clump of red ocher. Cloth and wood were preserved as fragments in association with the metal artifacts. All of these items were possibly contained within a wooden box and/or cloth bundle. A smoking kit containing a pewter pipe, an ember tender, and flint were located opposite the face of the skull (Figure 4.12). An iron axe head was found between the smoking apparatus and the southwest wall. Other grave associations consisted of a rum bottle positioned behind the skull and adjacent to the artifact bundle. Perishable items such as cloth or skins may have been placed on the ledge along the eastern edge at the bottom of the pit (the soil in this area was darker and more organic than the rest of the burial fill).

Burial 4 (Figures $4 \cdot 13-4.14$ )

Pit Morphology. This burial was visible at
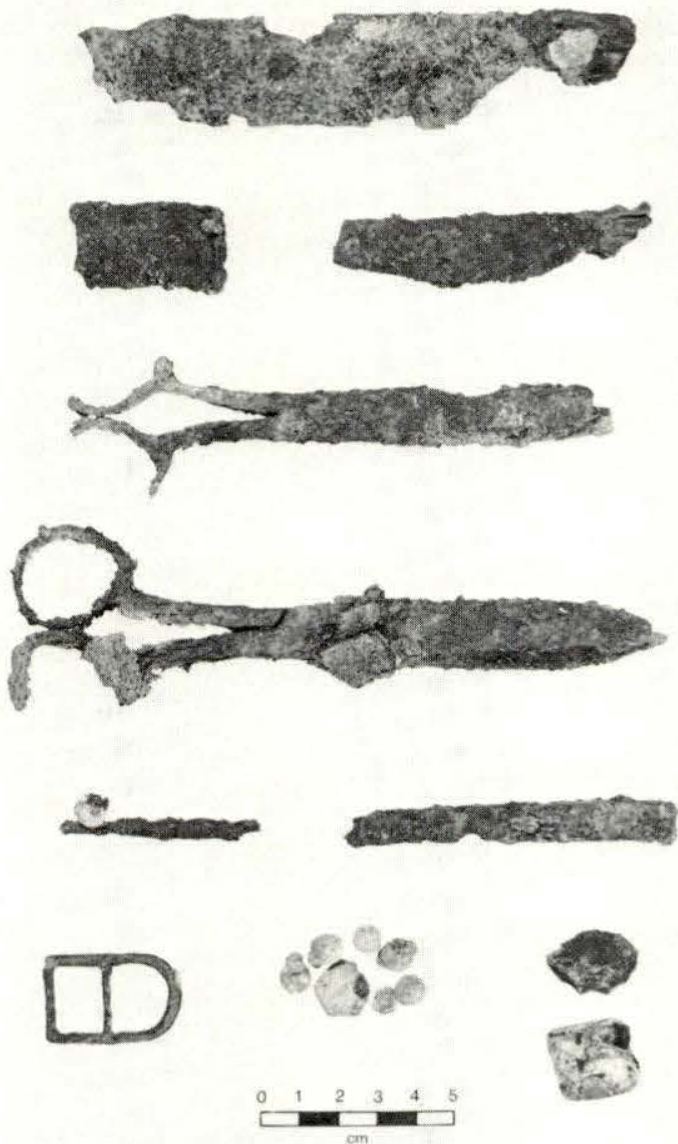

Figure 4.11

Artifact cluster from Burial 3 at the Fredricks site. 


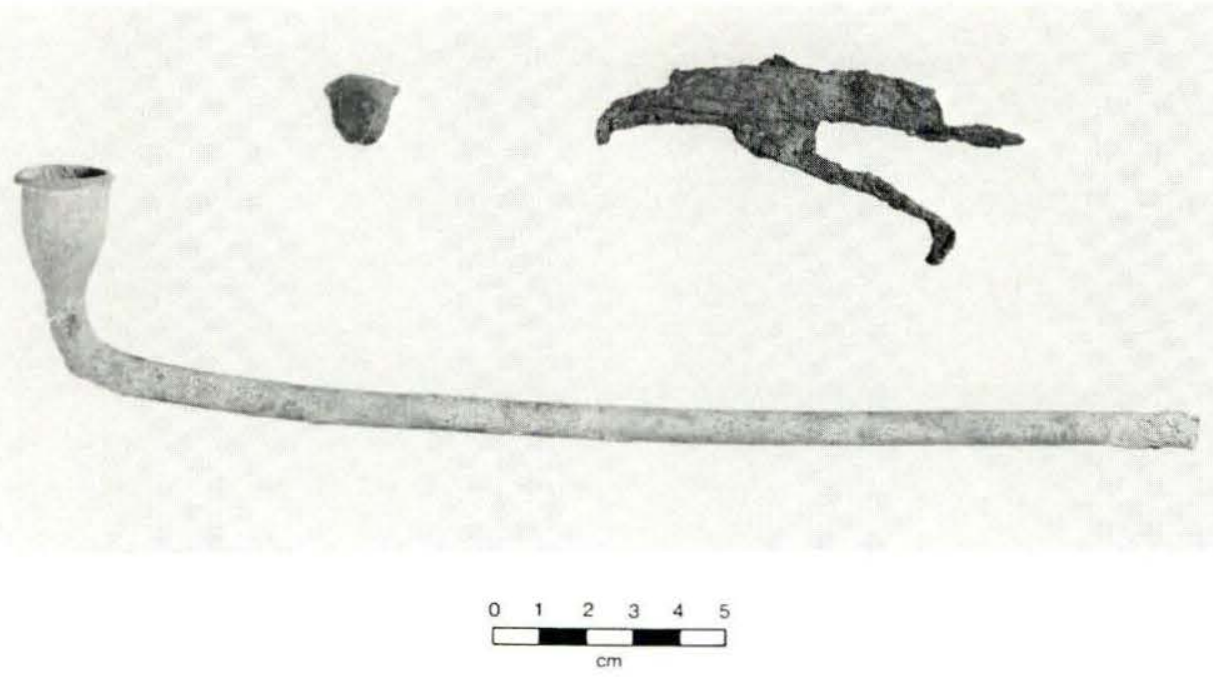

Figure 4.12

Smoking kit from Burial 3 at the Fredricks site.

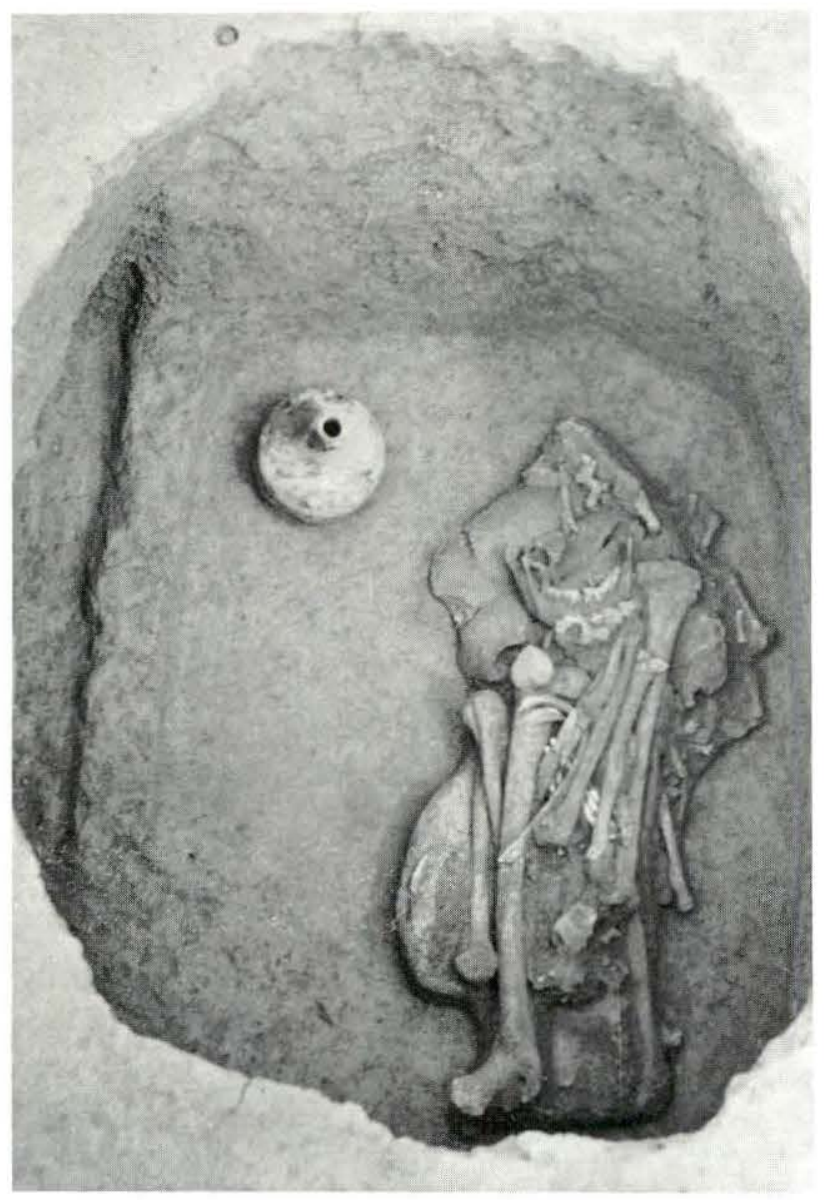

Figure 4.13

Burial 4 at the Fredricks site. the top of the subsoil as a rectangular stain with slightly rounded corners. The center portion of the pit was comprised of dark brown humic soil with charcoal, animal bone and potsherds. This fill is similar to the Zone $1 \mathrm{fill}$

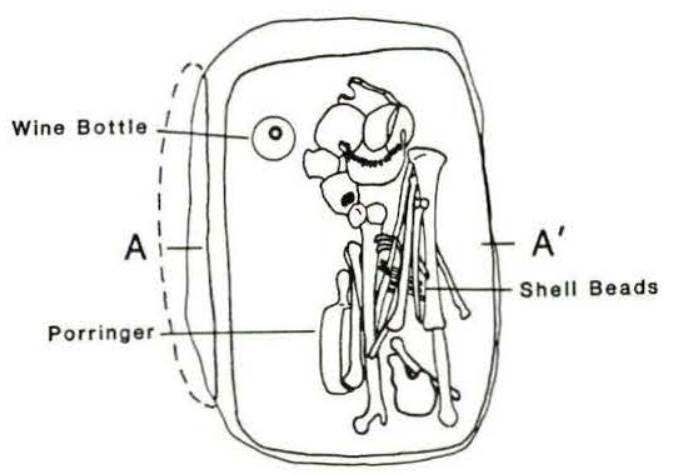

PLAN

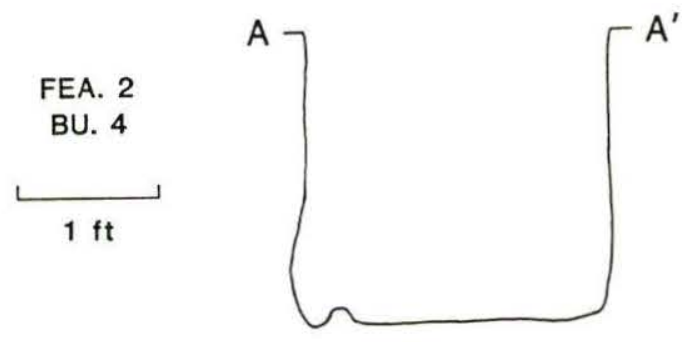

\section{PROFILE}

Figure 4.14

Plan and profile of Burial 4 at the Fredricks site.

of the other three burials; a mottled orange clay formed a band around the perimeter of the pit. The pit, whose main axis was northwestsoutheast, measured $3.2 \mathrm{ft}$ by $2.2 \mathrm{ft}$ across the 
top and was $2.1 \mathrm{ft}$ deep (Table 4.1). The pit walls were straight, and the bottom was flat except for a ledge along the northeast side. It is again proposed that metal implements were used to excavate this pit. A dark brown fill (Zone 1) extended unevenly across the middle portion of the pit to a depth of a little over one foot. This was underlain by a mottled orange clay fill (Zone 2) that contained some of the darker soil near the top.

Burial Deposition. One of two skeletons in the pit was that of an adult male between 25-30 years old that had been disarticulated and placed in a tight bundle lying against the southwest wall of the pit. The ledge at the bottom of the pit paralleled the orientation of the body. Cut marks around the cranium suggests this individual was scalped. Mixed with the bundle were the remains of an infant, which because they were not discovered until the adult burial was being cleaned in the laboratory, makes it impossible to know the precise spatial relationships of the two individuals.

Grave Goods. A cluster of 11 long tubular columella beads were located within the chest area of the bundle and unquestionably were

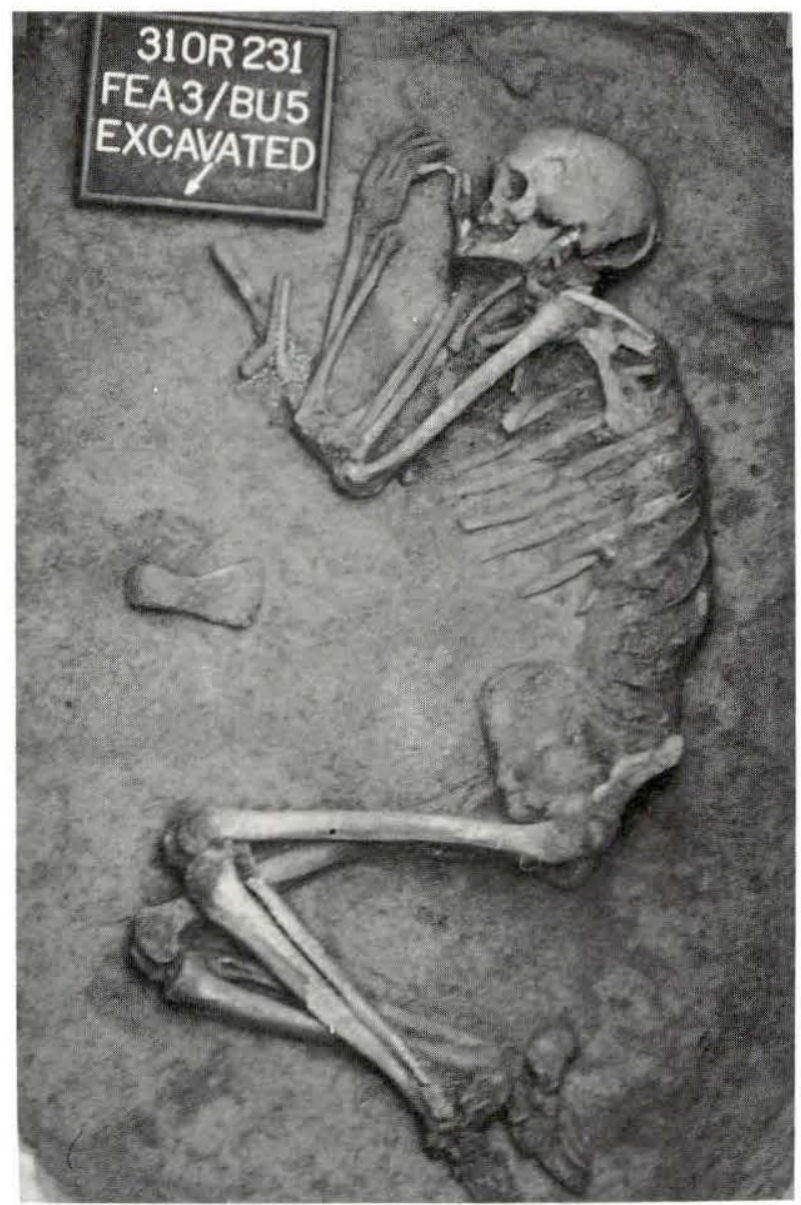

Figure 4.15

Burial 5 at the Fredricks site. associated with it. A wine bottle was found in the southeast corner of the pit next to the adult skull, and a pewter porringer was found at the foot of the bundle adjacent to and on the northeast side of the long bones. Based on the fact that a wine bottle was also found with Burial 3, an adult, it is probably that the bottle in Burial 4 was meant to accompany the adult. The porringer, on the other hand, may have been placed with the infant, because a similar specimen was found with Burial 2, a subadult.

Burial 5 (Figures 4.15-4.17)

Pit Morphology. The pit for Burial 5 was visible at the top of subsoil as a rectangular area of dark brown, charcoal-flecked fill surrounded by an irregular band of mottled orange fill. The configuration of this fill resembled in plan that of Burial 4. The pit was rectangular in outline, with its main axis oriented northwest-southeast. It measured $5.1 \mathrm{ft}$ in length, $2.9 \mathrm{ft}$ in width, and had a maximum depth of $2.0 \mathrm{ft}$ (Table 4.1). The pit corners were slightly rounded, and three of the walls were straight; the northeast wall, however, was undercut at the bottom of the pit creating a
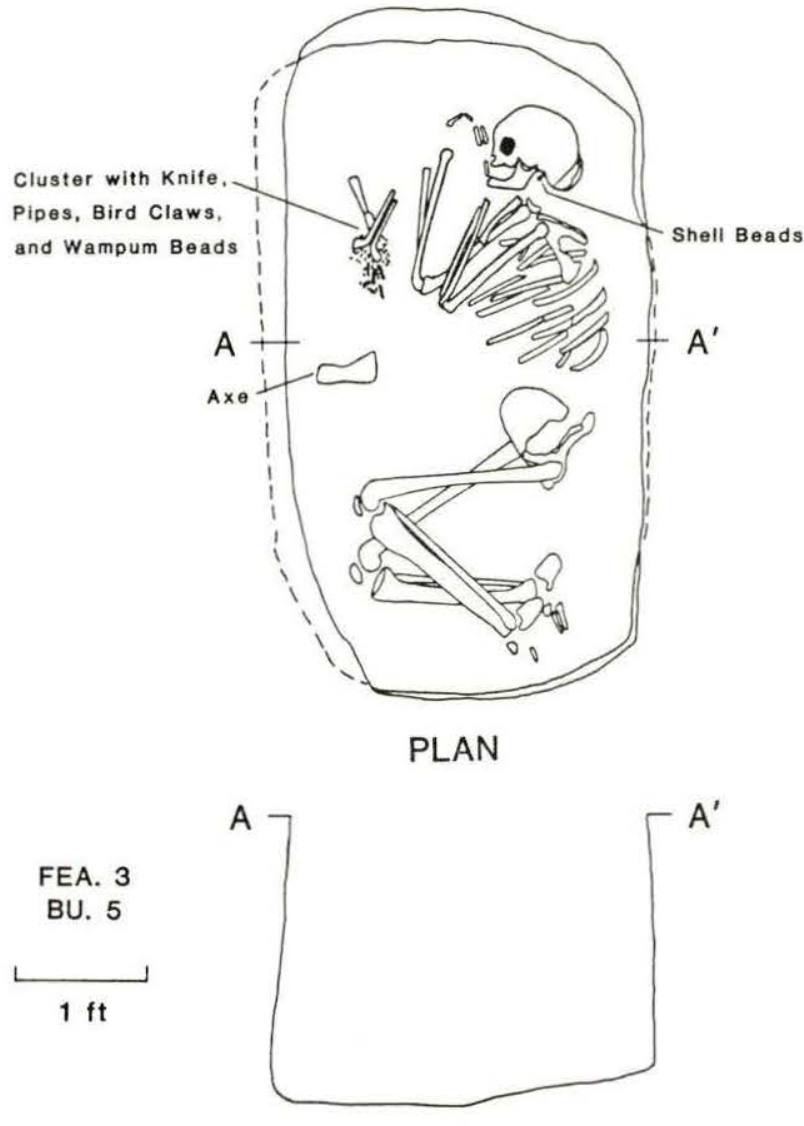

PROFILE

Figure 4.16

Plan and profile of Burial 5 at the Fredricks site. 


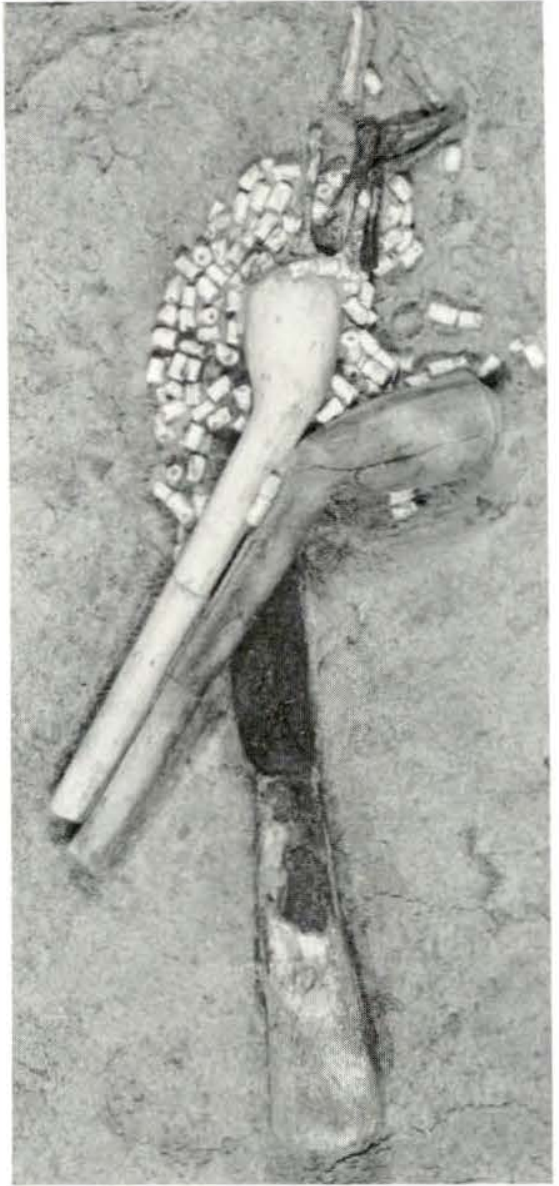

Figure 4.17

Artifact cluster from Burial 5 at the Fredricks site.

small side chamber. The bottom was flat and smooth indicating the use of iron tools.

The burial fill consisted of two primary zones. The upper zone (Zone 1), which comprised the central two-thirds of the pit, was composed of a dark brown loam with an ashy grey lens near the center that was nearly $1 \mathrm{ft}$ thick. Zone 1 contained charcoal, chunks of burned clay, shell, and burned and unburned animal bone. Although the dark fill was homogeneous, at the top, it became more mottled and blended into a zone of mottled orange clay (Zone 2) near the bottom. Zone 2 contained lens of dark organic fill and ashy deposits similar to Zone 1. These deposits extended down to the top of the ske1eton in the area between the arms and skull and near the thoracic vertebra. A group of large mammal bones in this fill made it difficult for the excavators to isolate the human skeletal remains. However, the mottled orange clay comprised the largest volume of fill in Zone 2 and extended over most of the skeleton. It also extended to the surface along the edges of the pit forming a broken ring around the dark organic fill at the subsoil surface of the pit.
Body Deposition. The body was that of an adult male who was over 50 years old at death. It was loosely flexed, lying on its right side with the skull to the southwest. The arms were bent with the hands in front of the face. The body was situated in the northwest half of the pit.

Grave Goods. The remains of a pouch decorated with wampum shell beads and a bird's clay contained two kaolin trade pipes and a bone-handled iron knife (Figure 4.17). The pouch lay adjacent to the elbow. An iron axe head lay in the area between the elbows and knees.

Burial 6 (Figures 4.18-4.19)

Evidence of this burial appeared in the plowzone as an area of dark brown soil containing animal bones, potsherds, and charcoal. At the top of the subsoil, the pit was rectangular in outline and measured $5.6 \mathrm{ft}$ by $4 \mathrm{ft}$ and extended to a depth of $2.3 \mathrm{ft}$ below the top of the subsoil (Table 4.1). The major axis was northwest-southeast. Three of the walls sloped slightly inward at the bottom. The northeast wall was undercut at the bottom, creating a long narrow chamber. As with the other burials, it was excavated with iron tools. When first observed at the top of the subsoil, the pit was rectangular in outline and had a central zone of dark brown humic loam with pebbles (Zone 1). The dark zone was surrounded by a collar of mottled orange and brown clay (Zone 2), which in turn, was encircled by more brown humic loam that contained some clay mottling (Zone 3). Beneath these fairly shallow zones with a combined thickness of $0.5 \mathrm{ft}$ was a mottled orange clay (Zone 4) similar to Zone 2 in the other burials. The final zone (Zone 5) lay over the body and consisted of a dark brown humus. The majority of food refuse was found in the west-central band of Zone 1 . In general, the zones of fill in this burial were more convoluted and harder to separate than those in the other pits.

Body Deposition. The skeleton was that of an adult male between 25 and 35 years old at the time of death. It was loosely flexed, lying on the right side with the elbows bent and the hands opposite the face. As with the other burials, the skull was pointing to the southwest.

Grave Goods. Accompanying the burial were an aboriginal pottery vessel lying behind the skull, a pewter pipe stem and bow1 rim between the face and hands, a large iron hoe near the feet, and a dog-lock musket lying parallel to the left side of the body. There was a leather covered copper wire bracelet around the left wrist. A pair of scissors and a lead shot were found beneath and immediately north of the pot. Glass beads were located under the pottery vessel and at each heel. Other glass beads were found under the copper bracelet. 


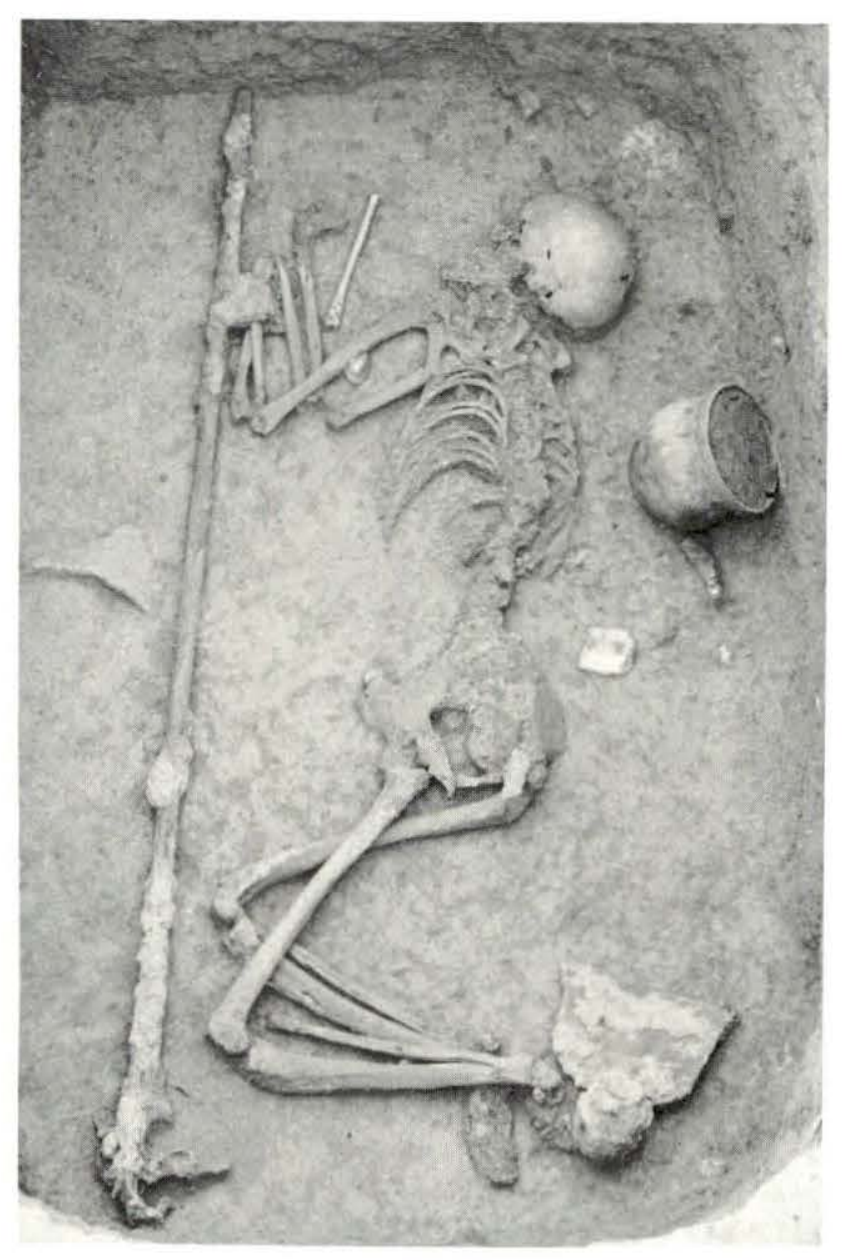

Figure 4.18

Burial 6 at the Fredricks site.

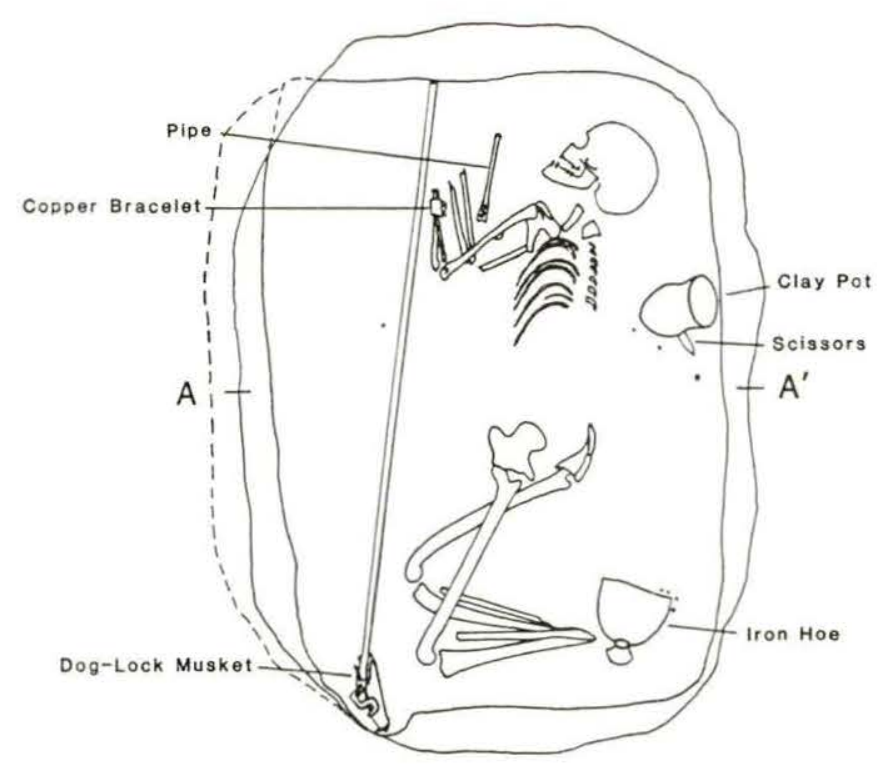

PLAN

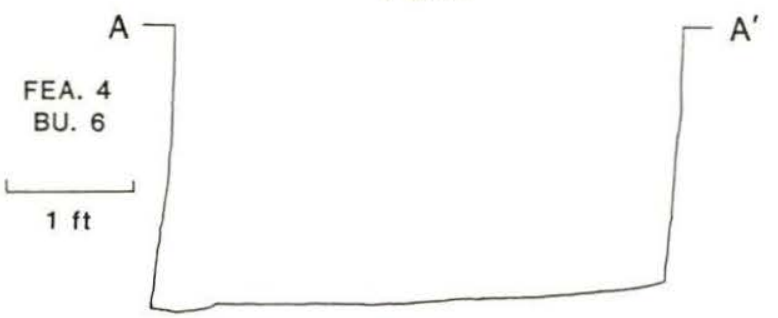

PROFILE

Figure 4.19

Plan and profile of Burial 6 at the Fredricks site.

observed in the pit. However, because of the alignment of this pit with the other burials and the inclusion of trade items, it is felt that the pit originally contained the remains of an infant or still-born.

Grave Goods. Two clusters of cast brass bells lay in the northwest half of the pit. Given the orientation of the other burials, they were probably associated with the legs of the individual. There were 10-15 incised bells in each cluster held together by leather thongs, portions of which were preserved, as well as fragments of wood or matting that had been on the floor of the pit.

Burial 8 (Figures 4.21-4.24)

Pit Morphology. When first observed at the top of the subsoil, this pit consisted of a rectangular stain of mostly brown loamy soil (Zone 1). The excavated pit measured $4.0 \mathrm{ft}$ by $2.5 \mathrm{ft}$ and was aligned with the long axis running northwest-southeast. A depth of $2.5 \mathrm{ft}$ made it the second deepest pit in the cemetery (Table 4.1). The sides were straight and not undercut to form a chamber as with many of

Body Deposition. No skeletal remains were 


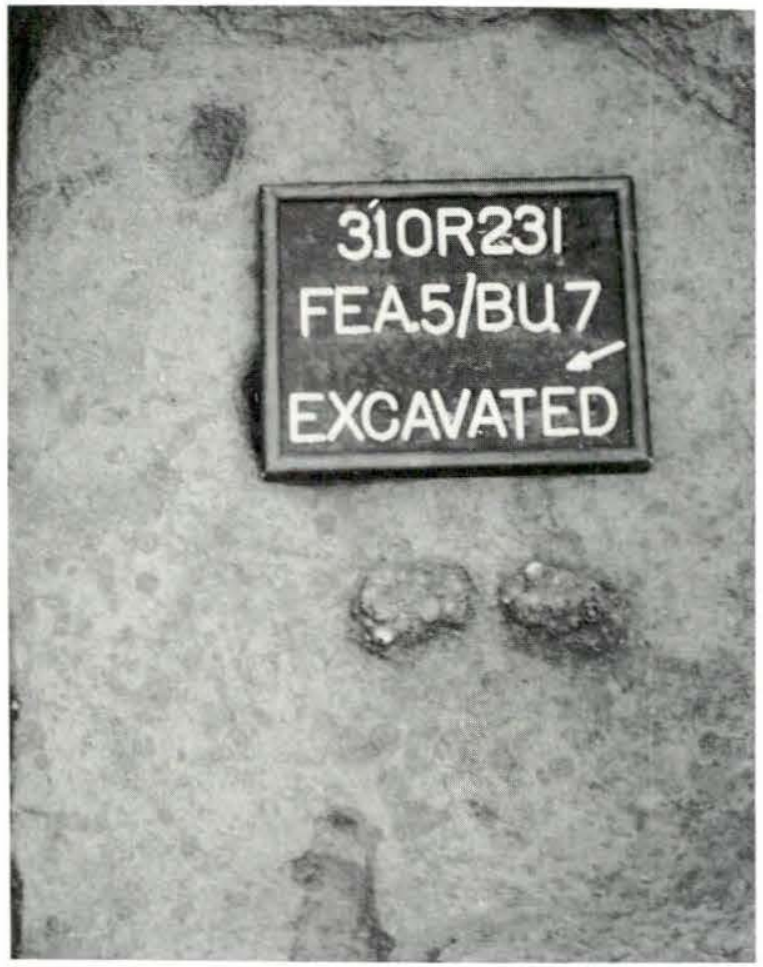

Figure 4.20

Bells associated with Burial 7 at the Fredricks site.

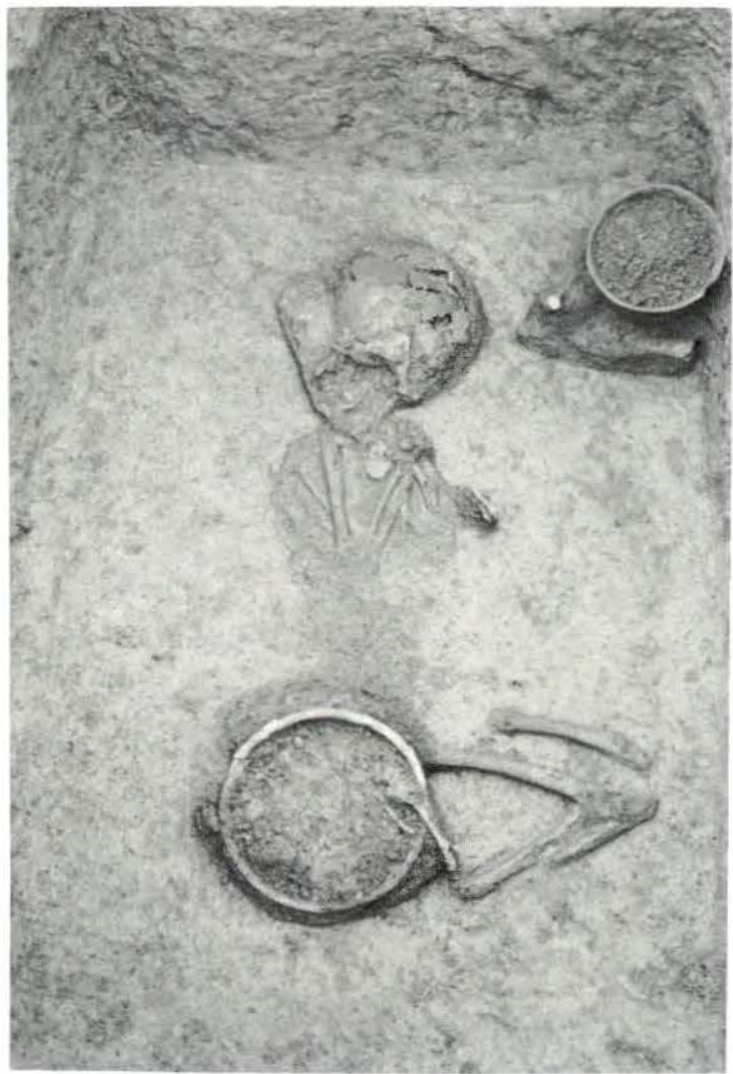

Figure 4.21

Burial 8 at the Fredricks site. the other burial pits. An uppermost brown loamy soil (Zone 1) contained numerous pebbles, and there was a dense concentration of animal bone and charcoal in the northwestern corner. A small patch of mottled orange and brown clay was located near the center of the top of the pit. The dark loamy soil, mixed with lenses of mottled clay comprising four zones, extended to a depth of approximately $1.0 \mathrm{ft}$. Below this was a thick zone of mottled orange clay that represented the original fill. Although this latter zone was fairly homogenous, a finger of the brown loam comprising Zone 1 continued to the floor of the pit along the southern wall.

Body Deposition. The pit contained the poorly preserved skeleton of a subadult, three to five years old at death. It was loosely flexed on its left side with the skull to the southeast. The hands lay on the chest.

Grave Goods. A copper kettle was positioned between the feet and pelvis. Several small lumps of vermillion or red ochre were found beneath the chin, and a brass buckle with part of a leather strap attached had been placed near the arms. Most interesting was a cluster of artifacts located in the southwest corner of the pit opposite the skull (Figure 4.23). This cluster consisted of a small check-stamped clay pot flanked by a bone-handled iron knife, a

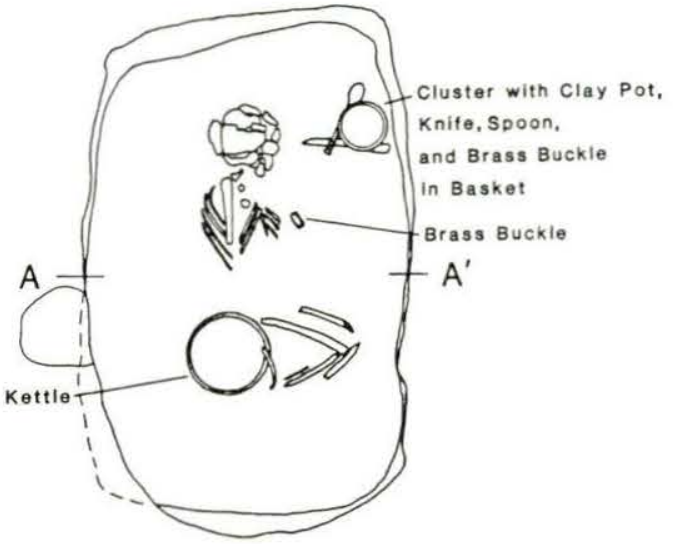

PLAN

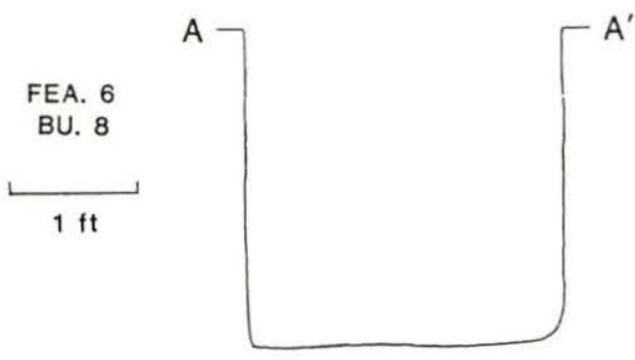

\section{PROFILE}

Figure 4.22

Plan and profile of Burial 8 at the Fredricks site. 

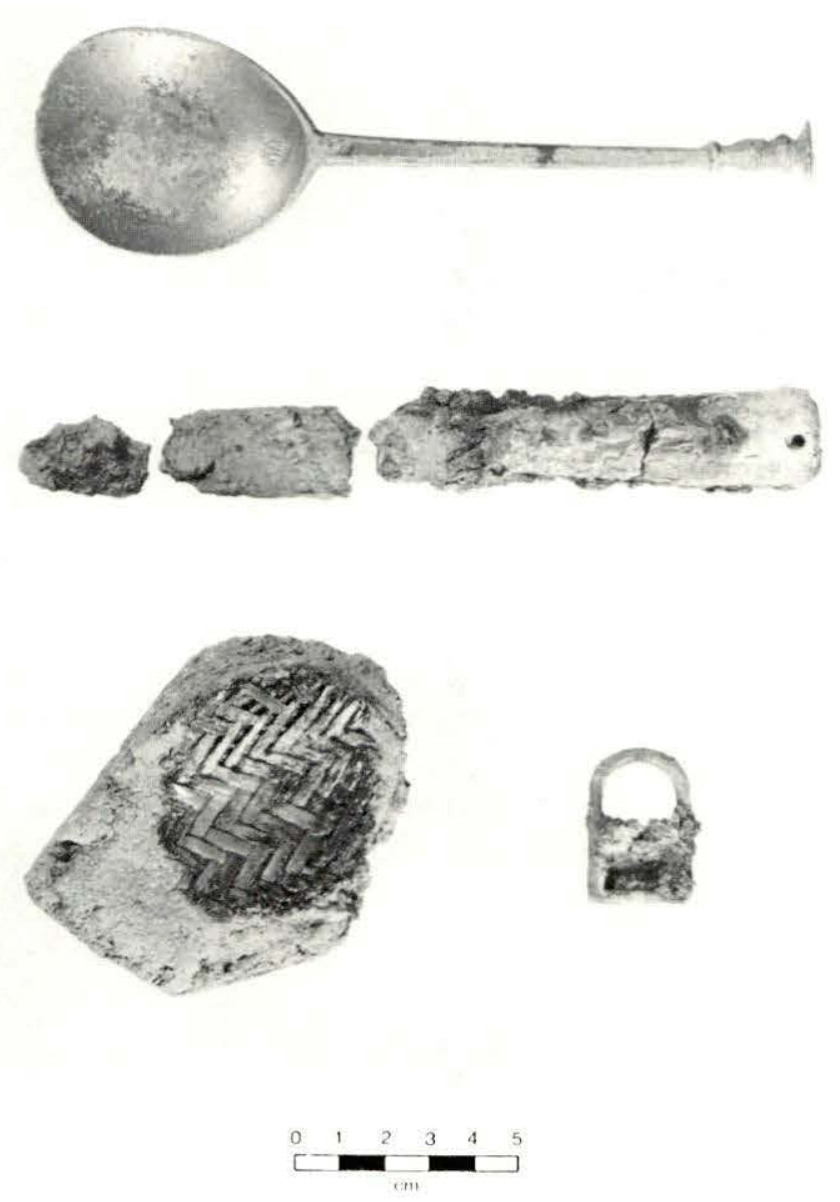

Figure 4.23

Artifact cluster from burial 8 at the Fredricks site.

latten spoon, and a brass buckle. All these objects had been placed in a twilled, split-cane basket, a portion of which was well preserved beneath the spoon bowl (Figure 4.24).

Burial 9 (Figures 4.25-4.26)

Pit Morphology. The fill of this burial was observed at the base of the plowzone as a rectangular stain whose long axis measured 5.1 $\mathrm{ft}$. It was $3.5 \mathrm{ft}$ wide, and extended to a depth of $2.3 \mathrm{ft}$ (Table 4.1). The sides of the pit were relatively straight, except the northeast side which was undercut at the bottom to create a slight $(0.5 \mathrm{ft})$ side chamber. The upper fill of the pit was comprised primarily of a brown loamy soil (Zone 1). Along the northwest side of the pit, this soil contained pebbles, animal bones, and charcoal. Along the northeast side, it was ashy in texture and not as rich. Patches of mottled clay were also noted in the center of the pit and across the southeast ends. The upper layer of refuse was approximately $1 \mathrm{ft}$ thick near the center of the pit. It rested upon a fairly homogeneous orange mottled clay which represented the original fill. This zone extended to the floor of the pit.

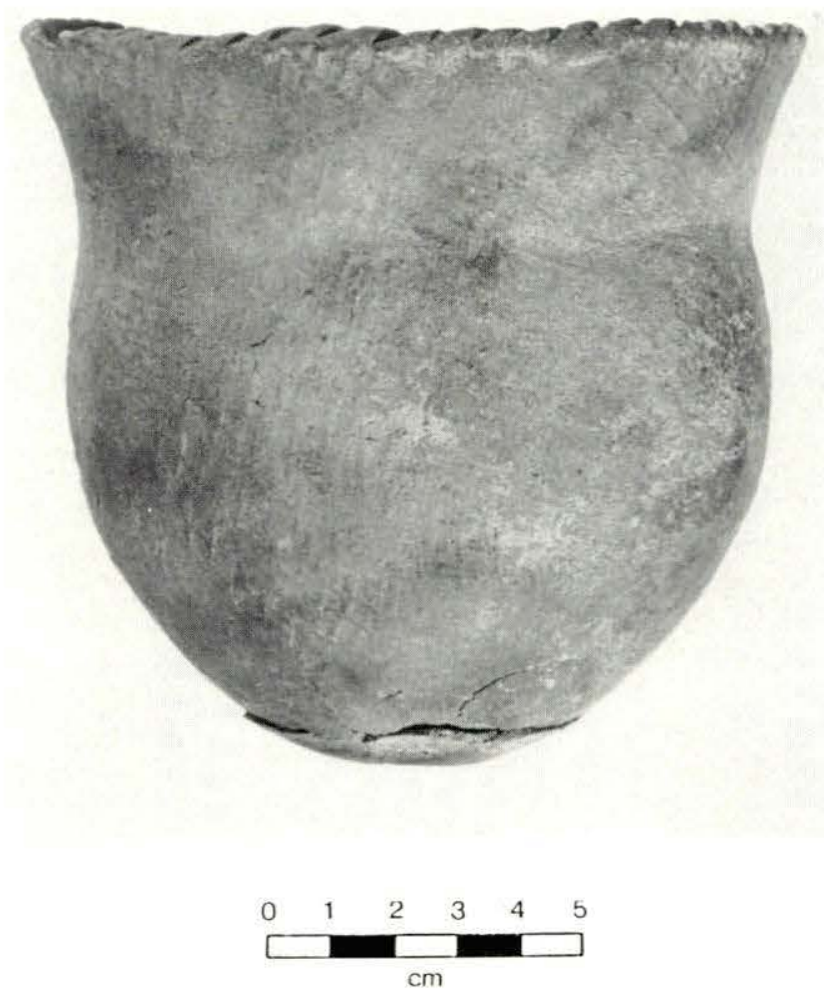

Figure 4.24

Pot with artifact cluster from Burial 8 at the Fredricks site.

Body Deposition. The poorly preserved skeleton was that of an adult female, 35-40 years old at death. It was loosely flexed on its right side. The skull was oriented to the southeast, and the hands were positioned on and in front of the face.

Grave Goods. Associated artifacts consisted of an iron hoe adjacent to and southwest of the skull (the blade end lay under the shoulder and occipital region of the skull). A bone-handled iron knife was under the right forearm. A possible violent death is indicated by a lead shot flattened against the left fibula. Another lead shot was recovered just above the pelvic area. Because of poor bone preservation, it was not possible to identify the effect of the shot on the fibula.

\section{DISCUSSION OF FREDRICKS SITE BURIALS}

Although there were differences in the content and complexity of fill in burial pits, some attributes were shared. In all but one case, there was an upper zone of brown loamy soil that contained relatively large amounts of animal bone, charred plant remains, pottery, and other refuse. This zone sometimes extended across the entire top surface of the pit, and in most instances, it contained considerable grit and/or pebbles. In some cases, this layer was underlain by or graded into a dark grey ashy 


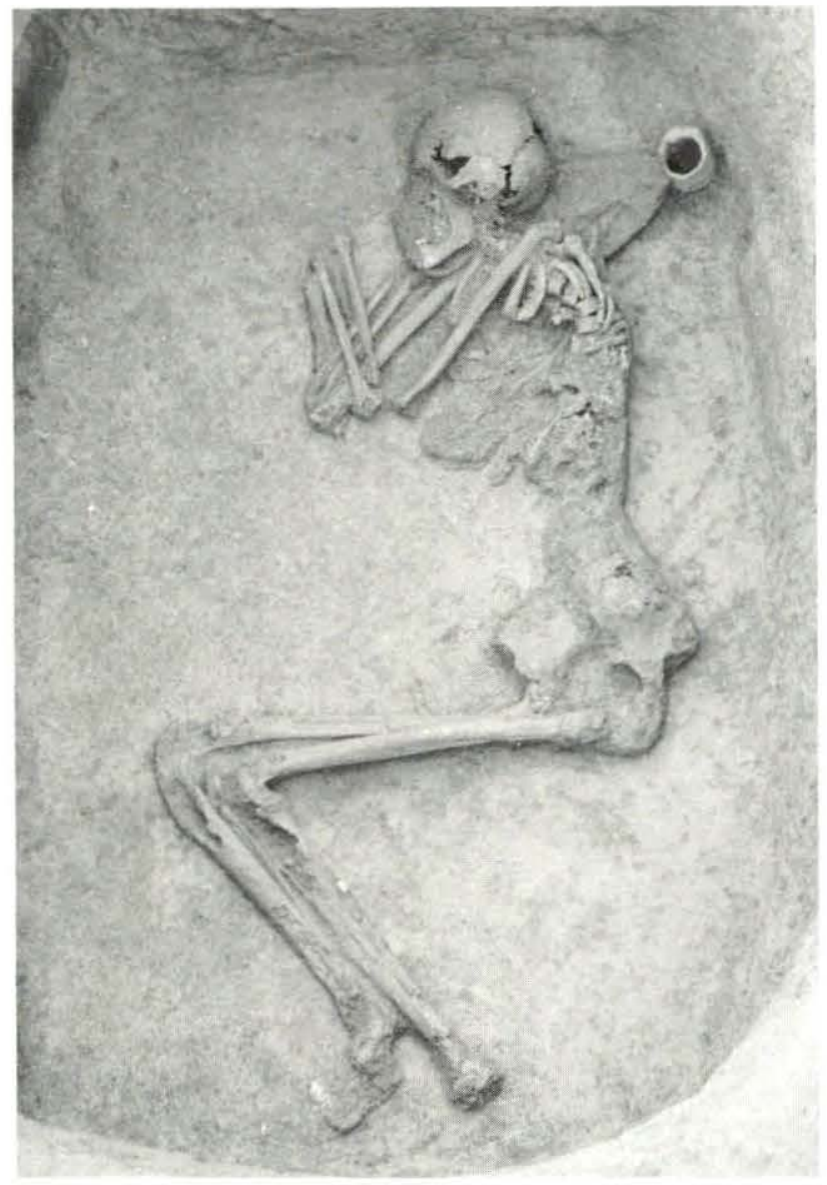

Figure 4.25

Burial 9 at the Fredricks site. layer, which was not as rich in refuse as the upper zone. Nonetheless, there were enough differences in the fill zones of the graves to separate the pits into four groups. The first group is represented by Burials 1,2 , and 3 . These pits were tightly grouped in the southeast end of the cemetery. Compared with the other burial pits, these three were rectangular and their edges were more sharply delineated. They also contained a rich dark brown loam that was usually homogeneous across the pit surface, extended to the pit edges, and had an average depth of one foot below the subsoil surface. This homogenous zone lay atop a grey ashy layer that in turn capped the typical mottled yellow burial fill (Figure 4.27).

Burials 4 and 5 comprise the second group (Figure 4.27). They are characterized by an upper fill zone that is slightly lighter brown in color and not as rich in refuse as the dark loamy fill of the first group. Neither was this zone homogeneous across the pit tops, as yellowish-orange clay formed a band around the pit edges. The pit outlines also were not as sharply delineated, and they were not as rectangular. The profiles of these burials show a semicircular zone of loamy fill sloping toward
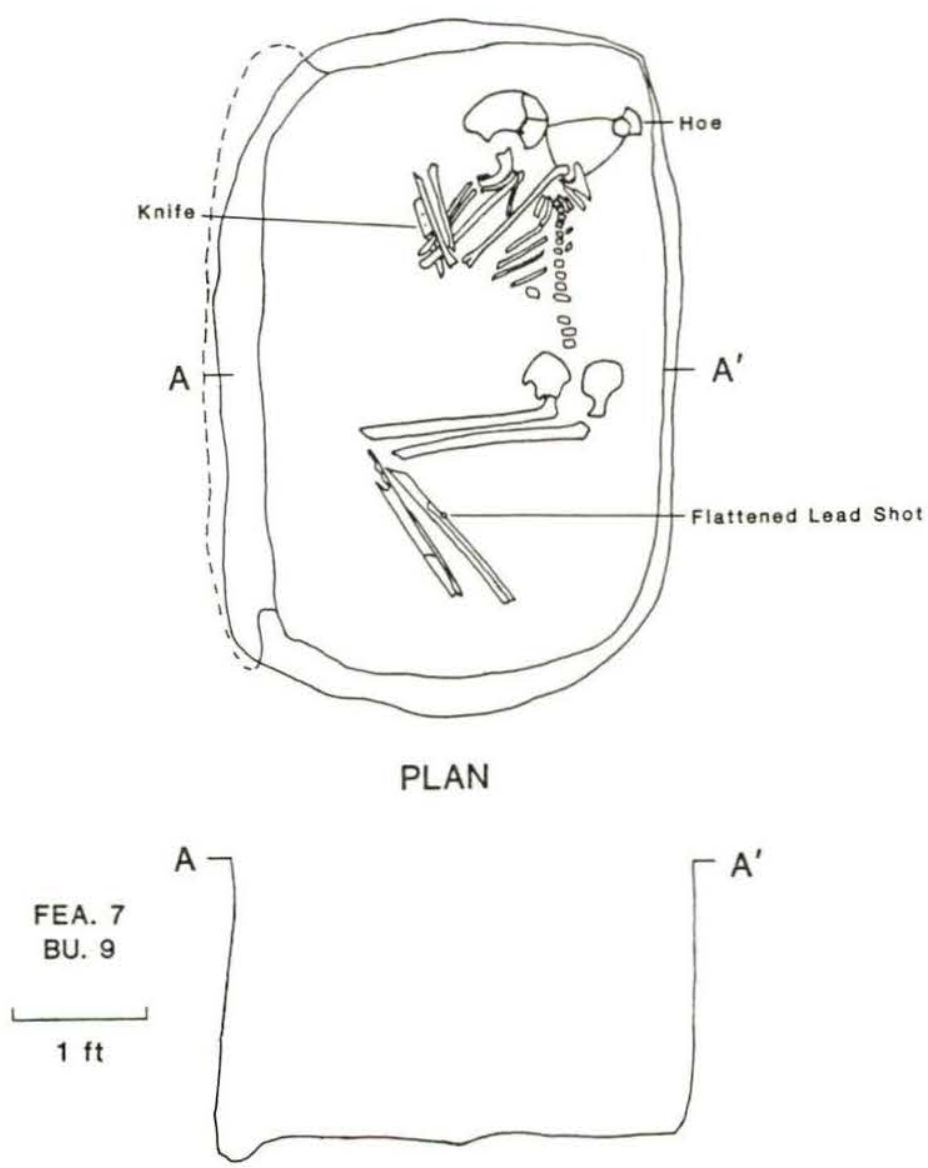

PROFILE

Figure 4.26

Plan and profile of Burial 9 at the Fredricks site.

the center of the pits that does not extend to the pit edges. It is instead surrounded by a mottled yellow clay collar which follows the perimeter of the pits.

The pit outlines of Group 3 (Burials 6, 8, and 9) were sharper than those in Group 2, but not as sharp as the first group of pits. The subsoil surface displayed a restricted zone of brown loam with orange clay present in the middle of the pits and around the edges. In profile, the brown humus formed a shallow depression that was surrounded by abundant orange clay (Figure 4.28). These soils sometimes overlay a fairly thick zone of brown humus that was lensed with orange clay. The latter rested on mottled orange clay burial fill.

The final category of burial fill was represented by a single burial, Burial 7 (Figure 4.28). An oval stain of light brown soil mottled with yellow clay was approximately 0.6 ft thick across the top of the pit and overlay a zone of mottled orange and brown clay. The pit edges of Burial 7 were not distinct from the subsoil and the fill was not as rich in cultural materials as the other burials. 


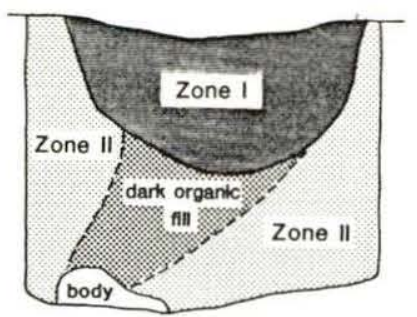

Zone I- brown loam

Zone II - mottled orange clay

FEA. 3/BU. 5

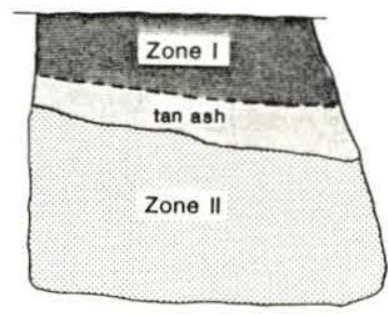

Zonel - brown loam

Zone II - mottled orange clay

\section{Figure 4.27}

Fill profiles of Burial Groups 1 (below) and 2 (above) at the Fredricks site.

The different categories of burial fill may reflect somewhat different behavioral activities in the final act of covering the bodies with soil. The first group of burials, those with the most distinctive fill profiles, suggest the following sequence of events. At death, the individuals were wrapped, placed in the pits, and covered initially by fill dug from the graves. (That the bodies were wrapped is suggested by the presence of a concentration of dark humic soil immediately over and around the skeletons.) There is no evidence that a vault as described by Lawson was ever constructed. The grey ashy soil overlying the initial burial fill indicates that ashes were cleaned from hearths and deposited in the pits. In situ fire is ruled out since there is no evidence of burning on the surface of the fill. After the ashes were thrown into the graves, domestic refuse was deposited on top to complete the filling of the pits. This final layer seems to represent the remains of feasts prepared and served at the time of death. As noted earlier, many ethnohistoric accounts of eastern North American Indians describe feasts as part of burial ritual. And, in the case of the Delaware and Shawnee, there are accounts of food and sometimes fire actually being placed on new graves.

It is surprising that there is no evidence of vaults or house-like structures constructed over the burials. There was, however, a small side chamber in Burials 2 and 3. Apparent1y, at the Fredricks site, the small chambers were used to hold perishable grave goods such as furs or cloth, which were mentioned as grave offering several times in the ethnohistoric literature. Such materials are indicated by the fact that all of the chambers contained a mottled humic soil, similar to that surrounding the bodies, of the type that would be formed by the decay of a large amount of organic matter.

A set of behaviors different from Burial Group 1 is indicated by the fill in the pits of Groups 2 and 3 . The primary differences lie in

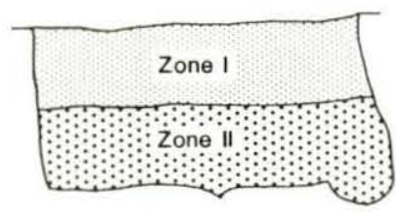

FEA. 5/BU.7
Zone 1 - mottled brown and yellow clay

Zone II - mottled orange and brown clay

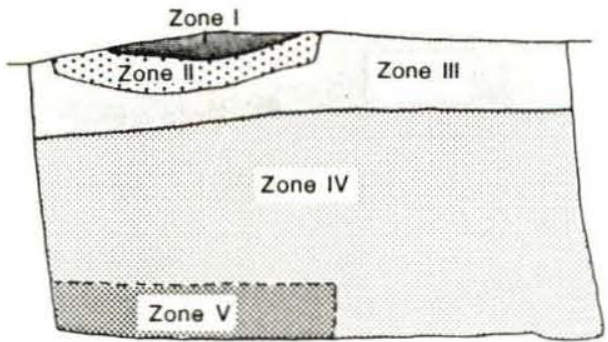

Zone I - brown loam

Zone II - mottled orange and brown clay

Zone III - brown loam and orange clay

Zone IV - mottled orange clay

Zone V - dark brown humus

FEA. 4/BU.6

Figure 4.28

Fill profiles of Burial Groups 3 (below) and 4 (above) at the Fredricks site. 
the fact that the top brown loam or humus layers contained fewer remains and were not homogeneous across the pits. In all cases, this zone(s) sloped inward towards the center of the pits and was partially or totally surrounded by mottled orange clay. In at least one instance (Burial 6), a zone of brown loam with lenses of orange clay overlay the typical mottled clay burial fill. The set of activities responsible for the filling of these pits is not as clear as those for the first group. More time seems to have elapsed during the filling process, as indicated by the lensing of the fill and the slumped, rather than sharp, profiles of the brown loam. A longer filling period is also indicated by the less distinct outlines of the pit walls. It almost seems as if the pits were originally only partly filled with soil added as previous layers settled. However, the last layer (brown loam) did contain refuse, although not as much as the first three burials. Perhaps the cleaning and feasting activities were delayed for a period of time after the pits were initially filled. It could be further suggested that the feasting rituals involved fewer individuals and were not as intense as those proposed for the first burial group. Three of the graves from Groups 2 and 3 did contain either ledges (Burial 4) or small side chambers (Burials 6 and 9) where organic materials had been placed.

Burial 7 contrasts markedly with the others in the simplicity of its fill. The pit was dug with a relatively deep side chamber which probably also contained organic remains such as cloth or furs. After the infant was placed in the pit, it was apparently quickly refilled, and there was little or no attendant ritual.

An examination of the associated grave goods reveals differences that parallel the spatial and fill clusters described above, and that indicate age and sex parameters. Using Brain's (1979) "acculturation index," the European artifacts were each assigned a value between 1 and 4 , and the values were then totaled for each burial (Table 4.2). If it is assumed that the numbers and kinds of trade artifacts associated with a burial are to some extent a reflection of access to such items and indirectly of status, then Brain's index should provide a means of numerically expressing the social dimensions of burial ceremonialism. Additionally, all burialassociated artifacts, European and aboriginal, were compared as to whether they were utilitarian or ornamental (Table 4.3). The glass and shell beads, which, when present, were in great quantities, were compared only on a presence or absence basis. Lead shot, buttons, and nails were treated in like fashion. Thus, it was assumed that 10 beads or nails were not 10 times more important than one such item. For the beads, in particular, a large group may reflect nothing more than a single decoration on an article of clothing.

Using Brain's index, Group 1 has the highest average at 25.3, followed by Group 3 at
Table 4.2

Acculturation indices for Fredricks site burlal grouplngs.

\begin{tabular}{|c|c|c|c|c|c|c|}
\hline \multicolumn{2}{|c|}{ Group 1} & \multirow{2}{*}{$\begin{array}{l}\text { Group } \\
\text { Burial }\end{array}$} & \multirow{2}{*}{$\begin{array}{l}2 \\
\text { Index }\end{array}$} & \multirow{2}{*}{$\begin{array}{l}\text { Group } \\
\text { Burial }\end{array}$} & \multirow{2}{*}{$\begin{array}{l}3 \\
\text { Index }\end{array}$} & \multirow{2}{*}{$\begin{array}{c}\text { Burlal } 7 \\
\text { Index }\end{array}$} \\
\hline Burial & Index & & & & & \\
\hline Bu. 1 & 18 & Bu. 4 & 4 & Bu. 6 & 13 & 4 \\
\hline Bu. 2 & 15 & Bu. 5 & 5 & Bu. 8 & 13 & \\
\hline Bu. 3 & 43 & & & Bu. 9 & 6 & \\
\hline Total & 76 & & 9 & & 32 & 4 \\
\hline Mean & 25.3 & & 4.5 & & 10.7 & - \\
\hline S.D. & 12.6 & & 0.5 & & 3.4 & - \\
\hline
\end{tabular}

Table 4.3

Distribution of artifacts associated with the Fredricks site burials by age categorles.

\begin{tabular}{|c|c|c|c|c|c|}
\hline Subadult & & Adult & & Shared & \\
\hline Spoons & U & Smok1ng P1pes & u & KnI ves & $\mathrm{v}$ \\
\hline Metal Buttons & 0 & Ember Tender & u & Sclssors & $\mathrm{u}$ \\
\hline Glass Buttons & 0 & Wine Bottle & u & Porringer & U \\
\hline Jews Harp & 0 & Gun & u & Lead Shot & u \\
\hline Kettle & u & Axe & U & Buckles & $\mathrm{U}$ \\
\hline Be11s & 0 & Hoe & $\mathrm{u}$ & Ochre & 0 \\
\hline Basket & u & Bracelet & 0 & Beads & 0 \\
\hline Shell Gorgets & 0 & B1rdelaw & 0 & Pots & $\mathrm{u}$ \\
\hline $\begin{array}{l}\text { She11-Beaded } \\
\text { Garments }\end{array}$ & 0 & & & & \\
\hline$U=3 ; \quad 0=6$ & & $U=6 ; \quad 0=2$ & & $U=6 ; 0=2$ & \\
\hline
\end{tabular}

$U=U t 111 \operatorname{tar} 1 \mathrm{an} ; \quad 0=$ Ornamental.

10.7 and Group 2 at 4.5. Group 1 also displays the highest standard deviation because of the extremely high innovative value for Burial 3, which is more than triple that of any other burial (Table 4.2). Although the overall burial sample is small, there appear to be clusters of artifact associations that parallel the groupings based on fill characteristics. Even if Burial 3 was removed from Group 1 , the remaining burials of that group still have the highest scores on the acculturation index. The uniqueness of Group 1 is further enhanced if the large numbers of shell and glass beads are considered by total numbers rather than only by presence or absence. The burials in the other groups contained very few beads. Originally, it was thought that large numbers of beads were a characteristic of subadult burials because the beads were sewn on burial garments special to children. This still may be the case for the subadults in Group 1, i.e., Burials 1 and 2, but Burial 8 (Group 3), also a subadult, had no associated beads, glass or shell. And in terms of fill attributes, Burial 8 was more like adult Burials 6 and 9 . 
There also appears to be a dichotomy of burial associations based on age. Most of the artifacts associated solely with subadults fall into the ornamental category, whereas the majority of the artifacts associated only with adults are utilitarian (Table 4.3). Those utilitarian artifacts associated with subadults are not tools and are associated with activities that are not technomic in nature. Spoons, kettles, and baskets (associated with children) are used for eating and for containers, which is in sharp contrast to the gun, gun parts, hoes, and axes (associated with adults), which are used in heavy labor and subsistence-related activities. The items shared by adults and subadults, such as knives, scissors, and beads, represent activities and items that probably would be shared by both age groups.

In summary, pitfill characteristics and associated artifacts suggest that at least two levels of treatment were accorded the Fredricks site burials. The first three burials are very distinctive. The upper zone of refuse-laden soil indicates a more intense burial ritual probably having to do with ritual feasting. Apparently similar, but less intense, ceremonies were conducted for the other burials, except Burial 7.

In general, children received the most attention. Burials 1,2 , and 8 all contained large numbers of European artifacts, and Burials 1 and 2 also contained shell gorgets and numerous shell beads. Although most of the beads were probably sewn on garments, the gorgets and some of the larger beads represent deposition of individual items having sociotechnic or ideotechnic meanings. Other historic cemetery sites have also shown a pattern of large numbers of beads and she 11 artifacts being associated with children (e.g., Witthoft et al. 1959:115).

Although children received much attention, neonates received almost none. Burial 7 contained only a few brass bells, and the infant associated with Burial 4 was accompanied probably by only a pewter porringer. Feature one probably also contained the remains of a small infant that was not accompanied by any nonperishable grave goods. The chamber of this burial, however, did contain a darker soil indicating that perishable artifacts such as blankets or furs may have been included.

Where children received elaborate treatment, Burial 3, a young adult male, contained the largest collection of burial furniture and richest upper fill of any burial in the cemetery. Burial 3, therefore, may represent the highest ranking individual in the cemetery. Burial 6, also a young adult male, appears to have occupied a social position akin to that of Burial 3. Both contained large numbers of primarily utilitarian artifacts, probably personal property. Burial 3 contained a smoking kit, scissors, knives, gun parts, as well as a rum bottle and an iron axe head. Burial 6 contained an almost comparable array of smoking artifacts along with a musket and a large iron hoe.

In contrast with Burials 3 and 6, Burial 4, an adult male of similar age, contained very few grave associations, only a group of tubular shell beads and a rum bottle. This burial was unique in the fact that it was bundled, which indicates that the individual died away from the village. Cut marks on the skull also indicate that he died a violent death.

In summary, the burials at the Fredricks site represent a portion of a cemetery that lies immediately northeast of a palisade surrounding at least part of the village. All of the burials seem either to have been made over a short interval of time or to have been precisely located by above-ground markers, or both. Only one burial pit, Burial 2, was intruded by posts which suggest they may have served as markers; however, smaller postholes were found near most of the pits. Interment over a short interval is indicated by the precise orientation of the skeletons along a northwest-southeast axis and the fact that the heads all point in the same southeastward direction. If a solar reference point was used to align the bodies, they must have been interred over a very brief period of time (cf. Gruber 1971).

\section{WALL SITE BURIALS $(1938,1940-41)$}

A total of five burials were excavated by Joffre Coe at the Wall site during the 1938, 1940, and 1941 field seasons. The descriptions of these burials will be abbreviated because data pertaining to spatial dimensions and pit fill morphology were not available.

Burial 1. This burial was located near the center of Structure $E$. The skeletal remains were that of an adult male, over 45 years old at the time of death. It was tightly flexed and placed in a circular pit on its left side. The axis of the body was east-west, with the head to the east. Grave goods consisted of several potsherds, representing two different vessels, and an aboriginal pipe. Burial 1 also contained a rich dark brown refuse layer in the upper level of the pit. In the field, it was originally given a feature number and designated as a "trash pit". Several large rocks separated the refuse zone from a lower zone of mottled yellow burial fill.

Burial 2. This pit contained the remains of a child between two and three years old at death. The burial pit was located in a line of posts forming the northern wall of Structure C. The skeleton was placed in a shaft-and-chamber pit in a flexed position on its left side. The long axis was northeast-southwest with the head to the northeast. In the grave with the body were one aboriginal pot, left and right arm 
bands comprised of over 1000 small shell disc beads, a headdress containing over 4000 small shell disc beads and four copper beads, and three shell gorgets.

Burial 3. This was an adult, about 50 years old at the time of death. The burial was located in a palisade line in the northwest corner of the excavations. The skeleton was placed in a shaft-and-chamber pit, lying on its left side. The axis of the body was northeast-southwest, and the head was to the northeast. Grave associations consisted of only two serrated mussel shells.

Burial 4. This shaft-and-chamber burial contained the remains of an adult male, 18-25 years old at the time of death. The skeleton was tightly flexed on its left side. The main axis of the burial was, again, northeastsouthwest, and the head was pointed to the northeast. It was located between Structures D and E. Associated artifacts included: one aboriginal pottery vessel, 52 large columella shell beads around the neck, over 4000 small shell disc beads (probably sewn to a garment) also in the neck area, 176 small shell disc beads (sewn to a band) around the left ankle, and 75 small shell disc beads comprising a band around the left wrist.

Burial 5. This burial was represented by a fetus that was placed in an unprovenienced posthole. No further information was available.

WALL SITE BURIALS (1983)

Burial 1-83 (Figure 4.29)

Pit Morphology. This burial pit was visible at the top of subsoil as a circular stain of dark brownish-black loam (Zone 1) that contained a large number of animal bones, potsherds, and shell fragments, including a complete shell scraper. The dark, rich fill averaged approximately $0.8 \mathrm{ft}$ thick. At the base of this zone were five large rocks (averaging roughly 30 lbs each) which rested on an almost sterile yellowish orange clay (Zone 2). Zone 2 extended to a depth of approximately three feet, where a brown loamy lens containing charcoal was encountered. Associated with this lens were two long brown log-like stains. The orientation of these stains suggests that the lenses and stains were the remains of a cover over the chamber. The lenses and stains were underlain by a mottled yellow clay that continued to the bottom of the pit and into the chamber. The total depth of the pit was $4 \mathrm{ft}$. Maximum width at the top of subsoil was $3 \mathrm{ft}$. The chamber extended $1.5 \mathrm{ft}$ under the southeast wall.

Burial Deposition. The burial was that of a subadult who was between four and six years old at the time of death. The skeleton was
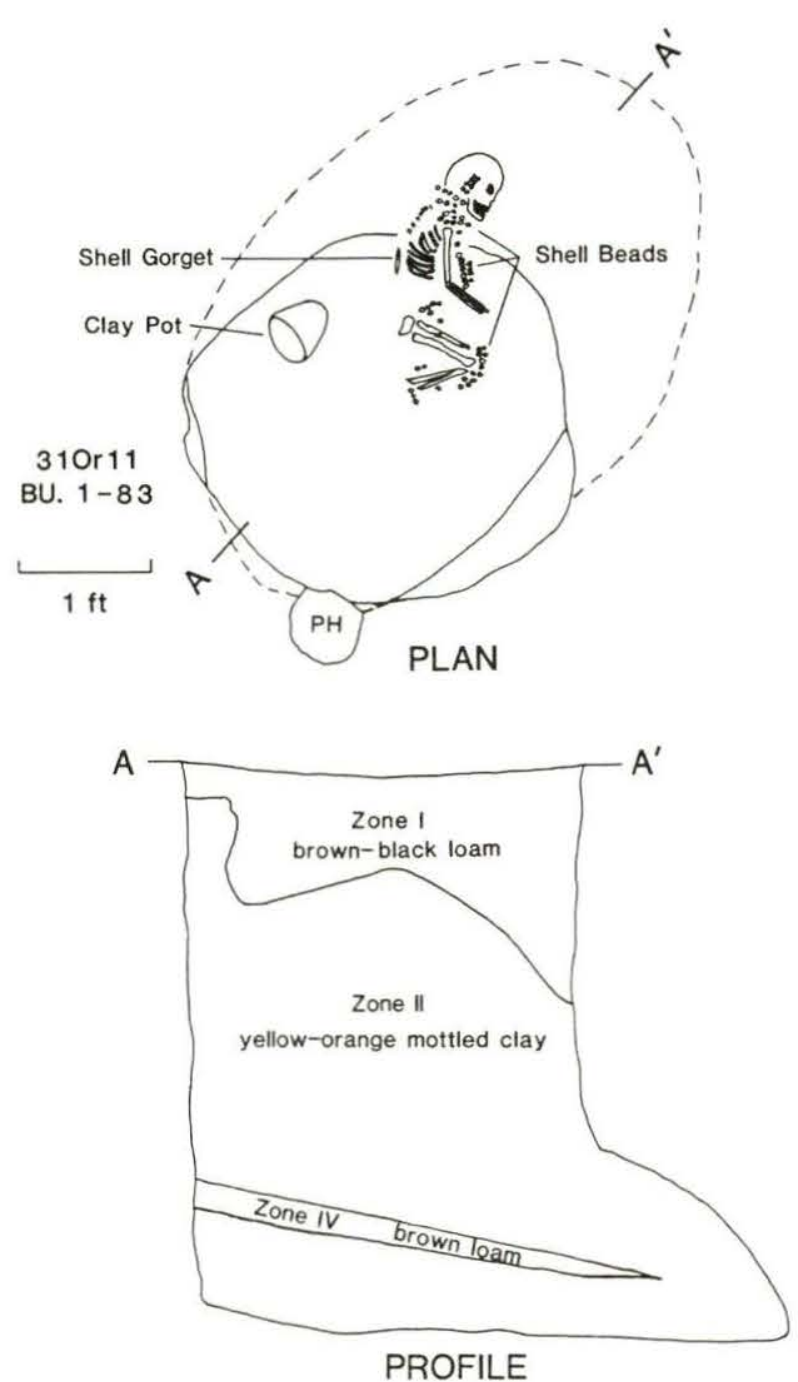

Figure 4.29

Burial $1-83$ at the Wall site.

loosely flexed on its left side with the head oriented to the southeast. The arms were slightly bent, and the hands lay above the knees. Rather than being tightly tucked into the chamber, the skeleton lay with the head and chest area within the chamber and the lower body on the floor of the pit. The pit and chamber were deep and large compared to other shaftand-chamber burials from Siouan sites.

Grave Goods. A band of marginella beads represented the remains of a cap or bonnet over the skull. Small shell disc beads and columella beads were strung around the neck. A single tubular copper bead was also found in the neck area. Additional columella beads were present in the leg and pelvic areas. A strand of marginella beads along the left leg may have been attached to leggings. A shell gorget located behind the neck probably had been worn around the neck. North of the feet was a small pottery vessel. When cleaned in the $1 \mathrm{ab}$, it was discovered that this vessel contained another smaller pot and an unmodified mussel shell. 


\section{Burial 2-83}

Pit Morphology. The upper fill in this burial consisted of two distinct soil zones. The first zone was a dark brown loam that covered the eastern third of the circular pit outline. The remainder of the pit appeared as a mottled orange clay. The configuration of the pit in plane is typical of shaft-and-chamber burials. The dark brown soil represents slump as the burial chamber collapsed, and the mottled clay is indicative of the original burial fill placed in the pit shaft. The shaft was dug to a depth of $1.8 \mathrm{ft}$. In the southeast corner the chamber undercut the pit wall and sloped to a depth of $2.4 \mathrm{ft}$. Maximum diameter of the top of the pit was $3.3 \mathrm{ft}$.

Burial Deposition. The skeletal remains were in extremely poor condition and appear to represent the remains of small a child 9 to 18 months old at the time of death. The head was oriented to the southwest and the body was flexed on its right side.

Grave Goods. The only burial association was a necklace of large columella beads. The skull was lying on a flat rock.

\section{Burial 3-83}

Pit Morphology. At the top of subsoil, this pit had an appearance very similar to Burial 2-83. The southern half of the fill was a dark loamy soil, whereas the northern half contained a mottled orange clay. The latter soil type defined the burial shaft; the former represented the slump of the chamber which only slightly undercut the southern wall of the pit. The pit measured $2.5 \mathrm{ft}$ wide and $2 \mathrm{ft}$ deep.

Burial Deposition. The burial was that of an infant or still-born. Because only a few small bone fragments were preserved, body position and orientation could not be discerned. It is notable, however, that the body stain was covered by a thin mantle of white clay.

Grave Goods. Two small ceramic vessels were found immediately west of the clay mantle and skeletal remains. The vessels were sideby-side, one upright and the other on its side.

\section{DISCUSSION OF WALL SITE BURIALS}

As was the case with the Fredricks site, children and some adult males seem to have received the most attention at death. Three status dimensions are indicated by artifact associations and grave preparation. Burials 1 and 1-83 represent the most elaborate treatment as both were capped by especially rich midden. In fact, both of these burials were originally designated "trash pits" in the field. In both, there were also large rocks separating the upper organic fill from the sterile mottled yellow burial fill. The similarity between these two burials and those at the Fredricks site is striking, particularly when compared with the first three Fredricks site burials. Apparently, these pits were also partially filled with residue from ritual cleaning/feasting activities. Although the activities directly responsible for the refuse filled zones cannot be reconstructed more specifically, it is obvious that these behaviors were similar at both the Wall and Fredricks sites. Burials 2 and 4, a subadult and adult respectively, may represent an intermediate status position. They have an array of grave goods, particularly shell artifacts, but lack a capping of midden. The other child burials only contained a small number of decorative or utilitarian artifacts and do not appear to have been accompanied by as elaborate mortuary ritual as the other burials.

The spatial distribution of the Wall site burials contrasts markedly with that of the Fredricks site. The Wall site graves are more dispersed, with some either inside or adjacent to house structures. Any hint of clustering into cemetery units is lacking. Another interesting contrast lies in the burial orientations. Most of the Wall site burials have their heads oriented to the northeast, whereas most of the Fredricks burials are oriented to the southeast. The Wall site also has more variability in orientation with one burial oriented to the southwest, one to the southeast, and one to the east. This variability may indicate that interments were separated by lengthy intervals or that orientation was not as important as at the Fredricks site. All of the Wall site burials were oval in plan and of the shaftand-chamber type, in contrast with the rectangular, simple-pit burials at the Fredricks site.

\section{THE MITCHUM SITE BURIAL}

\section{Burial 1 (Figure 4.30)}

Pit Morphology. At the top of subsoil, this circular pit was $2.9 \mathrm{ft}$ in diameter and had the configuration of most shaft-and-chamber burials. The western two-thirds was defined by a light brown sand that filled the cavity created by chamber collapse and slump, whereas, the eastern one-third contained a mottled light tan sand comprising original burial fill. The shaft extended to a depth of $2.2 \mathrm{ft}$ below subsoil, and the chamber undercut the west wall of the pit and sloped downward to a maximum depth of $2.8 \mathrm{ft}$.

Body Deposition. The skeleton was that of a subadult who was between seven and nine years old at death. It was lying within the chamber on its left side with the head oriented to the north. The legs were loosely flexed and the arms were bent with the hands beneath the chin. 


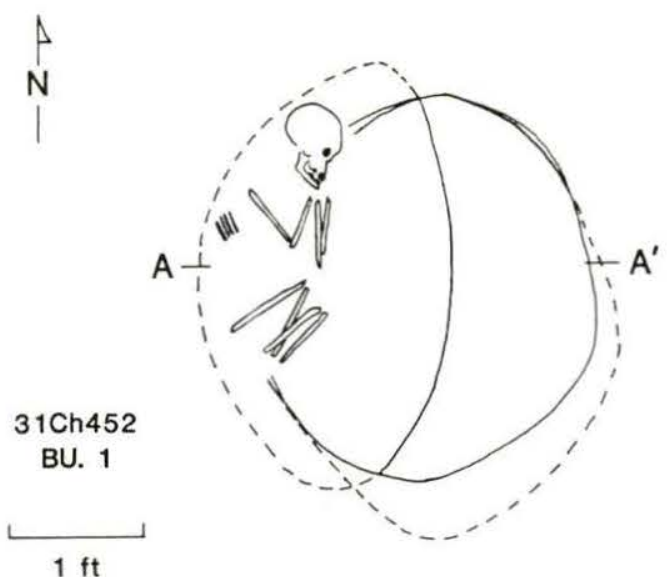

PLAN

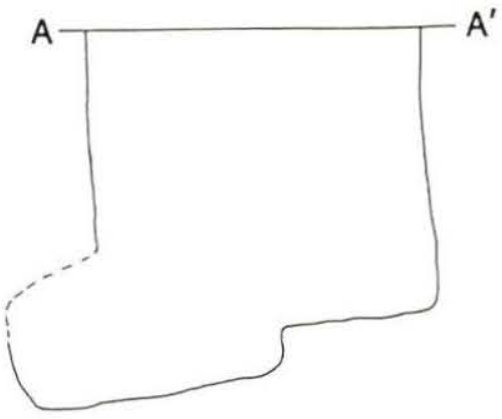

PROFILE

Figure 4.30

Burial 1 at the Mitchum site.

Grave Goods. Grave goods consisted of a necklace of hundreds of small glass trade beads and two copper or brass ear ornaments.

The single shaft-and-chamber burial at the Mitchum site appears to occupy an intermediate chronological position between the Wall and Fredricks sites. The shaft-and-chamber style of pit and the relatively large number of glass beads are characteristics similar to the Siouan burials at Upper Saratown.

\section{SUMMARY OF MORTUARY DATA}

During the Late Prehistoric and Protohistoric periods, as evidenced at the Wall site, burials were loosely arranged within a village, with placement usually being in or near a house (Figure 4.31). Burial pits were circular or oval shaft-and-chambers. Except for clay pots (probably food containers), grave offerings consisted almost entirely of shell ornaments. Shell beads either were sewn on clothing or strung as jewelry. Shell gorgets sometimes were included with children. There is little evidence in the data thus far for status differences in these burials.

In the early part of the Contact period, as evidenced at the Upper Saratown (Navey 1982; Wilson 1983) and Mitchum sites, villagers continued to place burials in and around houses, with no clear indication of clustering, and a common pit type still was the shaft-andchamber. At Upper Saratown, there is much greater burial density than at the equivalently-sized Wall site, probably evidence of the effects of European diseases (Figure 4.31). Also during this period, glass beads replaced those of shell, and copper or brass ornaments became popular. Children still received the most numerous and elaborate grave offerings, and there is still little evidence of different treatments according to status for adults.

Later in the Contact period, by the time of the Fredricks site, there is evidence for dramatic changes in the mortuary practices of the Piedmont Indians. Burials were clustered in cemeteries outside the village, rather than being loosely arranged within the village (Figure 4.31). Metal tools were used to dig rectangular, straight-sided pits. Muskets, hoes, knives, scissors, and spoons replaced many of the metal trinkets of the earlier period. Glass beads remained popular, but their use was primarily with children. Both children and adults occasionally received shell beads or a clay pot as burial offerings. At the Fredricks site, some individuals, usually young adult males, received greater attention at death through feasting rituals and elaborate burial offerings.

\section{SOCIO-POLITICAL IMPLICATIONS OF THE FREDRICKS SITE BURIALS}

During the Prehistoric and early Historic periods, mortuary beliefs and practices seem to have been shared by an entire village, which was composed of closely related unilineal descent groups. This pattern is reflected in the scattered placement of burials within villages, such as at the Wall site and Upper Saratown. During this time, the village itself was, in effect, a "cemetery". The lack of spatial segregation of burials at these villages suggests an egalitarian social structure and may also indicate that village membership was more important than clan or lineage affiliation (cf. Tainter 1978; Bartell 1982). These earlier villages probably represent exogamous residential components of lineal tribes as defined by Service (1964: 128-133).

From the ethnohistoric records, it is obvious that by 1700 disease and warfare had decimated Piedmont Indians' populations. Vi1lages had fragmented and remnants of once 


\section{LATE PREHISTORIC EARLY HISTORIC}

MIDDLE

HISTORIC
LATE

HISTORIC

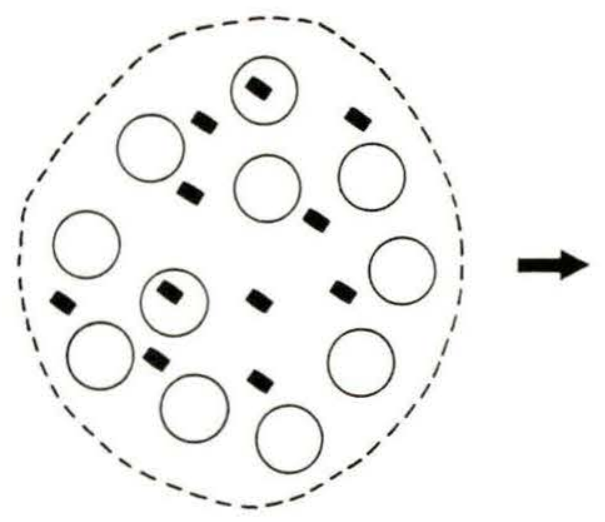

WALL SITE

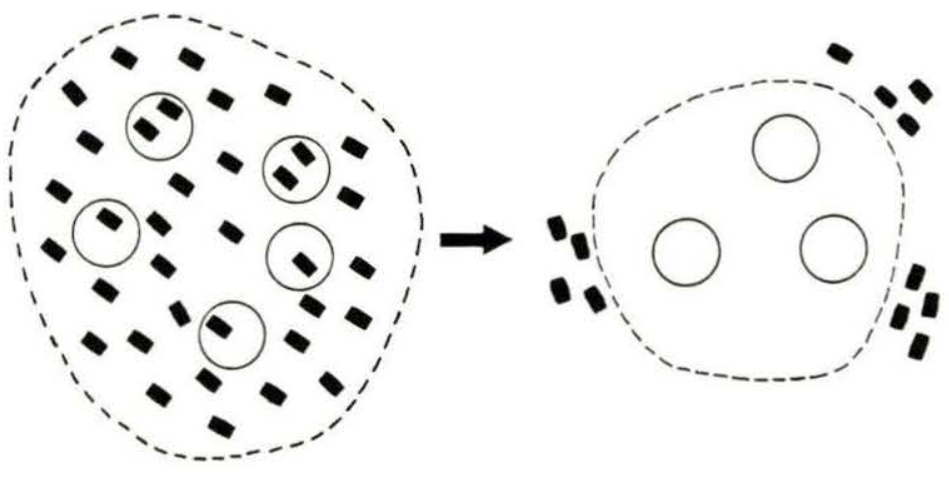

UPPER SARATOWN
FREDRICKS SITE

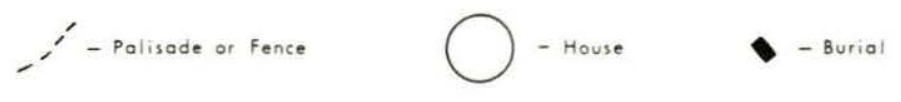

Figure 4.31

Schematic plan of village-burial spatial relationships. linguistically and politically distinct groups were forced to join together in an effort to cope with the constant pressures of colonial expansion and the perpetual hostilities from northern neighbors (cf. Dobyns 1983). During this time, the Keyauwee, Shoccoree, Saponi, and Tutelo combined with the Occaneechi to resist Iroquois raids. In fact, Lawson turned south of the Occaneechi trail because of the threat of an Iroquois attack in Virginia (Lefler 1967:61).

This process of decimation, fragmentation, and recombination of village groups necessitated structural changes in all components of Siouan culture. Large unilineal descent groups (1ineages or clans) and sodalities probably lost much of their social significance. The ceremonial and ritual behavior that sanctioned these groups also were lost or drastically modified. In short, villages and tribes that had been unified and held together by a deep traditional network of kinship and shared ideology probably vanished as early as 1670 . In their places were villages comprised of groups consolidated for expedience rather than on the bases of kinship and a shared system of beliefs. Within these villages, social segments were defined by ethnic and linguistic affiliation, not by unilineal kinship ties. Kindred-like social groups (cf. Speck 1938) formed the primary units of production and consumption, and mortuary ritual and ceremonial beliefs were held in common within these groups.
The cemetery at the Fredricks site may have resulted from the mortuary practices of one of these social units, in which individuals were differentiated by age and sex as well as personal achievement. Children were held in high esteem, and it was possible for adults to achieve positions of high status. By the late 1600 s, individuals may have risen to positions of prominence by developing special relationships with White traders.

Traditionally, tribes are led by "big men" who achieve a high status position by being successful warriors, magicians, and hunters. In short, they are individuals who excel in tribal society (Sahlins 1968:22). Since external political and economic dealings were left to the big men, those individuals who excelled in trade and other dealings with the colonists probably gained added respect, and through their generosity, a degree of social control that exceeded that they would have obtained within the traditional social structure. Based on the Fredricks site data, these big men were young adult males who probably replaced the more elder leaders who had been most influential prior to European contact.

Given the massive depopulation of the Piedmont over a short period of time, acculturation, in the traditional uses of that term (Beals 1962; Spicer 1961), did not take place. Certainly the social and ideological changes 
postulated above should not be seen simply as a borrowing of colonial customs. They were, instead, internal systemic adjustments made in an effort to adapt to and cope with a very destabilizing cultural environment. Obviously, the Piedmont Indians were borrowing material culture from the colonists. But as others have pointed out (e.g., Sheehan 1980:135; Merrel1 1983:4), the Indians were capable of absorbing great quantities of European trade goods without loosing the integrity of their native culture. On this point, it is interesting to note Service's comments on the evolution of composite tribes:

One salient consequence of civilization on a great many tribes has been depopulation through foreign disease, most usually car ried by Europeans; another is disturbance of the resource base by such things as economic exploitation and alienation of native lands or outright removal; still another but frequently overemphasized in studies of changes in social organization, is acculturation--direct borrowing from the invaders (Service 1964:136, emphasis added).

These comments could not be more applicable had they been directed specifically at the Piedmont Siouans. They suggest that the arrangement of burials in a cemetery at the Fredricks site does not mean that, by the end of the 17 th century, the Occaneechis were burying their dead like the colonists. To the contrary, this shift in mortuary behavior may be interpreted as a consequence of internal changes in Siouan social organization, changes expected in a society evolving from a lineal to a composite tribe. Perhaps if the bodies had been extended instead of flexed, or interred in coffins, an emulation of colonial mortuary practices might be postulated (cf. Axtell 1982:123-124). Such was not the case, however.

The rectangular shapes and straight walls of most of the burial pits at the Fredricks site do not represent mimicking of colonial burials; rather they were the result of the use of metal tools. Sharp corners and straight sides are a more likely consequence of the use of iron hoes and spades than of wood or stone implements. Though many of the grave goods from the Fredricks site burials are European in origin, almost all have aboriginal counterparts. Their incorporation in the mortuary complex seems to reflect only replacement of aboriginal items and not changes in native ritual and ideology.

In short, the mortuary evidence suggests that Siouan culture change should not be viewed as an increasing accommodation to European ways. Rather, these changes are better interpreted as adaptive responses within societies that remained, in many respects, resistant to change and that attempted to maintain their traditional cultural systems in the face of devastating pressures. 


\title{
Chapter 5
}

\section{Human Skeletal Remains from the Wall and Fredricks Sites}

\author{
by
}

\author{
Homes Hogue Wilson
}

\section{INTRODUCTION}

An investigation of the skeletal remains from the protohistoric Wall site and the historic Fredricks site was undertaken to provide biocultural information on the effects of European contact on the Native American cultures of the Carolina Piedmont. Four areas of inquiry comprise this investigation: demographic studies, analysis of pathologies, assessment of trace elements, and examination of biological continuity.

Demographic analyses are conducted to determine the overall health and fitness of the study populations. Changes in the mortality rates of certain groups, based on age and sex, would be expected among the study populations given the disruptions of the natural and cultural environments of the Piedmont Indian groups during the Historic period. Life expectancies would also be expected to change during the Historic period with the introduction of new stresses, such as European diseases, and an increase in existing stresses, such as warfare.

Although some diseases, e.g., mumps and measles, leave no marks on the skeleton, and only preliminary studies have dealt with the effects of smallpox on bone (Jackes 1983), the study of bone pathologies can provide corollary evidence of disease-induced stress. For example, changes in the frequencies of certain types of pathologies, such as cut and puncture wounds, might be expected in an Historic period population faced with a social environment in which warfare was prominent.

Assuming that the natural environment in the vicinity of the Wall and Fredricks sites remained approximately constant from the Protohistoric to the Historic period with respect to food resources, changes in the diet of individuals within the populations can be investigated through trace element analysis of the skeletal remains. Thus, by monitoring the concentrations of such elements as zinc, copper, magnesium, strontium, and vanadium, in the bone, it is possible to estimate the relative contributions of floral and faunal resources to the diet of an individual or group (Szpunar et al. 1978).
To study the possible biological connections between late prehistoric/protohistoric and historic populations, and the extent of population disruption and movement following prolonged contact, both metric and non-metric techniques are used on cranial remains. The non-metric traits are utilized in formulae for measuring population diversity (cf. Lieberson 1969) in order to evaluate the amount of variability within each population. Also, metric indices are compared with those from other populations to provide information on their similarities and differences.

The late prehistoric/protohistoric skeletal series comes from the Wall site (310r11). A total of eight burials comprise this sample population. These eight burials consist of five individuals excavated between 1938 and 1941, and three recovered during the 1983 excavations at the site. The historic skeletal remains consist of 13 burials excavated at the Fredricks site (310r231) in 1983, 1984, and 1985.

Because the skeletal samples from the two primary sites are so small, information from two additional samples is incorporated in this study. One sample of 88 burials comes from the Shannon site (44My8), a late prehistoric Siouan site in Montgomery County, Virginia (Benthall 1969). The second collection consists of 74 individuals from the Historic period Siouan site of Upper Saratown (31Skla), in Stokes County, North Carolina (Navey 1982). These two skeletal series are included in this study because they are the largest available documented populations from the Carolina and Virginia Piedmont that are comparable in age and general cultural affiliation to those from the Wall and Fredricks sites.

\section{DEMOGRAPHIC ANALYSIS}

In any study of the demography of an archaeological population, the first question that must be considered is the representativeness of the sample population. Demographic statements are based on the assumption that the numbers, ages, and sexes of the skeletal sample accurately reflect the original population, and that any biases can be recognized and considered in the analysis. Sources of error that can 
result in inaccurate interpretations include: 1) differential disposal of particular segments of a population, such as infants; 2) inadequate sampling of the overall mortuary complex; and 3) differential preservation of the skeletons (Ubelaker 1978:91-92). In addition, there are problems unique to each analytical technique utilized in the study of the biological aspects of burial data. These problems will be addressed when the appropriate technique is considered.

The first step in the demographic reconstruction of a population is to obtain the age at death and the sex of each individual. Usually, only adult remains are sexed, as individuals less than the age of 15 at the time of death cannot be accurately sexed (cf. Ubelaker 1978:42). In the present study, each skeleton in the sample population was subjected to the same set of aging and sexing techniques. All individuals were assigned to an age category, and all but one adult could be sexed.

\section{Age of Subadults}

The subadults were aged using dental eruption and development (cf. Ubelaker 1978: 112-113), longbone length (Ubelaker 1978:48-49), and epiphyseal closure (Bass 1971). Where only fragments of the longbones or cranium were present, the remains were compared with subadults of "known" age, and an age was assigned based on this comparison.

Subadults comprise $62.5 \%$ of the population at the Wall site, and $46.1 \%$ of the Fredricks site population. Table 5.1 presents the age at death for the subadults from both sites, as well as the average age of death for the subadults for each site.

Table 5.1

Age of subadults from the Wall and Fredricks sites.

\begin{tabular}{ccc}
\hline & & \\
\hline Site/Burial & Age in Years & Age in Months \\
& & \\
\hline Wall Site & & \\
Burial 2 & $1.5-3$ & $18-36$ \\
Burial 5 & $0-.67$ & $0-8$ \\
Burial 1-83 & $1.5-3$ & $18-36$ \\
Burial 2-83 & $.75-1.5$ & $9-18$ \\
Burial 3-83 & $0-.67$ & $0-8$ \\
Mean Age & 1.26 & 15.1 \\
Fredricks Site & & \\
Buria1 1 & $3-5$ & $36-60$ \\
Burial 2 & $6-8$ & $0-8$ \\
Burial 4a & $0-.67$ & $0-8$ \\
Burial 7 & $0-.67$ & $36-60$ \\
Burial 8 & $3-5$ & $48-60$ \\
Burial 10 & $4-5$ & 40.33 \\
Mean Age & 3.36 & 31.60 \\
Mean Age 0-5 years & 2.63 & \\
\hline
\end{tabular}

\section{Age and Sex of Adults}

Criteria used to age the adult individuals from the two sites are: 1) dental eruption of the third molar (Ubelaker 1978); 2) dental attrition (Molnar 1971); 3) endocranial suture closure (Krogman 1978); 4) epiphyseal closure (Bass 1971); and 5) erosion of the symphyseal face of the os pubis (McKern and Stewart 1957). Each of the adult individuals in the sample population possessed the skeletal elements necessary for these observations, except for the symphyseal face of the pelvis. Only Burials 1 and 3 from the Wall site and Burial 5 from the Fredricks site could be aged using the pubic symphysis. Whenever possible, the adult individuals were placed in five-year age categories.

The sex of the adult individuals was determined primarily from morphological examination of the cranium, mandible (cf. Bass 1971; Krogman 1978), and pelvis (cf. Bass 1971). Sex determination from metric data on each individual was incorporated to support the results of the morphological analyses. Metrical techniques included: 1) femur midshaft circumference (Black 1978); 2) ischium-pubis index (Bass 1971); 3) femur head diameter (Bass 1971); 4) humerus head diameter (Bass 1971); and 5) the angle of the sciatic notch (Ubelaker 1978). Table 5.2 presents the age and sex of the adult individuals from both sites.

An examination of the age of all of the individuals and the sex of the adults clearly indicates that a representative population is not present at either site. This is not unexpected given the small number of individuals present in each sample. At the Wall site, very young individuals and very old individuals predominate in the burial population; only one individual is between the ages of 10 and 20 years, and there is none between ages 20 and 45 . Furthermore, all the adults are males. Similar

Table 5.2

Age of adults from the Wall and Fredricks sites.

\begin{tabular}{ccc}
\hline Site/Burial & Age in Years & Sex \\
\hline Wall Site & & \\
Burial 1 & $45+$ & M \\
Burial 3 & $50+$ & M \\
Burial 4 & $18-22$ & M \\
Mean Age & 38.3 & \\
Fredricks Site & & \\
Burial 3 & $25-35$ & M \\
Burial 4 & $25-30$ & M \\
Burial 5 & $50+$ & M \\
Burial 6 & $20-25$ & F \\
Burial 9 & $35-40$ & $?$ \\
Burial 11 & $15-20$ & M \\
Burial 13 & $35-40$ & \\
Mean Age & 33.5 & \\
\hline
\end{tabular}


biases can be seen in the burial population from the Fredricks site, where many individuals aged 0 to 5 years are present, but few old adults are present. The age group from 20 to 45 years comprises only four individuals, and one individual is present in the 10-to-20-year category. A bias for male sex is also evident at the Fredricks site, where there are five males and only one female.

Another problem that arises in the analysis of the two primary population samples is the spatial context from which they are drawn from within their respective sites. As Ward (this report) notes, the population from the Wall site is drawn from burials scattered throughout the site, whereas all of the burials from the Fredricks site were in a single "cemetery." Given the differences in age, sex, and placement of burials between the two populations, as well as the small sample size of each population, extreme caution should be exercised in drawing general conclusions from the demographic analysis.

\section{Demographic Profiles}

Frequent infant mortality probably characterized both the Late Prehistoric/Protohistoric and Historic periods because of the stresses associated with early life (cf. Buikstra 1976: 22-23). High infant mortality would be indicated by a large percentage of the population being between the ages of 0 and 5 years at death. Because of the high death risk associated with childbearing, adult females aged 18 to 30 years at death also should be relatively common at both sites. Males aged 18 to 40 years, who would have encountered stresses associated with hunting and warfare, probably would have had a moderately high mortality rate in the Late Prehistoric and Protohistoric periods, and a still higher rate after contact because of increased stress from competition induced by the deerskin trade and from the general animosity that marked relations between the Indians of the Northeast and those of the Virginia and Carolina Piedmont (cf. Lawson in Lefler 1967:49-50,233; and Ward, this report).

The segment of the late prehistoric/protohistoric population classified as old (age 45 years and above), should be comprised mostly of males, as they would have been more likely to survive the stress years of 20 to 40 . For Historic period populations, both males and females would endure increased stress from exposure to European diseases, increased warfare and competition, and disruption in the existing social system. Few of either sex would be likely to survive the stress years. However, females would probably have a better chance to survive to old age than males if there was a decrease in childbearing and its related stresses.

The presence of European diseases and other stresses might cause an increase in the number of deaths in all age groups among historic Indian populations. This would be marked particularly by an increase in the number of deaths in the 5-to-15-year age group (time of European induced childhood diseases), an age when mortality appears to decline in a normal population (Droessler 1981). Also, life expectancy at birth and for each age category should be lower for historic populations when compared to late prehistoric or protohistoric populations.

In summary, five general demographic trends should be noted when a late prehistoric/protohistoric population is compared with an historic population:

1. similar mortality profiles between the populations for infants and females;

2. higher mortality for males aged 20 to 40 years in the historic population;

3. more old individuals in the late prehistoric/protohistoric population and more young adults in the historic population;

4. higher mortality among individuals aged 10 to 20 years in the historic population; and

5. higher life expectancies for all age groups in the late prehistoric/protohistoric population.

\section{Demographic Propositions}

To investigate these five propositions, burial data from two sites with relatively large populations were utilized to construct demographic profiles against which the Wall site and the Fredricks site collections could be compared. The two sites are the Shannon site (44My8), a late prehistoric village site with 88 analyzed burials, and Upper Saratown (31Skla), an historic village site with 74 analyzed burials. Information on the age and sex of the Shannon site population is from Benthall (1969), and that for the Upper Saratown population is from Navey (1982). The demographic reconstructions for these two populations are based on several assumptions (cf. Ubelaker 1974:59). The first assumption, that the skeletal samples are complete, is not fully met, as both sites have been only partly excavated, and therefore only a portion of the total burial populations is represented. The second assumption, that the age at death can be accurately determined, is probably met. Although the age of each individual was carefully evaluated, there may be some bias present created by the fact that the two populations were aged by different observers. The final assumption is that the size of the living population and its death rate remained constant during the occupation of the site. This third assumption is problematic as regards historic populations where mortality probably fluctuated from year to year under the varying 
influences of epidemics of European disease and general warfare. Indian warriors also took captives to serve as slaves for the Europeans (Lefler 1967:208-212). This custom would probably cause population fluctuation from year to year. Given these assumptions and the limitations of all the populations used in this study, one can only view the results as preliminary and as a basis for developing future investigations.

The first step in this study is to construct mortality curves and life tables for the four sample populations. Mortality curves will also be constructed for the sexed adults in order to investigate the potential differences and similarities in the mortality of males and females in the Late Prehistoric/Protohistoric and Historic periods.

\section{Mortality Curves}

Mortality curves allow one to compare the percentages of a population that died in particular age groups. Although five-year categories provide the most information ( $\mathrm{cf}$. Ubelaker 1978: 93), ten-year categories are used here to accommodate Navey's (1982) age groups for Upper Saratown. Figure 5.1 illustrates the mortality curves for the four populations. The late prehistoric/protohistoric sites are represented by the Shannon site $(n=88)$ and the Wall site $(n=8)$. The Historic period sites are Upper Saratown $(n=74)$ and Fredricks $(n=13)$.

Several similarities can be seen when the two profiles of the late prehistoric/protohistoric sites are compared. First, there is a

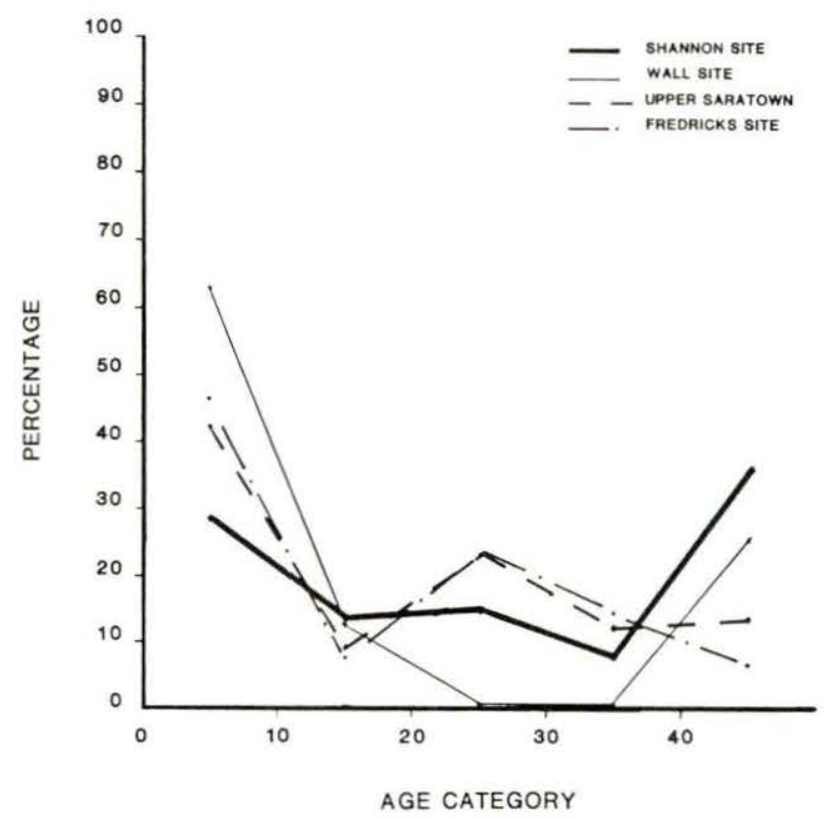

Figure 5.1

Mortality curves for the Fredricks, Wall, Upper Saratown, and Shannon skeletal samples. high frequency of deaths in the first 10 years, followed by a decline through childhood into adolescence as represented by the 10-to-20-year age category. A gradual increase in deaths is present in the 20-to-30-year age group at the Shannon site, which gradually declines in the 30-to-40 age group. As no individuals in these last two age groups were found at the Wall site, nothing more can be said about the mortality among the segment of the protohistoric population aged 20 to 40 years. From age 40 up, both populations show a sharp increase in mortality. A pattern of high infant mortality, followed by lower childhood mortality, and subsequent increase in adult mortality are similar to United Nations mortality model curves developed from 158 censuses in 50 countries and published in 1955 (Buikstra 1976:22-23). And, Buikstra (1976:22-24) found a similar pattern in a Middle Woodland skeletal series from the Gibson-Klunk sites in Illinois. Such a pattern is considered to approximate a "normal" mortality curve.

A similar pattern of high infant mortality and low childhood-adolescent mortality was noted for the populations from the Fredricks and Upper Saratown sites. In contrast to the late prehistoric/protohistoric sites, however, the two historic sites showed a significant increase in the percentage of deaths in the 20-to-30 age group. Mortality in the Upper Saratown population decreases in the subsequent 30-to-40 age group before increasing slightly in the over 40 category. The Fredricks site population showed a high mortality rate in the $30-t 0-40$ age group with a decrease in the over 40 group. This pattern of a higher mortality rate between the ages of 20 and 40 years, with less people surviving over 40, deviates from the United Nations model and that noted for the two late prehistoric/protohistoric populations.

The previous discussion and the data derived from the mortality curves tend to support two of the propositions stated earlier. First, there was a high rate of infant mortality in both late prehistoric/protohistoric and historic populations. This trend is not surprising given that such a pattern is typical of most Third World populations today (Droessler 1981:48). It is interesting that there is a lower percentage of infant deaths in the late prehistoric Shannon site population when compared with the historic Upper Saratown population. The mean age of the individuals in the Shannon site population from age 0 to 5 years is 1.5 years. In contrast, the mean age at Upper Saratown for this age category is 2.78. The mean ages for the protohistoric Wall site is 1.26 years, and that for the historic Fredricks site is 2.63. This suggests that neonates (individuals aged 0 to 12 months at death) are underrepresented at both historic sites. Either the neonates were buried in a different location, were disposed of without burial, or they survived longer in the Historic period.

The first two of these possibilities cannot 
be addressed. The third, however, can be investigated using the twin concepts of " $r$ and $K$ selection" developed by population biologists for the study of group formation (cf. Horn 1978; Pianka 1978). r- and K-strategies portray two poles of a continuum of strategies that a population can utilize to insure its survival. In population biology, r-strategy is denoted by large litters, small offspring, little parental care, low parental survival, and high survival of juveniles (Horn 1978:416). K-strategies, on the other hand, are characterized by small litters, large young, much parental care, high parental survival, and low survival of young (Horn 1978:416).

Although all the implications of the model for the study of the demographic changes between late prehistoric/protohistoric and historic populations have yet to be fully explored, the following proposition can be made about the observed differences in the average age of the subadults in the study populations. It is probable that in late prehistoric and protohistoric times, factors would have tended to favor a population's utilizing a reproductive strategy similar to r-selection. Under conditions where competition and stress are low, as generally would have been the case in the Late Prehistoric and Protohistoric periods, offspring are weaned at an early age, say between ages 0 and 24 months. In contrast, the "best" reproductive strategy for the Historic period, which would be characterized by increased competition and stress, might be one similar to $\mathrm{K}$-selection, where more energy is put into competition and maintenance, and into the production of offspring with enhanced survival abilities. One behavior trait that might have characterized the $\mathrm{K}$-selection strategy of the Historic period would be prolonged nursing of the individual offspring to insure its survival into childhood and adolescence. This suggestion is supported by Lawson (Lefler 1967:196), who noted that Indian women nursed until the children were "well grown":

neither does the youngest Wife ever fail of proving so good a Nurse, as to bring her Child up free from Rickets and Disasters that proceed from the Teeth, with many other Distempers which attack our Infants in England, and other Parts of Europe. They let their Children suck till they are well grown, unless they prove big with child sooner.

The second proposition supported by the mortality curves is that older individuals comprise a larger percentage of the late prehistoric/protohistoric populations than of the historic populations. Among the two late prehistoric/protohistoric populations, $35.7 \%$ of the Shannon site sample and $25 \%$ of the Wall site sample survived over age 40. In contrast, only $13 \%$ of the Upper Saratown sample and $7.7 \%$ of the Fredricks site sample survived to that age.
Related to the higher percentage of individuals aged over 40 being present at the late prehistoric/protohistoric sites is the fact that more individuals between age 20 and 40 died at the historic sites. Only $22.7 \%$ of the Shannon site population died between the ages of 20 and 40, whereas $0.0 \%$ died during this age at the Wall site. For the historic sites, $35.1 \%$ of the population at Upper Saratown and $38.5 \%$ at the Fredricks site was between 20 and 40 years at death.

A third proposition, that more individuals would be found in the 10-to-20-year age category of the mortality curve for the historic populations, is not supported by this study. Higher mortality for this age group is seen in the late prehistoric/protohistoric populations-- $13.6 \%$ of the Shannon site population and $12.0 \%$ of the Wa11 site population. For the Historic period, only $9.5 \%$ of the population at Upper Saratown and $7.7 \%$ of population at the Fredricks site are in the 10-to-20-year age group. An explanation for this pattern might be found in the earlier discussion of $r$ and $\mathrm{K}$-strategies. If extra care and protection from competition were afforded subadults in the Historic period, they would have tended to survive better to an age when they would normally be exposed to the stresses of childbirth, providing their own subsistence, hunting, warfare, etc.

\section{Mortality Curves by Sex}

Mortality curves by sex were also constructed for the Shannon and Upper Saratown populations (Figure 5.2). None were computed for the Wall and Fredricks sites because of the small size of the samples and the absence of females at the Wall site. For the late prehistoric Shannon site, $26.0 \%$ of the males and $48.0 \%$ of the females died between the ages of 20 and 30 years. For the age group 30 to 40 years, $9.0 \%$ of the males and $15.0 \%$ of the females died. Males over 40 years at death represented $65.0 \%$ of the male population, and females over 40 years represented $37.0 \%$ of the female population.

For the Shannon site, a Chi-square test was used to determine whether the number of males and females in two age categories, 20 to 40 years and age 40+, were significantly different. The results were: $\mathrm{X}^{2}=4.79 ; \mathrm{df}=1 ; \mathrm{p}<.025$ (Table 5.3). This indicates that the differences noted for the two age groups by sex are statistically significant. To further test the significance of the age/sex differences for adults aged 20 years and above, a Chi-square test utilizing three age categories--20 to 30 years, 30 to 40 years, and 40+ years--was conducted (Table 5.4). The results were: $\mathrm{X}^{2}=4.62 ; \mathrm{df}=2 ; \mathrm{p}<.10$. Although these results are not statistically significant, they do indicate a possible mortality pattern that should be explored when more data are available. 


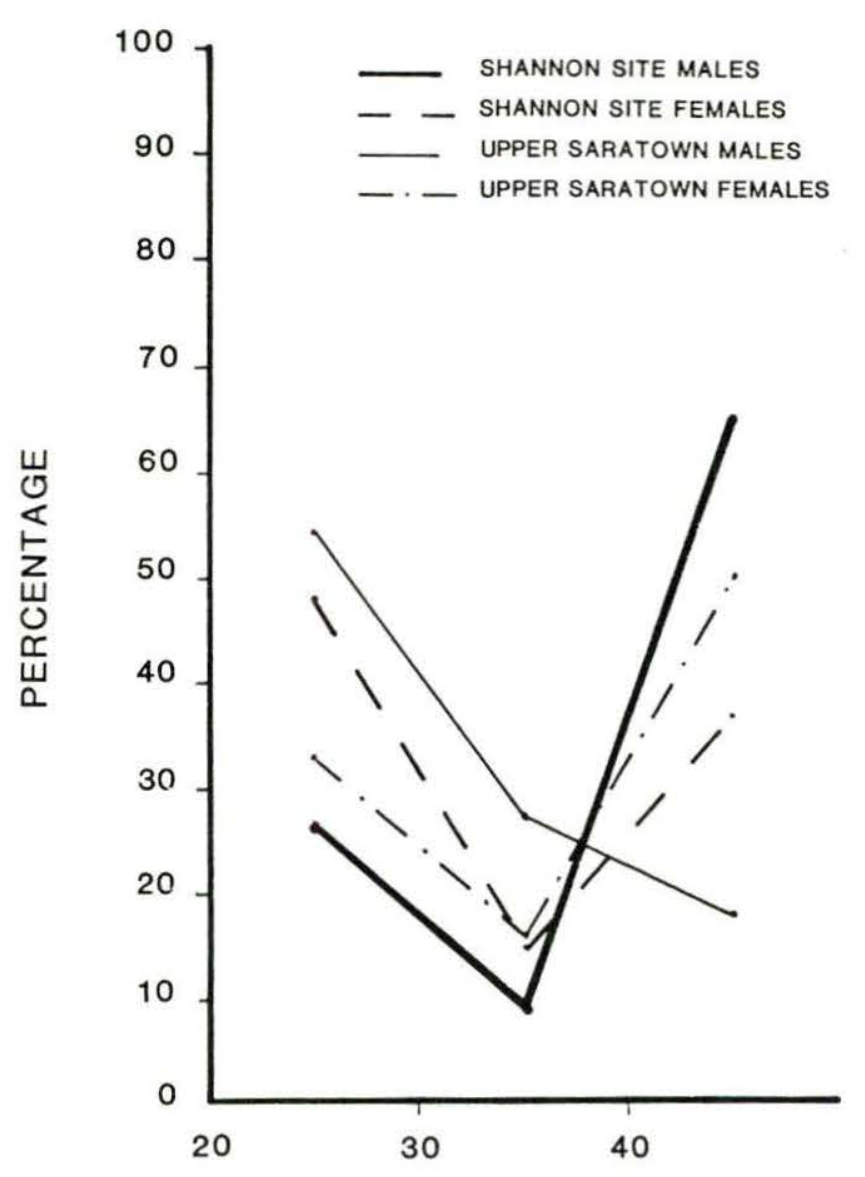

AGE CATEGORY

Figure 5.2

Mortality curves by sex for the Shannon and Upper Saratown skeletal samples.

The results of the Chi-square tests suggest that, at the Shannon site, there is a statistically significant pattern of mortality by sex for adults aged 20 to 40 years and for the adults aged $40+$. The pattern is evidenced by the higher mortality rate for females aged 20 to

Table 5.3

$2 \times 2$ contingency table to test the significance of male and female mortality at the prehistoric Shannon site.

\begin{tabular}{|c|c|c|c|c|c|}
\hline \multirow[b]{3}{*}{ Sex } & \multirow{2}{*}{\multicolumn{2}{|c|}{$20-40$}} & \multirow{2}{*}{\multicolumn{2}{|c|}{$40+$}} & \multirow[b]{3}{*}{ Total } \\
\hline & & & & & \\
\hline & $o_{i}$ & $e_{i}$ & $o_{i}$ & $e_{1}$ & \\
\hline Male & 12 & 16.16 & 22 & 17.84 & 34 \\
\hline Female & 17 & 12.84 & 10 & 14.16 & 27 \\
\hline Total & 29 & & 32 & & 61 \\
\hline
\end{tabular}

Table 5.4

$2 \times 3$ contingency table to test the significance of male and female mortality at the prehistoric Shannon site.

\begin{tabular}{|c|c|c|c|c|c|c|c|}
\hline \multirow[b]{2}{*}{ Sex } & \multicolumn{2}{|c|}{$20-30$} & \multicolumn{2}{|c|}{$\begin{array}{r}\text { Age } \\
30-40\end{array}$} & \multicolumn{2}{|c|}{$40+$} & \multirow[b]{2}{*}{ Total } \\
\hline & $o_{1}$ & $e_{1}$ & & $e_{1}$ & $o_{i}$ & $e_{1}$ & \\
\hline Male & 9 & 12.26 & 3 & 3.90 & 22 & 17.84 & 34 \\
\hline Female & 13 & 9.74 & 4 & 3.10 & 10 & 14.16 & 27 \\
\hline Tota1 & 22 & & 7 & & 22 & & 61 \\
\hline
\end{tabular}
$x^{2}=4.619, \quad d f=2, \quad p=.10$

40 years. This coincides roughly with the stress years for females associated with childbearing.

At Upper Saratown, a different relationship of age and sex is observed. A total of $54.5 \%$ of the male population is present in the 20-to-30-year group, as opposed to only $33.3 \%$ of the female population. In the 30-to-40-year category, $27.3 \%$ of the males are present, and $16.7 \%$ of the females. Finally, $18.2 \%$ of the males survived to age $40+$, whereas $50 \%$ of the females survived to that age. A $2 \times 2$ contingency table (Table 5.5) with two age categories, one of 20 to 40 years and the other of $40+$ years, was constructed. The results are: $X^{2}=1.3507$; $\mathrm{df}=1 ; \mathrm{p}<.30$. A Chi-square test (Table 5.6) on three age categories (20 to 30 years, 30 to 40 years, and $40+$ years) yielded: $x^{2}=2.56$; $d f=2$; $\mathrm{p}<.20$. Both tests indicate that the differences between male and female mortality at Upper Saratown are not statistically significant.

One more Chi-square test was conducted. Both $2 \times 2$ and $2 \times 3$ contingency tables (Tables 5.7-5.8) were constructed using the mortality data for just the males from the Shannon site and Upper Saratown. The age groups for the $2 \times 2$ test were $20_{2}$ to 40 years and $40+$ years. The result was $x^{2}=7.21 ; d f=1 ; p<.01$. For the $2 \times 3$ test, with age groups of 20 to 30 years, 30 to 40 years, and $40+$ years, the result was $x^{2}=7.44$;

Table 5.5

$2 \times 2$ contingency table to test the significance of male and female mortality at Upper Saratown.

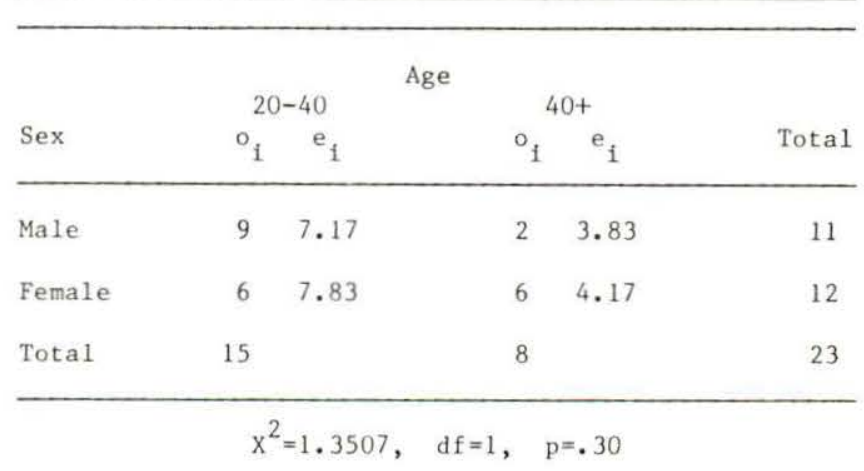


Table 5.6

$2 \times 3$ contingency table to test the significance of male and female mortality at Upper Saratown.

\begin{tabular}{|c|c|c|c|c|c|c|c|}
\hline \multirow[b]{2}{*}{ Sex } & \multicolumn{2}{|c|}{$20-30$} & \multicolumn{2}{|c|}{$\begin{array}{r}\text { Age } \\
30-40\end{array}$} & \multicolumn{2}{|c|}{$40+$} & \multirow[b]{2}{*}{ Total } \\
\hline & & $e_{1}$ & $o_{i}$ & $e_{1}$ & $o_{1}$ & $e_{1}$ & \\
\hline Male & 6 & 4.78 & 3 & 4.11 & 2 & 3.82 & 11 \\
\hline Female & 4 & 5.22 & 2 & 2.61 & 6 & 4.17 & 12 \\
\hline Total & 10 & & 5 & & 8 & & 23 \\
\hline
\end{tabular}

$x^{2}=2.564, \quad$ df $=2, \quad p=.20$

Table 5.7

$2 \times 2$ contingency table to test the significance of male mortality at the Shannon and Upper Saratown sites.

\begin{tabular}{|c|c|c|c|c|c|}
\hline \multirow[b]{2}{*}{ Site } & \multicolumn{2}{|c|}{$20-40$} & \multicolumn{2}{|c|}{ Age } & \multirow[b]{2}{*}{ Total } \\
\hline & $o_{i}$ & $e_{i}$ & $o_{i}$ & $e_{1}$ & \\
\hline Shannon & 12 & 15.86 & 22 & 18.12 & 34 \\
\hline Upper Saratown & 9 & 5.14 & 2 & 5.86 & 11 \\
\hline Total & 21 & & 24 & & 45 \\
\hline
\end{tabular}

Table 5.8

$2 \times 3$ contingency table to test the significance of male mortality at the Shannon and Upper Saratown Sites.

\begin{tabular}{|c|c|c|c|c|c|c|c|}
\hline \multirow[b]{2}{*}{ Site } & \multicolumn{2}{|c|}{$20-30$} & \multicolumn{2}{|c|}{$\begin{array}{c}\mathrm{Age} \\
30-40\end{array}$} & \multicolumn{2}{|c|}{$40+$} & \multirow[b]{2}{*}{ Total } \\
\hline & $o_{1}$ & $e_{1}$ & $o_{i}$ & $e_{1}$ & $o_{1}$ & $e_{1}$ & \\
\hline Shannon & 9 & 11.33 & 3 & 4.53 & 22 & 18.13 & 34 \\
\hline Upper Saratown & 6 & 3.67 & 3 & 1.47 & 2 & 5.87 & 11 \\
\hline Total & 15 & & 6 & & 24 & & 45 \\
\hline
\end{tabular}

$$
x^{2}=7.4437, \quad d f=2, \quad p=.05
$$

$\mathrm{df}=2 ; \mathrm{p}<.05$. Both tests indicate that the change in male mortality from the late prehistoric site to the historic site is statistically significant. Chi-square tests computed on mortality data for the adult females from Upper Saratown and the Shannon site (Tables 5.9-5.10) suggest that there are no statistically significant differences in age at death.

From these studies, the proposition that female mortality would remain constant in both the late prehistoric/protohistoric and historic populations is supported. Also, the proposition that higher mortality would be found in the historic population among males aged 20 to 40
Table 5.9

$2 \times 2$ contingency table to test the significance of female mortality at the Shannon and Upper Saratown sites.

\begin{tabular}{|c|c|c|c|c|c|}
\hline \multirow[b]{2}{*}{ Site } & \multicolumn{4}{|c|}{ Age } & \multirow[b]{2}{*}{ Tota] } \\
\hline & $o_{i}$ & $\mathrm{e}_{i}$ & $o_{i}$ & $e_{i}$ & \\
\hline Shannon & 17 & 15.92 & 10 & 11.07 & 27 \\
\hline Upper Saratown & 6 & 7.08 & 6 & 4.93 & 12 \\
\hline Total & 23 & & 16 & & 39 \\
\hline
\end{tabular}

Table 5.10

$2 \times 3$ contingency table to test the significance of female mortality at the Shannon and Upper Saratown sites.

\begin{tabular}{|c|c|c|c|c|c|c|c|}
\hline \multirow[b]{2}{*}{ Site } & \multicolumn{2}{|c|}{$20-30$} & \multicolumn{2}{|c|}{$\begin{array}{c}\text { Age } \\
30-40\end{array}$} & \multicolumn{2}{|c|}{$40+$} & \multirow[b]{2}{*}{ Total } \\
\hline & $o_{1}$ & $e_{1}$ & $o_{1}$ & $e_{1}$ & & $1 \quad e_{1}$ & \\
\hline Shannon & 13 & 11.77 & 4 & 4.15 & 10 & 11.07 & 27 \\
\hline Upper Saratown & 4 & 5.23 & 2 & 1.85 & 6 & 4.93 & 12 \\
\hline Total & 17 & & 6 & & 16 & & 39 \\
\hline
\end{tabular}

$$
x^{2}=0.769, \quad d f=2, \quad p=.70
$$

years is supported. Although little can be done at present to test these propositions using the small samples from the Wall and Fredricks sites, it appears likely that similar mortality patterns are present for at least the male populations at the two sites. At the protohistoric Wall site $(n=3)$, one male is between 17 and 20 years at death, and two are aged $40+$. This is similar to the ratio noted for the Shannon site. In contrast, at the historic Fredricks site $(n=5)$, four males are aged between 20 and 40 years at death, and only one is 40+, which is similar to the pattern found in the male population from Upper Saratown.

\section{Life Tables}

Using the information on age at death, life tables were constructed for the late prehistoric Shannon site (Table 5.11), protohistoric Wall site (Table 5.12), and the two historic sites of Upper Saratown (Table 5.13) and Fredricks (Table 5.14). These life tables were used to investigate the aforementioned proposition that the life expectancy of an individual would tend to be greater in late prehistoric/protohistoric populations than in historic populations. Life tables were determined from the same formulas used by Ubelaker (1974:62) in his analysis of the Nanjemoy ossuaries in Maryland. As noted earlier, 10-year categories had to be employed 
Table 5.11

Life table for the Shannon site.

\begin{tabular}{|c|c|c|c|c|c|c|c|}
\hline $\begin{array}{c}\text { Age } \\
\text { Interval } \\
(x)\end{array}$ & $\begin{array}{l}\text { No. of } \\
\text { Deaths } \\
(D x)\end{array}$ & $\begin{array}{c}\% \text { of } \\
\text { Deaths } \\
(\mathrm{dx})\end{array}$ & $\begin{array}{l}\text { Survivors } \\
\text { Entering } \\
\text { (1x) }\end{array}$ & $\begin{array}{l}\text { Probability } \\
\text { of Death } \\
(\mathrm{qx})\end{array}$ & $\begin{array}{l}\text { Total } \\
\text { Years Lived } \\
\text { Between X } \\
\text { and } X+10 \\
(\text { Lx })\end{array}$ & $\begin{array}{l}\text { Total Years } \\
\text { Lived After } \\
\text { Lifetime } \\
\text { (Tx) }\end{array}$ & $\begin{array}{c}\text { Life } \\
\text { Expectancy } \\
\text { (ex) }\end{array}$ \\
\hline $0-10$ & 25 & 28.4 & 100.0 & .2840 & 858.0 & 2581.0 & 25.8 \\
\hline $11-20$ & 12 & 13.6 & 71.6 & .1899 & 648.0 & 1723.0 & 24.1 \\
\hline $21-30$ & 13 & 14.8 & 58.2 & .2552 & 506.0 & 1075.0 & 18.5 \\
\hline $31-40$ & 7 & 7.9 & 43.2 & .5156 & 392.5 & 569.0 & 13.2 \\
\hline $40+$ & 31 & 35.3 & 35.3 & 1.0000 & 176.5 & 176.5 & 5.0 \\
\hline Total & 88 & & & & & & \\
\hline
\end{tabular}

Table 5.12

Life table for the Wall site.

\begin{tabular}{|c|c|c|c|c|c|c|c|}
\hline $\begin{array}{l}\text { Age } \\
\text { Interval } \\
\quad(x)\end{array}$ & $\begin{array}{l}\text { No. of } \\
\text { Deaths } \\
(\mathrm{Dx})\end{array}$ & $\begin{array}{c}\% \text { of } \\
\text { Deaths } \\
(\mathrm{dx})\end{array}$ & $\begin{array}{l}\text { Survivors } \\
\text { Entering } \\
\quad(1 \mathrm{x})\end{array}$ & $\begin{array}{c}\text { Probability } \\
\text { of Death } \\
(\mathrm{qx})\end{array}$ & $\begin{array}{c}\text { Total } \\
\text { Years Lived } \\
\text { Between X } \\
\text { and } X+10 \\
(L x)\end{array}$ & $\begin{array}{l}\text { Total Years } \\
\text { Lived After } \\
\text { Lifetime } \\
\text { (Tx) }\end{array}$ & $\begin{array}{c}\text { Life } \\
\text { Expectancy } \\
\text { (ex) }\end{array}$ \\
\hline $0-10$ & 5 & 62.5 & 100.0 & .6250 & 162.5 & 1625.0 & 16.2 \\
\hline $11-20$ & 1 & 12.5 & 37.5 & .3333 & 312.5 & 937.5 & 25.0 \\
\hline $21-30$ & 0 & 0.0 & 25.0 & .0000 & 250.0 & 625.0 & 25.0 \\
\hline $31-40$ & 0 & 0.0 & 25.0 & .0000 & 250.0 & 375.0 & 15.0 \\
\hline $40+$ & 2 & 25.0 & 25.0 & 1.0000 & 125.0 & 125.0 & 5.0 \\
\hline Total & 8 & & & & & & \\
\hline
\end{tabular}


Table 5.13

Life table for the Upper Saratown site.

\begin{tabular}{|c|c|c|c|c|c|c|c|}
\hline $\begin{array}{l}\text { Age } \\
\text { Interval } \\
(x)\end{array}$ & $\begin{array}{l}\text { No. of } \\
\text { Deaths } \\
(D x)\end{array}$ & $\begin{array}{c}\% \text { of } \\
\text { Deaths } \\
(\mathrm{dx})\end{array}$ & $\begin{array}{l}\text { Survivors } \\
\text { Entering } \\
\quad(1 \mathrm{x})\end{array}$ & $\begin{array}{l}\text { Probability } \\
\text { of Death } \\
(q x)\end{array}$ & $\begin{array}{c}\text { Total } \\
\text { Years Lived } \\
\text { Between X } \\
\text { and X }+10 \\
(\text { Lx })\end{array}$ & $\begin{array}{l}\text { Total Years } \\
\text { Lived After } \\
\text { Lifetime } \\
(\mathrm{Tx})\end{array}$ & $\begin{array}{c}\text { Life } \\
\text { Expectancy } \\
\text { (ex) }\end{array}$ \\
\hline $0-10$ & 31 & 41.9 & 100.0 & .4190 & 790.5 & 1958.0 & 19.6 \\
\hline $11-20$ & 7 & 9.5 & 58.1 & .1635 & 533.5 & 1167.5 & 20.1 \\
\hline $21-30$ & 17 & 23.0 & 48.6 & .4732 & 371.0 & 634.0 & 13.0 \\
\hline $31-40$ & 9 & 12.1 & 25.6 & .4726 & 195.5 & 263.0 & 10.3 \\
\hline $40+$ & 10 & 13.5 & 13.5 & 1.0000 & 67.5 & 67.5 & 5.0 \\
\hline Total & 74 & & & & & & \\
\hline
\end{tabular}

Table 5.14

Life table for the Fredricks site.

\begin{tabular}{|c|c|c|c|c|c|c|c|}
\hline $\begin{array}{l}\text { Age } \\
\text { Interval } \\
(x)\end{array}$ & $\begin{array}{l}\text { No. of } \\
\text { Deaths } \\
(\mathrm{Dx})\end{array}$ & $\begin{array}{c}\% \text { of } \\
\text { Deaths } \\
(\mathrm{dx})\end{array}$ & $\begin{array}{l}\text { Survivors } \\
\text { Entering } \\
\quad(1 \mathrm{x})\end{array}$ & $\begin{array}{c}\text { Probability } \\
\text { of Death } \\
(q x)\end{array}$ & $\begin{array}{c}\text { Total } \\
\text { Years Lived } \\
\text { Between X } \\
\text { and X }+10 \\
(\mathrm{Lx})\end{array}$ & $\begin{array}{c}\text { Total Years } \\
\text { Lived After } \\
\text { Lifetime } \\
\text { (Tx) }\end{array}$ & $\begin{array}{c}\text { Life } \\
\text { Expectancy } \\
(\mathrm{ex})\end{array}$ \\
\hline $0-10$ & 6 & 46.0 & 100.0 & .4600 & 770.0 & 1810.0 & 18.1 \\
\hline $11-20$ & 1 & 8.0 & 54.0 & .1481 & 500.0 & 1040.0 & 19.3 \\
\hline $21-30$ & 3 & 23.0 & 46.0 & .4000 & 540.0 & 540.0 & 11.7 \\
\hline $31-40$ & 2 & 15.0 & 23.0 & .6522 & 155.0 & 195.0 & 7.6 \\
\hline $40+$ & 1 & 8.0 & 8.0 & 1.0000 & 40.0 & 40.0 & 5.0 \\
\hline Total & 13 & & & & & & \\
\hline
\end{tabular}


here instead of the recommended five-year intervals.

Life expectancy at birth for the late prehistoric Shannon site population was 25.81 years (Table 5.11). If an individual reached the age of 10 , the life expectancy was 35.06 years. At age 20, this figure was 38.47 years. The Wall site provided a life expectancy at birth of 16.25 years (Table 5.12). This figure, which is extremely low when compared with the Shannon site and other late prehistoric sites in the Southeast, is probably an artifact of sample bias. A comparison of the life expectancies at each of the remaining age categories shows that the Wall site figures are consistently higher than those of the Shannon site.

Life expectancies for the two historic sites are similar. The life expectancy at Upper Saratown is 19.58 years at birth, increases to 30.10 years at age 10 , and to 33.04 years at age 20. Life expectancy at birth for the Fredricks site is 18.10 years, 29.30 years at age 10 , and 31.70 years at age 20 .

It appears that life expectancies are generally higher for the late prehistoric/protohistoric populations when compared with the historic populations (Tables 5.11-5.14). Although the population samples from the Wall and Fredricks sites are too small to permit reliable comparisons, the general trend seems to be for a longer life expectancy in the late prehistoric/protohistoric groups. It should be noted here that because the Shannon and Upper Saratown samples were not aged and sexed by this author, the results of the comparisons should be viewed as preliminary.

\section{Crude Mortality Rates}

The crude mortality rate of a population represents the number of individuals per 1000 that die in a year. Assuming that the death rate is constant, the crude mortality rate of a population can be computed by dividing the life expectancy at birth into 1000 (Ubelaker 1978: 96). For the Wall site a life expectancy at birth of 16.2 yielded a crude mortality rate of 61.73. A crude mortality rate of 38.76 was calculated for the late prehistoric Shannon site. Due to the problems of sample size of the Wall site discussed above, it is felt that the Shannon site estimate best represents the crude mortality rate for a late prehistoric population used in this study.

For the historic sites, the Fredricks population had a crude mortality rate of 55.25 and the Upper Saratown sample yielded a crude mortality rate of 51.02 . Therefore, as expected, there is an increase in the number of deaths per year per 1000 individuals at the historic sites when compared with the late prehistoric Shannon site.

\section{Population Estimates for the Fredricks site}

Since the 13 burials from the Fredricks site represent a spatially distinct cemetery estimates of population size were computed. The crude mortality rate can be used to reconstruct the size of a population if the length of time the site was occupied can be determined (Ubelaker 1978:96), or in this case, the length of time the cemetery was used. Since it is uncertain if the Fredricks site cemetery is the only cemetery used by the inhabitants of the site, a number of temporal periods are used to reconstruct the population. Table 5.15 presents the population size that can be reconstructed for the Fredricks site depending on whether the cemetery was used for $1,2,3,4,5,10$, or 15 years. This provided a population range of 232 individuals if the cemetery represented 1 year of interments to 15 if the cemetery was used for 15 years. It seems likely that the cemetery would not have been used for more than about 5 years if it were the only cemetery in use and thus represented the entire burial population for the temporal span. It should be noted that individuals may have been buried in another cemetery at the site that has yet to be discovered, or that individuals may have died and/or been buried away from the village proper, either of which would result in a skewed population estimate. However, the data presented in Table 5.15 can be used in conjunction with other population estimates for the Fredricks site (see Petherick, this report) to provide as accurate a population estimate as is currently possible.

\section{Summary of the Demographic Studies}

The information on age-specific mortality and life expectancy presented here has proved valuable in evaluating the overall health and

Table 5.15

Estimated population size of the Fredricks site.
Number of Years Cemetery Was in Use
Estimated Population Size 
fitness of the four Piedmont Indian populations. Several propositions were introduced and tested using techniques of demographic analysis. The relatively large skeletal samples from the late prehistoric Shannon site and historic Upper Saratown site were used to develop "models" against which the smaller (and probably biased) samples from the protohistoric Wall site and historic Fredricks site could be compared. of the five propositions originally set forth in this section, four were supported by data from the Shannon site and Upper Saratown, with similar patterns being documented for the Wall and Fredricks sites. Support was provided for the propositions that there will be: 1) similar mortality profiles for infants and females at the late prehistoric/protohistoric and historic sites; 2) higher mortality for young males at the historic sites; 3) more older individuals in the late prehistoric/protohistoric sites; and 4) higher life expectancies for the late prehistoric/protohistoric populations. The fifth proposition, that higher mortality among individuals aged 10 to 20 years would exist in the historic sample, was not supported. Hopefully, larger and more representative samples from the Wall and Fredricks sites will be forthcoming. This will permit a more thorough study of the propositions and suggested patterns.

\section{PATHOLOGIES}

The above demographic analysis suggests that European contact led to a more stressful environment for the aboriginal populations, especially for young males. Examination of the skeletal remains for pathological lesions can furnish information about the susceptibility of a population to stress and disease. When used in conjunction with data from other sites, such information can provide insights into stressrelated and disease-related changes in behavior patterns. Unfortunately, most disease states, including those of European origin, do not affect the bone, and in those cases where the bone is affected, specific diseases cannot always be associated with the pathology (Buikstra and Cook 1980:439-444). Before considering the pathologies present in the skeletal series from the Wall and Fredricks sites, it is necessary to briefly discuss the diseases, both endemic and European-introduced, that may have acted upon the Indian groups of the Carolina Piedmont during the Historic period.

\section{Epidemic Diseases in the Southeast, 1680-1710}

According to Dobyns (1983:8), diseases introduced from the old World caused numerous epidemics of lethal pathogens in the New World between A.D. 1520 and 1918. The most fatal of these 0ld World pathogens for Native Americans was smallpox. Following its introduction, smallpox spread rapidly throughout Native American populations, who possessed no immunity to the disease (Dobyns 1983:11). The first smallpox epidemic, which lasted from about 1520 to 1524 , was more deadly than any of the later epidemics. Between 1665 and 1667, a second virulent smallpox epidemic struck the Southeast from Florida to Virginia. Another epidemic broke out in 1696 that decimated the Southeastern and Gulf Coast chiefdoms before it ended in 1699 (Dobyns 1983:15).

The smallpox epidemic of 1696-1699 is mentioned by John Lawson during his travel through the Carolina hinterland in the winter of 1701. Lawson (Lefler 1967:231-232) notes that "The Smal1-Pox has been fatal to them; they do not often escape, when they are seiz'd with that Distemper, which is a contrary Fever to what they ever knew." He (Lefler 1967:232) goes further and proposes that smallpox had probably not been in America before European contact as "it destroy'd whole Towns, without leaving one Indian alive."

The only other European disease that may have affected the Indian groups of the Carolinas and Virginia is influenza, which may have been a component of the smallpox epidemic of 1696 (Dobyns 1983:18). Dobyns (1983:18-19) suggests that the effects of influenza on Native American populations is probably unreported in the historic records, and therefore underestimated, because its symptoms are not readily visible. Influenza may have been the third most lethal disease after smallpox and measles among Indian populations (Dobyns 1983:19). Lawson does not specifically identify influenza as being present among the aboriginal people with whom he was familiar, but he does identify several medicines made of roots, herbs and/or snake-skins used by the Indians to treat "fever" (Lefler 1967:17), one of the symptoms of influenza, smallpox, and measles. There is no historical evidence to support the presence of measles in the Carolina back country.

The final disease discussed in this section is syphilis, although the question of whether it is of 01d or New World origin is still debated. Several theories, variously based on biological, environmental, and paleopathological evidence, have been proposed for explaining the presence of syphilis in the New World (Steinbock 1976: 87-97). Fortunately, the question of origin does not have to be addressed here, for whatever its origin, syphilis is considered to have been one of the major diseases responsible for population decline among North American Indians during the Protohistoric and early Historic periods (Dobyns 1983:35). Lawson (Lefler 1967: $25,231)$ makes several references to individuals that have been affected by the "Pox" (syphilis) and had lost their noses. He (Lefler 1967:25) also notes that yaws, as well as syphilis (both of which are treponemal infections), is present among the Indians and that both have similar symptoms :

Yawes... is...tattended with nocturnal Pains in the Limbs, and commonly makes such a 
Progress, as to vent Part of the Matter by Botches, and several Ulcers in the Body, and other Parts; oftentimes Death ensuing. I have known mercurial Unguents and Remedies work a Cure, following the same Methods as in the Pox.

Osteological indicators are also similar for both syphilis and yaws, which makes it difficult to positively identify one or the other from only skeletal evidence. The osteological indicators include cranial lesions and nasal destruction, and lesions and swelling of the lower limbs (Steinbock 1976:102-105).

In summary, there are at least three major diseases--smallpox, treponemal infections (yaws and syphilis), and influenza--that may have acted on the Indian groups of the CarolinaVirginia Piedmont during the Historic period. Since influenza leaves no bone lesions to indicate its presence, it cannot be positively identified using only skeletal material. Smallpox (variola osteomyelitis) deforms the bones of the upper extremities, especially in the area of the elbow, although any joint can be affected (Ortner and Putshar 1981:227-228). At the Grimsby site in Ontario, Canada, deformities of the bones that comprise the elbow are identified as having been caused by variola osteomyelitis (Jackes 1983). This Neutral Indian cemetery dates to about 1650, and provides the first archaeological evidence of smallpox in the New World. Treponemal infections have been documented archaeologically through the presence of lesions particular to this type disease on cranial bones and longbones (e.g., Bullen 1972; Steinbock 1976:86-160).

No evidence of smallpox or treponemal infection is found in the skeletal population from the Fredricks site. Therefore, the presence of these three diseases as additional stresses during European contact can only be inferred from the ethnohistorical accounts of their occurrence, and not directly from the existing skeletal remains.

\section{Pathologies at the Wall and Fredricks Sites}

The ultimate goal of the study of the skeletal pathologies at the Wall and Fredricks sites is the comparison of the frequencies of the types of pathologies. Six major categories of pathologies are present: 1) traumatic or violent pathologies; 2) degenerative pathologies; 3) tumors; 4) general stress-indicating pathologies; 5) indicators of dietary stress; and 6) general osteitic pathologies.

Traumatic/Violent Pathologies. Pathologies that are considered to be evidence of trauma and/or violence include fractures, cuts, and piercing wounds. A higher incidence of such pathologies would be expected in Historic period populations when compared with late prehistoric/ protohistoric populations because of the increased amount of warfare, such as Lawson describes (Lefler 1967:49-50,233), and a possible increase in hunting accidents resulting from participation in the European skin and fur trade.

At the Wall site, there is only one instance of traumatic/violent pathology, a healed fracture on the left humerus of a male aged over 45 in Burial 1 (Figure 5.3). This totals $3.8 \%$ of all the pathologies from the Wall site, where $12.5 \%$ of the population exhibits evidence of pathologies.

In contrast to the Wall site, $15.4 \%$ of the
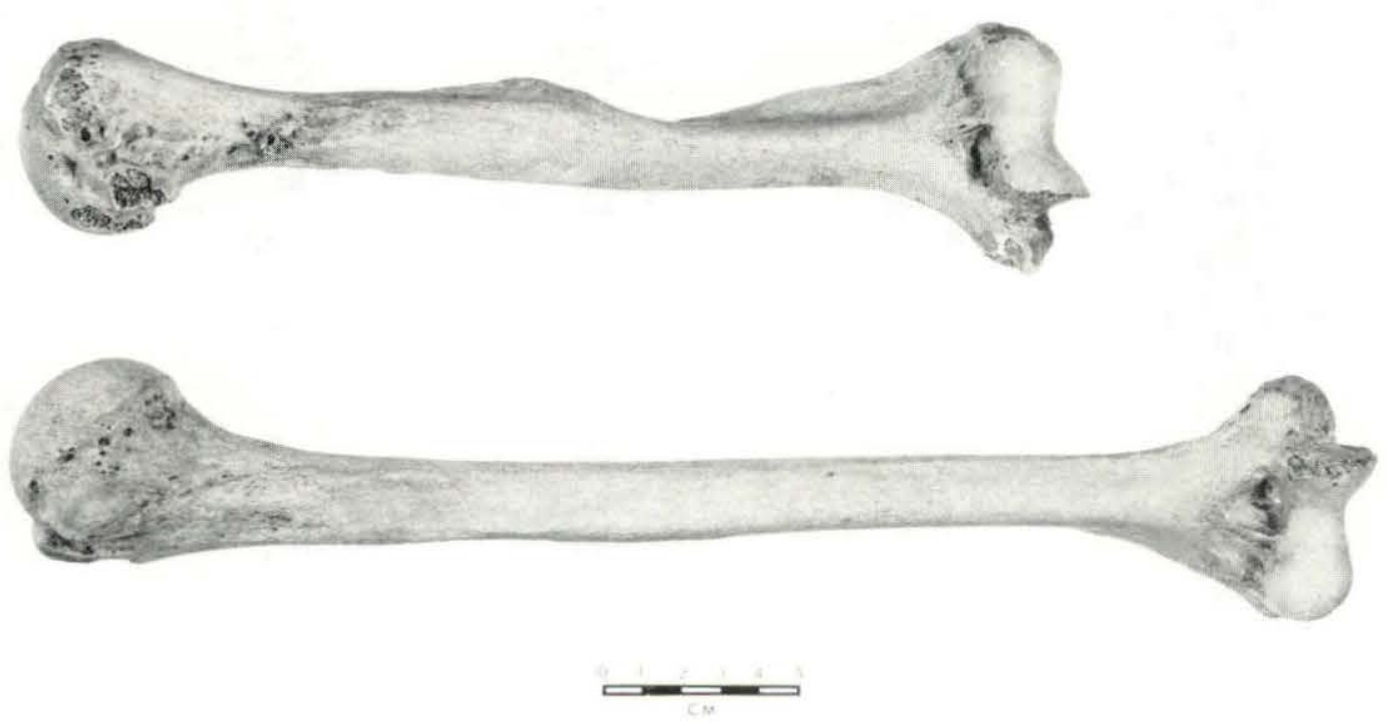

Figure 5.3

Left humerus (top) compared with right humerus (bottom) of Burial 1 at the Wall site. 
population from the Fredricks site show evidence of traumatic/violent pathologies, which comprises $5.7 \%$ of the total number of pathologies present in the population. Burial 9, a female aged 35 to 40 , has a possible pierce wound of the left fibula caused by a gun shot. Although the fibula is extremely fragmented, there is no evidence of bone reconstruction or osteitis, which means that death probably occurred soon after the wound was inflicted. Of special interest are the pathologies exhibited by Burial 4. This male, aged 25 to 30 years at death, had sutures to the right temporal bone (Figure 5.4). The depth of the cuts through the periosteal layer of the cranium intimates that a very sharp instrument was used with much force to inflict the injury. Although scalping is a sound possibility for these injuries, the irregular cuts are not those associated with the usual scalping method whereby the frontal region is cut and the scalp pulled towards the back of the head where it is cut away, sometimes with part of the sku1l (cf. Lefler 1967:207). Rather, it appears that the individual may have been struck repeatedly with a sharp weapon, possibly a light weight sword. This interpretation is supported by the what appears to be the presence of a separate pierce wound on a segment of the frontal above the left orbit. As the individual had been buried in a disarticulated, bundled state, and had probably suffered (and died) from violent trauma, it is proposed that Burial 4 represents the remains of a victim of warfare or some other violent act, and that death occurred away from the village. The body was later gathered and returned to the village for burial. In his journals on his life among the "Chikkasahs" [Chickasaw] in 1744, Adair (Williams 1930:181-182) refers to the Choctaw and Creek treatment of war victims:

Thus all numerous nations of Indians perform the like friendly office to every deceased person of their respective tribe, in so much that those who lose their people at war, if they have not corrupted their primitive customs, are so observant of this kindred duty as to appropriate some time to

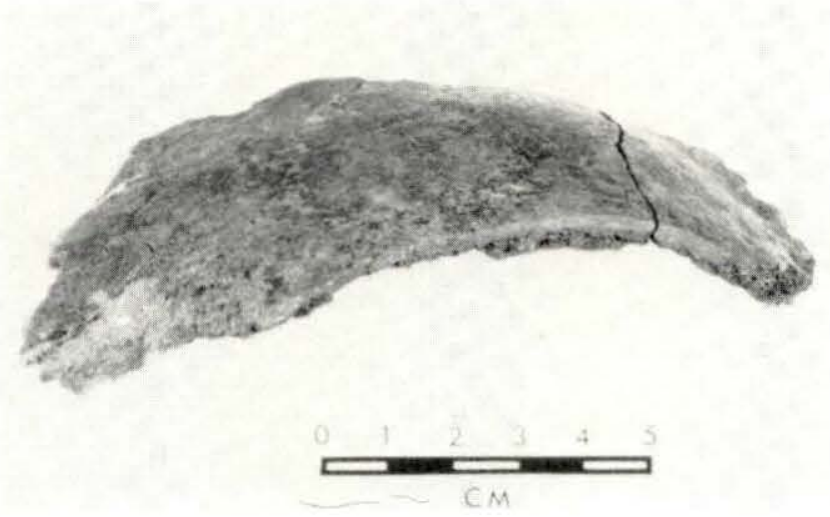

Figure 5.4

Right parietal fragment of Burial 4 from the Fredricks site, showing the cut mark or traumatic injury. collect the bones of their relations; which they call bone gathering...

It is posited that Burial 4 had probably been exposed to the effects of the environment for an unknown amount of time (possibly several weeks) before the remains were finally buried in the village. No defleshing marks, which would suggest intentional disarticulation and secondary burial of the individual, are present.

In summary, there is, as originally postulated, an increase in the occurrence of traumatic/violent pathologies from the protohistoric Wall population to the historic Fredricks population. One extreme case of violence is documented by the skeletal material from Burial 4 at the Fredricks site. However, given the small samples, little more can be said at this time.

Degenerative Pathologies. Since more individuals survived to old age at the Wall site, it is proposed that more degenerative pathologies will be present in this protohistoric population when compared with the historic population from the Fredricks site. Burials 1 and 3, which comprise $25 \%$ of the Wall site population, exhibit evidence of two degenerative pathologies, arthritis (Figure 5.5) and tendonitis (Figure 5.6). These degenerative pathologies total $11.4 \%$ of all the pathologies present at the Wall site. From the Fredricks site, two burials, 5 and 9, display arthritic lipping of the vertebral column. This represents $3.8 \%$ of the pathologies and $15.4 \%$ of the population at the Fredricks site. Although the percentages of degenerative pathogens present and the population affected are not remarkably different, mors degenerative pathologies occur in the Wall site population. Thus, the postulated difference in the occurrence of degenerative pathologies between the late prehistoric/ protohistoric and historic populations may be substantiated by a larger sample.

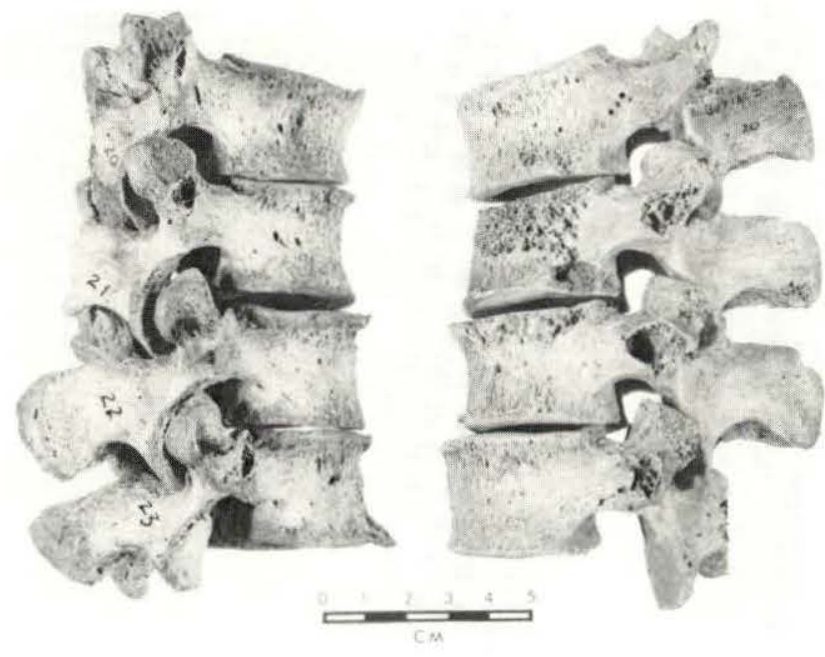

Figure 5.5

Vertebral 1ipping of Burials 1 (left) and 3 (right) from the Wall site. 


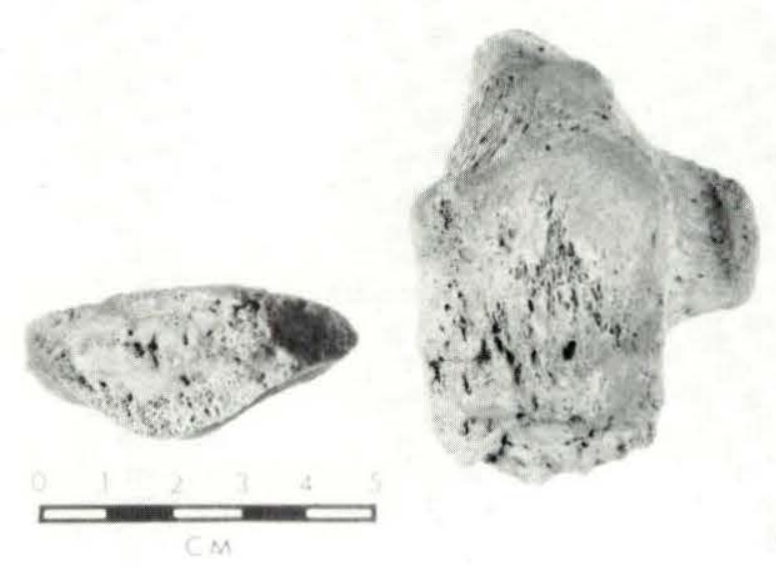

Figure 5.6

Patella and calcaneous of Burial 3 from the Wall site, showing possible tendonitis.

Tumors. No tumors or tumor-like lesions are present in the Wall site population. Two individuals, Burials 2 and 4, from the Fredricks site, however, have unusual bone formations that may be some form of osteoma, although such an identification is uncertain at present. The exterior right side of the mandible from Burial 2 , a subadult aged 6 to 8 years, exhibits swelling of the bone with some osteitis in the area below the first permanent molar and developing second molar. On the right interior of the mandible from Burial 4, an adult aged 25 to 30 years, is a small protrusion/lump that shows evidence of bone reconstruction. These examples of tumors tentatively identified as osteoma are manifested in $15.4 \%$ of the Fredricks site population, and they comprise $3.8 \%$ of the pathologies in the population.

Mechanical and General Stress. Schmorl's nodes and dental hypoplasia are two pathologies that indicate general stress in a population. Schmor1's nodes, or intervertebral disc hernia, are caused by pressure from constant elastic tension of the spine. Trauma, induced for example by mechanical stress, can cause the centrum to collapse in young individuals and produce Schmorl's nodes. In older individuals, Schmorl's nodes appear as a factor of degenerative change (Schmor1 1971:158-166). Given these causes of Schmorl's nodes, it is postulated that this pathology will afflict a greater percentage of the adult population between the age of 20 and 40 at the historic Fredricks site than at the protohistoric Wall site. This condition would be associated with an increase in the Historic period of mechanical stress related to obtaining, dressing, and transporting furs and skins for trade with the Europeans and from increased participation in warfare. Conversely, since the level of mechanical stress in the late prehistoric/ protohistoric young adult population would be less, this segment of the population at the Wall site should exhibit a low incidence of Schmorl's nodes. Most of the occurrences of Schmorl's nodes in the late prehistoric/protohistoric population should be among old adult individuals since more of this population would survive to old age and be subjected to degenerative stress. Because of the predicted presence of both mechanical and degenerative stress, those individuals that survive to old age in the historic population should also exhibit a relatively high level of occurrence of Schmor1's nodes.

In the protohistoric Wall site population, both of the old males (Burials 1 and 3 ) have Schmorl's nodes in the lumbar vertebra. Burial 1 also has Schmorl's nodes in the lower thoracic vertebra. Both individuals exhibit arthritic lipping of the lumbar and thoracic vertebra, which indicates that the herniated discs are probably degenerative and are not related directly to mechanical stress.

Burial 5, an old adult male aged over 50, and Burial 9, a female aged 35 to 40 years, are two of the four individuals from the Fredricks site that have Schmorl's nodes. The pathologies in these two cases are located in the middle to lower thoracic vertebra, and both have arthritic lipping. Burial 3, a young adult male aged 25 to 35 , and Burial 4, a young adult male aged 25 to 30 , both have a herniated disc in the lower thoracic area. It is suggested that the latter two cases are related to mechanical stress, whereas the former two cases, especially Burial 5 , are related to degenerative stress.

As originally postulated, the occurrence of Schmorl's nodes in the Wall site population is restricted to two individuals aged over 45 . These comprise $25 \%$ of the total population, and $7.6 \%$ of the total pathologies at the Wall site. The one young adult individual, Burial 4, a young adult male aged 18 to 22 , does not, as would be expected, exhibit Schmorl's nodes. In contrast, $30.8 \%$ of the Fredricks site population--one adult male aged over 50, one female aged 35 to 40 , one adult male aged 25 to 30 , and one adult male aged 25 to $35--$ possess herniated discs (Figure 5.7), which represents $7.5 \%$ of the total pathologies at the site. Thus, the original proposition that young adults in the historic population would exhibit a high incidence of Schmorl's nodes due to mechanical stress, whereas older adults in both historic and late prehistoric populations would exhibit the pathology because of degenerative stress, is supported.

Another indicator of stress in a population is enamel hypoplasia, a marker of physiological stress derived from the effects of disease and/or diet. It is defined as a deficiency in enamel thickness resulting from the slowing of enamel formation due to stress (Huss-Ashmore et a1. 1982:441). Transverse lines or rings are formed when the stress discontinues and normal development resumes. These transverse lines are not altered or otherwise affected by later events in life, thus they form a record of stress during developmental years (Huss-Ashmore 


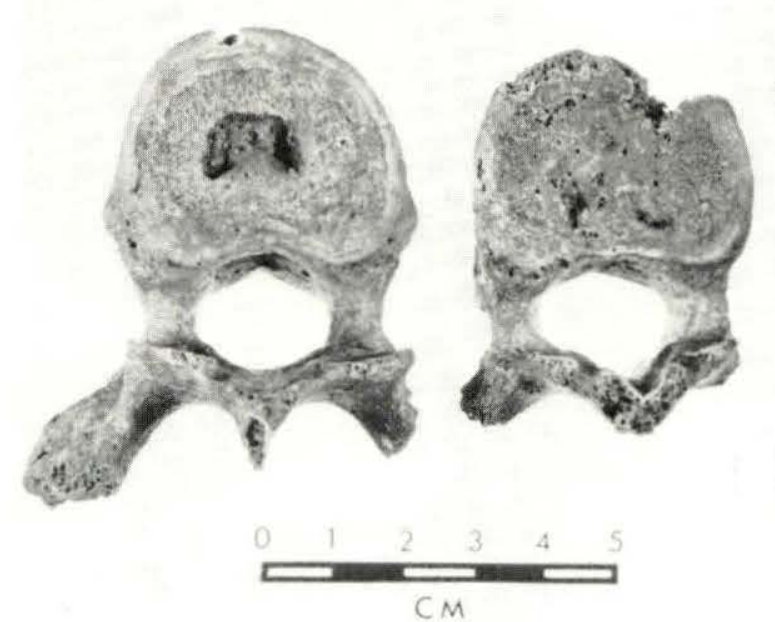

Figure 5.7

Thoracic vertebrae of Burials 4 (left) and 9 (right) from the Fredricks site, with herniated disc or Schmorl's nodes.

et al. 1982:441).

At both sites, where teeth are present (75\% of the Wall site population and $85 \%$ of the Fredricks site population), some amount of enamel hypoplasia is observed. This condition represents $23 \%$ of the pathologies present at the Wall site and $20.7 \%$ of the pathologies at the Fredricks site. Although these samples appear similar in the incidence of enamel hypoplasia and in the percentage of the population affected, Sorohan (1985:51) suggests that shorter periods of stress exhibited by more severe instances of hypoplasia are present in the Wall site population. In contrast, longer periods of less severe stress are noted for the Fredricks site. From the earlier discussion of $r$ and $K$ reproductive strategies the apparent decrease in the severity of enamel hypoplasia in the historic Fredricks site population may be due to the increased care and extended nursing of the young, assuming a move to more of a $\mathrm{K}$ reproductive strategy. Thus, whereas stress from disease may have been more prevalent in the Historic period, the young may have been shielded from its effects to some extent by the pattern of care associated with a $\mathrm{K}$ reproductive strategy.

Dietary Pathologies. Pathologies related to diet include cribra orbitalia, spongy hyperostosis, periodontal disease, and caries. Elsewhere (Wilson 1983:29), it has been hypothesized that nutritional diseases will be less common in late prehistoric populations given the general sociocultural stability present, and the presence of low levels of stress and competition relative to later periods. In contrast, diet related diseases will increase during the Historic period because of the increased incidence of disease in general, an increase in stress and competition, and the disruption of the sociocultural system (Wilson 1983:30-31).
Evidence of cribra orbitalia is manifested by lesions affecting the roof of the eye orbit. Although several conditions may cause such lesions, they are usually attributed to some type of nutritional stress related iron deficiency anemia (Steinbock 1976:244-246). Spongy hyperostosis is generally found on the parietal and occipital bones of the cranium, and is also related to iron deficiency anemia (Steinbock 1976:230). These two iron deficiency related diseases are exhibited by $25 \%$ of the Wall site population (Burial 4, an adult male aged 18 to 22 , and Burial $1-83$, a subadult aged 1.5 to 3 years at death), and they account for $11.4 \%$ of the total pathologies present. At the Fredricks site, cribra orbitalia is found in $30.8 \%$ of the population (Burial 1, a subadult aged 3-5 years; Burial 2, a subadult aged 6 to 8 years; Burial 6 , a young adult aged 20 to 25 years; and Burial 8 , a subadult aged 3 to 5 years) and represents $7.5 \%$ of the total pathologies present in the Historic period population. In all but one case, Burial 4 from the Wall site, only the porotic type of cribra orbitalia affects the eye orbits. This suggests that the iron deficiency anemia afflicting the other five individuals may have been mild in form. Burial 4, in contrast, possesses a more critical type of cribra orbitalia known as cribrotic (cf. Steinbock 1976:239). The presence of cribrotic cribra orbitalia (Figure 5.8), with severe spongy hyperostosis of the cranial vault (Figure 5.9), indicates that Burial 4 suffered from severe anemia that may have contributed to the individual's death. Spongy hyperostosis is found on both parietals of Burial 13, and represents $1.9 \%$ of the total pathogens at the Fredricks site. It is expected that the occurrence of dental caries and periodontal disease in individuals in Historic period populations will generally increase over that found in late prehistoric/protohistoric populations. The introduction of new European foods, such as peaches (Lefler 1967:115-116; Gremillion, this report), rum (Lefler 1967:232-233), and a possible increased reliance on maize (Gremillion, this report), resulted in an increase in the amount of sugar in the historic diet. This would have contributed to an increase in dental caries and periodontal disease during the Historic period.

Sorohan's (1985) preliminary study of the dental remains from the Wall and Fredricks sites tentatively supports this proposition. For the Wall site $37.5 \%$ of the population has some form of periodontal disease, which represents $11.5 \%$ of all the pathologies in this late prehistoric population. In contrast, periodontal disease affects $46 \%$ of the Fredricks site population, and it comprises $11.3 \%$ of the total pathologies at the site. Caries afflict $62.5 \%$ of the Wall site population, and account for $19.2 \%$ of the pathologies. Caries are exhibited in $85 \%$ of the Fredricks site population, where they account for $20.7 \%$ of the total pathologies. Sorohan (1985:56) proposes that the differences in the presence of caries and periodontal disease may 


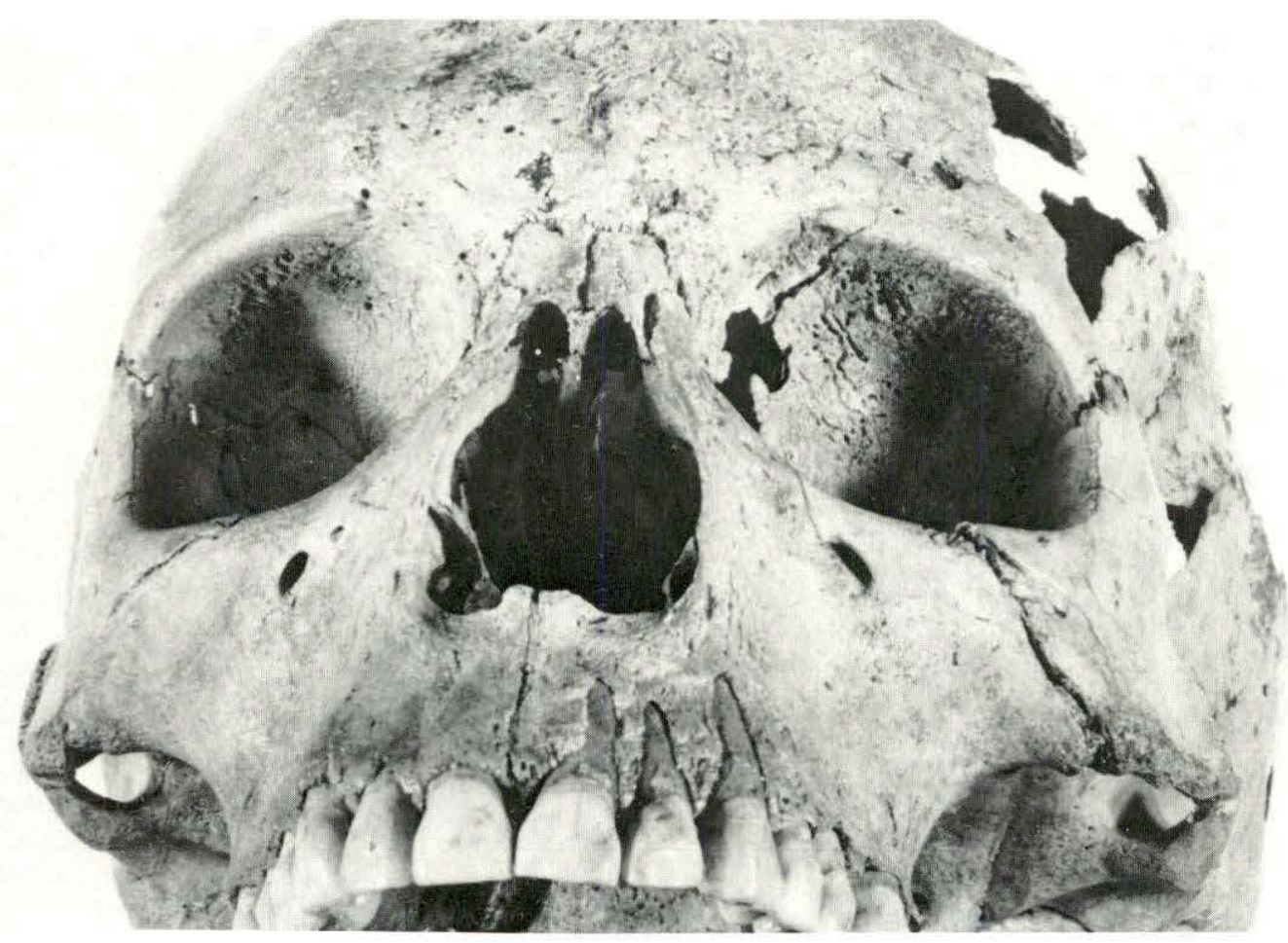

Figure 5.8

Close-up of Burial 4 skull from the Wall site, with orbits showing cribra orbitalia.

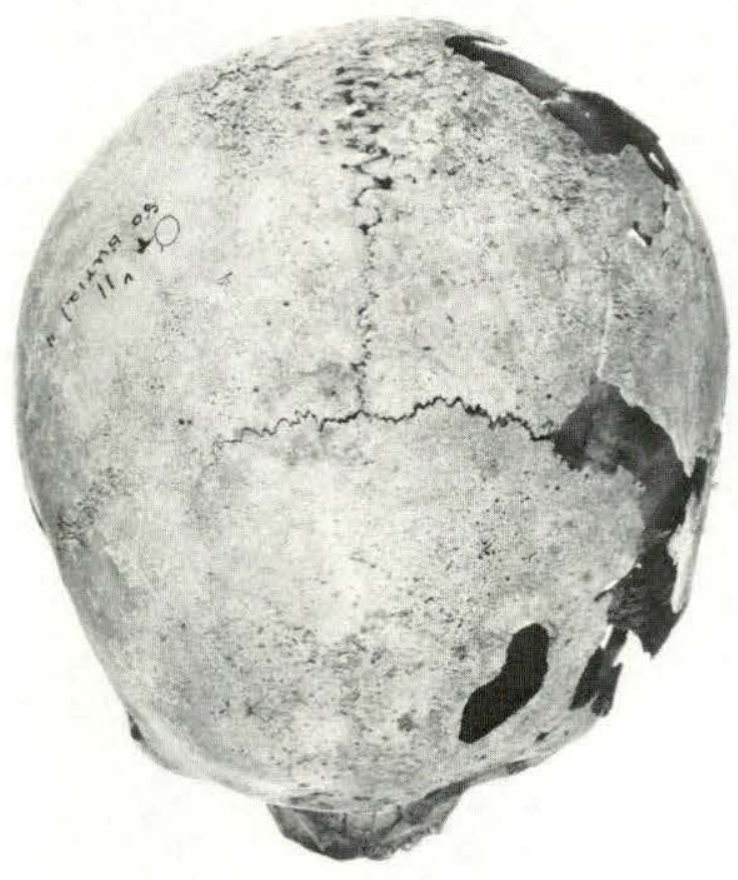

Figure 5.9

Top view of Burial 4 cranium from the Wall site, showing frontal and parietal pitting related to spongy hyperostosis. be indicative of a higher carbohydrate diet at the earlier Wall site.

It is interesting to note that whereas more individuals from the Fredricks site exhibit periodontal disease and caries (cf. Sorohan 1985:55-56), the percentage of the total pathologies is similar to that found at the Wall site. A similar trend is seen when the occurrences of all the pathologies (cribra orbitalia, spongy hyperostosis, caries, and periodontal disease) related to diet are examined. A total of $42.1 \%$ of the Wall site pathogens are diet related as compared to $41.4 \%$ of the Fredricks site pathologies. It would appear that both dietary stress and pathologies greatly affected the two populations, with more individuals at the Fredricks site being afflicted. This trend is certainly influenced by the different percentages of neonates present in the two populations $(37.5 \%$ at the Wall site and $15.3 \%$ at the Fredricks site), for whom dental remains are usually not carious and are generally not affected by periodontal disease. Although the percentages of the samples that possess diet-related diseases appear to be dramatically different, the two populations are similar when only those individuals with erupted dentition are considered. Therefore, it is difficult to say at this time whether dietary stress changed significantly from late prehistoric to historic times. This question will be considered again from a different perspective in the forthcoming section on trace element analysis. 
Osteitis. Osteitis, inflammation of the bone, is caused by trauma, infection, and/or disease (Steinbock 1976:60). It is expected that osteitis will increase in historic populations over that found in late prehistoric/ protohistoric populations. Factors favoring such a change include the presence of European diseases, increased nutritional stress, and the posited increase in overall levels of stress and competition.

In support of this proposition, more cases of osteitis are observed in the Fredricks population $(69 \%$ of the individuals in the sample) than in the protohistoric Wall site population (37.5\%). Also, the percentage of the total pathologies present increases to $17 \%$ for the Fredricks site from $11.5 \%$ for the Wall site. A11 cases of osteitis identified in both populations represent rather mild instances of remodeling in the long bones. Unfortunately, the direct cause or causes of these lesions cannot be determined.

\section{Summary of Pathologies}

Overall, the results of the analysis of pathologies show that there is a general decrease in the overall health of the historic Fredricks population from that enjoyed by the protohistoric Wall population. Assuming that variables of the natural environment remained relatively constant for both populations, given the proximity of the sites, the decline in health can be attributed to increased stresses related to European contact. One of these stresses is new diseases such as influenza, smallpox, and possibly treponemal infections, that, according to Lawson (Lefler 1967) and Dobyns (1983), are present in the Southeast at the time Occaneechi Town (Fredricks site) was occupied. Evidence of increased warfare and hunting associated with the European skin and fur trade is suggested by an increase in traumatic/violent pathologies at the Fredricks site. Also, the occurrence of severe mechanical stress among Historic period populations, perhaps related to increased warfare and hunting, is supported in part by the large percentage of young adults at the Fredricks site who were afflicted by Schmorl's nodes. Accompanying the decrease in number of individuals surviving to old age in the Historic period population is a decrease in the percentage of degenerative pathologies at the Fredricks site. The occurrence of pathologies related to disease and/or diet stress that is documented by linear enamel hypoplasia remains relatively constant in both the percentage of total pathologies it represents and in the percentage of population that it affects at both sites (see Sorohan 1985 for a more detailed discussion of the dental pathologies). Similar distributions are exhibited by pathologies, including cribra orbitalia and spongy hyperostosis, related to diet. Finally, there appears to be an increase among the Fredricks site population in general osteitis and tumors from that seen at the Wa11 site. Although the direct cause(s) for this increase cannot be determined, the change is possibly due, at least in part, to the general increase in stress brought about by European contact.

The increase in the presence of pathogens and in the percentage of the population affected by such pathogens at the historic Fredricks site is far from conclusive. Larger and more representative samples for both the Wall and Fredricks sites will be necessary before substantive conclusions can be put forth. For example, one question of great importance that cannot be addressed here concerns the differential incidence of pathologies by sex at the Fredricks site. Data for investigating this question would also provide a better understanding of the pattern of increased male mortality seen in the mortality curves for the historic Upper Saratown site. Therefore, until such time as better samples are available, the conclusions and trends documented in this discussion of pathologies have to remain strictly preliminary.

\section{DIET, HEALTH, AND TRACE ELEMENT ANALYSIS}

Trace element analysis is used to consider questions pertinent to the diet and general health of the protohistoric Wall site and the historic Fredricks site populations. Techniques that measure the relative concentrations of trace elements in human bone have come to enjoy increasing favor among anthropologists interested in the reconstruction of diet, disease states, and the general health of archaeological populations (Bahou 1975; Blakely and Beck 1981; Lambert, Szpunar, and Buikstra 1979). Another use of trace element analysis has been for determining differential access to certain foods, such as meat, or the relative contribution of meat resources and plant foods to the diet of various segments of a population, and how such a pattern relates to status (Brown 1974; Schoeninger 1979; Hatch and Guide1 1982).

For trace element analysis to be meaningful, it is essential that the subsistence patterns of the study population(s) be reconstructed from the archaeological record. The analysis of the faunal remains from the Wall and Fredricks sites by Holm (this report) suggests that the major animal resources for both sites were deer (Odocoileus virginianus) and catfish (Ictaluras sp.). The historic Fredricks site possesses the remains of European mammals including the horse (Equus caballus) and pig (Sus scrofa), but only in very small quantities. Rabbit (Sylvilagus $\mathrm{sp}_{\mathbf{c}}$ ) and beaver (Castor canadensis), present at the Wall site, are absent from the Fredricks site. Holm concludes that the role of faunal resources in the overall subsistence strategy at the Fredricks site is not significantly different from that at the protohistoric Wall site. 
Gremillion's study (this report) of the plant foods utilized at the two sites suggests a similar lack of change. Although peach is the only European-introduced plant food at the Fredricks site, the relative and actual amounts of acorn and hickory nut differs for the two sites also. Acorns, and all nuts in general including hickory, are present in greater quantities at the Wall site. In contrast, there are less acorns present in the later Fredricks site, and hickory nuts predominate. Differences in the contribution of maize to the overall subsistence is also suspected, as slightly larger amounts of this cereal are present at the Fredricks site. However, Gremillion (this report) feels that these differences cannot, at this time, be interpreted as representing a greater dependence on maize for the Fredricks site occupants. Both Holm and Gremillion caution that sample bias may affect their studies, as the remains from the Wall site are mostly from the site midden, whereas those from the Fredricks site are from the fill of features and burial pits.

Diet

Several propositions about possible changes in diet can be tested by identifying the relative and absolute amounts of zinc, copper, magnesium, strontium, and vanadium present in the skeletal series from the Wall and Fredricks sites. Zinc and copper are found to predominate in meats, seafood, and nuts (Gilbert 1977). Vanadium, strontium, and magnesium are highest in grains, cereals, and nuts (Gilbert 1977). High levels of strontium are also found in molluscs (Schoeninger and Peebles 1981).

The central proposition about diet to be considered at this time is that if there was little difference in the relative amounts of meat and plant foods consumed by the Wall and Fredricks populations, the relative amounts of trace elements will be similar in the two populations. If, however, more meat was available for consumption by the historic Fredricks site population as a consequence of increased hunting of fur and skin bearing animals for trade to the Europeans, there will be a relative increase at the Fredricks site in the amounts of zinc and copper and a corresponding decrease in the other three elements. The possible shift from a greater reliance on nuts, especially acorns, at the Wall site to less reliance at the Fredricks site may be documented by higher overall levels of all five elements at the earlier Wall site. If maize is a more important component in the subsistence of the Fredricks site population higher levels of magnesium, strontium, and vanadium, and lower levels of zinc and copper, will be present in the bones at the historic site.

The use of trace element analysis in the reconstruction of diet is fraught with difficulties. Although the trace elements usually can be associated with either plants or animals, only a few can be specifically restricted to being derived from a particular plant or animal food. Nuts are an excellent example of this problem, as, like meat resources from animals, nuts are high in zinc. Another problem encountered in the analysis of trace elements is the antagonistic characteristics of zinc and copper. When levels of zinc decrease, copper levels increase (Gilbert 1977:89-90). The interpretation of the levels of zinc present is uncertain as zinc decreases in the presence of increasing amounts of phylate, a component of grains and cereals (Sever 1975; Gilbert 1977). Also, levels of zinc are decreased by sweating and parasitic infestations (Sever 1975).

\section{Technique of Trace Element Analysis}

A total of $15 \mathrm{gm}$ of cortical bone from the femurs of the burials from both sites were assayed for levels of zinc, strontium, magnesium, copper, vanadium, and lead. Bone samples from Burials 2, 2-83, and 3-83 from the Wall site and Burials $4 \mathrm{a}$ and 7 from the Fredricks site could not be taken due to the poor preservation of the femurs. In each of the bone samples assayed, the levels of the trace elements were determined at the Horticulture Laboratory, the University of Georgia, Athens, using "inductively coupled argon plasma emission spectrometry," or ICP. The ICP technique is preferable to the more commonly used atomic absorption analysis as ICP reduces the potential for ion interference (Price and Kavanagh 1982), and allows for the assay of a number of trace elements at the same time (Gilbert 1977:95). Preparation of samples for ICP follows the procedure outlined below.

After cleaning the bone samples are weighed and placed in a beaker. The samples are then muffled at $500^{\circ} \mathrm{C}$ for eight hours. The residue is dissolved in agua regia and dried on a hot plate. The residue is then taken up in $50 \mathrm{ml}$ of acid and $20 \mathrm{ml}$ of the original sample is assayed on a plasma emission spectrometer (Long personal communication).

Bone samples from four Wall site burials and 11 Fredricks site burials were assayed for five elements--zinc, strontium, magnesium, copper, vanadium, and lead. Bone samples from a deer (Odocoileus virginianus) and raccoon (Procyon lotor) were also assayed to provide a scale against which the results of the human bone sample analyses could be compared. The inclusion of the bone from deer, a known herbivore, also provides a check on the reliability of the strontium assay for the human samples. For example, if the human bone samples possess levels of strontium higher than the deer bone samples, then it is possible that molluscs are an important part of the diet (cf. Schoeninger and Peebles 1981). When such elevated levels of strontium is documented in the bone, no conclusions based on the variation in strontium levels within the study populations can be reached. 


\section{Results of the Trace Element Analysis}

Tables 5.16 and 5.17 present the results (in parts per million or ppm) of the trace element analyses for the Wall site and Fredricks site respectively. In general, the trace element levels within the Wall site population (Table 5.16) show little variation between individuals. The levels of strontium (mean $=851.7 \mathrm{ppm}, \mathrm{sd}=94.6 \mathrm{ppm}$, range $=758-$ $982 \mathrm{ppm})$ are consistently lower than the deer $(1268 \mathrm{ppm})$ from the site, and are similar to that noted for the raccoon (844 ppm). This suggests a mixed diet for the Wall site population with both meat and plant foods being consumed. Magnesium levels are slightly lower than the $1100 \mathrm{ppm}$ that is considered a normal concentration for healthy bone (Gilbert 1977: 93). The mean human magnesium level is 787.3 ppm with a mean of 877.0 for the adults from the Wall site. Zinc averages $175.6 \mathrm{ppm}$ for all four burials and $174.3 \mathrm{ppm}$ for the adult burials. In modern populations, healthy bone contains approximately $181 \mathrm{ppm}$ of zinc (Gilbert 1977: 92). Levels of both lead and copper are too low in the bone samples from the Wall site to be detected.

In contrast to the Wall site results, there is more variation in the individual levels of strontium at the Fredricks site (Table 5.17), with values ranging from $143.2 \mathrm{ppm}$ to $595.2 \mathrm{ppm}$ $($ mean $=836.6, \quad \mathrm{sd}=166.2)$. Al1 strontium levels for the Fredricks site bone samples are consistently lower than that calculated for a mature deer femur ( $1190 \mathrm{ppm})$ form the site. Two subadults, Burials 1 and 2, have unusually high levels of strontium (1143.2 ppm and $1084.4 \mathrm{ppm}$ respectively). Two explanations for this pattern can be considered at this time. First, both individuals may have been from another area/region where strontium levels in the environment were naturally higher than at the Fredricks site. Schoeninger (1979:306) notes that one of stcontium's empirical properties is its uneven geographical distribution. An alternative cause for the high strontium levels in Burials 1 and 2 may be that molluscs, which are strontium rich, comprised an inordinate percentage of the diet of the two individuals (cf. Schoeninger and Peebles 1981:391-397). Schoeninger and Peebles (1981) found that Archaic period populations from northern Alabama, who consumed molluscs as an integral part of their diet, had higher levels of strontium than the agricultural Mississippian population that later inhabited the same area. Burials 1 and 2 are also noteworthy among the Fredricks site burials as they both (especially Burial 1) have an abundance of shell artifacts as grave accompaniments when compared with the other burials. Only two (Burials 3 and 4) of the other eleven burials had shell associations. The presence of ornamental shell artifacts in Burials 1 and 2 may indicate a coastal affiliation for the two, as well as for Burials 3 and 4 .

Magnesium levels for the Fredricks site
Table 5.16

ICP of trace elements in bones from the Wall site (parts per million, ppm).

\begin{tabular}{lrrrrrrr}
\hline $\begin{array}{l}\text { Sample } \\
\text { Descr1pt1on }\end{array}$ & $\mathrm{Sr}$ & $\mathrm{Mg}$ & \multicolumn{2}{c}{ Trace Element } & $\mathrm{Zn}$ & $\mathrm{V}$ & $\mathrm{Pb}$ \\
\hline Bur1a1 1 & 758.0 & 951.2 & 210.2 & - & 5.4 & - \\
Bur1al 3 & 842.0 & 728.0 & 146.2 & - & - & - \\
Bur1a1 4 & 982.8 & 952.0 & 166.6 & - & 1.3 & - \\
Bur1al 1-83 & 824.0 & 518.0 & 179.4 & - & - & - \\
Procyon 1otor & 844.0 & 1126.4 & 231.7 & - & 3.4 & - \\
\hline Odoco11eus v1rg1n1anus & 1268.0 & 695.2 & 154.9 & - & 9.9 & - \\
\hline
\end{tabular}

(-) level too low to detect element.

Table 5.17

ICP of trace elements in bones from the Fredricks site (parts per million, ppm).

\begin{tabular}{|c|c|c|c|c|c|c|}
\hline $\begin{array}{c}\text { Sample } \\
\text { Description }\end{array}$ & $\mathrm{Sr}$ & $\mathrm{Mg}$ & $\begin{array}{l}\text { Trace } \\
\mathrm{Zn}\end{array}$ & $\begin{array}{c}\text { Element } \\
\mathrm{Cu}\end{array}$ & v & $\mathrm{Pb}$ \\
\hline Burfal 1 & 1143.2 & 733.2 & 167.2 & 1.6 & 10.6 & 72.0 \\
\hline Burial 2 & 1084.0 & 823.2 & 209.5 & 1.6 & 5.8 & 4.0 \\
\hline Burial 3 & 773.2 & 436.4 & 179.8 & - & - & - \\
\hline Burlal 4 & 888.8 & 498.0 & 215.6 & - & 15.4 & 106.0 \\
\hline Burial 5 & 900.8 & 763.2 & 196.1 & - & 8.8 & - \\
\hline Burial 6 & 799.6 & 495.2 & 475.6 & - & 14.6 & 14.0 \\
\hline Burial 8 & 595.2 & 555.6 & 2296.9 & 264.4 & - & - \\
\hline Burfal 9 & 738.4 & 467.2 & 312.5 & - & - & - \\
\hline Burial 10 & 640.0 & 500.4 & 3964.8 & 1058.4 & 1.9 & - \\
\hline Burial 11 & 848.0 & 400.0 & 229.5 & - & - & - \\
\hline Burial 13 & 792.0 & 535.2 & 400.0 & - & - & - \\
\hline Procyon 1otor & 1138.4 & 871.2 & 184.9 & - & - & - \\
\hline Odocolleus v1rginfanus & 1190.0 & 1013.2 & 159.5 & - & - & - \\
\hline
\end{tabular}

(-) level too low to detect element.

population have a mean of 564.3 ppm with a standard deviation of $142.1 \mathrm{ppm}$ for a range of 400 to $763.2 \mathrm{ppm}$. This level is somewhat lower than that calculated for the Wall site population. Zinc levels for the Fredricks site population are also higher (mean $=786.1 \mathrm{ppm}$, $\mathrm{sd}=1221.59$, range $=167.2-3964.8)$ when compared with the Wall site population. Deleting the zinc levels determined for the subadults, of which two (Burials 8 and 10) have extremely high zinc levels, in the Fredricks population reduces the mean zinc level to $287.0 \mathrm{ppm}$ with a standard deviation of $113.4 \mathrm{ppm}$ given a revised range of 196.1 to $312.5 \mathrm{ppm}$ for the adults. This is slightly higher than the zinc level noted for the Wall site adults (mean $=174.3 \mathrm{ppm}, \mathrm{sd}=$ $32.7 \mathrm{ppm}$, range $=146.2-210.2 \mathrm{ppm})$.

Because the Wall and Fredricks sites are located within $200 \mathrm{yds}$ of each other within the same bend of the Eno River, it is possible to compare the results of the trace element assays conducted for the two sites. Given the close proximity of the two sites, it can be assumed that the potential occurrence of trace elements 
in the natural environment is not biased by differing environmental conditions, and that any differences in trace element levels in the human bone samples will probably be due to cultural factors.

Comparing different levels of strontium, magnesium, and zinc in the adult bone samples from the Wall and Fredricks sites indicates that the two populations had slightly differing diets. The earlier Wall site population may have had a diet higher in cereals and grains given the higher strontium and magnesium levels present. A diet that included more meat would provide a pattern of lower strontium and magnesium levels coupled with higher zinc levels as the Fredricks site population evidenced. This does not negate the proposition put forth earlier that more meat was probably available for consumption by the historic Fredricks site population, due to the increased hunting of fur-bearing animals for trade with the Europeans. The levels of vanadium, which is derived from such protein sources as nuts, average $8.3 \mathrm{ppm}$ for the Wall site adults and 12.9 ppm for the Fredricks site adults. The small increase in vanadium levels tentatively supports a hypothesized increase in the amount of protein-rich nuts, such as hickory nuts, consumed by the historic population at the Fredricks site (see Gremillion, this report).

As would be expected, the introduction of lead-bearing European artifacts, such as lead shot, vermillion, and pewter utensils, into the material culture of the Fredricks site population elevated the level of lead in the population's environment and, hence, the bone samples of the populations as well. A total of four of the 11 Fredricks site burials had levels of lead high enough to be detected, giving a mean of $49 \mathrm{ppm}$ and a standard deviation of 48.4 ppm. The two Fredricks site adults had a lead mean of $60 \mathrm{ppm}$ and a standard deviation of 65 ppm.

\section{Summary of the Trace Element Analysis}

The results of the trace element analyses provide information important to addressing questions concerning diet and status among the Wall and Fredricks populations. Differing levels of trace elements that suggest an increased utilization of animal protein and of protein-rich nuts such as hickory, at the Fredricks site support the conclusion that there were differences between the two populations in diet. Likewise, differences in the range of strontium levels in the later Fredricks site population may reflect an influx of people into the area from other environments where strontium levels were different, such as the coast, or of individuals form regions where molluscs were an important food resource.

In comparing the results of the trace element assays with the study of the floral and faunal remains, several convergencies with the above findings can be seen. First, as Gremillion (this report) notes, there is a higher percentage of acorn nut in the floral samples from the earlier Wall site. The occurrence of acorn decreases at the Fredricks site, and is accompanied by an increase in the hickory nut. The higher zinc and vanadium levels at the Fredricks site may be due, at least in part, to the increased utilization by the inhabitants of this site of protein rich nuts such as hickory. Furthermore, the increase in zinc at the Fredricks over that noted at Wall site may document an increased use of meat foods at the historic site. This finding is not unexpected given that meat may have been more accessible due to the increase in hunting of fur-and-skin bearing animals for trade with the European.

Unfortunately, this discussion of diet, status, and trace element analysis has to remain incomplete at this time because of the small skeletal samples available for study. Some differences in the diet and status within and between the two sample populations, however, are suggested. With the recovery of a larger sample a more indepth study of the questions and hypotheses raised in this section can be conducted. Comparison of age and sex groups by different levels of each trace element with amounts and types of grave associations is just one example of the numerous investigations that could be undertaken. In closing this section, it is again stressed that the findings presented here are preliminary and serve more as a focus for future research than as answers to any particular questions.

\section{BIOLOGICAL DISTANCE AND DIVERSITY}

Biological distance is the expression of the genetic similarities between two or more populations. In biological distance studies one assumes that among a collection of individuals, those displaying the most similarities are more closely related. Thus, it should be possible to investigate biological relationships between the Wall and Fredricks site populations through the analysis of biological distance. Were the Historic period Fredricks site occupants descendants of the earlier Wall site occupants? Since the two sites are located in the same bend of the Eno River, and studies of their respective pottery assemblages (Davis 1984 and this report) have revealed that potters of both sites participated in a common ceramic tradition, we might expect there to be little biological distance, and thus little diversity, between the two populations.

Conversely, the Fredricks site inhabitants may represent a population from a foreign area, or a mixture of different populations, some local and some foreign. The first case could be marked by either high or low within-group diversity, depending on the amount of preexisting biological diversity. If the Fredricks 
site population were a mixture of peoples, then diversity would be expected, and the amount of similarity to the Wall site population would depend to some extent on whether descendants of the Wall site occupants were included in that mixture.

Direct evidence of combining of previously separate Indian groups in the Middle Atlantic area during the Historic period is provided by the ethnohistoric record. For example, at the time of Bacon's Rebellion in Virginia in 1676, reports show that several Indian groups resided with the Occaneechis at their stronghold on the Roanoke River (Sainesbury 1893:414; Billings 1975:267-269). Among these groups were the Manakins, Annalectins, and Susquehannocks. The Susquehannocks were Iroquoian speakers from the upper Chesapeake Valley, and the Manakins are thought to have been remnants of a Piedmont Virginia Siouan group (J. Wilson 1984:102-105). The Annalectins cannot be identified with any known group at this time. A similar pattern of amalgamation probably characterized the Occaneechis after their move to the Eno, especially given the casualties inflicted on them by Bacon's forces (J. Wilson 1984:104-105, 112114).

A third question to be considered is how do the Wall and Fredricks site populations compare with other populations from the Piedmont region of North Carolina that have been previously identified as "Siouan." No biological distance information is available for the late prehistoric population from the Shannon site, identified with the Siouan-speaking Tutelo Indians of Virginia (Benthal1 1968). However, a limited amount of biological/genetic information is available for the skeletal collection from Upper Saratown, a Siouan site located on the Dan River in the northern Piedmont of North Carolina (Navey 1982; J. Wilson 1984).

The accuracy with which biological relationships can be explored is dependent on the representativeness of the skeletal samples and the selection of traits that are purely genetic and that are not affected by environment, or by age or sex of the individuals (Ubelaker 1978:87). The first requirement presents a problem, as the two samples from the Wall and Fredricks sites are not representative of a normal (i.e., both sexes represented by all age groups) population. Ubelaker (1978:87) states that a sample of 100 or more adults is sufficient for study, given that no biases are present in the selection of the sample. As only four crania from the Wall site and eight crania from the Fredricks site comprise the study sample, the results of this biological distance study are highly preliminary, and can be used only to suggest directions for future research.

Two techniques are used here to determine the degree of similarity between the Wall and Fredricks site samples. One involves combining a non-metric trait study similar to that employed by Buikstra (1976) in her investigation of prehistoric Illinois River population samples with a formula for measuring population diversity (Lieberson 1969). The other technique consists of comparing selected cranial and postcranial indices and stature estimates in order to determine similarities and differences. Information useful to this study is provided by the Upper Saratown skeletal samples, where four crania from burials provide comparative cranial indices and non-metric traits, and 14 burials have data from which post-cranial indices and stature estimates can be obtained.

\section{Non-Metric Traits and Diversity}

The analysis of the non-metric data involves coding the presence/absence of six discrete traits suggested by Buikstra (1976:53, 84). A total of eight variants can be extracted using the six discrete traits: 1) asterionic bone, 2) parietal notch bone, 3) supraorbital foramen, 4) mylohyoid arch, 5) divided hypoglassal canal, 6) multiple zygomatic facial foramina, 7) ossicle at lambda, and 8) superior sagittal sulcus flexes right. These traits are used, as they have been shown to be unaffected by age, sex, trait intercorrelation, or cultural deformation (Buikstra 1976:49-51). As the population samples are too small to compute Chi-square and the mean measure of divergence values similar to those used by Buikstra (1976:53-54), another measure of diversity, Lieberson's (1969) diversity formula, is utilized to explore the degree of similarity within the study populations. Lieberson's measure of diversity, a variation on Simpson's formula (Lieberson 1969), describes the position of a population along a continuum from homogeneous to heterogeneous with respect to the trait under study. The formula for calculating the within-sample diversity is expressed as

$$
D_{\mathrm{w}}=1-\mathrm{S}=1-\left(\left(\mathrm{x}_{1}\right)^{2}+\left(\mathrm{x}_{2}\right)^{2}+\left(\mathrm{x}_{3}\right)^{2}+\ldots+\left(\mathrm{x}_{\mathrm{n}}\right)^{2}\right),
$$

where $D_{\text {}}$ is the diversity within the population, and $X{ }^{W}$ is the percentage of a particular trait in the population. The sum of the squared percentages (S) is subtracted from 1 to give the probability that two randomly paired traits of the population will be different. For example, if $D_{W}=0.375$, there is only a $37.5 \%$ chance that

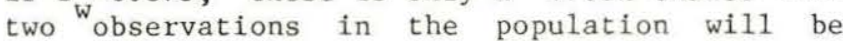
different. Conversely, a diversity index of 0.875 is very high and indicates that there is a $87.5 \%$ probability that two observations will be different.

\section{Biological Diversity within the Study Populations}

Within-sample diversity indices calculated for each population using the eight discrete biological variants noted above indicates that the most diverse sample is from the Fredricks site $(\bar{X}=.408)$, and that the Upper Saratown sample has the least diversity $(\bar{X}=.229)$. The within-sample diversity index for the Wall site population is $\overline{\mathrm{X}}=.317$. The greater diversity at 
the Fredricks site may be an indication of the increased interaction between and admixture of groups during the Historic period. The index for the protohistoric Wall site is, as would be expected, lower than that of the Fredricks site, as there was less interaction and admixture with other populations during protohistoric times. What is not expected is the low within-sample diversity index $\left(D_{=}=.229\right)$ for the Upper Saratown sample. As the Upper Saratown population was also disrupted by European contact and enjoyed increased interaction with other Indian groups during the early Historic period, a higher diversity value, similar to that computed for the Fredricks site, was expected. This may indicate that Upper Saratown is a late protohistoric and/or early historic site.

\section{Metric Analyses}

Cranial and postcranial indices determined for the three study populations are presented in Tables 5.18-5.19. The mean cranial indices indicate that the Fredricks site population is more similar to the Upper Saratown population than to the Wall site population. It is interesting to note that, of the three standard deviations of the means for the cranial indices, the one calculated for the Upper Saratown population is greater than the other two. That there is more diversity in the cranial indices of the Upper Saratown sample is somewhat unexpected given the low within-sample diversity index calculated for the non-metric cranial traits.

Table 5.18

Mean cranial indices.

\begin{tabular}{|c|c|c|c|c|c|c|c|c|c|c|c|c|c|}
\hline Population & Sex & No. & $\begin{array}{l}\text { Cranial } \\
\text { Index }\end{array}$ & No. & $\begin{array}{l}\text { Cranial } \\
\text { Module }\end{array}$ & No. & $\begin{array}{l}\text { Cranial } \\
\text { Length- } \\
\text { Height } \\
\text { Index }\end{array}$ & No. & $\begin{array}{l}\text { Cranial } \\
\text { Breadth } \\
\text { Height } \\
\text { Index }\end{array}$ & h- & $\begin{array}{l}\text { Mean } \\
\text { Height }\end{array}$ & No. & $\begin{array}{l}\text { Fronto- } \\
\text { Parietal } \\
\end{array}$ \\
\hline Wall Site & M & 3 & $\begin{array}{l}88.05 \\
(5.748)\end{array}$ & 3 & $\begin{array}{l}152.67 \\
(4.839)\end{array}$ & 3 & $\begin{array}{l}83.78 \\
(3.416)\end{array}$ & 3 & $\begin{array}{l}95.26 \\
(2.738)\end{array}$ & 3 & $\begin{array}{l}89.09 \\
(1.218)\end{array}$ & 3 & $\begin{array}{l}64.98 \\
(.834)\end{array}$ \\
\hline \multirow[t]{2}{*}{ Fredricks Site } & M & 2 & $\begin{array}{l}78.97 * \\
(4.88)\end{array}$ & 2 & $\begin{array}{c}156.66 * \\
(2.355)\end{array}$ & 2 & $\begin{array}{l}72.47 * \\
(.848)\end{array}$ & 2 & $\begin{array}{l}91.92 * \\
(4.603)\end{array}$ & )$^{2}$ & $\begin{array}{l}81.01 * \\
(1.258)\end{array}$ & 2 & $\begin{array}{l}68.14 \\
(1.153)\end{array}$ \\
\hline & $\mathrm{F}$ & 1 & 74.46 & 1 & 152.67 & 1 & 69.15 & 1 & 92.86 & 1 & 79.27 & 0 & - \\
\hline \multirow[t]{2}{*}{ Upper Saratown } & M & 3 & $\begin{array}{l}80.47 \\
(11.86)\end{array}$ & 2 & $\begin{array}{l}151.50 \\
(.707)\end{array}$ & 2 & $\begin{array}{l}73.36 \\
(2.527)\end{array}$ & 2 & $\begin{array}{c}96.03 \\
(15.634)\end{array}$ & 2 & $\begin{array}{l}82.78 \\
(4.281)\end{array}$ & 3 & $\begin{array}{l}66.52 \\
(5.461)\end{array}$ \\
\hline & F & 1 & 77.14 & 1 & 149.33 & 1 & 78.86 & 1 & 102.22 & 1 & 89.03 & 1 & 69.63 \\
\hline Iswanid $\mathrm{d}^{1}$ & M & 33 & 76.25 & 33 & 150.23 & 33 & 78.27 & 33 & 102.89 & 33 & 89.00 & 0 & - \\
\hline Lenapid ${ }^{2}$ & M & 24 & 75.39 & 24 & 153.50 & 24 & 77.76 & 24 & 103.65 & 0 & - & 0 & - \\
\hline \multirow[t]{2}{*}{ Munsee ${ }^{3}$} & M & 4 & 73.90 & 7 & 155.60 & 4 & 73.10 & 4 & 98.90 & 4 & 83.86 & 0 & - \\
\hline & $\mathrm{F}$ & 5 & 75.80 & 9 & 147.50 & 5 & 73.10 & 5 & 96.40 & 5 & 83.23 & 0 & - \\
\hline
\end{tabular}

${ }^{1}$ Neumann 1952:17-19, 32.

${ }^{2}$ Neumann 1952:23, 24, 32 .

${ }^{3}$ Hrdlicka 1916:21, 22, 26, 31 .

* Estimated. " ( )" Standard Deviation. 
Table 5.19

Post-cranial indices.

\begin{tabular}{|c|c|c|c|c|c|c|}
\hline & & $\begin{array}{c}\text { Humerus } \\
\text { Robusticity }\end{array}$ & $\begin{array}{c}\text { Femur } \\
\text { Platymeric }\end{array}$ & $\begin{array}{c}\text { Femur } \\
\text { Pilastric }\end{array}$ & $\begin{array}{c}\text { Femur } \\
\text { Robusticity }\end{array}$ & $\begin{array}{c}\text { Femur } \\
\text { Platycnemic }\end{array}$ \\
\hline Population & Sex & Index & Index & Index & Index & Index \\
\hline
\end{tabular}

Wall Site

\begin{tabular}{|c|c|c|c|c|c|c|c|c|c|c|}
\hline M & 3 & $\begin{array}{l}18.45 \\
(1.56)\end{array}$ & 2 & $\begin{array}{c}81.54 \\
(12.84)\end{array}$ & 3 & $\begin{array}{l}111.29 \\
(4.74)\end{array}$ & 3 & $\begin{array}{l}11.07 \\
(.511)\end{array}$ & 3 & $\begin{array}{l}64.53 \\
(6.65)\end{array}$ \\
\hline
\end{tabular}

Fredricks Site

$\begin{array}{lllllllllll}\text { M } & 2 & \begin{array}{l}20.74 \\ (.179)\end{array} & 4 & \begin{array}{l}86.86 \\ (2.90)\end{array} & 4 & \begin{array}{l}117.32 \\ (6.340)\end{array} & & \begin{array}{l}12.46 \\ (.923)\end{array} & 3 & \begin{array}{l}65.98 \\ (5.07)\end{array} \\ \text { F } & 1 & 16.98 & 1 & 92.86 & 1 & 121.74 & 1 & 10.74 & 0 & -\end{array}$

Upper Saratown

\begin{tabular}{|c|c|c|c|c|c|c|c|c|c|c|}
\hline M & 3 & $\begin{array}{l}18.06 \\
(1.39)\end{array}$ & 7 & $\begin{array}{l}78.03 \\
(4.90)\end{array}$ & 7 & $\begin{array}{l}118.87 \\
(8.656)\end{array}$ & 4 & $\begin{array}{l}12.24 \\
(.712)\end{array}$ & 4 & $\begin{array}{l}60.72 \\
(5.27)\end{array}$ \\
\hline F & 0 & - & 4 & $\begin{array}{l}75.94 \\
(4.72)\end{array}$ & 5 & $\begin{array}{l}107.67 \\
(3.345)\end{array}$ & 2 & $\begin{array}{l}12.36 \\
(1.40)\end{array}$ & 3 & $\begin{array}{l}60.66 \\
(3.65)\end{array}$ \\
\hline
\end{tabular}

"( )" Standard Deviation.

Comparison of the postcranial measurements from the three sites does not yield any identifiable patterns of similarities or differences. In general, the males from all three sites are similar in robusticity but differ when other indices are compared.

Stature estimates, calculated using Genoves (1967) formula, for males from the three sites are very similar. At the Wall site, males range in height from $162.4+3.4$ to $175.1+33.4 \mathrm{~cm}$, with an average of $169.8^{-} \mathrm{cm}\left(5^{\prime} 6^{\prime \prime}\right)$. The four males from the Fredricks site for whom measurements

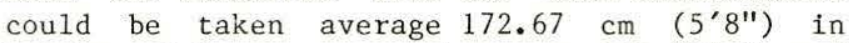
height with a range between $170.2+3.4$ and $176.7+3.4 \mathrm{~cm}$. The one female from the Fredricks site, Burial 9, is estimated to have been $171.5+3.8 \mathrm{~cm}\left(5^{\prime} 7^{\prime \prime}\right)$ tall. Similar heights are seen for the males from Upper Saratown, with four femurs providing a stature range between $165.2+3.4$ and $175.1+3.4 \mathrm{~cm}$, and an average of $170.7^{-} \mathrm{cm}\left(5^{\prime} 7^{\prime \prime}\right)$. The average female at Upper Saratown is estimated to have been $163.2 \pm 3.8 \mathrm{~cm}$ (5'4") tall.

\section{Summary of Biological Distance Study}

The following interpretations of biological distance between the Wa11, Fredricks, and Upper Saratown sites are based on the non-metrical and metrical analyses of the skeletal series from the three sites. Several patterns are recognized. First, the Wall and Fredricks site populations appear to be the least similar of the three. It is felt that biological continuity between the two is not supported at this time, although the effects of admixture on the historic Fredricks site population and small sample size may be masking such a relationship. Figures 5.10-5.12 compare two of the old males from these two sites. Second, the Fredricks and Upper Saratown populations seem to be the most similar of the three (Figures 5.13-5.15). This may be due to interaction of these two groups with similar populations during the Historic period. Alternately, this similarity between the Fredricks site and Upper Saratown may document the effects of interaction and admixture between two related Siouan populations at different points in the Historic period. The populations from the Wall and Upper Saratown sites are less similar. Skulls from these two sites are compared in Figures 5.16-5.18. In general, the Fredricks site population is similar to the more robust, long-headed populations (the Algonquians and the Iroquois) of the Coast than the Siouan populations of the Piedmont (see Table 5.18). This is somewhat unexpected given that the Occaneechi Indians have long been considered Siouan (cf. J. Wilson 1984). What may be represented here are the results of interaction and admixture of the 


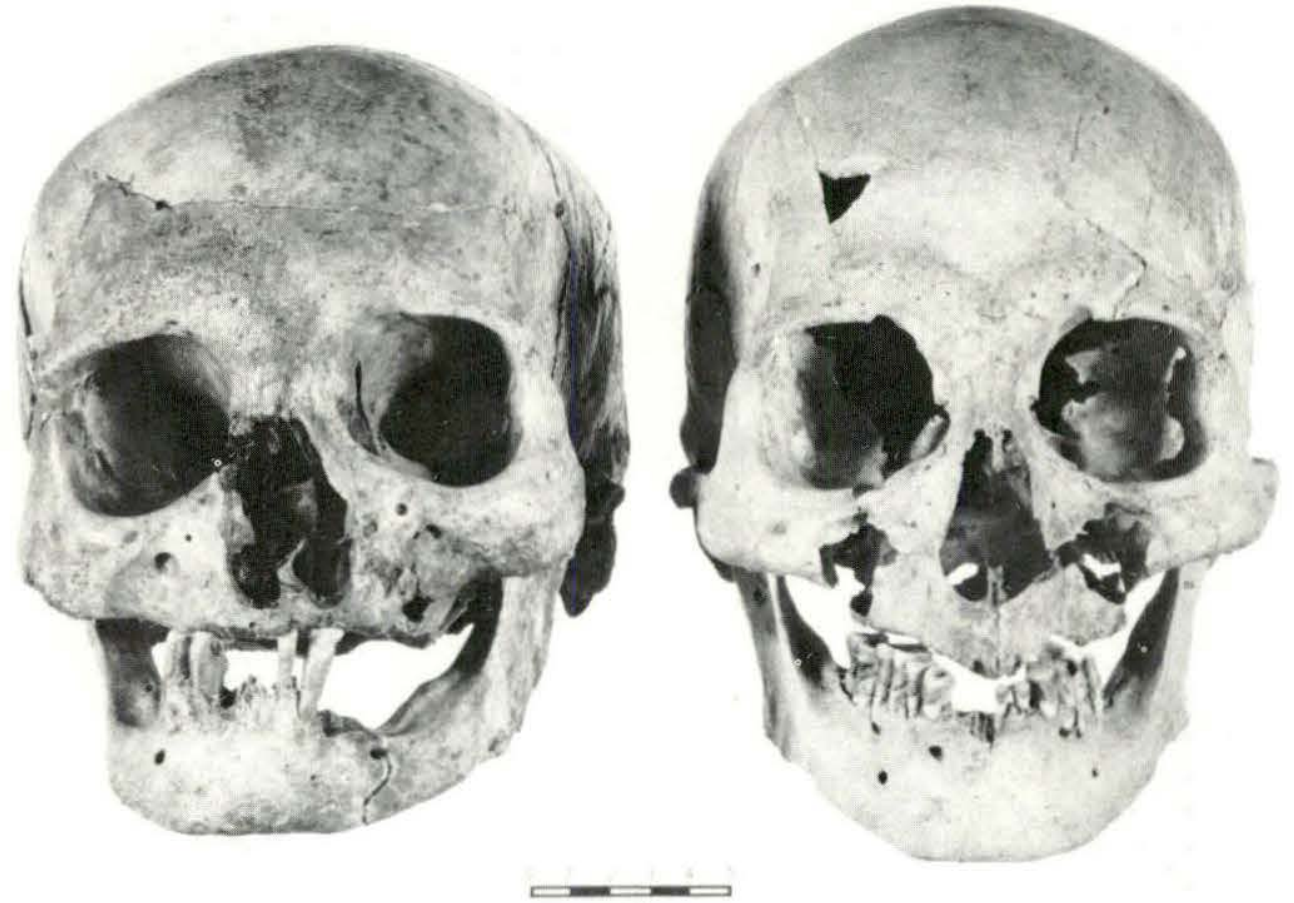

Figure 5.10

Front view of crania from Fredricks site Burial 5 (left) and Wall site Burial 1 (right).

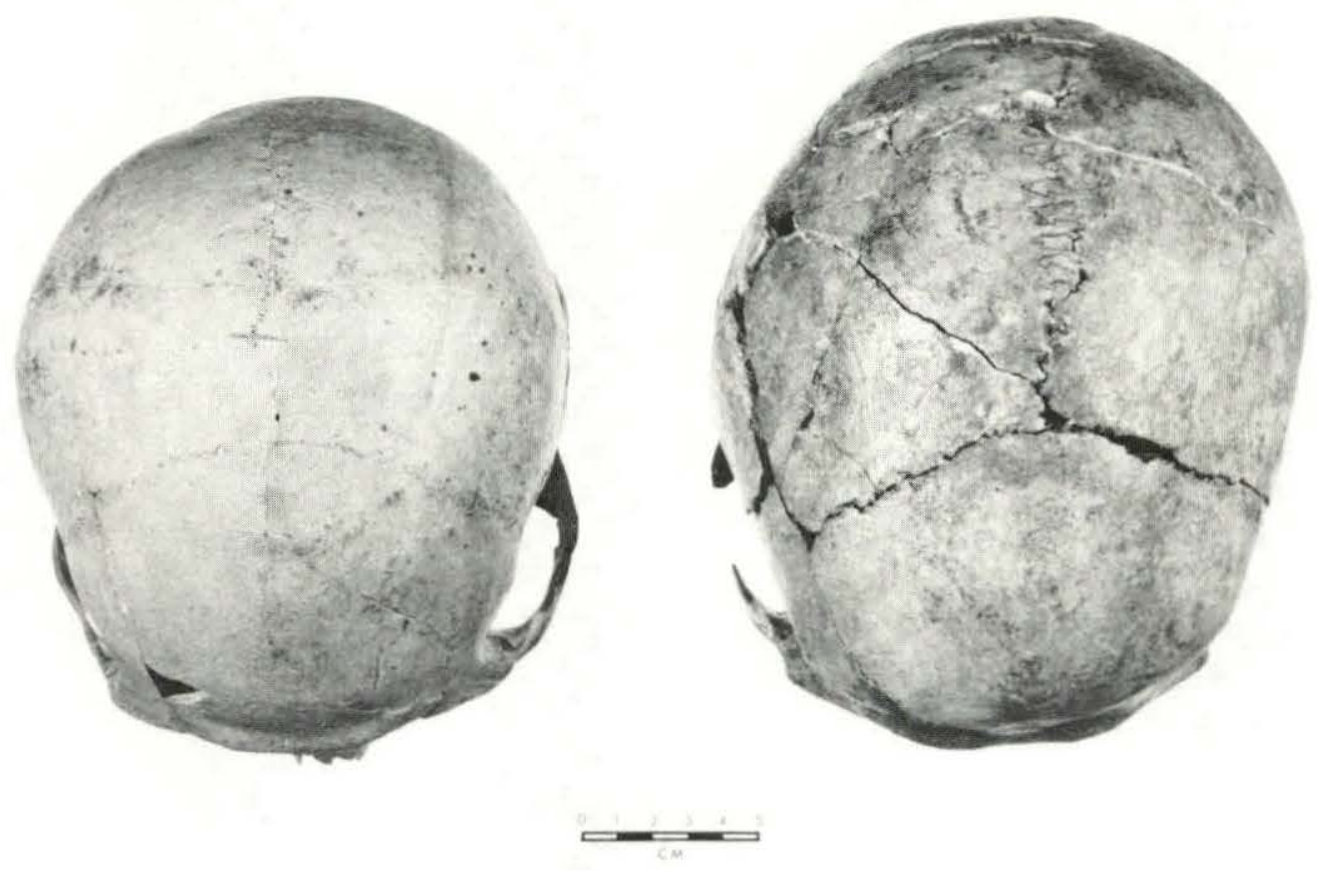

Figure 5.11

Top view of crania from Wall site Burial 1 (left) and Fredricks site Burial 5 (right). 


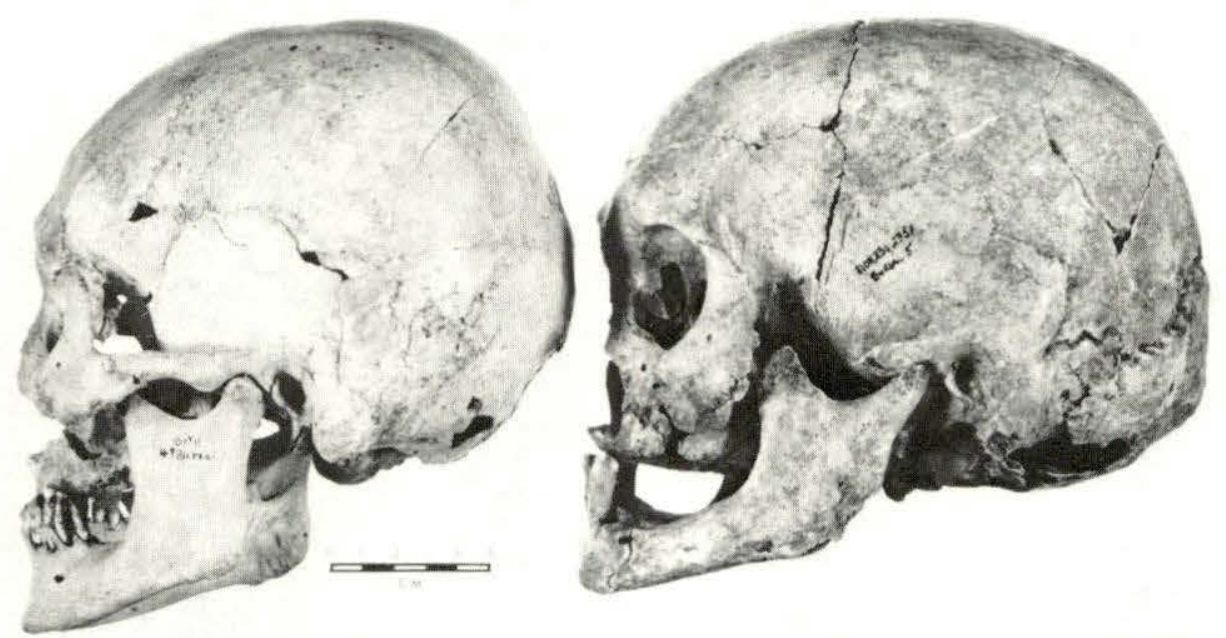

Figure 5.12

Side view of crania from Wall site Burial 1 (left) and Fredricks site Burial 5 (right).

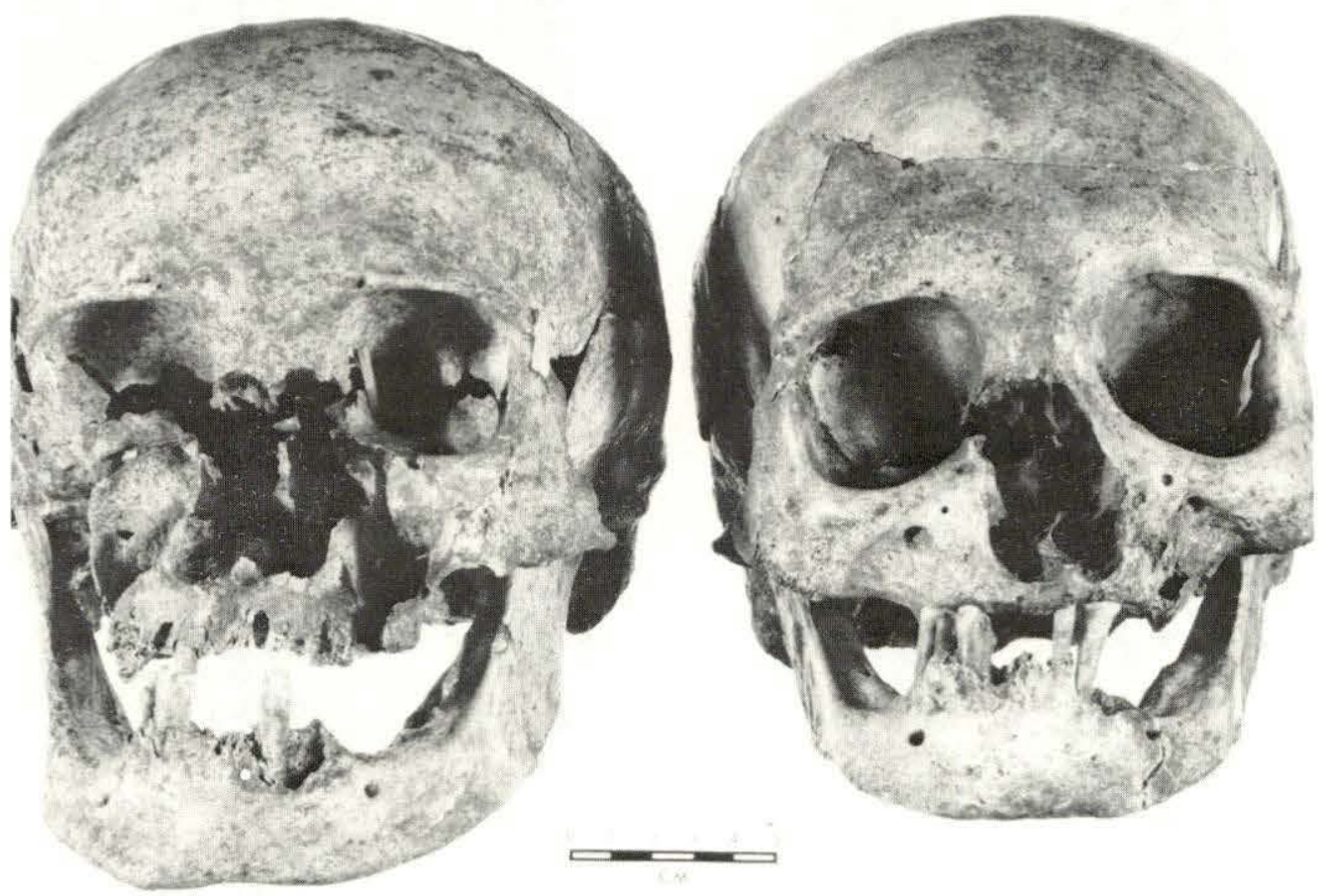

Figure 5.13

Front view of crania from Upper Saratown Burial 73

(left) and Fredricks site Burial 5 (right). 


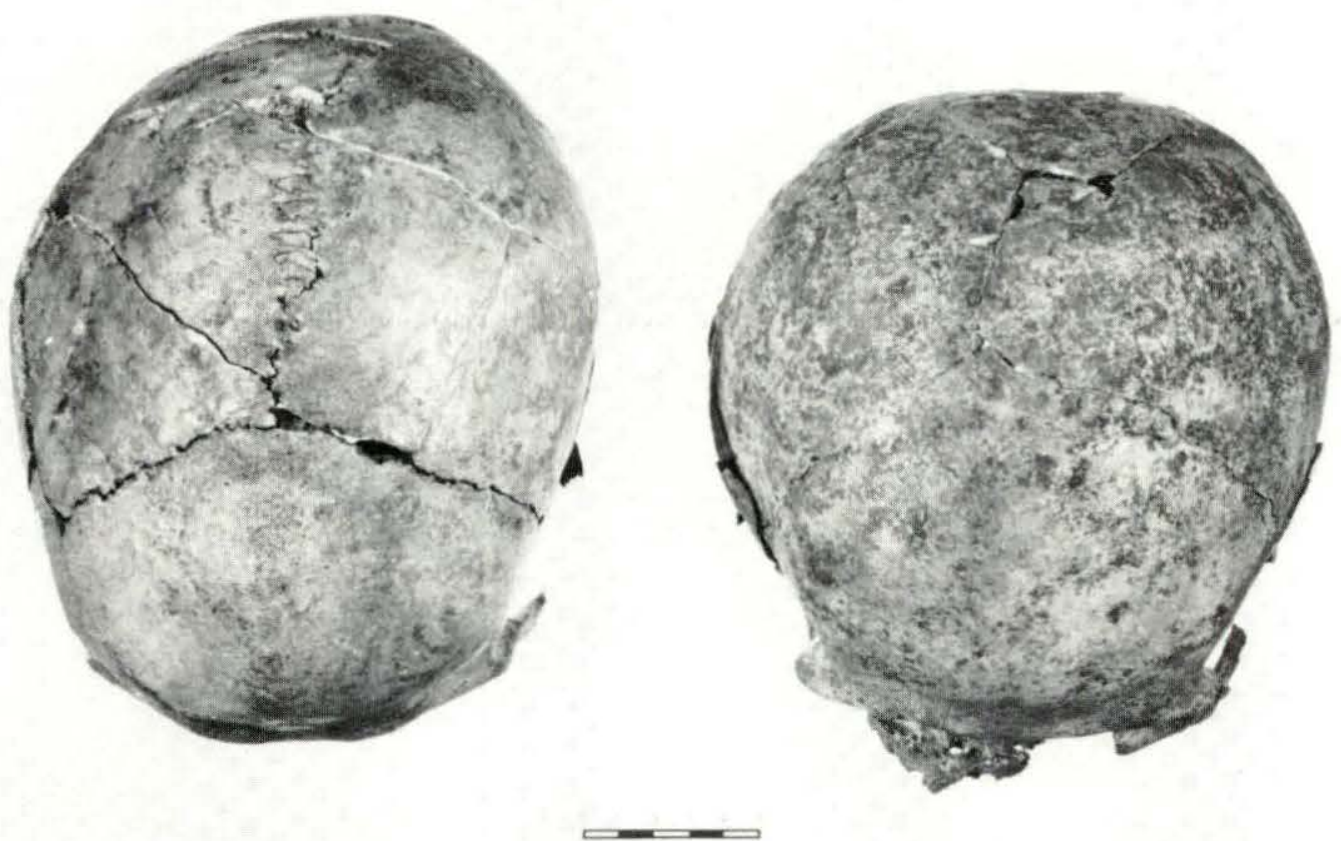

Figure 5.14

Top view of crania from Fredricks site Burial 5 (left) and Upper Saratown Burial 73 (right).

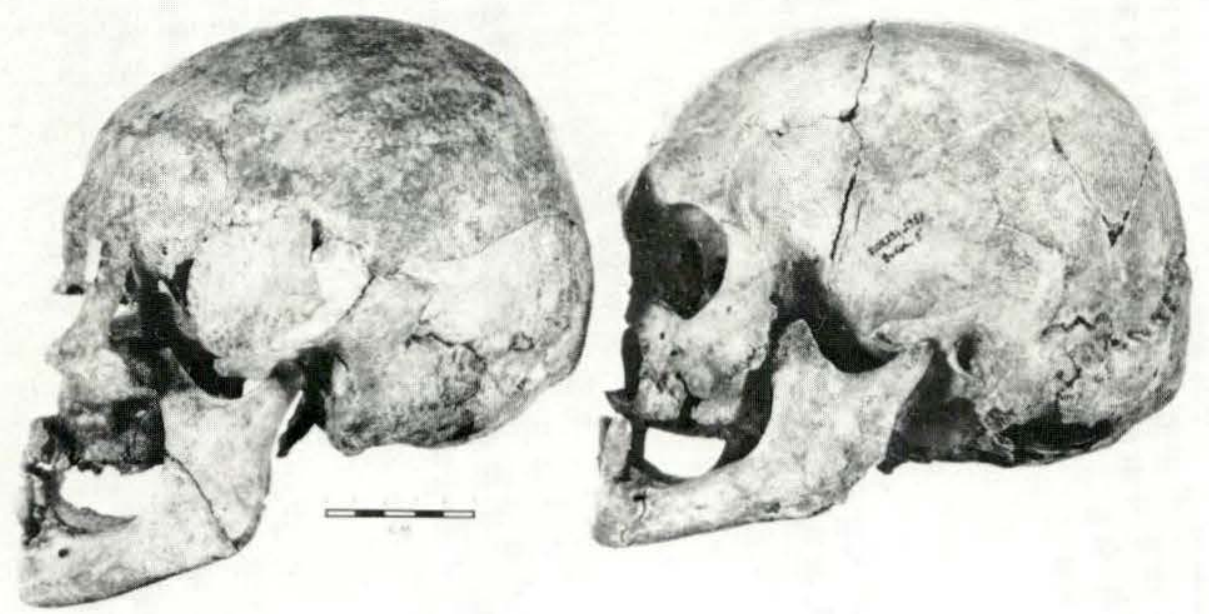

Figure 5.15

Side view of crania from Upper Saratown Burial 73 (left) and Fredricks site Burial 5 (right). 


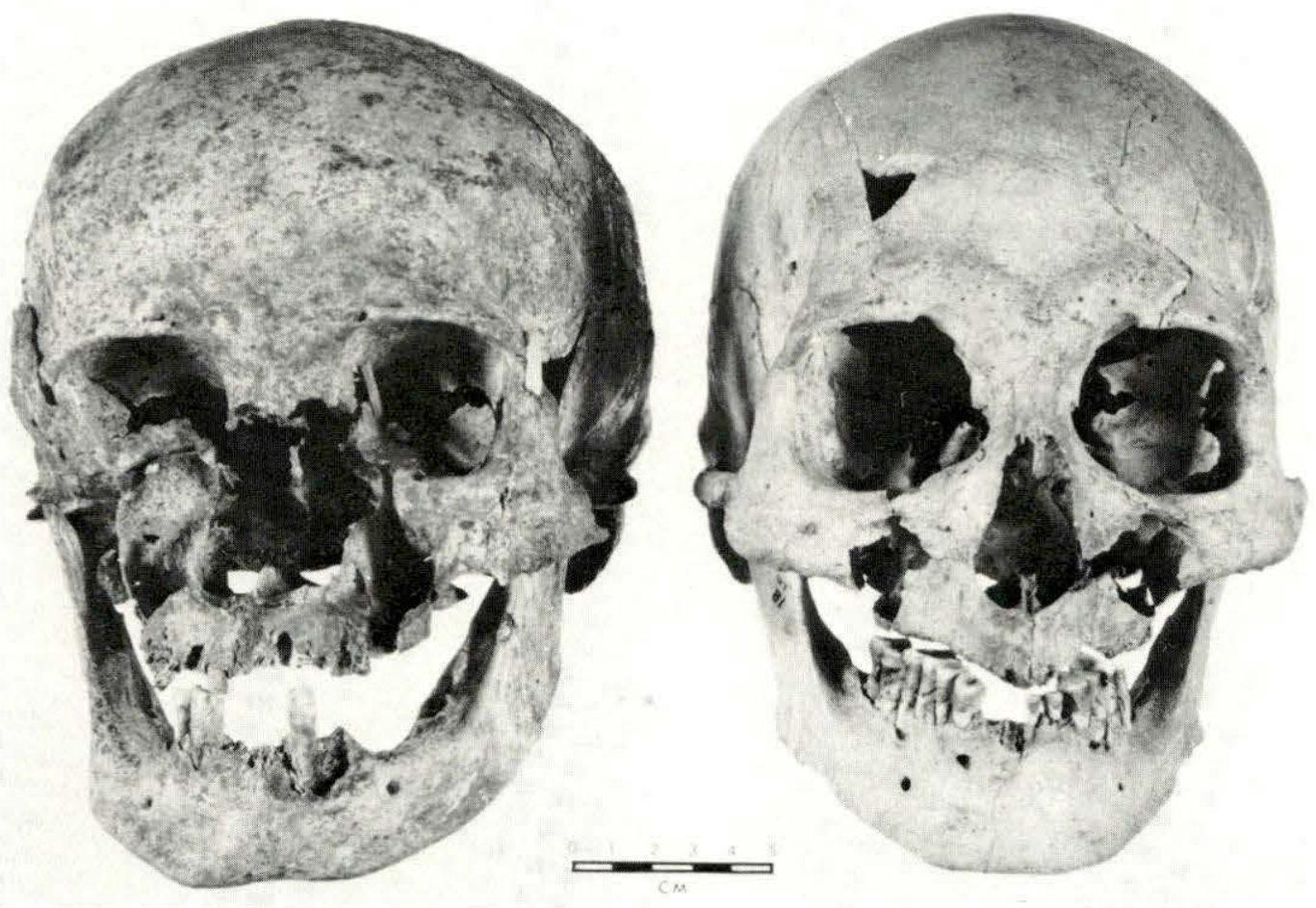

Figure 5.16

Front view of crania from Upper Saratown Burial 73 (left) and Wall site Burial 1 (right).
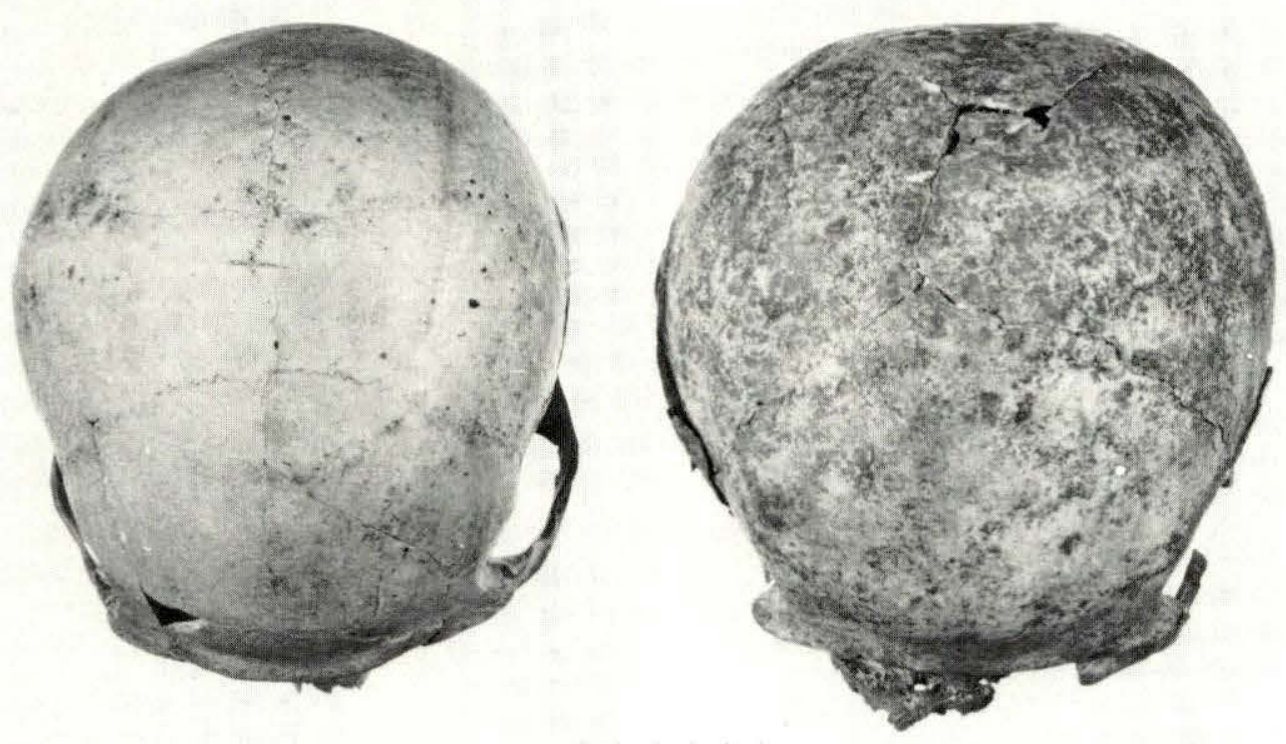

Figure 5.17

Top view of crania from Wall site Burial 1 (left) and Upper Saratown Burial 73 (right). 


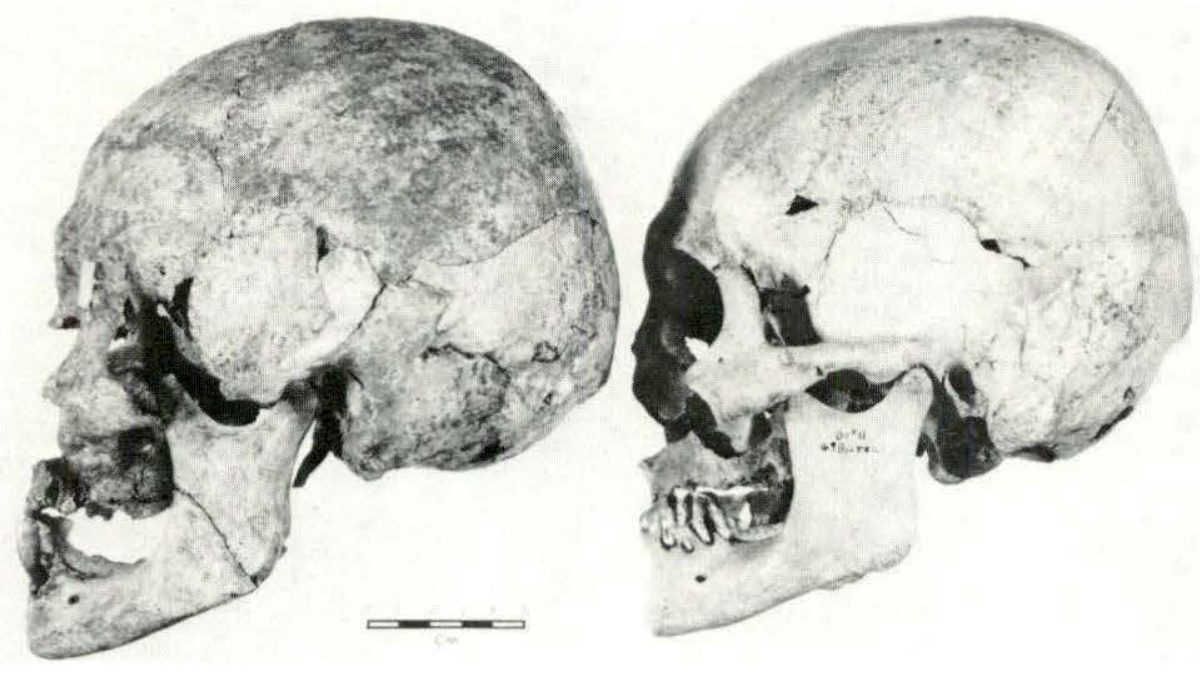

Figure 5.18

Side view of crania from Upper Saratown Burial 73 (left) and Wall site Burial 1 (right).

Siouan Occaneechi with Algonquian-speaking and Iroquoian-speaking groups of the Coastal plain and the Middle Atlantic Piedmont during the Historic period. There is also the possibility that the Occaneechis, or the Indians residing at the Fredricks site, were not Siouan.

As with the other sections of this study, it has to be borne in mind that patterns suggested here must be treated as non-conclusive given the very small size of the skeletal samples from the three sites under consideration. The patterns discussed in this section will be supported, modified, and/or discarded as larger samples are made available for study by future research.

\section{CONCLUSIONS}

This study of the human skeletal remains from the Wall and Fredricks sites has utilized demographic profiles, pathologies, trace element assays, and biological data. Information from two other sites, Upper Saratown in North Carolina and the Shannon site in Virginia, has been used for comparative purposes. The samples have also been examined for adaptive trends using the model of $\mathrm{r}$ and $\mathrm{K}$ reproductive strategies and within-site and between-site diversity.

The most important considerations for each of the four areas of inquiry can be summarized as follows. At the Wall site, there is a high frequency of deaths in the first ten years, followed by a decline through childhood and into adolescence. A pattern of high infant mortality and low childhood-adolescent mortality appears to be present in the Fredricks site population. The increase in the average age of death for young subadults from 1.26 years at the Wall site to 2.63 years at the Fredricks site may indicate a change in reproductive strategy from the Late Prehistoric/Protohistoric period to the Historic period. In the former, a more r-oriented reproductive strategy was marked by low levels of stress and competition and an early weaning of offspring. A $\mathrm{K}$ reproductive strategy, where more energy was put into competition and maintenance and the production of better survival abilities, which may have been marked by extended nursing of the young, seems to be characteristic of the Historic period. The propositions concerning $\mathrm{r}$ and $\mathrm{K}$ reproductive strategies will be more fully developed in future work now planned for the burial material from the Shannon site and Upper Saratown.

Also, the demographic data suggest that older individuals may have comprised a larger percentage of the population at the protohistoric Wall site than at the Fredricks site. Related to this is the fact that more individuals aged 20 to 40 years died at the Historic sites. This is probably due to the increased stress and competition, including increased warfare and hunting, and the presence of European-introduced diseases, among the Indian groups of the Historic period.

A total of six general categories of pathologies were considered in this analysis: traumatic or violent pathologies, degenerative pathologies, tumors, general stress-indicating pathologies, indicators of dietary stress, and general osteitic pathologies. It was hypothesized that the occurrence of traumatic or violent pathologies, including fractures, cuts, and piercing wounds, would increase in the Historic period population at the Fredricks site when compared with the protohistoric population at the Wall site. This was supported by the fact that only $3.8 \%$ of the Wall site pathologies, which afflicted $12.5 \%$ of the population, were categorized as violent. Traumatic pathol- 
ogies increased to $5.7 \%$ among $15.4 \%$ of the population at the Fredricks site. Again, the increased stress and competition that marked the Historic period provides a reasonable explanation for this increase.

For the mechanical and general stress pathologies, it was postulated that these would afflict a greater percentage of the adult population between the age of 20 and 40 at the Fredricks site than at the Wall site. Most of the occurrences of Schmorl's nodes, caused by the collapse of the centrum of the vertebra, at the Wall site would be in the population aged over 40 years, although the old adult population segment at the Fredricks site would also possess this pathology related to stress. As postulated, Schmor1's nodes occur only in individuals aged over 45 years at the Wall site. And, most of the occurrence of mechanical stress pathologies at the Fredricks site are among individuals aged 25 to 40, with the one individual aged $50+$ also afflicted.

For dietary pathologies--cribia orbitalia, spongy hyperostosis, dental caries, and periodontal disease--it was thought that nutritional disease would be less common in late prehistoric and protohistoric populations given the general sociocultural stability present, and the presence of low levels of stress and competition when compared with the Historic period. This proposition was supported by an increase from the protohistoric Wall site to the Fredricks site in the occurrence and percentage of population affected for the four diseases.

For the final pathology, osteitis, it was hypothesized that a general increase in the incidence of this disease would be noted in the Historic period. As expected, more cases of osteitis were observed in the Fredricks site sample than in the Wall site sample. Factors that favored such an increase included the presence of European diseases, increased nutritional stress, and the overall increase in physical stress and competition that marked the period.

One of the more informative analyses conducted for this study involved the assay, by burial, of the trace elements strontium, magnesium, vanadium, zinc, and copper. This investigation is, as with the use of $r$ and $K$ reproductive strategies, still at a very preliminary stage. The results of the assays conducted on the small skeletal samples from the Wall and Fredricks sites do, however, indicate promising directions for future research. The differences noted within and between the levels of trace elements at the Wall site and Fredricks site suggest that there was a change in diet from the Protohistoric period to the Historic period, with animal protein and possibly high protein nuts (such as hickory) being more important in the Historic period. Also, differences in the levels of strontium at the Fredricks site indicate possible migration into the area from the coastal region. The possibility of identifying individuals from other Indian groups of the Middle Atlantic Piedmont and Coastal Plain is another research potential for trace element analysis.

This last question also served as the major focus of the biological distance/diversity investigation. Besides comparing the general morphological characteristics of the two populations, the question of the ethnic-linguistic affiliation of the Fredricks site population was considered. Analysis of the skeletal remains was conducted utilizing the same non-metric traits used by Buikstra (1976). Variability in the occurrence of these non-metric traits within the sample was investigated using Lieberson's (1969) diversity measure. The historic Fredricks sample displays the most within-site variability when compared with the Wall and Upper Saratown sites. This may indicate that this population experienced greater admixture during the Historic period. A similar pattern of increased interaction, however, is not supported by the historic Upper Saratown sample where a low diversity index for non-metric traits was calculated. A greater amount of diversity is seen in the results of the metrical analysis of Upper Saratown. The metrical studies also suggest that Fredricks and Upper Saratown are the most similar of the three sites. Possible explanations for this similarity are that the Fredricks and Upper Saratown populations represent different ethniclinguistic groups who interacted with similar populations during the Historic period, or that they represent two genetically similar populations. Lastly, the morphological traits indicate that either the Occaneechi population at the Fredricks site interacted and intermarried with Algonquian- and Iroquoian-speaking groups, or that the Occaneechi were not Siouan people. 


\title{
Euroamerican Artifacts from the Fredricks, Wall, and Mitchum Sites
}

\author{
by
}

Linda F. Carnes

\section{INTRODUCTION}

\begin{abstract}
In this section, 10,182 artifacts of European or Euroamerican origin from the Fredricks (310r231), Wall (310r11), and Mitchum (31Ch452) sites are described, dated (where possible), and organized typologically and functionally. The descriptive analysis is followed by a discussion of the distributions of these artifacts at each site and an interpretation of the trade assemblage at the Fredricks site.
\end{abstract}

South's (1977:95-96) functional scheme is employed to provide an organizational framework for comparative purposes and is not intended to suggest that the objects in question functioned in the aboriginal context in the same way as they were intended to function in the European context. It was necessary to modify South's original scheme in order to incorporate categories for glass trade beads, construction materials, metal resources, and some ethnobiological items such as carved wood and leather. Modifications of South's format have been used to study successfully other aboriginal-European Contact assemblages from sites in Tennessee (Carnes 1983; Ford 1979; Newman 1977; and Polhemus 1984).

\section{METHODS}

A11 artifacts of European and Euroamerican origins recovered from the 1983 and the 1984 excavations at Fredricks and Wall sites, and the 1983 excavations at the Mitchum site were analyzed. In addition, aboriginal pipes believed to date to the Historic period from the Fredricks and Mitchum sites were also examined. Four separate coding formats were created to facilitate computerized data manipulation: 1) aboriginal and historic pipes, 2) historic ceramics, 3) glass beads, and 4) a general format for all other historic items (e.g., metal, glass, non-vessel ceramics, and cloth). This analysis included measurements, functional classification, quantification by type, and dating (where possible). Context categories included: plowzone from 29 excavation units at the Wall site; plowzone from eight excavation units, one burial, 14 features, and a few postholes from the Mitchum site; and surface collections, plowzone from 35 excavated units, six features, nine burials, and several postholes from the Fredricks site.

Of the 255 historic artifacts from the Wall site, all but three were from the plowzone; the other three were from the top of the midden and probably were intrusive from the plowzone. of the 1,990 European artifacts from the Mitchum site, $1,960 \quad(98 \%)$ were glass trade beads recovered from six features and one burial. From the Fredricks site there were 7,937 Euroamerican items, of which 6,632 (84\%) were glass trade beads, the majority of those from Burial 3 (5,686 total). Most of the Euroamerican artifacts or artifact fragments were identifiable. Only a small number of items (i.e., ceramics, some beads, gun parts, pipe stems, whole bottles, and spoons), however, could be accurately dated; others could only be assigned a broad temporal range of manufacture and/or utilization (i.e., kettle, knives, scissors, iron nails, and iron implements).

To follow is a description of each artifact by type, group, and class. These descriptions are by: 1) functional group (i.e., Architecture, Arms, Clothing, Food Preparation/Consumption, Personal, Construction Tools, Farm Tools, Miscellaneous Hardware, Metal Resource, Other, and Indeterminate); 2) condition (whole, broken, corroded, cut, drilled, etc.); 3) period of manufacture/use (pre-1730, post-1680, etc.); and 4) archaeological interpretive significance in the overall cultural assemblage for that site. There is also a discussion of the recycled or aboriginally modified artifacts from the Fredricks site.

Table 6.1 is a compilation of Euroamerican trade items and their value in deerskins at the Fredricks and Mitchum sites that appear on trade records of the Contact period. For comparison, a list of trade goods from the Upper Saratown site, located on the upper Dan River, is also included. Perishable items, which appear in great quantities on the trade lists but are not often recovered archaeologically, include cloth items (such as blankets, shirts, hats), leather straps, rum (which was usually sold from a keg), snuff and tobacco, gun powder, and sometimes paints and pigments such as vermillion and red lead. Though the trade good inventories provide 
Table 6.1

Euroamerican trade items from trade lists (with deerskin values) for the Fredricks, Mitchum, and Upper Saratown sites.

\begin{tabular}{|c|c|c|c|c|c|}
\hline Trade List Item & Value in Skins* & Fredricks & Mitchum & Upper & Saratown \\
\hline Gun & $25-35$ & + & - & & + \\
\hline Pistol & $12-20$ & - & - & & - \\
\hline Powder & 1-"as you can" & - & - & & - \\
\hline Bullets & 1 & + & - & & + \\
\hline F1ints & 1 & + & + & & + \\
\hline Steel & 1 & - & - & & - \\
\hline Hatchet & $2-3$ & + & - & & - \\
\hline Cut lass & 8 & - & - & & - \\
\hline Sword & 10 & - & - & & - \\
\hline Knife & 1 & + & - & & - \\
\hline Hanger & $7-10$ & - & - & & - \\
\hline Scissors & 1 & + & - & & - \\
\hline Axe & $4-6$ & + & - & & + \\
\hline Hoe (Narrow) & $2-3$ & + & - & & - \\
\hline Hoe (Broad) & $4-6$ & + & - & & - \\
\hline Kettles (Brass) & "as you can" & + & - & & - \\
\hline Looking Glasses (Mirror) & "as you can" & + & - & & - \\
\hline Pipes & $11 b$ & + & - & & + \\
\hline Rum (Bottles) & $41 \mathrm{bs}$. & + & - & & + \\
\hline Beads & $1 / 31 \mathrm{~b}$ & + & - & & + \\
\hline Sa1t & "as you can" & - & - & & - \\
\hline Vermillion (Red Ocher) & 201 bs., if mixed & + & - & & - \\
\hline Red Lead & 201 bs., if mixed & - & - & & - \\
\hline
\end{tabular}

*after Crane (1928:Appendix B, pp. 332-333).

Note: $(+)=$ Present; $(-)=$ Not Recovered.

an indication of the availability, shipping modes, quantities, and values of some trade items, the Indians' personal choices must be recognized as a critical factor in trade-good selection. Wholesale substitution of European trade items for their native counterparts was probably more conservative among the traditional Southeastern Indians groups than among Northern tribes such as the Susquehannock Indian groups or the Iroquois (Watts 1985:1). James Merre11 (see Watts 1985:4), in a discussion of the influence of trade on the Catawba Indians, cautions that

all of these products were less disruptive to native life, more enjoyable than we might think; in part at least, this was because the Indians did not immediately or abruptly abandon their own goods or skills. In some cases, the Catawba simply placed new merchandise into old, familiar slots.

\section{EUROAMERICAN ARTIFACTS BY FUNCTIONAL GROUP}

Table 6.2 summarizes the Euroamerican artifacts from the Fredricks, Wall, and Mitchum sites, and relates them to the modified version of South's functional scheme.

\section{Architecture Group}

Items belonging to this functional group were subdivided into two classes, Construction Fasteners which consist of iron nails, tacks, nuts, bolts/spikes, and Construction Materials which consist of brick, glazed brick, flat glass (pane ?), and mortar. Flat glass fragments were distinguished from mirror glass by several criteria: 1) the absence of silver backing, which, according to Polhemus (1984:1189), "is very fugitive and is frequently eliminated during water screening or artifact processing"; 2) thickness of the glass (panes usually are thinner); 3) surface finish of the glass (po1ished or not); 4) edge color (typically smokey gray for mirror and more green to yellow-green for pane); and 5) edge treatment (typically mirrors have ground or smoothed edges).

Bricks also needed careful examination. During preliminary laboratory processing, daub, fired clay (possibly chinking), rocks, and even unglazed or slightly glazed coarse red earthenware sherds were inadvertently classified as "bricks." During analysis, however, care was taken to discriminate real brick fragments from these other items. Attributes for brick identification included: 1) composition of paste (iron and quartz inclusions) for brick as 
Table 6.2

Frequency of historic artifacts by site, context, and functional classiftcation.

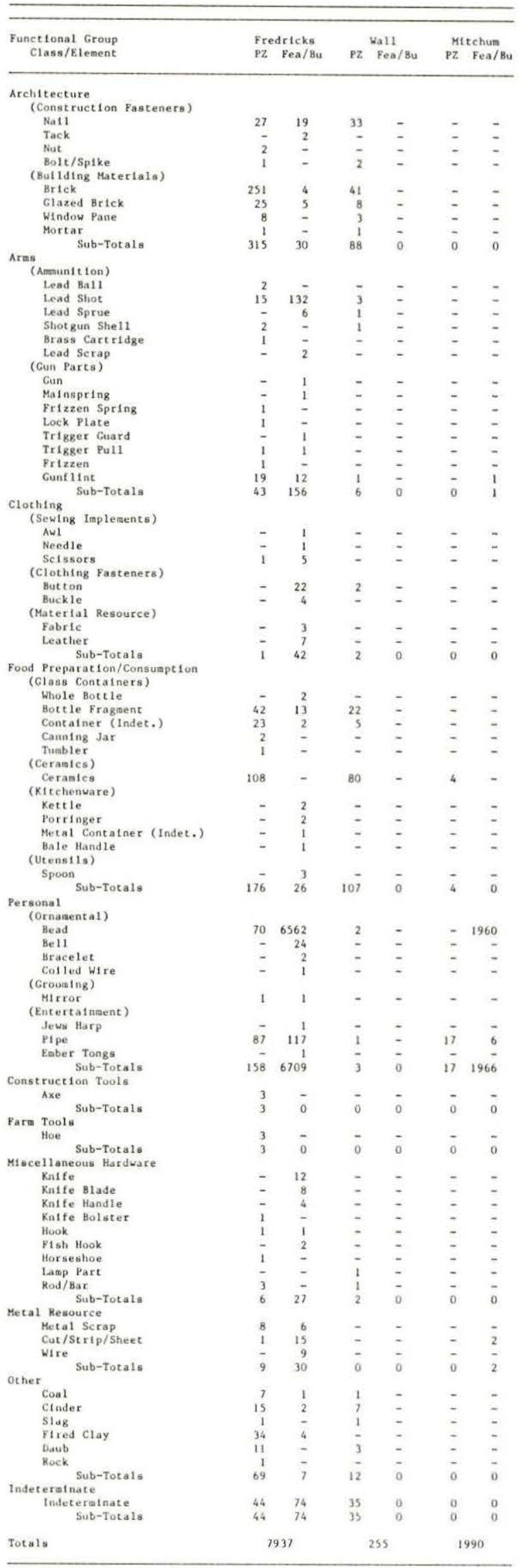

opposed to a typically gritty or sandy paste for daub; 2) edge treatment (most bricks of this period were hand-made in a press-mold box and smoothed over the top edge with a board or trowel, which resulted in linear striations and some edge lipping); and 3) weight (typically bricks are slightly heavier per cubic inch than daub or pottery). Glazed brick fragments were readily identifiable with all specimens exhibiting at least one flat surface. The glaze appeared to be a thin salt glaze, which is more likely to have resulted from hearth burning than from kiln burning. Historically, salt has been used to clean out built-up residue in a chimney, with the result being a thin salt-glaze deposit on the interior bricks of the hearth and chimney.

A11 nails, tacks, bolts, and nuts were made of iron, and most were extremely corroded which restricted identification by type and manufacture (wrought cut, machine-made, etc.). Despite this fact, all nails recovered from undisturbed contexts (i.e., burials and features at the Fredricks site) were wrought and possessed " $T$ ", "L", or "Rose" heads. Each type of nail was manufactured to serve a particular purpose. Rose-head nails, for example, were employed as an all-purpose nail; L-head nails were used to fasten trim and flooring; and T-head nails, usually also used for flooring, had a flattened disc head hammered over opposite sides of the shaft (Noel Hume 1969:252). Wrought nails date from the beginning of European contact to as late as 1790, when cut nails became popular. The presence of wrought nails in undisturbed contexts at the Fredricks site suggests that they were available as a trade good by at least 1700. Another explanation for the presence of these nails would be that they functioned as primary fasteners on wooden crates or boxes used to ship trade goods. It is unlikely that the Indians used these nails as construction fasteners; rather, they probably used them as punches for leather working, or as engraving tools. Later varieties of nails (machine-cut and wire nails) were recovered from plowzone contexts at both the Fredricks and Wall sites. Spatial distribution of the architectural items recovered from plowzone will be discussed in the second part of this section.

In summary, there was a total of 433 items in this functional category--345 (80\%) from the Fredricks site, and $88(20 \%)$ from the plowzone at the Wall site.

\section{Arms Group}

Ammunition, gun parts, and gunflints comprised the Arms artifact group. Trade inventories and ethnohistorical accounts suggest that trade guns were an important and valuable trade item (France 1985). Guns were used for defense, as status symbols, and to increase hunting efficiency. They were probably the Euroamerican trade item most prized by the Indians. 
One hundred fifty-six pieces of ammunition, nine pieces of lead sprue, 33 gunflints, one nearly complete gun, and seven other gun parts compose the Arms artifact group. Lead ammunition was divided into three size categories: 1) balls ( $15 \mathrm{~mm}$ in diameter or .59 caliber) (Figure $6.1: \mathrm{a})$; 2) buck shot (7-9 $\mathrm{mm}$ in diameter or .30 caliber) (Figure 6.1:b); and 3) swan shot (4-6 $\mathrm{mm}$ diameter or .20 caliber) (Figure 6.1:c). Three brass shotgun shell cartridges were recovered from plowzone context (two from Fredricks and one from Wall) and were identified as 12 gauge Winchester shells. One small .22 caliber, rimfire, brass cartridge was also found in plowzone at the Fredricks site. All 132 pieces of lead ammunition retrieved from burial or feature context at the Fredricks site were of the buck shot variety, averaging $7.5 \mathrm{~mm}$ in diameter. Most of these exhibited stem remnants typical of shot manufactured in a gang mold (Figure $6.1: \mathrm{b}$ ). Also, most of the recovered shot were unfired or undeformed, with prominent equatorial mold seams. Lead shot this size would be packed into the gun barrel and fired as multiple projectiles. It was confirmed by an expert on colonial weaponry (John Bivins, personal communication) that this size shot would be suitable for hunting turkey, other fowl, deer, and rabbit.

Nine pieces of lead sprue or cut-lead scrap were recovered: eight pieces from burial/feature contexts at the Fredricks site, and one piece from the plowzone at the Wall site. The sprue pieces provide some evidence for on-site ammunition casting. The cut-lead pieces may represent sprue, or trimming from hand-carved lead pipe stems, such as the one recovered from Burial 6 at the Fredricks site.

Thirty-three gunflints were also part of this artifact group. A sample of these flints is illustrated in Figure 6.2. Of the total, 31 came from the Fredricks site (19 from plowzone and 12 from burial/feature contexts), one from the plowzone at the Wall site, and one from a posthole at the Mitchum site. Seven aboriginally manufactured gunflints were also found at the Fredricks site. Morphologically, aboriginal gunflints are square to rectangular in shape, thin in cross section, and bifacially worked on al1 four edges (Hamilton 1960:73) (Figure 6.2:a). Tippitt (personal communication) has identified the lithic resource for these gunflints as local (i.e., Orange County). Average measurements for the aboriginal flints were 20.9 $\mathrm{mm}$ long, $21.9 \mathrm{~mm}$ wide, and $6.8 \mathrm{~mm}$ thick. Twenty-one of the European gunflints could be measured by length, width, and thickness. All were of the spall-shaped variety (Figure 6.2:b). The average length was $18.6 \mathrm{~mm}$, average width was $21.1 \mathrm{~mm}$, and average thickness was $7.1 \mathrm{~mm}$. Many of these exhibited "exhausted" edges. Three "chip-shaped" gunflints were also recovered from the Fredricks site (Figure 6.2:c). Kent (1984:248) speculates that these chip-style chunks of chert may have been the first type of European gunflint to arrive with the earliest

trade guns. He (Kent 1984:250) has recovered both spall and chip-type gunflints from early Susquehannock Indian sites dating to the late 1600 s. The single gunflint recovered from plowzone at the Wall site (Sq. 350R640) was also identified as an aboriginal gunflint of local chert (17 mm long, $28 \mathrm{~mm}$ wide, and $8.1 \mathrm{~mm}$ thick). A single gunflint of honey-colored chert was recovered from a posthole ( $\mathrm{Sq}$. 300R650) at the Mitchum site; it measured $16 \mathrm{~mm}$ long, $20.5 \mathrm{~mm}$ wide, and $8 \mathrm{~mm}$ thick.

Eight gun parts, including one nearly whole dog-lock, long-fowler musket, were found at the Fredricks site. All gun parts appear to be iron. Dog-locks were a transitional design between the snaphaunce and flint-lock. A "dog"

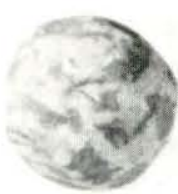

a

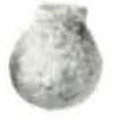

b
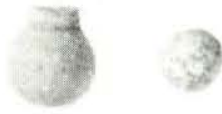

C

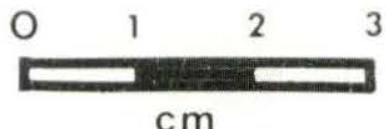

$\mathrm{cm}$

Figure 6.1

Sample of spherical lead ammunition from the Fredricks site.

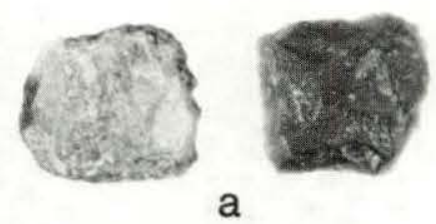

a

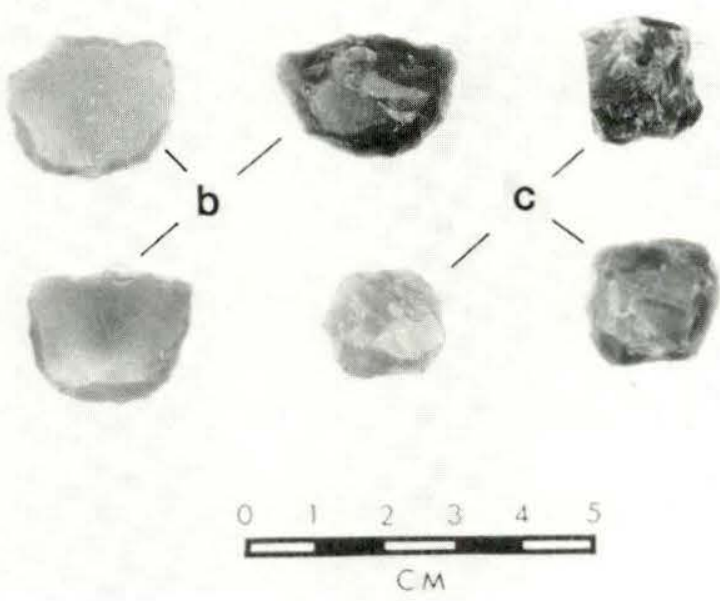

Figure 6.2

Gunflints from the Fredricks site: aboriginal

(a); European spa11 type (b); and European "chip" style (c). 
or safety catch was engaged to hold the heel of the haunce in a half-cock position (Peterson 1956:23). Long-fowler refers to an early style of hunting weapon with a very long, round barrel. The nearly whole gun was found in Burial 6 at the Fredricks site. Observations in the field and subsequently in the laboratory revealed that the butt-end of the gun stock had been broken off prior to its placement in the burial. The lock plate was severely bent and the butt-end of the trigger guard broken off. Also, both ends of the gun abutted the walls of the burial pit leaving no room for a butt stock. Some wood from the barrel stock was preserved and identified as maple (Yarne11, personal communication). The hardware (firing mechanism and barrel) were identified as British and dated to 1625-1640 (John Bivins, personal communication). Other sources (Peterson 1956:31, and Neumann 1967:10) confirm this temporal placement for such an early dog-lock style of gun. Peterson (1956:31) states that this was the most popular trade gun during the 1625 to 1675 period. Dog-1ocks were sometimes used by the British army until after 1700. These later dog-1ocks had vertically attaching sear springs, and often the tumbler had notches for half and full-cock positions. The cock (or hammer) was long and slender in style. Two hammers of nearly identical shape to the Fredricks site specimen were recovered from excavations at Upper Saratown, located on the Dan River in North Carolina and believed to date 1660-1680 (Figure 6.3). Early dog-lock long-fowlers were introduced to North American Indians with the first white settlers in Ralph Lane's Company in 1586 and with the P1ymouth and Jamestown settlements in the early 1600s (Peterson 1956: 42-44). Peterson also suggests that during the last half of the seventeenth century, longfowlers were frequently assembled in America using barrels and locks made in Holland or England and stocks of American curly maple. Although botanical analysis of the wood preserved on the gun barrel from the Fredricks site was identified as maple, a determination of origin (Europe or America) was not possible.

Measurements on the gun from the Fredricks site are as follows: the barrel is $55-3 / 4$ inches (141.6 cm) 1ong; base diameter is calibrated at .55; the lock plate measures 6 inches $(15.2 \mathrm{~cm})$ long; and the hammer (or cock) is $2-3 / 4$ inches $(7.0 \mathrm{~cm})$ high. As shown in Figure 6.4, the hammer is frozen in an engaged position, the frizzen extended, and the pan exposed. A flint clamped in the jaws of the hammer appears to be of the spall type. A small brass "butterfly" rear sight is located on the top rear of the barrel. The mainspring, lateral sear spring, and "dog" are all intact. The trigger pull and a portion of the trigger guard are also present. No manufacturer's marks are visible on the weapon.

A frizzen, frizzen spring, lock plate, and trigger pull, all of which appear to be from a dog-lock musket were recovered from the plowzone at the Fredricks site. One other gun part, a mainspring fragment, was found in a "bundle" associated with Burial 3. The context of this item suggests that it was curated by its owner. No aboriginal modification of this gun part was observed. No gun parts were recovered from the Wa11 or Mitchum sites.

In summary, this functional artifact group comprised $25 \%$ of the total artifacts from the Fredricks site, $2.3 \%$ of the total from the Wall site, and $.05 \%$ of the total from the Mitchum site.

\section{Clothing Group}

This artifact group is divided into three classes: 1) Clothing Fasteners, 2) Sewing Implements for the construction and repair of clothing articles, and 3) Material Resources used for clothing construction. Artifacts related to this functional group appear frequently on trade lists and were popular commodities among the Indians throughout the Historic period. France (1985) has also discussed the prevalence on trade lists of yard goods and ready-made clothing, which sometimes comprised nearly half of the total inventories. Since textiles are not often preserved archaeologically, their importance as trade items can easily be underestimated.

Clothing Fasteners consisted of four brass buckles and 24 buttons, a sample of which is illustrated in Figure 6.5. Two of the buttons are from the plowzone at the Wall site; the remaining 22 are from Burial 1 (19) and Burial 2 (3) at the Fredricks site. The two buttons from the Wall site were identified and dated by reference to South's $(1964: 115)$ button classification scheme. One was South's Type 32, which dates 1837-1865 (Mean = 1851); the other was identified as South's Type 18, which dates 1815-1830 (Mean $=1822.5)$. Combining the mean dates for these two items, a date of 1836 is obtained for the plowzone context at the Wall site. The buttons recovered from Burial 1 consisted of 12 black glass buttons with iron wire eyelets (some missing) (Figure 6.5:b), and seven solid cast pewter buttons (Figure 6.5:a). The black glass buttons (not typed by South) measure 11.6 to $14 \mathrm{~mm}$ in diameter. Ten of the 12 black glass buttons were found in the neck area of Burial 1 which suggests three possible functions: 1) strung on a necklace like beads, 2) sewn onto a garment for decoration, or 3) used as fasteners on a European trade garment. The other two black glass buttons were found in the fill of Burial 1. The seven solid cast pewter buttons, also from Burial 1, measure 12 $\mathrm{mm}$ in diameter and have the dome and eyelet cast as one piece. They were found in association with a "bundle" of artifacts interred with Burial 1, and were possibly used as ornamentation sewn to the outside of this bundle (see Ward, this report). All of these buttons appear to have a tri-part floral motif on the "face" or dome of the button. A similar decorative motif 

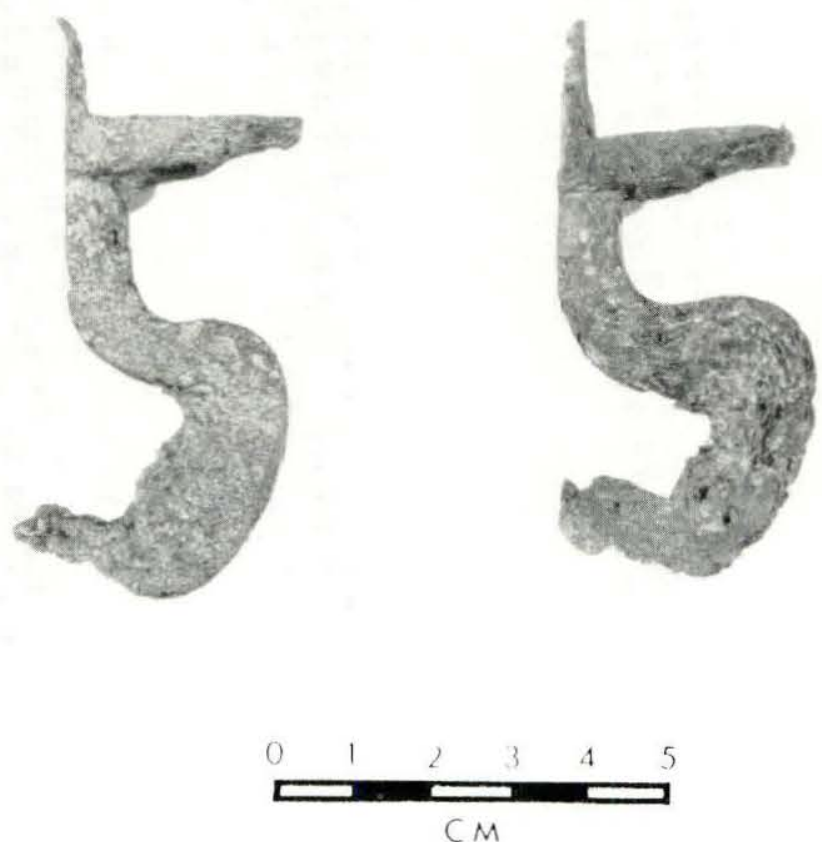

Figure 6.3

Dog-lock hammers from Upper Saratown that are similar in style to the gun from Burial 6 at the Fredricks site.
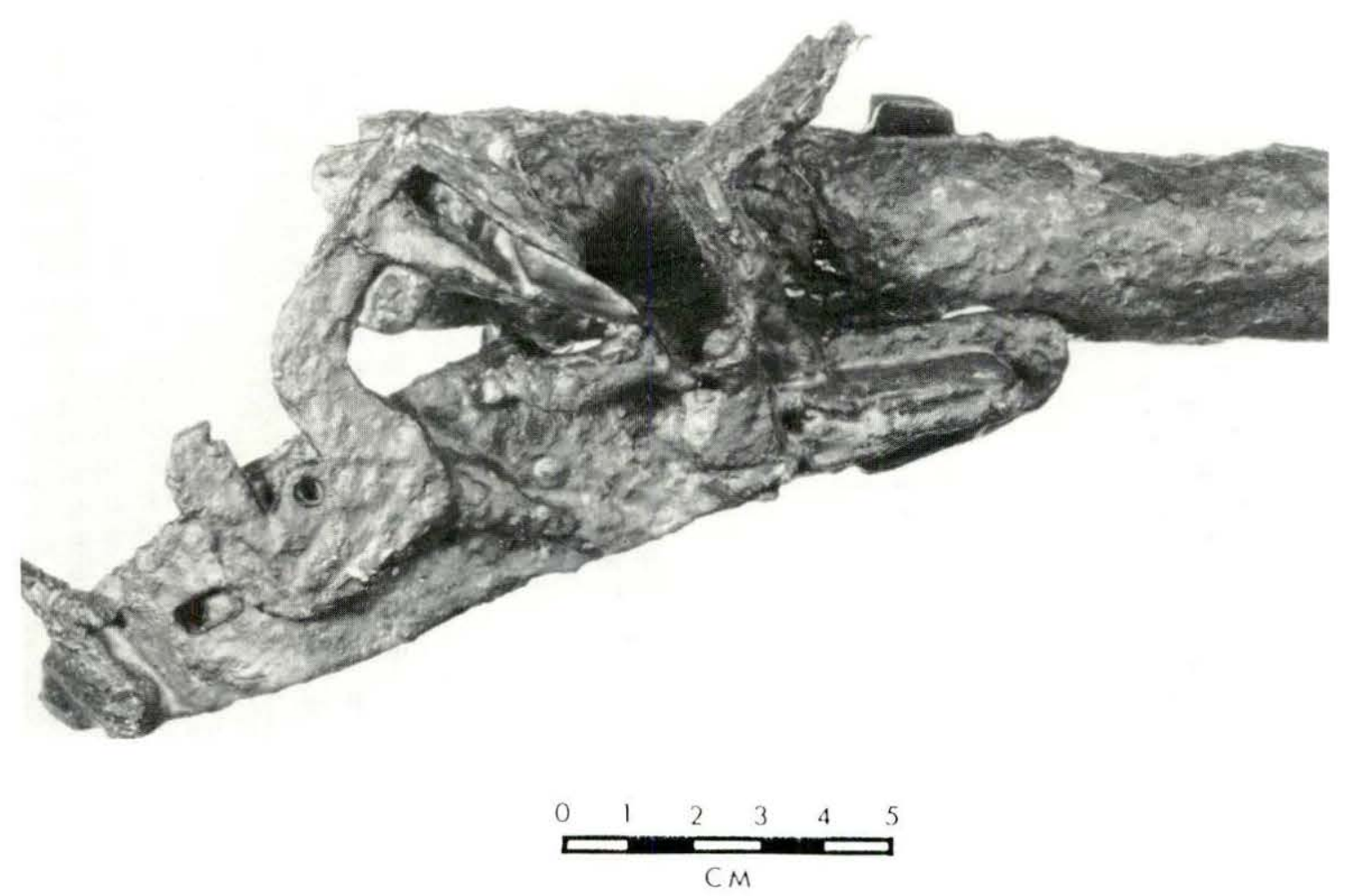

Figure 6.4

Close-up of dog-lock firing mechanism from Burial 6 at the Fredricks site (Note gunflint in situ in jaws of hammer). 


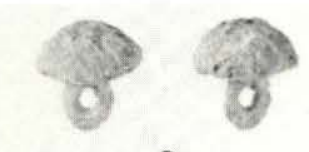

a

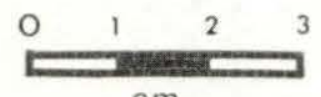

$\mathrm{cm}$

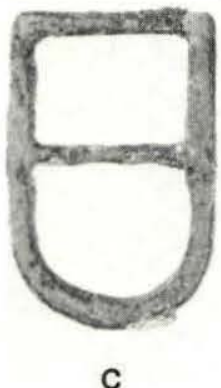

C

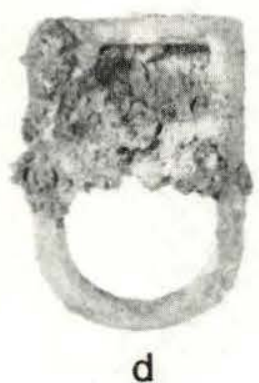

Figure 6.5 b
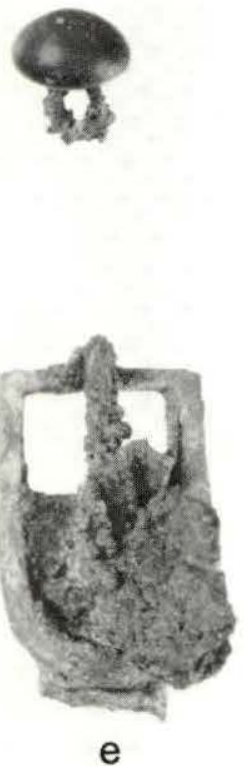

Sample of clothing fasteners from the Fredricks site. has been found on slightly larger buttons (14 to $16 \mathrm{~mm}$ diameter) from the Fort Michilimackinac site and have been dated by Stone to the early 1700s (1974:46-48). Cast pewter buttons, most of which date to the 1720 s, have also been recovered at many Overhill Cherokee sites in Tennessee (Carnes 1983:192).

Three hollow-cast pewter buttons were found in Burial 2. Noel Hume (1982:89) suggests that hollow-cast buttons, of either white metal (pewter) or brass, were common during the early 18th century. A floral motif is slightly visible on these three specimens, which are more eroded than the solid cast pewter buttons previously described. These buttons were also associated with a "bundle" of other artifacts and may have been, along with numerous glass beads, used to decorate a bag.

Four brass buckles ( 3 complete and 1 partial) comprise the other clothing fasteners. All of these buckles were recovered from the Fredricks site and all are of standard brass construction, have a D-shaped frame, and iron tangs (missing on two specimens). Three buckles are illustrated in Figure 6.5, the two on the right from Burial 8 (Figure $6.5: \mathrm{d}-\mathrm{e}$ ) and the one on the left from Burial 3 and associated with a "bundle" (Figure 6.5:c). The two from Burial 8 have the remnants of leather adhering to the metal, which makes them belt or strap fasteners. One buckle fragment was recovered from the plowzone at the Fredricks site. Brass buckles of a similar style were recovered from Fort Michilimackimac where they were interpreted as being of French or British military issue (Stone 1974:32-34) and given a broad temporal range.

Sewing Implements from the Fredricks site include: one iron awl from Burial 3; one iron needle from Burial 1 ; and six pairs of steel scissors, two pairs from Burial 1, two from Burial 3, one from Burial 6, and a scissor handle fragment from the plowzone. The iron awl from Burial 3 was found in association with a bundle of other artifacts. Following Stone's (1974:155) criteria for identification of awls, this specimen is pointed on one end, has the remains of a wooden handle on the other end, and appears to have a squarish shank when viewed in cross-section. Awls were popular leather working tools and appear on trade lists of the period (France 1985). Often, iron nails were modified to be used for the same purposes.

The iron needle recovered from Burial 1 was missing the end with the "eye" and, therefore, could not be identified as a specific variety (Stone 1974:159). Although needles appear on trade 1 ists, they are not often recovered from archaeological context because of their fragile nature and small size. Needles, probably used for clothing construction and beadwork, were popular among the Overhill Cherokee in the early 1700s (Newman 1977:45).

The five pairs of scissors from burial contexts at the Fredricks site appear to be of the same style, with wide blades, equal-sized oval-shaped eyes, and a central rivet well below the handle branches. As shown in Figure 6.6, the blade tips and eye loops of these specimens were not well preserved. In both Burial 1 and Burial 2, two pairs of scissors were found in association with "bundles." The pair of scissors in Burial 6 did not appear to be in a bundle. The individuals in Burials 1,3 , and 6 all were adult males, which may suggest sex or status indicators for these scissors. Scissors appear frequently on early trade inventories (France 1985). Considering the quantities of yard goods and clothing traded to the Indians, the presence of these scissors as clothing
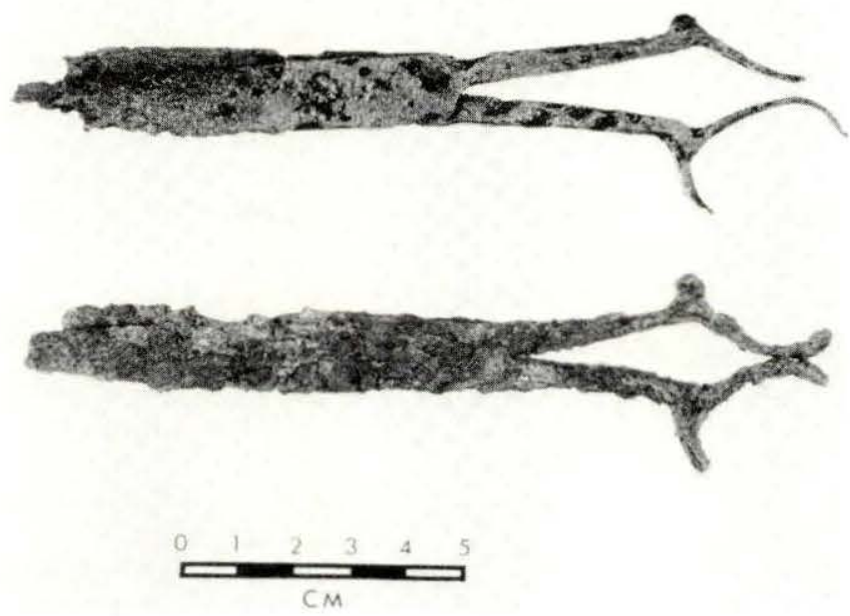

Figure 6.6

Scissors from burials at the Fredricks site (Top pair has been cleaned and treated). 
construction implements is not surprising. Brain (1979:274), in his discussion of acculturation by technological innovation, has placed a high "value" on scissors as tools because they have no native counterpart and represent a "new technique of use."

The Material Resources class of the Clothing group is represented by seven pieces of leather (or hide), three pieces of a coarsewoven fabric, and two leather strap fragments fastened to the brass buckles, all recovered from burial contexts at the Fredricks site. The leather fragments were preserved by being in contact with two copper wire bracelets found on the left arm of Burial 6. The fabric was preserved by being in contact with a pair of scissors in Burial 3. Although the fiber of this fabric could not be positively identified, the weave appears to be a simple one-over and one-under pattern, and the texture resembles a coarse canvas or duck type of cloth. It is suggested that this cloth, found in association with a "bundle" of artifacts, represents the remains of a bag or tote sack, rather than an article of clothing.

In summary, the clothing group artifacts comprise $0.5 \%$ of the Fredricks site assemblage and $0.8 \%$ of the Wall site total.

\section{Food Preparation and Consumption Group}

Items related to food preparation, serving, and storage comprise this artifact group. Classes consist of Glass Containers, Nonaboriginal Ceramics, Metal Containers, and Utensils. Included in the Glass Container class were two whole wine bottles, 77 bottle glass fragments, 30 indeterminate glass container fragments, two canning jar pieces, and one tumbler fragment. A total of 192 non-aboriginal (mostly European) ceramic fragments were analyzed, 108 from plowzone at the Fredricks site, 80 from plowzone at the Wall site, and four from plowzone at the Mitchum site. Two brass kettles (one nearly whole with an iron handle, and one small fragment), two badly decomposed pewter porringers, one indeterminate metal container fragment comprise the Metal Container class. Two latten spoons (an alloy of copper, zinc, and iron), and one broken spoon handle represent the Utensils class in the Food Preparation/Consumption artifact group.

The two dark green English wine bottles from the Fredricks site were found in burial contexts--one in Burial 3 (Figure 6.7) and one in Burial 4 (Figure 6.8). Both bottles were analyzed using Carrillo's (1974) statistical model, Baker's (1974) modification of Carrillo's mode1, Dumbre11's (1938) descriptive information, and Noel Hume's (1974) comparative summary. A series of 32 measurements was recorded for each specimen, including basal ring width, mouth radius, height, width, and height of kickup. A vessel profile of each bottle was constructed to facilitate comparison (Burial 4

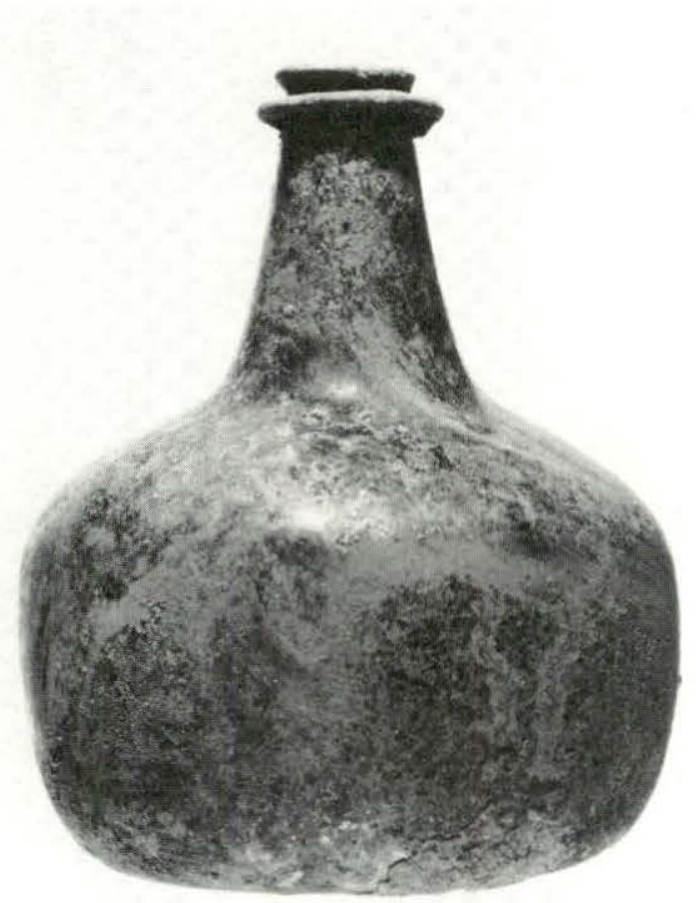

Figure 6.7

Wine (rum) bottle from Burial 3 at the Fredricks site.

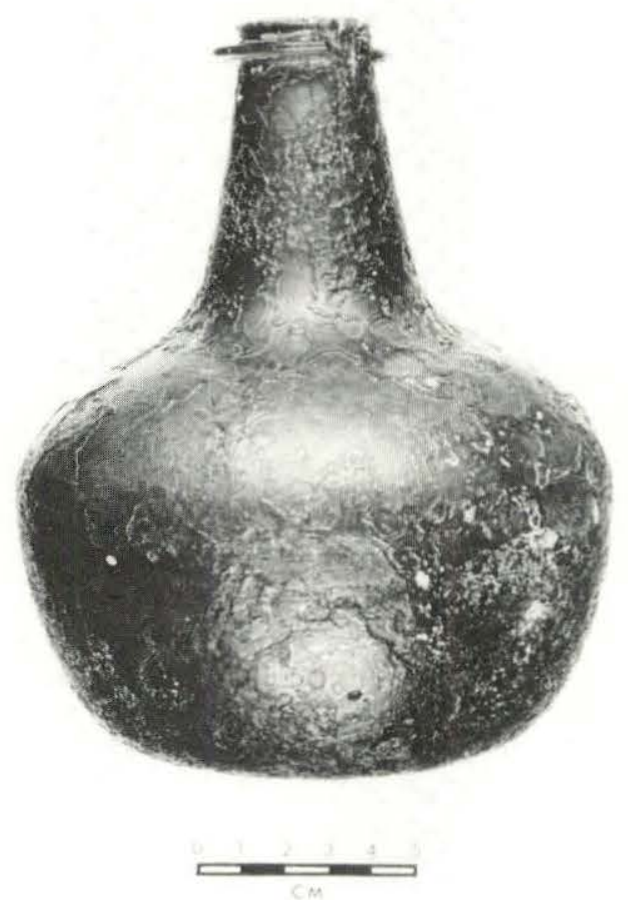

Figure 6.8

Wine (rum) bottle from Burial 4 at the Fredricks site. 
bottle is shown in Figure 6.9 ). In sumnary, the measurements produced a date of 1688-1700 (Mean $=1694$ ) for the Burial 4 bottle and a date of 1700-1704 (Mean $=1702$ ) for the Burial 3 bottle.

The Burial 3 bottle, analyzed originally by Wilson (1984:7), measures $16.5 \mathrm{~cm}$ in height, with a maximum diameter of $43 \mathrm{~cm}$. The shape is short and squat with straight sides, narrow neck, and a broad, slightly domed kickup. An incised mark (possibly the initial "M" or " $W "$ ) is present on the shoulder of this bottle. It could not be determined if this initial was of European or aboriginal origin. Wilson (1984:7) proposes that "this symbol may have been a trader's mark, or the mark of a European who could not afford a proper seal."

Visual comparison of the Burial 4 bottle with examples illustrated by Dumbrell (1983:36) suggested a 1680 date for this specimen. Also, lip and neck profiles were compared to those illustrated by Noel Hume (1974:195), and these provided a date of 1685 . Compared to the Burial 3 bottle, this specimen is slightly shorter (15.8 $\mathrm{cm}$ or $6-1 / 4$ inches) with more rounded sides and a thicker, shorter neck, and an overall width of $13.7 \mathrm{~cm}$ or $53 / 8$ inches. Whole bottles, as containers for rum and other

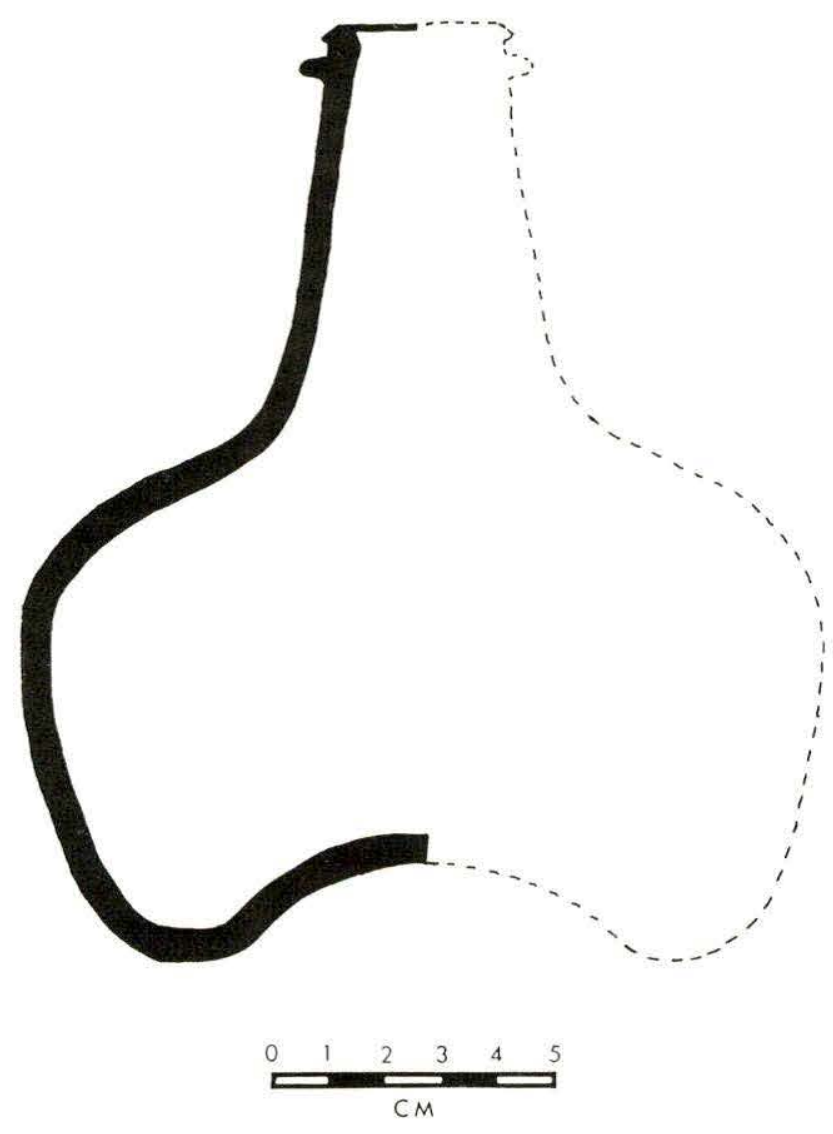

Figure 6.9

Profile of whole wine bottle from Burial 4 at the Fredricks site. liquids, appear on trade lists of the period (France 1985). These bottles probably also served as water containers when rum was not available.

Fifty-five glass bottle fragments were recovered from the Fredricks site ( 42 from the plowzone and 13 from feature/burial contexts), and 22 pieces came from plowzone at the Wa11 site. Bottle glass was determined by the presence of diagnostic attributes, i.e., lip, rim, shoulder, base, kickup, wall thickness, and color. At the Fredricks site, Features 1, 9, 10 and 13 and Burials 3 and 5 contained fragments of dark green bottle glass. In Burial 3, two thick basal fragments were found in a "bundle" association. Whereas these two glass fragments represent curated items, their exact function remains undetermined. Twenty-five glass container fragments from the Fredricks site and five fragments from plowzone at the Wall site could not be attributed to any specific type of bottle. Among the artifacts from the plowzone at the Fredricks site were two canning jar fragments (aqua in color and with threaded rims) and one clear press-molded, hexagonal tumbler. The jar and tumbler date to the mid-nineteenth century.

A total of 192 sherds of non-aboriginal ceramics were analyzed according to South's $(1977: 210,217)$ ceramic typology and mean ceramic dating formula. All non-aboriginal ceramics were recovered from the plowzone; 108 from the Fredricks site, 80 from the Wall site, and 4 from the Mitchum site. Of the 108 sherds from the Fredricks site, only 64 (59\%) could be positively identified and assigned a manufacturing range and median date. The resulting mean ceramic date was 1810.48. From the Wall site, only $56(70 \%)$ of the 80 sherds could be identified and assigned a manufacturing date range, which produced a mean of 1811.87. The four specimens from the Mitchum site were identified but not assigned a mean ceramic date because of the small size of the sample.

One crushed but nearly whole sheet brass kettle was found in Burial 8 at the Fredricks site. The diameter of this piece was $19.5 \mathrm{~cm}$, and its estimated height was 12 to $14 \mathrm{~cm}$. Six fragments of an iron bail handle go with this kettle. The bail "ears" were of sheet brass and were attached to the kettle walls with paired rivets. The rim appears to have been rolled over a circular iron wire. Similar kettles have been found at the Guebert site in Illinois, dating 1670-1730 (Good 1972:166), the Tunica site (Type A, Variety 2) dating 1718 (Brain 1979:173), and the Conestoga site in Pennsy1vania, dating 1676-1680 (Kent 1984:209). One kettle patch, identified by the presence of rivets, was found in the fill of Feature 1 at the Fredricks site. No type could be determined for this fragment; however, it appears to have been aboriginally modified or cut. Sheet brass scraps without diagnostic attributes (i.e., patches, rolled rims, or "ears", etc.) were 
placed in the Metal Resource artifact category and will be described later. For some Southeastern Indians, it has been found that kettles were a primary source of sheet brass from which to make items of personal adornment or metal projectile points (Carnes 1983:199). Kettle parts are more common at early 18th-century sites than late 18th-century sites, which may reflect a decline in the availability of brass kettles and probably some replacement of them by tin containers (Carnes 1983:199; Newman 1977:32; and Polhemus 1984:1206).

Two badly decomposed pewter porringers were also included in the metal container subgroup. Both of these items were recovered from burial contexts at the Fredricks site. The porringer from Burial 2 was analyzed by Wilson in 1984 (Wilson 1984:8), and described as circular in shape with a single pierced handle; measuring 14 $\mathrm{cm}$ in diameter, a depth of $4 \mathrm{~cm}$, and a $3.8 \mathrm{~mm}$ handle. The porringer from Burial 4 appeared to have a tapered scallop-shaped handle and measured $14.5 \mathrm{~cm}$ in diameter. Although both specimens probably are of British origin, no positive identification could be made. Similar porringers have been recovered from the Tunica site (Brain 1979:160), Fort Michilimackinac (Stone 1974:192), and Jamestown (Cotter and Hudson 1957:45), all dating from the early 1600s to early 1700s. Unlike brass kettles, pewter porringers are not often listed on trade inventories; however, they were popular domestic items among the White settlers in the Coastal Plain region according to personal estate records (Brad Rauschenburg, personal communication). For the inhabitants of the Fredricks site, these porringers may have served as liquid or food containers.

Three latten spoons (two whole specimens and one handle) represent the Utensils category of artifacts from the Fredricks site. Latten, which is composed of $73 \%$ copper, $25 \% \mathrm{zinc}$, and $2 \%$ iron, was not made in England until the latter part of the sixteenth century (Raymond 1952:228). The latten spoon recovered from Burial 1 has a round bowl with an unadorned straight handle that is hexagonal in crosssection. There is residue of tin plating on the bowl and a small circular maker's mark on the concave bowl portion of the spoon. The mark appears to consist of a circular cartouche surrounding three spoons (the outer two pointing in the opposite direction from the center one) flanked by two indistinquishable initials. Price (1908:35-37) contends that the three-spoon mark with initials is a common trade mark. Unfortunately, however, no guild for whitesmiths had yet been established in England in the 17th and early 18th century (Merry Outlaw, personal communication). A second complete latten spoon was found in Burial 8. Stylistically, it is different from the one described above, in that it has a seal-top finial on the handle and a fig-shaped (or oval) bow1 (Figure 6.10). It also exhibited traces of tin plating. The touch mark, clearly visible on this specimen, consisted of a circle surrounding three spoons and the initials $\mathrm{T}$ and $\mathrm{S}$ (Figure 6.11). Again, no maker or exact date could be determined for this spoon. The bowl of the spoon had aboriginal modification in the form of incised geometric designs. The interior designs resembled cursive "L"s, arranged in a series around the bowl rim. The exterior patterns (not visible in Figure 6.10) appeared to be a stylized chevron, again arranged in a series around the bowl perimeter. Similar geometric patterns were noted on a shell gorget from Burial 2 at the Fredricks site (see Hammett, this report). Whether this spoon was worn as an ornament or was just a vehicle for artistic expression is problematic. Similar spoons with similar touch marks are reported for Susquehannock sites in Pennsylvania and are believed to date to 1660-1700 (Kent 1984:287-293). Noel Hume (1982:180-181) proposes that tin plating replaced silver plating around 1650 .

Finally, the mid-section of a hexagonallyshaped latten spoon handle was found in the fill of Feature 13 at the Fredricks site. The finial and bowl were missing, and the handle was bent, which suggests aboriginal modification.

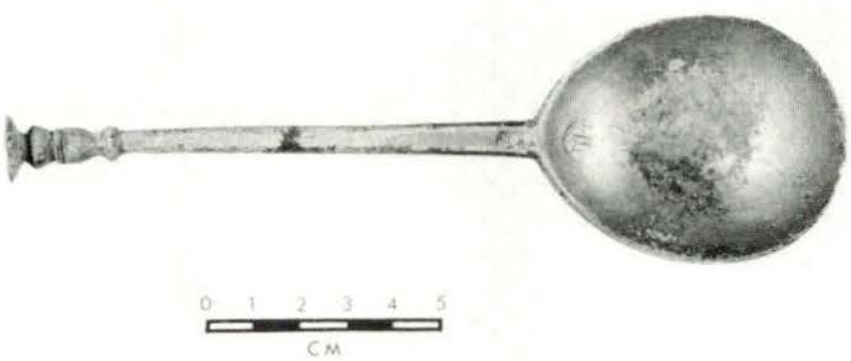

Figure 6.10

Latten spoon from Burial 8 at the Fredricks site.

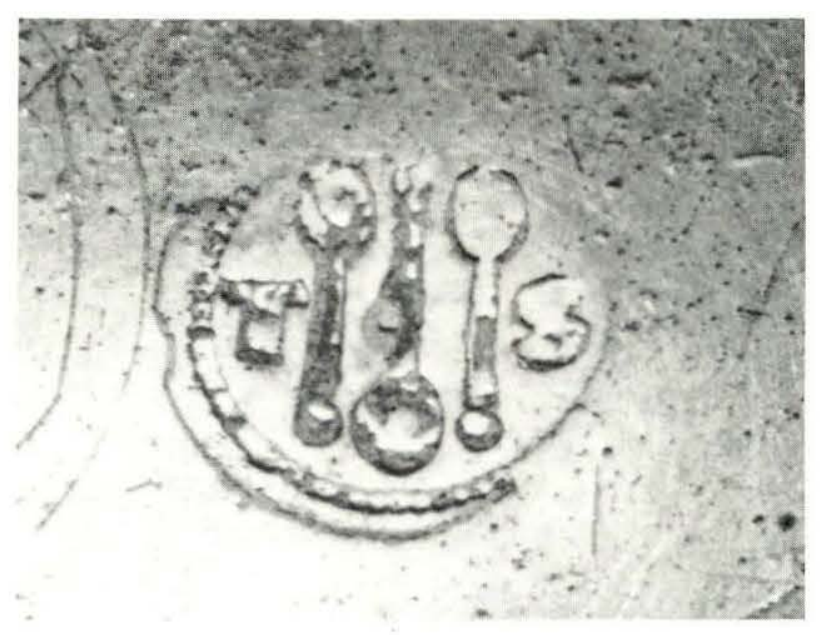

Figure 6.11

Close-up of touch mark on Burial 8 spoon bowl. 
In summary, the Food Preparation and Consumption artifact group comprised $2.5 \%$ of the total Euroamerican artifacts from the Fredricks site, $41.9 \%$ of the artifacts from $310 \mathrm{r} 11$, and $0.2 \%$ of the artifacts from the Mitchum site.

\section{Personal Group}

This artifact group contained the greatest number and variety of Euroamerican trade items. The Personal group was divided into three classes: 1) Ornamental; 2) Grooming; and 3) Entertainment. The Ornamental class, which represented the largest class at the Fredricks and Mitchum sites, consisted of jewelry (e.g., glass beads, brass bells, wire bracelets, and coiled wire used for ornamentation or as hair tweezers). A few of the artifacts from the Personal group were datable.

There were 8,594 glass trade beads from the three sites: 6,632 from the Fredricks site, 1,960 from the Mitchum site, and two from the plowzone at the Wall site. There also were three ivory beads from the Fredricks site. Wampum beads and other aboriginally manufactured shell beads were analyzed by Hammett (this report) and will not be discussed in this section. A sample of the trade beads is illustrated in Figure 6.12.

The three ivory beads were found, one each, in Burial 2, Burial 3, and Feature 13 fill. All three of the specimens are $6 \mathrm{~mm}$ in diameter and $5 \mathrm{~mm}$ long, spherical, and have polished exteriors (Figure $6.12: \mathrm{h}$ ). All were yellowed with age. Beads of this type, often called "rosary beads," and have been found at Fort Michilimackimac (Stone 1974:114-115), the Tunica site (Brain 1979:221), the site of the Cherokee town of Tomotley (Carnes 1983:202), and the Guebert site (Good 1972:123). Stone (1974:115) suggests that these beads were traded individually as well as being attached to religious apparel (1974:115), and that they were present as early as 1680 and lasted through the mid-1700s.

Glass beads were present in most contexts at the Fredricks site (none were recovered from Feature 8): 32 were from surface collections, 6,146 from nine burials, 416 from six features, and 38 from 15 units of plowzone. At the Mitchum site, glass beads were found in Burial 1 $(1,775)$ and six features (185). A special coding format created for historic bead analysis combined information from Kidd and Kidd's (1970) typology, Karklins (1982) analysis format, and Carnes (1983:203) analysis of beads from the site of the Cherokee town of Tomotley. Using this combined format, all beads were examined for: 1) manufacturing techniques (drawn, wound, molded, carved, etc.); 2) size (very small - <2 mm dia., small - 2-4 mm dia., medium - 4-6 mm dia., large - 6-10 m dia., very large - 10-17 $\mathrm{mm}$ dia., and very, very large - >17 mm); 3) diaphaneity (opaque, transparent, translucent, burned, etc.); and 4) color (an attempt was made to match color with Kidd and Kidd's color chart
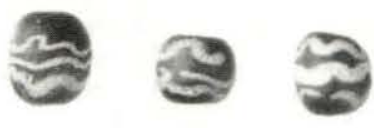

a
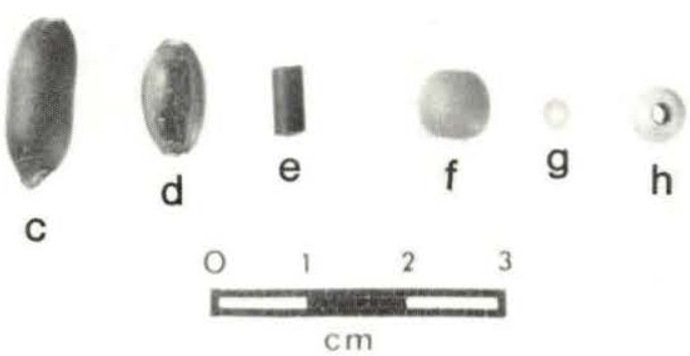

Figure 6.12

Sample of glass (a-g) and ivory (h) beads from the Fredricks site.

for consistency). Bead types were assessed as to compound or simple construction, and surface decoration (stripes, glass inlay, etc.). Color was difficult to determine in cases where patination was extreme. Following a technique described by Polhemus (1984:1180), bead clarity was determined by dampening each specimen and placing it under a consistent light source. This technique reduces the opacity created by weathering and patina and allows true (or nearly true) bead color to be revealed. Once dry, the protective patina returns. The "original" condition of the glass, whether opaque or translucent, presented another critical attribute for color determination. As pointed out by Carnes (1983:203), "true" black beads are opaque in diaphaneity, whereas translucent black beads actually appear to be a dark burgundy or dark rose brown when held under illumination. "White" beads also vary in shades and opacity. Heavy patination observed on a few colored beads, namely bright mint green, light gold, and some turquoise, suggests a rapid decomposition of the glass due to the particular coloring agents through chemical reaction to acidic soils.

Seed beads (ranging from 2-4 $\mathrm{mm}$ in diameter) were the most abundant size and probably sewn on articles of clothing, as well as strung for necklaces. For comparative purposes, all beads were regrouped and quantified into the four most popular colors--white, black, blue, and redwood over green. Of the total beads from the Fredricks site, the breakdown was: 6,111 ( $92 \%)$ white; 210 (3\%) redwood over green/gray core; $189(3 \%)$ black (transparent and opaque combined); 81 ( $1 \%$ ) blue (al1 shades); $22(0.5 \%)$ were fancy (striped, inlaid, etc.); and 19 $(0.5 \%)$ other colors. Obviously, white beads were, by far, the most abundant. For the Mitchum site the breakdown was: 1,894 (97\%) blue (a11 shades); 40 (2\%) white; $12(0.7 \%)$ black (transparent and opaque); $9(0.3 \%)$ redwood over green/gray core; and $5(0.1 \%)$ yellow. No fancy 
beads were found at this site. Kent (1984: 211-223) has placed the peak popularity for blue seed beads at 1575 to 1760 , with a major increase from 1600 to 1665. The popularity of white beads seems to have remained fairly consistent throughout the Historic period. For the most part, however, the trade records lack details of glass beads. With only the beads from the Fredricks and Mitchum sites to compare, a more detailed trade bead chronology for this region could not be attempted. Comparative bead studies on other Piedmont North Carolina Contact period sites hopefully will be the focus of future research.

A few of the fancy beads did provide additional chronological information. "Roman" beads (Figure 6.12:a) recovered from Burial 1 at the Fredricks site were opaque black with yellow glass inlaid lines, and of wire-wound construction (Kidd and Kidd's Type IIj). Brain (1979:113) contends that these beads originated in Amsterdam, and he reports dates of 1669 to 1799 for the type. They have been found at the Tunica site (Brain 1979:113), the Guebert site (Good 1972:115), Fort Michilimackimac (Stone 1974:99), the Tomotley site (Carnes 1983:206), and Susquehannock sites in Pennsylvania (Kent 1984:214-215). A large blue bead (translucent) (Figure 6.12:c), identified as Kidd and Kidd's Type IIa 55 was found in Burial 6 at the Fredricks site. These are dated $1600-1800$ by Brain (1979:112). An opaque turquoise bead with a compound white/red stripe, found in Burial 2 at the Fredricks site, is dated 1680-1836 (Brain 1979:104). It is Kidd and Kidd's Type IIb 7. Several varieties of Cornaline de Allepo beads, identified as Kidd and Kidd's Type IVa were found at both the Fredricks and Mitchum sites. In general, these beads have a redwood colored opaque slip over a gray, green, clear, or white core and occur in all sizes (Figures 6.12:b). They range in date from 1600 to 1836 (Brain 1979:106). A single dark red-purple tubular bead, found in Feature 13 at the Fredricks site, may represent glass wampum (Figure 6.12:e). Good (1972:120) found similar beads at the Guebert site, and assigns them a date of 1660-1677. Three translucent navy blue beads with alternating red and white stripes (Figures 6.12:d) were found in Features 9 and 13 at the Fredricks site and can be dated to 1700-1740 (Good 1972:109).

Finally, Kent (1984:213) offers a few critical observations on the use of bead types as chronological indicators. At Susquehannock sites, straw beads (tubular or cane beads) had their greatest popularity between 1630 and 1670; sites dating after 1670 contained fewer straw beads and more tumbled seed beads. Also, numbers of wire-wound beads (all sizes) increased on sites dating to the mid to late 18 th century. Polhemus' (1984:1187) observations at Overhill Cherokee town sites confirm these temporal trends in bead popularity. The only wirewound bead found at the Fredricks site were the "Roman" beads previously mentioned. Based on the above information, a date between 1670 and 1740 for the historic occupation of the Fredricks site is reasonable.

Twenty-four sheet brass bells were also included in the Personal artifact group. Twenty-three of these be11s were found in Burial 7 at the Fredricks site. The bells appear to have been sewn together on straps (Figure 6.13:c) or on a garment and fastened around the knees of the individual, 12 around one knee and 11 around the other. A sample of these bells, along with a single bell found in Feature $13 \mathrm{fill}$, is shown in Figure 6.13. The bells from Burial 7 are identified as flush-edge type, made of stamped sheet brass by flush-loop construction, and measure $15.5 \mathrm{~mm}$ in diameter (Figure $6.13: \mathrm{a}, \mathrm{c}$ ). A small iron sphere was visible through the holes of the lower hemisphere of each bell. Brain (1979:197) reported finding identical bells at the Tunica site that date 1699-1730. The single bel1 from Feature 13 is also made from stamped sheet brass, but the equatorial seam is flanged rather than flush (Figure $6.13: \mathrm{b}$ ). It measures $18 \mathrm{~mm}$ in diameter and has a flush loop for attachment. This variety, which is often referred to as a "Saturn Be11," has been found at the Fatherland site (1699-1730), Fort Michilimackimac (1700-1730), and other sites dating 1659-1681 (Brain 1979: 202). Bells appear frequently on trade inventories and apparently were popular ornamental items to the Indians. A chronology for bells in the Piedmont region of North Carolina could be attempted by incorporating bells from Upper Saratown (31Skla) and western Cherokee sites into a comparative framework.

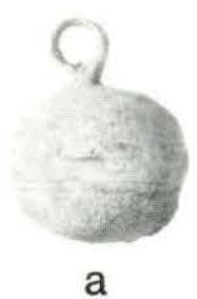

a

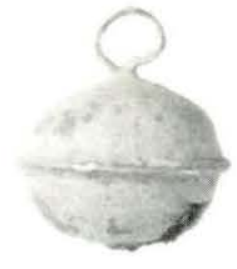

b
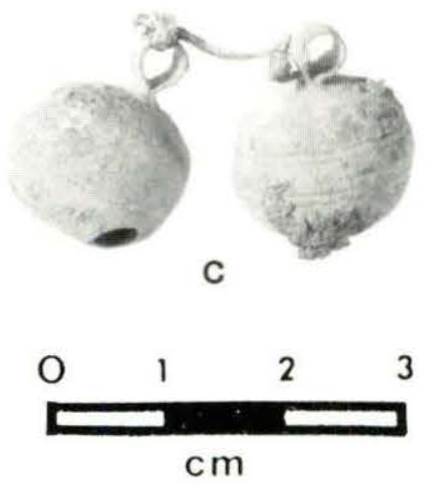

Figure 6.13

Sample of brass bells from Burial 7 (a, c) and Feature 13 (b) at the Fredricks site. 
Two copper wire bracelets were found in Burial 6 at the Fredricks site. Both bracelets are of the same style and gauge of wire but are of slightly different size (Figure 6.14). These compound $\mathrm{C}$-shaped bracelets were found on the left forearm of the individual and appeared to be partially covered with leather (possibly the remains of a garment lying on the bracelets). Bracelets of a similar style but constructed of iron wire were found at the Upper Saratown site ( $31 \mathrm{Skla}$ ) and one made of brass wire was found at the Madison site (31Rk6). A11 of these others were found in burial contexts. C-Bracelets are diagnostic of the early to middle period of Indian trade (Brain 1979:193), when they were common ornamental items. Often bulk quantities of metal wire (brass, iron, copper, etc.) were traded to the Indians specifically intended for modification into ornaments, fishhooks, or coils. A single brass wire coil was found in Feature 13 fill at the Fredricks site (Figure 6.15:d). Similar coils have been found at the Tunica site (Brain 1979:196) and at many of the
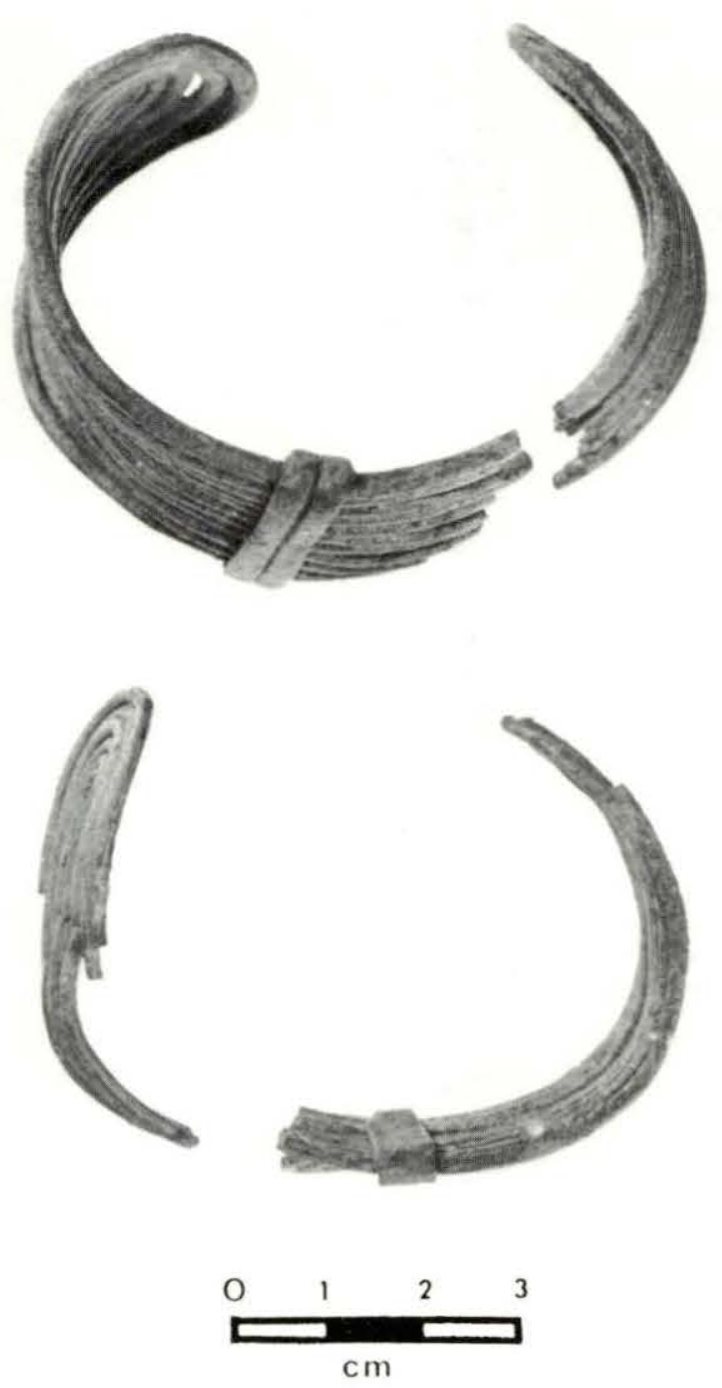

Figure 6.14

C-shaped wire bracelets from Burial 6 at the Fredricks site (after cleaning).

Cherokee sites in Tennessee (Carnes 1983:208; Polhemus 1984:1206), again mostly in burial contexts. It has been suggested that these coils functioned either as ornaments or sometimes as hair pluckers (tweezers) or ear ornaments (Brain 1979:196). A specific function of this coil from the Fredricks site, however, could not be determined from its context or appearance.

Two pieces of flat glass, both identified as mirror fragments, were found at the Fredricks site, one from the plowzone and another from the fill of the wall trench of Structure 1. No mirror fragments were recovered from burial contexts. These mirror fragments comprise the Grooming Implement class of Personal artifacts. Mirrors, or looking glasses, show up frequently on trade lists (France 1985). It has been noted at Cherokee sites, however, that mirrors sometimes functioned as personal adornment, suspended from the neck or sewn on clothing, rather than as grooming implements (Carnes 1983:208).

The third class of Personal artifacts have been collectively termed Entertainment and include smoking pipes, ember tongs, and an iron Jew's harp. In the category of smoking pipes, there were 204 pipe fragments (103 European or kaolin, and 101 of aboriginal manufacture) from the Fredricks site. From the Mitchum site, there were 23 pipe fragments ( 1 European and 22 aboriginal). One molded, stub-stemmed pipe fragment from the plowzone at the Wall site probably dates to the early 19th century. From the Fredricks site, in addition to the Kaolin pipes, there were two pewter pipes, a whole pipe from Burial 3 and a carved pewter stem from Burial 6. A sample of pipes from the Fredricks site, to illustrate bowl shape, stem size, and decorative elements, is provided in Figure 6.16 .

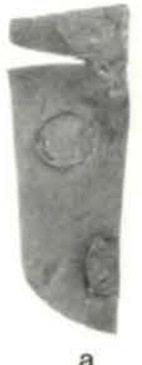

a

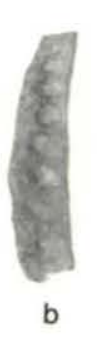

b

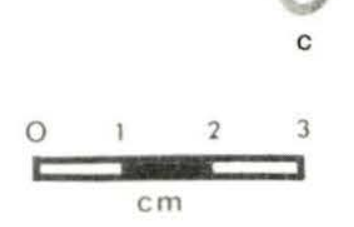

Figure 6.15

Sample of aboriginally-modified metal artifacts from the Fredricks site: brass kettle fragments $(a-b)$; brass wire fish hook (c); and brass wire coil (d). 


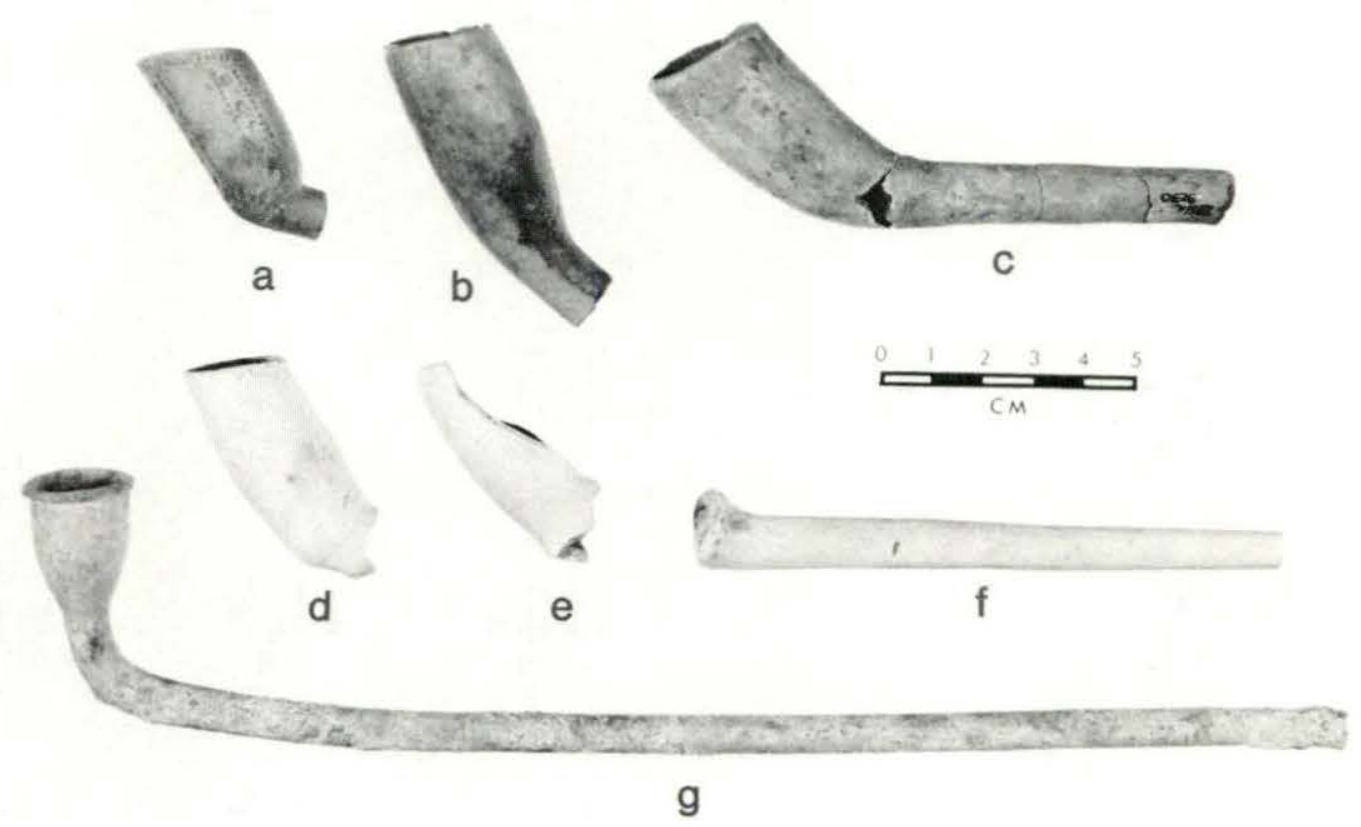

Figure 6.16

Sample of pipes from the Fredricks site: aboriginal pipes (a-c); European kaolin pipes $(d-f)$; and European pewter pipe $(g)$.

Stem fragments of kaolin pipes from the Fredricks site were sorted by context and by bore diameter (Table 6.3). A general period of manufacture was calculated for each bore diameter using the formula of Harrington (1954: 6-8). Of 42 measurable mid-section fragments, $55 \%$ belong to a $1650-1680$ period of manufacture, whereas 33\% date to 1680-1710. When Binford's (1962:19-21) regression formula was applied to the sample, a date of 1678.95 was determined for plowzone pipe stems, and a date of 1683.16 was calculated for pipe stems from burial/feature contexts. This finding strongly suggests that the pipe stems recovered in the plowzone can be identified with the same occupation that is responsible for the burials and features. Because of the small sample ( 18 stems from plowzone and 24 from burial/feature contexts), and criticisms of the validity of pipe-stem dating, however, caution must be exercised in the interpretation of these dates.

Aboriginally-manufactured clay pipes from the Fredricks and Mitchum sites were analyzed for form, decoration, composition, completeness, and dimensions of stem length, bore diameter, and bowl-to-stem angle. A single reconstructible pipe was recovered in the fill of Feature 13 (Figure 6.16:c). Decorations on a few of the bowls and stem sections included incised bands, roulettes, punctations, and dentate designs. Paste consisted of micaceous clays, sometimes with fine-grain grit tempering. Although none was glazed, many exhibited evidence of burning through soot deposits. A few specimens, like the one shown in Figure 6.16:a, revealed marks of metal tools. An attempt was made to corre- late bore diameters of the finer-made, or tooled, aboriginal pipes to the bore diameters of European kaolin pipes. Using the same gauges as were used on the aboriginal specimens, an average bore diameter of $8 / 64$ in $(3.7 \mathrm{~mm}$ ) was obtained from 26 specimens. Binford's (1962: 19-21) regression formula was used on 13 specimens to obtain a date of 1673.21 for burial/ feature contexts.

Table 6.3

Kaolin pipe stem fragments by context and bore diameter.

\begin{tabular}{lccccc}
\hline & \multicolumn{5}{c}{ Bore Diameter } \\
\hline Context & $5 / 64$ th & $6 / 64$ th & $7 / 64$ th & $8 / 64$ th & Tota1 \\
\hline Plowzone & 0 & 8 & 9 & 1 & 18 \\
Bu. 1 & - & 1 & - & - & 1 \\
Bu. 3 & - & 2 & - & - & 2 \\
Bu. 4 & - & - & 1 & - & 1 \\
Bu. 5 & - & 1 & 3 & - & 4 \\
Bu. 9 & - & 1 & - & - & 1 \\
Fea. 1 & - & 1 & - & - & 1 \\
Fea. 9 & 2 & 1 & 2 & - & 5 \\
Fea. 10 & - & - & 3 & - & 3 \\
Fea. 11 & - & - & 2 & - & 2 \\
Fea. 12 & - & 1 & - & - & 1 \\
Fea. 13 & - & - & 3 & - & 3 \\
Total & 2 & 16 & 23 & 1 & 42 \\
Percent & 5 & 38 & 55 & 2 & 100 \\
Period & $1710-1750$ & $1680-1710$ & $1650-1680$ & $1620-1659$ & \\
\hline
\end{tabular}


The cast pewter pipe recovered from Burial 3 at the Fredricks site was identified as a tulip-bowl style, possibly of Dutch or British origin (Figure 6.16:g and Figure 6.17). Although cast pewter pipes could not be found on any of the trade lists reviewed (France 1985), they have been found archaeologically at Iroquois sites in New Jersey dating to 1650-1687 (Heye and Pepper 1915:50), and Kent (1984:287) reported a pewter pipe stem from the Conestoga site (ca. 1680s). It is also possible that this pipe could be of Anglo-American origin and was produced specifically for trade among the Indians. Noel Hume (1982:308) has noted that metal pipes were popular items among White hunters and travelers in the latter part of the 18 th century because clay pipes tended to be too fragile for travel. Archaeological remains of lead ammunition manufacturing at the Fredricks site suggests that the natives themselves were knowledgeable about casting methods and could have made this pipe. A second, hand-carved pewter pipe stem found in Burial 6 strengthens this observation (Figure 6.18). The stem consists of a hollow tube of pewter encased in an outer sleeve with geometric cut-outs decorating the bowl end. Three small spurs were noticed at this end and may have served to attach a wooden bowl (not archaeologically preserved). Observations in the field during excavation of this object suggested the deteriorated remains of a metal bowl rim or liner were present. A funnel-shaped, hand-made pewter object was found in Feature 13 which has been tentatively identified as a pipe bowl liner for a wooden bowl. This artifact is shown in Figure 6.18. A similar pipe bowl liner made of copper was found at an historic Indian cemetery known as the Grimsby site (Kenyon 1982:108). It seems likely that a bowl liner of this type would have been used with the carved pipe stem from Burial 6. An artist's reconstruction (Figure 6.19) helps to visualize this possible combination, although no direct archaeological association of these two specimens is proposed.

An iron ember tong (Figure 6.20), also known as a "smoker's companion" or spring tong, was found in Burial 3 at the Fredricks site. Wilson (1984:4) has suggested that this item was associated with a smoker's kit or bundle in this burial. Ember tongs or "tobacco tongues" are described in ethnohistorical accounts of this
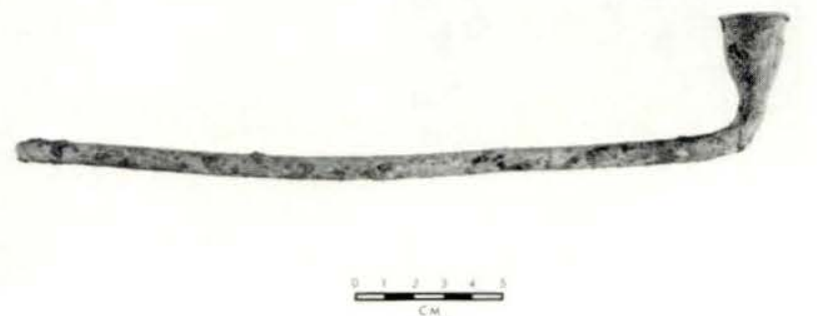

Figure 6.17

Cast pewter tulip bowl style pipe from Burial 3 at the Fredricks site.

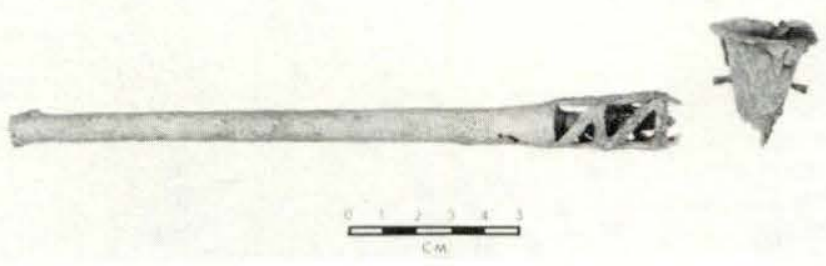

Figure 6.18

Hand-carved pipe stem from Burial 6 (left) and pewter pipe bowl liner from Feature 13 (right) at the Fredricks site.

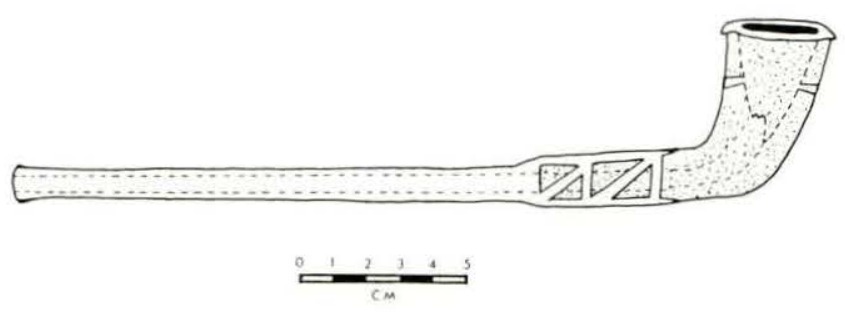

Figure 6.19

Artist's reconstruction of pewter pipestem with wooden bowl and pewter bowl liner (based on artifacts illustrated in Figure 6.18).

period and appear on trade inventories (France 1985). Noel Hume (1982:309) suggests that ember tongs were used throughout the 17 th and 18 th century. This tong is made of iron and has rounded pincer arms and flattened handle ends for easy gripping. The spring clip found with the specimen was broken off.

The last item in the Personal artifact group to be discussed is an iron Jew's harp recovered from a bundle of artifacts in Burial 2 at the Fredricks site. This item was very
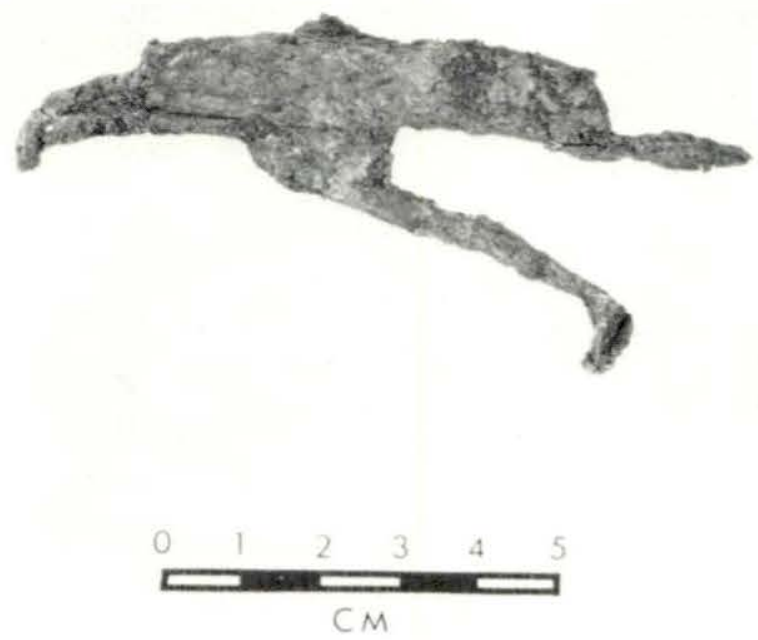

Figure 6.20

Iron ember tongs from Burial 3 at the Fredricks site. 
corroded and fragmentary and had to be drawn in situ and then removed in pieces. Jew's harps have been found archaeologically at other contact Indian sites, i.e., the Guebert site (Good 1972:132).

In summary, the Personal group of Euroamerican artifacts comprised $86.5 \%$ of the total assemblage from the Fredricks site, $1.1 \%$ of the artifacts from the Wall site, and $99.6 \%$ of the artifacts from the Mitchum site, with glass trade beads being the most abundant artifact in this category.

\section{Construction Tool Group}

Three iron axe heads comprise this artifact group. The specimens are from Burial 3, Burial 5, and the bottom of Feature 9 at the Fredricks site. The specimen from Feature 9 was the blade portion only and may have been discarded when it broke and could not be repaired. The two axes from Burials 3 and 5 were complete and nearly identical in style. Figure 6.21 shows the axe from Burial 5 after cleaning. The broken, discarded axe fragment indicates that recycling of metal was not always practiced, and may further indicate that replacement tools were readily available from traders. The two intact specimens have oval eyes, and the blades have a maximum width of approximately 3 inches $(7.7 \mathrm{~cm})$ and length of $5-1 / 2$ inches $(14 \cdot 3 \mathrm{~cm})$.

Axes of this type were made by bending a thick sheet of iron around a mandrel to form the eye of the haft, then forging the two ends of the sheet together into a blade, and finally spreading and thinning the blade toward the bit which was ground to a sharp edge. Sometimes a steel bit was added to the working edge to prolong sharpness. Similar axes have been found at the Tunica site (Brain 1979:140), the Guebert site (Good 1972:162), Fort Michilimackinac (Stone 1974:301), and Susquehannock sites in Pennsylvania (Kent 1984:236). Also called hatchets or tomahawks on trade inventories, small axes of this variety were popular

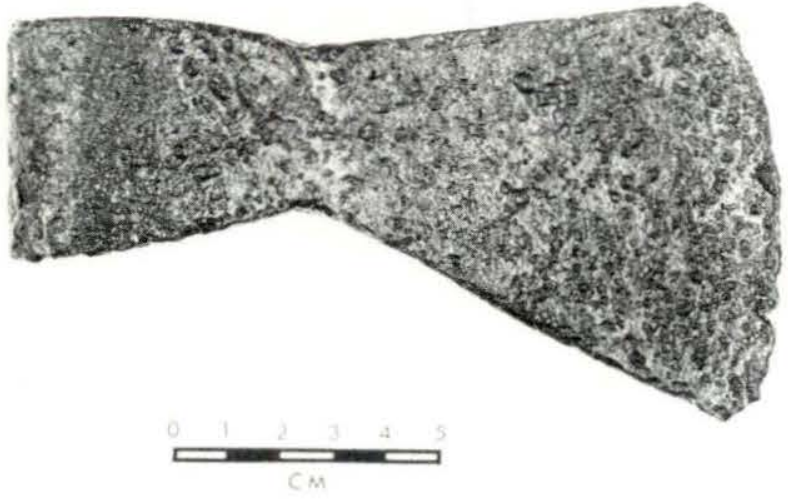

Figure 6.21

Cleaned and treated iron axe head from Burial 5 at the Fredricks site. commodities in the Indian trade (France 1985). Brain (1979:140) notes that "while axes were distributed widely throughout the Historic period there was little or no change in basic styles, which renders them of little use for dating or other correlations."

The Construction Tool category comprised less than $.03 \%$ of the total assemblage from the Fredricks site, and was missing from the other two sites.

\section{Farm Too1 Group}

Three iron hoe blades from the Fredricks site comprise this artifact group. There were two complete blades, one each from Burials 6 and 9 , and a broken blade from the bottom of Feature 13. The specimen from Burial 6 is a broad-blade variety with a blade width of 8 inches $(20 \mathrm{~cm})$ and blade length of $6-1 / 2$ inches $(16.5 \mathrm{~cm})$. As shown in Figure 6.22, this piece has a cylindrical haft which is heavily reinforced by a strong ridge at ridge at the bottom, at which point a prominent tang extends approximately half-way down the inner surface of the blade. The blade has rounded shoulders and a flaring bit. This hoe is identical to the type found at the Tunica site and classified by Brain (1979: 144) as Type A, Variety 1. Broad hoes have also been recovered from many of the Overhill Cherokee towns (Carnes 1983:176), where they replaced stone and wooden tools for digging and farming. On period trade lists, broad hoes appear to have a higher value in deerskins than narrower hoes (France 1985).

The second intact iron hoe was recovered from Burial 9 and is a narrow-blade variety (Figure 6.23). This type of hoe has been described at the Tunica site by Brain (1979:146) as Type C. It was constructed in a similar fashion to the Type A hoe except that the haft was not reinforced and the central tang was not pronounced. The blade is six inches $(15 \mathrm{~cm}$ ) wide and eight inches $(20 \mathrm{~cm})$ long. Another hoe of this type was found at Jamestown (Cotter and Hudson 1957:74) and dates to the late 17th century.

The broken hoe from Feature 13 seems to be the broad-blade variety, but the blade is missing. No evidence of retouch or reuse was noted on this hoe fragment.

Farm Tools comprised less than $0.03 \%$ of the total artifact assemblage at the Fredricks site, and was missing at the Wall and Mitchum sites.

\section{Miscellaneous Hardware Group}

Several miscellaneous hardware items were included in this artifact group: 25 knife parts, two iron hooks, two brass wire fishhooks, two iron horseshoes, one lamp part, and three unidentified rod/bar fragments. All items in this group came from the Fredricks site, except one horseshoe and the lamp part which were found 


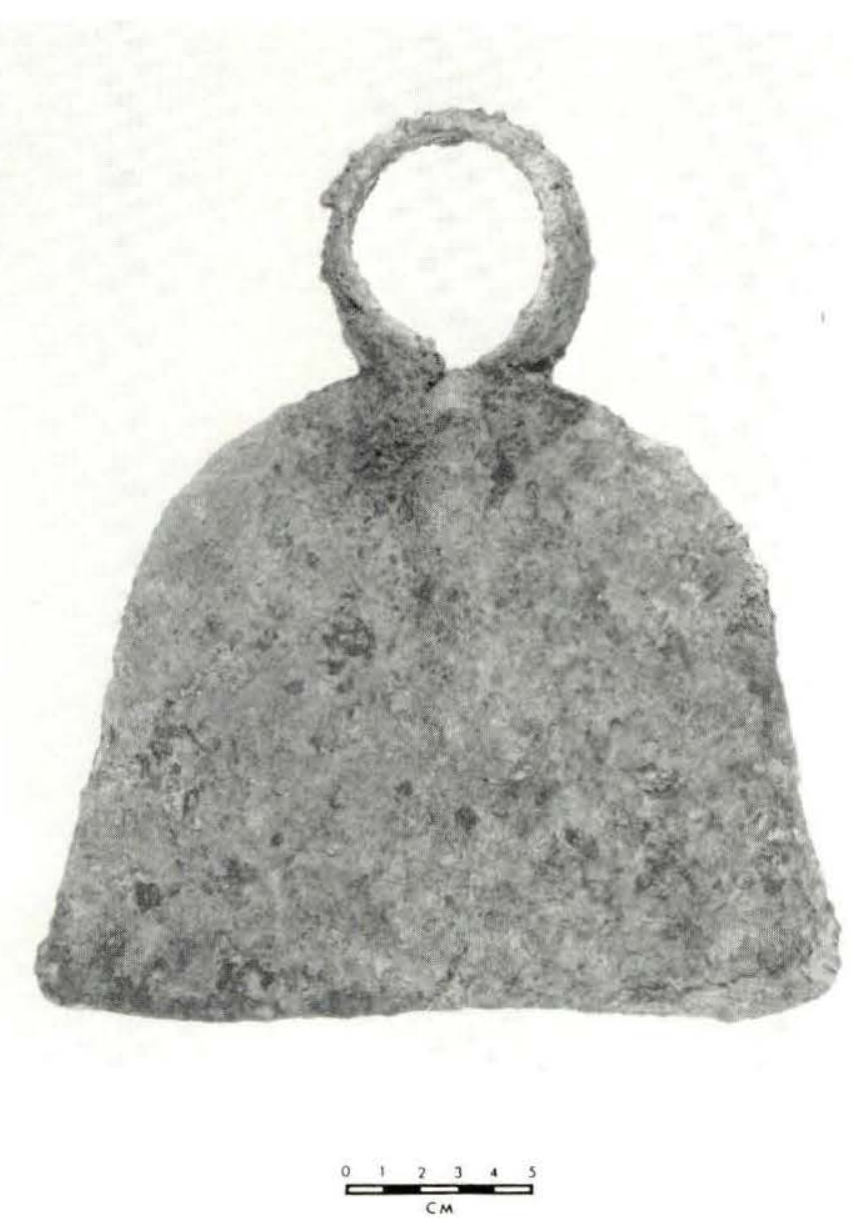

Figure 6.22

Broad-bladed iron hoe from Burial 6 at the Fredricks site.
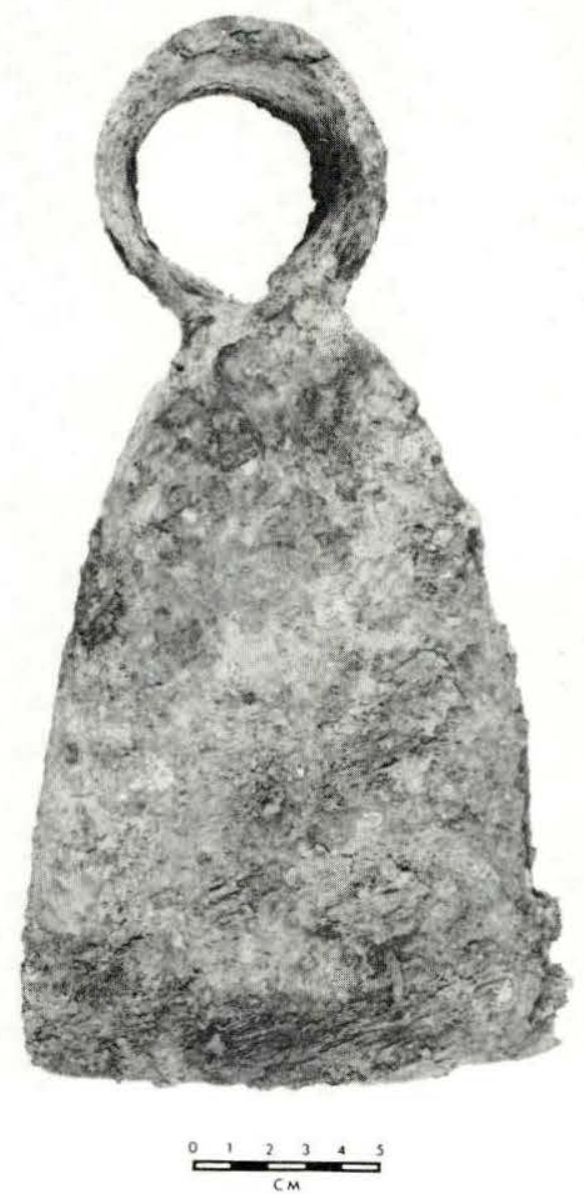

Figure 6.23

Narrow-bladed iron hoe from Burial 9 at the Fredricks site. in the plowzone at the Wall site. Most numerous in this group were knives and knife parts: 12 whole (or nearly whole) case knives, eight case knife blade fragments, four handle fragments, and one possible bolster fragment. All of these items were found in feature and burial contexts, except the bolster which was found in the plowzone. All knives were the case knife type, with steel blades and handle sidings of bone or wood. Pairs of knives were recovered from Burial 1 (Figure 6.24) and Burial 3. Two knives were found in Burial 2, but not together; also a single knife was found in Burial 5. Seven other knife parts, each representing a single knife, were found in Burial 8 and Burial 9. The two knives from Burial 3 appear to have wooden handle sidings. Measurements taken on the more complete knives indicate that blade lengths ranged from 3 to 5 in $(8-12 \mathrm{~cm})$, and blade width from $1 / 2$ to $1-1 / 4$ in $(1.5-3 \mathrm{~cm})$. Handle shape also varied from a rounded end to a beveled apex. Most blade tips were missing. Knives were common trade items, typically sold or traded in bulk quantity (France 1985). Brain (1979:153) states that metal knives were the first Euroamerican item to replace a native counterpart (stone knives).

Two brass wire fishhooks were recovered from undisturbed contexts at the Fredricks site, one from the upper fill of Burial 1 and the other from the fill of Feature 13. The specimen from Feature 13 is shown in Figure 6.15c. Both fishhooks had tapered tips and flattened tops for attachment. European-made fishhooks appear on trade inventories and were sold by the dozen (France 1985). One iron snap hook, made to be attached to a leather strap, was recovered from the plowzone at the Fredricks site. Another nonspecific iron hook was found in the fill of Feature 12 .
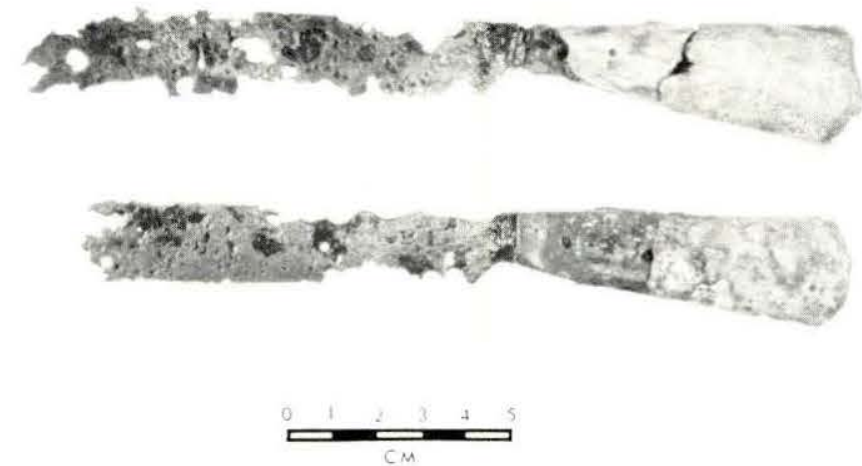

Figure 6.24

Bone-handled, steel-bladed case knives from Burial 1 at the Fredricks site. 
Miscellaneous Hardware items comprised . $4 \%$ of the Euroamerican artifact assemblage from the Fredricks site, less than $0.8 \%$ of the assemblage from the Wall site, and none of the assemblage from the Mitchum site.

\section{Metal Resource Group}

A Metal Resource group was created for this analysis to include all functionally indeterminate metal artifacts from the excavations at the Fredricks and Mitchum sites. Two sheet copper ear spools, probably of aboriginal manufacture, and constructed of native materials, are included in this category from the Mitchum site and, therefore, are not considered Euroamerican in origin. The remaining artifacts, all from the Fredricks site, consist of nine pieces of wire, 14 pieces of metal scrap (lead and iron), and 16 pieces of cut sheet-metal strips, mostly of brass. Many of these pieces probably represent by-products of aboriginally modified Euroamerican artifacts. The Metal Resource category made up $0.5 \%$ of the Euroamerican artifacts from the Fredricks site, and $0.1 \%$ of the artifacts from the Mitchum site.

\section{"Other" Group}

This artifact group was created to contain by-products of heat-generating activities and construction debris (e.g., coal, slag, cinders, daub, fired clay, and rock) that are of both Euroamerican and aboriginal origin. Most of these items occurred in plowzone context at the Fredricks site and the Wall site. Three pieces of coal and cinders were recovered from the upper mixed fill of features and burials.

In total, 76 pieces of by-products/debris came from the Fredricks site representing $(0.9 \%$ of the total Euroamerican artifacts), and 12 pieces came from plowzone at Wall site $(4.7 \%$ of the total); there were no such items found at the Mitchum site.

\section{Indeterminate Group}

This group mostly consisted of corroded metal objects, fragments, and "globs" that could not be identified. A total of 118 pieces make up this group at the Fredricks site ( $1.4 \%$ of the total), and 35 pieces from the plowzone at the Wall site ( $13.7 \%$ of the total), and none at the Mitchum site.

\section{INTERPRETATIONS}

\section{Spatial Distributions}

Archaeological evidence, to date, suggests that the aboriginal occupants at the Wall site lived there in the Protohistoric period (ca. 1545). Historical documentation indicates that activities associated with Anglo-American settlement could be expected in the vicinity of this site by the mid 1700s. A 1768 map of the town of Hillsborough, drawn by C. J. Sauthier, shows an oval "race ground" in the area of the Wall site. No specific archaeological materials or features, however, could be positively identified with this activity. Euroamerican ceramic sherds, glass fragments, and architectural debris indicate farming and/or domestic activities on or near the site by the first quarter of the $1800 \mathrm{~s}$. All historic artifacts from this site were recovered in the plowzone. Generally, brick fragments and iron nails, representing architectural debris, tended to concentrate in the northern portion of the site. Bottle glass and ceramics appear to cluster in the eastern portion of the site. No discernible pattern was noted for the other artifact groups. In view of known historic disturbances of this site (e.g., erosion, plowing, and previous archaeological excavations), patterns of spatial distribution of historic artifacts at the Wall site should be cautiously considered.

At the Mitchum site, four Euroamerican ceramic sherds, one piece of glass, one cinder, and a few possible brick fragments were found in the plowzone. These artifacts probably can be attributed to date 19th to 20th century activities related to farming and/or to a house in the immediate area. All other historic artifacts recovered from this site came from buried, undisturbed contexts and are related to the aboriginal occupation. From the small sample of early Euroamerican artifacts, especially the trade beads, a tentative placement of the aboriginal occupation at 1660-1680 is proposed.

At the Fredricks site, three contexts must be examined: plowzone, features, and burials. Artifacts from the plowzone were combined into nine general categories to investigate spatial distributions. These were iron nails (a11 types), brick fragments (glazed and unglazed), glass (container fragments, plus eight flatglass pieces), historic ceramics, miscellaneous metal artifacts (lead shot, four gun parts, horseshoe fragment, etc.) gunflints, other byproducts (coal, slag, cinders, etc.), Euroamerican trade pipes, and aboriginal pipes. Figures 6.25-6.33 illustrate the frequency and spatial distribution of historic artifacts recovered from plowzone at the Fredricks site.

The plowzone distribution of iron nails (Figure 6.25) was uniform, with a slight increase in density in the south half of the site. Iron nails also were recovered from buried contexts at this site, which suggest that some of the plowzone specimens could be from the aboriginal occupation. The corroded nature of most plowzone nails, however, prevented a positive identification of type or variety. Also, wrought nails remained stylistically similar from the early 1600 s to about 1790 . Thus, the plowzone nails could represent either aboriginal or early Euroamerican activities at this site. 


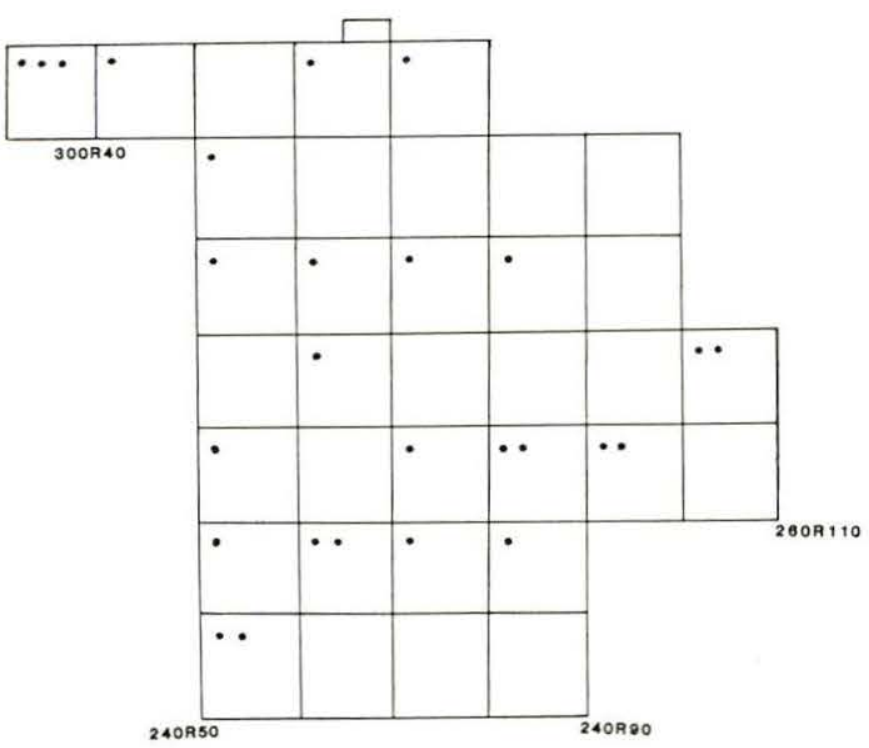

- artifact

Figure 6.25

Spatial distribution of iron nails at the Fredricks site.

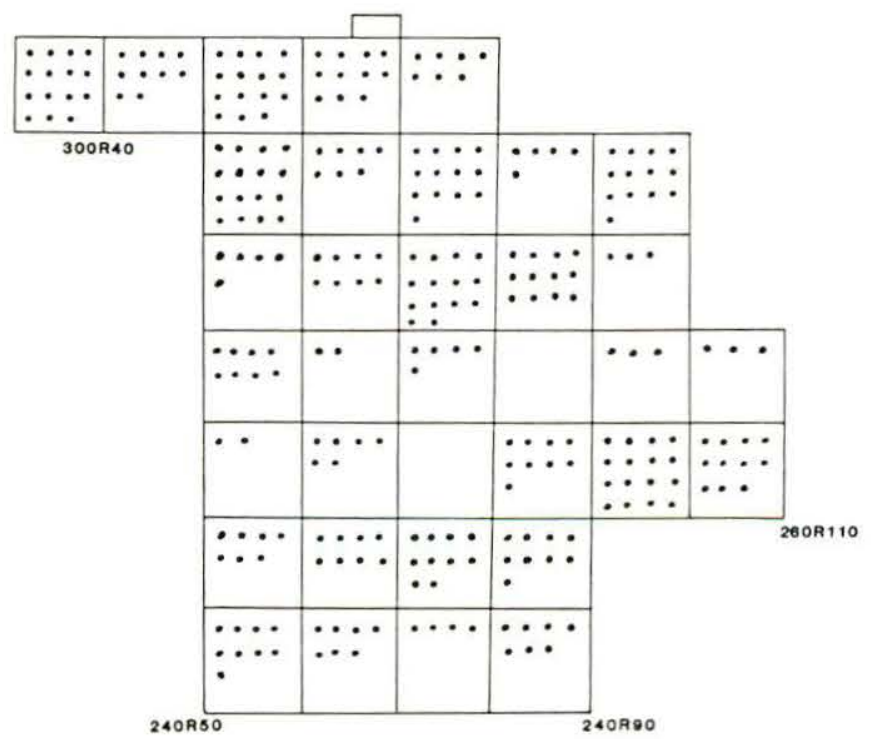

- artitact

Figure 6.26

Spatial distribution of brick fragments at the Fredricks site.

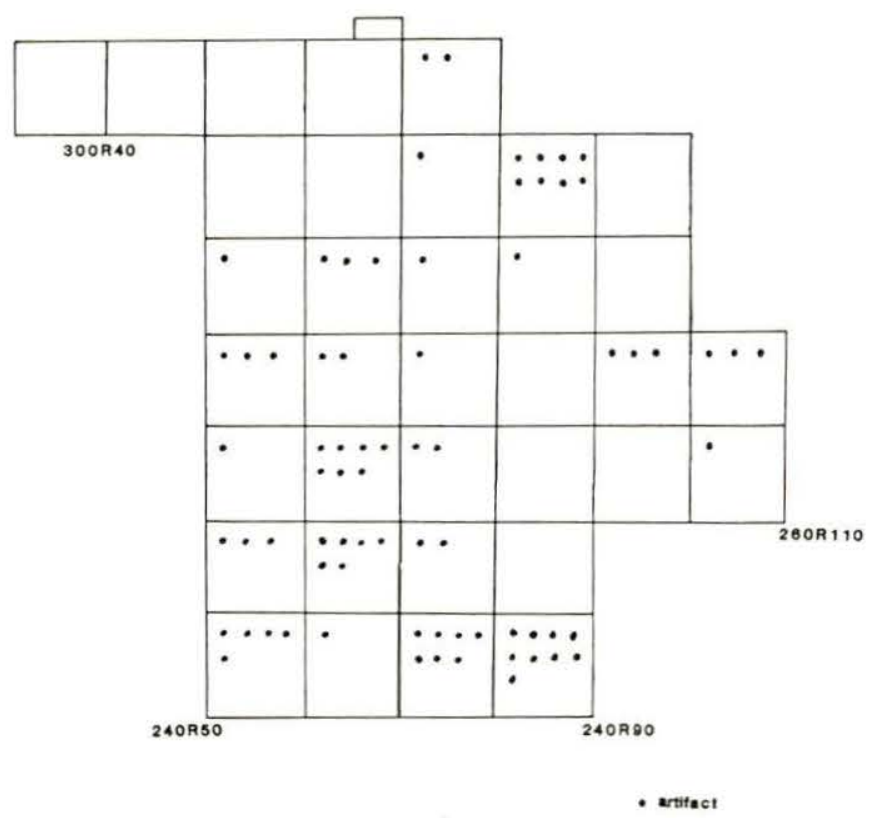

Figure 6.27

Spatial distribution of glass artifacts at the Fredricks site.

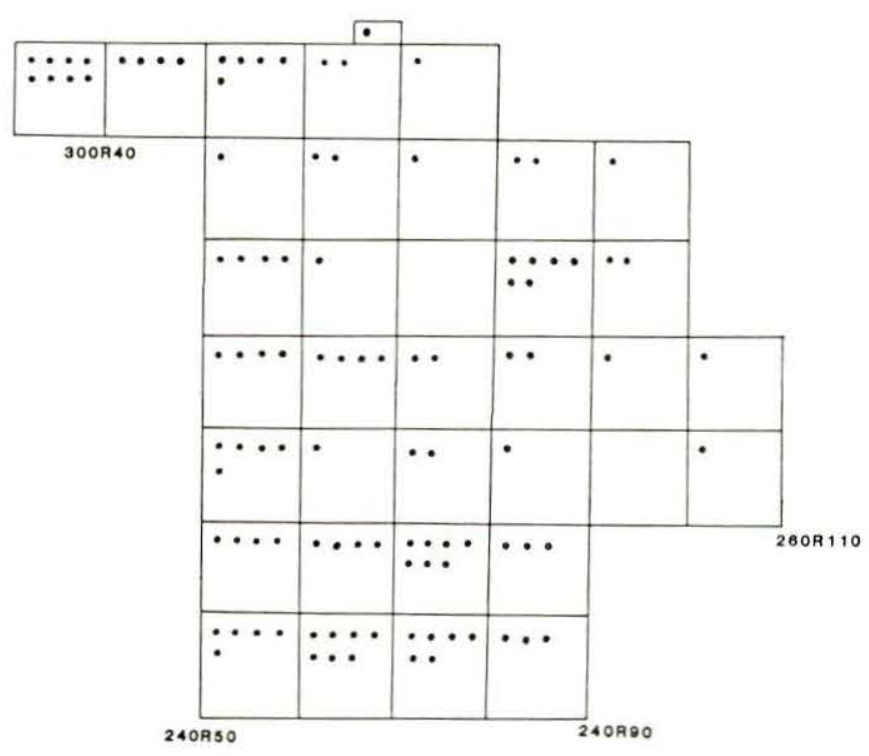

- ertifact

Figure 6.28

Spatial distribution of European ceramics at the Fredricks site. 


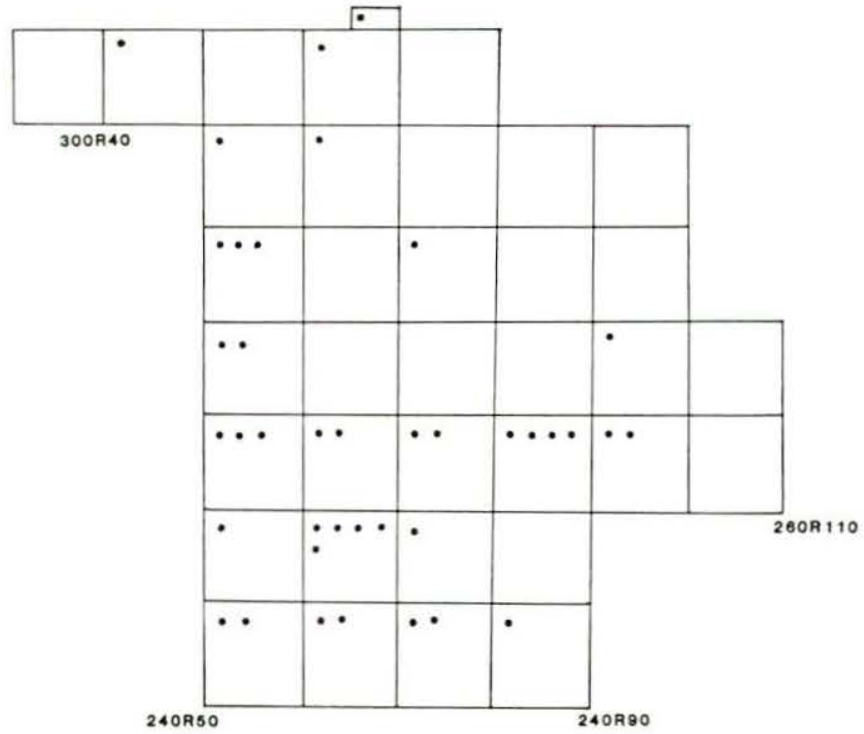

- artifact

Figure 6.29

Spatial distribution of metal artifacts at the Fredricks site.

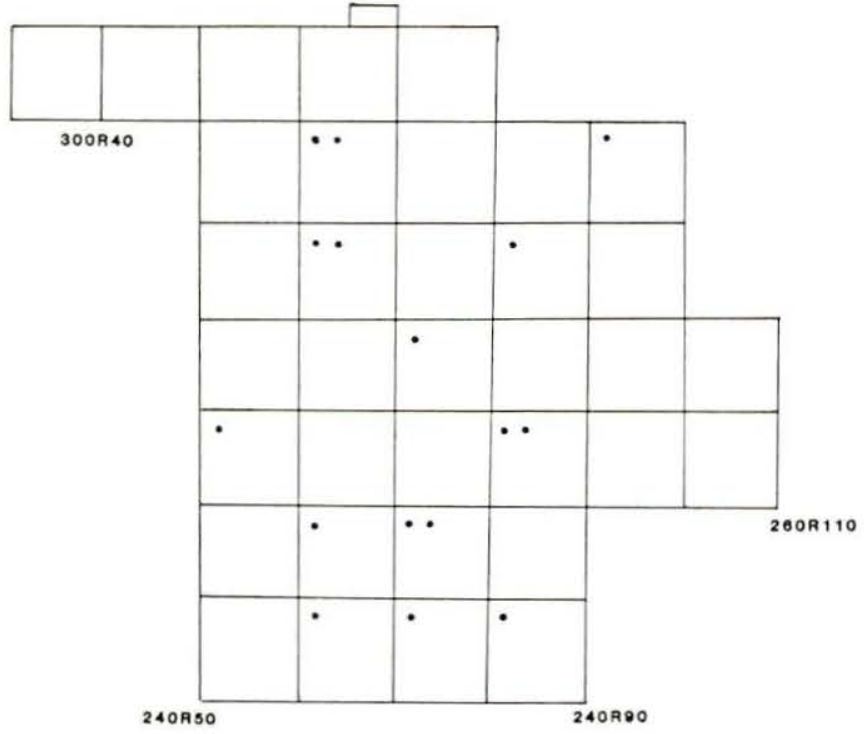

Figure 6.30

Spatial distribution of gunflints at the Fredricks site.

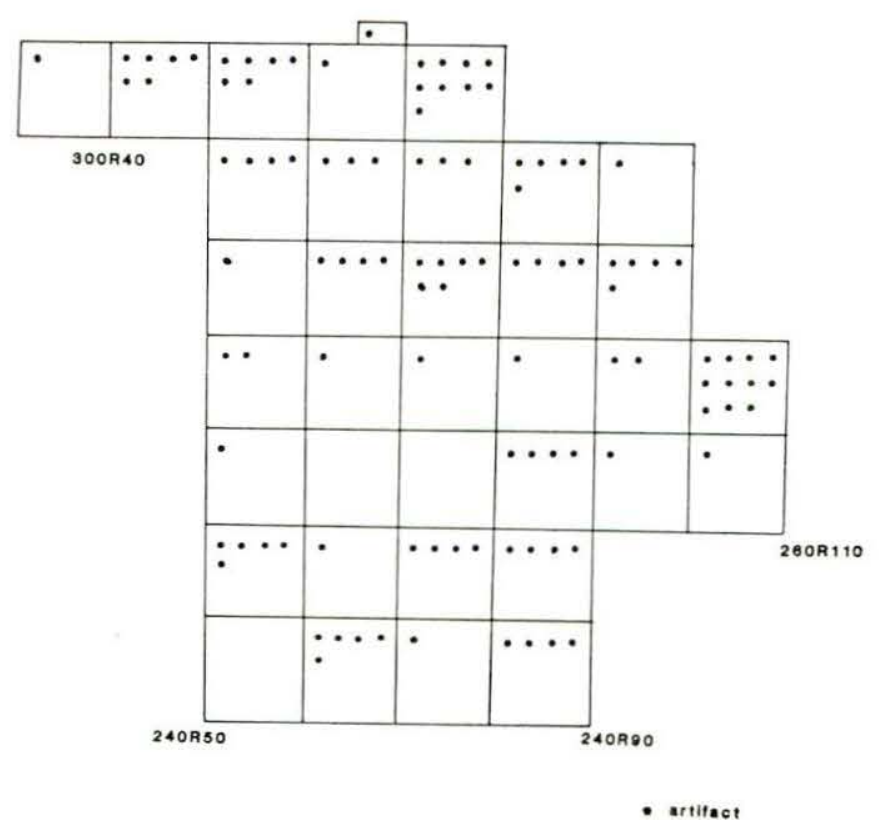

Figure 6.31

Spatial distribution of miscellaneous historic artifacts at the Fredricks site.

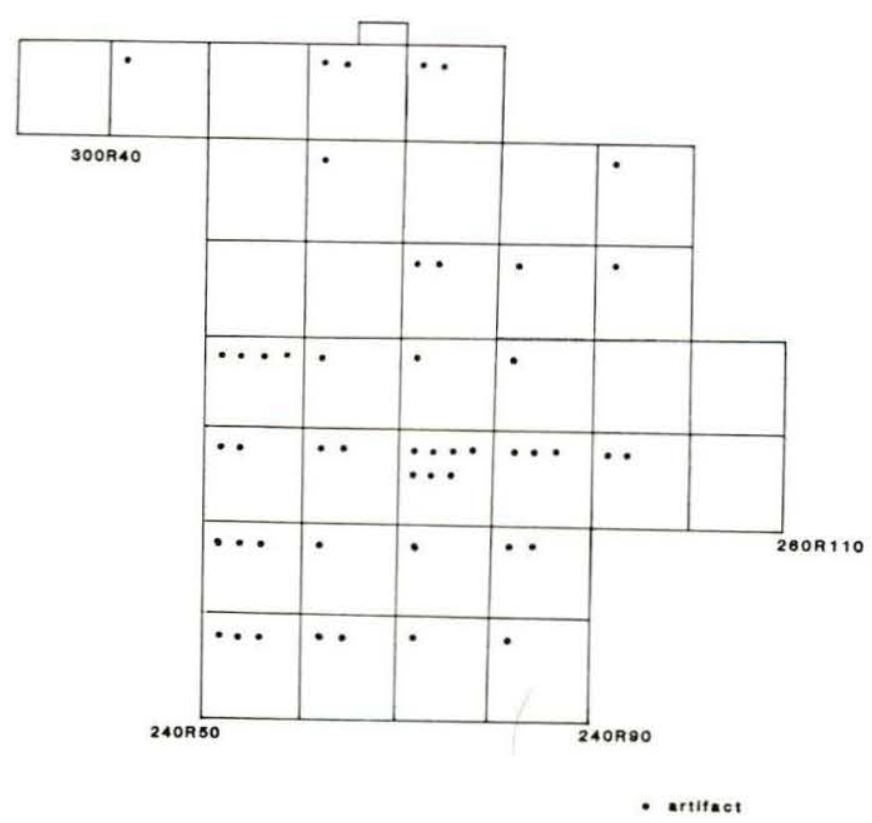

Figure 6.32

Spatial distribution of European trade pipes at the Fredricks site. 


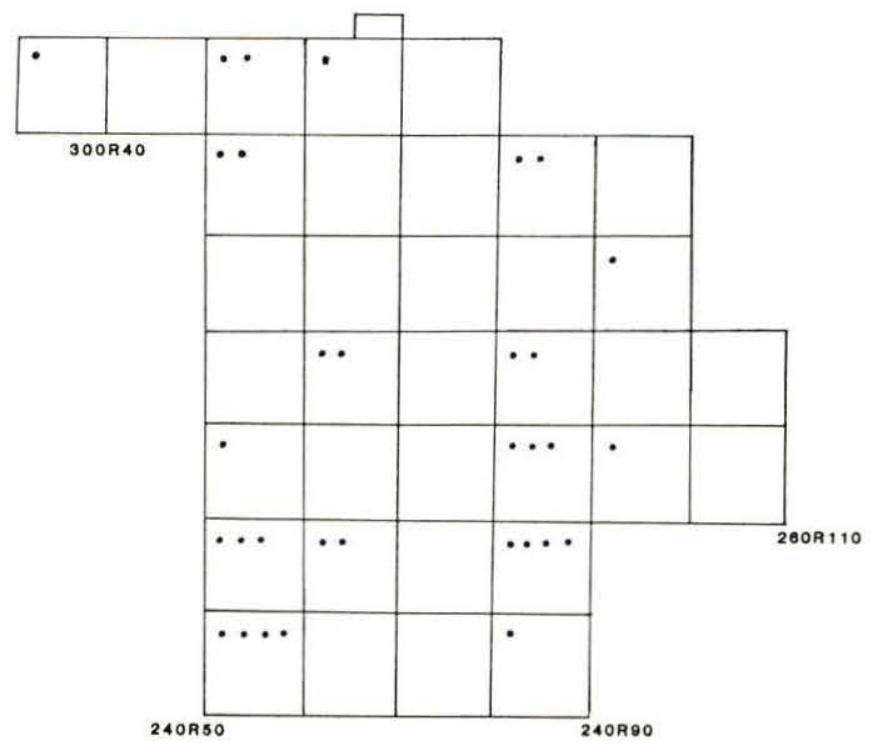

Figure 6.33

Spatial distribution of aboriginal pipes at the Fredricks site.

Brick fragments were more numerous in the plowzone (Figure 6.26) than any other category of historic artifact. There were concentrations in the northwest corner and the southern half of the excavation. As architectural remnants, these brick fragments strongly suggest a nearby structure. The brick may have been used for a chimney, hearth, or piers for a log structure. The frequency of glazed brick fragments indicate the presence of a hearth. Without additional architectural features (foundation walls, chimney base, etc.), it would be difficult to propose a date or cultural affiliation for the structure. It should be noted that glazed-brick fragments were also recovered from buried contexts at this site (one from the fill of Feature 1, five from the fill of Burial 4, one from the fill of Burial 5, and two from the fill of Feature 9). These remains may have been derived from an Indian structure or a trader's house, of they could represent recycling activities by the Indians of discarded architectural materials from a nearby trading post or cabin. Future excavations hopefully will help to clarify this aspect of the artifact assemblage.

Glass artifacts tended to concentrate in the southern half of the excavation (Figure 6.27). Eighteenth-19th-20th-century glass fragments were mixed in the plowzone. Dark green bottle glass fragments, similar to the pieces recovered from buried contexts were noted in the plowzone. Dark green glass of this type, however, has a broad temporal span of manufacture, and without diagnostic attributes (e.g., lip, kickup, or moldseam) it is difficult to segregate 18th-century specimens from those of the 19 th century.
Euroamerican ceramics were recovered from plowzone context only and are, therefore, attributed to post-aboriginal activities on or near the site. Spatially, there was a fairly even scattering, with two possible concentrations in the northwest corner and south end of the excavation (Figure 6.28). As previously noted, 64 of the 108 ceramic sherds (59\%) could be identified by type and assigned a mean manufacture date of 1810.48 , which suggest early 19 th-century Anglo-American activities on or near the site.

Miscellaneous metal artifacts in the plowzone also tended to cluster in the southern half of the excavation (Figure 6.29). Since the gun parts date to the historic aboriginal occupation, their plowzone distribution may be important in interpreting village patterns. The same is true for the gunflints, which also have a distributional trend toward the southern end of the excavation (Figure 6.30).

The "other" by-products category of plowzone artifacts have a fairly uniform distribution (Figure 6.31), with a possible concentration in the northeast portion of the excavation. Dating of this material is difficult; however, the coal, cinders, and slag probably are associated with 19th-century and 20th-century activities on or near the site. The daub and fired clay could be either aboriginal of Anglo-American.

Pipe fragments (Figures 6.32-6.33) show a concentration in the southern end of the excavation. As previously discussed in the artifact analysis section, 18 of the 48 kaolin pipe fragments recovered from plowzone were datable stem pieces. A date of 1678.95 was derived, which suggests that the pipe fragments are associated with the 17 th-century Indian occupation at this site.

Overa11, plowzone distributions of Euroamerican artifacts that definitely or probably date to the historic aboriginal occupation of the Fredricks site tend to show concentrations in the southern, and secondarily in the western, portions of the present excavations. Such distribution are not surprising, since other evidence strongly suggests that the present excavations lie on the northeast edge of the village. As more of the domestic area is revealed, the plowzone artifact distributions may begin to show spatial patterning more detailed than just the direction of greatest general activity. In short, the larger distributions may reveal areas of specialized activities such as tool repair, metal working, ammunition manufacture, and merchandising of trade goods.

\section{The Trade Assemblage from the Fredricks Site}

Schiffer (1972, 1975, 1977) has described the cultural and natural processes by which objects and materials are transformed from the 
systemic context to the archaeological context, and he has noted that a particular context and its artifact associations reflect the behaviors that contributed to the transformation. In order to provide an initial interpretation of the functions of Euroamerican trade items and groups of items in late 17 th-early 18 th-century Occaneechi society, a comparison is now made of these items by archaeological context at the Fredricks site.

At the Fredricks site, there are three basically different archaeological contexts in which Euroamerican trade artifacts are found. There are those items that occur as burial associations, those that are found as part of the refuse fill of pits, and those found in the plowzone. Most of the latter items probably were originally in pitfill and have been pulled into the topsoil zone by modern plowing. The pitfill context can be subdivided into burial pitfill and non-burial pitfill.

The burial associations constitute objects and materials that were intentionally selected from the systemic context to be deposited with the dead. It should be noted, however, that in the minds of the participants, these items probably were considered to continue to function in the system of the afterlife (Ward, this report). In contrast to the burial associations, most objects and materials that reached pitfill contexts got there by having been lost, discarded, or abandoned. These objects, therefore, represent both intentional (discarded and abandoned) and unintentional (lost) deposition, probably far more of the former than the latter (except for glass beads).

First, frequencies of artifacts occurring as burial associations are compared with frequencies of the same artifacts occurring in combined pitfill-and-plowzone contexts (Table 6.4). The artifacts are grouped by the same functional categories used earlier in this section. (Most Euroamerican artifacts--gunflints, pipes, musket shot, beads, etc.--from the plowzone have already been demonstrated to be contemporaneous with the Occaneechi occupation of the site.) This comparison is designed to determine what, if any, categories of trade goods were more likely to have been selected from the overall trade assemblage to become burial inclusions, and, conversely, which items were more likely to be retained in day-to-day use and ultimately to be lost, discarded, or abandoned. In making this comparison, it is recognized that the relative frequency of whole items within an assemblage (i.e., burial associations) may not correspond well with a similar assemblage comprised of mostly fragmentary items (i.e., pitfill).

A Chi-square test on the overall 22-cell matrix, with 10 degrees of freedom, yielded a Chi-square statistic of 1915.0, significant at $\mathrm{p}<.001$. Eight of the 10 functional groups showed significant differences; only the Con-
Table 6.4

Frequency of Euroamerican artifacts as burlal association and in pitfill-plowzone contexts.

\begin{tabular}{|c|c|c|}
\hline Functional Group & Burial Associations & Pitfill-Plowzone \\
\hline Architecture & 11 & 323 \\
\hline Arms & 108 & 112 \\
\hline Food Preparation/Consumption & 17 & $13 *$ \\
\hline Clothing & 41 & 3 \\
\hline Personal & 5799 & 1019 \\
\hline Construction Tools & 2 & 1 \\
\hline Farm Tools & 2 & 1 \\
\hline Miscellaneous Hardware & 22 & 16 \\
\hline Metal Resources & 12 & 39 \\
\hline Other (By-products) & 7 & 73 \\
\hline IndetermI nate & 38 & 93 \\
\hline Total & 6042 & 1680 \\
\hline
\end{tabular}

* Plowzone itens for this group excluded $(n=176)$.

struction Tool and Farm Tool groups were not significantly different between the two contexts, and these two groups had only 3 items each for the combined contexts.

Architectural items (nails, bricks, flat glass, etc.) were significantly associated more with pitfill-plowzone than with burials. A11 eleven of the "architectural" items found with burials were iron nails, which probably were used by the Indians as engravers or punches rather than as construction fasteners.

Arms-related artifacts (shot, gun flints, gun parts, etc.) overall were associated significantly more with pitfill than with burials. However, musket shot were associated significantly with burials. The relative scarcity of shot in non-burial contexts, together with the fact that most of the shot from both contexts are unfired, suggests that the dog-lock trade weapon, because of its length, weight, noise, awkward loading features, and probable overall poor quality, was not a popular hunting weapon among the Indians. Such an interpretation is further supported by the numbers of traditional chipped-stone arrow points from all contexts at the Fredricks site (Tippitt and Daniel, this report). Also, it should be noted that Burials 2 and 6 contained most of the arms-related items from burial contexts--77 lead shot with Burial 2 and an almost complete gun with Burial 6. Only discarded (broken or worn out) gun parts and lost or abandoned lead shot were found in pitfill contexts.

Food Preparation and Consumption items were associated significantly with pitfill rather than burials (items in this category from the plowzone were excluded from the Chi-square test because most of them, especially ceramics and 
glass, date to the 19th and early 20th centuries). Although the only whole items in this category--two rum bottles, two pewter porringers, two latten spoons, and one brass kettle--were found in burials, there were fragmentary examples (bottle sherds, brass kettle scraps, pieces of spoons, etc.) in pitfill. Obviously these items were highly prized (see value in deer skins, Table 6.1) and were not often selected for inclusion in burials. They were only discarded after they were broken beyond repair and had been fully recycled.

The Clothing group, represented by buttons, buckles, scissors, and material resources, were significantly associated with burial contexts. In fact, all of the scissors, buckles, and leather straps, and most of the buttons were found as burial associations. Such a distribution suggests that clothing items were not commonly lost, abandoned, or discarded, but were valuable possessions that were highly curated and deliberately placed with deceased individuals. As proposed earlier in this section, the buttons and buckles seem to have functioned as ornamentation on "bundles" or on necklaces (buttons), rather than as clothing fasteners in the Euroamerican style.

Although Food Preparation and Consumption artifacts were omitted from the Chi-square test because of the presence of post-aboriginal ceramics and glass in the plowzone, a few comments about the distributions of these items in undisturbed contexts are in order. The only whole specimens in this category--two rum bottles, two pewter porringers, two latten spoons, and one brass kettle--were found as burial associations. All other examples were fragmentary (bottle sherds, brass scraps and cut fragments, pieces of spoons, etc.) and were found in pitfill and plowzone contexts. Obviously, these items were highly prized and were not discarded or abandoned until they were broken beyond repair and the materials recycled.

The Personal Artifact group (beads, bells, tobacco pipes, etc.) was the most numerous at the site. Although there were significantly more of these items in burials than pitfill, the expected frequencies were closer to the actual frequencies than most of the other groups. The proportionally greater number of glass beads in burials probably also was enhanced by the fact that burial and pitfill soils were all waterscreened, whereas, plowzone soils were dry-screened through 1/2-inch mesh screen. Nevertheless, personal items stood a somewhat better chance of reaching burial contexts than being lost, discarded, or abandoned. This was especially true of bells and whole pipes. A greater number of beads, because of their small size and presence en masse on clothing, were lost and thus became incorporated into pitfill. It is also worth noting that the Occaneechi personal assemblage of ca. 1680-1710 1acks some of the items common in other Southeastern trade assemblages. Conspicuous by their absence, for example, are the silver ornaments of the early to middle 18th-century Cherokee. Such assemblage differences may reflect different preferences, different sources (temporal and spatial) of trade goods, or different functions of trade items in the respective social structures. In the latter case, there may have been a need among the Cherokee for high-status ornaments (e.g., silver earrings, armlets, brooches, gorgets) that was lacking among the tribal Siouans.

Construction Tools and Farm Tools were rare in both contexts. This absence of items commonly reported on trade lists (France 1985) suggests that their route through the systemic context to the archaeological context had some distinctive features. Only two whole iron axes and two whole iron hoes were found in burial contexts, and one identifiable fragment each of an axe and a hoe was found in pitfill contexts. It can be suggested that hoes and axes were highly valuable working tools (second only to weapons in deerskin value), providing considerable advantages over their stone counterparts, and that they would have been maintained in the systemic context for as long as possible. They seldom were selected out of the system for burial (except possibly with influential adults), and they were not discarded until they were worn or broken beyond further use. Even in the latter cases, it is probable that the remaining iron was recycled, thus making the material unidentifiable as to its original form and function.

Miscellaneous Hardware, which consisted mostly of knives and knife parts, were associated significantly with pitfill contexts rather than burials. Nevertheless, all whole items in this category were found as burial associations (Burials 1, 2, 3, 5, 8, and 9 all contained one or two knives). Pitfill and plowzone contexts contained broken knife parts and two brass fishhooks. As with the axes and hoes, knives were valuable tools in day-to-day situations and probably would have been maintained as long as possible in the systemic context.

Items in the Metal Resource group, represented by aboriginally modified cut metal scrap and brass wire, were associated significantly with pitfill and plowzone. This distribution suggests that these materials were important to maintaining the technological system and were seldom used as grave goods.

Of artifacts in the Other group, only vermillion was present in burials. All other items in this group--coal, slag, and cinders, were found in pitfill and plowzone (although most of these materials in the plowzone probably are post-aboriginal, some examples of each were found in undisturbed pitfil1). Vermillion probably would not have reached the archaeological context unless it was placed in a 
burial. Otherwise, it would have been ground and mixed as paint, which seldom survives archaeologically.

Finally, items in the Indeterminate category (unidentifiable metal objects, mostly iron) had a significant association with nonburial contexts. These objects probably represent mostly recycled pieces of axes, hoes, knives, gun parts, and the like. Their distribution is not surprising given previous observations about their functions in the systemic context.

A second comparison, this time between pitfill contexts, is in order. The frequencies of items in all groups, except those having no examples in one or the other context (Table 6.5), are compared between burial pitfill and feature pitfill (burial associations and plowzone artifacts are not included). This comparison is made to determine if there are significant differences in the kinds of items occurring in one kind of pitfill as opposed to the other. Such a comparison is warranted given Ward's (this report) suggestion that the upper fill of the burial pits was associated with death feasting and intentional deposition over graves.

A Chi-square test on the overal1 16-cel1 matrix, with seven degrees of freedom, yielded a Chi-square statistic of $10.56, p<.20$. Thus, overall, the two pitfill assemblages are not significantly different. Two categories--Architecture and Food Preparation and Consumption-that have divergent actual and expected frequencies also have too few examples to warrant interpretation. Thus, it appears from the present data that activities producing fill for burial pits were essentially the same as those for other types of pits.

In order to determine if the Euroamerican assemblage at the Fredricks site would be different from other historic Piedmont sites, a comparison was made, by functional category, between the burial assemblage at the Fredricks site and the Upper Saratown site. The latter site appears to represent a slightly earlier period than the former and is located in a position about 60 miles more remote from trade sources. To obtain a comparative sample from Upper Saratown, five adults and four subadults from 87 burials previously analyzed by Navey (1982) were randomly selected. The distributions by age category and the total number match those of the Fredricks sample.

Three of the Upper Saratown burials (two adult and one subadult) contained no artifacts. The remaining six burials contained a total of 13,391 Euroamerican trade items. These were 13, 345 glass beads, 21 brass (or copper) beads, 18 brass "hairpins" (tubular beads), three brass bells, one clay pipe bowl, and one iron axe head. When grouped by the same functional categories used to analyze the Fredricks site
Table 6.5

Frequency of Euroamerican artifacts in burial pitfill and non-burial pitfill.

\begin{tabular}{lcc}
\hline Functional Group & Burial Pitfil1 & Non-Burial Pitfil1 \\
\hline Architecture & 9 & 3 \\
Arms & 19 & 25 \\
Food Preparation/Consumption & 3 & 10 \\
Personal & 25 & 448 \\
Miscellaneous Hardware & 8 & 3 \\
Metal Resources & 3 & 4 \\
Other (By-products) & 20 & 16 \\
Indeterminate & 509 & 510 \\
$\quad$ Total & & 1 \\
\hline
\end{tabular}

assemblage, the total was 13,390 artifacts in the Personal group and one (iron axe) in the Construction Tool group. This comparison clearly shows that at the Upper Saratown site far more items of personal (mostly ornamental) use were selected for inclusion in burials than items in other categories, as compared with a more varied assemblage at the Fredricks site. A cursory examination of the artifact catalog for the Upper Saratown site indicates that a bias for personal items would also be found in non-burial contexts.

\section{CONCLUSIONS}

In summary, this research has examined the Euroamerican artifacts from three archaeological sites in the Piedmont region of North Carolina, the Fredricks site (310r231), Wall site (310r11), and Mitchum site (31Ch452). The distribution of various classes of historic material from the Fredricks site indicates that the aboriginal occupants of the site were the recipients of Euroamerican trade goods that date from the mid-17th century to the first decade of the 18th century. This dating strongly supports an interpretation of the Fredricks site as John Lawson's Occaneechi Town. An analysis of datable Euroamerican artifacts from burial and feature contexts suggests that the trade good assemblage remained consistent throughout the probable 20 to 30-year occupation of the site. A more secure interpretation of possible temporal variation, however, will have to await excavation of larger portions of the site.

A comparison of the trade items by functional category between types of pitfill (burial fill vs. feature fill) at the Fredricks site did not reveal significant differences. The same comparison between the contexts of burial association vs. pitfill (burial and feature combined), however, did show several significant differences. If one ignores the problem of comparing mostly whole items in the burials with mostly fragmented items in the 
pitfill, a statement can be made that items in the Personal and Clothing categories were more often selected for inclusion in burials, relative to their frequency in the overall site assemblage, than items in other categories.

When the Fredricks site burial assemblage was compared to a sample burial assemblage from the Upper Saratown site, it was discovered that almost no non-personal items were present in the latter assemblage. This finding suggests that more utilitarian (i.e., construction, farming, food preparation, architectural, and arms) items were traded to the Occaneechi in ca. 1700 than to the Sara in ca. 1675. At least, more utilitarian items reached burial contexts at the later Fredricks site.

At the Wall site, the historic artifacts suggest a late occupation, probably early 19th century, of white settlers who farmed in the area of the river bend. Some plowzone items (e.g., Euroamerican ceramic sherds, glass fragments, and nails) from the Fredricks site also indicate some 19th-century activities on or near this site. Based on the historic artifact analysis, the Mitchum site $(31 \mathrm{Ch} 452)$ seems to represent an early period of Euroamerican trade (1660-1680) and/or a smaller settlement geographically removed from major trade routes.

A broader data base is needed to study the effects of Euroamerican contact among the Piedmont Indian groups and changes in their material culture. As Kent (1984:293) points out, culture contact and its results-acculturation and culture change--can cause very abrupt shifts in the frequencies of certain artifacts, especially when compared to systems in which there are no major outside influences. The measurement and display of these changes can be illuminated through intersite patterning within a larger geographical region. 


\title{
Chapter 7
}

\section{Shell Artifacts from the Carolina Piedmont}

\author{
by
}

\author{
Julia E. Hammett
}

\section{INTRODUCTION}

The shell artifacts from the Wall site (310r11) and the Fredricks site (310r231) are made up of two basic kinds: tools and ornaments. Only one tool type, the serrated shell, has been identified in the artifacts recovered thus far, and it will be discussed at the end of this section. Because of their greater number and complexity, the ornaments will be discussed at length.

Anthropologists have long known that human ornamentation reflects economic, political and other types of social information (e.g., Spencer 1886; Bunzel 1933). More recently, Binford (1972) has addressed the potential for studying the relationships of artifacts to the maintenance of social relationships. He defined "sociotechnic" artifacts as "material elements having their primary functional context in the social subsystems of the total cultural system" (Binford 1972:24).

In western North America, shell beads and other ornaments recovered from archaeological contexts have proven useful not only for dating purposes (Bennyhoff and Heizer 1958), but they have also produced important information on intergroup and intragroup dynamics and more generally on the evolution of social organization in that region (King 1982). Although shell ornaments are a fairly common occurrence in burials at late prehistoric, protohistoric and historic Indian sites in North Carolina, little attention has been devoted to these artifacts. Recently, pioneer work to establish a bead typology for the Tennessee mountain area was initiated by Polhemus (1983).

The present study is devoted to establishing shell ornaments as a significant class of artifacts for addressing questions about changing social interactions from the Late Woodland period until historic times in the Carolina Piedmont area. Following the presentation of a preliminary bead typology, several issues regarding the changing relationships between shell ornaments, their makers, traders and holders will be addressed. These issues will be approached through the examination of: 1) beadmaking techology; and 2) distributions of various ornament types and styles within specific site contexts and between sites.

Ornaments from the Fredricks and the Wall sites will be used as the focus of this study, to provide a qualitative and quantitative characterization of the ornament types and their uses and to ascertain differences between prehistoric and historic social contexts. Finally, ornaments from other sites in western North Carolina will be examined and compared with the ornaments from the two Piedmont sites to determine the place of shell ornaments in social interactions on a regional scale.

\section{BEADMAKING TECHNOLOGY}

At the Wall site, a protohistoric village, many ornaments, (e.g., segments, tubes and spherical beads) were made from the columella of large marine gastropods, probably of the Melogenidae (Crown Conch) family which occur along the Atlantic seaboard from Massachusetts to Florida (Percy 1972). Other items, consisting of small disks, gorgets and pendants, were made from the outer whorl of these large univalves. A small univalve called marginella (of the Marginellidae family) which occurs along the coast from the West Indies to the southern beaches of North Carolina (Percy 1972) was also used as a form of ornament.

On the whole the coastal univalves were the source material for the greatest proportion of ornaments. To a much lesser extent bivalve shells (presumably mussel) and stone fragments were made into small disk beads, and native copper and mica were used for other types of ornaments.

By the time the Fredricks site was occupied, many of the previously available bead forms (pendants, tubes, and sphericals) had been modified and others (columella segments and marginellas) had been all but dropped from use. Several new types--runtees and cylinder/barrels made from columella, and wampum made from quahogs (Mercenaria mercenaria)--had appeared. Quahogs occur from the Gulf of St. Lawrence to Florida (Percy 1972). Although small disk beads appear to have retained both their form and function through this time, they too show indi- 
cations of the impact of European influence. Aboriginal beadmaking and its transformation through the introduction of European technology is a topic worthy of lengthy consideration. For purposes here, however, discussion will be confined to ethnohistorical accounts of beadmaking and the use of this information for 1) characterizing aboriginal beadmaking kits and beadmaking detritus, and 2) the identification of changes in the technology that may be manifest in the differences between the two Piedmont Carolina sites.

Even though many of the early historical descriptions of beadmaking focus on wampum, and thus are most relevant to the New England area, these accounts can serve as a general model for approaching beadmaking technology. In 1634, William Wood called the Naragansett the "mintmasters" of "wampompeag and mowhacheis, which they form out of the inmost wreaths of periwinkle shells." This group appears to have been the traditional providers of money beads in New England (Vaughan 1977:81). In 1643, Roger Williams of New England published A Key Into the Language of America which was primarily devoted to the language of the Naragansett. In his key he gave a list of Indian words pertaining to bead money:

\section{Naragansett Word}

Wauompeg

Wauompesichick-mesim

Assawompatittea

Anawsuck

Meteauhock

Suckauanausuck

Suckauaskeesaquash

Puckwheganash

Mucksuck

Papuckakiuash

Natouwompitea

Nnanatouwompiteem

Natouwompitees

Puckhummin

Puckwhegonnautick

Tutteputch anawsin

Qussuck-anash

Cauompsk

Nickautick

Enomphommin

Aconaqunnauog
Williams' Translation

Give me white

Give me white

Come, let us change

Shells

The Periwinckle

The blacke Shells

The blacke eyes, or

that part of the shel-fish called Poquauhock (or Hens) broken out neere the eyes, of which they make the blacke.

Awl blades

Awl blades

Britle, or breaking, which they desire to be hardened to a britle temper.

A coyner or Minter

I cannot coyne

Make money or Coyne

To bore through

The awl blade sticks

To smooth them, which they doe on stones.

Stone, Stones

A Whetstone Pincers or Vice

To thread or string

Thread the Beads
A kinde of wooden

Williams (1936:156-157) added this observation to his list of words:
Before ever they had awle blades from Europe they made shift to bore this their shell money with stone and so fell their trees with stone set in a wooden staff, and used woden howes: which some old \& poore women (fearfull to leave the old tradition) use to this day.

In 1709 John Lawson (Lefler 1967:204), who had travelled through North Carolina described Indian beadmaking:

This [the shell preform] the Indians grind on Stones and other things, till they make it current but the Drilling is the most difficult to the Englishmen, which the Indians manage with a Nail stuck in a Cane or Reed. Thus they roll it continually on their Thighs, with their Right-Hand holding the Bit of Shell with this Left, so in time they drill a Hole quite through it, which is a very tedious Work; but especially in making their Roanoak, four of which will scarce make one Length of Wampum.

Based of the above ethnohistorical information, we can expect to see several manifestations in the archaeological record. The basic beadmaking process appears to have involved first obtaining a preform from the shell by breaking or the groove-and-snap technique, then reducing the piece, and finally drilling, grinding, and smoothing. Prehistoric tool types for this work would have included hammerstones, stone drills, burins, chisels, and anvils, grinding stones, and stone or pottery abraders. At historic beadmaking stations one should find many of the components of the prehistoric tool kit along with the replacement or addition of metal tools such as awls/drills/needles, pincers/tongs/vices for holding preforms, and perhaps also hammers and saws for reducing the shells. Where the purple wampum were made, we should expect to find Quahog shells (Mercenaria mercenaria). Where other types of beads and ornaments were manufactured, there should be refuse from univalve shells (called periwinkles in the 1600-1700s).

Beadmaking was probably limited to only a few sites within a region. It is also probable that in areas where beads and other ornaments were used as a medium for exchange, there would have been standardization of bead forms and specialization of manufacture by only a few groups or even individuals within a group. Such was the case not only for the Naragansett in New England, but also in the western United States where the Chumash were the major producers of beads for a region that extended from the California coast to the Great Basin and into the Southwest (Bennyhoff and Heizer 1952; King 1982). One might hypothesize that the degree of beadmaking specialization, may reflect the extent of devotion to a money-type economy. It would follow that the fewer the sites of manufacture and the greater the standardization of a form or style, the greater the investment 
into this type of economic system. These suggestions will be considered when reviewing the data from the Siouan Project area.

\section{ORNAMENTS FROM THE SIOUAN PROJECT}

\section{Pendants}

Pendants were made from either shell or mica. Some of the she11 pendants from the Siouan Project have previously been described by Sizemore (1984), and her work is extensively drawn upon here. All the shell pendants in the present study sample are of a ground circular discoidal form, and have either a single central perforation or a pair of central perforations. The double holed style has been called elsewhere "gorget" (e.g., Benthall 1969; Polhemus 1981). The shell gorgets from the Wall site were found with two subadults (Burials 2 and 1-83). Burial 2 (1940-41 excavations) had three relatively small pendants, two having the double perforation of gorgets and one having a single hole. Burial 1-83 (1983 excavations) had one larger pendant. All of these are plain, meaning that they bear no evidence of any superficial design (Figure $7.1: \mathrm{a}-\mathrm{e})$. They are strikingly similar to other pendants and gorgets found at two sites in southwestern Virginia, the Shannon site (44My8) (Benthal1 1969) and RLA-Vir196 (Figure 7.1:b).

The two pendants from the Fredricks site were found with Burial 1 in 1983. Both of these pendants have single holes (Figure 7.2). The hole in the larger one is slightly elongated and appears to have once been two holes so close together that after some wear they broke through, making the two holes one. The designs on both of the pendants are of the drilled dot technique. Sizemore (1984) gave the following description of the design on the larger pendant:

Beginning from the center are six punctated lines, each with a short segment hooking to the left at the line's end, thereby making a swastika-like design. The swastika is included within the Cross design category of Fundaburk (1957:39), and is one of the most common designs on Southeastern gorgets. It is usually enclosed within a "Sun Circle" design. The swastika on this gorget is closely surrounded by a punctated circle, making up the inner border of a band of punctated chevrons or triangles with their apices pointing inward. Fifteen chevrons are visible; the rest have eroded. The bases of these figures rest on another punctated circle which almost completely encircles the gorget, with on segment in the top right portion having been eroded away. Outside of this circle there are three distinct punctated triangles similar to the others, and what seem to be parts of other triangular designs that are mostly eroded.
Sizemore also described the smaller pendant :

On the concave side, and emanating from the central perforation, is a six-pronged star design made of punctations. Encircling the end of these prongs seems to be two, possible three, circles of punctations, the inner circle(s) made up of larger depressions than the outer and partially eroded circle of small dot-like punctations.

Sizemore noted that a pendant with a design very similar to the smaller pendant was found at the Irene Mound Site near Savannah, Georgia (Caldwe11 and McCann 1941:Plate XIX). Other pendants of the drilled dot technique were also found there, as well as specimens of the incised (and sometimes painted) rattlesnake style of gorget (Figure $7.1: \mathrm{h}-\mathrm{i}$ ), which was found at Early Upper Saratown (31Sk1). The latter style has been found a numerous sites to the south and west from Tennessee to St. Catherine's Island off the coast of Georgia (Ann Tippitt, personal communication). The drilled dot pendants, on the other hand, have been found exclusively to the north, primarily in Virginia, with the exception of the Irene Mound specimens (Sizemore 1984). The distribution of these pendants suggests that the area of the Siouan Project may have been at or near the boundary between southern and northern spheres of influence.

A badly corroded design resembling the one on the larger of the two pendants, was scratched or incised onto the back of the metal spoon from Feature 6/Burial 8, another subadult (Figure 7.3). Other examples of pendants with similar designs made by this drilled dot technique were recovered from the Potomac Creek site, in northeastern Virginia (Stephen Potter, personal communication), and the Irene Mound site near Savannah, Georgia (Caldwell and McCann 1941). None of the pendants from the Wall or Fredricks sites are of the incised and painted "rattlesnake" style mentioned above.

In 1701, John Lawson (Lefler 1967:204) noted that:

they oftentimes make...a sort of Gorge, which they wear about their neck on a string; so it hangs on their collar, whereon sometimes is engraven a Cross, or some odd sort of Figure, which comes next in their Fancy.

If his observation is taken literally, one would expect there to be no limits to the stylistic variation on these pendants, except those bounded by the individual's imagination, yet there do seem to be set styles. If pendant blanks were being traded than people at specific localities could have individualized their ornaments within the limits ascribed by their group, be they techniques, style elements or motifs. It remains unclear whether or not specific elements or motifs were owned or controlled by 


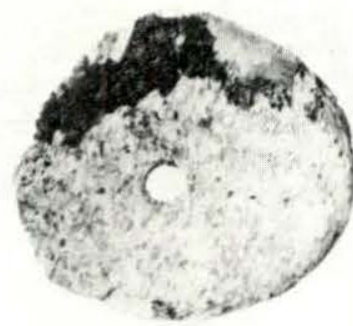

a

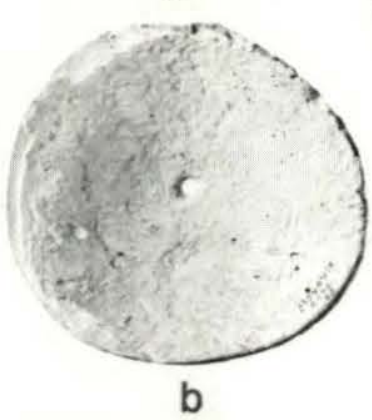

b
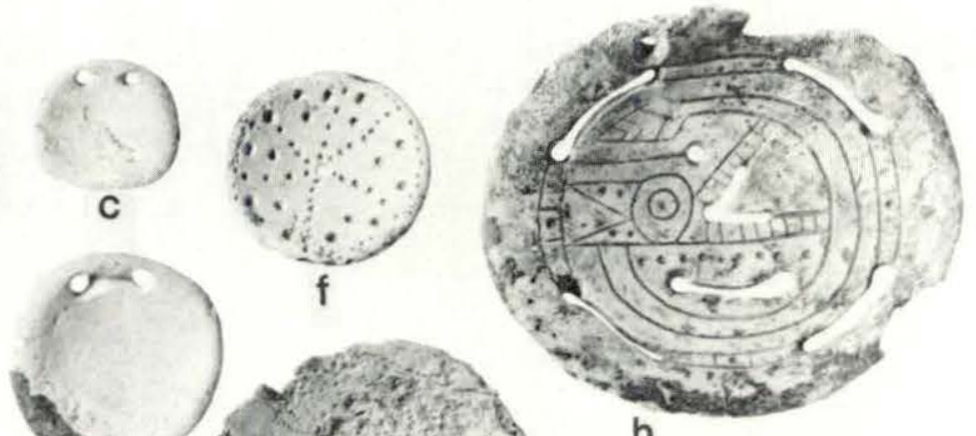

$\mathrm{h}$

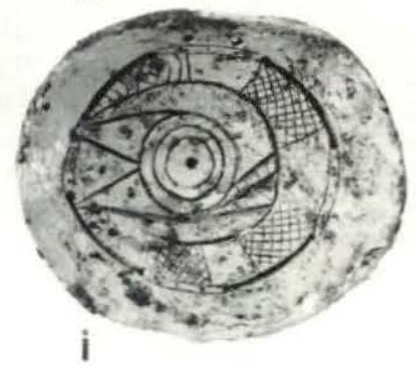

Figure 7.1

Pendants and gorgets: plain pendants from Burial $1-83$ at the Wall site (a), RLA-Vir196 (b), and Burial 2 at the Wall site (c-e); drilled dot pendants from Burial 1 at the Fredricks site $(\mathrm{f}-\mathrm{g})$; rattlesnake gorgets from Upper Saratown $(h-i)$.
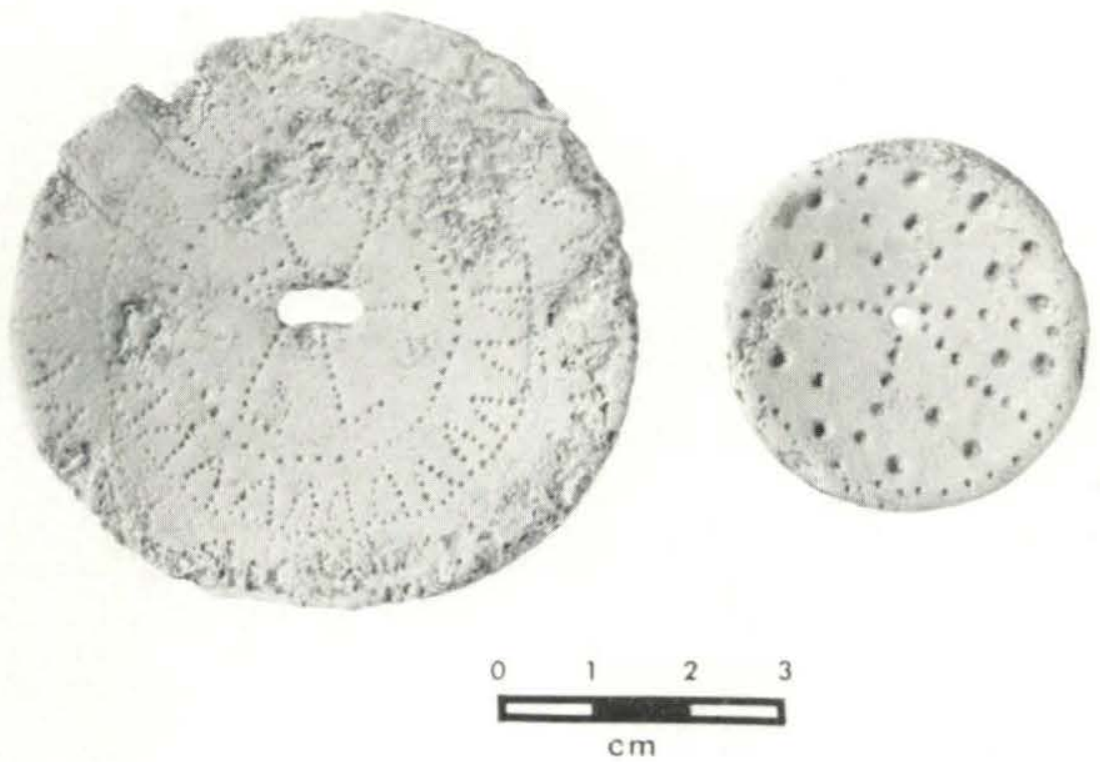

Figure 7.2

Close-up of pendants from Burial 1 at the Fredricks site. 

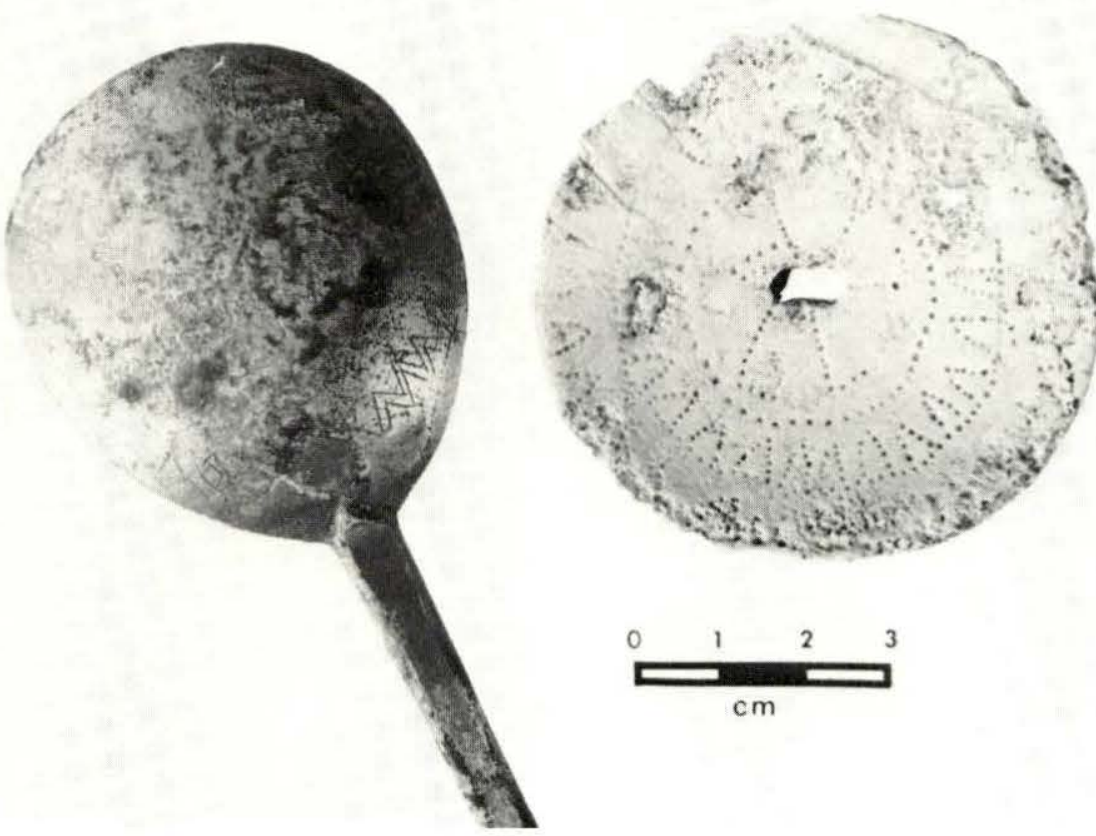

$\mathrm{cm}$

Figure 7.3

Comparison of designs on large pendant from Burial 1 and on spoon from Burial 8 at the Fredricks site.

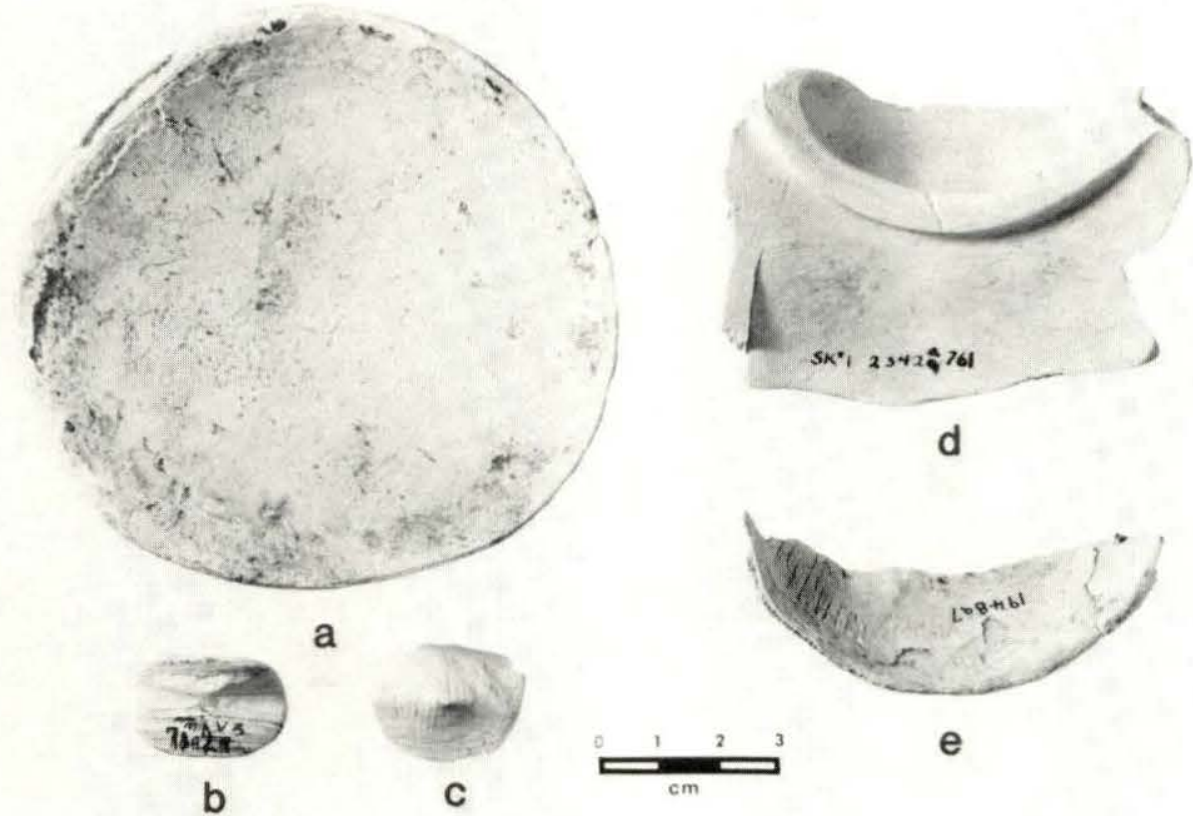

Figure 7.4

Evidence of beadmaking: pendant blank (a); ground fragments of large univalve shell (b, d-e); and reground segment bead (c). 
groups or even individuals, yet these pendants may well have served as social identity markers that would probably have been worn while traveling. This would have allowed the wearer to transmit information about their cultural affiliations, place of origin and quite possibly any associated allegiances as well.

Overall there appear to have been standard forms of shell pendants in the region, the plain discoidal shape being the most common. A pendant blank found at the Sharp site (31Rk12) may indicate they were traded in this unfinished form (Figure 7.4:a). The residents of the Wall site shared in this regional exchange of gorgetstyle pendants prehistorically. The subadult from the Fredricks site (or his or her kin) either had access to even longer distance trading, or perhaps originally resided outside the local area.

\section{Tube Beads}

Tube beads have been found only at the Fredricks site. Tubes made from columellas have been recovered from other sites in the region sharing many other bead forms with the Wall site such as Early Upper Saratown (31Sk1) to the northwest and two southwestern Virginia sites, the Shannon site and RLA-Virl96 (Table 7.1). The absence of this type at the Wall site may be more a result of small sample size than the lack of use of this form. The tubes from both of the Virginia sites were drilled down the center of the tube from either end of the long axis. Two of the tubes from RLA-Vir196 demonstrate the difficulty of drilling a narrow hole through these tubes. Where the columella is at its narrowest, the artisan's drill came through the tube along the silca groove. One of the tubes from this site was broken diagonally at some point. The well ground edges of this break suggest that this did not deter its owner from continuing to use the ornament (Figure 7.5). This suggests there was a high value placed upon the ornament form despite any imperfections. At Early Upper Saratown all the finished tubes were drilled at an angle from the end of the tube to a side. This corner drilled technique would have drastically reduced the time and energy investment and risk of drilling out the long axis.

The eleven tubes from the Fredricks site were all recovered from the chest area of Feature 2/Burial 4 which was a bundle burial containing a male adult and an infant (Wilson, this report). The tubes were found lying together in parallel order on the adult's chest. The nine tubes that were complete ranged in size from $105-123 \mathrm{~mm}$ in length and $6.0-7.4 \mathrm{~mm}$ in diameter. Their hole diameters ranged from $2.0-2.7 \mathrm{~mm}$. These measurements demonstrate the uniformity of these tubes. On none of them is the silca groove very apparent, unlike all the tubes from earlier contexts. No doubt the use of a metal drill enabled the $\operatorname{artisan}(\mathrm{s})$ of these historic tubes to produce a much more refined product with much less energy expenditure, however, even so some evidence of the groove should be apparent no matter how narrow the tube given the depth of this groove on other tubes from the area.

These historic tubes actually resemble the hair pipes used by Plains Indians which were manufactured commercially by Dutch settlers in Bergen County, New Jersey, from the West Indian conch, Strombus gigas (Ewers 1957). These tubes were made from the thick lip of the outer whorl of the conch. If the tubes from Fredricks site were made from this thicker lipped West Indian conch, it would explain the absence of a silca grove which only occurs on the inner columella part of the she11. Although the evidence suggests that commercial manufacture of these articles by the Dutch was begun between 1776 and 1798 (Ewers 1957:42), it is possible that the tubes at the Fredricks site represent a somewhat earlier example of trade for this distant source of shell. If this is true, then tubes, like the pendants, indicate a longer distance trade network historically than there is evidence for prehistorically at this locality.

\section{Colume11a Segment Beads}

Segment beads were made from the columella of large univalves by cutting or by a "grooveand-snap" technique. The resulting beads were then smoothed, drilled and strung. This bead type varies considerably in size and shape within specific contexts at each site, and to an even greater extent between sites. Their overall shape maintains the basic form of the columella although they are variable in size and length; hence they range from almost a tubular shape to a disk shape. As segments of quite different forms were worn together (Figure 7.6:a) with no indication of shape distinction, they were lumped under the single class of "segment," with only a size distinction of smal1, medium and large noted.

At the Wall site, medium segments ranged in length from $4.7-13.9 \mathrm{~mm}$, in width from $10.7-18.7$ $\mathrm{mm}$, and in thickness from $7.4-15 \mathrm{~mm}$. The small-sized segments ranged in length from 2.2-6.5 mm, in width from 4.9-7.7 mm, and in thickness from $4.0-6.5 \mathrm{~mm}$. No segments of this "primary" style were recovered from the Fredricks site. A majority (75\%) of the large segments and all of the small segments came from Burial 1-83 at the Wall site. For the most part, the segments occurred in long rows (presumably strings) around the neck as a necklace and around the arms and legs as bracelets and anklets. The remainder of the segments from the Wall site, which were recovered from Burial 2-83 and Burial 4 (1940-41 excavations), were also worn as necklaces.

The preform nature of this basic bead type allowed these segments to be modified into more finished forms of beads, some of which also were found at the Fredricks site. Also, the larger 
Table 7.1

Preliminary 1nventory of shell artifacts from selected Southeastern sites.

\begin{tabular}{|c|c|c|c|c|c|c|c|c|c|c|c|c|c|c|c|c|c|c|c|}
\hline Site & $\begin{array}{c}\text { She11 } \\
\text { Pendants }\end{array}$ & $\begin{array}{l}\text { Tube } \\
\text { Beads }\end{array}$ & $\begin{array}{l}\text { Barrels/ } \\
\text { Cylinders }\end{array}$ & $\begin{array}{c}\text { Spherical } \\
\text { Beads }\end{array}$ & $\begin{array}{c}\text { Ear } \\
\text { Ornaments }\end{array}$ & $\begin{array}{c}\text { Col un } \\
\text { Large }\end{array}$ & $\begin{array}{l}\text { ella Seg } \\
\text { Medfum }\end{array}$ & $\begin{array}{l}\text { ints } \\
\text { Sma11 }\end{array}$ & $\begin{array}{l}\text { Olive } \\
\text { Shells }\end{array}$ & $\begin{array}{l}\text { Beadmaking } \\
\text { Evidence }\end{array}$ & $\begin{array}{l}\text { Smal1 } \\
\text { Disk } \\
\text { Beads }\end{array}$ & $\begin{array}{l}\text { Large } \\
\text { Disks }\end{array}$ & Wampum & Runtees & Marg1nella & $\begin{array}{l}\text { Native } \\
\text { Copper }\end{array}$ & Mica & $\begin{array}{l}\text { Serraced } \\
\text { She11 }\end{array}$ & other \\
\hline \multicolumn{20}{|c|}{ North Carolina Sites } \\
\hline $\begin{array}{l}\text { Warren w11son } \\
\qquad(31 \mathrm{Bn} 29)\end{array}$ & $\mathrm{x}$ & - & $\mathrm{x}$ & $\mathrm{x}$ & $\mathrm{x}$ & $\mathrm{x}$ & - & - & - & - & $x$ & - & - & - & - & - & $\mathrm{x}$ & - & - \\
\hline $31 \mathrm{Cy} 42$ & - & - & - & - & - & - & - & - & - & $\begin{array}{l}\text { univalve } \\
\text { detritus }\end{array}$ & - & - & - & - & - & - & - & - & - \\
\hline $\begin{array}{l}\text { Town Creek } \\
\text { (31Mg 3) }\end{array}$ & - & - & $\mathrm{x}$ & - & - & 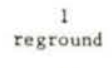 & - & - & - & $\begin{array}{l}\text { univalve } \\
\text { detritus }\end{array}$ & - & - & - & - & $\mathrm{x}$ & - & - & - & - \\
\hline $\begin{array}{l}\text { Wa11 } \\
\qquad(310 \mathrm{r} 11)\end{array}$ & $x$ & - & - & - & - & $\mathrm{x}$ & $\mathrm{x}$ & $\mathrm{x}$ & - & - & $\mathrm{x}$ & $\mathrm{x}$ & - & - & $\mathrm{x}$ & $\mathrm{x}$ & $\mathrm{x}$ & $\mathrm{x}$ & $\begin{array}{c}\text { perforated } \\
\text { clan } \\
\text { shell }\end{array}$ \\
\hline $\begin{array}{l}\text { Fredricks } \\
\quad(310 \mathrm{r} 231)\end{array}$ & $\mathrm{x}$ & $\mathrm{x}$ & $\mathrm{x}$ & $x$ & $\mathrm{x}$ & - & $\mathrm{x}$ & - & - & - & $x$ & $\mathrm{x}$ & $\mathrm{x}$ & $\mathrm{x}$ & - & - & - & - & - \\
\hline $\begin{array}{l}\text { Sharp } \\
\quad\left(31 R_{k} 12\right)\end{array}$ & 1 blank & - & $\mathrm{x}$ & - & - & - & - & - & - & - & $\mathrm{x}$ & $\mathrm{x}$ & - & - & - & - & - & - & - \\
\hline $\begin{array}{c}\text { Early Upper } \\
\text { Saratown } \\
(315 k 1)\end{array}$ & $\begin{array}{c}\text { X } \\
\text { "ratt1esnake" } \\
\text { gorgets }\end{array}$ & $\begin{array}{c}x \\
\text { 1ncluding } \\
\text { I blank }\end{array}$ & $\mathrm{x}$ & $\mathrm{x}$ & - & - & - & - & - & $\begin{array}{l}\text { univalives: } \\
\text { detritus: } \\
\text { whole (hoes?) }\end{array}$ & $x$ & $\mathrm{x}$ & - & - & $\mathrm{x}$ & - & - & $\mathrm{x}$ & - \\
\hline $\begin{array}{l}\text { Upper Saratown } \\
\quad(31 \mathrm{Sk} 1 \mathrm{a})\end{array}$ & n & - & $\mathrm{x}$ & - & $\mathrm{x}$ & - & $\mathrm{x}$ & - & - & - & $\mathrm{x}$ & - & $\mathrm{x}$ & - & - & $\mathrm{x}$ & - & $\mathrm{x}$ & - \\
\hline \multicolumn{20}{|l|}{ Virginia Sites } \\
\hline RLA-V1r150 & $\begin{array}{c}15 \\
\text { triangular }\end{array}$ & - & $\mathrm{x}$ & $\mathrm{x}$ & - & - & - & - & - & - & $\mathrm{x}$ & $\mathrm{x}$ & - & - & $x$ & $x$ & - & - & - \\
\hline $\begin{array}{l}\text { Leatherwood } \\
\text { Creek } \\
(44 \mathrm{Hr} 1)\end{array}$ & $\mathrm{x}$ & $\mathrm{x}$ & $\mathrm{x}$ & $\mathrm{x}$ & - & - & $\mathrm{x}$ & - & - & - & - & - & - & - & $x$ & - & - & - & - \\
\hline $\begin{array}{l}\text { Shannon * } \\
\quad(44 \text { My } 8)\end{array}$ & - & - & - & - & - & $x$ & $x$ & - & $x$ & - & $x$ & - & - & - & $x$ & - & - & - & - \\
\hline \multicolumn{20}{|c|}{ South Carolina Sites } \\
\hline RLA-SoC28 & - & - & - & - & - & - & - & - & - & $\begin{array}{l}\text { univalve } \\
\text { detritus }\end{array}$ & - & - & - & - & - & - & - & - & - \\
\hline Georgia Sites & & & & & & & & & & & & & & & & & & & nins. \\
\hline Irene Mound * & $\mathrm{x}$ & - & $x$ & - & - & - & - & - & $x$ & $\begin{array}{l}\text { untvalves } \\
\text { (hoes?) }\end{array}$ & $x$ & - & - & - & $x$ & - & - & - & $\begin{array}{c}\text { awls } \\
\text { ladle(?) }\end{array}$ \\
\hline
\end{tabular}

- Shannon S1te: Bentha11 (1969)
Irene Mound: Caldwel1 and McCann (1941) 

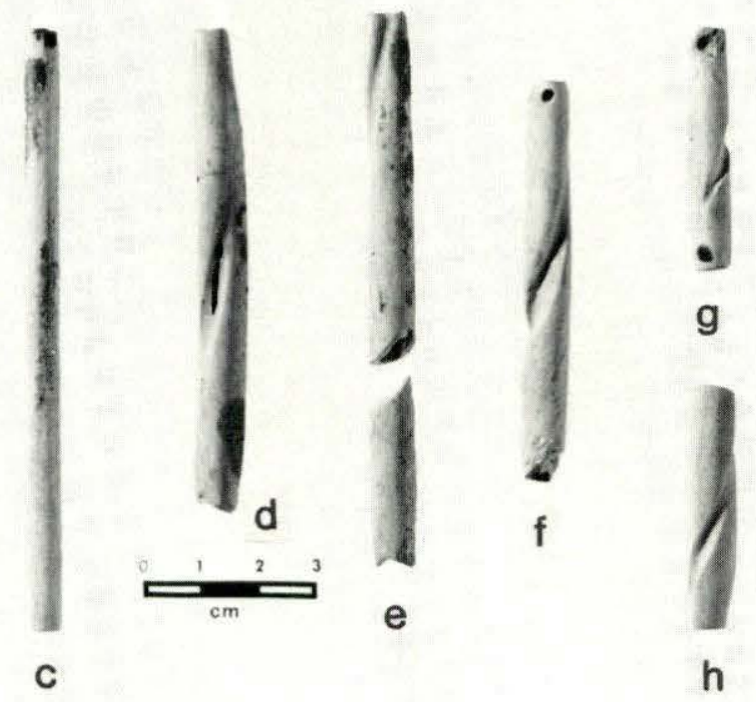

Figure 7.5

Tube beads from the Fredricks site (a-c), RLA-Vir196 (d-e), and Upper Saratown $(f-g)$; tube blank from Upper Saratown (h).

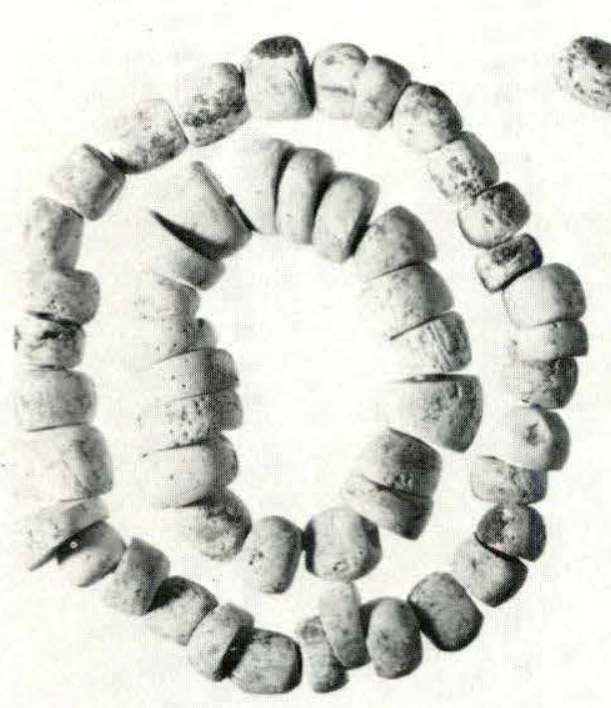

a
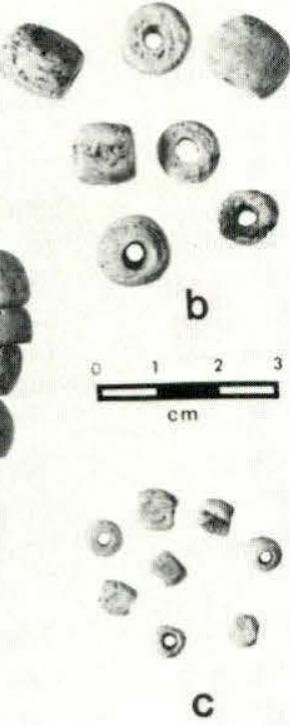

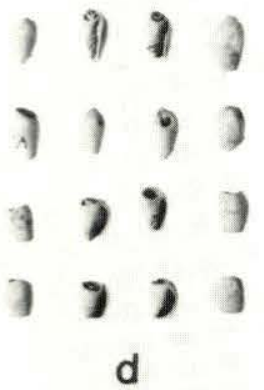

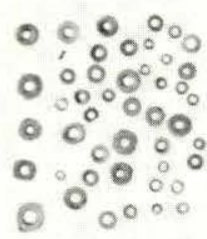

e

Figure 7.6

Bead types from the Wall site: columella segments from Burial 1-83

(a); medium-sized (b) and small (c) loose segments; marginellas

(from top to bottom: shoulder ground, spire ground, and spire and base ground) (d); and disks (e). 
segments could be ground down and reworked into a smaller form if they were broken. This was exhibited in a reground large segment fragment from Town Creek (31Mg3) (Figure 7.4:c). Two beads made from segments having a more finished, symmetrical form and leaning toward the discoidal end of this bead form's spectrum were present in Feature 3/Burial 5. These beads were found above the left temporal, next to the left ear and are believed to have been a form of ear ornament.

\section{Spherical Columella Beads}

Spherical columella beads were present in burial contexts at both sites. The specimens from the Wall Site appear to have been made by roughly grinding a segment bead into a fairly spherical shape. These occurred with marginella ornaments on the headdress of Burial 2 (1940-41 excavations).

At the Fredricks site spherical beads occurred with barrel/cylinders, runtees, and wampum (all discussed below) around the neck and chest area of Burial 2. All of these spherical beads were very badly preserved (Figure 7.7:d). It appeared from the few intact surfaces, that they were of a more finished quality (i.e. smoother and more polished) than those from the Wall site. Measurements ranged from $4 \cdot 6-7.2 \mathrm{~mm}$ in length and from $5.4-7.3 \mathrm{~mm}$ in diameter.

\section{Barre1/Cylinder Beads}

Like the other beads discussed so far, barrel/cylinder beads were made from the columella. They vary from segments in that they are characteristically longer than wide, and they have been ground along their long axis so that they are more symmetrical than the segments. For the Tennessee area, Richard Polhemus has outlined several discrete classes including, cylinders, barrels, elongate, flattened elongate, which he considers to have specific temporal and contextual placements (Polhemus 1981); however, a preliminary study of beads from North Carolina mountain sites (Hammett 1983) indicated that while these morphological attributes did occur in these Carolina sites, the measurements of these bead types overlapped too much to be quantified. Fifty-four barrel/cylinder beads were recovered from around the chest and neck of Burial 2 at the Fredricks site. They range in length from $12.2-15.4 \mathrm{~mm}$, in diameter from $5 \cdot 0-8.5 \mathrm{~mm}$, and thickness from $4.9-7.5 \mathrm{~mm}$. All are badly damaged.

\section{Sma11 Disks}

Small disk beads, most of which were probably made from the wall sections of large univalves, occurred at both sites in large numbers, comprising approximately $90 \%$ of each site's ornament assemblage. Small white beads made up $90 \%$ of the total glass bead assemblage at Fredricks site as well (Carnes, this report). Apparently there was a continuous use and demand for this general bead form (first shell and then of glass and shell) throughout the period under consideration. Only two small disks were made from a dark stone, possibly argillite. Both occurred in contexts with shell disk beads, suggesting they were a rare variation on the more commonly occurring type.
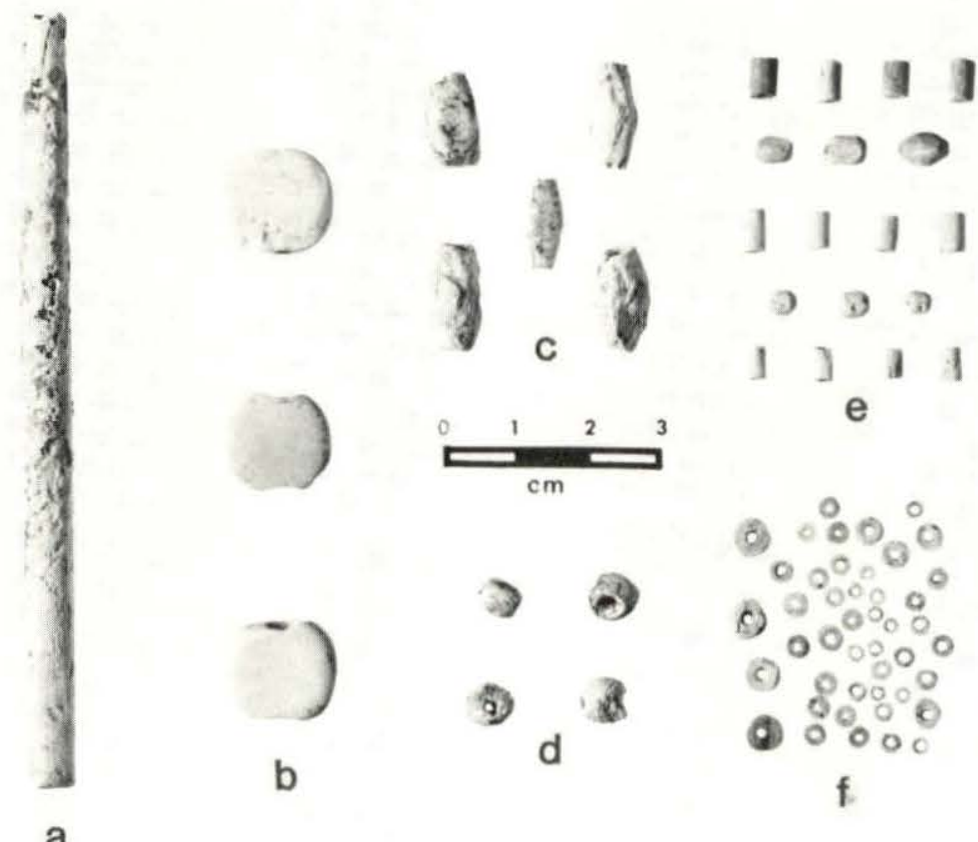

e
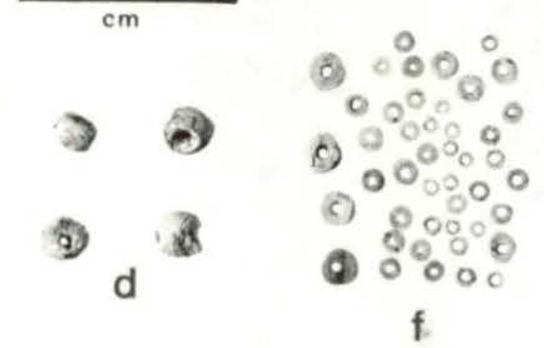

a

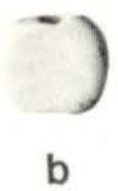

Figure 7.7

Bead types from the Fredricks site: tube bead (a); runtees (b); barrel/cylinders (c); spheres (d); wampum (e); and disks (f). 
In studies of shell disks in California, Chester King (1982) observed that, through prehistoric times, a gradual reduction in shell disk in size were correlated with increased social status differentiation. He explained this change with a cost/benefit model in which a greater energy investment was demonstrated by reduced bead size, and a higher the value was placed on the item and more prestige accorded to it. Shortly after European contact the Spanish disrupted the traditional beadmaking and exchange system. For example, manufacture, which had formerly been limited to residents of Santa Cruz Island, became a more widespread craft. This dispersion of the craft resulted in a drastic reduction in the standardization of this medium of exchange. There was also a decline in bead value. Disk beads, which had been getting smaller and smaller, suddenly became larger and cruder.

Using King's model, a hypothesis was developed for the Siouan Project disk beads, stating the expectation that they would pass through changes similar to the California beads. Preliminary observations of disks revealed that the Wall site disks were all stone drilled. Many of the Fredricks site disks, on the other hand, were drilled or punched with a small cylindrical object, presumably a metal nail, needle, or awl. The use of metal tools was suggested by the sharp sides of the bead holes as opposed to the hourglass concavity present on beads which had been slowly drilled from both sides (Figure 7.8). Also, a few of the Fredricks site beads revealed a larger, seemingly "unfinished" form similar to the rough disks from historic contexts in California. Several also revealed a triangular or quadrangular shaped hole which suggested they were not drilled but driven through by a very hard object, again a sign of the use of metal tools.

To test the King hypothesis, disk beads from each site were sorted by size using U.S. standard screens with mesh diameters of $4.0 \mathrm{~mm}$, $2.83 \mathrm{~mm}, 2.38 \mathrm{~mm}, 2.0 \mathrm{~mm}$, and $1.0 \mathrm{~mm}$. Beads caught in each screen were counted and the condition of each bead was noted. For each sample, the number of complete beads (with all edges complete), the number of damaged beads (that may have fallen through the screen catching complete beads of the same size range), and the number of fragments (fragments which were $1 / 2$ of a complete bead were given a MNI of .5; fragments which were at least $3 / 4$ of a bead were given an MNI of 1) were quantified. Complete beads were distinguished from fragments and damaged beads to determine if the condition of the bead skewed their distributions by screen sizes. Total counts of mussel disk beads were tabulated separately because beads of this material tended to separate in layers which distorted their total numbers.

The bar graphs in Figure 7.9 show the results of the disk bead test. The top graph gives frequencies for only the complete
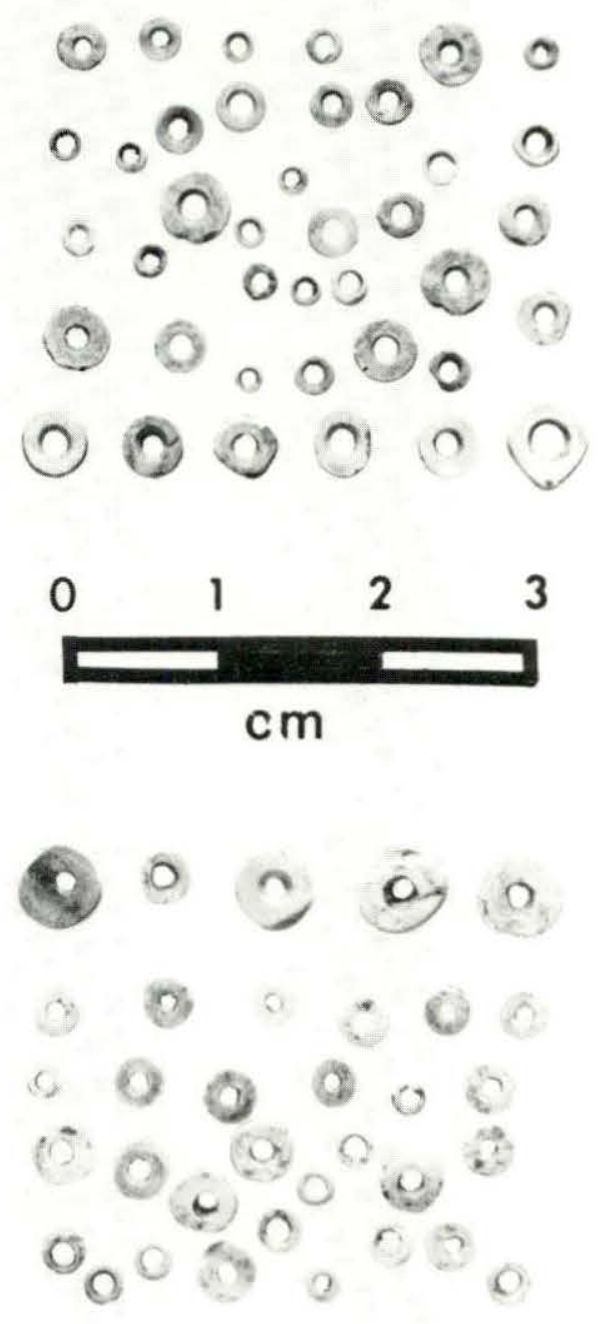

Figure 7.8

Shell disk beads from the Wall site (top) and Fredricks site (bottom).

specimens; the bottom graph gives frequencies for all specimens no matter what their condition. It is evident from these graphs that with a sufficiently large sample the condition of the beads does not significantly skew their distribution. The graphs demonstrate that a1though there does appear to be a slight trend toward smaller bead size over time, the differences are fairly negligible. At both sites, there does appear to be a standardization of disk size at around $2 \mathrm{~mm}$ to $3 \mathrm{~mm}$, (but one should keep in mind that screen sizes are based on the diameter length between mesh wires and beads of a slightly greater size can slip through diagonally). This evidence suggests that energy/time costs may not have been as critical to these people as they were to the Californian beadmakers and traders.

\section{Marginella Ornaments}

Marginella shell ornaments were found primarily in two types of contexts at the Wall site, burials and the midden. No marginella 


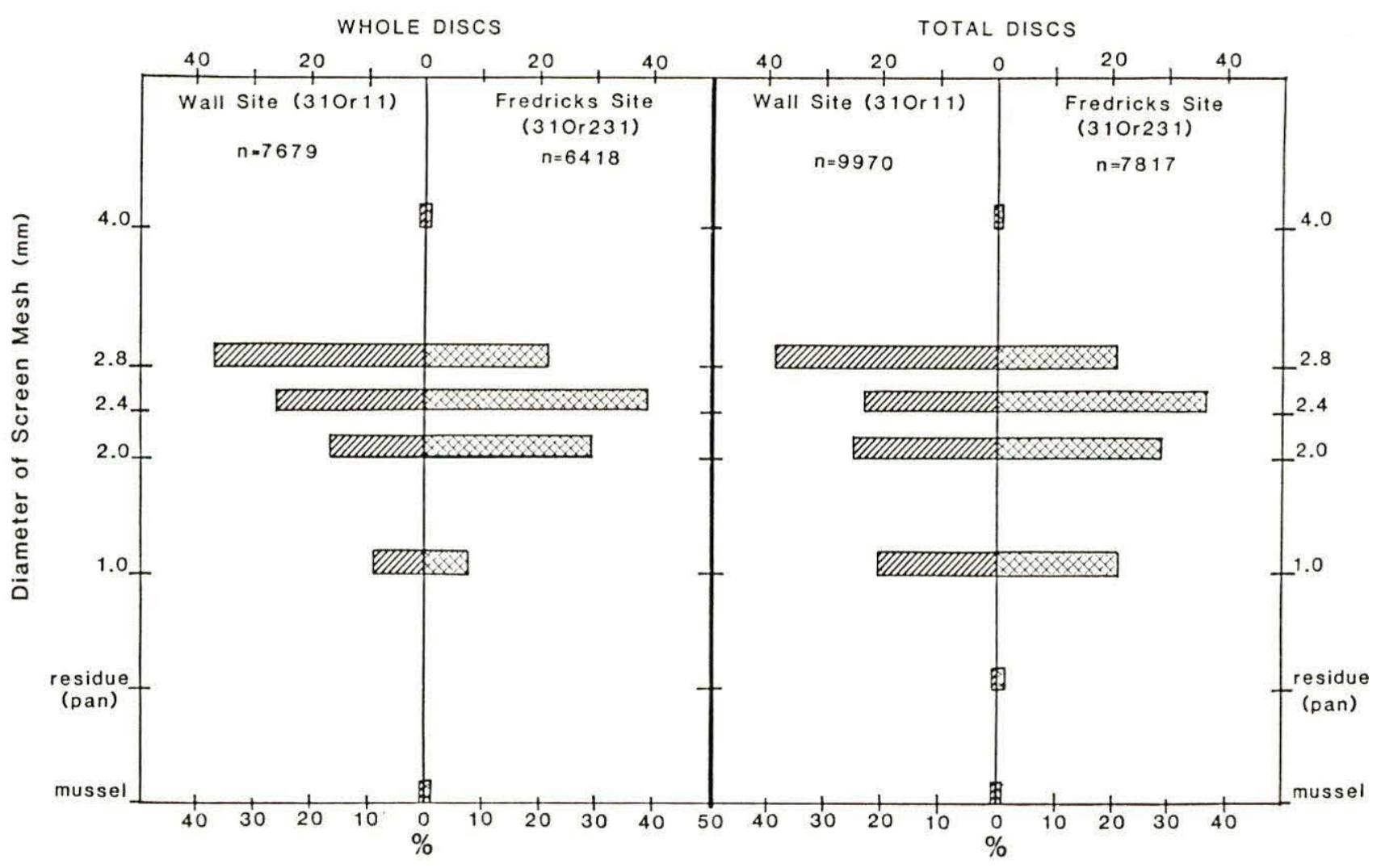

Figure 7.9

Frequency distribution of shell disk beads by screen size.

shells were recovered from the Fredricks site. This suggests that either this type was more accessible or more desirable to the Wall site inhabitants. All marginella ornaments occurring with burials were ground near the spire at the top of the shell (Table 7.2). The most common place for grinding was on the shoulder of the whorl adjacent to the canal opening and a couple of millimeters down from the spire. A few more were ground on different horizontal plains, but keeping the same relationship to the spire tip. Only a very few (.01\%) were spire ground, i.e., ground from the spire tip at a perpendicular angle to the long axis of the she11. Presumably this latter technique would be preferable if the shells were to be worn as beads. The former technique would be preferable for making ornaments to be sewn onto garments. By grinding the surface nearest the natural slit in the shell, the artisan allowed the greatest unmarred surface area to be exposed after the ground side had been sewn to a backing (Figure 7.6:d, outer beads). At the Wall site, it appears that the ornaments were sewn on articles of clothing, since, for the most part, they occurred over chest areas (shirts), around the head (headbands), around the waists (girdles or belts), and over legs (leggings) (Table 7.3).

Conversely, the majority of marginella shell from midden contexts were either unmodified shells or broken shells. The broken shells may represent disposal from manufacture or breakage from wear. The presence of unmodified whole shells suggests that either: 1) they were traded whole and were then ground into ornaments in the village; or 2) a few unground shells were found among shipments of the finished ornaments, which were then discarded along with the fragments broken during the process of sewing the finished ornaments onto garments. Why the whole shells were not ground remains a mystery since the only tool necessary for this procedure would have been some form of grinding slab.

\section{Wampum}

Wampum has been treated in great detail elsewhere (Speck 1919; Orchard 1929; Brown 1948). For the Siouan Project, a study of the wampum recovered in 1983 was previously initiated (Sizemore 1984). So far wampum have only been recovered from the Fredricks site. Two basic types of wampum have been identified: 1) a cylindrical form, which has frequently been described in the 1iterature; and 2) a morphological variant on the former type which will be called oval wampum (Figure 7.7:e). The oval wampum were made by grinding the sharp corners from the ends of the cylinders. Both purple and white varieties of both types have been identified, although only five purple oval wampum have been recovered so far from this site. Overall, the wampum range in length from 4.8-7.0 $\mathrm{mm}$ and in diameter from $3 \cdot 2-4.6 \mathrm{~mm}$, with the white wampum making up a higher proportion 
Table 7.2

Summary of marginella ornaments from the Wall site.

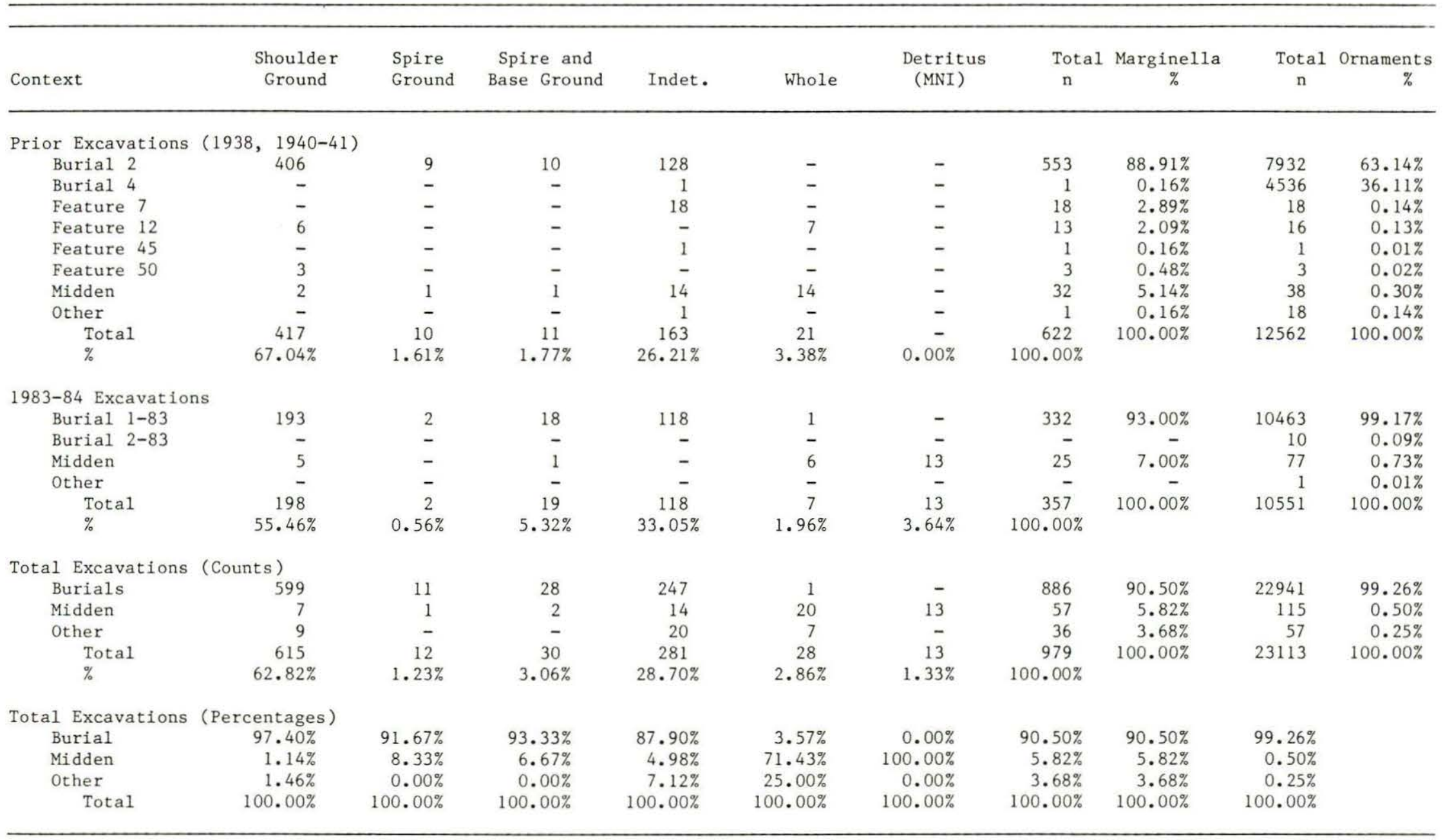


Table 7.3

Inventory of shell ornaments from the Wall site.

\begin{tabular}{|c|c|c|c|c|c|c|c|c|c|c|c|}
\hline Proventence & $\begin{array}{l}\text { Pendant } 5 / \\
\text { Gorgets }\end{array}$ & $\begin{array}{c}\text { Large } \\
\text { Segments }\end{array}$ & $\begin{array}{l}\text { Smal1 } \\
\text { Segwent } 8\end{array}$ & $\begin{array}{c}\text { Rough } \\
\text { Sphericals }\end{array}$ & $\begin{array}{c}\text { Other } \\
\text { Ornawents }\end{array}$ & $\begin{array}{c}\text { Mica } \\
\text { Pendants }\end{array}$ & $\begin{array}{l}\text { Nat 1 ve } \\
\text { Copper }\end{array}$ & $\begin{array}{c}\text { Total } \\
\text { Marginella }\end{array}$ & $\begin{array}{l}\text { Total } \\
\text { Disks }\end{array}$ & $\begin{array}{c}\text { Total } \\
\text { Ornaments }\end{array}$ & $\begin{array}{l}\text { Percent } \\
\text { Ornaments }\end{array}$ \\
\hline
\end{tabular}

1938 Excavat1ons

Feature 7

Feature 12
Stake $60 \quad$ N3'10",W1"; LI

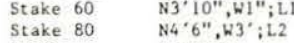

$\begin{array}{ll}\text { Stake } 80 & \mathrm{~N}^{\circ} \cdot 6^{\prime \prime}, \mathrm{W}^{\prime} \text { '; } \\ \text { Sq.90 } & \text { Level } 2\end{array}$

1940-41 Excavations

\begin{tabular}{|c|c|}
\hline Burial 2 & FS $980-118$ \\
\hline Burial 2 & Headdress/FS $\quad 80-117$ \\
\hline Burfal 2 & Right Arm Band/FS $080-116$ \\
\hline Burfal 2 & Left Arm Band/FS $880-115$ \\
\hline Burial 2 & Girdle \\
\hline Burial 2 & \\
\hline Burfal 4 & Neck Area/FS $080-139$ \\
\hline Burial 4 & Left Wrist \\
\hline Burial 4 & Left Ank1e/FS $880-140$ \\
\hline Burial 4 & Neck Area/FS $\quad 080-138$ \\
\hline Feature 1 & FS 128 \\
\hline Feature 2 & \\
\hline Feature 2 & \\
\hline Feature 4 & FS 1131 \\
\hline Feature 5 & FS 1141 \\
\hline Sq.0R50 & Plowzone \\
\hline Sq.0R60 & Plowzone \\
\hline Sq. $60 \mathrm{R} 70$ & Undisturbed Soll \\
\hline Sq.70R50 & Undisturbed Soll \\
\hline Sq. 75 & Level 2 \\
\hline $\mathrm{Sq} .90 \mathrm{R} 50$ & Undisturbed Soll \\
\hline Sq. $90 R 60$ & Und1sturbed Soli \\
\hline $\mathrm{Sq} .90 \mathrm{R} 70$ & Und1sturbed Soll \\
\hline & Undisturbed Soll \\
\hline
\end{tabular}

\begin{tabular}{|c|c|c|c|c|c|c|c|c|c|c|}
\hline - & - & - & - & - & - & - & 18 & - & 18 & $0.08 z$ \\
\hline - & - & - & - & - & - & - & 13 & 3 & 16 & $0.07 z$ \\
\hline - & - & - & - & - & - & - & 1 & - & 1 & $<0.012$ \\
\hline - & - & - & - & - & - & - & 1 & - & 1 & $<0.012$ \\
\hline - & - & - & - & - & - & - & - & 1 & $i$ & $<0.01 z$ \\
\hline
\end{tabular}

1983-84 Excavations

\begin{tabular}{|c|c|c|c|c|c|c|c|c|c|c|c|c|}
\hline Sq. 330 R520 & 01d Backf 111 & - & - & - & - & - & - & - & - & 1 & 1 & $<0.01 z$ \\
\hline Burial $1-83$ & Zone 1 ; NE $1 / 2$ & - & - & - & - & - & - & - & 1 & 4 & 5 & $0.02 z$ \\
\hline Bur1al $1-83$ & zone $i$ & - & - & - & - & - & - & - & $i$ & 2 & 3 & 0.012 \\
\hline Burlal $1-83$ & Around Bone & - & - & - & - & - & - & - & 41 & 739 & 780 & 3.372 \\
\hline Burial 1-83 & Cleaning Pit & - & - & 1 & - & - & - & - & - & 10 & 11 & $0.05 z$ \\
\hline Burial 1-83 & Assoctated $\sim /$ Pot OI & - & - & - & - & 1 & - & - & - & - & 1 & $<0.017$ \\
\hline Burfal $1-83$ & Around Head (Hand) & - & - & - & - & - & - & - & 74 & - & 74 & $0.32 z$ \\
\hline Burial $1-83$ & Behind Skull & - & 9 & - & - & - & - & - & 14 & 289 & 312 & $1.35 z$ \\
\hline Burfal $1-83$ & Front Lower Skull Area & - & - & - & - & - & - & - & - & 170 & 170 & $0.74 z$ \\
\hline Burial $1-83$ & In Sku11 & - & - & - & - & - & - & - & 4 & 105 & 109 & $0.47 \%$ \\
\hline Burfal $1-83$ & Below Skul1 & - & 3 & - & - & - & - & - & 4 & 197 & 204 & $0.88 \%$ \\
\hline Bur1al 1-83 & Skull Area & - & - & - & - & - & - & 1 & 9 & 323 & 333 & $1.44 \%$ \\
\hline Burtal $1-83$ & Around Neck & - & 32 & - & - & - & - & - & - & 49 & 81 & $0.35 \%$ \\
\hline Bur1al 1-83 & Head/Chest Area & - & 2 & - & - & - & - & - & 45 & 2018 & 2065 & $8.93 \%$ \\
\hline Burial $1-83$ & Around Chest Area & - & 26 & - & - & - & - & 1 & 1 & 957 & 985 & $4.26 \%$ \\
\hline Bur1al $1-83$ & Back Area & 1 & - & - & - & - & - & - & - & - & 1 & $<0.01 \%$ \\
\hline Burial $1-83$ & Near Gorget & - & 19 & - & - & - & - & - & - & - & 19 & $0.08 \%$ \\
\hline Burial 1-83 & Upper Arm Area & - & 1 & - & - & - & - & - & 9 & 308 & 318 & $1.38 z$ \\
\hline Burial $1-83$ & Ara Area & - & 40 & 20 & - & - & - & 1 & - & 1840 & 1901 & 8.222 \\
\hline Burfal 1-83 & Lower Ara & - & 10 & - & - & - & - & - & 1 & 1304 & 1315 & $5.69 z$ \\
\hline Burfal $1-83$ & Below R1bs 6 Aras & - & - & - & - & - & - & - & - & 620 & 620 & $2.68 z$ \\
\hline Bur1al $1-83$ & Ribs o Vertebra Area & - & 7 & - & - & - & - & - & - & 73 & 80 & $0.35 \%$ \\
\hline Bur1al $1-83$ & H1p Area & - & 12 & - & - & - & - & - & 1 & - & 13 & $0.06 z$ \\
\hline Burial $1-83$ & Lower Back/Leg Area & - & - & - & - & - & - & - & - & 168 & 168 & $0.73 z$ \\
\hline Burfal $1-83$ & DIrt Around Legs & - & 15 & - & - & - & - & - & 47 & 38 & 100 & $0.43 \%$ \\
\hline Burial $1-83$ & Left Tibia/Etbula & - & 3 & - & - & - & - & - & 59 & 2 & 64 & $0.28 z$ \\
\hline Burial $1-83$ & Below Lower Legs & - & 7 & - & - & - & - & - & 14 & - & 21 & $0.09 \%$ \\
\hline Burfal $1-83$ & General Cleaning & - & - & - & - & - & - & 1 & 7 & 702 & 710 & $3.07 \%$ \\
\hline Bur1al 2-83 & Around Neck & - & 10 & - & - & - & - & - & - & - & 10 & $0.04 \%$ \\
\hline $\mathrm{Sq} .360 \mathrm{R} 530$ & Midden Level 1 & - & - & - & - & - & - & - & 17 & 39 & 56 & $0.24 z$ \\
\hline Sq. $360 R 530$ & Midden Level 1; NW Quad & - & - & - & - & - & - & - & - & 2 & 2 & $0.01 \%$ \\
\hline Sq. $360 R 530$ & Midden Level 1 ; SH Quad & - & - & - & - & - & - & - & - & $i$ & $i$ & $<0.01 \%$ \\
\hline Sq. 350 R 500 & MIdden Level i & - & - & - & - & - & - & - & - & i & $i$ & $<0.01 \%$ \\
\hline Sq. $370 \mathrm{R} 540$ & Midden Level i & - & - & - & - & - & 1 cut frag. & - & - & - & - & $0.00 \%$ \\
\hline Sq. $370 \mathrm{R} 540$ & Midden Leve1 2 & - & - & - & - & - & i cut frag. & - & - & - & - & $0.00 z$ \\
\hline $\mathrm{Sq} .370 \mathrm{R} 560$ & Midden Level 2 & - & - & - & - & - & - & - & 4 & 3 & 7 & $0.03 z$ \\
\hline Sq. $360 R 530$ & Midden Levei 2 & - & - & - & - & - & - & - & 4 & 3 & 7 & $0.03 z$ \\
\hline $\mathrm{Sq} .360 \mathrm{R} 530$ & Midden Leve1 2; NE Quad & - & - & - & - & - & - & - & - & 2 & 2 & $0.01 \%$ \\
\hline Sq. 320 R620 & Plowzone & - & - & - & - & - & - & - & - & - & - & $0.00 z$ \\
\hline Sq. $320 \mathrm{R} 620$ & Flatshoveling & - & - & - & - & - & 1 & - & - & - & 1 & $<0.01 \%$ \\
\hline Sq. 320R620 & Flatshoveling Subsoll & - & - & - & - & - & - & - & - & - & - & $0.00 z$ \\
\hline Total $(1983$ & 3-84 Excavatfons) & $\begin{array}{c}1 \\
0.017\end{array}$ & $\begin{array}{c}196 \\
1.86 z\end{array}$ & $\begin{array}{c}21 \\
0.20 z\end{array}$ & $\begin{array}{c}0 \\
0.00 z\end{array}$ & $\begin{array}{c}1 \\
0.01 z\end{array}$ & $\begin{array}{c}1 \\
0.01 \%\end{array}$ & 0.047 & $\begin{array}{c}357 \\
3.387\end{array}$ & $\begin{array}{c}9970 \\
94.49 z\end{array}$ & $\begin{array}{c}10551 \\
100.00 z\end{array}$ & $45.65 z$ \\
\hline Total (1938- & 8-41 Excavations) & $\begin{array}{c}3 \\
0.02 z\end{array}$ & $\begin{array}{c}53 \\
0.42 z\end{array}$ & $\begin{array}{c}0 \\
0.00 z\end{array}$ & $\begin{array}{c}119 \\
0.95 \%\end{array}$ & $\begin{array}{c}0 \\
0.00 z\end{array}$ & $\begin{array}{c}0 \\
0.00 z\end{array}$ & $0.03 z$ & $\begin{array}{c}622 \\
4.957\end{array}$ & $\begin{array}{l}11761 \\
93.62 z\end{array}$ & $\begin{array}{c}12562 \\
100.00 z\end{array}$ & $54.35 z$ \\
\hline Total (A11 & Excavat 1ons) & $0.02 z$ & $\begin{array}{c}249 \\
1.08 z\end{array}$ & $\begin{array}{c}21 \\
0.09 z\end{array}$ & $\begin{array}{r}119 \\
0.51 z\end{array}$ & $<0.01 z$ & $\left\langle\begin{array}{c}1 \\
0.01 z\end{array}\right.$ & $\begin{array}{c}8 \\
0.032\end{array}$ & $\begin{array}{c}979 \\
4.247\end{array}$ & $\begin{array}{l}21731 \\
94.027\end{array}$ & $\begin{array}{c}23113 \\
100.00 z\end{array}$ & $100.00 z$ \\
\hline
\end{tabular}


of the smaller sizes. All of the white oval wampum were made from smaller white wampum, and they occurred exclusively with this small white cylindrical form. All the purple wampum, including the oval form, were larger than the white oval wampum.

The majority of the wampum ( $68 \%)$ was found with Burial 1 (Table 7.4), occurring around the neck, chest, and the lower arms areas where they were arranged in closely spaced parallel rows. The wampum with Burial 2 were found around the neck and chest in a similar pattern. The wampum with Burial 1 appeared to have been strung around the neck. The rest of the wampum were found with Feature 3/Burial 5. These latter specimens, probably decoration on a small bag or pouch, were lying over a cluster of two ceramic pipes, a knife and a bird claw. The contextual relationship of the wampum at this site suggest they were used as ornaments sewn onto garments and other personal gear, and were strung as necklaces worn around the neck.

\section{Runtees}

In 1722, Robert Beverley of Virginia described this type of bead as being made "of the Conch Shell, as the Peak is, only the Shape is flat and like a Cheese, and drill'd Edgeways" (1722:145). Runtees from the Siouan Project ranged from $12.8-17.2 \mathrm{~mm}$ in length, $12.8-17.5 \mathrm{~mm}$ in diameter, and $4.1-6.4 \mathrm{~mm}$ in thickness. They occurred in very small numbers $(n=21)$ and only at the Fredricks site. They were found exclusively around the neck and chest areas of Burials 1 and 2. Those with Burial 2 occurred with spherical and barrel/cylinder beads (Table 7.5). In both instances, they occurred in association with wampum. No other runtees are known to have been found at Piedmont sites.

\section{Clam Shell Artifact}

One possible shell ornament is left for consideration. It is a clam shell with a perforation in its cup which exhibits wear on two sides of the hole. This shell may have been worn as a pendant. On the other hand it may have been affixed to a wooden handle and used as a spoon. It was found within a pot associated with Burial $1-83$ at the Wall site. At this time the function of this artifact remains uncertain.

\section{Non-She11 Ornaments}

The only non-shell ornaments, aside from the stone disk beads discussed above, were small tubes made from copper and a single mica pendant. A few tubes of native copper were identified from the Wall site, four from Burial 1-83 and four from Burial 2 (1940-41 excavations). All were in poor condition. The tubes were approximately 1-2 $\mathrm{mm}$ in diameter and were all less than $5 \mathrm{~mm}$ 1ong. Their short lengths were at least partially due to poor preservation. All were of a rolled technique, a form which has also been found at Late Upper Saratown and RLA-Vir150. They appeared to have occurred with strands of shell disks beads and were probably strung with them.

One mica hexagonal pendant, measuring $40 \mathrm{~cm}$

Table 7.4

Sumnary of wampum from the Fredricks site.

\begin{tabular}{|c|c|c|c|c|c|c|c|c|}
\hline & \multicolumn{4}{|c|}{ Cylindrical } & \multicolumn{2}{|c|}{ Oval } & \multirow{2}{*}{$\begin{array}{l}\text { Tota1 } \\
\text { Wampum }\end{array}$} & \multirow{2}{*}{$\begin{array}{c}\text { Total } \\
\text { Ornaments }\end{array}$} \\
\hline & Purple & White & Indet. & Sub-Total & Purple & White & & \\
\hline \multirow{2}{*}{\multicolumn{9}{|c|}{$\frac{\text { Counts }}{1983 \text { Excavations }}$}} \\
\hline $\begin{array}{l}1983 \text { Excavations } \\
\text { Burial } 1\end{array}$ & & & 76 & 499 & 3 & 57 & 559 & 6315 \\
\hline Burial 2 & 101 & 9 & 6 & 116 & 2 & - & 118 & 2112 \\
\hline Burial 3 & - & 2 & 18 & 20 & - & - & 20 & 48 \\
\hline Other & - & - & - & - & - & - & - & 1 \\
\hline \multicolumn{9}{|l|}{1984 Excavations } \\
\hline Feature 3/Burial 5 & - & 126 & - & 126 & - & - & 126 & 174 \\
\hline Other & - & - & - & - & - & - & - & 97 \\
\hline Total & 307 & 354 & 100 & 761 & 5 & 57 & 823 & 8747 \\
\hline \% Wampum & $37.30 \%$ & $43.01 \%$ & $12.15 \%$ & $92.47 \%$ & $0.61 \%$ & $6.93 \%$ & $100.00 \%$ & \\
\hline \multirow{2}{*}{\multicolumn{9}{|c|}{$\frac{\text { Percentages }}{1983 \text { Excavations }}$}} \\
\hline & & & & & & & & \\
\hline Burial 1 & $67.10 \%$ & $61.30 \%$ & $76.00 \%$ & $65.57 \%$ & $60.00 \%$ & $100.00 \%$ & $67.92 \%$ & $72.20 \%$ \\
\hline Burial 2 & $32.90 \%$ & $2.54 \%$ & $6.00 \%$ & $15.24 \%$ & $40.00 \%$ & $0.00 \%$ & $14.34 \%$ & $24.15 \%$ \\
\hline Burial 3 & ERR & $0.56 \%$ & $18.00 \%$ & $2.63 \%$ & $0.00 \%$ & $0.00 \%$ & $2.43 \%$ & $0.55 \%$ \\
\hline Other & $0.00 \%$ & $0.00 \%$ & $0.00 \%$ & $0.00 \%$ & $0.00 \%$ & $0.00 \%$ & $0.00 \%$ & $0.01 \%$ \\
\hline \multicolumn{9}{|l|}{1984 Excavations } \\
\hline Feature 3/Burial 5 & $0.00 \%$ & $35.59 \%$ & $0.00 \%$ & $16.56 \%$ & $0.00 \%$ & $0.00 \%$ & $15.31 \%$ & $0.00 \%$ \\
\hline Other & $0.00 \%$ & $0.00 \%$ & $0.00 \%$ & $0.00 \%$ & $0.00 \%$ & $0.00 \%$ & $0.00 \%$ & $1.99 \%$ \\
\hline Total & $100.00 \%$ & $100.00 \%$ & $100.00 \%$ & $100.00 \%$ & $100.00 \%$ & $100.00 \%$ & $100.00 \%$ & $0.00 \%$ \\
\hline
\end{tabular}


Table 7.5

Inventory of shell ornaments from the Fredricks site.

\begin{tabular}{|c|c|c|c|c|c|c|c|c|c|c|c|c|}
\hline Provenience & & Gorgets & $\begin{array}{l}\text { Tube } \\
\text { Beads }\end{array}$ & $\begin{array}{c}\text { Cylinder/ } \\
\text { Barrels }\end{array}$ & Runtees & $\begin{array}{c}\text { Medium } \\
\text { Sphericals }\end{array}$ & $\begin{array}{c}\text { Ear } \\
\text { Ornaments }\end{array}$ & $\begin{array}{l}\text { Columella } \\
\text { Segments }\end{array}$ & $\begin{array}{l}\text { Total } \\
\text { Wampum }\end{array}$ & $\begin{array}{l}\text { Total } \\
\text { Disks }\end{array}$ & $\begin{array}{c}\text { Total } \\
\text { Ornaments }\end{array}$ & $\begin{array}{c}\text { Percent } \\
\text { Ornaments }\end{array}$ \\
\hline \multicolumn{13}{|c|}{1983 Excavations } \\
\hline Fea. 1 & Finding edge & - & - & - & - & - & - & _ & - & 1 & 1 & $0.01 \%$ \\
\hline Bu. 1 & Zone 1 ; NW $1 / 2$ & - & - & - & - & - & - & - & 2 & 6 & 8 & $0.09 \%$ \\
\hline $\mathrm{Bu} .1$ & Around Bone & - & - & - & - & - & - & - & 60 & 113 & 173 & $3.64 \%$ \\
\hline $\mathrm{Bu} .1$ & Skull Area & - & - & - & - & - & - & - & 8 & 452 & 460 & $5.26 \%$ \\
\hline $\mathrm{Bu} .1$ & West of Mandible & - & - & - & - & - & - & - & 1 & 8 & 9 & $0.10 \%$ \\
\hline $\mathrm{Bu} .1$ & Around Right Shoulder & - & - & - & - & - & - & - & - & 22 & 22 & $0.25 \%$ \\
\hline $\mathrm{Bu} .1$ & Around Right Scapula & - & - & - & - & - & - & - & - & 1 & 1 & $0.01 \%$ \\
\hline $\mathrm{Bu}, 1$ & Around Neck/Chest Area & - & - & - & 8 & - & - & 1 & 162 & 4332 & 4503 & $51.48 \%$ \\
\hline $\mathrm{Bu} .1$ & Cleaning Bone, Chest Area & 2 & - & - & 4 & - & - & - & 76 & 511 & 593 & $6.78 \%$ \\
\hline $\mathrm{Bu} .1$ & Around Ribs & - & - & - & - & - & - & - & 1 & 27 & 28 & $0.32 \%$ \\
\hline $\mathrm{Bu} .1$ & Over Right Elbow & - & - & - & - & - & - & - & 3 & 11 & 14 & $0.16 \%$ \\
\hline $\mathrm{Bu} .1$ & Around Lower Right Arm & - & - & - & - & - & - & - & 129 & - & 129 & $1.47 \%$ \\
\hline $\mathrm{Bu}, 1$ & Around Right Hand & - & - & - & - & - & - & - & 97 & 242 & 339 & $3.88 \%$ \\
\hline $\mathrm{Bu} .1$ & Around Left Arm & - & - & - & - & - & - & - & 20 & 8 & 28 & $0.32 \%$ \\
\hline $\mathrm{Bu} .1$ & Cleaning Around Scissors & - & - & - & - & - & - & - & - & 2 & 2 & $0.02 \%$ \\
\hline $\mathrm{Bu} .1$ & Zone $3 \mathrm{~A}$; Under Beads & - & - & - & - & - & - & - & - & 2 & 2 & $0.02 \%$ \\
\hline $\mathrm{Bu} .1$ & Zone 3A; Under But tons & - & - & - & - & - & - & - & - & 4 & 4 & $0.05 \%$ \\
\hline $\mathrm{Bu} .2$ & Cleaning Around Bone & - & - & - & - & - & - & - & 2 & 3 & 5 & $0.06 \%$ \\
\hline $\mathrm{Bu} .2$ & Around Neck/Chest Area & - & - & 54 & 9 & 16 & - & - & 115 & 1 & 195 & $2.23 \%$ \\
\hline $\mathrm{Bu} .2$ & Right Arm Area & - & - & - & - & - & - & - & - & 1889 & 1889 & $21.60 \%$ \\
\hline $\mathrm{Bu} .2$ & Right Arm Area & - & - & - & - & - & - & - & 1 & - & 1 & $0.01 \%$ \\
\hline $\mathrm{Bu} .2$ & At Left Radius/U1na & - & - & - & - & - & - & - & - & 3 & 3 & $0.03 \%$ \\
\hline $\mathrm{Bu} .2$ & Right Femur Area & - & - & - & - & - & - & - & - & 2 & 2 & $0.02 \%$ \\
\hline $\mathrm{Bu} .2$ & Beaded Area Beside Body & - & - & - & - & - & - & - & - & 10 & 10 & $0.11 \%$ \\
\hline $\mathrm{Bu} .2$ & With Knife, East of Body & - & - & - & - & - & - & - & - & 7 & 7 & $0.08 \%$ \\
\hline $\mathrm{Bu} .3$ & Zone 1 & - & - & - & - & - & - & - & 2 & 28 & 30 & $0.34 \%$ \\
\hline $\mathrm{Bu} .3$ & Strung Around Neck & - & - & - & - & - & - & - & 16 & - & 16 & $0.18 \%$ \\
\hline $\mathrm{Bu} .3$ & With Knife, Under Mandible & - & - & - & - & - & - & - & 2 & - & 2 & $0.02 \%$ \\
\hline \multicolumn{13}{|c|}{1984 Excavations } \\
\hline $\mathrm{Fea} \cdot 2 / \mathrm{Bu} \cdot 4$ & Chest Area & - & 11 & - & - & - & - & - & - & - & 11 & $0.13 \%$ \\
\hline $\mathrm{Fea} .3 / \mathrm{Bu} .5$ & Trowelling Sq. 290R70 & - & - & - & - & - & - & - & - & 13 & 13 & $0.15 \%$ \\
\hline $\mathrm{Fea} \cdot 3 / \mathrm{Bu} .5$ & Trowelling Sq.290R80 & - & - & - & - & - & - & - & - & 6 & 6 & $0.07 \%$ \\
\hline $\mathrm{Fea} \cdot 3 / \mathrm{Bu} .5$ & Zone 1 & - & - & - & - & - & - & - & - & 4 & 4 & $0.05 \%$ \\
\hline $\mathrm{Fea} .3 / \mathrm{Bu} .5$ & Zone 2 & - & - & - & - & - & - & - & 1 & 18 & 19 & $0.22 \%$ \\
\hline $\mathrm{Fea} .3 / \mathrm{Bu} .5$ & Zone 3 & - & - & - & - & - & - & - & 3 & 3 & 6 & $0.07 \%$ \\
\hline $\mathrm{Fea} \cdot 3 / \mathrm{Bu} .5$ & Left Temporal (near ear) & - & - & - & - & - & 2 & - & - & - & 2 & $0.02 \%$ \\
\hline $\mathrm{Fea} .3 / \mathrm{Bu} .5$ & East of Arms (in situ) & - & - & - & - & - & - & - & 103 & - & 103 & $1.18 \%$ \\
\hline $\mathrm{Fea} \cdot 3 / \mathrm{Bu} .5$ & Cleaning Artifact Cluster & - & - & - & - & - & - & - & 19 & 2 & 21 & $0.24 \%$ \\
\hline $\mathrm{Fea} .4 / \mathrm{Bu} .6$ & Zone 1 , NW $1 / 2$ & - & - & - & - & - & - & - & - & 1 & 1 & $0.01 \%$ \\
\hline $\mathrm{Fea} .4 / \mathrm{Bu} .6$ & Zone 5 & - & - & - & - & - & - & - & - & 4 & 4 & $0.05 \%$ \\
\hline $\mathrm{Fea} .4 / \mathrm{Bu} .6$ & w/Bracelet on Wrist & - & - & - & - & - & - & - & - & 71 & 71 & $0.81 \%$ \\
\hline $\mathrm{Fea}$. 4/Bu. 6 & Bracelet Area & - & - & - & - & - & - & - & - & 6 & 6 & $0.07 \%$ \\
\hline $\mathrm{Fea} .6 / \mathrm{Bu} .8$ & Trowelling & - & - & - & - & - & - & - & - & 1 & 1 & $0.01 \%$ \\
\hline Fea.13 & Zone 1 & - & - & - & - & - & - & - & - & 3 & 3 & $0.03 \%$ \\
\hline Total & & $\begin{array}{c}2 \\
0.02 \%\end{array}$ & $\begin{array}{c}11 \\
0.13 \%\end{array}$ & $\begin{array}{c}54 \\
0.62 \%\end{array}$ & $\begin{array}{c}21 \\
0.24 \%\end{array}$ & $\begin{array}{c}16 \\
0.18 \%\end{array}$ & $\begin{array}{c}2 \\
0.02 \%\end{array}$ & $\begin{array}{c}1 \\
0.01 \%\end{array}$ & $\begin{array}{l}823 \\
9.41 \%\end{array}$ & $\begin{array}{c}7817 \\
89.37 \%\end{array}$ & $\begin{array}{c}8747 \\
100.00 \%\end{array}$ & $100.00 \%$ \\
\hline
\end{tabular}


between opposite sides, was found in the plowzone over Structures G-H at the Wall site along with several pieces of mica that were apparently unmodified. Two cut fragments were also recovered from the midden. No other mica has been found at these two sites thus far.

\section{A REGIONAL PERSPECTIVE}

Most of the major bead types at the Wall site have been recovered from other sites in the general region (Table 7.1). Their distribution suggests a degree of continuity of the exchange of ideas and goods through late prehistoric times between groups. Evidence of fragments of the large univalve used for manufacturing many of the beads and other ornaments has been found at only two sites in the area, Early Upper Saratown and Lower Sauratown, both excavated by the Research Laboratories of Anthropology. of these, only the fragments of she11 from Early Upper Saratown can really be considered reasonably reliable indications of onsite beadmaking. Here, several pieces of univalves and whole univalves were found as well as pieces of shell indicative of partial ornament preforms (or blanks). The whole shells were perforated in a fashion similar to the shells recovered from the Irene Mound on the coast of Georgia (Caldwell and McCann 1941) which have been posited elsewhere to be some form of digging tool, such as hoes. Broken and ground fragments of large univalve shells, presumably shell artifact manufacture detritus, were also found at the Irene Mound site. The archaeological work at Lower Sauratown produced one large rounded blank (undrilled) which could have been traded from a village such as Upper Saratown or even the Irene Mound. Upper Saratown is equidistant between the Wall site and the Shannon site, located in Montgomery County, Virginia. Early historic records suggest that the Upper Saratown sites were on a major north/south trail which intersected a east/west trail that extended from the western foothills to the coast via Occaneechi (at or near the Wall and Fredricks sites [Simpkins 1984]). From the ornament distribution it appears likely that by late prehistoric times sites in southwestern Virginia and northwest and north central North Carolina were already connected by such trails.

Historically, the introduction of wampum and other bead types in this region is an indication an expansion of trade networks. It is evident from the literature that the beadmakers of New England had taken care to standardize their medium of exchange and had been able to put a high value on their beads. This is demonstrated by the continued use of these beads well into the contact period. Yet it is not entirely clear what part, if any, that the aboriginal New England economic system played in bead manufacture and exchange in the Carolina area.
Between the times of occupation of Wall and Fredricks sites we see the addition of several bead types (wampum, runtees, barrel/cylinders) and the disappearance of others (marginellas and segments). Certainly these changes indicate increasing ties to the north and lessening ties southward. Also, however, a comparison of their contextual relationships suggests that some of the new types also served as replacements for previous types. It appears from the record that marginellas and wampum functioned quite similarly, in terms of their placement on similar parts of the body and with similar types of individuals (subadults and to a lesser extent adult males; see Wilson, this report). The columella segments appear to have been replaced by runtees and barrel/cylinders. Disks, however, seem to have maintained a similar function through time, based on the distribution and frequencies of both shell and glass forms.

At a regional level, there are indications that shell artifacts were manufactured prehistorically at only a few villages and were traded from these centers to other villages throughout the Piedmont and western Appalachian foothills. This is a pattern seen elsewhere prehistorically in North America, although in the other areas the beadmaking centers were along the coast. The present study suggests that the raw materials were being traded to interior villages where beadmaking occurred. Another difference is that the Piedmont forms appear to have been larger and overall less finished than the standardized trade products from the other beadmaking centers. This information, along with the comparison of the disks discussed above, may indicate that a time/energy constraint was less critical in the Carolina interior than some other constraint, such as for example access to resources. It would seem that access to coastal resources, which is implied symbolically by the material traded, would be assumed where the beadmakers were coastal residents. Perhaps there was a secondary criterion for transmitting information regarding access restrictions was necessary for this medium of exchange. In the interior, access to coastal resources alone might have been sufficient to allow a standardization of exchange values, without the additional expenditure of reducing these ornaments.

An alternative explanation is that the Piedmont residents were less directly tied into a money economy than the California Chumash or the Naragansett of the Northeast. In the Carolina Piedmont, populations were relatively small and not very dense and there was less social ranking than in the coastal areas. To the Piedmont residents, transmission of information about access to rare resources may have been more crucial than demonstrating control over human labor. Such a distinction may be reflected in the large and unfinished size of many of the Piedmont ornaments. Also the large original size of some forms would have allowed for reuse through reduction after breakage, a 
practice observed for the columella tubes and segments. To have a large, less finished product would symbolically affirm more direct access to the rare commodity than a reduced more refined product. There is a small amount of evidence to suggest that blanks rather than the finished products were traded. This would allow purchasers to customize their own ornaments and wearers to transmit the information that they had access to a larger trade network, while at the same time having allegiances to a more specific group. In any event it is evident that a fairly standardized medium of exchange existed aboriginally in the Piedmont. It is noteworthy that the one beadmaking center that has been isolated for the Piedmont region so far is near the territory of the more populated groups of the mountains.

\section{SERRATED SHELLS}

The serrated shell tools from the Wall site were made from a freshwater bivalve (Cheryl Clausen, personal communication), which had been notched along their edges to produce a cutting edge. The specific use for these shells is not fully understood. There is ethnographic information that suggests they may have been used to scrape excess clay out of the interior of ceramic vessels prior to firing (Figure 7.10). The problem with this explanation is that scrape marks produced from such tools rarely occur on the ceramics from this site (Davis, this report) although they do occur from earlier sites in the region. The use of these tools may have been followed by a smoothing shell which would have erased the traces of the serrated shells. Smoothing shells would show little or no evidence of manufacture and would be difficult to identify in the archaeological record. Other fresh water shells were collected from the Wall site but so far none have been found that reveal evidence of wear or modification perhaps partly due to their poor preservation.

It is quite possible that these serrated shells had another purpose or that they were multi-use tools, however, this remains to be demonstrated. Their absence thus far from the remains recovered at the Fredricks site indicates that after contact they were either replaced by another tool or tools, or the function that was performed with them no longer occurred. Either way, it appears the need for this tool type ceased to exist.

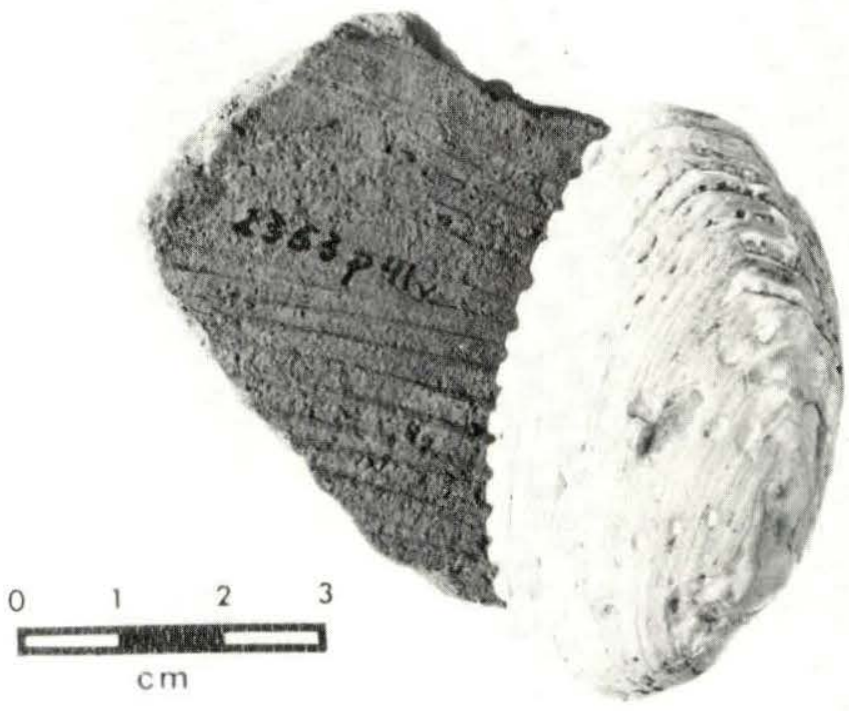

Figure 7.10

Serrated shells from the Wall site showing similarity of edge to scrape marks on inside of potsherd.

\section{CONCLUSIONS}

Shell artifacts from the Wall and Fredricks sites provide substantial information about changes and continuity in the region from protohistoric to historic times. A shell artifact classification has been developed and changes through time have been demonstrated. The evidence indicates that a regional trade network with fairly standardized forms of ornaments serving as mediums of exchange was present in the area prehistorically. The impact of European contact resulted in a longer range trade network with increasing ties northward and decreasing ties southward. Overall, there is evidence for reuse and curation of shell ornaments and for replacement of traditional types with newer, similar types during the Historic period. The latter observation leaves the question of whether there were changes in ethnicity or exchange networks, or both. It could be that the same ethnic groups imposed their preconceived models on new items, or that new people brought in similar models of function for similar forms. 


\title{
Pottery from the Fredricks, Wall, and Mitchum Sites
}

by

\author{
R. P. Stephen Davis, Jr.
}

\section{INTRODUCTION}

One of the most perplexing problems in North Carolina archaeology has been the identification and interpretation of the historic Piedmont Siouan cultures. Despite almost fifty years of relatively continuous scientific archaeological study within the region, much of which has been specifically directed toward this problem, the Historic period remains poorly understood. Moreover, very few of the several ethnohistorically-described villages of the Piedmont Siouans have been positively identified; and most of those located by early research during the 1930 s and 1940 s can now be shown to predate the Historic period. Given this situation, the methodological problem of recognizing Historic period sites remains one of fundamental importance to addressing other questions of culture change and adaptation. The problem of temporal placement is equally relevant to studies of earlier occupations as well.

The usefulness of ceramics as a basis for establishing cultural relationships has long been appreciated by archaeologists working in the eastern United States (Holmes 1903:18-19). This usefulness derives from three primary qualities which ceramics possess: 1) their relative ubiquity on Woodland and Mississippian period habitation sites; 2) their durability; and 3) their amenability to variable, culturally prescribed stylistic and technological expression. Consequently, it is not surprising that previous studies of ceramics from Woodland period sites in the Siouan Project study area have focused on using ceramics to establish culture-historical relationships. The present study also has a similar focus in that it seeks to define the configuration of particular ceramic assemblages and to determine their relationships to other assemblages. However, unlike previous work, which considered ceramic variability from a purely typological perspective (Coe and Lewis 1952; Coe 1964; Gardner 1980; Smith 1965), the present study approaches variability from the attribute level rather than from the artifact level (see Clarke 1968 for an elaboration of this distinction). A recent and similar study by Wilson (1983) has made substantial progress toward a synthetic, regional depiction of Late Woodland ceramic traditions of the North Carolina and southern Virginia Pied- mont and an interpretation of their historical relationships. The present study adds new and significant information for advancing our understanding within one area of this region.

During 1983-1984, archaeological excavations were undertaken by the Research Laboratories of Anthropology at the Fredricks (310r231) and Wall (310r11) sites near Hillsborough in Orange County and at the Mitchum site (31Ch452) in Chatham County, North Carolina. These investigations document the protohistoric and historic Indian occupation of the Eno and Haw River valleys and provide an empirical basis for studying synchronic and diachronic variability in Piedmont Siouan ceramic technology (e.g., Davis 1984). Specifically, the primary components at the three sites represent villages that were occupied from the Protohistoric (Wall site) to the Middle Contact (Fredricks site) period. Based upon historic artifact assemblages, the Mitchum site represents an Early Contact period village that immediately preceded the major occupation of the Fredricks site.

From the three sites, a total of 33,033 ceramic artifacts (excluding clay pipes and daub) have been recovered and analyzed. Most of these represent non-conjoining fragments or sherds of fired-clay storage, cooking, or eating vessels. Because of a paucity of whole vessels and reconstructible sections of broken vessels, this analysis is based primarily upon potsherd assemblages.

Three questions will be addressed by the following analysis. All of these questions focus on the interpretation of the Fredricks site artifacts and how they compare with the other two analyzed samples. First, which artifacts comprise the ceramic assemblage associated with the Middle Contact period Indian occupation at the Fredricks site, and how can this assemblage be defined in terms of stylistic/technological/morphological attributes? Second, is there any evidence for other Woodland components at the Fredricks site? Finally, what are the relationships between the Historic period ceramic assemblage at the Fredricks site and other late prehistoric/historic ceramic assemblages identified within the study area, and what implications do they have for interpreting the Fredricks site as an intrusive Occaneechi vil- 
lage? The first two questions will be considered by an analysis of covariability among ceramic attributes and their intrasite spatial patterning. The last question will be examined through comparative analyses of the Fredricks site collection with ceramic collections from the Wall and Mitchum sites, as well as from several other Woodland sites in piedmont North Carolina and Virginia, including sites located in the area of Occaneechi Island where the Occaneechis resided prior to 1676 (Alvord and Bidgood 1912).

\section{ANALYTIC METHODS}

Analysis of ceramic artifacts from the Fredricks, Wall, and Mitchum sites was accomplished within the context of a computerized data recording system developed by the Research Laboratories of Anthropology. Other comparative data used to evaluate the Fredricks site assemblage were derived from published and unpublished site reports. Ceramic artifacts were computer-coded according to seven contextual/ provenience variables, four morphological attributes, two technological attributes, three stylistic attributes, and size. Vessels and reconstructible vessel sections were further coded for additional morphological attributes and size measurements. Measured sherd attributes and attribute states relevant to the

\section{REFERENCE POINT}
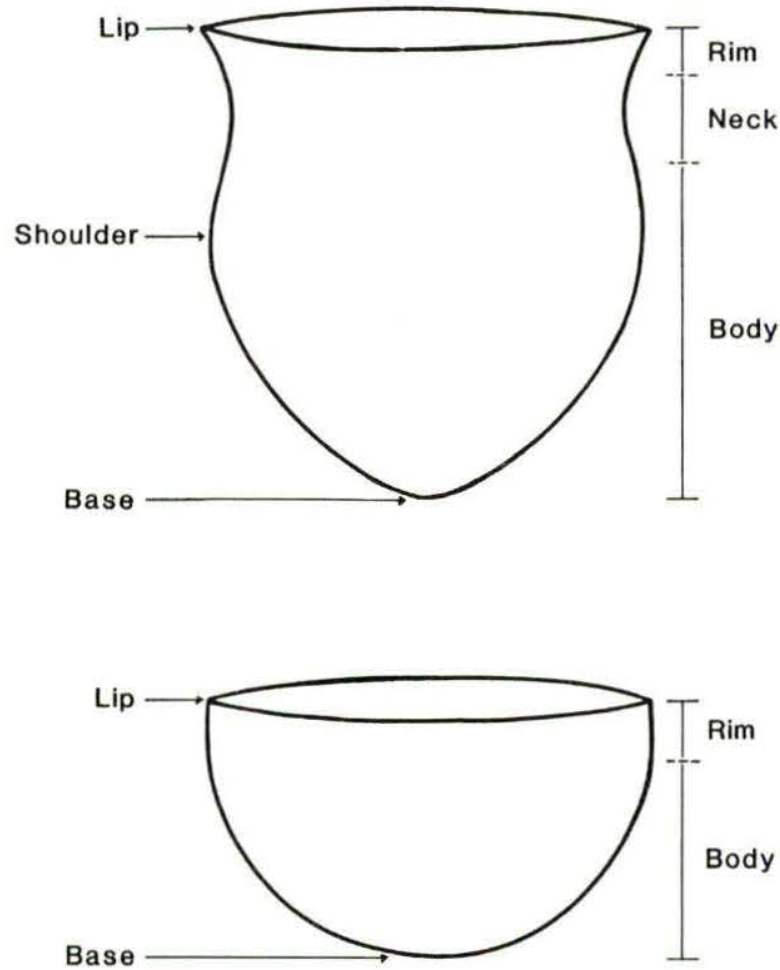

Figure 8.1

Definition of vessel portion attribute states. present study are briefly described below.

\section{Morphological Attributes}

Four separate attributes, which provide information about morphological characteristics of the parent vessel assemblage, were coded. First, the portion or portions of a vessel represented by a sherd was identified. Three vessel portions--rim, neck, and body--are recognized and defined in Figure 8.1. Classification by vessel portion is considered important since it provides an indirect measure of similarity and difference in vessel morphology within and between ceramic assemblages in the absence of sufficiently large vessel samples. Second, sherds were measured according to average thickness. Six thickness attribute states are used and include: $\langle 4 \mathrm{~mm},>4-6 \mathrm{~mm},>6-8 \mathrm{~mm},>8-10$ $\mathrm{mm}$, and $>10 \mathrm{~mm}$. Thickness was measured using a block of wood which had been slotted to the appropriate thickness sizes. As with vessel portion, thickness measurement provides an indirect means for assessing variability in vessel morphology. Two additional morphological attributes of rim sherds--rim form and lip form--were measured. Rim form refers to the configuration of the upper portion of a vessel, as viewed in profile and terminated at the lip, and provides direct information about rim morphology. Lip form refers to the configuration in profile of the lip itself. Attribute states for rim and lip forms are defined in Figure 8.2.
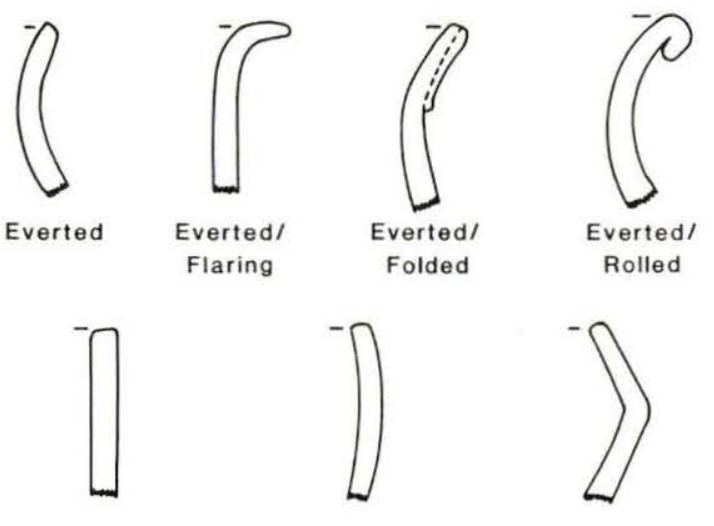

Straight

Inverted

(Simple)

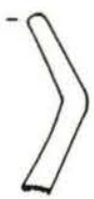

Inverted

(Carinated)

\section{RIM FORMS}

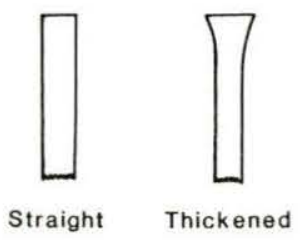

Flat

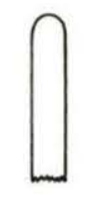

Straight

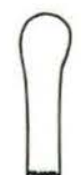

Thickened
Rounded

\section{Pointed}

\section{LIP FORMS}

Figure 8.2

Definition of rim and lip form attribute states. 


\section{Technological Attributes}

Observations of vessel breakage patterns made during the course of analysis indicate that the majority of vessels at all three sites were constructed by a coiling method. Coils were subsequently welded together by scraping, pinching, or by use of a malleating paddle and an anvil. Two technological attributes that relate to the method of vessel manufacture were coded. First, sherds were classified according to the type of temper or aplastic material which was added to the clay in order to provide strength and control shrinkage during firing. Attribute states identified during the analysis include: coarse quartz sand (water-rounded particles up to $2 \mathrm{~mm}$ in dia.), medium quartz sand (visible particles $\leq 1 \mathrm{~mm}$ in dia.), fine quartz sand (sandy textūre but no visible particles), coarse crushed quartz (angular particles $>5 \mathrm{~mm}$ in dia.), medium crushed quartz (particles 2.5-5.0 $\mathrm{mm}$ in dia.), fine crushed quartz (particles $<2.5$ $\mathrm{mm}$ in dia.), coarse crushed feldspar (angular particles usually 2-6 mm in dia.), fine crushed feldspar (finely pulverized particles or feldspar sand usually $<2 \mathrm{~mm}$ in dia.), mixed crushed quartz and feldspar (not differentiated by particle size), coarse grit (misc. crushed rock particles $\geq 2 \mathrm{~mm}$ in dia.), and fine grit (misc. crushed rock particles $\langle 2 \mathrm{~mm}$ in dia.). Ethnohistoric and ethnographic accounts of potterymaking among Southeastern Indians clearly indicate that temper selection varied according to culturally-prescribed rules and was not a random process (Swanton 1946:549-555; Stern 1951). Moreover, temper has proven to be an extremely useful attribute in general ceramic classification in the North Carolina Piedmont (Coe $1964: 26-34$ ).

The second technological attribute considered is interior surface treatment. This attribute refers to the method by which a vessel interior was modified prior to firing rather than the damage to the interior resulting from use. Interior surface treatment attribute states include: plain (uniformly smoothed), scraped (displaying shallow to deep, wide, parallel striations, possible reflecting the use of a serrated shell for thinning the vessel wal1), smoothed/scraped (a scraped interior which was subsequently but incompletely smoothed), and burnished (a highly smoothed surface produced by rubbing with a slick stone). Previous archaeological studies of ceramic variability in piedmont North Carolina suggest that, during the Late Woodland period (after ca. A.D.1000), interior scraping was gradually replaced by smoothing (Coe and Lewis 1952). Coe (1952:308, 310) considers interior scraping with a serrated-edged tool to be a diagnostic characteristic of his Uwharrie and Dan River ceramic series.

\section{Stylistic Attributes}

Three stylistic attributes were coded. The first, exterior surface treatment, represents the predominant type of exterior surface modifi- cation prior to firing. The specific type of surface treatment used is considered here to reflect the stylistic expression of the potter; however, it is recognized that the general process of modifying the exterior surface has certain technological benefits for vessel construction using the techniques of coiling and paddle-and-anvil. In an experimental study to determine the function of textiles in potterymaking, William Holmes (1903:73) observed that

the imprintings were in many cases not made by textiles used as supports, but were applied wrapped about the hand or a mode1ing tool as a means of knitting or welding together the clay surface. Experiment shows that the deeper and more complex the imprintings, if properly managed, the more tenacious becomes the clay.... Scarifying, combing, pinching with the fingernails, or malleating with engraved paddles, served the same purpose.

It is also likely that wrapping or carving of malleating paddles facilitated the removal of the impressed paddle without damaing the exterior surface through adhesion to the clay.

Eight surface treatment attribute states reflecting four separate methods of treatment-smoothing or scraping, impressing, stamping with a wrapped wooden paddle, and stamping with a carved wooden paddle--are represented in the sherd samples. Because of the importance of exterior surface treatment to existing typological studies of ceramics, these attribute states are more fully described below.

Plain. The exterior surface has been smoothed, obliterating any evidence of previous stamping to shape the vessel or weld adjoining coils. Although the majority of the plain sherds analyzed were completely smoothed (Fredricks - 93.2\%, Wall - 90.1\%, Mitchum $97.3 \%)$, a small number of sherds were only roughly smoothed and even fewer $(<0.5 \%$ at each site) were burnished.

Brushed. The exterior surface has been scraped with a stiff twig brush or possibly a serrated shell following stamping and prior to firing, producing parallel but irregular lands and grooves. Brushing or scraping may have served to weld coils and help thin and even out the vessel wall in addition to producing a textured exterior (Holmes 1903:54; also see Harrington 1908:404).

Cob Impressed. The surface has been impressed with a dried corncob, applied by rolling across the vessel exterior. Although one completely-impressed vessel has been reported from the early excavations at the Wall site (Wilson 1983:Plate XVIII), this type of surface treatment appears to be associated more specifically with the rim and neck areas of later vessels from the Mitchum and Fredricks sites. 
Cord Marked. The exterior surface has been stamped with a cord-wrapped paddle. Direction of cordage twist ( $S$ or $Z$ ) was coded during initial analysis; however, this information is not used in this study due to the small number of cord-marked sherds recovered. Cord marking is a predominant surface treatment in Coe's (1952:306-308; 1964:27-32) Badin, Yadkin, and Uwharrie ceramic series, all of which predate the primary occupations at the Wall, Mitchum, and Fredricks sites.

Fabric Marked. The exterior surface has been stamped with a fabric-wrapped paddle. Sherds having simple-plaited fabric impressions comprise major ceramic types within the Badin (Early Woodland) and Yadkin (Middle Woodland) series (Coe 1964:28-32).

Net Impressed. The exterior surface has been stamped with a net-wrapped paddle. Both loop and knotted nets are represented in the samples. As a technique, net impressing has been previously associated with the Uwharrie and Dan River ceramic series (Coe and Lewis 1952). Although net-impressed sherds comprise a significant portion of the Mitchum and Fredricks site samples, their association with the historic occupations at these sites is uncertain. This question is explored in greater detail later.

Simple Stamped. The exterior surface has been stamped with a carved paddle containing a pattern of parallel lands and ooves. Simple stamping is identified by $\mathrm{Cr}$ 1952:311) as the predominant surface treatm . of his Hillsboro series, defined from a ceramic sample recovered at the Wall site between 1938 and 1941 (more fully described by Wilson 1983:342-366). This surface treatment also characterizes the only recognized type within the late prehistoric Gaston series (South 1959:62-84; Coe 1964: 105-106), prevalent along the middle Roanoke River valley northeast of the study area. As expected, simple stamping was the most frequent1y observed ( $58 \%$ ) surface treatment in the Wall site sample. Simple stamping was also well represented within the Mitchum site sample $(11 \%$ of sample total). Whereas the Mitchum vessels were stamped in a manner which produced a linear pattern of lands and grooves parallel or slightly oblique to the rim, the simple stamps observed on vessels and rim sherds from the Wall site were invariably placed diagonal to the rim and perpendicular to one another, producing either a distinctive herringbone pattern or checkered pattern (when overstamped).

Check Stamped. The exterior surface has been stamped with a carved paddle possessing a square to diamond-shaped grid pattern comprised of parallel grooves cut perpendicular or nearly perpendicular to one another. Check stamping represents a major treatment type at both the Wall and Fredricks sites, being represented on $11 \%$ and $27 \%$, respectively, of the sherds analyzed. Stamp motifs at the Wall site are usually bold and deeply impressed, whereas those observed at the Fredricks site are consistently shallow and faint. This latter pattern may reflect either the condition of the paddles being used or the dryness of the clay at time of final stamping. Because these check stamps were unclear, it is likely that several sherds from check stamped vessels were misclassified as plain.

Complicated Stamped. The exterior surface has been stamped with a carved paddle containing a more intricate design than defined for either simple or check stamped treatments. Only a small number of sherds with this type of treatment were recovered from the Fredricks and Mitchum sites. All of them represented curvilinear designs but were too small to ascertain the specific design motif. Complicated stamping is a more common surface treatment at late prehistoric sites of the Pee Dee focus (Coe 1952: 309) and at protohistoric/historic sites of the lower Yadkin and Catawba River drainages (Wilson 1983:486-488).

In addition to these eight treatment types, a ninth category--indeterminate--was used to classify those sherds whose surface treatments were either unidentifiable or ambiguous.

The remaining two stylistic attributes considered by the analysis consist of the type of decoration and location of the decorative motif on the vessel. The frequency of decoration, method of decoration, and placement of decorative motifs varied considerably among the three analyzed assemblages. Decoration, as distinguished from surface treatment, was usually applied by a technique which is distinctively different from the one used to treat the exterior surface of the same vessel and is confined to a specific portion of the vessel. Eighteen decoration attribute states were observed among the three sites; these can be reduced to five separate decorative modes. These modes are briefly summarized below.

Incised. Decoration consists of one or more lines scratched into the exterior vessel surface (while still wet) with a sharp, pointed instrument. Specific attribute states representing this decorative mode and observed in the analyzed samples include: a) inter-connecting "V"s circumscribing the vessel exterior; b) parallel lip incisions placed oblique to the rim edge; c) short incised lines placed perpendicular to the rim edge; d) a single, straight line circumscribing the vessel exterior; and e) lines of varying direction representing an indeterminate decorative motif.

Punctated. Decoration consists of one or more small, deep concavities on the exterior surface, formed by impressing the damp clay with the end of a reed, stick, or other pointed instrument. Attribute states representing punctated decorations include: a) circular; b) circular reed; c) oblong; and d) U-shaped. 
Notched. Decoration consists of notches placed along one of the rim edges. Most notches observed in the analyzed samples appear to have been produced using the edge of the malleating paddle and are oriented perpendicular to the rim edge; however, a few notches were probably produced by impressing a fingertip into the damp clay. Notching was the most frequently observed mode of vessel decoration.

Finger Impressed. Decoration consists of impressing the fingernail or fingertip into the damp clay either singularly or linearly. Finger impressions are applied to the vessel body and thus are distinguished from fingertip notching. Both notching and finger impressing have been recognized as common decorative modes of the Dan River series (Coe and Lewis 1952; Gardner 1980).

Applique. Decoration consists of attaching modeled clay elements to the vessel exterior. Only three such treatments were observed in the Fredricks and Wall site samples and consist of two podes or vessel feet and one simple node.

In addition to the kinds of decoration discussed above, two other types of vessel/sherd modification were observed. First, nine sherds from the Wall site and two sherds from the Mitchum site had been recycled into disks by edge grinding or chipping. Second, incidents of vessel repair were found at all three sites in the form of drilled holes on sherds. The interpretation of these holes as mends rather than suspension holes is supported by the facts that the drill holes frequently occur on non-rim portions of vessels and are located near edges that represent vertical vessel cracks.

All decorative motifs were additionally coded as to their location on the vessel. Recognized locations include: $1 \mathrm{ip}, 1 \mathrm{ip} / \mathrm{rim}, \mathrm{rim}$, rim fold, lower edge of rim fold, neck, neck/ shoulder, and body. Exterior surface treatments and decorations are illustrated later for each of the three analyzed samples.

\section{Size}

Sherd size was coded for all of the specimens analyzed. Size was measured using a template of concentric circles representing categories defined by specific maximum diameter measurements. Those categories are: $\leq 2 \mathrm{~cm}$ dia., $>2-4 \mathrm{~cm}$ dia., $>4-6 \mathrm{~cm}$ dia., >6-8 cm dia., >8-10 $\mathrm{cm}$ dia., and $>10 \mathrm{~cm}$ dia.

Size is considered an important attribute for two reasons. First, sherd size can be used to explore behavioral questions related to discard modes. For example, whether a sherd sample is derived from a primary versus secondary context has specific implications for the expected size distribution of those sherds (see Schiffer 1976:30-34). In a somewhat related manner, size can be used as a criterion for evaluating the probable contemporaneity of two or more sherd types recovered from the same archaeological context (e.g., feature fill). Given that breakage rates are essentially similar for all periods, sherds from an earlier occupation and mixed with later material should be identifiable by a distinctively different size distribution profile which is skewed toward smaller sherd size. Such a pattern can be expected because of the greater cumulative effect of cultural and natural transformations upon the older artifacts (see Schiffer 1976:14-16 for a discussion of transformation processes).

Second, size measurement provides a basis for correcting the relative frequency estimates of sherd types within an assemblage when significant differences in size distribution can be shown to exist. This is important in any attempt to quantify the relationships among sherd types relative to vessel surface area.

\section{FREDRICKS SITE}

Archaeological excavations at the Fredricks site (310r231) recovered 11,203 aboriginal potsherds and three complete vessels. All vessels were recovered in primary context as burial associations; sherds were recovered in secondary context and occurred in all plowzone and sub-plowzone excavation units. All sherds $>2 \mathrm{~cm}$ in diameter were classified according to the attributes discussed above; sherds $\langle 2 \mathrm{~cm}$ in diameter were counted but not classified since their very small size made classification by most attributes either impossible or unreliable. These latter sherds comprise $67 \%$ of the total sample (Table 8.1). The remaining 3698 ceramic artifacts, including vessels, are summarized by temper and exterior surface treatment in Tables $8 \cdot 2-8 \cdot 3$. Frequency distributions of other measured attributes, according to specific temper and surface treatment combinations, are presented in Tables $8.4-8.11$.

Table 8.1

Frequency of Fredricks site sherds and vessels by size.

Size (diameter)

$\mathrm{n}$

$\%$

$\begin{array}{lrr}\leq 2 \mathrm{~cm} & 7508 & 67.00 \\ >2-4 \mathrm{~cm} & 3426 & 30.57 \\ >4-6 \mathrm{~cm} & 210 & 1.87 \\ >6-8 \mathrm{~cm} & 42 & .38 \\ >8-10 \mathrm{~cm} & 13 & .12 \\ >10 \mathrm{~cm} \text { (sherds) } & 4 & .04 \\ >10 \mathrm{~cm} \text { (vessels) } & 3 & .02 \\ \text { Totals } & 11,206 & 100.00\end{array}$


Table 8.2

Frequency of Fredricks site sherds by temper and exterior surface treatment (excluding sherds $\leq 2 \mathrm{~cm}$ dia.).

\begin{tabular}{|c|c|c|c|c|}
\hline Temper & Surface & $\mathrm{n}$ & $\%$ & $\begin{array}{c}\% \\
\text { (of identifiable } \\
\text { sherds) }\end{array}$ \\
\hline \multirow[t]{9}{*}{ Coarse } & Sand & & & \\
\hline & Plain & 13 & .35 & .44 \\
\hline & Brushed & 2 & .05 & .07 \\
\hline & Cord Marked & 1 & .03 & .03 \\
\hline & Net Impressed & 63 & 1.71 & 2.14 \\
\hline & Simple Stamped & 6 & .16 & .20 \\
\hline & Check Stamped & 8 & .22 & .27 \\
\hline & Indeterminate & 38 & 1.03 & - \\
\hline & Sub-Totals & 131 & 3.55 & 3.15 \\
\hline \multirow[t]{11}{*}{ Medium } & Sand & & & \\
\hline & Plain & 883 & 23.88 & 29.93 \\
\hline & Brushed & 61 & 1.65 & 2.07 \\
\hline & Cob Impressed & 9 & .24 & .31 \\
\hline & Cord Marked & 54 & 1.46 & 1.83 \\
\hline & Net Impressed & 469 & 12.68 & 15.90 \\
\hline & Simple Stamped & 76 & 2.06 & 2.58 \\
\hline & Check Stamped & 954 & 25.80 & 32.34 \\
\hline & Complicated Stamped & 2 & .05 & .07 \\
\hline & Indeterminate & 601 & 16.25 & - \\
\hline & Sub-Totals & 3109 & 84.07 & 85.03 \\
\hline \multicolumn{5}{|c|}{ Fine Sand } \\
\hline & Plain & 30 & .81 & 1.02 \\
\hline & Cordmarked & 1 & .03 & .03 \\
\hline & Sub-Totals & 31 & .84 & 1.05 \\
\hline \multicolumn{5}{|c|}{ Coarse Crushed Quartz } \\
\hline & Net Impressed & 5 & .14 & .17 \\
\hline & Indeterminate & 2 & .05 & - \\
\hline & Sub-Totals & 7 & .19 & .17 \\
\hline \multicolumn{5}{|c|}{ Medium Crushed Quartz } \\
\hline & Plain & 2 & .05 & .07 \\
\hline & Brushed & 2 & .05 & .07 \\
\hline & Cord Marked & 1 & .03 & .03 \\
\hline & Net Impressed & 37 & 1.00 & 1.25 \\
\hline & Indeterminate & 7 & .19 & - \\
\hline & Sub-Totals & 49 & 1.32 & 1.42 \\
\hline \multicolumn{5}{|c|}{ Fine Crushed Quartz } \\
\hline & Plain & 40 & 1.08 & 1.36 \\
\hline & Brushed & 8 & .22 & .27 \\
\hline & Cob Impressed & 1 & .03 & .03 \\
\hline & Cord Marked & 5 & .14 & .17 \\
\hline & Net Impressed & 65 & 1.76 & 2.20 \\
\hline & Simple Stamped & 7 & .19 & .24 \\
\hline & Check Stamped & 4 & .11 & .14 \\
\hline & Indeterminate & 54 & 1.46 & - \\
\hline & Sub-Totals & 184 & 4.99 & 4.41 \\
\hline \multicolumn{5}{|c|}{ Coarse Crushed Feldspar } \\
\hline & Plain & 9 & .24 & .31 \\
\hline & Brushed & 1 & .03 & .03 \\
\hline & Net Impressed & 1 & .03 & .03 \\
\hline & Simple Stamped & 7 & .19 & .24 \\
\hline & Check Stamped & 1 & .03 & .03 \\
\hline & Indeterminate & 2 & .05 & - \\
\hline & Sub-Totals & 21 & .57 & .64 \\
\hline \multicolumn{5}{|c|}{ Fine Crushed Feldspar } \\
\hline & Plain & 62 & 1.68 & 2.10 \\
\hline & Cord Marked & 4 & .11 & .14 \\
\hline & Net Impressed & 4 & .11 & .14 \\
\hline & Simple Stamped & 31 & .84 & 1.05 \\
\hline & Check Stamped & 21 & .57 & .71 \\
\hline & Indeterminate & 44 & 1.19 & - \\
\hline & Sub-Totals & 166 & 4.50 & 4.14 \\
\hline Tot: & als & 3698 & 100.03 & 100.01 \\
\hline
\end{tabular}


Table 8.3
Frequency of Fredricks site sherds from Feature and Burial contexts.

\begin{tabular}{|c|c|c|c|c|c|c|c|c|c|c|c|c|c|c|c|c|c|c|c|c|}
\hline Tempe & $\begin{array}{l}\text { er } \\
\text { Surface. }\end{array}$ & Bu. 1 & $\mathrm{Bu} .2$ & $\mathrm{Bu} .3$ & Fea.1 & $\begin{array}{l}\mathrm{Fea} .2 \\
\mathrm{Bu} .4\end{array}$ & $\begin{array}{l}\mathrm{Fea} .3 \\
\mathrm{Bu} .5\end{array}$ & $\begin{array}{l}\mathrm{Fea} .4 \\
\mathrm{Bu} .6\end{array}$ & $\begin{array}{l}\text { Fea. } 5 \\
\text { Bu. } 7\end{array}$ & $\begin{array}{l}\text { Fea. } 6 \\
\text { Bu. } 8\end{array}$ & $\begin{array}{l}\text { Fea. } 7 \\
\text { Bu. } 9\end{array}$ & Fea. 8 & Fea.9 & Fea.10 & Fea.11 & Fea.12 & Fea.13 & Total & $z$ & $\%^{*}$ \\
\hline \multicolumn{21}{|c|}{ Coarse Sand } \\
\hline & Plain & - & - & - & - & - & 1 & 1 & - & - & - & - & - & - & - & - & - & 2 & .11 & .28 \\
\hline & Brushed & - & - & - & - & - & - & 1 & - & - & - & - & - & - & - & - & - & 1 & .06 & .14 \\
\hline & Net Impressed & - & - & - & - & 2 & 1 & - & 1 & 1 & 2 & - & 1 & 2 & 1 & - & - & 11 & .61 & 1.53 \\
\hline & Simple Stamped & 2 & - & - & - & - & - & 1 & - & 1 & - & - & - & - & - & - & - & 4 & .22 & .56 \\
\hline & Check Stamped & - & - & - & - & - & - & $\overline{-}$ & - & - & 1 & - & 1 & - & - & - & - & 2 & .11 & .28 \\
\hline & Indeterminate & 1 & 1 & 1 & - & - & - & 1 & - & - & - & - & - & 1 & - & - & - & 5 & .28 & - \\
\hline \multirow{2}{*}{\multicolumn{21}{|c|}{ Medium Sand }} \\
\hline & & & & & & & & & & & & & & & & & & & & \\
\hline & Plain & 31 & 8 & 69 & 11 & 14 & 2 & 4 & - & 26 & 4 & 2 & 11 & 4 & 2 & 11 & 10 & 209 & 11.60 & 29.07 \\
\hline & Brushed & 1 & - & 1 & - & 1 & 4 & 1 & - & 4 & 1 & - & 6 & 10 & - & - & - & 29 & 1.61 & 4.03 \\
\hline & Cob Impressed & - & - & - & - & - & - & 1 & - & - & 3 & - & - & - & - & - & - & 4 & .22 & .56 \\
\hline & Cord Marked & - & - & 1 & - & 3 & 1 & 1 & - & - & - & - & 1 & - & - & - & - & 7 & .39 & .97 \\
\hline & Net Impressed & 2 & 2 & 21 & 3 & 1 & 4 & 1 & 2 & 3 & 6 & 1 & 17 & 3 & 1 & 1 & 4 & 71 & 3.94 & 9.87 \\
\hline & Simple Stamped & 1 & - & 4 & 2 & 2 & 2 & 1 & - & 1 & 2 & $\overline{-}$ & - & - & - & - & 1 & 16 & .89 & 2.23 \\
\hline & Check Stamped & 25 & 9 & 62 & 15 & 29 & 29 & 8 & 2 & 15 & 16 & 2 & 9 & 9 & - & 6 & 23 & 259 & 14.37 & 36.02 \\
\hline & Indeterminate & 4 & 1 & 3 & 2 & 3 & 3 & 6 & - & 3 & 5 & - & 5 & - & - & - & - & 35 & 1.94 & - \\
\hline & Sub-Totals & 64 & 20 & 161 & 33 & 53 & 45 & 23 & 4 & 52 & 37 & 5 & 49 & 26 & 3 & 18 & 37 & 630 & 34.96 & - \\
\hline \multirow{2}{*}{ Fine } & Sand & & & & & & & & & & & & & & & & & & & \\
\hline \multirow{2}{*}{\multicolumn{2}{|c|}{ Coarse Crushed Quartz }} & - & - & 5 & 1 & 2 & 1 & 1 & - & - & 2 & - & 2 & 2 & - & - & - & 16 & .89 & 2.23 \\
\hline & & & & & & & & & & & & & & & & & & & & \\
\hline & Net Impressed & - & - & - & - & - & - & - & - & - & 2 & - & - & - & - & - & - & 2 & .11 & .28 \\
\hline & Indeterminate & - & - & - & - & - & - & 1 & - & - & - & - & - & - & - & - & - & 1 & .06 & - \\
\hline \multirow{2}{*}{\multicolumn{2}{|c|}{$\begin{array}{c}\text { Sub-Totals } \\
\text { Medium Crushed Quartz }\end{array}$}} & - & - & - & - & - & - & 1 & - & - & 2 & - & - & - & - & - & - & 3 & .17 & - \\
\hline & & & & & & & & & & & & & & & & & & & & \\
\hline & Plain & - & - & 1 & - & - & - & - & - & - & - & - & - & - & - & - & - & 1 & .06 & .14 \\
\hline & Brushed & - & - & - & - & - & - & - & - & 1 & - & - & - & - & - & - & - & 1 & .06 & .14 \\
\hline & Cord Marked & - & - & - & - & - & 1 & - & - & - & - & - & - & - & - & - & - & 1 & .06 & .14 \\
\hline & Net Impressed & 1 & 1 & - & - & - & - & - & - & - & 1 & - & - & 2 & - & - & - & 5 & .28 & .69 \\
\hline & Sub-Totals & 1 & 1 & 1 & - & - & 1 & - & - & 1 & 1 & - & - & 2 & - & - & - & 8 & .44 & - \\
\hline \multirow{8}{*}{ Fine } & Crushed Quartz & & & & & & & & & & & & & & & & & & & \\
\hline & Plain & 4 & - & 2 & - & - & 1 & - & - & - & - & 1 & - & 1 & 2 & 3 & 1 & 15 & .83 & 2.09 \\
\hline & Brushed & - & - & - & - & 1 & - & - & - & - & - & - & - & - & - & - & 1 & 2 & .11 & .28 \\
\hline & Net Impressed & 1 & - & - & - & - & - & 1 & - & 1 & 2 & 1 & - & - & - & - & 1 & 7 & .39 & .97 \\
\hline & Simple Stamped & - & - & - & 1 & - & 1 & - & - & 1 & 1 & - & - & - & - & - & - & 4 & .22 & .56 \\
\hline & Check Stamped & - & - & - & - & - & - & - & - & - & - & - & - & - & 1 & - & - & 1 & .06 & .14 \\
\hline & Indeterminate & - & - & - & - & - & - & 1 & - & 1 & - & - & - & - & - & - & - & 2 & .11 & - \\
\hline & Sub-Totals & 5 & - & 2 & 1 & 1 & 2 & 2 & - & 3 & 3 & 2 & - & 1 & 3 & 3 & 3 & 31 & 1.72 & - \\
\hline \multirow{2}{*}{\multicolumn{2}{|c|}{$\begin{array}{l}\text { Coarse Crushed Feldspar } \\
\text { Plain }\end{array}$}} & & & & & & & & & & & & & & & & & & & \\
\hline & & - & - & - & - & - & - & - & - & - & - & 2 & 1 & 2 & - & - & - & 5 & .28 & .69 \\
\hline & Check Stamped & - & - & - & - & - & - & - & - & - & 1 & - & - & - & - & - & - & 1 & .06 & .14 \\
\hline \multirow{7}{*}{ Fine } & Sub-Totals & - & - & - & - & - & - & - & - & - & 1 & 2 & 1 & 2 & - & - & - & 6 & .33 & - \\
\hline & Crushed Feldspar & & & & & & & & & & & & & & & & & & & \\
\hline & Plain & 1 & 1 & 2 & - & 3 & 1 & 3 & - & 8 & 2 & 2 & 5 & - & - & - & 6 & 34 & 1.89 & 4.73 \\
\hline & Simple Stamped & - & - & - & - & 1 & - & - & - & - & 1 & - & 1 & - & - & - & 1 & 4 & .22 & .56 \\
\hline & Check Stamped & - & - & 1 & - & - & - & 1 & - & - & 3 & - & - & - & - & - & - & 5 & .28 & .69 \\
\hline & Indeterminate & - & - & - & - & 1 & - & - & - & - & 2 & - & - & - & - & - & 1 & 4 & .22 & - \\
\hline & Sub-Totals & 1 & 1 & 3 & - & 5 & 1 & 4 & - & 8 & 8 & 2 & 6 & - & - & - & 8 & 47 & 2.61 & - \\
\hline \multicolumn{2}{|c|}{ Other $(\leq 2 \mathrm{~cm}$ dia. $)$} & 116 & 17 & 249 & 38 & 77 & 87 & 80 & 8 & 79 & 94 & 13 & 52 & 21 & 2 & 32 & 71 & 1036 & 57.49 & - \\
\hline \multicolumn{2}{|c|}{ Totals } & 190 & 40 & 422 & 73 & 140 & 139 & 115 & 13 & 145 & 151 & 24 & 114 & 55 & 9 & 53 & 119 & 1802 & 100.00 & 100.01 \\
\hline
\end{tabular}

* of identifiable sherds. 
Table 8.4

Sumnary of coarse sand-tempered sherds from the Fredricks site.

\begin{tabular}{|c|c|c|c|c|c|c|}
\hline $\begin{array}{l}\text { Attribute } \\
\text { Attribute State }\end{array}$ & Plain & Brushed & $\begin{array}{l}\text { Exterfor } \\
\text { Cord } \\
\text { Marked }\end{array}$ & $\begin{array}{c}\text { Surface } \\
\text { Net } \\
\text { Impressed }\end{array}$ & $\begin{array}{l}\text { Simple } \\
\text { Stamped }\end{array}$ & $\begin{array}{l}\text { Check } \\
\text { Stamped }\end{array}$ \\
\hline \multicolumn{7}{|l|}{ Portion } \\
\hline $\mathrm{Rim}$ & 0 & 0 & 0 & 1 & 0 & 0 \\
\hline Neck & 2 & 0 & 0 & 4 & 2 & 0 \\
\hline Body & 11 & 2 & 1 & 58 & 4 & 8 \\
\hline \multicolumn{7}{|l|}{ Th1ckness } \\
\hline$\leq 6 \mathrm{~mm}$ & 2 & 0 & 0 & 1 & 3 & 1 \\
\hline $56-8 \mathrm{~mm}$ & 7 & 1 & 1 & 27 & 3 & 4 \\
\hline$>8 \mathrm{~mm}$ & 4 & 1 & 0 & 34 & 0 & 3 \\
\hline \multicolumn{7}{|l|}{ Interfor Surface } \\
\hline Plain & 11 & 1 & 1 & 16 & 6 & 8 \\
\hline Scraped & 2 & 1 & 0 & 39 & 0 & 0 \\
\hline \multicolumn{7}{|l|}{ Rim Form } \\
\hline Everted & 0 & 0 & 0 & 1 & 0 & 0 \\
\hline \multicolumn{7}{|l|}{ Lip Form } \\
\hline Flat & 0 & 0 & 0 & 1 & 2 & 0 \\
\hline \multicolumn{7}{|l|}{ Decoration/Location } \\
\hline $\begin{array}{l}\text { V-shaped Notches } \\
\text { (on } 11 \mathrm{p})\end{array}$ & 0 & 0 & 0 & 1 & 0 & 0 \\
\hline $\begin{array}{l}\text { F1ngernail Impress. } \\
\text { (on neck) }\end{array}$ & . 0 & 0 & 0 & 2 & 0 & 0 \\
\hline \multicolumn{7}{|l|}{ size } \\
\hline$>2-4 \mathrm{~cm}$ & 13 & 1 & 1 & 56 & 5 & 8 \\
\hline$>4-6 \mathrm{~cm}$ & 0 & 1 & 0 & 6 & 1 & 0 \\
\hline
\end{tabular}

Table 8.5

Sumary of medtum sand-tempered sherds from the Fredricks aite.

\begin{tabular}{|c|c|c|c|c|c|c|c|c|}
\hline $\begin{array}{l}\text { Actribute } \\
\text { Attribute State }\end{array}$ & Plain & Brushed & $\begin{array}{c}\text { Cob } \\
\text { Impressed }\end{array}$ & $\begin{array}{l}\text { Exterfor } \\
\text { Cord } \\
\text { Marked }\end{array}$ & $\begin{array}{c}\text { I Surface } \\
\text { Net } \\
\text { Impressed }\end{array}$ & $\begin{array}{l}\text { Simple } \\
\text { Stamped }\end{array}$ & $\begin{array}{l}\text { Check } \\
\text { Stamped }\end{array}$ & $\begin{array}{l}\text { Comp. } \\
\text { Stamped }\end{array}$ \\
\hline \multicolumn{9}{|l|}{ Portion } \\
\hline Rim & 85 & 6 & 2 & 3 & 18 & 7 & 79 & 0 \\
\hline Neck & 128 & 8 & 2 & 6 & 24 & 21 & 168 & 1 \\
\hline Body & 689 & 49 & 5 & 45 & 430 & 49 & 718 & 1 \\
\hline \multicolumn{9}{|l|}{ Th1ckness } \\
\hline$\leq 6 \mathrm{~mm}$ & 300 & 6 & 3 & 10 & 22 & 16 & 552 & 0 \\
\hline $56-8 \mathrm{~mm}$ & 477 & 48 & 6 & 43 & 262 & 47 & 346 & 2 \\
\hline$>8 \mathrm{~mm}$ & 104 & 7 & 0 & 1 & 184 & 13 & so & 0 \\
\hline \multicolumn{9}{|l|}{ Intertor Surface } \\
\hline Plain & 869 & 35 & 6 & 44 & 118 & 73 & 937 & 2 \\
\hline Scrnped & 11 & 26 & 2 & 7 & 283 & 2 & 6 & 0 \\
\hline \multicolumn{9}{|l|}{ Rin Form } \\
\hline Everted & 32 & 4 & 1 & 0 & 9 & 6 & 27 & 0 \\
\hline Stralght & 13 & 1 & 0 & 0 & 4 & 0 & 9 & 0 \\
\hline Inverted & 3 & 0 & 0 & 0 & 0 & 0 & 4 & 0 \\
\hline \multicolumn{9}{|l|}{ Lip Form } \\
\hline Fist & 45 & 2 & 0 & 1 & 1 & 4 & 71 & 0 \\
\hline Rounded & 28 & 4 & 2 & 2 & 13 & 3 & 11 & 0 \\
\hline Polnted & 10 & 0 & 0 & 0 & 4 & 0 & 0 & 0 \\
\hline \multicolumn{9}{|l|}{ Decoration/Location } \\
\hline $\begin{array}{l}\text { Inc1sed (oblique } \\
\text { on 11p) }\end{array}$ & 3 & 0 & 0 & 1 & 0 & 0 & 18 & 0 \\
\hline $\begin{array}{l}\text { Incised "v"s (on } \\
\text { neck/shoulder) }\end{array}$ & 1 & 0 & 0 & 0 & 0 & 0 & 0 & 0 \\
\hline $\begin{array}{l}\text { oblong Punctations } \\
\text { (on 11p) }\end{array}$ & 1 & 0 & 0 & 0 & 0 & 0 & 0 & 0 \\
\hline $\begin{array}{l}\text { V-Shaped Notches } \\
\text { (on 1ip) }\end{array}$ & 0 & 0 & 1 & 0 & 1 & 1 & 0 & 0 \\
\hline $\begin{array}{l}\text { V-Shaped Notches } \\
\text { (on } 11 \mathrm{p} / \mathrm{r} 1 \mathrm{~m})\end{array}$ & 0 & 0 & 0 & 0 & 6 & 0 & 0 & 0 \\
\hline $\begin{array}{l}\text { Fingertip Notches } \\
\text { (on } 11 p)\end{array}$ & 0 & 2 & 0 & 0 & 0 & 0 & 0 & 0 \\
\hline $\begin{array}{l}\text { Fingernali Impress. } \\
\text { (on neck) }\end{array}$ & 1 & 0 & 0 & 0 & 0 & 0 & 0 & 0 \\
\hline \multicolumn{9}{|l|}{ S1ze } \\
\hline$>2-4 \mathrm{~cm}$ & 817 & 49 & 9 & 51 & 433 & 67 & 861 & 1 \\
\hline $34-6 \mathrm{~cm}$ & 46 & 10 & 0 & 2 & 32 & 9 & 66 & 1 \\
\hline$>6 \mathrm{~cm}$ & 20 & 2 & 0 & 1 & 4 & 0 & 27 & 0 \\
\hline
\end{tabular}

Table 8.6

Summary of fine sand-tempered sherds from the Fredricks site,

\begin{tabular}{|c|c|c|}
\hline \multirow{3}{*}{$\begin{array}{l}\text { Attribute } \\
\text { Attribute State }\end{array}$} & \multicolumn{2}{|c|}{ Exterior Surface } \\
\hline & & Cord \\
\hline & Plain & Marked \\
\hline \multicolumn{3}{|l|}{ Portion } \\
\hline RIm & 10 & 0 \\
\hline Neck & 2 & 0 \\
\hline Body & 19 & 1 \\
\hline \multicolumn{3}{|l|}{ Thickness } \\
\hline$\leq 6 \mathrm{~mm}$ & 18 & 0 \\
\hline$\overline{>}_{6}-8 \mathrm{~mm}$ & 9 & 0 \\
\hline$>8 \mathrm{~mm}$ & 3 & 1 \\
\hline Interior Surface & & \\
\hline Pla1n & 25 & 1 \\
\hline \multicolumn{3}{|l|}{ Rim Form } \\
\hline Everted & 2 & 0 \\
\hline Straight & 1 & 0 \\
\hline Inverted & 4 & 0 \\
\hline \multicolumn{3}{|l|}{ Lip Form } \\
\hline Flat & 1 & 0 \\
\hline Rounded & 6 & 0 \\
\hline Pointed & 3 & 0 \\
\hline Decoration/Location & - & - \\
\hline \multicolumn{3}{|l|}{ Size } \\
\hline$>2-4 \mathrm{~cm}$ & 26 & 1 \\
\hline$>4-6 \mathrm{~cm}$ & 3 & 0 \\
\hline$>6 \mathrm{~cm}$ & 1 & 0 \\
\hline
\end{tabular}

Table 8.7

Summary of coarse crushed quartz-tempered sherds from the Fredricks site.

\begin{tabular}{lc}
\hline & \\
\hline $\begin{array}{c}\text { Attribute } \\
\text { Attribute State }\end{array}$ & $\begin{array}{c}\text { Exterior Surface } \\
\text { Net } \\
\text { Impressed }\end{array}$ \\
\hline $\begin{array}{l}\text { Portion } \\
\text { Neck } \\
\text { Body }\end{array}$ & 1 \\
Thickness & 4 \\
$>6-8$ mm & \\
$>8$ mm & 1 \\
Interior Surface & 4 \\
P1ain & 1 \\
Scraped & 3 \\
Rim Form & - \\
Lip Form & - \\
Decoration/Location & - \\
S1ze $>2-4 \mathrm{~cm}$ & 4 \\
$>4-6 \mathrm{~cm}$ & 1 \\
\hline
\end{tabular}

Table 8.8

Sumnary of medium crushed quartz-tempered sherds from the Fredricks site.

\begin{tabular}{|c|c|c|c|c|}
\hline $\begin{array}{l}\text { Attribute } \\
\text { Attribute State }\end{array}$ & Plain & $\begin{array}{l}\text { Extertor } \\
\text { Brushed }\end{array}$ & $\begin{array}{c}\text { Surface } \\
\text { Cord } \\
\text { Marked }\end{array}$ & $\begin{array}{c}\text { Net } \\
\text { Impressed }\end{array}$ \\
\hline \multicolumn{5}{|l|}{ Portion } \\
\hline Rim & 0 & 0 & 0 & 1 \\
\hline Neck & 0 & 0 & 0 & 2 \\
\hline Body & 2 & 2 & 1 & 34 \\
\hline \multicolumn{5}{|l|}{ Thickness } \\
\hline$\leq 6 \mathrm{~mm}$ & 0 & 0 & 0 & 1 \\
\hline $56-8 \mathrm{~mm}$ & 1 & 1 & 1 & 17 \\
\hline$>8 \mathrm{~mm}$ & 1 & 1 & 0 & 19 \\
\hline \multicolumn{5}{|l|}{ Interfor Surface } \\
\hline Pla1n & 2 & 0 & 0 & 7 \\
\hline Scraped & 0 & 2 & 0 & 22 \\
\hline \multicolumn{5}{|l|}{ Rin Form } \\
\hline Everted & 0 & 0 & 0 & 1 \\
\hline \multicolumn{5}{|l|}{ Lip Form } \\
\hline Rounded & 0 & 0 & 0 & 1 \\
\hline Decoration/Location & - & - & - & - \\
\hline \multicolumn{5}{|l|}{ Size } \\
\hline$>2-4 \mathrm{~cm}$ & 2 & 2 & 1 & 36 \\
\hline$>4-6 \mathrm{~cm}$ & 0 & 0 & 0 & 1 \\
\hline
\end{tabular}


Table 8.9

Summary of fine crushed quartz-tempered sherds from the Fredricks site.

\begin{tabular}{|c|c|c|c|c|c|c|c|}
\hline \multirow[b]{2}{*}{$\begin{array}{l}\text { Attribute } \\
\text { Attribute State }\end{array}$} & \multicolumn{6}{|c|}{ Exterior Surface } & \multirow[b]{2}{*}{$\begin{array}{l}\text { Check } \\
\text { Stamped }\end{array}$} \\
\hline & Plain & Brushed & $\begin{array}{c}\text { Cob } \\
\text { Impressed }\end{array}$ & $\begin{array}{l}\text { Cord } \\
\text { Marked }\end{array}$ & $\begin{array}{c}\text { Net } \\
\text { Impressed }\end{array}$ & $\begin{array}{l}\text { S1mple } \\
\text { Stamped }\end{array}$ & \\
\hline \multicolumn{8}{|l|}{ Portion } \\
\hline Rim & 0 & 0 & 1 & 0 & 4 & 0 & 0 \\
\hline Neck & I & 1 & 0 & 0 & 3 & 1 & 0 \\
\hline $\begin{array}{l}\text { Body } \\
\text { Thickness }\end{array}$ & 39 & 7 & 0 & 5 & \multirow{2}{*}{ Thickness } & 6 & 4 \\
\hline$\leq 6 \mathrm{~mm}$ & 2 & 0 & 0 & 0 & & 1 & 1 \\
\hline$>6-8 \mathrm{~mm}$ & 14 & 6 & 1 & 4 & 31 & 6 & 0 \\
\hline$>8-10 \mathrm{~mm}$ & 24 & 2 & 0 & 1 & 32 & 0 & 3 \\
\hline \multicolumn{8}{|l|}{ Intertor Surface } \\
\hline Plain & 37 & 3 & 1 & 3 & 12 & 7 & 2 \\
\hline Scraped & 2 & 5 & 0 & 2 & 42 & 0 & 2 \\
\hline \multicolumn{5}{|l|}{ Rim Form } & 0 & 0 & 0 \\
\hline \multicolumn{8}{|l|}{ L1p Form } \\
\hline Flat & 0 & 0 & 0 & 0 & 1 & 0 & 0 \\
\hline \multicolumn{8}{|l|}{ Decorat Ion/Locat ton } \\
\hline $\begin{array}{l}\text { Short Inc1sions } \\
\text { (on neck) }\end{array}$ & 0 & 0 & 0 & 0 & 1 & 0 & 0 \\
\hline $\begin{array}{l}\text { V-Shaped Notches } \\
\text { (on 11p) }\end{array}$ & 0 & 0 & 1 & 0 & 0 & 0 & 0 \\
\hline $\begin{array}{l}\text { Fingerna11 Impress. } \\
\text { (on 11p) }\end{array}$ & 0 & 0 & 0 & 0 & 1 & 0 & 0 \\
\hline \multicolumn{8}{|l|}{ Size } \\
\hline$>2-4 \mathrm{~cm}$ & 31 & 2 & 1 & 5 & 59 & 5 & 3 \\
\hline$>4-6 \mathrm{~cm}$ & 6 & 3 & 0 & 0 & 5 & 2 & 1 \\
\hline$>6 \mathrm{~cm}$ & 3 & 3 & 0 & 0 & 1 & 0 & 0 \\
\hline
\end{tabular}

Table 8.10

Summary of coarse crushed feldspar-tempered sherds from the Fredricks site.

\begin{tabular}{|c|c|c|c|c|c|}
\hline \multirow[b]{2}{*}{$\begin{array}{l}\text { Attribute } \\
\text { Attribute State }\end{array}$} & \multicolumn{5}{|c|}{ Exterior Surface } \\
\hline & Plain & Brushed & $\begin{array}{l}\text { Net } \\
\text { Impressed }\end{array}$ & $\begin{array}{l}\text { Simple } \\
\text { Stamped }\end{array}$ & $\begin{array}{l}\text { Check } \\
\text { Stamped }\end{array}$ \\
\hline \multicolumn{6}{|l|}{ Portion } \\
\hline $\mathrm{Rim}$ & 1 & 0 & 0 & 0 & 0 \\
\hline Neck & 1 & 1 & 0 & 2 & 1 \\
\hline Body & 8 & 0 & 1 & 5 & 0 \\
\hline \multicolumn{6}{|l|}{ Thickness } \\
\hline$>6-8 \mathrm{~mm}$ & 6 & 0 & 1 & 3 & 1 \\
\hline$>8 \mathrm{~mm}$ & 3 & 1 & 0 & 4 & 0 \\
\hline \multicolumn{6}{|l|}{ Interfor Surface } \\
\hline Plain & 9 & 1 & 1 & 7 & 1 \\
\hline \multicolumn{6}{|l|}{ Rim Form } \\
\hline Everted & 1 & 0 & 0 & 0 & 0 \\
\hline \multicolumn{6}{|l|}{ Lip Form } \\
\hline Flat & 1 & 0 & 0 & 0 & 0 \\
\hline Decoration/Location & - & - & - & - & - \\
\hline \multicolumn{6}{|l|}{ S1ze } \\
\hline$>2-4 \mathrm{~cm}$ & 7 & 1 & 1 & 7 & 1 \\
\hline$>4-6 \mathrm{~cm}$ & 2 & 0 & 0 & 0 & 0 \\
\hline
\end{tabular}

Table 8.11

Sumary of fine crushed feldspar-tempered sherds from the Fredricks site.

\begin{tabular}{|c|c|c|c|c|c|}
\hline \multirow[b]{2}{*}{$\begin{array}{l}\text { Attribute } \\
\text { Attribute State }\end{array}$} & \multicolumn{4}{|c|}{ Exterior Surface } & \multirow[b]{2}{*}{$\begin{array}{l}\text { Check } \\
\text { Stamped }\end{array}$} \\
\hline & Plain & $\begin{array}{l}\text { Cord } \\
\text { Marked }\end{array}$ & $\begin{array}{c}\text { Net } \\
\text { Impressed }\end{array}$ & $\begin{array}{l}\text { S1mple } \\
\text { Stamped }\end{array}$ & \\
\hline \multicolumn{6}{|l|}{ Portion } \\
\hline R1m & 6 & 0 & 0 & 1 & 0 \\
\hline Neck & 4 & 0 & 1 & 9 & 2 \\
\hline Body & 55 & 4 & 3 & 22 & 19 \\
\hline \multicolumn{6}{|l|}{ Th1ckness } \\
\hline$\leq 6 \mathrm{~mm}$ & 12 & 0 & 1 & 0 & 6 \\
\hline $56-8 \mathrm{~mm}$ & 41 & 1 & 2 & 18 & 10 \\
\hline$>8 \mathrm{~mm}$ & 9 & 3 & 1 & 13 & 5 \\
\hline \multicolumn{6}{|l|}{ Interior Surface } \\
\hline Plain & 58 & 3 & 3 & 30 & 20 \\
\hline Scraped & 2 & 1 & 1 & 1 & 1 \\
\hline \multicolumn{6}{|l|}{ Rim Form } \\
\hline Everted & 3 & 0 & 0 & 1 & 0 \\
\hline \multicolumn{6}{|l|}{ Lip Form } \\
\hline Flat & 4 & 0 & 0 & 0 & 0 \\
\hline Rounded & 1 & 0 & 0 & 1 & 0 \\
\hline Pointed & 1 & 0 & 0 & 0 & 0 \\
\hline \multicolumn{6}{|l|}{ Decoration/Location } \\
\hline $\begin{array}{l}\text { V-Shaped Notches } \\
\text { (on 11p) }\end{array}$ & 3 & 0 & 0 & 1 & 0 \\
\hline \multicolumn{6}{|l|}{ Size } \\
\hline$>2-4 \mathrm{~cm}$ & 59 & 4 & 4 & 29 & 20 \\
\hline$>4-6 \mathrm{~cm}$ & 3 & 0 & 0 & 2 & 1 \\
\hline
\end{tabular}

Two primary goals of the Fredricks site ceramic analysis were to define the ceramic assemblage associated with the Middle Contact period occupation at the site and to evaluate the possibility of other cultural components. This was accomplished by evaluating the frequency distributions of sherd categories (temper and surface treatment combinations) for the whole site and for plowzone and sub-plowzone contexts, and by examining patterns of attribute associations. Since all excavated features and burials date to the Middle Contact period (recognized by the presence of historic trade artifacts), it was expected that most sherds from those contexts could also be associated with that occupation. This expectation is based on the premise, supported by field observation, that the feature and burial fill largely represents episodes of intentional filling with general and activity-specific refuse by the occupants of the site. Plowzone sherds, conversely, represent artifacts that have been plowed from the tops of features and burials as well as artifacts which gradually accumulated on the living surface of the site. Given the absence of nonhistoric features within the excavation area, ceramic evidence of earlier cultural components (if present) should occur primarily within the plowzone and secondarily as minor contaminants within features and burials. Earlier ceramic artifacts also should be smaller in size when compared with the later historic assemblage, assuming similar breakage rates (see above).

Eight separate temper types and exterior surface treatments are recognized within the sample, defining 38 distinct sherd categories. Thirteen of these categories are represented by more than $1.00 \%$ of the total sample of identifiable sherds $(n=2893)$. Of these, medium sandtempered plain (29.93\%), check-stamped $(32.34 \%)$, and net-impressed $(15.90 \%)$ sherds comprise $78.17 \%$ of the identifiable sherd sample. Given similarities to the three whole vessels recovered, medium sand-tempered plain and checkstamped ceramics are clearly associated with the historic occupation. The relationship of the net-impressed sherds, however, is less obvious. Since these three sherd categories comprise the majority of the sample, they are considered in greater detail below.

Evaluation of the relationships between medium sand-tempered plain, check-stamped, and net-impressed ceramics initially involved the use of a Chi-square test of association to identify significant differences among the three sherd categories with respect to various measured attributes. Given that all three categories comprise the same assemblage, general similarity can be anticipated for other attributes reflecting technological, morphological, and stylistic dimensions. Conversely, overall attribute dissimilarity can be expected if these sherd categories represent either temporally or culturally distinct contexts. Although functional variability may be offered as a possible 
explanation for certain differences, significant difference among a majority of attributes more likely can be considered as an indicator of separate ceramic traditions.

Attributes examined for the three sherd categories include: vessel portion, thickness, interior surface treatment, size, rim form, lip form, and decoration/location. All but the last attribute were represented in samples large enough to be evaluated statistically; these results are presented in Figure 8.3. Data used for the Chi-square tests were taken from Table 8.5. Significant differences were observed for all attributes except size and rim form. Additionally, substantial differences in decoration also exist between the net-impressed sherds and sherds of the other two categories.

Medium sand-tempered plain and checkstamped sherds reflect a ceramic technology where vessels interiors were invariably smoothed. Morphologically, they reflect thinwalled jars and, less frequently, bowls. Relative frequency profiles for vessel portion suggest that restricted vessel forms comprise a majority of the overall assemblage, as indicated by the comparatively high proportion of neck sherds. This is also evidenced by the predominance of everted rims, most of which have flattened lips. The major type of decoration observed for both plain and check-stamped sherds was oblique incising of the flattened lip surface. Although this decoration was common on check-stamped rims $(22.8 \%)$, it was rare on plain rims $(3.5 \%)$.

Medium sand-tempered net-impressed sherds, conversely, reflect moderately thick-walled vessels that usually were boldly scraped on the interior surface. The relatively low frequency of rim and neck sherds, when compared with the plain and check-stamped sherds, suggests that net-impressed vessels probably were significantly larger, had little neck constriction, and had a substantially greater ratio of body area to rim circumference. Everted rims on netimpressed sherds appear to represent simple flaring of the vessel lip. In contrast to the other two sherd categories, net-impressed lips were predominantly rounded and notched. Notching was observed on $38.9 \%$ of all rim sherds.

It was anticipated that a significant difference between net-impressed and other sherd categories would also be manifested in sherd size. Specifically, interior scraping on netimpressed ceramics was seen as a probable indicator of temporal priority over the plain and check-stamped categories; consequently, it was expected that net-impressed sherds would be generally smaller in size, reflecting a greater cumulative effect of naturally and culturally induced weathering processes on this sherd category. When examined, however, it was found that all categories were similarly distributed by size. Given the observation that netimpressed sherds are significantly thicker, it is likely that breakage rates among the three sherd categories are not equal. As a consequence, the analysis of size is inconclusive.

At this point, it can be reasonably concluded that net-impressed ceramics and plain and check-stamped ceramics are products of two separate traditions; however, the question of their contemporaneity still remains. In order to address this question, sherd frequencies for the three categories were compared from plowzone and feature/burial contexts. As a method of analysis, 95\% confidence intervals were calculated for the relative frequency (percent) of each category for each context. Confidence intervals were also calculated for all other temper/surface treatment categories comprising more than one percent of the total identified sherd sample. It was concluded that the distributions were significantly different if the confidence intervals did not intersect. The interpretation of potential analysis results are as follows:

1. Significantly higher percent within features/burials indicates a likelihood that the sherd category and features/burials are associated (i.e., are a product of the historic occupation);

2. Significantly lower percent within features/burials indicates a likelihood that the sherd category and features/burials are not associated; and

3. No significant difference between contexts indicates that a sherd category may or may not be associated with the historic occupation.

The results of this analysis are presented in Table 8.12.

of the 13 sherd categories considered, six displayed significantly different distributions. Medium sand-tempered brushed, fine sand-tempered plain, and fine crushed feldspar-tempered plain were significantly more frequent within features and burials, indicating an historic association for these sherds. Distributions of other sherd attributes for these three categories are generally consistent with this interpretation. On the other hand, three sherd categories-including medium sand-tempered net-impressed-displayed significantly lower percentages for feature/burial contexts, suggesting their probable association with other site occupations (i.e., either spatially or temporally distinct). The other two sherd categories--medium sandtempered cord-marked and fine crushed quartztempered net-impressed--are similar to medium sand-tempered net-impressed in that they reflect moderately thick-walled, rounded lip vessels that were often scraped on the interior vessel surface. Finally, medium sand-tempered plain and check-stamped, along with five other categories, were similarly distributed (in a statistical sense) between plowzone and feature/ burial contexts. The plain and check-stamped categories, however, were the major constituents 

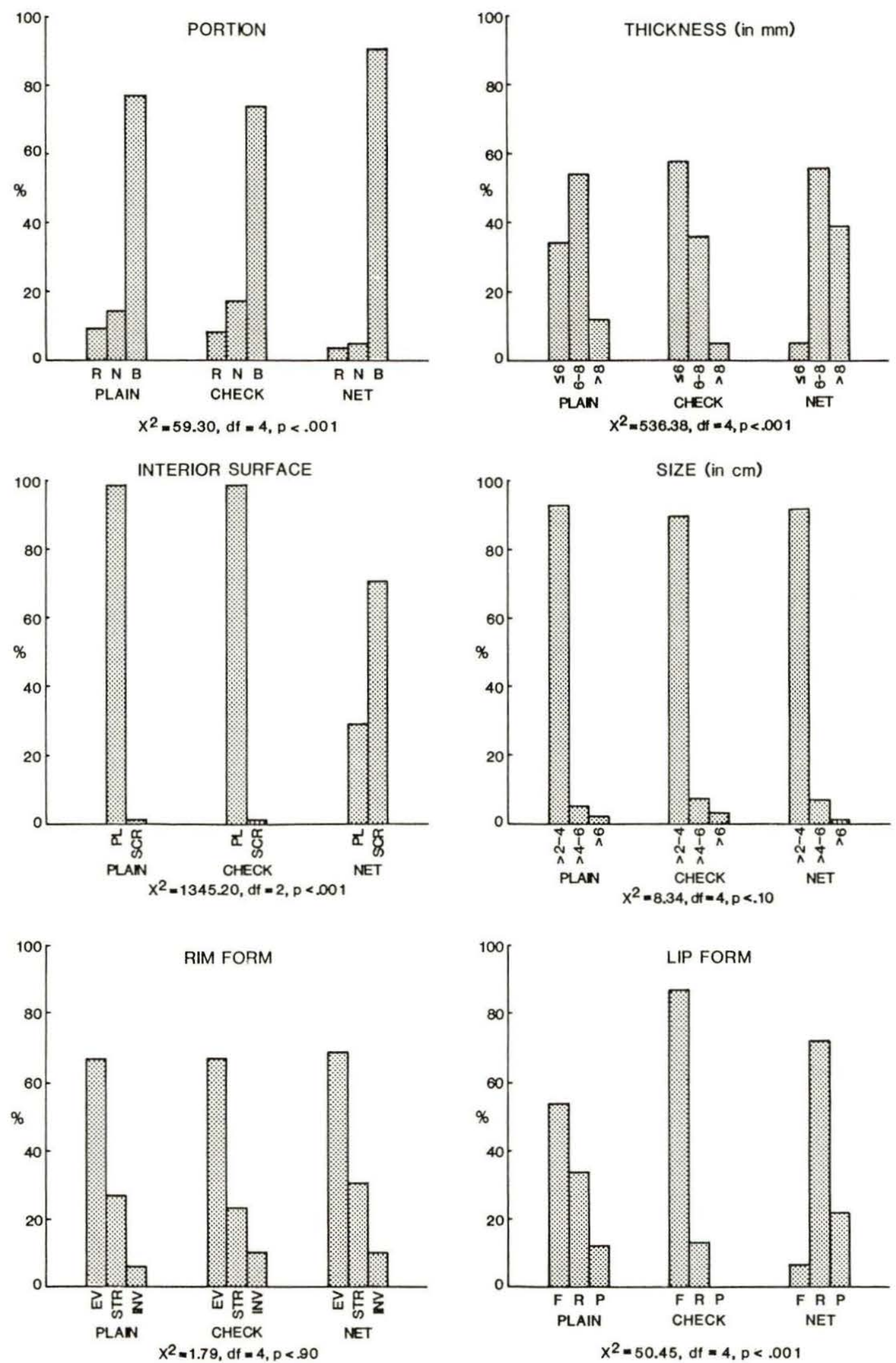

Figure 8.3

Relative frequency distributions and statistical evaluation of selected attributes for medium sand-tempered plain, checkstamped, and net-impressed sherds from the Fredricks site. 
Table 8.12

Evaluation of differences in spatial distribution of selected sherd categorles at the Fredricks site.

\begin{tabular}{|c|c|c|c|c|c|c|c|}
\hline \multirow[b]{2}{*}{$\begin{array}{l}\text { Temper } \\
\quad \text { Surface Treatment }\end{array}$} & \multirow[b]{2}{*}{$\mathrm{n}$} & \multicolumn{2}{|c|}{ Plowzone } & \multicolumn{3}{|c|}{ Features/Burfals } & \multirow[b]{2}{*}{$\begin{array}{l}\text { S1gniftcant ly } \\
\text { Different }\end{array}$} \\
\hline & & & $\begin{array}{l}952 \text { c. } 1 . \\
(+1-)\end{array}$ & n & 3 & $\begin{array}{c}953 \text { c. } 1 . \\
(+/-)\end{array}$ & \\
\hline Coarse Sand & & & & & & & \\
\hline & 49 & 2.25 & 0.52 & 11 & 1.53 & 0.76 & \\
\hline $\begin{array}{l}\text { Medium Sand } \\
\text { Plafn }\end{array}$ & 657 & 30.22 & 1.63 & 209 & 29.70 & 2.79 & \\
\hline Brushed & 31 & 1.43 & .42 & 29 & 4.03 & 1.21 & * \\
\hline Cord Marked & 46 & 2.12 & .51 & 7 & .97 & .60 & * \\
\hline Net Impressed & 387 & 17.80 & 1.35 & 71 & 9.87 & 2.04 & * \\
\hline Stmple Stamped & 57 & 2.62 & .57 & 16 & 2.23 & .91 & \\
\hline Check Stamped & 687 & 31.60 & 1.65 & 259 & 36.02 & 2.95 & \\
\hline Fine Sand & & & & & & & \\
\hline $\begin{array}{l}\text { Plain } \\
\text { Medtum Crushed Ouart }\end{array}$ & 14 & .64 & .28 & 16 & 2.23 & .91 & * \\
\hline $\begin{array}{l}\text { Medfum Crushed Quartz } \\
\text { Net Impressed } \\
\text { Fine Crushed Quartz }\end{array}$ & 31 & 1.43 & .42 & 5 & .69 & .51 & \\
\hline $\begin{array}{l}\text { Fine Crushed Quartz } \\
\text { Plaln }\end{array}$ & 24 & 1.10 & .37 & 15 & 2.09 & .88 & \\
\hline $\begin{array}{l}\text { Net Impressed } \\
\text { Ftne Crushed Feldspar }\end{array}$ & 56 & 2.58 & .56 & 7 & .97 & .60 & * \\
\hline $\begin{array}{l}\text { Fine Crushed Feldspar } \\
\text { Plain }\end{array}$ & 28 & 1.29 & .40 & 34 & 4.73 & 1.31 & * \\
\hline Simple Stamped & 25 & 1.15 & .38 & 4 & .56 & .46 & \\
\hline N & 2174 & & & 719 & & & \\
\hline
\end{tabular}

Note: Confldence Intervals are calculated (following Hays 1973:379) as:

$$
\begin{aligned}
\text { C.1. = } & \pm=\sqrt{\frac{(p q)}{N}}, \\
\text { where } & p=2 / 100 \\
z & =1.65 \text { (for } 95 \% \text { c. } 1 .) \\
p & =P \\
q & =(1-P) \\
N & =\text { Total number of Identifiable sherds. }
\end{aligned}
$$

within both contexts, comprising a total of $61.82 \%$ and $65.09 \%$ of those samples, respectively.

By considering the contextual relationships among these sherd categories, as well as attribute associations and covariability, three separate ceramic groups that represent potentially distinct cultural components can be identified at the Fredricks site (Table 8.13). The first group, comprising $69.29 \%$ of all identifiable sherds, is associated with the historic occupation of the site. Group I sherds are tempered mostly with medium sand (95.50\%), but also with fine sand $(1.47 \%)$ and fine crushed feldspar (3.03\%); they have plain, checkstamped, brushed, and cord-marked exteriors. The first two surface treatments comprise over $94 \%$ of the group sample (Figures 8.4-8.7). These sherds and the three whole vessels represent thin-walled, restricted jars and bowls that were invariably smoothed on the interior surface. Lips were predominantly flattened and, when decorated, were obliquely incised. Other vessel areas were only rarely decorated. All three whole vessels were check-stamped, restricted, sub-conical jars with everted rims (two with obliquely incised lips) and range in size from $12-18 \mathrm{~cm}$ in height and $14-17 \mathrm{~cm}$ in orifice diameter (Figure 8.8). Four other vessel sections were recovered which were sufficiently large to determine certain vessel attributes. Three of these represent undecorated check-stamped vessels with orifice diameters of 16,28, and $34 \mathrm{~cm}$. Although this sample is admittedly small, the orifice data suggest two distinct vessel size categories--

\begin{tabular}{|c|c|c|c|c|c|}
\hline Group & $\begin{array}{l}\text { Exterior } \\
\text { Surface }\end{array}$ & Temper & n & $z$ & $\begin{array}{l}\text { Overall } \\
\quad z\end{array}$ \\
\hline \multirow[t]{7}{*}{ I } & P1ain & Medlum Sand & 883 & 43.20 & \\
\hline & Plain & Fine Sand & 30 & 1.47 & \\
\hline & Plaln & FIne Crustied Feldspar & 62 & 3.03 & \\
\hline & Brushed & Medfum Sand & 61 & 2.99 & \\
\hline & Cord Marked & Medtum Sand & 54 & 2.64 & \\
\hline & Check Stamped & Medfum Sand & 954 & 46.67 & \\
\hline & Sub-Totals & & 2044 & 100.00 & $69.29 \%$ \\
\hline \multirow[t]{8}{*}{ II } & Brushed & Medfum Crushed Quartz & 2 & .31 & \\
\hline & Brushed & Fine Crushed Quartz & 8 & 1.23 & \\
\hline & Net Impressed & Coarse Sand & 63 & 9.71 & \\
\hline & Net Impressed & Medtum Sand & 469 & 72.27 & \\
\hline & Net Impressed & Coarse Crushed Quartz & 5 & .77 & \\
\hline & Net Impressed & Medfum Crushed Qunrtz & 37 & 5.70 & \\
\hline & Net Impressed & Fine Crushed Quartz & 65 & 10.01 & \\
\hline & Sub-Totals & & 649 & 100.00 & $22.00 \%$ \\
\hline \multirow[t]{12}{*}{ III } & Platn & Coarse Sand & 13 & 5.93 & \\
\hline & Plain & Fine Crushed Quart? & 40 & 18.26 & \\
\hline & Plain & Coatse Crushed Feldspar & 9 & 4.11 & \\
\hline & Simple Stamped & Coarse Sand & 6 & 2.74 & \\
\hline & Simple Stamped & Medium Sand & 76 & 34.70 & \\
\hline & Simple Stamped & Fine Crushed Quartz & 7 & 3.20 & \\
\hline & Simple Stamped & Coarse Crushed Feldspar & $?$ & 3.20 & \\
\hline & Simple Stamped & Fine Crushed Feldspar & 31 & $14 \cdot 16$ & \\
\hline & Check Stamped & Coarse Sand & 8 & 3.65 & \\
\hline & Check Stainped & Coarse Crushed Feldspar & 1 & .46 & \\
\hline & Check Stamped & Fine Crushed Feldspar & 21 & 9.59 & \\
\hline & Sub-Totals & & 219 & 100.00 & $7.42 \%$ \\
\hline \multirow[t]{15}{*}{ UnGrouped } & Plain & Medfum Crushed Quartz & 2 & 5.26 & \\
\hline & Brushed & Coarse Sand & 2 & 5.26 & \\
\hline & Brushed & Coarse Crushed Feldspar & 1 & 2.63 & \\
\hline & Cob Impressed & Medlum Sand & 9 & 23.68 & \\
\hline & Cob Impressed & F1ne Crushed Quartz & 1 & 2.63 & \\
\hline & Cord Marked & Coarse Sand & 1 & 2.63 & \\
\hline & Cord Marked & Fine Sand & i & 2.63 & \\
\hline & Cord Marked & Medfum Crushed Quartz & $i$ & 2.63 & \\
\hline & Gord Marked & FIne Crushed Quartz & 5 & 13.16 & \\
\hline & Cord Marked & Fine Crushed Feldspar & 4 & 10.53 & \\
\hline & Net Impressed & Coarse Crushed Feldspar & 1 & 2,63 & \\
\hline & Net Impressed & Fine Cruslied Feldspar & 4 & 10.53 & \\
\hline & Check Stamped & F1ne Crushed Quartz & 4 & & \\
\hline & Comp. Stamped & Medium Sand & 2 & 5.26 & \\
\hline & Sub-Totals & & 38 & 99.99 & $1.29 \%$ \\
\hline Totals & & & 2950 & & $100.00 \%$ \\
\hline
\end{tabular}
small (ca. $15 \mathrm{~cm}$ dia.) and large (ca. $30 \mathrm{~cm}$
Table 8.13

Definition of ceranle groups, based on atcribute simflarities, at the Fredicks site.

dia.). The remaining vessel section represents a small (16 cm dia.), hand-modeled, unrestricted vessel comprised of a fine sand tempered, loosely compacted paste and having a plain exterior surface. Several check-stamped sherds had been drilled (Figure 8.5), reflecting attempts to extend usage of cracked vessels through mending. The use of both plain and check-stamped vessels in cooking is indicated by the presence of carbonized organic residue on the interior surface of several sherds.

Group II sherds comprise $22.00 \%$ of the total sample. These sherds are tempered with coarse sand $(9.71 \%)$, medium sand $(72.27 \%)$, coarse quartz $(0.77 \%)$, medium quartz $(6.01 \%)$, and fine quartz $(11.24 \%)$, and have brushed (1.54\%) and net-impressed ( $98.46 \%)$ exterior surfaces (Figure 8.6). These sherds have interior surfaces which are predominantly scraped, and they represent relatively thick-walled vessels. Rim decoration is more common and mostly consists of V-shaped notches along the lip/rim edge. The single vessel section within the sample, recovered from Feature 8 , represents an unusual vessel form with the orifice diameter being substantially smaller than the maximum body diameter (Figure $8.8)$.

Analyses discussed earlier indicate that this group represents a ceramic tradition that is distinctively different from the one reflected by Group I and that shows strong relationships to Coe and Lewis' (1952) Dan River 

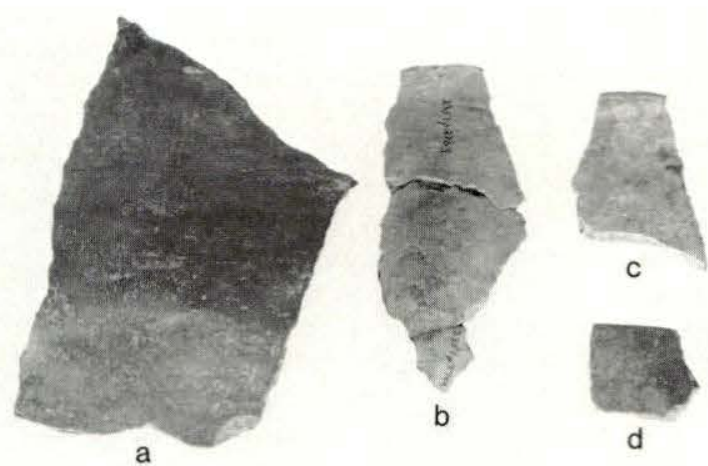

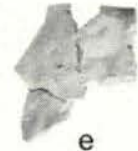

e

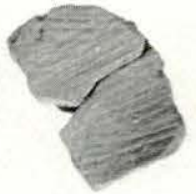

h

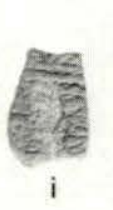

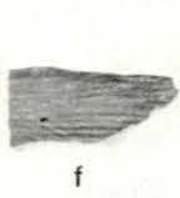

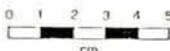

Figure 8.4

Plain (a-e), brushed ( $\mathrm{f}-\mathrm{h})$, and cord-marked $(i-j)$ sherds from the Fredricks site.
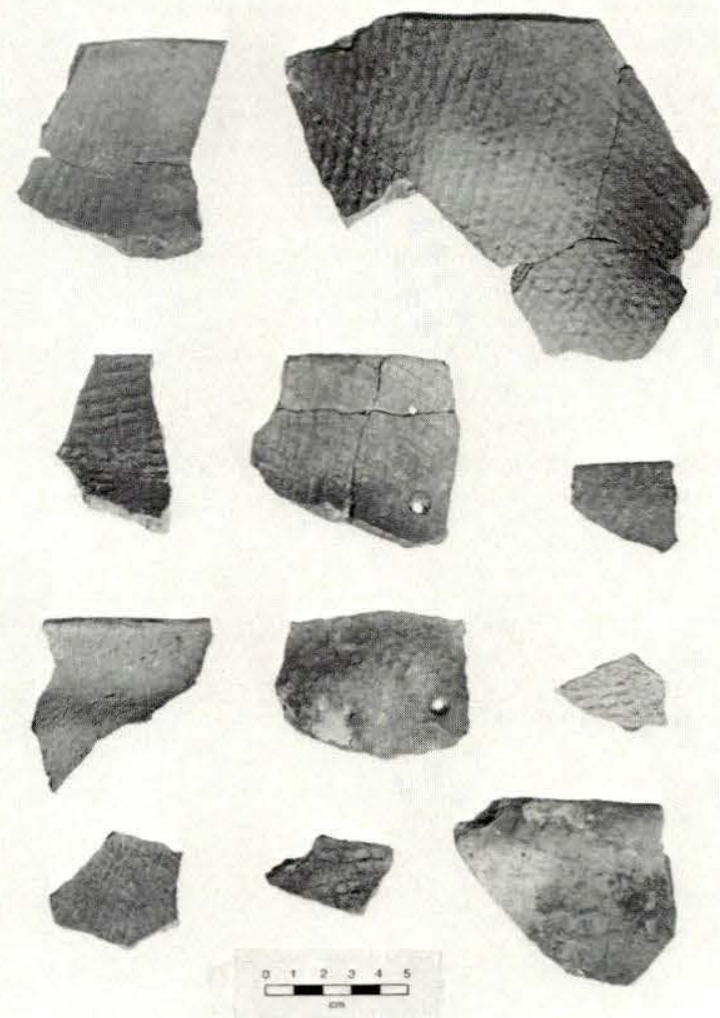

Figure 8.5

Selected check-stamped sherds from the Fredricks site.

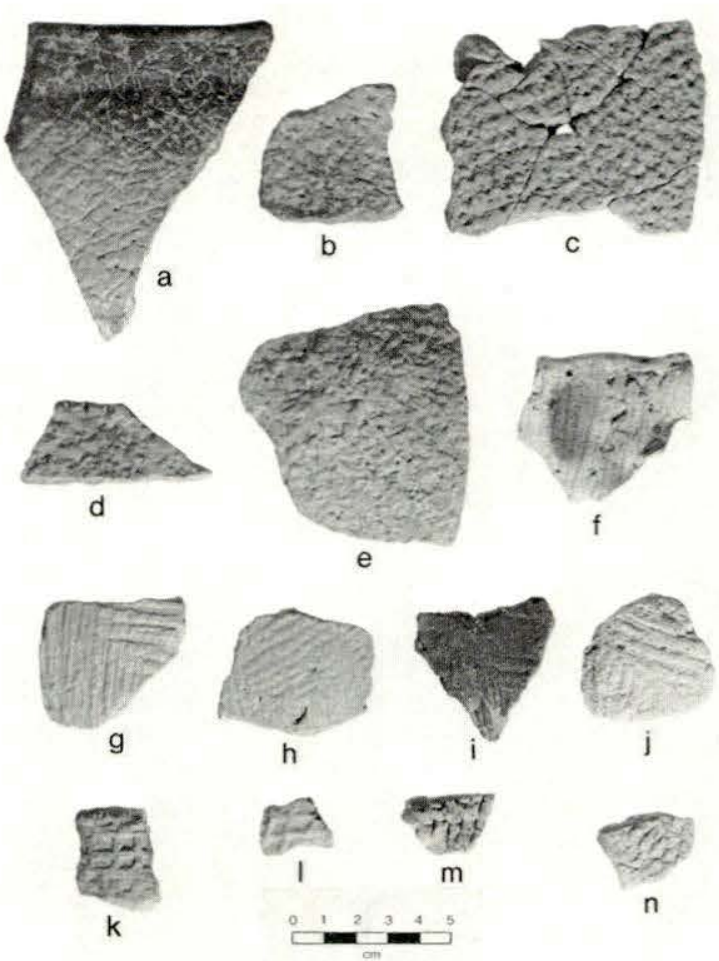

Figure 8.6

Net-impressed (a-e), brushed (f), simple-stamped $(\mathrm{g}-\mathrm{i})$, check-stamped $(\mathrm{k}-1)$, and cob-impressed $(m-n)$ sherds from the Fredricks site.

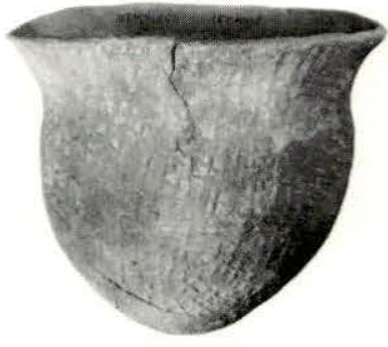

a

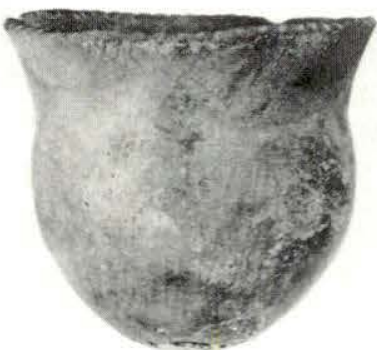

b

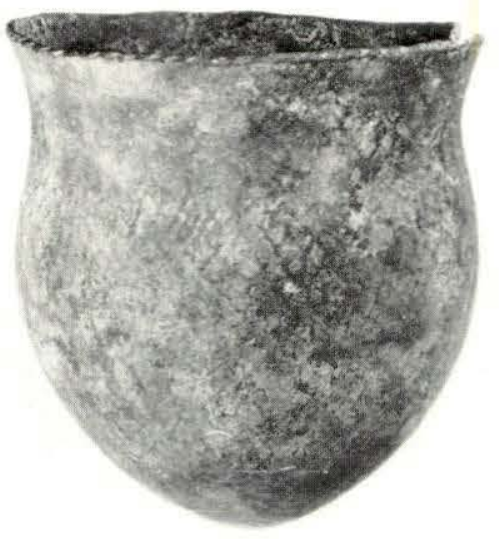

C

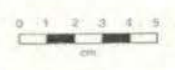

Figure 8.7

Whole vessels from Burial 2 (a), Burial 8 (b) and Burial 6 (c) at the Fredricks site. 

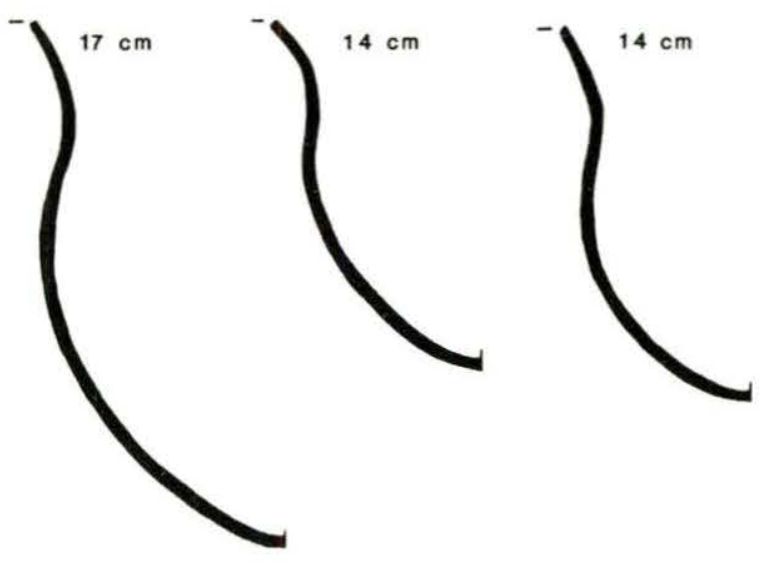

CHECK STAMPED

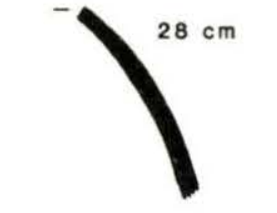

CHECK STAMPED

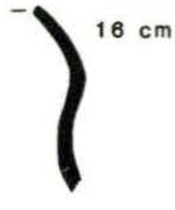

PLAIN

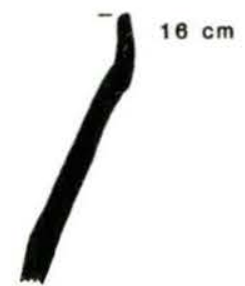

NET IMPRESSED
Figure 8.8

Profiles of vessels and selected vessel fragments from the Fredricks site.

series. Furthermore, Group II sherds generally do not appear to be directly associated with any of the excavated features or burials at the Fredricks site. Three possible interpretations of these ceramics can be offered, none of which can be fully confirmed or rejected by the available data. First, these sherds may represent the material remains of a contemporary but ethnically distinct social/ethnic group residing at the site. Given abundant ethnohistoric accounts of population movement and amalgamation during this period, such a potential explanation is not unreasonable. Second, Group II sherds may represent a mixture of chronologically separate materials, only part of which are associated with the historic site occupation. This explanation is also reasonable in the absence of good contextual data for these sherds. Third, these sherds may simply relate to an earlier occupation of the Fredricks site. Although a few large net-impressed sherds were recovered from Features $8-9$ and in association with check-stamped pottery, this latter explanation is the most plausible since a majority of other net-impressed sherds from features and burials, while similar with respect to all other attributes, typically have highly eroded edges and surfaces. These conditions contrast with the usually crisp edges of plain and checkstamped sherds from the same contexts.

Finally, Group III sherds comprise $7.42 \%$ of the ceramic sample and are tempered with coarse sand $(12.32 \%)$, medium sand $(34.70 \%)$, fine quartz $(21.46 \%)$, coarse feldspar (7.77\%), and fine feldspar (23.75\%). Exterior surfaces consist of plain $(28.30 \%)$, simple-stamped (58.00\%), and check-stamped (13.70\%) treatments (Table 8.13). Sherds are thick, have predominantly smoothed interiors, and represent vessels with everted rims. Decoration consists of $V$-shaped notches applied to the lip. As will be seen later, these sherds are most likely associated with the protohistoric occupation at the nearby Wall site and may represent an isolated house or peripheral village debris. Additional potential sites of this type have been identified by survey and testing elsewhere within 400 yards of the site.

Fourteen other sherd categories, comprising a total of 38 sherds ( $1.29 \%$ of the overall sample), cannot be placed into any of these groups because of small sample size or ambiguous attribute associations.

\section{WALL SITE}

Excavations at the Wall site (310r11) during 1983-1984 produced 18,426 potsherds and four complete vessels. A majority of the sherds were recovered from the midden deposit at the northern edge of the site and from plowdisturbed soil. The four complete vessels are artifacts associated with Burials $1-83$ and 3-83. As with the Fredricks site sample, detailed analysis was limited to sherds greater than $2 \mathrm{~cm}$ in diameter (Table 8.14) and are summarized in Tables 8.15-8.16. Additional information about vessel morphologies represented by the Wall site ceramic assemblage was obtained through a brief examination of vessels and vessel fragments recovered from excavations conducted at the site by the Research Laboratories of Anthropology between 1938 and 1941 .

Table 8.14

Frequency of Wall site sherds and vessels by size.

\begin{tabular}{lrr}
\hline Size (diameter) & $\mathrm{n}$ & $\%$ \\
\hline$\leq 2 \mathrm{~cm}$ & 14,018 & 76.06 \\
$>2-4 \mathrm{~cm}$ & 3890 & 21.11 \\
$>4-6 \mathrm{~cm}$ & 420 & 2.28 \\
$>6-8 \mathrm{~cm}$ & 71 & .39 \\
$>8-10 \mathrm{~cm}$ & 22 & .12 \\
$>10 \mathrm{~cm}$ & 5 & .03 \\
$>8 \mathrm{~cm}$ (vesse1s) & 4 & .02 \\
Totals & 18,430 & 100.01 \\
\hline
\end{tabular}


Table 8.15

Frequency of Wall site sherds by temper and exterior surface treatment (excluding sherds $\leq 2 \mathrm{~cm}$ dia.).

\begin{tabular}{|c|c|c|c|c|}
\hline Temper & Surface & $\mathrm{n}$ & $\%$ & $\begin{array}{c}\% \\
\text { (of identifiable } \\
\text { sherds) }\end{array}$ \\
\hline \multirow[t]{8}{*}{ Coarse } & Sand & & & \\
\hline & Plain & 7 & .16 & .20 \\
\hline & Brushed & 1 & .02 & .03 \\
\hline & Net Impressed & 2 & .05 & .06 \\
\hline & Simple Stamped & 9 & .20 & .26 \\
\hline & Check Stamped & 7 & .16 & .20 \\
\hline & Indeterminate & 7 & .16 & - \\
\hline & Sub-Totals & 33 & .75 & .75 \\
\hline \multirow[t]{9}{*}{ Medium } & Sand & & & \\
\hline & P1ain & 205 & 4.65 & 5.86 \\
\hline & Cob Impressed & 10 & .23 & .29 \\
\hline & Cord Marked & 2 & .05 & .06 \\
\hline & Net Impressed & 14 & .32 & .40 \\
\hline & Simple Stamped & 1345 & 30.49 & 38.44 \\
\hline & Check Stamped & 207 & 4.69 & 5.92 \\
\hline & Indeterminate & 452 & 10.24 & - \\
\hline & Sub-Totals & 2235 & 50.67 & 50.97 \\
\hline \multicolumn{5}{|c|}{ Fine Sand } \\
\hline & Plain & 12 & .27 & .34 \\
\hline & Simple Stamped & 1 & .02 & .03 \\
\hline & Sub-Totals & 13 & .29 & .37 \\
\hline \multirow[t]{5}{*}{ Coarse } & Crushed Quartz & & & \\
\hline & Plain & 1 & .02 & .03 \\
\hline & Cord Marked & 2 & .05 & .06 \\
\hline & Indeterminate & 5 & .11 & - \\
\hline & Sub-Totals & 8 & .18 & .09 \\
\hline \multirow[t]{5}{*}{ Medium } & Crushed Quartz & & & \\
\hline & Net Impressed & 3 & .07 & .09 \\
\hline & Simple Stamped & 6 & .14 & .17 \\
\hline & Indeterminate & 8 & .18 & - \\
\hline & Sub-Totals & 17 & .39 & .26 \\
\hline \multicolumn{5}{|c|}{ Fine Crushed Quartz } \\
\hline & Plain & 11 & .25 & .31 \\
\hline & Cob Impressed & 2 & .05 & .06 \\
\hline & Fabric Marked & 2 & .05 & .06 \\
\hline & Net Impressed & 3 & .07 & .09 \\
\hline & Simple Stamped & 48 & 1.09 & 1.37 \\
\hline & Check Stamped & 58 & 1.31 & 1.66 \\
\hline & Indeterminate & 56 & 1.27 & - \\
\hline & Sub-Totals & 180 & 4.09 & 3.55 \\
\hline \multirow[t]{7}{*}{ Coarse } & Crushed Feldspar & & & \\
\hline & Plain & 2 & .05 & .06 \\
\hline & Cord Marked & 1 & .02 & .03 \\
\hline & S1mple Stamped & 30 & .68 & .86 \\
\hline & Check Stamped & 9 & .20 & .26 \\
\hline & Indeterminate & 16 & .36 & - \\
\hline & Sub-Totals & 58 & 1.31 & 1.21 \\
\hline \multirow[t]{11}{*}{ Fine $\mathrm{Cr}$} & rushed Feldspar & & & \\
\hline & P1ain & 147 & 3.33 & 4.20 \\
\hline & Brushed & 1 & .02 & .03 \\
\hline & Cob Impressed & 4 & .09 & .11 \\
\hline & Cord Marked & 4 & .09 & .11 \\
\hline & Fabric Marked & 2 & .05 & .06 \\
\hline & Net Impressed & 16 & .36 & .46 \\
\hline & Simple Stamped & 1085 & 24.59 & 31.01 \\
\hline & Check Stamped & 198 & 4.49 & 5.66 \\
\hline & Indeterminate & 356 & 8.07 & - \\
\hline & Sub-Totals & 1813 & 41.09 & 41.64 \\
\hline \multicolumn{5}{|c|}{ Quartz \& Feldspar } \\
\hline & Plain & 5 & .11 & .14 \\
\hline & Net Impressed & 1 & .02 & .03 \\
\hline & Simple Stamped & 25 & .57 & .71 \\
\hline & Check Stamped & 11 & .25 & .31 \\
\hline & Indeterminate & 13 & .29 & - \\
\hline & Sub-Totals & 55 & 1.24 & 1.19 \\
\hline \multicolumn{2}{|l|}{ Totals } & 4412 & 100.01 & 100.03 \\
\hline
\end{tabular}


Table 8.16

Summary of Wall site sherds by exterior surface treatment.

\begin{tabular}{|c|c|c|c|c|c|c|c|c|}
\hline $\begin{array}{l}\text { Attribute } \\
\text { Attribute State }\end{array}$ & Plain & Brushed & $\begin{array}{l}\text { Cob } \\
\text { Impressed }\end{array}$ & $\begin{array}{c}\text { Exterior } \\
\text { Cord } \\
\text { Marked }\end{array}$ & $\begin{array}{l}\text { Surface } \\
\text { Fabric } \\
\text { Marked }\end{array}$ & $\begin{array}{l}\text { Net } \\
\text { Impressed }\end{array}$ & $\begin{array}{l}\text { Simple } \\
\text { Stamped }\end{array}$ & $\begin{array}{l}\text { Check } \\
\text { Stamped }\end{array}$ \\
\hline \multicolumn{9}{|l|}{ Portion } \\
\hline Rim & 42 & 1 & 1 & 0 & 0 & 5 & 210 & 34 \\
\hline Neck & 33 & 0 & 2 & 1 & 1 & 8 & 343 & 63 \\
\hline Body & 322 & 1 & 13 & 7 & 3 & 32 & 2030 & 398 \\
\hline \multicolumn{9}{|l|}{ Thickness } \\
\hline$\leq 6 \mathrm{~mm}$ & 109 & 0 & 5 & 0 & 0 & 2 & 391 & 78 \\
\hline$\overline{>}_{6}-8 \mathrm{~mm}$ & 231 & 2 & 7 & 5 & 0 & 22 & 1757 & 337 \\
\hline$>8 \mathrm{~mm}$ & 50 & 0 & 4 & 4 & 0 & 15 & 401 & 75 \\
\hline \multicolumn{9}{|l|}{ Interior Surface } \\
\hline Plain & 384 & 2 & 16 & 7 & 0 & 31 & 2537 & 480 \\
\hline Scraped & 2 & 0 & 0 & 2 & 0 & 5 & 10 & 6 \\
\hline \multicolumn{9}{|l|}{ Rim Form } \\
\hline Everted & 18 & 1 & 0 & 0 & 0 & 4 & 131 & 22 \\
\hline Everted/Flaring & 3 & 0 & 0 & 0 & 0 & 0 & 1 & 0 \\
\hline Everted/Folded & 0 & 0 & 0 & 0 & 0 & 1 & 29 & 8 \\
\hline Everted/Rolled & 0 & 0 & 0 & 0 & 0 & 0 & 1 & 0 \\
\hline Straight & 1 & 0 & 0 & 0 & 0 & 0 & 4 & 0 \\
\hline Inverted & 10 & 0 & 0 & 0 & 0 & 0 & 5 & 0 \\
\hline \multicolumn{9}{|l|}{ Lip Form } \\
\hline Flat & 14 & 0 & 0 & 0 & 0 & 2 & 112 & 24 \\
\hline Rounded & 27 & 1 & 0 & 0 & 0 & 0 & 91 & 10 \\
\hline Pointed & 4 & 0 & 0 & 0 & 0 & 3 & 10 & 1 \\
\hline \multicolumn{9}{|l|}{ Decoration/Location } \\
\hline $\begin{array}{l}\text { V-Shaped Notches } \\
(\text { on lip) }\end{array}$ & 1 & 1 & 0 & 0 & 0 & 0 & 35 & 2 \\
\hline $\begin{array}{l}\text { V-Shaped Notches } \\
\quad(\text { on } 1 \mathrm{ip} / \mathrm{rim})\end{array}$ & 11 & 0 & 0 & 0 & 0 & 4 & 117 & 18 \\
\hline $\begin{array}{l}\text { Fingertip Notches } \\
(\text { on } 1 \mathrm{ip} / \mathrm{rim})\end{array}$ & 0 & 0 & 0 & 0 & 0 & 0 & 2 & 1 \\
\hline $\begin{array}{l}\text { Incised "V"s } \\
\text { (on neck) }\end{array}$ & 2 & 0 & 0 & 0 & 0 & 0 & 0 & 0 \\
\hline $\begin{array}{l}\text { Incised "V"s } \\
\text { (on neck/shoulder) }\end{array}$ & 2 & 0 & 0 & 0 & 0 & 0 & 0 & 0 \\
\hline $\begin{array}{l}\text { Incised "V"s } \\
\text { (on body) }\end{array}$ & 1 & 0 & 0 & 0 & 0 & 0 & 0 & 0 \\
\hline $\begin{array}{l}\text { Misc. Incisions } \\
\quad \text { (ail vessel areas) }\end{array}$ & 7 & 0 & 0 & 0 & 0 & 0 & 0 & 0 \\
\hline $\begin{array}{l}\text { Circular Punctations } \\
\quad(\text { on } 1 \text { ip) }\end{array}$ & 1 & 0 & 0 & 0 & 0 & 0 & 0 & 0 \\
\hline $\begin{array}{l}\text { Circular Punctations } \\
\text { (on shoulder) }\end{array}$ & 10 & 0 & 0 & 0 & 0 & 0 & 0 & 0 \\
\hline $\begin{array}{l}\text { Circular Punctations } \\
\text { (on body) }\end{array}$ & 2 & 0 & 0 & 0 & 0 & 0 & 0 & 0 \\
\hline $\begin{array}{l}\text { U-Shaped Punctations } \\
\text { (on body) }\end{array}$ & 3 & 0 & 0 & 0 & 0 & 0 & 0 & 0 \\
\hline $\begin{array}{l}\text { Fingernail Impressions } \\
\quad(\text { on neck) }\end{array}$ & 0 & 0 & 0 & 0 & 0 & 0 & 0 & 1 \\
\hline Pinched Rim Fold & 0 & 0 & 0 & 0 & 0 & 0 & 0 & 1 \\
\hline \multicolumn{9}{|l|}{ Size } \\
\hline$>2-4 \mathrm{~cm}$ & 334 & 2 & 13 & 7 & 3 & 31 & 2183 & 420 \\
\hline$>4-6 \mathrm{~cm}$ & 38 & 0 & 3 & 1 & 1 & 7 & 289 & 64 \\
\hline$>6 \mathrm{~cm}$ & 17 & 0 & 0 & 1 & 0 & 1 & 77 & 6 \\
\hline
\end{tabular}


Examination of covariability among sherd attributes suggests that most of the Wall site sherds are probably associated with the major occupation of the site; consequently, they are treated as a single assemblage in the following discussion. Wall site pottery is predominantly tempered with medium sand $(50.67 \%)$, fine crushed feldspar ( $41.09 \%)$, and (to a much lesser extent) fine crushed quartz $(4.09 \%)$. Other temper categories comprise less than five percent of the overall sample. Exterior surfaces are largely simple stamped ( $72.85 \%)$; however, significant numbers of check-stamped $(14.00 \%)$ and plain (11.15\%) sherds are also present. Other surface treatments represented in the sample are: net impressed $(1.11 \%)$, cob impressed $(0.46 \%)$, cord marked $(0.26 \%)$, fabric marked $(0.11 \%)$, and brushed $(0.06 \%)$ (Figures 8.9-8.12). Although some of these sherds may in fact represent earlier or later occupations, this cannot be demonstrated because of inadequate sample size. With one exception, temper and exterior surface treatment vary independently of one another. Fine crushed quartz temper is associated largely with check stamping. Possible implications of this pattern are considered below.

As with the Fredricks site sherd analysis, Chi-square tests of association were used to evaluate attribute differences among the three major sherd categories (i.e., surface treatments) represented within the sample (Figure 8.13). These tests are based on data presented in Table 8.16. Significant differences (at $\mathrm{p}=.01$ ) among plain, simple-stamped, and checkstamped sherds were observed for only two attributes--thickness and rim form. Both of these attributes monitor vessel morphology. Whereas simple-stamped and check-stamped sherds usually represent moderately thick-walled, constricted jars with everted or everted/folded rims, plain sherds appear to be associated more with unrestricted bowls which have relatively thin walls. This pattern is also reflected by the frequency distribution of vessel portions represented by plain sherds, with rim sherds outnumbering neck sherds. Plain sherds also display considerably more variability in decoration than the other two sherd categories. Decoration of simple-stamped and check-stamped vessels is almost solely restricted to the rim area and usually consists of $V$-shaped notches along either the $1 \mathrm{ip}$ or $1 \mathrm{ip} / \mathrm{rim}$ edge. Whereas similar decorations were observed on plain rim sherds, other decorative modes involving incising and punctating seem to have been more common on plain vessels. Additionally, these latter decorations frequently occur on the vessel shoulder and body.

In order to assess potential diachronic variability in the Wall site sample, the relative frequency distributions of two attributes-exterior surface treatment and temper--were examined from plowzone and midden contexts in four $10 \times 10-f t$ squares excavated along the northern edge of the site. The squares included in the analysis are 360R530, 370R530, 370R540, and
370R560. The plowzone within these squares is $.65-.75 \mathrm{ft}$ thick whereas the midden zone, excavated in two levels, ranges from $.40-.90 \mathrm{ft}$ thick. Midden levels were distinguished in the field by soil color and artifact content. Midden Level 1 is interpreted as a sealed refuse deposit; Level 2 appears to represent an earlier, buried humus zone. Plowzone artifact content is derived in part from the uppermost portion of Midden Leve1 1. Consideration of differences in sherd samples among the plowzone and midden levels is important since it provides a rough assessment of site duration and a means for detecting sources of ceramic variability that may be temporally significant. Distributions of exterior surface treatments and temper types are provided in Table 8.17 .

Potentially significant variability in surface treatment is reflected by a slight decrease in simple stamping and corresponding increase in check stamping from Midden Level 1 to Midden Leve1 2. The strength of this pattern was evaluated using a Chi-square test comparing major surface treatment (plain, simple stamped, check stamped) with context. The test results $\left(\mathrm{X}^{2}=15.28, \mathrm{df}=4, \mathrm{p}<.005\right)$ indicate a statistically significant difference, and suggest the possibility that check stamping may have slight temporal priority over simple stamping. This potential trend may also be reflected by the association (noted earlier) of fine crushed quartz temper with check stamping. Beyond this, the three contexts are generally similar in sherd content and support the hypothesis that the Wall site does not represent a lengthy occupation.

Temper distributions among the plowzone and midden levels are also similar. Statistical comparison of the three most frequent temper types (medium sand, fine feldspar, fine quartz) failed to reveal any significant differences $\left(x^{2}=2.97, d f=4, p<.75\right)$. Again, the data do not suggest any appreciable time depth for the analyzed stratigraphic contexts.

In summary, the vessel assemblage represented by the Wall site sherd sample can be characterized as follows. Jar forms are predominant. Most are simple stamped but some have check-stamped and plain surfaces. When stamped, the stamp motif is boldly applied and covers the entire vessel exterior. Simple stamps are typically applied at right angles to one another, producing a distinctive herringbone effect. Check-stamped designs are similar to those observed within the Fredricks site sample, consisting of large squares or diamonds; however, they are much more heavily stamped. Vessel interiors were almost always uniformly smoothed.

Most jars have constricted necks and everted rims, some of which also have rim folds. Rim profiles for these jar forms are illustrated in Figure 8.14:1-aa. Decoration is common and consists of notching the lip or lip/rim edge. 

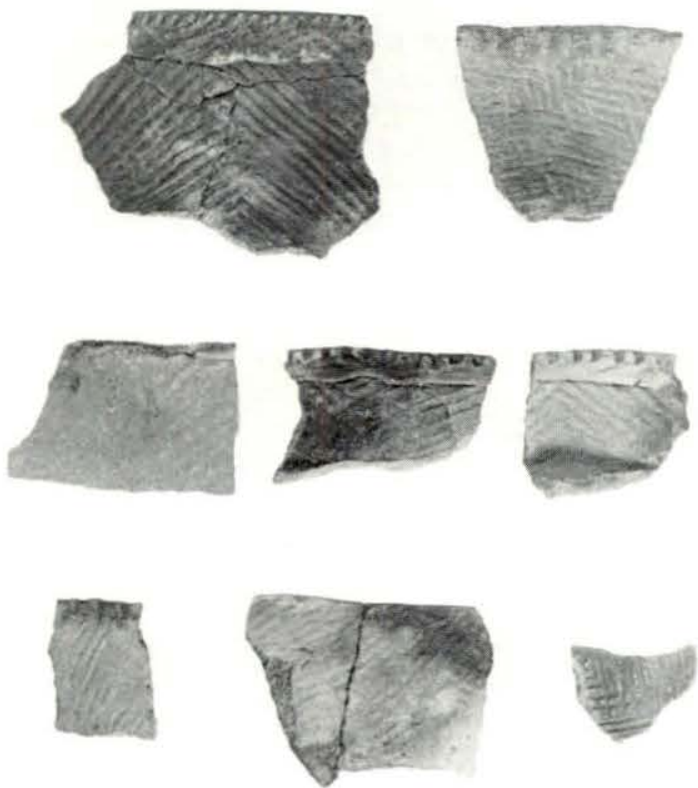
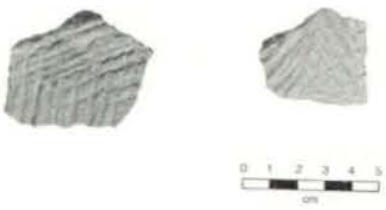

Figure 8.9

Simple-stamped sherds from the Wall site.
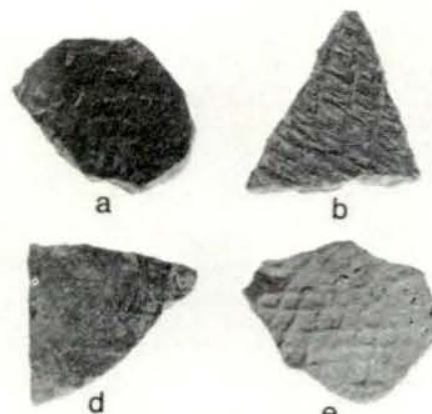

b
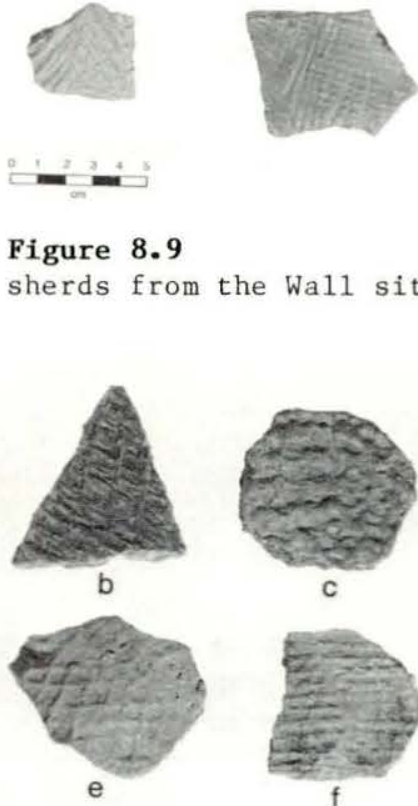

c
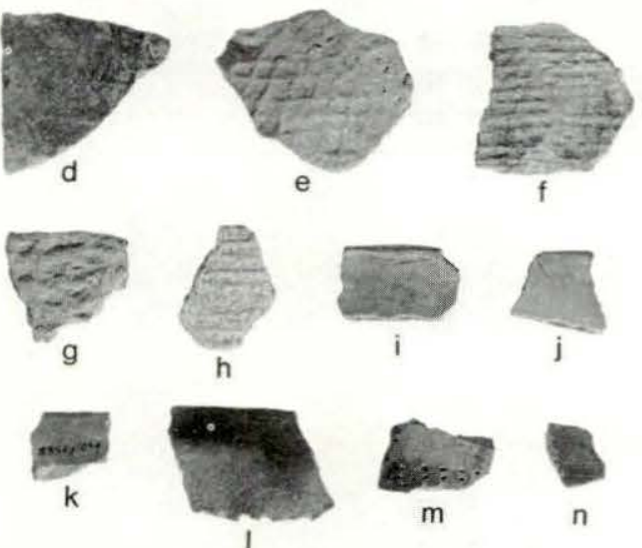

$\mathrm{m}$
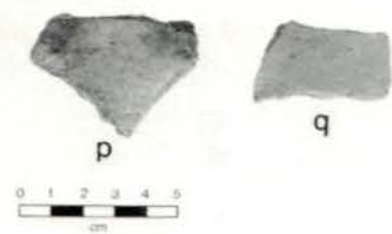

Figure 8.10

Check-stamped $(\mathrm{a}-\mathrm{h})$ and plain sherds from the Wall site.

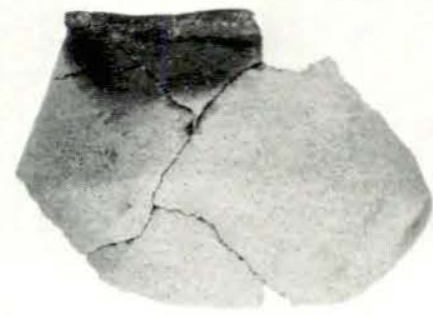

a
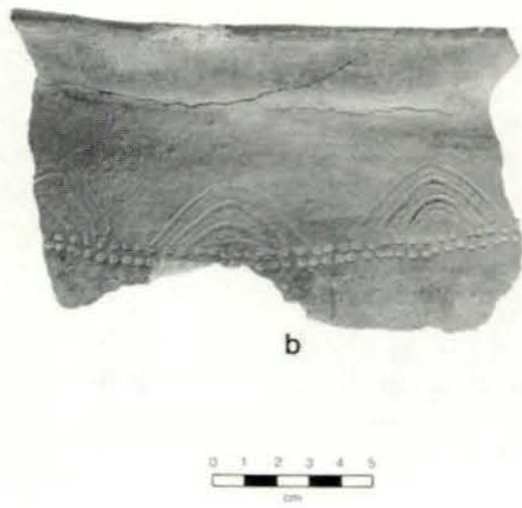

Figure 8.11

Undecorated (a) and decorated (b) plain vessel sections from the Wall site.

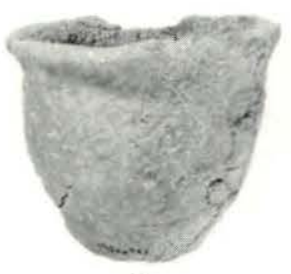

a
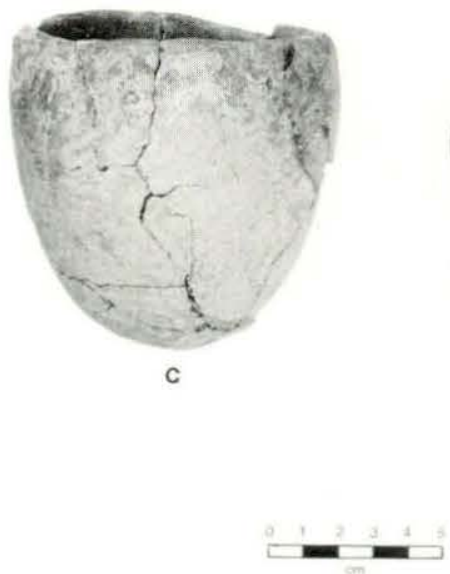

Figure 8.12

Whole vessels from Burial 3-83 (a-b) and Burial 1-83 (c-d) at the Wall site. 

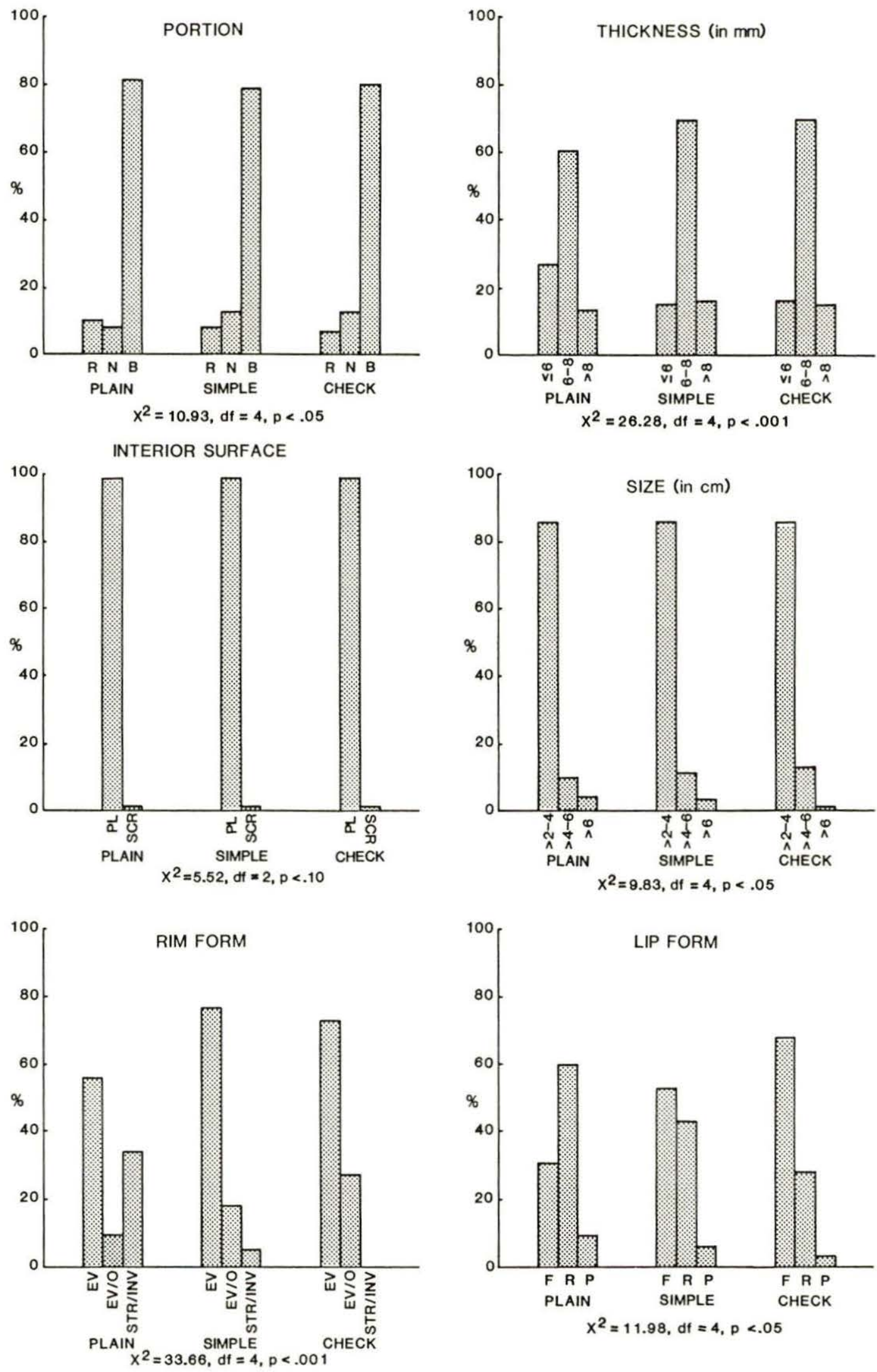

Figure 8.13

Relative frequency distributions and statistical evaluation of selected attributes for plain, simple-stamped, and checkstamped sherds from the Wall site. 
Table 8.17

Comparison of selected attributes for plowzone and midden contexts at the Wall site.

\begin{tabular}{|c|c|c|c|c|c|c|}
\hline \multirow{2}{*}{$\begin{array}{l}\text { Attribute } \\
\text { Attribute State }\end{array}$} & \multicolumn{2}{|c|}{ Plowzone } & \multirow{2}{*}{$\underset{n}{\text { Midden }}$} & \multirow{2}{*}{$\underset{z}{\text { Level } 1}$} & \multirow{2}{*}{$\begin{array}{c}\text { M1dden } \\
n\end{array}$} & \multirow{2}{*}{$\begin{array}{c}\text { Level } \\
z\end{array}$} \\
\hline & $\mathrm{n}$ & & & & & \\
\hline \multicolumn{7}{|l|}{ Exterior Surface } \\
\hline Plain & 29 & 13.68 & 94 & 11.72 & 73 & 11.39 \\
\hline Brushed & 1 & .47 & 0 & .00 & 0 & .00 \\
\hline Cob Impressed & 0 & .00 & 2 & .25 & 6 & .94 \\
\hline Cord Marked & 0 & .00 & 4 & .50 & 2 & .31 \\
\hline Fabric Marked & 0 & .00 & 2 & .25 & 0 & .00 \\
\hline Net Impressed & 3 & 1.42 & 8 & 1.00 & 3 & .47 \\
\hline Simple Stamped & 156 & 73.58 & 606 & 75.56 & 446 & 69.58 \\
\hline Check Stamped & 23 & 10.85 & 86 & 10.72 & 111 & 17.31 \\
\hline Totals & 212 & 100.00 & 802 & 100.00 & 641 & 100.00 \\
\hline \multicolumn{7}{|l|}{ Temper } \\
\hline Coarse Sand & 1 & .38 & 5 & .55 & 8 & 1.11 \\
\hline Medium Sand & 151 & 57.85 & 474 & 52.15 & 384 & 53.04 \\
\hline Fine Sand & 0 & .00 & 5 & .55 & 3 & .41 \\
\hline Coarse Crushed Quartz & 0 & .00 & 1 & .11 & 5 & .69 \\
\hline Medium Crushed Quartz & 0 & .00 & 3 & .33 & 6 & .83 \\
\hline Fine Crushed Quartz & 6 & 2.30 & 28 & 3.08 & 24 & 3.31 \\
\hline Coarse Feldspar & 3 & 1.15 & 10 & 1.10 & 8 & 1.11 \\
\hline Fine Feldspar & 100 & 38.31 & 377 & 41.47 & 270 & 37.29 \\
\hline Quartz \& Feldspar & 0 & .00 & 6 & .66 & 16 & 2.21 \\
\hline Tota1s & 261 & 99.99 & 909 & 100.00 & 724 & 100.00 \\
\hline
\end{tabular}
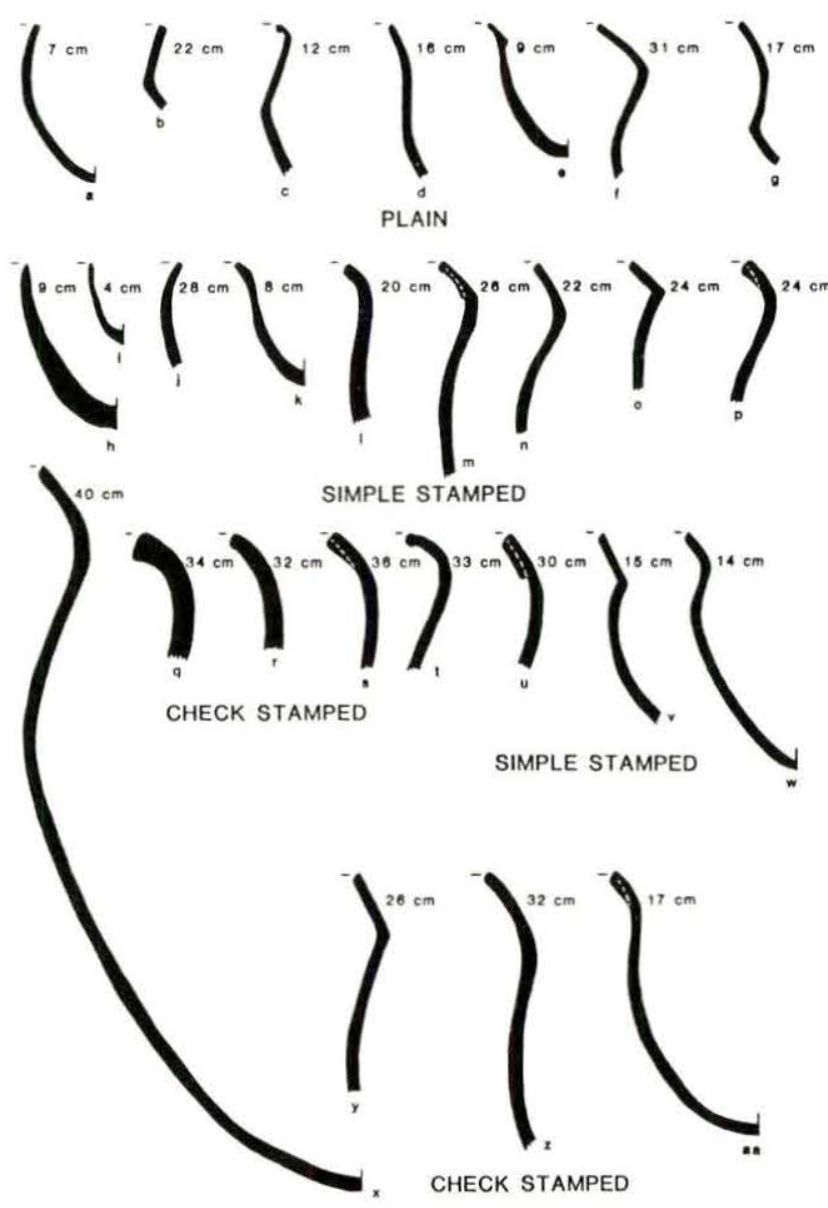

Figure 8.14

Profiles of vessels and selected vessel fragments from the Wall site.

Although morphologically similar, check-stamped jars are generally larger than simple-stamped jars, ranging from $17-40 \mathrm{~cm}$ in orifice diameter $(\vec{x}=31.0 \mathrm{~cm}, s d=7.06, n=7)$. Simple-stamped jars range from $14-33 \mathrm{~cm}$ in diameter $(\bar{x}=23.1, \mathrm{sd}=$
6.27, $n=9)$. Both categories of jars have slightly conical to rounded bases.

A small number of jars, including the four vessels recovered from burial contexts, represent an unrestricted jar form, i.e., they lack a constricted neck (Figure 8.14:a,e,h-i,1). These jars have incurvate, straight, or slightly flaring rimprofiles and are either plain or simple stamped. Unrestricted jars are significantly smaller than restricted jars, and range from 4-9 cm in orifice diameter $(\bar{x}=7.4, s d=2.07$, $\mathrm{n}=5$ ). Only two of the vessels analyzed are decorated. One plain vessel section, recovered in 1938, has several randomly placed pairs of short, curved incisions on the exterior body surface. The other vessel, one of two associated with Burial 3-83, is also plain, has a notched lip, and was painted with red ochre on both the exterior and interior surfaces. A11 four vessels recovered from burial contexts in 1983 are hand-modeled and poorly fired. This contrasts sharply with the remainder of the ceramic sample which represent well-fired vessels constructed by coiling. The paired vessels within both Burial $1-83$ and Burial 3-83 are morphologically similar to each other; and each pair appears to have been made by the same individual.

Finally, several sherds and vessel sections have been recovered from the Wall site which represent bow1 forms (i.e., having orifice diameters which exceed vessel height). Three distinct bow1 types are identified: unrestricted (Figure $8.14: \mathrm{d}, \mathrm{j}$ ), unrestricted carinated (Figure $8.14: b$ ), and restricted (Figure $8.14: c, f-g$ ). With the exception of one unrestricted simplestamped vessel, all of the bowls examined have smoothed plain interiors and exteriors. Vessel size, significantly greater than that observed for unrestricted jars, ranges from $12-18 \mathrm{~cm}$ in orifice diameter $(\bar{x}=21.00, s d=7.38, n=6)$. A11 of these vessels are well-made and most are decorated. Decoration consists primarily of a single or double line of circular reed punctations along the shoulder, sometimes accompanied by a band of incised "V"s just above the shoulder. Vessel sections were also observed from the 1938-1941 excavations which had punctated "V"s and incised "V"s just below the shoulder.

\section{MITCHUM SITE}

During 1983, limited testing was undertaken by the Research Laboratories of Anthropology at the Mitchum site ( $31 \mathrm{Ch} 452)$, an historic village site located along the Haw River about 17 miles southwest of the Hillsborough sites. The primary cultural component at the Mitchum site apparently post-dates the Wall site occupation and precedes the final historic Indian occupation at the Fredricks site. This interpretation is based upon the limited occurrence of historic trade material (mostly glass beads) 
within several archaeological features. A total of 3397 potsherds were recovered from plowzone and sub-plowzone strata, as well as from features $(n=14)$, one human burial, and postholes associated with an oval house pattern (Structure 1). This discussion is limited to the 1351 sherds that were greater than $2 \mathrm{~cm}$ in diameter (Table 8.18). Consideration of the Mitchum site sherds is important to earlier interpretations of attribute variability within the Fredricks site sample since they were also derived from an Historic period context within which plain, stamped, and net-impressed surface treatments are apparently associated. Because of the small number of identifiable sherds that were recovered from features and burials $(n=96)$, the following analysis is limited to a general statement about the ceramic assemblage at the site and a more detailed examination of the relationship of net-impressed sherds to other predominant modes of surface treatment.

Aboriginal pottery from the Mitchum site is tempered primarily with medium sand (36.55\%), coarse crushed feldspar $(19.50 \%)$, and fine crushed feldspar $(32.29 \%)$. Other temper types recognized within the sample, in descending order of frequency, are fine crushed quartz, medium crushed quartz, quartz and feldspar, coarse sand, coarse grit, coarse crushed quartz, fine grit, and fine sand (Table 8.19). Exterior surface treatments are predominantly plain $(40.07 \%)$, net impressed $(30.26 \%)$, simple stamped $(14.73 \%)$, and brushed $(8.06 \%)$; other minority treatments such as cob impressed, check stamped, cord marked, and complicated stamped occur on less than seven percent of the Mitchum sherds (Figures 8.15-8.16). Both plain and simplestamped surface treatments are equally associated with all three major temper types. Net-impressed and brushed sherds are predominantly tempered with fine feldspar and medium sand. This differential association of major temper and surface treatment types is statistically significant $\left(X^{2}=129.41, d f=6, p<.001\right)$; however, it is not known to what extent these differences reflect temporal or functional variability.

Table 8.18

Frequency of Mitchum site sherds by size.

\begin{tabular}{lcc}
\hline & $\mathrm{n}$ & $\%$ \\
\hline Size (diameter) & 2046 & 60.23 \\
$\leq 2 \mathrm{~cm}$ & 1202 & 35.38 \\
$>2-4 \mathrm{~cm}$ & 134 & 3.94 \\
$>4-6 \mathrm{~cm}$ & 14 & .41 \\
$>6-8 \mathrm{~cm}$ & 1 & .03 \\
$>8-10 \mathrm{~cm}$ & 0 & .00
\end{tabular}

Table 8.19

Frequency of Mitchum site sherds by temper and exterior surface treatment (excluding sherds $\leq 2 \mathrm{~cm}$ dia.).

\begin{tabular}{|c|c|c|c|c|}
\hline Temper & Surface & $\mathrm{n}$ & $\%$ & $\begin{array}{c}\% \\
\text { (of identifiable } \\
\text { sherds) }\end{array}$ \\
\hline \multirow[t]{8}{*}{ Coarse } & Sand & & & \\
\hline & Plain & 1 & .07 & .10 \\
\hline & Brushed & 1 & .07 & .10 \\
\hline & Cob Impressed & 1 & .07 & .10 \\
\hline & Net Impressed & 4 & .30 & .39 \\
\hline & Simple Stamped & 1 & .07 & .10 \\
\hline & Indeterminate & 3 & .22 & - \\
\hline & Sub-Totals & 11 & .80 & .79 \\
\hline \multirow[t]{11}{*}{ Medium } & Sand & & & \\
\hline & Plain & 126 & 9.32 & 12.22 \\
\hline & Brushed & 26 & 1.92 & 2.52 \\
\hline & Cob Impressed & 13 & .96 & 1.26 \\
\hline & Cord Marked & 2 & .15 & .19 \\
\hline & Net Impressed & 160 & 11.83 & 15.52 \\
\hline & Simple Stamped & 45 & 3.33 & 4.36 \\
\hline & Check Stamped & 3 & .22 & .29 \\
\hline & Comp. Stamped & 2 & .15 & .19 \\
\hline & Indeterminate & 93 & 6.88 & - \\
\hline & Sub-Totals & 470 & 34.76 & 36.55 \\
\hline \multicolumn{5}{|c|}{ Fine Sand } \\
\hline & P1a1n & 1 & .07 & .10 \\
\hline & Sub-Totals & 1 & .07 & .10 \\
\hline \multirow[t]{6}{*}{ Coarse } & Crushed Quartz & & & \\
\hline & Brushed & 3 & .22 & .29 \\
\hline & Net Impressed & 3 & .22 & .29 \\
\hline & Simple Stamped & 1. & .07 & .10 \\
\hline & Indeterminate & 1 & .07 & - \\
\hline & Sub-Totals & 8 & .58 & .68 \\
\hline \multirow[t]{9}{*}{ Medium } & Crushed Quartz & & & \\
\hline & Plain & 7 & .52 & .68 \\
\hline & Brushed & 3 & .22 & .29 \\
\hline & Cord Marked & 1 & .07 & .10 \\
\hline & Net Impressed & 13 & .96 & 1.26 \\
\hline & Simple Stamped & 1 & .07 & .10 \\
\hline & Check Stamped & 1 & .07 & .10 \\
\hline & Indeterminate & 8 & .59 & - \\
\hline & Sub-Totals & 34 & 2.50 & 2.53 \\
\hline Fine $\mathrm{Cr}$ & rushed Quartz & & & \\
\hline & Plain & 20 & 1.48 & 1.94 \\
\hline & Brushed & 10 & .74 & .97 \\
\hline & Cob Impressed & 3 & .22 & .29 \\
\hline & Net Impressed & 15 & 1.11 & 1.45 \\
\hline & Simple Stamped & 5 & .37 & .48 \\
\hline & Check Stamped & 3 & .22 & .29 \\
\hline & Indeterminate & 13 & .96 & - \\
\hline & Sub-Totals & 69 & 5.10 & 5.42 \\
\hline Coarse & Crushed Feldspar & & & \\
\hline & Plain & 130 & 9.62 & 12.61 \\
\hline & Brushed & 8 & .59 & .78 \\
\hline & Cob Impressed & 15 & 1.11 & 1.45 \\
\hline & Net Impressed & 8 & .59 & .78 \\
\hline & Simple Stamped & 39 & 2.88 & 3.78 \\
\hline & Check Stamped & 1 & .07 & .10 \\
\hline & Indeterminate & 64 & 4.73 & - \\
\hline & Sub-Totals & 265 & 19.59 & 19.50 \\
\hline Fine $\mathrm{Cr}$ & rushed Feldspar & & & \\
\hline & Plain & 126 & 9.32 & 12.22 \\
\hline & Brushed & 42 & 3.11 & 4.07 \\
\hline & Cob Impressed & 3 & .22 & .29 \\
\hline & Cord Marked & 3 & .22 & .29 \\
\hline & Net Impressed & 97 & 7.17 & 9.41 \\
\hline & Simple Stamped & 55 & 4.07 & 5.33 \\
\hline & Check Stamped & 7 & .52 & .68 \\
\hline & Indeterminate & 130 & 9.62 & - \\
\hline & Sub-Totals & 463 & 34.25 & 32.29 \\
\hline Quartz & $\&$ Feldspar & & & \\
\hline & Plain & 1 & .07 & .10 \\
\hline & Net Impressed & 7 & .52 & .68 \\
\hline & Indeterminate & 8 & .59 & - \\
\hline & Sub-Totals & 16 & 1.18 & .78 \\
\hline Coarse & Grit & & & \\
\hline & Net Impressed & 2 & .15 & .19 \\
\hline & Simple Stamped & 5 & .37 & .48 \\
\hline & Comp. Stamped & 2 & .15 & .19 \\
\hline & Indeterminate & 1 & .07 & - \\
\hline & Sub-Totals & 10 & .74 & .86 \\
\hline Fine Gr & int & & & \\
\hline & Plain & 1 & .07 & .10 \\
\hline & Net Impressed & 3 & .22 & .29 \\
\hline & Comp. Stamped & 1 & .07 & .10 \\
\hline & Sub-Totals & 5 & .36 & .49 \\
\hline Totals & & 1352 & 99.93 & 99.99 \\
\hline
\end{tabular}



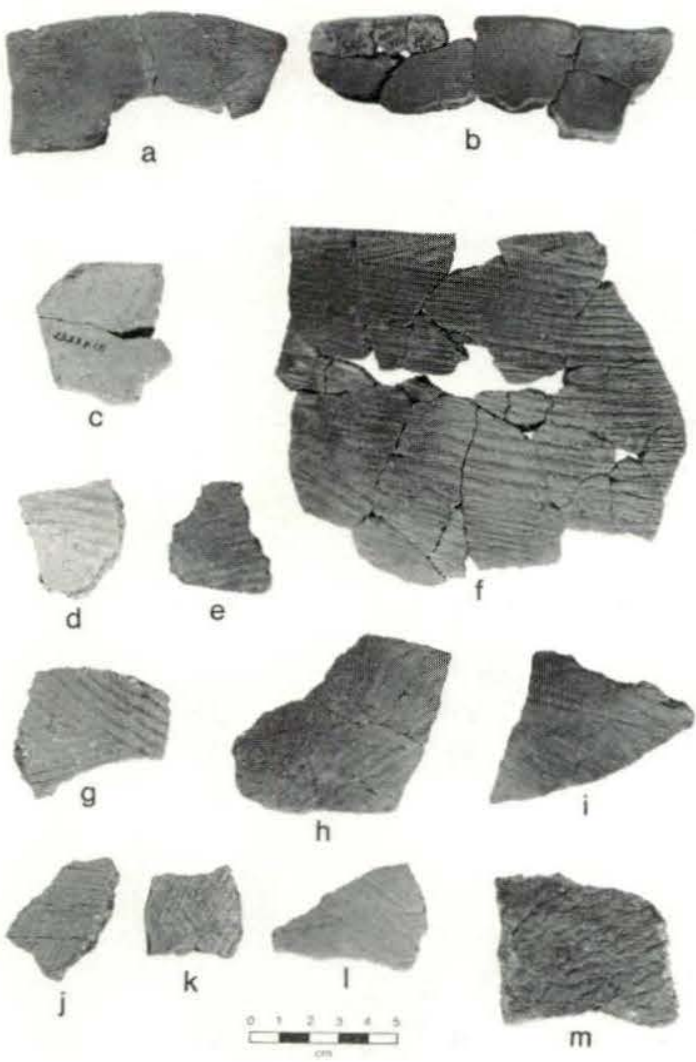

Figure 8.15

Plain $(a-c)$, simple-stamped $(d-i)$, brushed $(j-1)$, and cob-impressed $(m)$ sherds from the Mitchum site.
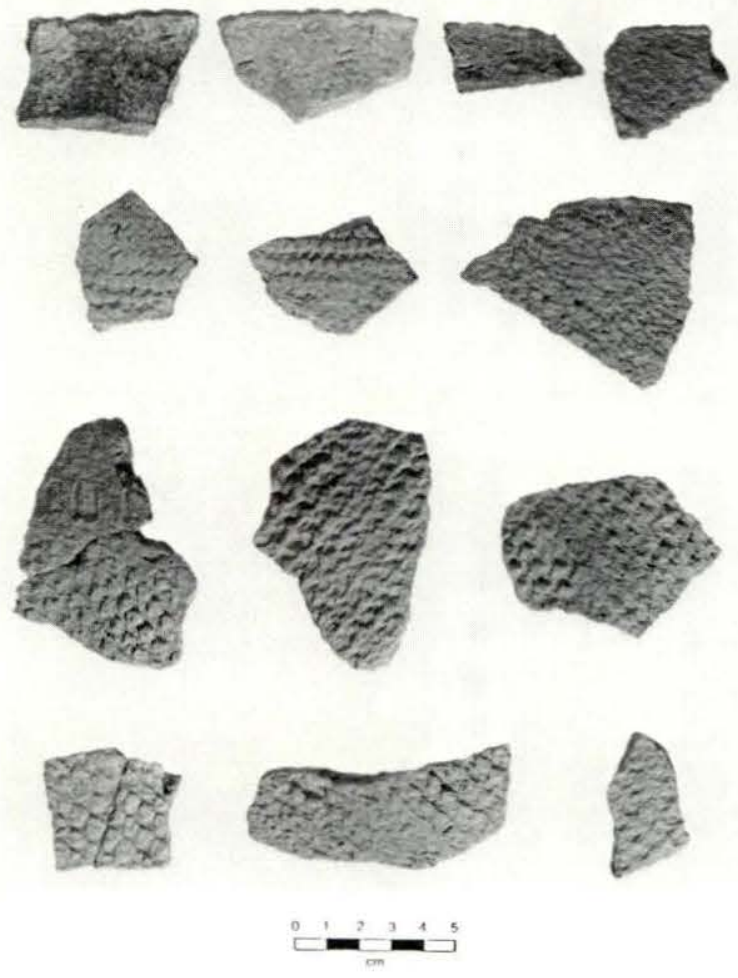

Figure 8.16

Net impressed sherds from the Mitchum site.
Statistical evaluation of other selected attributes for plain, brushed, simple-stamped, and net-impressed sherds also indicates certain significant differences. Their implications are briefly considered below. Attribute summaries for major temper/surface treatment categories are presented in Table 8.20; attribute distributions by surface treatment are shown in Figure 8.17 .

Plain sherds can be clearly distinguished from other sherd categories by a high proportion of rim sherds. This pattern also contrasts with vessel portion distributions observed at the Fredricks and Wall sites, and suggests the predominance of a shallow, wide-mouthed vessel form (i.e., with a high rim-to-body ratio). Unfortunately, this possibility cannot be evaluated further because of an insufficient sample of reconstructible vessels. The majority of plain vessels also have everted (or flaring) rims and either flat or rounded lips. Decoration of plain vessels consists primarily of V-shaped notches placed on the lip. Other treatments observed in the sample include incising, punctating, and impressing with a corncob.

Both brushed and simple-stamped sherds appear to represent similar vessel morphologies; however, simple-stamped vessel walls are substantially thicker. Simple-stamped and brushed surfaces were difficult to distinguish and in both cases lands and grooves were usually applied parallel to the vessel rim. This is markedly different from simple-stamped sherds at the Wall and Fredricks sites where stamping was applied either perpendicular or oblique to the rim. Although brushed and simple-stamped rim sherd samples are too small to provide reliable information about rim configuration, it is likely that a majority of these vessels had slightly everted rims with flattened lips. Notching of the lip is the sole mode of decoration that was observed on these sherds.

Finally, the sample of net-impressed sherds collected from the Mitchum site indicates a moderately low ratio of rim circumference to body area. The relatively low proportion of neck sherds, also reflected in the high percentage of straight rims, suggests a distinctly different vessel shape than that inferred for either the plain or simple-stamped/brushed categories. This contrast is further magnified by the predominance of interior scraping on net-impressed sherds. This type of interior surface treatment was rarely found on sherds other than net impressed. Differences between net-impressed sherds and other major sherd categories are also manifested in decoration. Notching of the vessel lip is still a principal decorative treatment; however, an equal number of sherds also showed evidence of decoration along the vessel neck. Neck treatments recognized on net-impressed sherds include a single band of vertical fingertip notches or short incised lines and incised "V"s. These methods of decoration, as well as other attributes such 
Table 8.20

Summary of selected sherd categories from the Mitchum site.

\begin{tabular}{|c|c|c|c|c|c|c|c|c|c|c|}
\hline $\begin{array}{l}\text { Attribute } \\
\text { Attribute State }\end{array}$ & Plain & $\begin{array}{r}\text { Medi } \\
\text { Brushed }\end{array}$ & $\begin{array}{l}\text { ium Sand } \\
\text { Net } \\
\text { Impressed }\end{array}$ & $\begin{array}{l}\text { Simple } \\
\text { Stamped }\end{array}$ & $\begin{array}{l}\text { Coarse } \\
\text { Plain }\end{array}$ & $\begin{array}{l}\text { Feldspar } \\
\text { Simple } \\
\text { Stamped }\end{array}$ & Plain & $\begin{array}{r}\text { Fine } \\
\text { Brushed }\end{array}$ & $\begin{array}{l}\text { Feldspar } \\
\quad \text { Net } \\
\text { Impressed }\end{array}$ & $\begin{array}{l}\text { Simple } \\
\text { Stamped }\end{array}$ \\
\hline \multicolumn{11}{|l|}{ Portion } \\
\hline $\operatorname{Rim}$ & 23 & 1 & 16 & 7 & 22 & 5 & 19 & 3 & 3 & 2 \\
\hline Neck & 15 & 3 & 9 & 11 & 16 & 7 & 12 & 10 & 18 & 14 \\
\hline Body & 91 & 22 & 136 & 30 & 93 & 27 & 95 & 29 & 76 & 39 \\
\hline \multicolumn{11}{|l|}{ Thickness } \\
\hline$<6 \mathrm{~mm}$ & 60 & 15 & 33 & 7 & 5 & 8 & 18 & 2 & 8 & 5 \\
\hline $56-8 \mathrm{~mm}$ & 61 & 10 & 102 & 35 & 89 & 12 & 92 & 28 & 62 & 31 \\
\hline$>8 \mathrm{~mm}$ & 5 & 1 & 24 & 3 & 35 & 19 & 15 & 12 & 27 & 19 \\
\hline \multicolumn{11}{|l|}{ Interior Surface } \\
\hline Plain & 124 & 24 & 39 & 44 & 127 & 37 & 124 & 41 & 42 & 54 \\
\hline Scraped & 2 & 2 & 119 & 1 & 1 & 2 & 1 & 0 & 51 & 1 \\
\hline \multicolumn{11}{|l|}{ Rim Form } \\
\hline Everted & 13 & 1 & 5 & 6 & 15 & 3 & 7 & 0 & 0 & 0 \\
\hline Everted/Folded & 0 & 0 & 0 & 0 & 0 & 0 & 1 & 0 & 0 & 0 \\
\hline Straight & 0 & 0 & 4 & 0 & 0 & 0 & 0 & 0 & 0 & 0 \\
\hline Inverted & 1 & 0 & 0 & 0 & 0 & 2 & 0 & 2 & 0 & 0 \\
\hline \multicolumn{11}{|l|}{ Lip Form } \\
\hline Flat & 11 & 1 & 11 & 2 & 12 & 5 & 8 & 2 & 2 & 2 \\
\hline Rounded & 11 & 0 & 4 & 1 & 10 & 0 & 9 & 0 & 1 & 0 \\
\hline Pointed & 1 & 0 & 0 & 4 & 0 & 0 & 1 & 1 & 0 & 0 \\
\hline \multicolumn{11}{|l|}{ Decoration/Location } \\
\hline $\begin{array}{l}\text { Incised } \\
\text { (oblique on 1ip) }\end{array}$ & 0 & 0 & 1 & 0 & 0 & 0 & 0 & 0 & 0 & 0 \\
\hline $\begin{array}{l}\text { Incised "V"s } \\
\text { (on neck) }\end{array}$ & 0 & 0 & 0 & 0 & 0 & 0 & 0 & 0 & 1 & 0 \\
\hline $\begin{array}{l}\text { Short Vertical Incised } \\
\text { Lines (on neck) }\end{array}$ & 0 & 0 & 2 & 0 & 0 & 0 & 0 & 0 & 1 & 0 \\
\hline $\begin{array}{l}\text { Misc. Incisions } \\
\text { (on body) }\end{array}$ & 0 & 0 & 0 & 0 & 0 & 0 & 1 & 0 & 0 & 0 \\
\hline $\begin{array}{c}\text { Circular Reed Punc- } \\
\text { tations (on lip) }\end{array}$ & 0 & 0 & 0 & 0 & 1 & 0 & 0 & 0 & 0 & 0 \\
\hline $\begin{array}{l}V \text {-Shaped Notches } \\
\text { (on } 1 \text { ip) }\end{array}$ & 4 & 0 & 6 & 5 & 1 & 4 & 1 & 2 & 0 & 2 \\
\hline $\begin{array}{l}\text { F1ngert1p Impressions } \\
\text { (on neck) }\end{array}$ & 0 & 0 & 1 & 0 & 0 & 0 & 0 & 0 & 2 & 0 \\
\hline $\begin{array}{l}\text { Corncob Impressions } \\
\text { (on shoulder) }\end{array}$ & 0 & 0 & 0 & 0 & 1 & 0 & 0 & 0 & 0 & 0 \\
\hline \multicolumn{11}{|l|}{ Size } \\
\hline$>2-4 \mathrm{~cm}$ & 114 & 24 & 129 & 35 & 119 & 30 & 117 & 39 & 80 & 51 \\
\hline$>4-6 \mathrm{~cm}$ & 10 & 2 & 23 & 10 & 11 & 8 & 8 & 3 & 16 & 4 \\
\hline$>6 \mathrm{~cm}$ & 2 & 0 & 7 & 0 & 0 & 1 & 1 & 0 & 1 & 0 \\
\hline
\end{tabular}



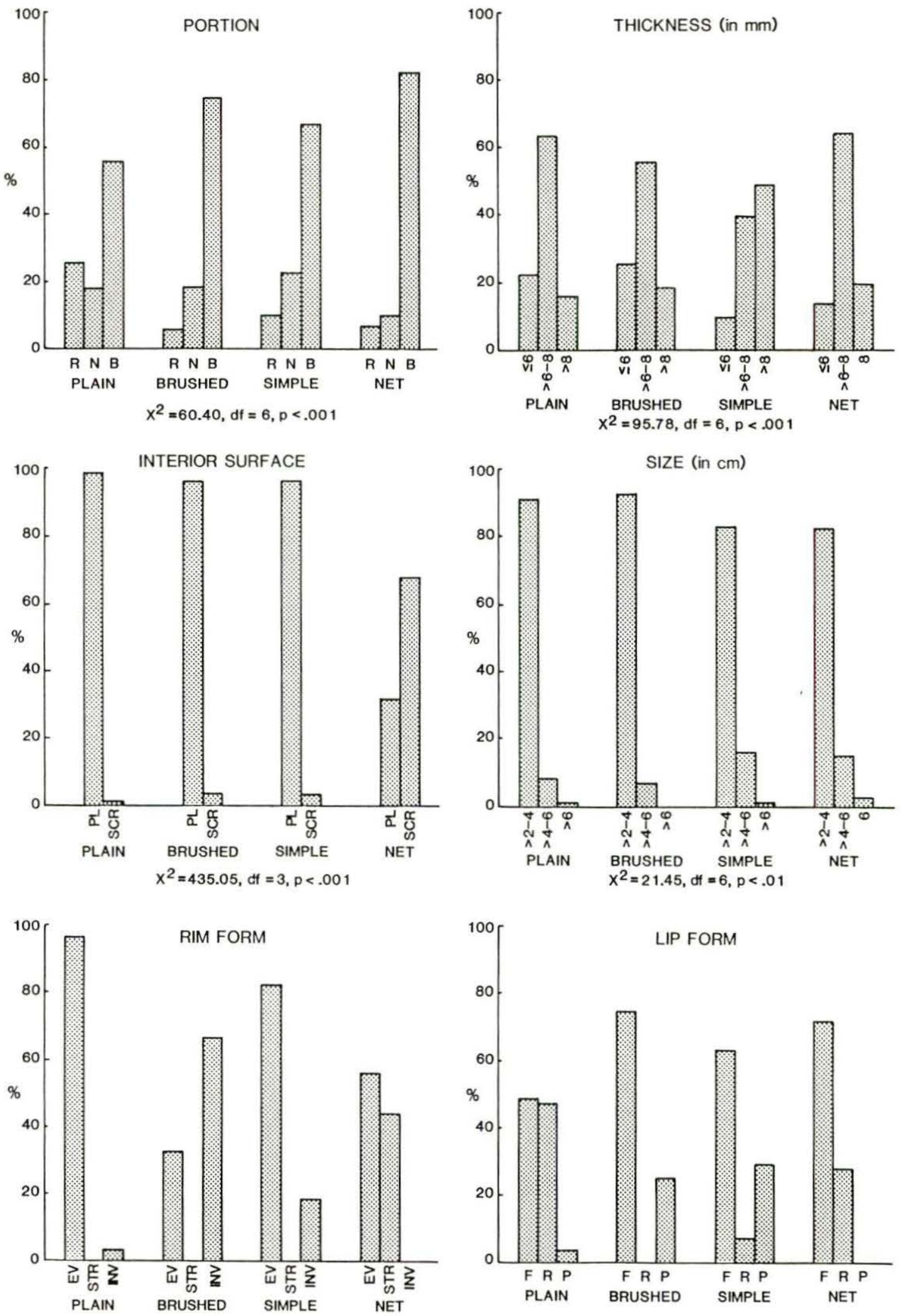

Figure 8.17

Relative frequency distributions and statistical evaluation of selected attributes for plain, brushed, simple-stamped, and net-impressed sherds from the Mitchum site. 
as prevalent rim form and interior surface treatment, conform well to Dan River ceramic series descriptions (Coe and Lewis 1952).

As with the Fredricks ceramic sample, significant attribute differences can be shown to exist between net-impressed sherds and sherds with other frequently observed surface treatments. Recognition of attribute variability at each site indicates certain distinctive intersite differences in the overall ceramic samples; however, vessels represented by net-impressed sherds appear to be essentially similar. Because a significantly greater proportion of large net-impressed sherds (i.e., greater than 4 $\mathrm{cm}$ in dia.) were recovered from the Mitchum site, many of which had uneroded edges, the possibility of their being associated with the remainder of the ceramic assemblage was considered to be greater. This potential association was examined in two ways, and produced mixed results. First, the distribution of plain, brushed, simple-stamped, and netimpressed sherds was examined within plowzone and sub-plowzone strata for a $700 \mathrm{ft}^{2}$ excavation area $(n=727$ sherds). The sub-plowzone stratum, Zone II, was approximately $0.2 \mathrm{ft}$ thick and represents an organically stained zone at the top of subsoil which was skim-shoveled to expose intrusive pits and postholes. It was assumed that if the net-impressed sherds were the product of an earlier occupation at the site, this situation might be reflected by a significantly greater frequency of those sherds within a sub-plowzone stratigraphic context. A Chisquare test of the sherd distributions within these two zones, however, ${ }_{2}$ does not indicate any significant difference $\left(x^{2}=0.97, \quad d f=3, p<.90\right)$. A second test comparing the combined strata with excavated features, most of which produced historic artifacts, does indicate statistically significant differences $\left(x^{2}=34.40, \quad d f=3\right.$, $\mathrm{p}<.001)$. Specifically, simple-stamped sherds occur in greater association with features whereas net-impressed sherds only rarely occurred in such contexts. Both plain and brushed sherds are randomly distributed within both features and excavated strata. These results, although far from conclusive given the limited sample, suggest that the net-impressed pottery may not be associated with the historic features excavated at the Mitchum site. This conclusion is also supported by the fact that the few large net-impressed sherds from undisturbed contexts were all recovered from postholes not associated with Structure 1. As with the Fredricks site, the available ceramic data suggest the presence of at least two separate cultural components; however, additional samples are clearly needed from each site to evaluate this possibility further.

\section{INTERSITE COMPARISONS}

The preceding analyses have provided a detailed examination of intrasite ceramic variability at the Fredricks, Wall, and Mitchum sites in order to delineate assemblages of culturally and temporally related sherds, and to provide an empirical basis for evaluating the position of the Fredricks site (via ceramics) relative to two other cultural occupations which are proximate in both time and space. Clarification of what occurred prior to the Fredricks site occupation is important in identifying this site as early 18th-century Occaneechi Town, since it can be historically documented that this settlement represented an intrusion (after 1676) from the Roanoke River (ca. $50 \mathrm{mi}$ NE of Hillsborough). Unfortunately, no 17 th-century Occaneechi sites have been identified along the Roanoke, and probably will not be since much of that valley (particularly the Occaneechi Island area at the confluence of the Dan and Roanoke Rivers) is now inundated by Kerr Reservoir. Archaeological sites identified and sampled prior to inundation document only prehistoric occupations (Miller 1962).

In order to consider more fully the position of the Fredricks site ceramics and their external relationships, a regional database was compiled which includes ceramic information for 25 assemblages from 17 sites (including the Fredricks, Wall, and Mitchum sites) in piedmont North Carolina and Virginia (Figure 8.18). These data, derived from published and unpublished reports and theses on file at the Research Laboratories of Anthropology, represent a significant portion of the quantified ceramic information presently available for this region (Table 8.21). With the exception of three assemblages, all represent Late Woodland-Historic period cultural components. Because the database encompasses studies conducted by several researchers over a period of nearly 35 years, much of the available data are too incompatible to be useful for intersite comparison; consequently, only relative frequency distributions of exterior surface treatments are considered (Table 8.22). In addition, it appears likely that at least some of the assemblages included in this study represent multiple components. Any conclusions drawn from these data must, therefore, be regarded as tentative and will be subject to change as more and better-analyzed assemblages become available.

The three goals of the following analysis are to: 1) examine the underlying structure of Woodland ceramic variability (with respect to surface treatment) within the region, focusing upon the Late Woodland-Historic periods (after A.D. 1000); 2) define broad spatio-temporal patterns of ceramic variability (e.g., reflecting separate traditions); and 3) examine the external and internal relationships among the Fredricks, Wall, and Mitchum sites with respect to those patterns. This was accomplished by performing a principal component factor analysis (with VARIMAX rotation) of the surface treatment data, and using Ward's minimum variance hierarchical cluster analysis to group assemblages 


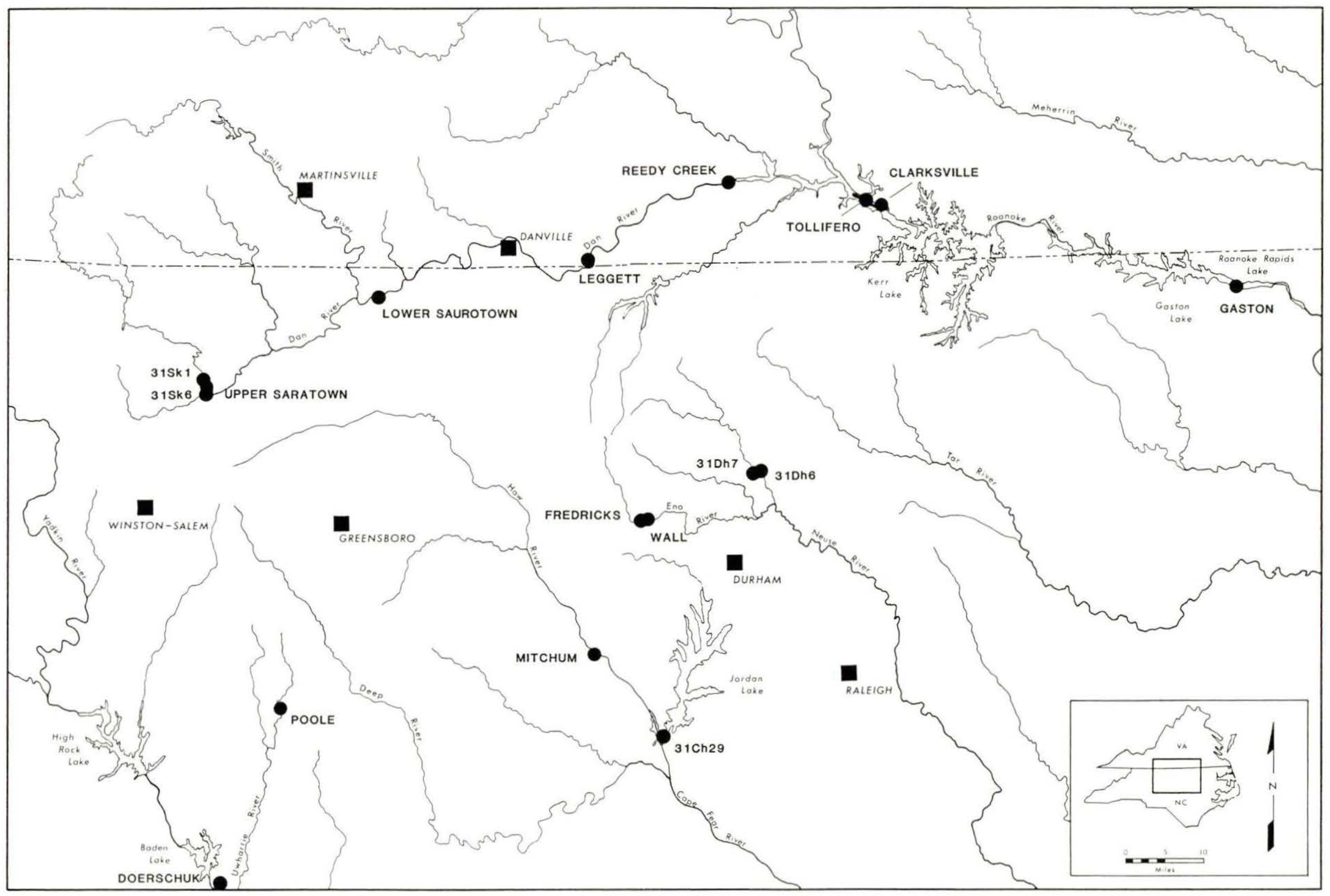


Table 8.21

Summary of assemblages used in intersite ceramic analysis.

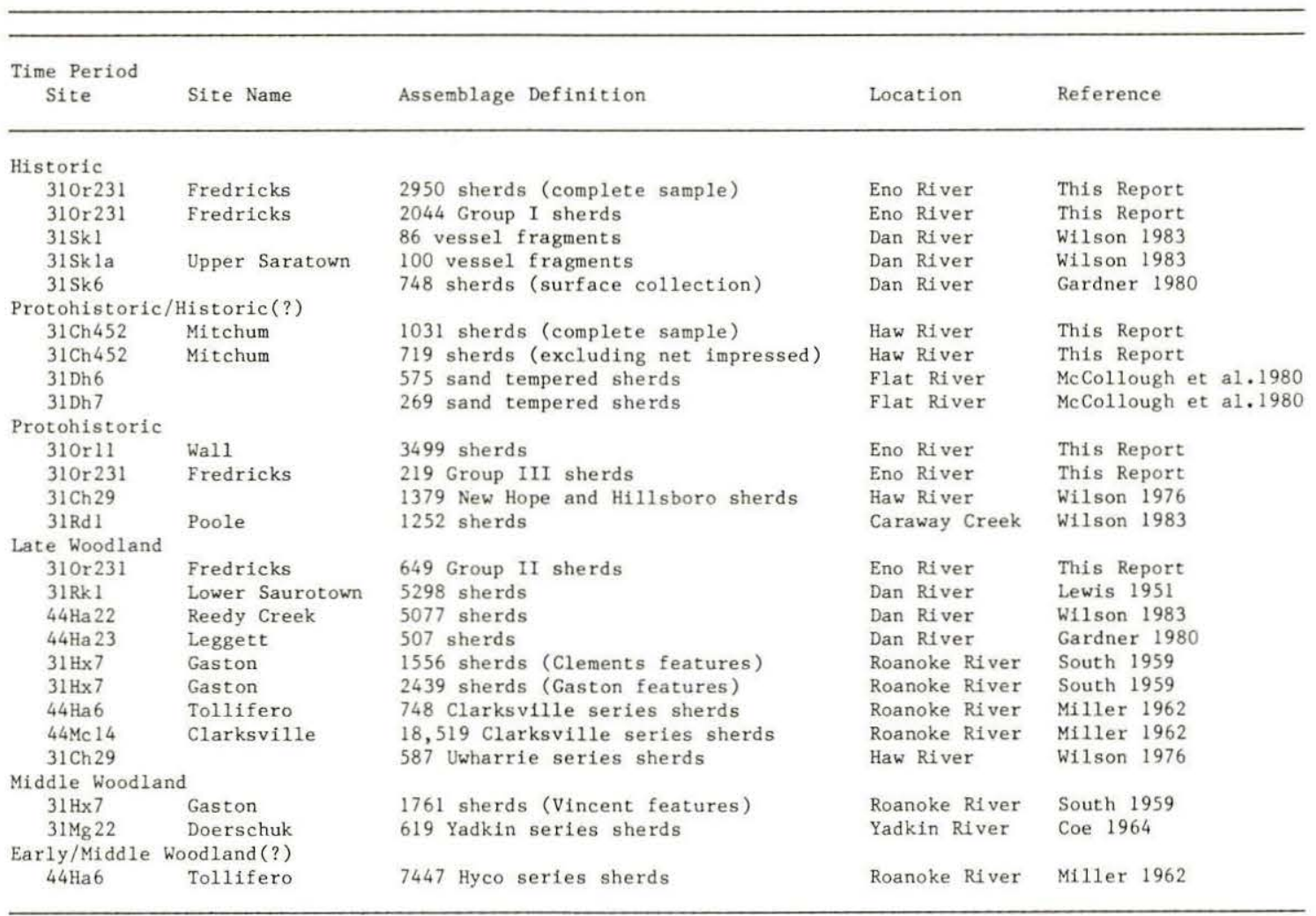

Table 8.22

Surface treatment percentages for analyzed ceramic assemblages.

\begin{tabular}{|c|c|c|c|c|c|c|c|c|c|}
\hline Ceramic Assemblage & Plain & Brushed & $\begin{array}{c}\text { Cob } \\
\text { Impressed }\end{array}$ & $\begin{array}{c}\text { Cord } \\
\text { Marked }\end{array}$ & $\begin{array}{c}\text { Net } \\
\text { Impressed }\end{array}$ & $\begin{array}{l}\text { Fabric } \\
\text { Marked }\end{array}$ & $\begin{array}{l}\text { Simple } \\
\text { Stamped }\end{array}$ & $\begin{array}{l}\text { Check } \\
\text { Stamped }\end{array}$ & $\begin{array}{l}\text { Comp. } \\
\text { Stamped }\end{array}$ \\
\hline \multicolumn{10}{|l|}{ Historic } \\
\hline $310 \mathrm{r} 231$ (A11) & 35.22 & 2.51 & .34 & 2.24 & 21.83 & .00 & 4.31 & 33.49 & .07 \\
\hline $310 \mathrm{r} 231(\mathrm{I})$ & 47.70 & 2.99 & .00 & 2.64 & .00 & .00 & .00 & 46.67 & .00 \\
\hline $31 \mathrm{Sk} 1$ & 43.02 & 1.16 & 5.81 & 2.33 & 38.37 & .00 & 8.14 & .00 & 2.33 \\
\hline $31 \mathrm{Skla}$ & 54.00 & 12.00 & 2.00 & 1.00 & 23.00 & .00 & 2.00 & 3.00 & 3.00 \\
\hline $31 \mathrm{Sk} 6$ & 26.20 & 4.14 & .00 & 15.51 & 51.34 & .00 & .00 & 1.07 & 1.74 \\
\hline \multicolumn{10}{|c|}{ Protohistoric/Historic(?) } \\
\hline $31 \mathrm{Ch} 452(\mathrm{All})$ & 40.06 & 9.02 & 3.39 & .58 & 30.26 & .00 & 14.74 & 1.45 & .48 \\
\hline $31 \mathrm{Ch} 452(\mathrm{w} / 0 \mathrm{Net})$ & 57.44 & 12.93 & 4.87 & .83 & .00 & .00 & 21.14 & 2.09 & .70 \\
\hline 31 Dh6 & 14.09 & 9.57 & 1.22 & 24.87 & 46.43 & .00 & .52 & .17 & 3.13 \\
\hline $31 \mathrm{Dh} 7$ & 23.79 & 1.12 & .00 & 5.95 & 69.14 & .00 & .00 & .00 & .00 \\
\hline \multicolumn{10}{|l|}{ Protohistoric } \\
\hline $310 \times 11$ (A11) & 11.15 & .06 & .46 & .26 & 1.11 & .11 & 72.85 & 14.00 & .00 \\
\hline $310 \times 231$ (III) & 28.30 & .00 & .00 & .00 & .00 & .00 & 58.00 & 13.70 & .00 \\
\hline $31 \mathrm{Ch} 29(\mathrm{NH} / \mathrm{H})$ & 87.60 & 1.38 & .51 & 4.28 & 3.19 & 3.05 & .00 & .00 & .00 \\
\hline 31Rd1 & 62.00 & 8.00 & 5.00 & 2.00 & 9.00 & .00 & .50 & 7.00 & 7.00 \\
\hline \multicolumn{10}{|l|}{ Late Woodland } \\
\hline $310 \mathrm{r} 231$ (II) & .00 & 1.54 & .00 & .00 & 98.46 & .00 & .00 & .00 & .00 \\
\hline $31 \mathrm{Rk} 1$ & 23.97 & 1.13 & 3.53 & 2.93 & 67.92 & .00 & .06 & .00 & .47 \\
\hline $44 \mathrm{Ha} 22$ & 21.06 & 2.19 & 1.77 & 7.19 & 67.54 & .00 & .26 & .00 & .00 \\
\hline $44 \mathrm{Ha} 23$ & 4.34 & 1.58 & 2.56 & 24.06 & 67.46 & .00 & .00 & .00 & .00 \\
\hline $31 \mathrm{Hx} 7(\mathrm{Clem})$ & 2.10 & .00 & .10 & 68.10 & .20 & 25.40 & 3.80 & .20 & .00 \\
\hline $31 \mathrm{Hx} 7$ (Gast) & 2.50 & .00 & .10 & 13.40 & .20 & 21.70 & 61.80 & .30 & .00 \\
\hline 44Ha6(Clark) & 20.72 & 8.42 & .00 & .00 & 70.86 & .00 & .00 & .00 & .00 \\
\hline $44 \mathrm{Mc} 14$ & $10 \cdot 36$ & 4.00 & .72 & 2.72 & 81.89 & .25 & .05 & .00 & .00 \\
\hline $31 \mathrm{Ch} 29($ Uwh $)$ & 35.95 & .00 & .00 & 5.11 & 53.15 & 5.79 & .00 & .00 & .00 \\
\hline \multicolumn{10}{|l|}{ Middle Woodland } \\
\hline $31 \mathrm{Hx} 7$ (Vinc) & 2.70 & .00 & .00 & 42.40 & .70 & 51.90 & 2.40 & .00 & .00 \\
\hline $31 \mathrm{Mg} 22$ (Yad) & .00 & .00 & .00 & 38.93 & .00 & 50.73 & .00 & 10.34 & .00 \\
\hline \multicolumn{10}{|c|}{ Early/Middle Woodland(?) } \\
\hline $44 \mathrm{Ha} 6$ (Hyco) & .16 & .00 & .00 & 15.48 & .13 & 84.22 & .00 & .00 & .00 \\
\hline
\end{tabular}


based on factor scores (SAS Institute, Inc. 1982).

The factor analysis produced a 3-factor solution, which accounts for almost $70 \%$ of the total variance (Tables 8.23-8.24). Factor 1 is defined by high factor loadings for plain, brushed, cob-impressed, and complicated-stamped surface treatments and reflects the patterned co-occurrence of these treatments within Protohistoric and Historic assemblages. Factor 2 is defined by high positive loadings for cord-marked and fabric-marked treatments and a high negative loading for net-impressed, and monitors a gradual shift toward net impressing during the Late Woodland period. Factor 3 is also defined by a high negative factor loading for net-impressed and high positive loadings for simple-stamped and check-stamped treatments. This factor recognizes a significant inverse relationship between these surface treatments and provides empirical support for the decision to exclude net-impressed sherds from Historic period assemblages at the Fredricks and Mitchum sites.

The cluster analysis of factor scores for the 25 assemblages defined four distinct groups (Figure 8.19), all of which correspond well with ceramic traditions previously recognized within the region (see Coe 1952, 1964; Wilson 1983). Cluster 1 is defined by high percentages of cord-marked and fabric-marked sherds. All preLate Woodland assemblages are contained within this cluster, as well as the Clements assemblage from the Gaston site. Although this assemblage has been classified within the Late Woodland period based on radiocarbon dates (Coe 1964: 118), strong relationships exist with the earlier Vincent assemblage from the same site. The overall similarity between these assemblages and the Yadkin series material from the Doerschuk site suggest a pre-Late Woodland ceramic tradition within piedmont North Carolina and Virginia that was of relatively long duration and spatially extensive.

Cluster 2 assemblages are characterized by a high percentage of net-impressed sherds and a lesser but significant percentage of plain sherds. With the exception of $31 \mathrm{Sk} 6$ and possibly $31 \mathrm{Dh} 7$, all of these assemblages date to the Late Woodland period. The high number of net-impressed sherds within the 31Sk6 sample suggest that this site probably contains an earlier cultural component (see Gardner $1980: 12,84)$. This may also be the case at 31Dh7. Cluster 2 represents the Uwharrie-Dan River ceramic tradition recognized by Coe and Lewis (1952), as well as materials previously classified as Clarksville series (Miller 1962). In fact, two of the sites within this cluster-$31 \mathrm{Rk} 1$ and 44Mc14--are type sites for the Dan River and Clarksville ceramic series, respectively. As with Cluster 1, Cluster 2 sites are widely dispersed and appear to represent a relatively long span of time (see Gardner 1980: 80-83).

Cluster 3 is comprised of assemblages that date to the Protohistoric and Historic periods and that contain high percentages of plain sherds. Other predominant surface treatments include net impressed and brushed. The six sites included within this cluster are widely distributed throughout the Dan, Haw, Yadkin, and upper Neuse river drainages, and appear to represent a development out of the preceding Uwharrie-Dan River tradition. Variability among Cluster 3 assemblages is, to a certain degree, spatially patterned. Assemblages most similar to one another were all obtained from sites

Table 8.23

Correlation matrix for data used in the intersite ceramic analysis $(n=25)$,

\begin{tabular}{|c|c|c|c|c|c|c|c|c|c|}
\hline & Plain & Brushed & $\begin{array}{c}\text { Cob } \\
\text { Impressed }\end{array}$ & $\begin{array}{c}\text { Cord } \\
\text { Marked }\end{array}$ & $\begin{array}{c}\text { Net } \\
\text { Impressed }\end{array}$ & $\begin{array}{l}\text { Fabric } \\
\text { Marked }\end{array}$ & $\begin{array}{l}\text { Simple } \\
\text { Stamped }\end{array}$ & $\begin{array}{c}\text { Check } \\
\text { Stamped }\end{array}$ & $\begin{array}{l}\text { Comp. } \\
\text { Stamped }\end{array}$ \\
\hline Plain & 1.00 & & & & & & & & \\
\hline Brushed & .45 & 1.00 & & & & & & & \\
\hline Cob Impressed & .45 & .47 & 1.00 & & & & & & \\
\hline Cord Marked & -.51 & -.31 & -.28 & 1.00 & & & & & \\
\hline Net Impressed & -.22 & .06 & .02 & -.28 & 1.00 & & & & \\
\hline Fabric Marked & -.47 & -.38 & -.32 & .55 & -.44 & 1.00 & & & \\
\hline Simple Stamped & -.13 & -.18 & -.08 & -.18 & -.42 & .08 & 1.00 & & \\
\hline Check Stamped & .20 & -.08 & -.20 & -.18 & -.37 & -.13 & .12 & 1.00 & \\
\hline Comp. Stamped & .41 & .52 & .57 & -.13 & -.08 & -.22 & -.17 & -.08 & 1.00 \\
\hline
\end{tabular}


Table 8.24

Factor loading matrix.

\begin{tabular}{|c|c|c|c|}
\hline Surface Treatment & Factor 1 & Factor 2 & Factor \\
\hline Plain & .68 & -.38 & .30 \\
\hline Brushed & .73 & -.25 & -.13 \\
\hline Cob Impressed & .77 & -.16 & -.14 \\
\hline Comp. Stamped & .84 & .04 & -.10 \\
\hline Fabric Marked & -.25 & .85 & .01 \\
\hline Cord Marked & -.20 & .83 & -.20 \\
\hline Net Impressed & -.23 & -.58 & -.77 \\
\hline Simple Stamped & -.20 & .08 & .65 \\
\hline Check Stamped & -.07 & -.16 & .72 \\
\hline $\begin{array}{l}\text { E1genvalue } \\
\text { \% Total Vartance }\end{array}$ & $\begin{array}{r}3.05 \\
33.91\end{array}$ & $\begin{array}{r}1.71 \\
18.95\end{array}$ & $\begin{array}{r}1.47 \\
16.32\end{array}$ \\
\hline \% Common Variance & 49.02 & 27.39 & 23.59 \\
\hline
\end{tabular}

along the Haw and upper Dan Rivers. Conversely, both $31 \mathrm{Dh} 6$ and 31Rdl--sites whose ceramic assemblages are most divergent from other group constituents--are more distantly located on the periphery of the Haw-Dan River area. Although 31Dh6 may reflect a mixture of sherds from different site occupations, the predominant assemblage composition at $31 \mathrm{Rdl}$ is perhaps better explained by its proximity to a more southerly ceramic tradition characterized by plain, burnished, and complicated-stamped wares (see Wilson 1983:368).

Finally, Cluster 4 contains all of the assemblages from the Hillsborough area (except Group II at the Fredricks site) and the Gaston assemblage from the Roanoke River valley. Predominant surface treatments include simple stamped, check stamped, and plain. Temporally, these assemblages represent the Late Woodland, Protohistoric, and Historic periods, and probably encompass about 200 years. The fact that these assemblages comprise a single cluster is significant. Despite differences noted earlier between the Fredricks and Wall site ceramics, these differences are minor when viewed within

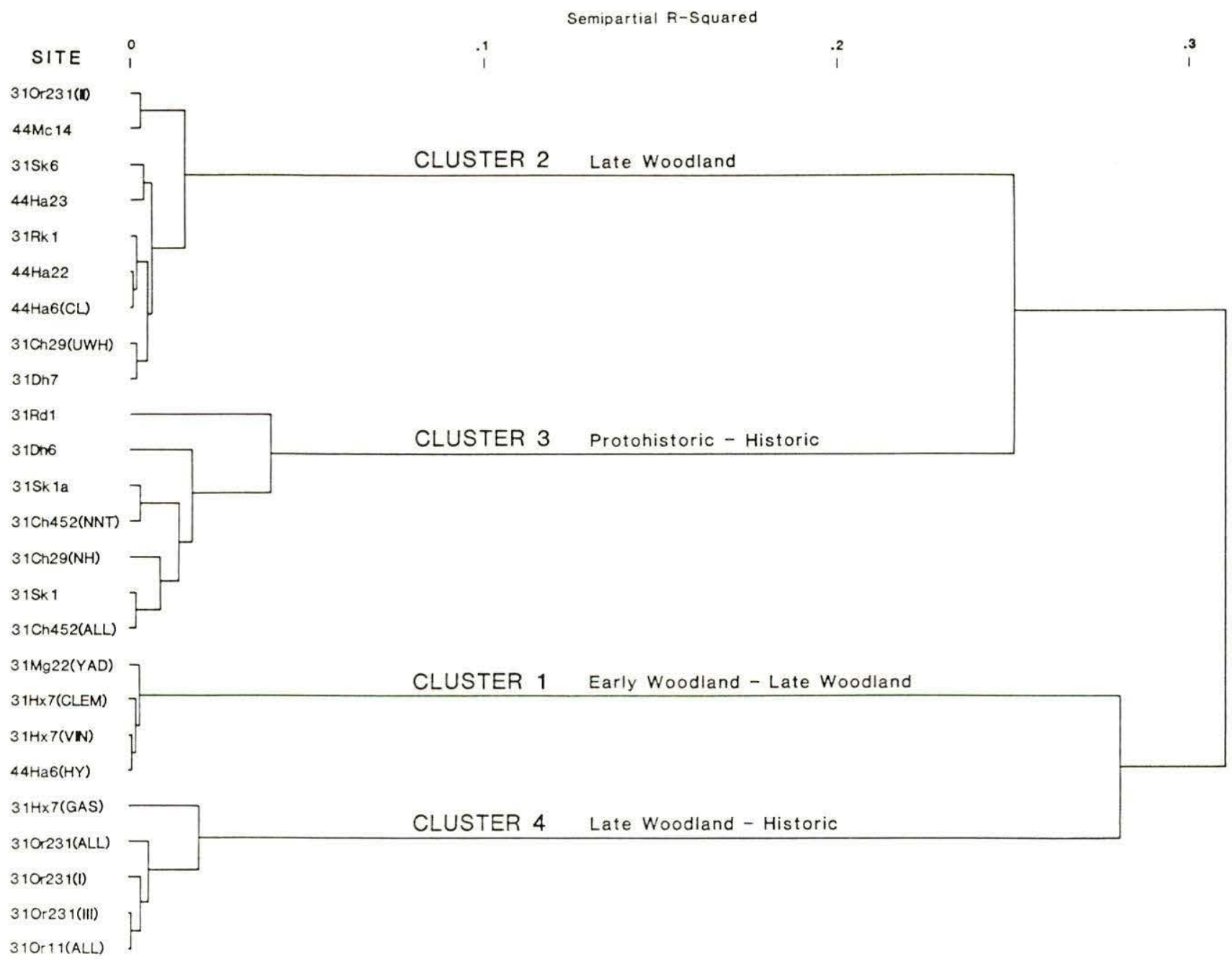

Figure 8.19

Dendrogram showing results of ceramic cluster analysis. 
the broader context of regional ceramic variability. These results provide compelling evidence for a close cultural relationship between the Fredricks and Wall sites, and the participation by potters at both sites in a ceramic tradition that was distinctively different from the one embraced by neighboring piedmont groups. Wilson (1983:369) has suggested that differences between the Wall site ceramics and other contemporary assemblages may reflect more basic linguistic differences, with the Wall site occupants possibly being Iroquoian-speakers. In the absence of good ceramic data from Historic period Iroquoian sites (e.g., Tuscarora), however, this possibility cannot be carefully evaluated. Nevertheless, the inclusion of the Gaston assemblage within this cluster strongly suggests that ceramic relationships of the Wall and Fredricks sites are probably to be found to the northeast rather than toward the south or west. Such directionality certainly strengthens any argument for an Iroquoian affiliation.

The overall dissimilarity between Cluster 3 (particularly the Mitchum site) and Cluster 4 assemblages further suggests the existence of two separate and distinct ceramic traditions within the study area during the Late Prehistoric, Protohistoric, and Historic periods. The boundary for these traditions generally lay between the Haw and upper Neuse drainages. However, to what extent this boundary shifted over time remains to be shown. In addition, the origins of the tradition represented by Cluster 4 remain obscure, as well as the degree to which these ceramic differences reflect larger cultural/1inguistic differences.

\section{SUMMARY AND CONCLUSIONS}

At the beginning of this section, three research questions were posed concerning the composition of the historic aboriginal ceramic assemblage at the Fredricks site, the recognition of additional cultural components at the site, and the relationship of the Fredricks site ceramics to other ceramic assemblages within the study area. In addition to examining materials from the Fredricks site, samples were also analyzed from the Wall and Mitchum sites. All three questions were considered within the context of an attribute analysis to discover quantifiable patterns of ceramic variability that could be empirically tested. Particular attention was also given to the evaluation of sample context in order to draw informed conclusions about probable cultural and temporal associations among recognizable categories of ceramic artifacts. As a consequence of this analysis, the following observations can be made.

Although future research may show that some of the variability within the Fredricks ceramic sample is a consequence of ethnic diversity during the same historic occupation, most of the variability is more likely due to the presence of temporally separate cultural components. From the available ceramic data, three separate Woodland occupations can be postulated for the Fredricks site. Remains of the first postulated occupation comprise $22 \%$ of the sherd sample and were recovered most $1 y$ from plowzone contexts. Sherds representing this occupation are tempered with sand and crushed quartz and have netimpressed and brushed surfaces. These materials show strong stylistic and technological relationships to net-impressed pottery from the Mitchum site and generally conform to descriptions of the Dan River ceramic series, which is prevalent along the Dan River drainage during the Late Woodland period.

A second occupation at the Fredricks site is represented by sand-tempered and crushed quartz and feldspar-tempered simple-stamped, plain, and check-stamped sherds, which comprise approximately $7 \%$ of the Fredricks sample. These sherds were also recovered primarily from plowzone contexts and are related to the Late Woodland occupation at the nearby Wall site.

Finally, the majority of ceramic artifacts (ca. 70\%) from all contexts at the Fredricks site are associated with the historic occupation and therefore are thought to be the material remains of the Occaneechi Indians. These materials consist of sand-tempered and fine crushed feldspar-tempered check-stamped, plain, brushed, and cord-marked pottery. Although check stamping is represented in other Historic period assemblages within the study area, it is always a minority surface treatment. At the Fredricks site, however, it was the predominant type of treatment. Check stamping is also a major type of surface finish in the Wall site assemblage.

The analysis of the Wall site ceramic sample produced little evidence to indicate more than a single occupation. Moreover, examination of stratigraphic contexts suggests that the Wall site occupation was relatively brief. Sherds from the Wall site are tempered predominantly with medium sand and fine crushed feldspar, and have simple-stamped, check-stamped, and plain exteriors. The simple stamped sherds bear a strong relationship to Late Woodland Gaston series pottery from the middle Roanoke River; however, the exact nature of this relationship is unclear.

Conversely, the Mitchum site sample appears to represent two separate occupations. A small amount of net-impressed pottery, mostly tempered with sand and fine crushed feldspar, conforms to the Dan River ceramic series and appears to represent a Late Woodland occupation. The majority of the sherds, however, are associated with the historic occupation at the site. These materials were recovered from all excavated contexts and are more closely related to historic ceramic traditions recognized to the west and northwest. Sherds are mostly tempered 
with sand and crushed feldspar and have plain, simple-stamped, and brushed surfaces.

Finally, comparisons with additional samples from Woodland sites within piedmont North Carolina and southern Virginia indicate a significant discontinuity in the spatial distribution of ceramics at the end of the Late Woodland period. This discontinuity appears to indicate the development of distinct, local ceramic traditions that persisted into the Historic period; however, the cultural processes that underly them remain obscure. It is hoped that as research continues and as additional samples are analyzed, these patterns of regional ceramic variability will become more precisely defined and will permit further insight into the processes of culture change that were well underway by the time John Lawson visited Occaneechi Town in 1701. 


\title{
Lithic Artifacts from the Fredricks, Wall, and Mitchum Sites
}

by

\author{
V. Ann Tippitt \\ I. Randolph Daniel, Jr.
}

\section{INTRODUCTION}

In this chapter, questions about the nature and structure of change in lithic technology from the Late Prehistoric to Historic periods are addressed. Although the analysis focuses upon the Fredricks (310r231), Wall (310r11), and Mitchum (31Ch452) sites, artifact samples from Early Upper Saratown (31Sk1) and Upper Saratown (31Skla) are also analyzed and described to provide comparative data. Collectively, these sites span the period from A.D. 1500-1700. An additional Late Woodland assemblage from Forbush Creek (31Yd1), analyzed and described by McManus (1985), is also used for comparative purposes. The main objectives of this study are to produce a technological/functional characterization of the lithic assemblage at each site and to investigate change within and between artifact classes through time.

The specific research questions to be addressed are:

1. What was the impact of European contact and the introduction of metal tools on the native lithic technology, and how are these factors reflected in the archaeological record?

2. Are there changes in the morphology and construction of triangular projectile points from the Protohistoric to Historic period and, if so, are these changes temporally diagnostic?

3. Are there patterns in lithic raw material utilization, and, if so, what do these patterns reveal about extents and directions of mobility?

It is first necessary to characterize the protohistoric and historic assemblages in order to make areal and regional comparisons, to assess the impact of contact as reflected in the lithic technology, and to provide density and spatial information on artifact classes.

\section{ANALYTIC METHODS}

In order to characterize the lithic arti- facts from the five sites and to provide information necessary to address the above research questions, data were generated on: 1) tool assemblage organization; 2) stages of manufacture; 3) raw materials used in tool construction; 4) tool categories (e.g., formalized and expedient); and 5) the overall morphology of formalized tools.

Specifically, observations were made along four dimensions: formal, functional, technological, and geological. It was recognized that these dimensions have both spatial and temporal aspects. The analytic methods used to gather these data are discussed in three sections: 1) observations made on lithic artifacts and how they were recorded; 2) descriptions of the relevant geologic data and raw material descriptions; and 3 ) methods used to analyze the structure of formalized tools.

Once the lithic artifacts were cataloged, an analysis format and attribute coding sheet were designed for computer data storage and retrieval. The 23 attributes used in the analysis consist of blank category, working edge category, raw material type, tool condition, artifact size, and a series of measurements made on complete tools and projectile points.

The debitage and tools from each excavation provenience were sorted first by reduction stage (i.e., blank category), then by working edge, raw material, and size.

\section{ARTIFACT TYPES}

Lithic tool and debitage types represented in the analyzed assemblages are briefly defined below.

\section{Debitage}

Primary Decortication Flake. Primary decortication flakes are usually large, broad flakes removed during the initial reduction stage. These flakes have cortex on the dorsal face and the platform.

Secondary Decortication Flake. These are flakes detached in the early stages of reduc- 
tion. Cortex is present on at least half of the dorsal surface, but there is no cortex on the platform.

Interior Flake. Interior flakes are usually thin, exhibit no cortex on the dorsal surface, and have scars of previous flakes removed from the dorsal surface. These flakes are usually produced during the thinning or shaping of tools and bifaces.

Bifacial Thinning Flake. Bifacial thinning flakes (BTF) have a number of distinctive characteristics that allow their separation from interior flakes. Bifacial thinning flakes are removed during the thinning or resharpening of bifaces. These flakes are relatively flat, have broad, shallow flake scars (produced by the detachment of previous thinning flakes from the dorsal face), and tend to exhibit a feathering out of lateral margins. The proximal end of the flake often retains the edge of the biface and, if the platform is retained, it often exhibits a low angle and evidence of crushing or grinding (i.e., platform preparation).

Shatter Fragment. These pieces of manufacturing debris are blocky, angular fragments that do not exhibit bulbs of percussion, striking platforms, or dorsal flake scars.

\section{Chipped-Stone Tools}

Biface. Bifaces are blanks that exhibit flake removal scars on both surfaces. These artifacts are usually irregular in outline and vary in thickness and size. Many artifacts classified as bifaces are probably tools that broke during manufacture, or bifaces that could not be thinned and were discarded. Several specimens assigned to this category are represented only by small edge fragments that cannot be further identified.

Preform. The bifaces in this category are well thinned but do not have well-shaped or retouched lateral margins. Many of these represent unfinished hafted bifaces. Some appear to have been biface blanks that were discarded during manufacture because of a flaw or an inclusion in the raw material. Others are blanks that were broken during the final stages of thinning.

Drill. A drill displays alternate bifacial retouch along the major portion of the working edge resulting in a rod-like projection that is usually biconvex or diamond-shaped in cross section.

Graver/Perforator. A graver is a tool with intentional retouch that results in a small triangular-shaped projection, whereas a perforator exhibits intentional retouch that results in a converging point that is larger than a graver. The retouch forming a graver or perforator may be either unifacial or bifacial but is more often unifacial.
Scraper. A scraper (end or side) is defined as a flake which displays regularized edge retouch to produce a uniform and continuous edge. In the assemblages under consideration, the scrapers also appeared to have been made primarily on flakes. The morphological differences between retouched flakes and scrapers may be more of a matter of degree rather than kind.

Denticulate. Denticulates exhibit regularly spaced, fine retouch resulting in a series of small sharp projections or serrations along the working edge.

Utilized/Retouched Flake. A retouched flake is defined as a flake which displays edge modification resulting from use or intentional retouch with flake scars that extend at least 2 $\mathrm{mm}$ from the edge of the tool. A utilized flake is defined as a flake with flake scars resulting from use that extend less than $2 \mathrm{~mm}$ from the tool edge. It is presumed that tools exhibiting retouched edges were utilized, however, no microwear analysis was performed during the study to verify this. Usually such modification occurs on flakes and is confined to the flake edge.

Pièce Esquillée. A pièce esquillée is a piece of stone which exhibits repeated bipolar percussion blows and is characterized by crushed working edges with sharp perpendicular corners. Presumably these tools would have been used to slot dense material such as bone and antler.

Core. Cores are nodules or chunks of raw material from which one or more flakes have been detached (leaving more than one negative bulb of percussion), and whose flake scars seem to indicate removal for the production of flake blanks or the initial stages of biface production.

Chipped Hoe. Hoes are large, roughly chipped stone tools with a bifacially produced convex working edge perpendicular to the long axis of the tool. It tapers from the working edge to a rounded distal end which gives it a wedge-shaped appearance. Such tools are assumed to have been used as digging implements.

Chipped Axe. An axe is a large, roughly chipped stone tool with primary and secondary bifacially-flaked working edges transverse to the long axis of the tool. This tool was probably hafted and used to chop dense material such as wood.

Chopper. A chopper is a heavy-duty tool, roughly chipped to produce an angular working face. Chipping on these tools may be either bifacial or unifacial but usually only slightly alters the natural shape of the stone.

Utilized Cobble. These specimens are cobbles that exhibit flake removal scars and/or utilized edges indicative of chopping or scraping activities. 
Chipped-Stone Disk. A chipped-stone disk is a flat, circular piece of stone whose edges have been flaked into a disk shape. The function of these artifacts is unknown.

\section{Projectile Points}

Projectile points are finished bifaces with lateral edges that converge to a point and have been modified at the proximal end to facilitate hafting. These artifacts were assigned either to a specific historical type (see Coe 1964) or to a general form, such as "projectile point, probably Woodland." In addition, six blade and base shapes were used to describe the specific edge configuration of small triangular projectile points.

\section{Ground-Stone Tools}

Hammerstone. Hammerstones are cobbles or cobble fragments that exhibit battered and pitted edges resulting from use as a percussor.

Mano. A mano is a cobble or cobble fragment with one or more flat surfaces produced by abrasion and grinding. Such tools were presumably used for food processing.

Anvil. An anvil is a cobble or large stone that displays one or more crushed, depressed, or slightly concave areas, presumably from use in lithic reduction or nut cracking.

Grinding Stone. Grinding stones are large stones that display smoothed or ground, flattened surfaces resulting from the processing of plant and animal foods.

Polished Cobble. Polished cobbles are cobbles or cobble fragments with smoothed or polished areas resulting from scraping or grinding activities.

Abrader. Abraders are lithic artifacts with ground, grooved, or striated surfaces resulting from the abrasion of stone, bone, or wooden implements.

Pitted Cobble. A pitted cobble is a cobble or cobble fragment that displays battered depressions on one or more faces. Although the traditional interpretation of these tools is that they are a product of nut processing, they probably also functioned as anvils.

Ground Celt. This ground stone axe form exhibits a bi-convex working edge, is subtriangular in form, and has a tapered poll end. Celts were probably manufactured by percussion, pecking, or grinding. Moreover, the bit end was polished to produce a sharp working edge.

Ground-Stone Disk. A ground-stone disk is a flat, circular piece of stone whose edges have been flaked and then ground into a disk shape. The function of these artifacts is unknown.
Chunkey Stone. These artifacts are circular in plan view and plano-convex in cross section. They are finely ground or polished.

Ground Gorget. Gorgets are bilaterally symmetrical, flattened, ground-stone artifacts with two (usually) drilled holes for attachment.

Ground-Stone Pendant. A pendant is a small tear-drop to oval-shaped ground stone artifact with a drilled hole or groove at one end for attachment.

\section{RAW MATERIALS}

As archaeologists have become more interested in the relationships between settlementsubsistence systems and mobility, and more concerned with the behavioral aspects of technology, there has been an increase in studies of lithic raw materials. These studies (e.g., Gould 1979; Klippe1 1971; Charlton 1978; Meyers 1970; and Novick 1978) have demonstrated that prehistoric peoples selectively utilized a wide variety of lithic materials. This variety supposedly reflects decisions on the part of prehistoric peoples to select raw materials based on physical characteristics of the rock (e.g., fracture properties, hardness, and how long the material would hold an edge), relative to the properties of the tool that was to be manufactured. However, Gould (1979) and Flannery (1976) have pointed out that not only should the physical properties of the material and tool structure be considered, but the cognitive or behavioral information that might be reflected in raw material selection also should be considered. Several ethnoarchaeologists have included this consideration in their studies of raw materials used by hunters and gatherers (Lee and DeVore 1976; Binford 1979). In his discussion of "righteous rocks" among Australian Aborigines, Gould (1979) points out that raw material variation can also carry religious and social meanings.

The three river drainages under study in the Siouan project lie within the geological province known as the Carolina Slate Belt. In 1822 , the rocks of the Carolina Slate Belt were described by Denison 01mstead as part of what he called the "Great Slate Formation" (Allen and Wilson 1968). With further geologic investigation and formation mapping, it has become clear that the Carolina Slate Belt contains both sedimentary and volcanic rocks. Although it is commonly called the Carolina Slate Belt, slate is not the major rock type in the zone, and the formation is not a single continuous belt (Butler 1963:169). The major rock units of the Carolina Slate Belt are argillite, slate, phyllite, greenstone, metamorphosed lithiccrystal tuff, devitrified glassy rocks, breccia, and volcanic conglomerate (Butler 1963:167). These rock units generally show the 
effects of low grade metamorphism, and their mineral assemblages are typical of the cholorite zone of regional metamorphism (Butler 1963:169).

As detailed by Allen and Wilson (1968), the stratigraphic succession in Orange County contains four major rock units which are summarized below in ascending order.

Unit I: This unit contains predominantly amygdaloidal basalt flows intercalated with basalt porphyries and lithic and crystal tuffs.

Unit II: Andesitic to dacitic lithic and crystal tuffs characterize this unit.

Unit III: This unit is composed predominantly of felsic tuffs and lithic and crystallithic tuffs, with occasional flows of rhyolite and rhyolite porphyries.

Unit IV: Epiclastic argillites, laminated argillites, and graywacke predominate in this zone. Metavolcanic-metasedimentary rocks are intrusive in some areas, and rocks of Triassic age can be found in a down-faulted basin in the extreme southeastern corner of the county.

There is a wide variety of materials that could be worked into lithic tools. The research questions on raw materials being addressed here required both identification of the raw materials used in the construction of artifacts and tools, and the field locations of the major rock types used. The rock types recorded for all three sites are chert (local and non-local), quartzite, vein quartz, crystal quartz, rhyolite, argillite, other metavolcanic, welded tuff, vitric tuff, felsic tuff, basalt, and silicate. In the recording process, several of these categories were subdivided into smaller groups by color, texture, and inclusions. With more field investigation, it may be possible to use some of these visual characteristics to identify source areas. Raw material studies are more profitably approached by focusing on the macroscopic qualities that guided raw material selection (e.g., inclusions, color, mineral structure, hardness, and fracturing qualities). In order to understand the impact of mobility and procurement strategy on the tool kit organization, it is necessary to understand the nature and distribution of various rock types over the landscape.

\section{ASSEMBLAGE DESCRIPTIONS}

The results of the artifact analysis are presented below for each of the three sites. Each site discussion contains the results of the debitage and chipped-stone tool analyses and raw material investigations.

\section{Fredricks Site}

A total of $3510 \times 10-\mathrm{ft}$ units, eight burials, and six features were excavated at the Fredricks site during 1983-84. Lithic debitage and chipped-stone artifacts were recovered from the plowzone of each unit and from all features and burials. In addition, 14 features, three burials and two structures from the 1985 excavations contained lithic remains and are also included in the analysis.

Debitage. All stages of the manufacturing process are represented in the debitage from the Fredricks site. The distribution of debitage by reduction stage is presented in Table 9.1. Although all classes are present, interior flakes predominate, and most of the flakes from all contexts are small, being less than $4 \mathrm{~cm}$ in size.

Chipped-Stone Tools. The chipped-stone tool assemblage also contains a variety of tool classes, including small triangular projectile points, drills, perforators, retouched flakes, utilized flakes, scrapers, choppers, and chipped stone disks (Table 9.2).

Table 9.1

Distribution of debitage by site.

\begin{tabular}{|c|c|c|c|c|c|c|c|c|c|c|c|c|c|}
\hline \multirow[b]{2}{*}{ Site } & \multicolumn{2}{|c|}{ Primary } & \multicolumn{2}{|c|}{ Secondary } & \multicolumn{2}{|c|}{$\begin{array}{l}\text { Flake } \\
\text { Interior }\end{array}$} & \multicolumn{2}{|l|}{ Type } & \multicolumn{2}{|c|}{ Shatter } & \multicolumn{2}{|c|}{ Misc. } & \multirow[b]{2}{*}{ Total } \\
\hline & $\mathrm{n}$ & $\%$ & $\mathrm{n}$ & $\%$ & $\mathrm{n}$ & $\%$ & $\mathrm{n}$ & $\%$ & $\mathrm{n}$ & $\%$ & $\mathrm{n}$ & $\%$ & \\
\hline Wall & 22 & 0.73 & 275 & 9.14 & 1212 & 40.29 & 151 & 5.02 & 1347 & 44.78 & 1 & 0.03 & 3008 \\
\hline Early Upper Saratown & 18 & 3.02 & 34 & 5.70 & 516 & 86.58 & 4 & 0.67 & 23 & 3.86 & 1 & 0.17 & 596 \\
\hline Mitchum & 14 & 3.28 & 82 & 19.20 & 211 & 49.41 & 6 & 1.40 & 114 & 26.70 & 0 & - & 427 \\
\hline Upper Saratown & 19 & 3.36 & 26 & 4.60 & 482 & 85.31 & 1 & 0.18 & 37 & 6.55 & 0 & - & 565 \\
\hline Fredricks & 35 & 1.65 & 267 & 12.58 & 1331 & 62.72 & 97 & 4.57 & 390 & 0.22 & 1 & 18.38 & 2122 \\
\hline
\end{tabular}

\footnotetext{
* (includes retouched and utilized flakes).
} 
Table 9.2

Distribution of tool types by site.

\begin{tabular}{|c|c|c|c|c|c|c|c|c|c|c|c|c|}
\hline \multirow[b]{2}{*}{ Type } & \multicolumn{2}{|c|}{$\begin{array}{l}\text { Forbush } \\
\text { Creek }\end{array}$} & \multicolumn{2}{|c|}{ Wal1 } & \multicolumn{2}{|c|}{$\begin{array}{l}\text { Early Upper } \\
\text { Saratown }\end{array}$} & \multicolumn{2}{|c|}{ Mitchum } & \multicolumn{2}{|c|}{$\begin{array}{c}\text { Upper } \\
\text { Saratown }\end{array}$} & \multicolumn{2}{|c|}{ Fredricks } \\
\hline & $\mathrm{n}$ & $\%$ & $\mathrm{n}$ & $\%$ & $\mathrm{n}$ & $\%$ & $\mathrm{n}$ & $\%$ & $\mathrm{n}$ & $\%$ & $\mathrm{n}$ & $\%$ \\
\hline PPt. (Archaic) & 0 & - & 3 & 0.53 & 2 & 2.50 & 0 & - & 1 & 0.83 & 3 & 0.69 \\
\hline PPt. (Woodland) & 20 & 2.87 & 2 & 0.36 & 0 & - & 0 & - & 0 & - & 2 & 0.46 \\
\hline PPt. (Sm. Triangular) & 184 & 26.36 & 113 & 20.10 & 43 & 53.75 & 34 & 31.48 & 67 & 55.37 & 90 & 20.64 \\
\hline PPt. (Other) & 5 & 0.72 & 0 & - & 0 & - & 0 & - & 1 & 0.83 & 0 & - \\
\hline PPt. (Frags.) & 61 & 8.74 & 84 & 14.94 & 2 & 2.50 & 18 & 16.66 & 9 & 7.44 & 60 & 13.76 \\
\hline Biface & 19 & 2.72 & 43 & 7.65 & 9 & 11.25 & 10 & 9.26 & 3 & 2.48 & 40 & 9.17 \\
\hline Preform & 7 & 1.00 & 18 & 3.20 & 3 & 3.75 & 1 & 0.93 & 3 & 2.48 & 12 & 2.75 \\
\hline Dri11 & 11 & 1.58 & 2 & 0.36 & 0 & - & 4 & 3.70 & 6 & 4.96 & 4 & 0.92 \\
\hline Perforator & 15 & 2.15 & 6 & 1.06 & 2 & 2.50 & 0 & - & 1 & 0.83 & 5 & 1.15 \\
\hline Graver & 3 & 0.43 & 7 & 1.24 & 0 & - & 0 & - & 0 & - & 4 & 0.92 \\
\hline Scraper & 7 & 1.00 & 8 & 1.42 & 0 & - & 1 & 0.93 & 0 & - & 6 & 1.38 \\
\hline Denticulate & 10 & 1.43 & 0 & - & 1 & 1.25 & 0 & - & 3 & 2.48 & 0 & - \\
\hline Ut1./Ret. Flake & 311 & 44.55 & 170 & 30.24 & 11 & 13.75 & 20 & 22.22 & 14 & 11.57 & 127 & 29.13 \\
\hline Pièce Esquillée & 1 & 0.14 & 5 & 0.89 & 0 & - & 1 & 0.93 & 0 & - & 2 & 0.46 \\
\hline Core & 3 & 0.43 & 80 & 14.23 & 3 & 3.75 & 14 & 12.96 & 0 & - & 20 & 4.59 \\
\hline Hammerstone & 14 & 2.01 & 7 & 1.25 & 4 & 5.00 & 1 & 0.93 & 8 & 6.61 & 7 & 1.60 \\
\hline Chopper & 2 & 0.29 & 5 & 0.89 & 0 & - & 0 & - & 0 & - & 18 & $4 \cdot 13$ \\
\hline Chipped Hoe & 5 & 0.72 & 0 & - & 0 & - & 0 & - & 0 & - & 0 & - \\
\hline Chipped Axe & 1 & 0.14 & 0 & - & 0 & - & 0 & - & 0 & - & 0 & - \\
\hline Chipped-St one Disk & 0 & - & 0 & - & 0 & - & 0 & - & 0 & - & 6 & 1.38 \\
\hline Ground-St one Disk & 0 & - & 0 & - & 0 & - & 0 & - & 2 & 1.65 & 5 & 1.15 \\
\hline Ground-Stone Celt & 2 & 0.29 & 1 & 0.18 & 0 & - & 0 & - & 0 & - & 1 & 0.23 \\
\hline Polished Cobble & 0 & - & 0 & - & 0 & - & 0 & - & 0 & - & 4 & 0.92 \\
\hline Pitted Cobble & 2 & 0.29 & 2 & 0.36 & 0 & - & 0 & - & 0 & - & 2 & 0.46 \\
\hline Utilized Cobble & 0 & - & 4 & 0.71 & 0 & - & 0 & - & 0 & - & 3 & 0.69 \\
\hline Chunkey Stone & 0 & - & 1 & 0.18 & 0 & - & 0 & - & 0 & - & 2 & 0.46 \\
\hline Mano & 0 & - & 1 & 0.18 & 0 & - & 0 & - & 1 & 0.83 & 0 & - \\
\hline Hammerstone/Anvil & 0 & - & 0 & - & 0 & - & 0 & - & 1 & 0.83 & 0 & - \\
\hline Mano/Hammerstone & 1 & 0.14 & 0 & - & 0 & - & 0 & - & 0 & - & 0 & - \\
\hline Anvi1/Hammerstone/Mano & 4 & 0.57 & 0 & - & 0 & - & 0 & - & 1 & 0.83 & 0 & - \\
\hline Ground Gorget & 2 & 0.29 & 0 & - & 0 & - & 0 & - & 0 & - & 0 & - \\
\hline Ground-St one Pendant & 2 & 0.29 & 0 & - & 0 & - & 0 & - & 0 & - & 0 & - \\
\hline Grinding Stone & 0 & - & 0 & - & 0 & - & 0 & - & 0 & - & 3 & 0.69 \\
\hline Ground-Stone Indet. & 0 & - & 0 & - & 0 & - & 0 & - & 0 & - & 10 & 2.29 \\
\hline Abrader & 1 & 0.14 & 0 & - & 0 & - & 0 & - & 0 & - & 0 & - \\
\hline Miscellaneous & 5 & 0.72 & 0 & - & 0 & - & 0 & - & 0 & - & 0 & - \\
\hline Total & 698 & 100.00 & 562 & 100.00 & 80 & 100.00 & 108 & 100.00 & 121 & 100.00 & 436 & 100.00 \\
\hline
\end{tabular}


Table 9.3

Distribution of utilized/retouched flakes by site.

\begin{tabular}{|c|c|c|c|c|c|c|c|c|c|c|c|c|c|}
\hline \multirow[b]{2}{*}{ Site } & \multicolumn{2}{|c|}{ Primary } & \multicolumn{2}{|c|}{ Secondary } & \multicolumn{2}{|c|}{$\begin{array}{l}\text { Flake } \\
\text { Interior }\end{array}$} & \multicolumn{2}{|c|}{ BTF } & \multicolumn{2}{|c|}{ Shatter } & \multicolumn{2}{|c|}{ Misc. } & \multirow[b]{2}{*}{ Total } \\
\hline & $\mathrm{n}$ & $\%$ & $\mathrm{n}$ & $\%$ & $\mathrm{n}$ & $\%$ & $\mathrm{n}$ & $\%$ & $\mathrm{n}$ & $\%$ & $\mathrm{n}$ & $\%$ & \\
\hline Wall & 2 & 0.62 & 56 & 17.39 & 98 & 30.43 & 5 & 1.55 & 0 & - & 0 & - & 161 \\
\hline Early Upper Saratown & 0 & - & 2 & 18.18 & 9 & 81.82 & 0 & - & 0 & - & 0 & - & 11 \\
\hline Mitchum & 0 & - & 10 & 41.67 & 14 & 58.33 & 0 & - & 0 & - & 0 & - & 24 \\
\hline Upper Saratown & 0 & - & 4 & 28.57 & 10 & 71.43 & 0 & - & 0 & - & 0 & - & 14 \\
\hline Fredricks & 3 & 2.36 & 42 & 33.07 & 71 & 55.90 & 5 & 3.94 & 3 & 2.36 & 3 & 2.36 & 127 \\
\hline
\end{tabular}

Of the 2,231 flakes analyzed, 5.7\% $(n=127)$ showed evidence of use along at least one edge. The edge damage on the flakes indicated that most of these flakes had been used for scraping or cutting. Secondary decortication (33\%) and interior flakes ( $56 \%$ ) were most often selected for retouch or use (Table 9.3).

A large number of other flakes also exhibit retouch along at least one edge. Because retouch is confined to the flake edges, flake morphology is evident on these tools. Similarly, a few scrapers, characterized by formalized retouch, are also present. This retouch usually resulted in shaping the tool so that it has a symmetrical convex working edge. These tools apparently were used in scraping and cutting.

The few drills and perforators present in the assemblage were constructed on flakes and are not highly formalized tools (Figure 9.1). One exception is a drill that appears to have been recycled from a small triangular projectile point. Overall, these tools appear to have been

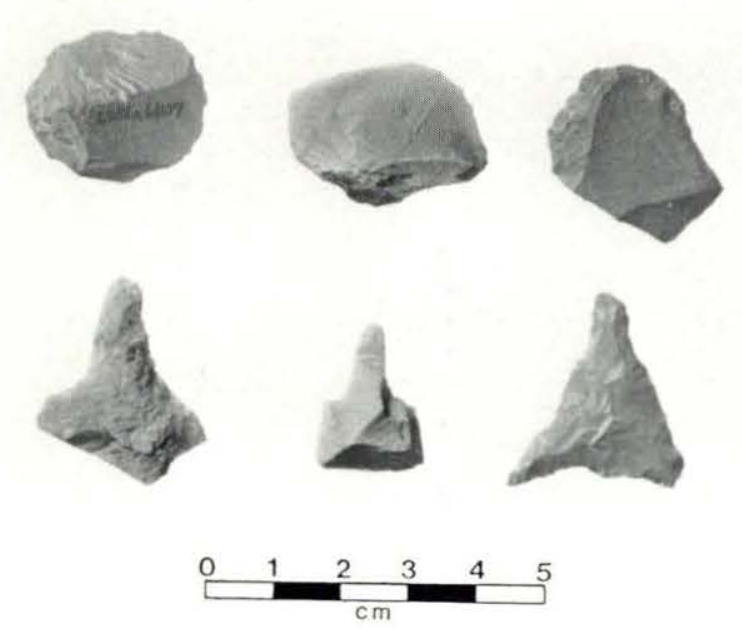

Figure 9.1

Flake tools from the Fredricks site. expediently manufactured on flakes. Flaking is primarily unifacial, and restricted largely to the bit end. Some of these tools also exhibit flaking along the base, presumably to shape the tool for hafting. These tools were probably used to punch or bore holes.

Other chipped-stone tools include several large choppers (Figure 9.2a-b) ranging from 6 to $22 \mathrm{~cm}$ in length. These tools were made from local, platy phyllite that had been roughly chipped along the edge to only slightly alter the natural shape of the stone. The shape and edge characteristics of these tools suggest that they were used in heavy duty tasks such as digging or chopping.

Seven chipped-stone disks (Figure 9.2c-d) were recovered from five features. The raw materials from which they were manufactured include fine-grained granite, argillite, soapstone, and schist. They range in diameter from
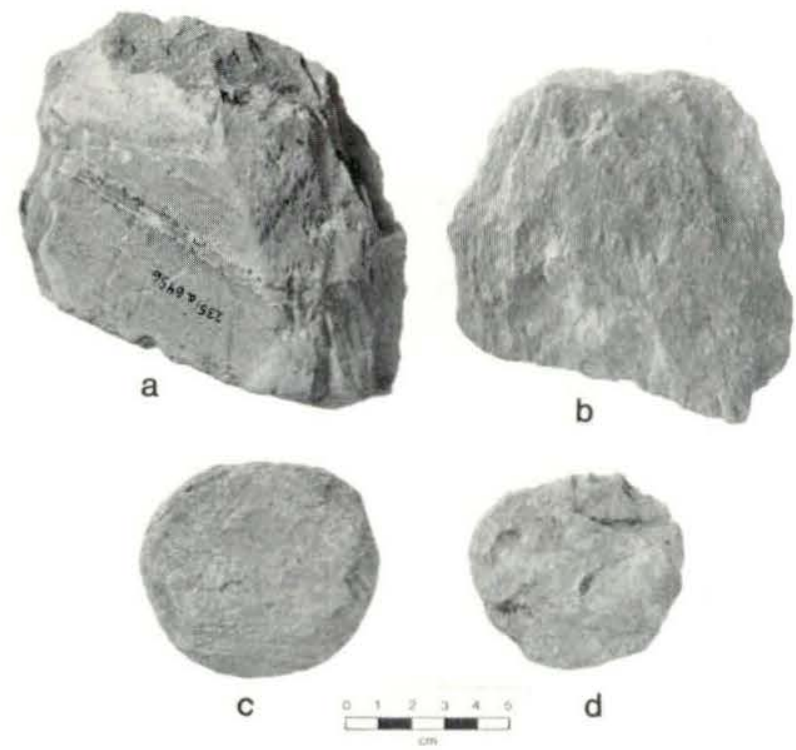

Figure 9.2

Choppers $(a-b)$ and chipped-stone disks $(c-d)$ from the Fredricks site. 
about 40 to $100 \mathrm{~mm}$, are made on non-cryptocrystalline stone, and are roughly chipped into a circular shape. Several other broken and unidentified ground-stone specimens and polished cobbles are also present. All were recovered from feature context, and the function(s) of these artifacts is unknown.

Projectile Points. The majority of the chipped-stone tools recovered are small triangular projectile points and point fragments. Of the 90 points recovered, only 30 were found in feature fill (Table 9.4).

The small triangular points from the Fredricks site range from 13 to $33 \mathrm{~mm}$ in length. The variation in size and blade form is illustrated in Figure 9.3. The presence of cortex, patination, or a curved surface indicate that most of these points were constructed by bifacially retouching small to medium-sized flakes as opposed to the use of a biface preform. Many of the smal!, equilateral triangular points were made on small flakes that were altered only to shape the lateral margins. Frequently, the flake platform formed one of the basal corners (or tangs), making it easier to shape the thinner part of the flake into a point.

Woodland and Archaic projectile points were recovered from the plowzone (Figure 9.4). With the exception of a possible Badin point recovered from Feature 28 (Zone 1), there were no Woodland or Archaic artifacts in any features or burials.

Ground-Stone Tools. Several types of ground-stone tools are also present in the assemblage. Included are several ground-stone
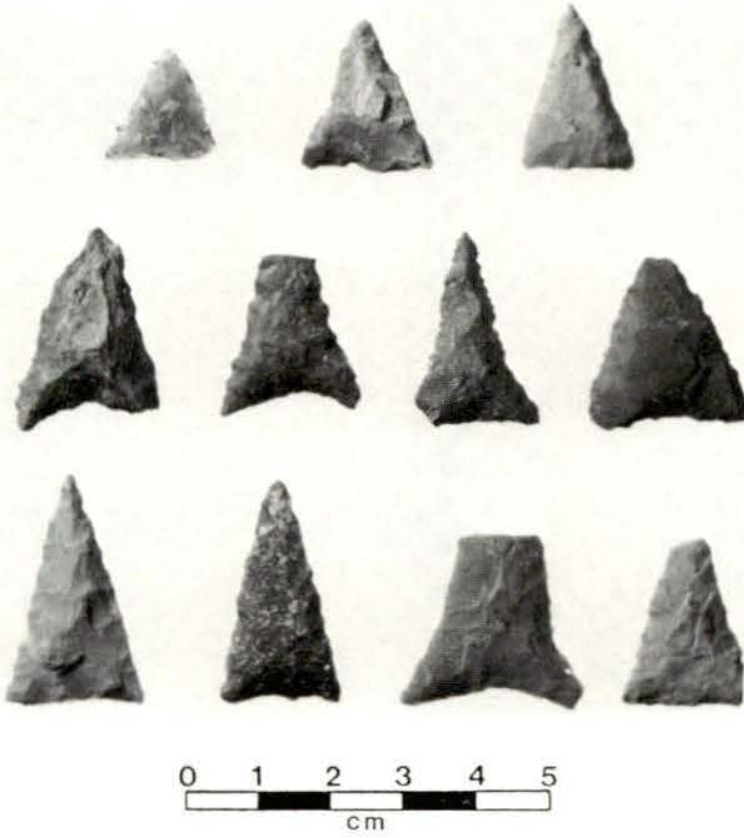

Figure 9.3

Triangular projectile points from the Fredricks site.

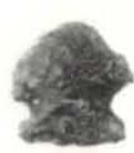

a

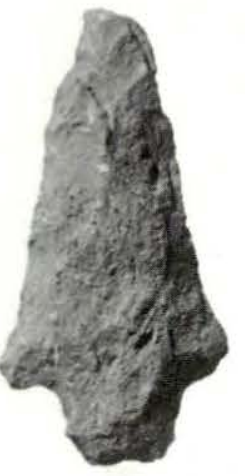

e

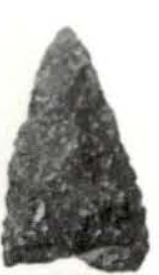

b

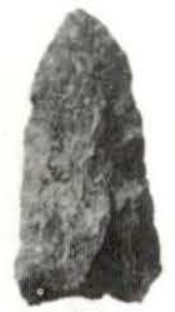

C
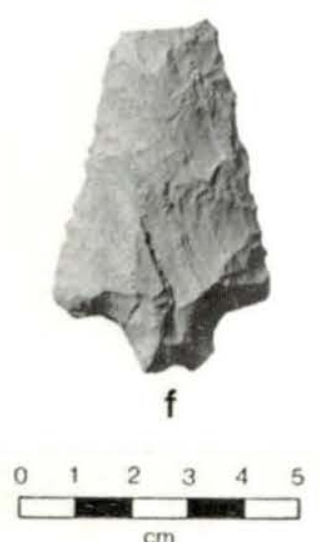

Figure 9.4

Archaic and Woodland projectile points from the Fredricks site. disks similar in size and shape to the chipped disks described above. Several other broken unidentified ground-stone specimens are also present. Identifiable raw materials from which these artifacts were made include fine-grained granite, schist, igneous, felsic tuff, and some type of metavolcanic rock. Four broken polished cobbles are present from feature contexts. Two chunkey stones--one complete and one broken-were also recovered from feature context (Figure 9.5a-b). The complete specimen, of fine-grained

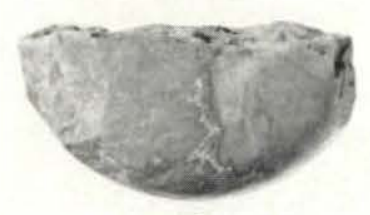

a

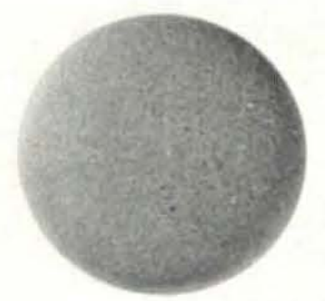

b
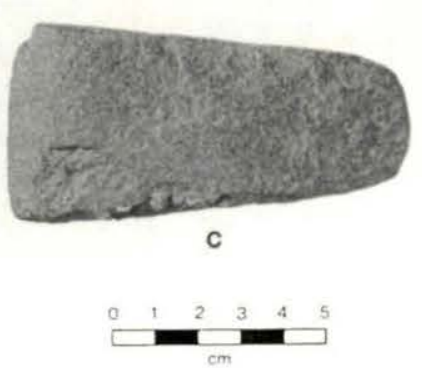

Figure 9.5

Ground-stone artifacts from the Fredricks site. 
Table 9.4

Fredricks site lithic artifact distribution by context.

\begin{tabular}{|c|c|c|c|c|c|c|c|c|c|c|c|c|c|c|c|c|c|c|}
\hline Type & Fea, 1 & $\begin{array}{c}\text { Fea. } 2 / \\
\text { Bu. } 4\end{array}$ & $\begin{array}{c}\text { Fea. 3/ } \\
\text { Bu. } 5\end{array}$ & $\begin{array}{c}\text { Fea. } 4 / \\
\text { Bu. } 6\end{array}$ & $\begin{array}{l}\text { Fea. } 5 / \\
\text { Bu. } 7\end{array}$ & $\begin{array}{c}\text { Fea.6/ } \\
\text { Bu. } 8\end{array}$ & $\begin{array}{c}\text { Fea.7/ } \\
\text { Bu.9 }\end{array}$ & Fea. 8 & Fea. 9 & Fea. 10 & Fea.11 & Fea. 12 & Fea.13 & $\begin{array}{c}\text { Fea. } 14 / \\
\text { Bu. } 11\end{array}$ & Fea. 15 & Fea. 17 & Fea. 18 & Fea.19 \\
\hline PPt. (Archaic) & - & - & - & - & - & - & - & - & - & - & - & - & - & - & - & - & - & - \\
\hline PPt. (Woodland) & - & - & - & - & - & - & - & - & - & - & - & - & - & - & - & - & - & - \\
\hline PPt. (Sm. Triangular) & r) - & 2 & 2 & 1 & - & - & 1 & - & 6 & - & - & - & 2 & 2 & 1 & - & - & 2 \\
\hline PPt. (Frags.) & 1 & - & - & 1 & 1 & 1 & 1 & - & 4 & - & - & - & - & - & - & - & - & 2 \\
\hline Biface & 1 & - & - & 3 & - & - & 1 & - & 2 & - & - & - & - & - & - & - & - & - \\
\hline Preform & - & - & - & - & - & - & - & - & - & - & - & - & - & - & - & - & - & 1 \\
\hline Drill & - & - & - & - & - & - & - & - & - & - & - & - & - & - & - & - & - & - \\
\hline Perforator & - & - & - & - & - & - & - & - & 1 & - & - & - & - & - & - & - & - & - \\
\hline Graver & - & - & - & - & - & - & - & - & - & - & - & - & - & - & - & - & - & - \\
\hline Scraper & - & - & - & - & - & - & - & - & - & - & - & - & - & - & - & - & - & - \\
\hline Ut1./Ret. Flake & - & 2 & 1 & 1 & - & 1 & 3 & - & 4 & - & - & - & - & - & - & 1 & - & 1 \\
\hline Pièce Esquillée & - & - & - & - & - & - & 2 & - & - & - & - & - & - & - & - & - & - & - \\
\hline Core & - & - & - & - & - & - & - & - & 1 & 1 & - & - & - & - & - & 1 & - & - \\
\hline Hammerst one & - & - & - & - & - & - & - & - & 2 & - & - & - & - & - & - & 2 & - & - \\
\hline Chopper & - & - & - & 1 & - & 1 & - & - & 2 & 1 & - & 1 & 1 & - & - & - & - & 1 \\
\hline Chipped-Stone Disk & - & - & - & - & - & - & - & - & - & - & - & - & - & - & - & 2 & - & 1 \\
\hline Ground-Stone Disk & - & - & - & - & - & - & - & - & - & - & - & - & - & - & - & - & - & 3 \\
\hline Ground-Stone Celt & - & - & - & - & - & - & - & - & - & - & - & - & - & - & - & - & - & - \\
\hline Polished Cobble & - & - & - & - & - & - & - & - & - & - & - & - & - & - & - & - & - & - \\
\hline Pitted Cobble & - & - & - & - & - & - & - & - & - & - & - & - & - & - & - & - & - & - \\
\hline Utilized Cobble & - & - & - & - & - & - & - & - & - & - & - & 1 & - & - & - & - & - & - \\
\hline Chunkey Stone & - & - & - & - & - & - & - & - & - & - & - & - & - & - & - & 2 & - & - \\
\hline Grinding Stone & - & - & - & - & - & - & - & - & 1 & - & - & - & 1 & - & - & - & 1 & - \\
\hline Ground-St one Indet. & - - & - & - & - & - & - & - & - & - & - & - & - & - & - & - & - & - & 1 \\
\hline $\begin{array}{l}\text { Flake } \\
\text { Primary }\end{array}$ & - & - & 1 & - & - & - & - & 1 & - & - & - & & & & & - & - & - \\
\hline Secondary & 1 & 2 & 5 & 10 & 1 & 2 & 11 & 3 & 18 & - & $\overline{1}$ & $\overline{-}$ & 1 & $\begin{array}{l}4 \\
4\end{array}$ & $\overline{-}$ & $\overline{6}$ & $\overline{2}$ & $\overline{3}$ \\
\hline Interior & 10 & 22 & 32 & 65 & 8 & 15 & 51 & 9 & 52 & 10 & 3 & 3 & 16 & 99 & 4 & 20 & $i$ & 56 \\
\hline BTF & 3 & 1 & 6 & 10 & - & - & - & 1 & 20 & 1 & - & - & 1 & 2 & 1 & - & - & 1 \\
\hline Shatter & 2 & 18 & 17 & 20 & 1 & 6 & 31 & 3 & 30 & 1 & - & - & 5 & 1 & - & - & - & - \\
\hline Misc. & - & - & - & - & - & - & - & - & - & - & - & - & - & - & - & - & - & - \\
\hline Total & 18 & 47 & 64 & 112 & 11 & 26 & 101 & 17 & 143 & 14 & 4 & 5 & 27 & 112 & 6 & 34 & 4 & 74 \\
\hline
\end{tabular}


Table 9.4 Continued.

\begin{tabular}{|c|c|c|c|c|c|c|c|c|c|c|c|c|c|c|c|c|c|}
\hline Type & Fea.20 & Fea.23 & Fea.24 & Fea. 25 & $\begin{array}{c}\text { Fea.26/ } \\
\text { Bu.13 }\end{array}$ & $\begin{array}{c}\text { Fea.27/ } \\
\text { Bu.10 }\end{array}$ & Fea. 28 & Fea. 29 & Fea. 30 & Fea. 33 & Fea. 35 & Fea. 38 & Fea. 39 & Fea. 41 & $\begin{array}{l}\text { Misc. } \\
\text { Fea. }\end{array}$ & $\begin{array}{l}\quad \text { PZ } \\
\text { Misc. }\end{array}$ & Total \\
\hline PPt. (Archaic) & - & - & - & - & - & - & - & - & - & - & - & - & - & - & - & 3 & 3 \\
\hline PPt. (Woodland) & - & - & - & - & - & - & 1 & - & - & - & - & - & - & - & - & 1 & 2 \\
\hline PPt. (Sm. Triangular) & ) 1 & 1 & - & - & - & 1 & 2 & 1 & 1 & 1 & - & - & - & 2 & 1 & 60 & 90 \\
\hline PPt. (Frags.) & - & 1 & - & - & 1 & 1 & 2 & - & 1 & - & - & - & - & 2 & 2 & 39 & 60 \\
\hline Biface & - & - & - & - & - & - & - & - & - & 1 & - & - & - & - & - & 32 & 40 \\
\hline Preform & - & - & - & - & - & 1 & - & 1 & 1 & - & - & - & - & - & - & 8 & 12 \\
\hline Drill & - & - & - & - & - & - & - & - & - & - & - & - & - & - & - & 4 & 4 \\
\hline Perforator & - & - & - & - & - & - & - & - & - & - & - & - & - & - & - & 4 & 5 \\
\hline Graver & - & - & - & - & - & - & - & - & - & - & - & - & - & - & - & 4 & 4 \\
\hline Scraper & - & - & - & - & - & - & - & - & - & - & - & - & - & - & - & 6 & 6 \\
\hline Ut 1./Ret. Flake & - & 1 & - & - & - & - & 1 & - & 1 & 2 & - & - & - & 2 & - & 106 & 127 \\
\hline Pièce Esquillée & - & - & - & - & - & - & - & - & - & - & - & - & - & - & - & - & 2 \\
\hline Core & - & - & - & - & - & - & - & - & - & - & - & - & 1 & - & - & 16 & 20 \\
\hline Hammerstone & - & - & - & - & - & - & - & - & - & - & - & - & - & 1 & - & 2 & 7 \\
\hline Chopper & - & - & - & - & - & - & - & - & 1 & 1 & - & - & - & 2 & - & 6 & 18 \\
\hline Chipped-Stone Disk & - & - & - & - & - & - & - & 1 & - & - & - & - & - & 2 & - & - & 6 \\
\hline Ground-Stone Disk & - & - & - & - & - & - & - & - & - & - & - & - & - & 2 & - & - & 5 \\
\hline Ground-Stone Celt & - & - & - & - & - & 1 & - & - & - & - & - & - & - & - & - & - & 1 \\
\hline Pollshed Cobble & - & - & - & - & - & - & - & - & - & 1 & - & - & - & 3 & - & - & 4 \\
\hline Pitted Cobble & - & - & - & - & - & - & - & - & - & - & - & - & - & - & - & 2 & 2 \\
\hline Utilized Cobble & - & - & - & - & - & - & - & - & - & - & - & - & - & - & - & 2 & 3 \\
\hline Chunkey Stone & - & - & - & - & - & - & - & - & - & - & - & - & - & - & - & - & 2 \\
\hline Grinding Stone & - & - & - & - & - & - & - & - & - & - & - & - & - & - & - & - & 3 \\
\hline $\begin{array}{l}\text { Ground-Stone Indet. } \\
\text { Flake }\end{array}$ & 5 & - & - & - & - & - & 1 & 1 & - & - & - & - & - & 2 & - & - & 10 \\
\hline Primary & - & 1 & - & - & 4 & 1 & 1 & - & 7 & - & - & - & 1 & - & - & 11 & 35 \\
\hline Secondary & 2 & 3 & - & - & 4 & 5 & 21 & 8 & 17 & 2 & - & - & - & 1 & 5 & 130 & 267 \\
\hline Interior & 15 & 36 & 1 & 1 & 24 & 57 & 101 & 73 & 68 & 68 & 1 & 1 & 18 & 22 & 35 & 334 & 1331 \\
\hline BTF & - & 2 & - & 1 & - & 5 & 10 & - & - & - & - & - & - & - & - & 32 & 97 \\
\hline Shatter & - & - & - & - & - & 1 & 6 & 14 & 6 & 9 & - & - & 3 & 2 & 13 & 201 & 390 \\
\hline Misc. & - & - & - & - & - & - & - & - & - & - & - & - & - & - & - & 2 & 2 \\
\hline Total & 23 & 52 & 1 & 2 & 33 & 73 & 146 & 99 & 103 & 85 & 1 & 1 & 23 & 43 & 56 & 1005 & 2558 \\
\hline
\end{tabular}


granite, is well made and very smooth. It is approximately $65 \mathrm{~mm}$ in diameter and plano-convex in cross-section. The other specimen is a highly polished quartz cobble. Although broken, it appears to have been slightly larger than the complete specimen and also plano-convex in cross-section.

A single ground stone celt (Figure 9.5c), $95 \mathrm{~mm}$ in length, was recovered from Burial 10. Made of fine-grained granite, the celt has a bi-convex working edge that tapers slightly to a rounded distal end and which gives it a wedge-shaped appearance. This tool was probably used for chopping.

Other Stone Tools. The Fredricks site lithic assemblage also contains hammerstones, chunks of raw material, and cores used in the production of other stone tools. The hammerstones, represented by small quartz river cobbles, exhibit different amounts of battering along their edges.

Three of the features (Features 9, 13, and 18) contained large rocks that had been altered by chipping or grinding. Feature 9 contained a number of large rocks that were fire-cracked or have burned exterior surfaces (Petherick, this report). Two large fragments that were separated in the bottom of Feature 9 were refitted. When the two fragments were joined, worn and smoothed surfaces could be traced on both the upper and lower surfaces. The smoothing on one surface appears to be the result of use as a grinding stone, whereas the polish on the other surface may have been formed by the movement of the stone against the ground or other surface during its use as a grinding stone.

Feature 13 was a circular pit more than two feet deep (Petherick, this report). Two of the four large stones found in the bottom of Feature 13 had been altered. One of the rocks had been shaped by rough chipping to a point on one end. The other altered rock is large and flat (ca. $10-12 \mathrm{~cm}$ thick and $30 \mathrm{~cm}$ in diameter) and had been chipped into a circular form. None of the rocks from this feature appear to have been thermally altered.

Feature 18 was a circular, shallow pit that contained a heavy concentration of simple-stamped and check-stamped sherds. In addition, it contained a number of stone fragments from a partially reconstructible grinding stone. One of the surfaces of this tool appeared to have been flattened and smoothed from use.

Assemblage Characteristics. The small-tool kit includes projectile points, drills, perforators, retouched and utilized flakes, and scrapers. Retouched and utilized flakes, and possibly the few scrapers, are expedient tools manufactured on flakes. These items represent the generalized cutting and scraping tools in the assemblage. Some functional specialization is present in the small-tool kit in the form of projectile points, drills, and perforators, although these also were manufactured from flakes.

The large-tool kit represents tools made from cobbles and larger masses of stone raw material. These include choppers, hammerstones, and a variety of ground-stone and chipped-stone specimens. While the hammerstones were used in tool production, the majority of the large chipped-stone implements appear to have been used in chopping or digging. Three of the largest stones show evidence of use in food processing as grinding stones.

A number of ground-stone and a few polished-stone artifacts are also present in the assemblage. However most of these are fragments, and their exact function(s) are unknown. Several whole disks, both ground and chipped, are also present. Finally, two chunkey stones are included in the assemblage.

In sum, the Fredricks site assemblage is predominantly composed of small chipped-stone tools. The majority are cutting, scraping, or chopping tools made of local raw materials and are not highly formalized tools constructed for a long use life. The remaining portion of the assemblage includes ground-stone items, many of which are of indeterminate function.

As noted above, the majority ( $68 \%$ ) of the stone tools from the Fredricks site were recovered from the plowzone. The distribution of chipped-stone and ground-stone artifacts in burial, feature, and plowzone context is summarized in Table 9.4. It should be noted that while this table presents the totals for all stone tools recovered from feature/burial contexts, artifacts from the plowzone excavated in 1985 are not reflected in the tool or debitage categories. An examination of Table 9.4 reveals that the majority of the small chipped-stone tool assemblage is present within plowzone contexts. In contrast, larger stone tools (e.g., chipped-stone disks, ground-stone tools, and chunkey stones) were almost exclusively recovered from feature contexts. Of the 30 points and point fragments, nine were from burial contexts whereas the remaining specimens were from storage pits and shallow basins. The largest number of lithic artifacts, mainly debitage, points and point fragments, were contained within Feature 28. This feature is located outside the village palisade and is believed to date to the initial period of Occaneechi occupation.

\section{Wall Site}

Lithic artifacts from 25 10x10-ft excavation units, three burials, and one feature from the 1983 and 1984 excavations at the Wall site were analyzed. These consisted of tools, tool fragments, and debitage. 
Debitage. All stages of tool manufacture are represented in the debitage from the Wall site. The distribution of these artifacts by reduction class is shown in Table 9.1. Again, the largest reduction class was interior flakes.

Chipped-Stone Tools. Of the 3,008 flakes analyzed, $161(5.3 \%)$ are retouched or utilized. The distribution of these flakes by reduction class is summarized in Table 9.3. Secondary $(35 \%)$ and interior ( $61 \%$ ) flakes account for $96 \%$ of all retouched or utilized flakes.

Aside from utilized and retouched flakes, most of the chipped-stone tools from the Wall site are small triangular projectile points (Figure 9.6). The assemblage also contains drills, perforators, gravers, end scrapers, and side scrapers. Again, the scrapers constructed on flakes and retouched flakes are similar in morphology to those of the other sites. Drills, perforators, and gravers were also constructed on flakes with some shaping of the drill bases, presumably to facilitate hafting (Figure 9.7).

Only a few specimens are present in the large-tool assemblage. The majority of these are cores and hammerstones. Most of the cores are randomly flaked, and the hammerstones are al1 from quartz cobbles. The choppers were roughly chipped from large masses of noncryptocrystalline stone similar to those described from the Fredricks site (see Figure 9.2). One of the more formalized specimens is wedge shaped, bifacially chipped, and has a bi-convex working edge. The distribution of stone tools by category is summarized in Table 9.2. The majority of the chipped-stone artifacts was found in the midden, and very little

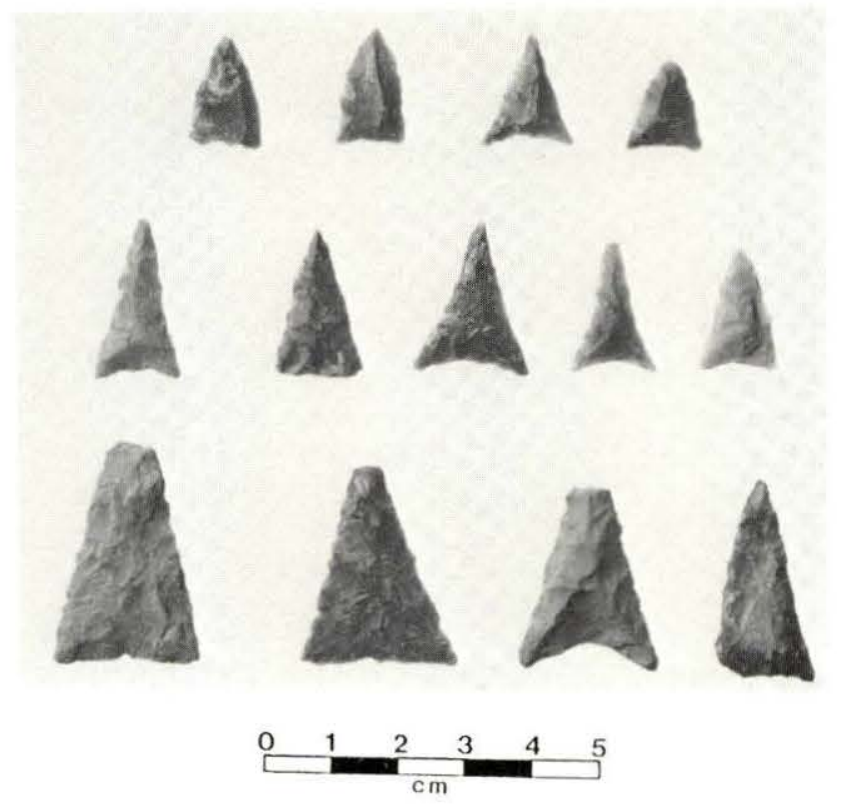

Figure 9.6

Triangular projectile points from the Wall site.

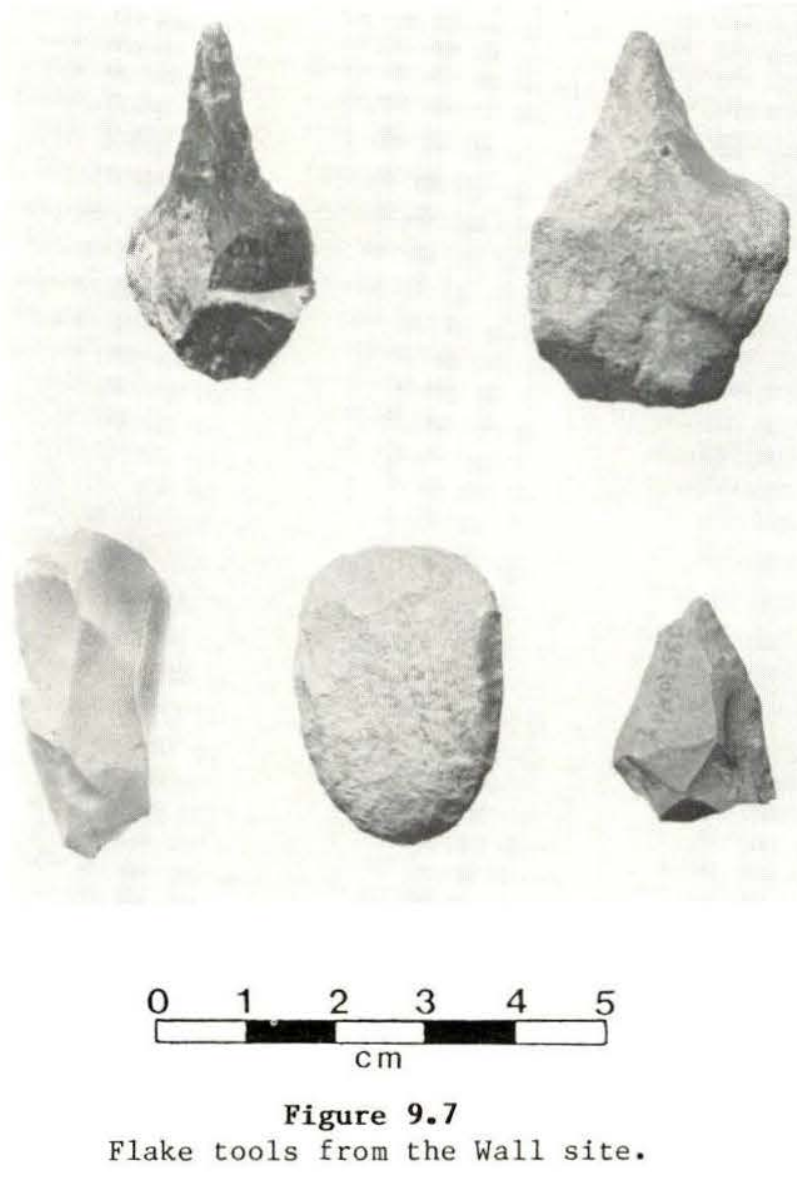

was recovered from feature or burial fill. of the features and burials only Feature 3 and Burial 1 contained chipped-stone artifacts.

Ground-Stone Tools. The sample of groundstone tools from the Wall site is very small. They include a mano manufactured from a cobble, a broken chunkey stone, and a portion of the bit end of a ground-stone celt.

As with the Fredricks site, Woodland and Archaic projectile points and other artifacts were found in the plowzone (Figure 9.8). In addition, one fluted Paleoindian point was recovered from the midden at the Wall site (Figure 9.8). This specimen, probably reworked by occupants of the Wall site village, shows retouching along most of the lateral margins. The steep lateral retouching created a fresh unpatinated surface which contrasts markedly with the patination rind covering the remainder of the point. This biface was made from a high quality, dark grayish-green welded tuff.

\section{Mitchum Site}

Limited test excavations were conducted at the Mitchum site during 1983. The lithic material analyzed from this Early Contact period site was recovered from six 10x10-ft excavation units, one burial, and seven features.

Debitage. As with the other sites, interior and secondary flakes predominate the 

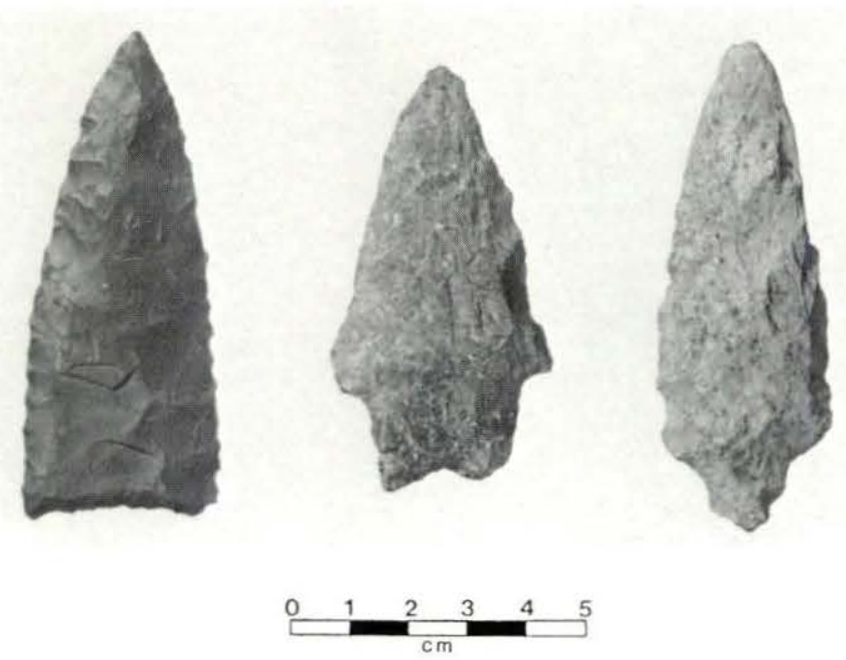

Figure 9.8

Paleoindian (a) and Archaic (b-c) projectile points from the Wall site.

debitage distribution (Table 9.1). Most of the debitage was contained in the plowzone.

Chipped-Stone Tools. A total of 427 flakes were recovered, of which $24(5.6 \%)$ are either utilized or retouched (Table 9.3). All of the retouched flakes are either secondary or interior flakes.

The categories and distribution of chipped-stone tools from the Mitchum site are listed in Table 9.2. The majority of the chipped-stone artifacts (other than utilized and retouched flakes) are small triangular projectile points (Figure 9.9). The chipped-stone assemblage also contains drills and scrapers (Figure 9.10). The drills from the Mitchum site are we11-made tools constructed on flakes. The single scraper exhibits consistent and patterned retouch, whereas the retouched flakes are similar in morphology to those from the other sites.

The remainder of the chipped-stone tool assemblage is dominated by cores and unidentified projectile point fragments (Table 9.2). No ground-stone artifacts were present in the sample analyzed.

\section{Early Upper Saratown}

Lithic artifacts from four refuse pits were analyzed from Early Upper Saratown. These features were arbitrarily chosen based on size and lithic artifact content. A total of 676 items was analyzed, consisting of tools, tool fragments, and debitage (Table 9.2). Reports on the excavations of Early Upper Saratown and Upper Saratown primarily deal with the feature types and ceramic assemblages and are presented elsewhere (see Ward 1980; J. Wilson 1983).

Debitage. The distribution of debitage by reduction class is shown is Table 9.1. A total

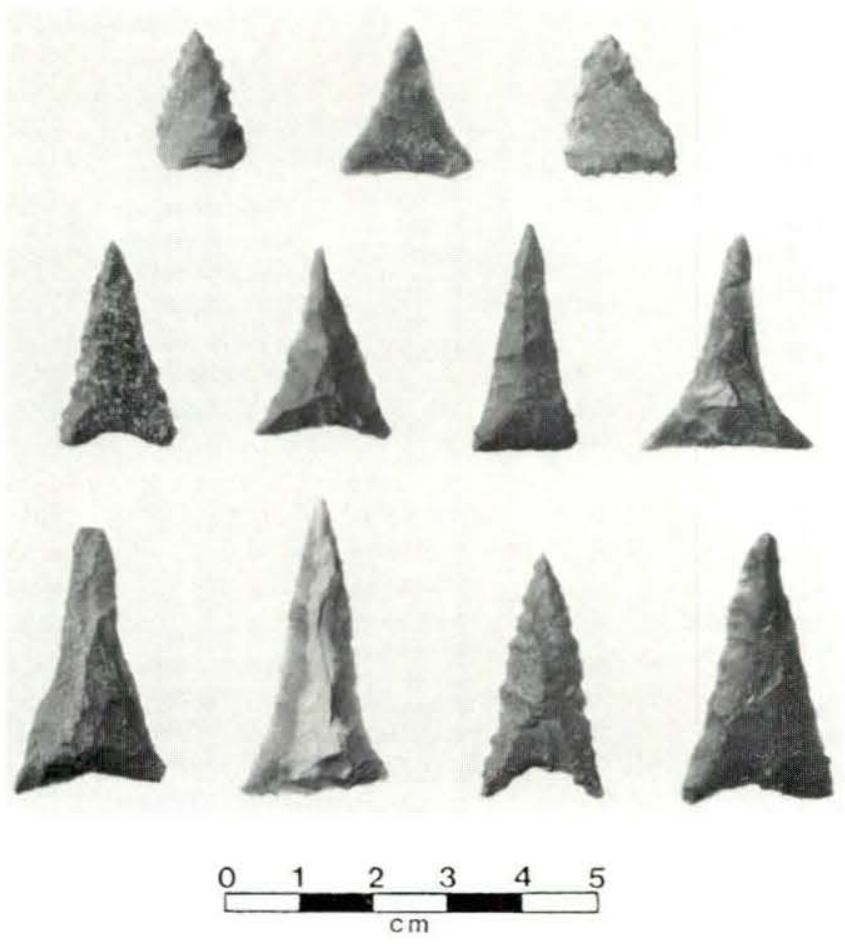

Figure 9.9

Triangular projectile points from the Mitchum site.
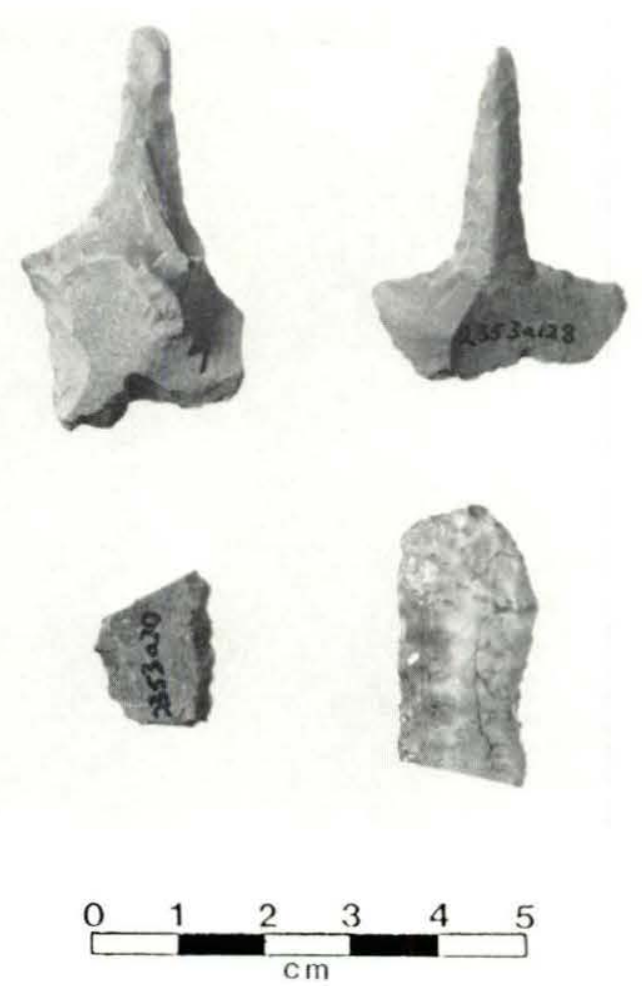

Figure 9.10

Flake tools from the Mitchum site. 
of 596 flakes were analyzed, most of which were interior flakes $(86.6 \%)$.

Chipped-Stone Tools. Only $11(1.8 \%)$ of the 596 flakes display evidence of use or retouch (Table 9.3). The majority of the chipped-stone tools are projectile points or bifaces (Figure 9.11). The distribution of stone tools by category is given in Table 9.2. Included in the projectile point category are one Kirk Corner Notched and one unidentified small stemmed point.

The small chipped-stone tool kit (Figure 9.12) is represented by retouched flakes, denticulates, perforators, and cores. Most of these are retouched flakes made on either interior or secondary decortication flakes. A single denticulate (Figure 9.12) was identified. This tool was made on a relatively large $(40 \mathrm{~mm})$ flake and exhibits retouch along one lateral edge, resulting in three to four projections. Two perforators, manufactured on flakes, are also present. Retouch is unifacial on one tool, bifacial on the other, and is primarily confined to the projection or working edge. Three small, randomly-flaked quartz cores are also included. Finally, three quartz cobble and one finegrained granite hammerstones are present in the assemblage.

Ground-Stone Tools. No ground-stone tools were present in the features analyzed.

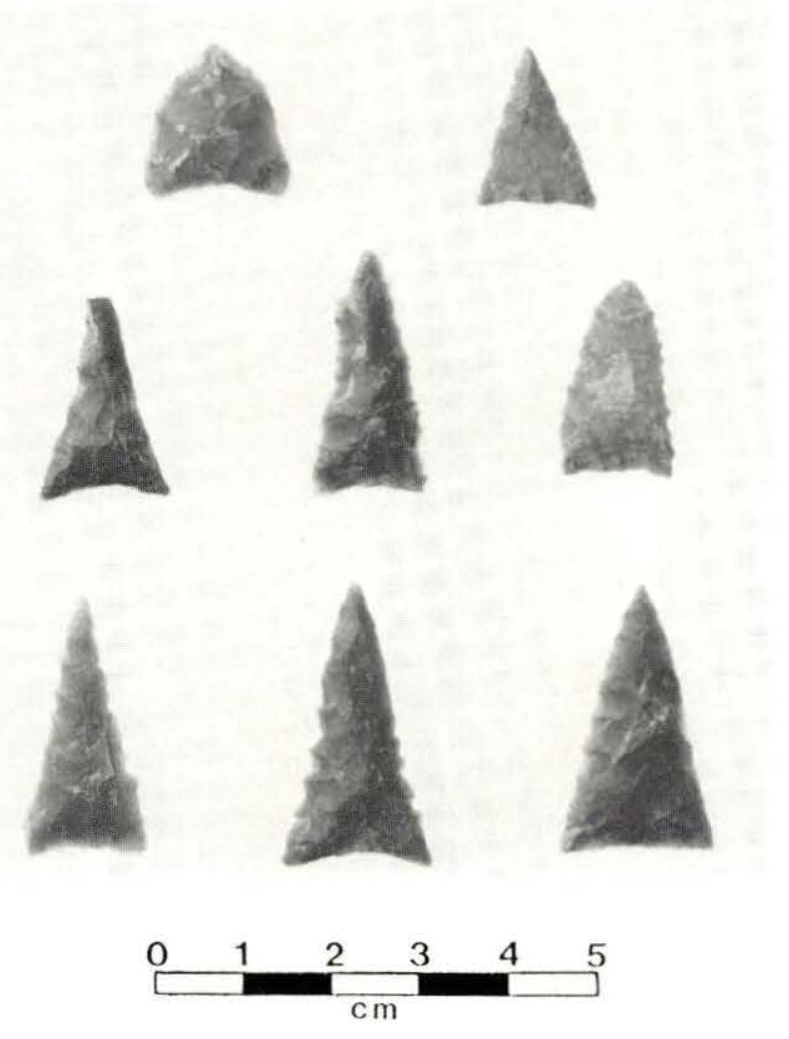

Figure 9.11

Triangular projectile points from Early Upper Saratown.

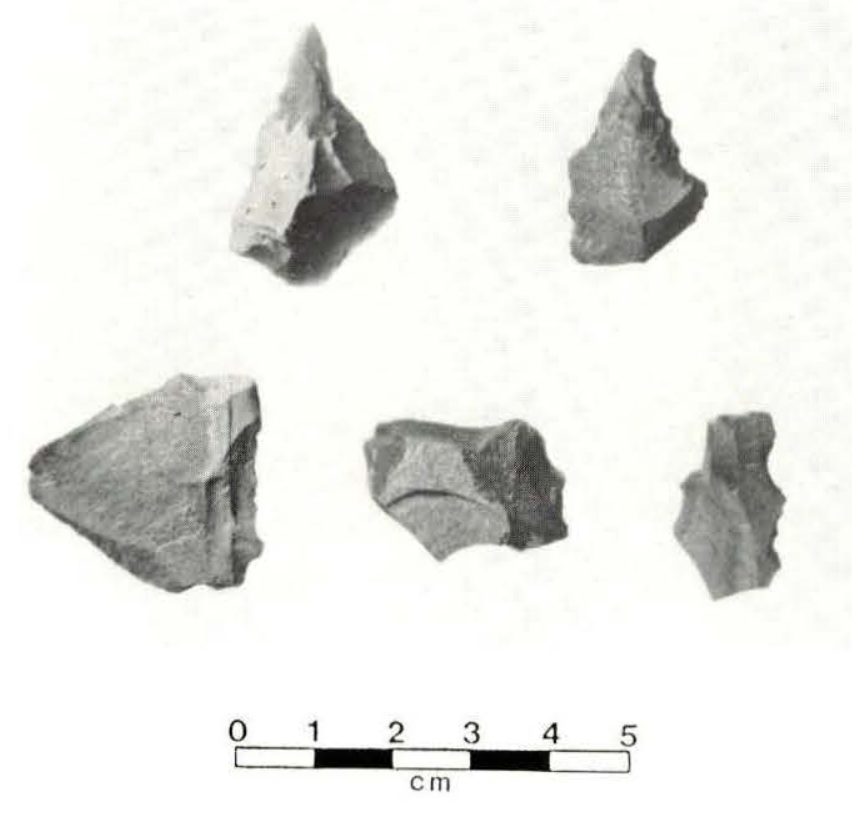

Figure 9.12

Flake tools from Early Upper Saratown.

\section{Upper Saratown}

Lithic items from 11 features, primarily associated with one structure, were analyzed. These included 686 artifacts representing tools, tool fragments, and debitage.

Debitage. Again, all stages of manufacture are present; however, interior flakes ( $85.3 \%)$ predominate (Table 9.1).

Chipped-Stone Tools. The majority of the chipped-stone tools are projectile points and bifaces (Figure 9.13). The distribution of al1 stone tool categories is given in Table 9.2.

The small chipped-stone tool (Figure 9.14) assemblage consists of retouched and utilized flakes, denticulates, drills, and perforators. of the 565 total flakes, only 14 (2.5\%) were identified as utilized or retouched (Table 9.3). As with other site assemblages, retouched and utilized flakes were predominantly made on interior flakes. Drills and perforators were also manufactured on flakes with the exception of one drill which was apparently recycled from a small triangular projectile point. The remaining specimens display some shaping of the base which suggests they were hafted. Four of the drills are broken and exhibit snapped projections near the base. The few denticulates present are also broken and made on flakes. Two of these display denticulate retouch on one lateral edge whereas the other displays retouch on portions of two sides and is "blade-like" in appearance.

Other Stone Tools. The large stone-tool assemblage is primarily composed of hammerstones. Single specimens from other classes 


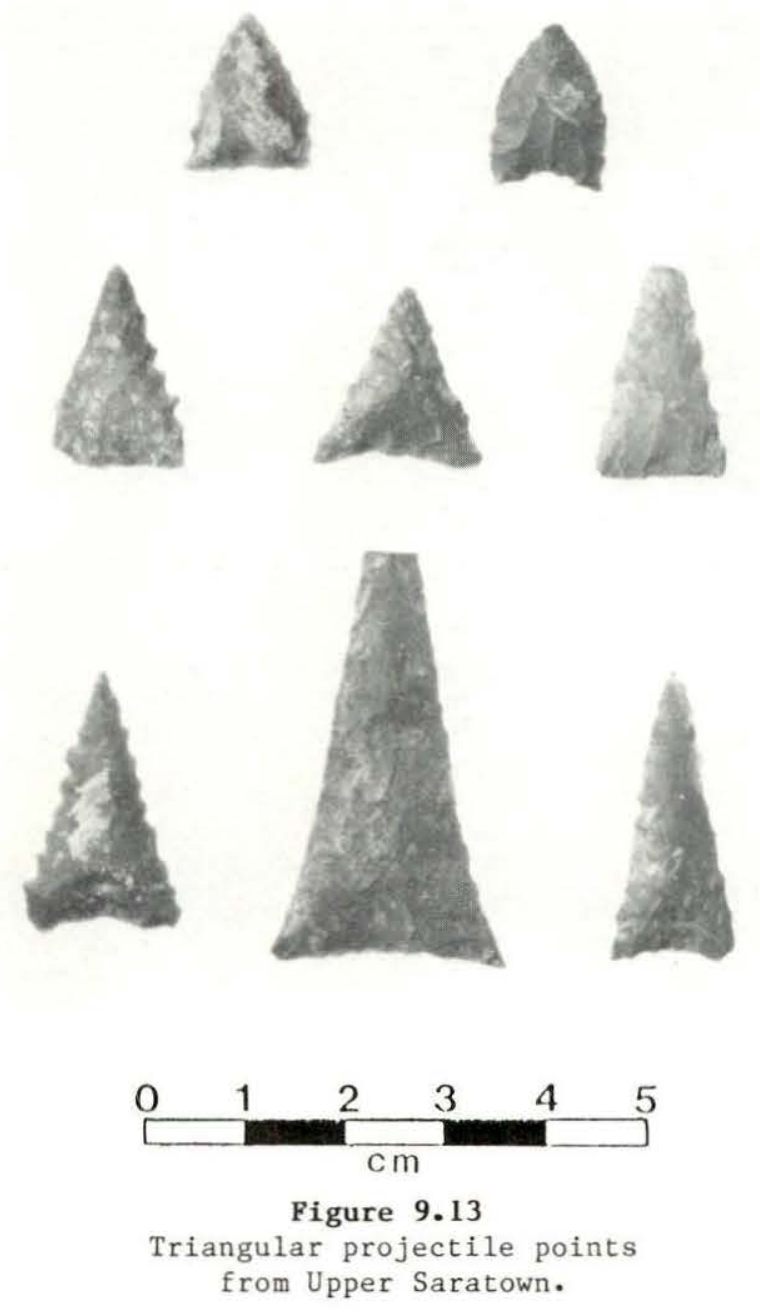

include a ground-stone disk, a perforated ground-stone disk, a hammerstone-anvil, and a mano.

The ground-stone disk is $55 \mathrm{~mm}$ in diameter and made of an unidentified, fine-grained, noncryptocrystalline material. A number of thin striations were lightly engraved across the face on one side of the disk. The perforated disk is smaller ( $30 \mathrm{~mm}$ in diameter) and made of soapstone. The hole, which lies in the approximate center of the specimen, appears to have been drilled from both sides. That is, the diameter of the hole is slightly larger $(9 \mathrm{~mm})$ on the surface and narrows toward the middle $(6-7 \mathrm{~mm})$. Approximately one dozen eroded notches appear to be evenly spaced along the edge of one face of the disk. The functions of these disks are unknown.

The anvil is a large piece of fine-grained granite, broken at two ends. Two of the surfaces are smoothed, with one surface exhibiting a small depressed area. The hammestone-anvil is a flattened quartz cobble which was battered around its edges from use as a percussor. In addition, both flattened surfaces display pitting which presumably resulted from use as an anvil. The mano, made of fine-grained granite,

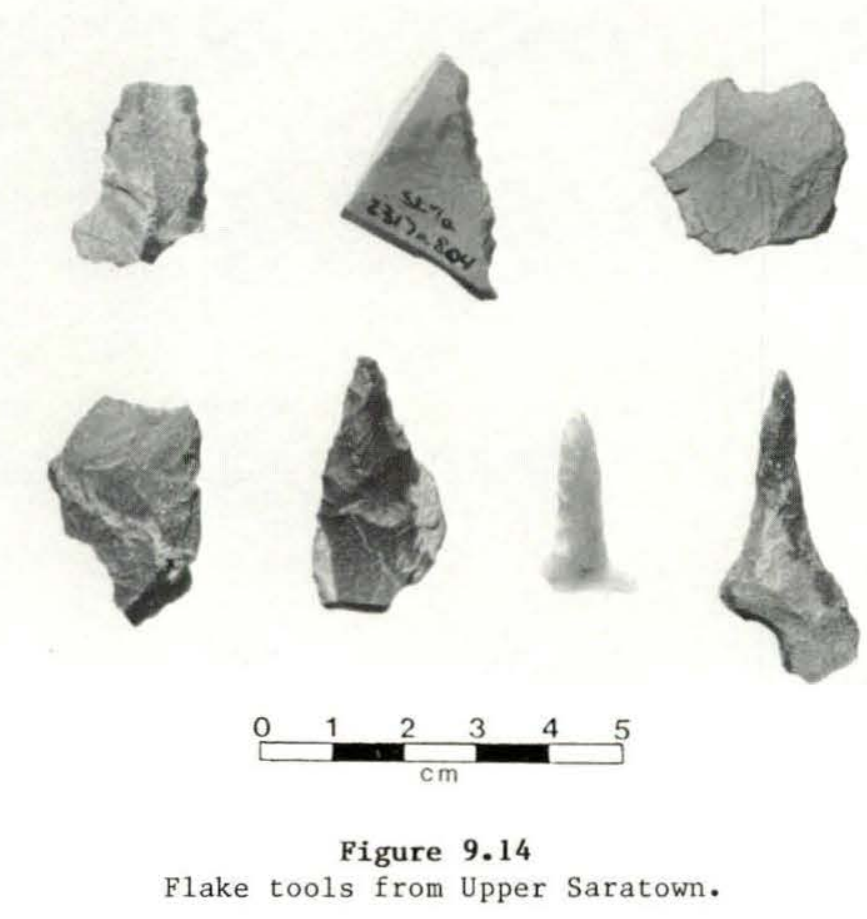

is wedge shaped and displays one flattened and smoothed surface worn from abrading. This tool may represent a food processing implement. The hammerstones are all made from quartz river cobbles with varying degrees of battering displayed along their edges. Finally, one very small unidentified ground-stone fragment is also present.

\section{COMPARISONS}

The first research question posed at the beginning of this section addressed the impact of European contact and the introduction of metal tools on the native lithic assemblage, and how such changes might be reflected in the archaeological record. A comparison of tool types from the sites is given in Table 9.2. Also included in this comparison is an assemblage from Forbush Creek, a Late Woodland period site located on the west bank of the Yadkin River, near Winston-Salem, North Carolina. The site was excavated by the Research Laboratories of Anthropology in 1972 (Coe 1972). The lithic artifacts from Features 43-126 were recently analyzed by McManus (1985) and are included here for comparison with the other analyzed assemblages.

European contact and the introduction of metal tools probably impacted the aboriginal lithic assemblage, as well as hunting and other subsistence activities. If so, a comparison of several sites from the Late Woodland through the Historic periods should reveal the disappearance of certain tools such as chipped hoes, chipped axes, and ground celts as they were replaced by metal axes and hoes. A general decrease in the 
overall number of items in the lithic assemblage might also be expected if rifles and other metal tools precipitated changes in hunting techniques and other subsistence practices.

Therefore, the impact of the introduction of metal tools on the composition of aboriginal lithic assemblages can be examined on two levels. The first is by a simple inspection of presence or absence of tool types among the sites. The second level is a functional comparison of tool types.

An intersite comparison of tool types indicates a total absence of ground-stone tools at Early Upper Saratown and Mitchum with a relative absence of ground-stone tools at Wall and Upper Saratown when compared to Forbush Creek and the Fredricks site. This absence of ground-stone tool types is most likely a function of the small sample sizes analyzed from these sites. Therefore, perhaps the best indicator of the influence of European metal tools can be seen from a comparison between the more complete assemblages analyzed from Forbush Creek and the Fredricks site.

A comparison of the small chipped-stone tool assemblage between the two sites reveals only one difference in tool types. Denticulates are present at Forbush Creek and are absent at the Fredricks site. If denticulates were primarily used to cut soft materials such as meat and vegetable matter, then their absence at Fredricks well indicate a replacement by metal knives. Another possible influence in the small chipped-stone tool assemblage might be reflected in the percentages of utilized/retouched flakes between the two sites. Utilized/retouched flakes comprise a significant amount of the stone tool assemblages at both sites; however, they comprise a greater percentage of the Forbush Creek (44\%) than the Fredricks site (29\%) assemblage. This decrease might represent an increased use of metal tools for cutting and scraping activities with a concomitant decrease in the use of stone tools. This interpretation, however, should be viewed with caution since no microscopic use-wear analysis was done on these specimens.

A similar comparison can be made in the large-tool kits. Formalized chipped hoes and axes are present at Forbush Creek but are absent at the Fredricks site. Perhaps metal hoes and axes replaced their chipped-stone counterparts. other presence/absence differences can be noted in the large-tool kit, primarily with groundstone tools (e.g., chunkey stones, ground stone disks, etc.). However, these do not have functional equivalents in metal tools.

If metal tools did replace certain stone tool types, this replacement may not have had a profound effect on the overall composition of the lithic assemblage. This possibility is suggested by a second level of comparison of functional categories of stone tool types from the Forbush Creek and Fredricks sites. Table 9.5 compares tool types and their inferred uses between the two sites. An examination of this table indicates that at least one tool type is present in each of the postulated activity sets. For example, although denticulates are not present in the butchering set at the Fredricks site, tools serving similar functions may be represented in the utilized/retouched flake category. That is, their function need not be exclusively replaced by a metal knife. Similarly, the chipped-stone hoe and axes present at Forbush Creek may not have been exclusively replaced by their metal counterparts at the Fredricks site, because both choppers and ground-stone celts are present and could represent the functional equivalent of these tool types.

If European introduced metal tools did not completely replace their stone tool counterparts, then the impact of metal tools on native lithic technologies might be viewed from a different perspective. Instead of tool types being replaced, the acquisition of metal tools may have had a greater impact on tool manufacturing techniques and technological organization. For example, the chipped-stone hoes from Forbush Creek are large and have a somewhat formalized triangular shape (McManus 1985:17). Although no similarly shaped tools are present at Fredricks, a functionally equivalent digging tool may be found in the slightly altered and crudely shaped choppers. Therefore, with the advent of metal hoes, the manufacture of more formalized chipped-stone hoes may have given way to the more expedient manufacture of crudely shaped choppers when metal tools were unavail-

Table 9.5

Comparison of Fredricks and Forbush Creek 11thic assemblages by inferred functional group.

\begin{tabular}{|c|c|c|}
\hline Activity & Forbush Creek & Fredricks \\
\hline Hunt1ng & Projectile Points & Projectile Points \\
\hline Butchering & $\begin{array}{l}\text { Ut1./Ret. Flakes } \\
\text { Scrapers } \\
\text { Dr11ls } \\
\text { Perforators } \\
\text { Dentlculates }\end{array}$ & $\begin{array}{l}\text { Ut1./Ret. Flakes } \\
\text { Scrapers } \\
\text { Dr1lis } \\
\text { Perforators }\end{array}$ \\
\hline Bone Working & $\begin{array}{l}\text { Pièce Esquiliée } \\
\text { Gravers }\end{array}$ & $\begin{array}{l}\text { P1èce Esquillée } \\
\text { Gravers }\end{array}$ \\
\hline Digging & $\begin{array}{l}\text { Choppers } \\
\text { Ch1pped Hoes }\end{array}$ & Choppers \\
\hline Woodworking & $\begin{array}{l}\text { Ground Celts } \\
\text { Chipped Axes }\end{array}$ & Ground Celts \\
\hline Flint Knapping & $\begin{array}{l}\text { Hammerstones } \\
\text { Cores } \\
\text { Abraders }\end{array}$ & $\begin{array}{l}\text { Hammerstones } \\
\text { Cores }\end{array}$ \\
\hline Food Processing & $\begin{array}{l}\text { Mano/Hammerstone } \\
\text { Anvil/Hammerstone/Hano } \\
\text { Pitted Cobble }\end{array}$ & $\begin{array}{l}\text { Grinding Stone } \\
\text { Pitted Cobble }\end{array}$ \\
\hline Miscellaneous & $\begin{array}{l}\text { Bead } \\
\text { Stone Pipe } \\
\text { Ground Gorget } \\
\text { Ground Pendant }\end{array}$ & $\begin{array}{l}\text { Ch1pped Disk } \\
\text { Ground Disk } \\
\text { Pol1shed Cobble } \\
\text { Ut1. Cobble } \\
\text { Chunkey Stone } \\
\text { Ground Stone (Indet.) }\end{array}$ \\
\hline
\end{tabular}


able. A similar situation might be reflected in the presence of chipped-stone axes at Forbush Creek and their absence at the Fredricks site. However, this interpretation is based solely on the presence of one chipped-stone axe at Forbush Creek. Also, ground-stone celts could have functioned as axes and are present (albeit in small numbers) at both sites.

Current data suggest that the impact of metal tools on native stone technologies resulted in a difference of degree rather than kind. Such an interpretation is reinforced by a comparison of the assemblages from all the sites. Ignoring the possible bias in the absence of ground-stone tools, the assemblages from all the sites can be characterized largely as small-tool kits (projectile points, drills, utilized/retouched flakes, and other small tools) made from flakes. The vast majority of these tools are not highly formalized and appear to have been expediently manufactured. Moreover, most of the small chipped-stone tool types are present at all six sites. In short, the organization of the lithic technologies are basically similar.

Based on these data, the introduction of guns and metal tools did not bring about major changes in the content or structure of aboriginal lithic assemblages characterized by small flake tools and projectile points. This is not to say, however, that European contact and the possession of trade goods did not change hunting techniques and tool use among Piedmont Indian groups.

There are several factors that could distort our view of the impact of European goods on the hunting methods of the Fredricks site inhabitants. There is considerable evidence elsewhere in the Southeast of changes in aboriginal hunting and food preparation methods caused by the introduction of European goods (e.g., Polhemus 1984). Perhaps in the Piedmont there was less time for the integration of European tools into the techno-economic system. Many European goods may have functioned in social and political rather than in utilitarian contexts where they would have replaced native tools. The character of the lithic assemblage at the Fredricks site also may reflect only a limited range of activities, with hunting and deerskin preparation being conducted away from the habitation site. As a consequence, European items would more likely be lost or discarded at other locations. This pattern also may be reflected in the distribution of bone elements at the Fredricks site (Holm, this report). In addition, there may be specialized activity areas yet to be discovered at the Fredricks site.

The second question addressed morphological changes in triangular projectile points from the Protohistoric to the Historic periods. The projectile point typology defined by Coe (1964) reflects a decrease in size of triangular pro- jectile points from the Middle Woodland to the Historic period. Preliminary analysis of the projectile points from the Wall, Mitchum, and Fredricks sites revealed a possible trend toward slightly larger points through the Historic period. As pointed out earlier, however, the majority of the projectile points from the Fredricks and Mitchum sites were recovered from the plowzone, and may represent earlier occupations of these sites. To examine the question of change in triangular point size through time and to address the problems of possible multiple components being within the plowzone, a comparative analysis of triangular points from feature/ burial context was undertaken. In addition to the Wall, Fredricks, and Mitchum sites, feature context materials from Upper Saratown, Early Upper Saratown, and the Forbush Creek sites were also included.

A large number of these points were made from flakes that were trimmed to a triangular shape by light retouch. The small, short retouch flake scars do not carry across the surface of the flake. The result is a small triangular point with the bifacial, and in many cases unifacial, retouch confined to the lateral and basal margins. Therefore, many of these points maintain both the curvature and striking platform of the flake from which they were constructed. It is clear in many cases that one of the major factors controlling point size was the size and thickness of the bulb of percussion of the flake.

Mean length, width, and thickness for triangular points is presented in Table 9.6. The standard deviation for each mean is also included. The greatest difference in mean length and width is between the Forbush Creek site and both Wall and Upper Saratown. Moreover, a cumulative frequency graph (Figure 9.15) indicates that while there is overlap in the variation of projectile point length among the six sites, the Forbush Creek assemblage contains a greater proportion of longer points with an overall range in length of 18 to $50 \mathrm{~mm}$. This is also reflected in the differences between the mean point length of Forbush Creek (30.2 mm) and the relatively tight clustering (19.8-23.6 mm)

Table 9.6

Table 9.6
Mean projectile point length, width and thickness by site.

\begin{tabular}{lccccccccccc}
\hline & \multicolumn{3}{c}{ Length } & \multicolumn{3}{c}{ W1dth } & \multicolumn{4}{c}{ Th1ckness } \\
S1te & $\mathrm{n}$ & $\overline{\mathrm{x}}$ & $\mathrm{s.d}$ & $\mathrm{n}$ & $\overline{\mathrm{x}}$ & $\mathrm{s.d}$. & $\mathrm{n}$ & $\overline{\mathrm{x}}$ & $\mathrm{s.d}$. \\
\hline Forbush Creek & 84 & 30.2 & 6.1 & 154 & 19.3 & 3.8 & 138 & 5.1 & 2.1 \\
Wal1 & 78 & 19.8 & 5.5 & 100 & 14.7 & 3.3 & 113 & 4.2 & 1.4 \\
Early Upper Saratown & 18 & 22.2 & 5.8 & 33 & 14.8 & 2.9 & 42 & 3.6 & 1.2 \\
Mitchum & 27 & 23.8 & 6.2 & 29 & 17.4 & 3.6 & 32 & 4.0 & 1.5 \\
Upper Saratown & 45 & 19.8 & 5.2 & 61 & 14.7 & 2.7 & 67 & 3.5 & 1.0 \\
Fredricks (Features) & 14 & 23.6 & 6.7 & 27 & 16.7 & 2.9 & 28 & 4.6 & 1.6 \\
Fredricks (Plowzone) & 26 & 29.8 & 10.6 & 50 & 18.7 & 4.1 & 58 & 4.6 & 1.6
\end{tabular}




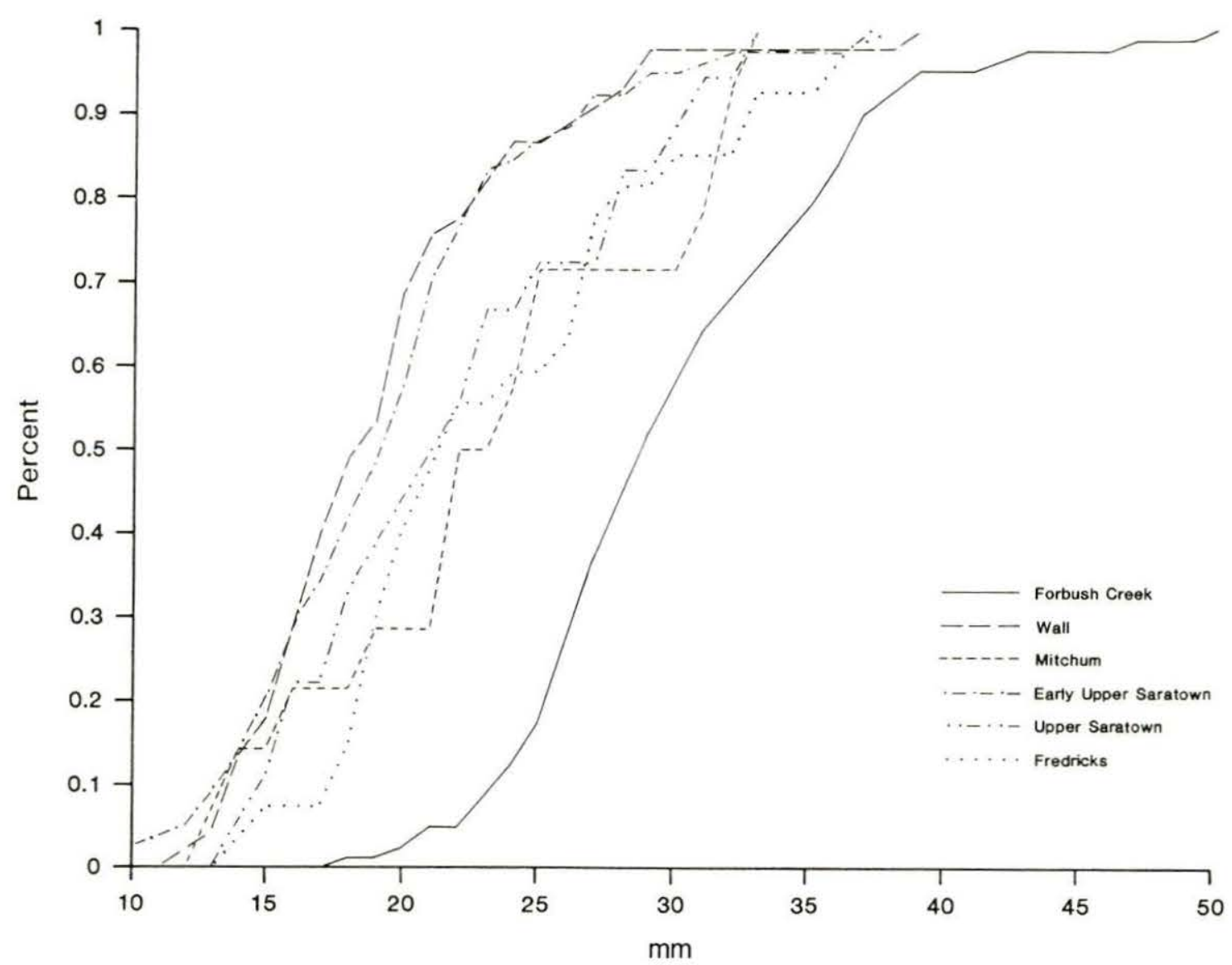

Figure 9.15

Cumulative frequency distribution of projectile point length.

of the mean lengths of the remaining five sites (Table 9.6).

An inspection of Figure 9.16, which is a cumulative frequency graph of point widths, reveals a pattern similar to that of the distribution of point lengths. Again, Forbush Creek is separated from the other sites by a relatively greater number of wider points. However, a possible clustering into two groups is indicated among the remaining five sites. One group includes Wa1l, Early Upper Saratown, and Upper Saratown, whereas the second includes the Mitchum and Fredricks sites. Since these two divisions do not represent chronological groupings, there does not appear to be any significant temporal differences reflected in the widths of projectile point from these five sites.

Projectile point sizes can also be compared by plotting length against width for the six site assemblages (Figure 9.17). Unlike the two previous cumulative frequency graphs, the Fredricks projectile points from feature and plowzone contexts were separated for this comparison in order to consider the potential for variability in point size due to multiple site occupations. Again, all sites except Forbush Creek and plowzone artifacts from
Fredricks cluster together. This latter observation supports the argument made using ceramic data (see Chapter 8) for an earlier cultural component at the Fredricks site.

Based on the comparative analysis of these six sites, the earliest site, Forbush Creek, contains wider, longer points. The Forbush Creek material also represents the largest sample included in this analysis; consequently, the possibility of bias being introduced by the small samples from Fredricks and Upper Saratown must be considered in the evaluation of the point size analysis.

The results of these mean length and width comparisons reveal a major difference in point size between Forbush Creek and the major occupations at the other five sites. There appears to be no clear pattern of reduction in point size through time within these five sites, even though they span approximately 200 years from the Protohistoric through Historic periods (Table 9.6). Instead, the pattern appears to be one of shifting size through time with subtle variations in the proportion of small to large points. These results indicate that the significant temporal information reflected by projectile point size is at the assemblage rather than individual artifact level, and tends to 


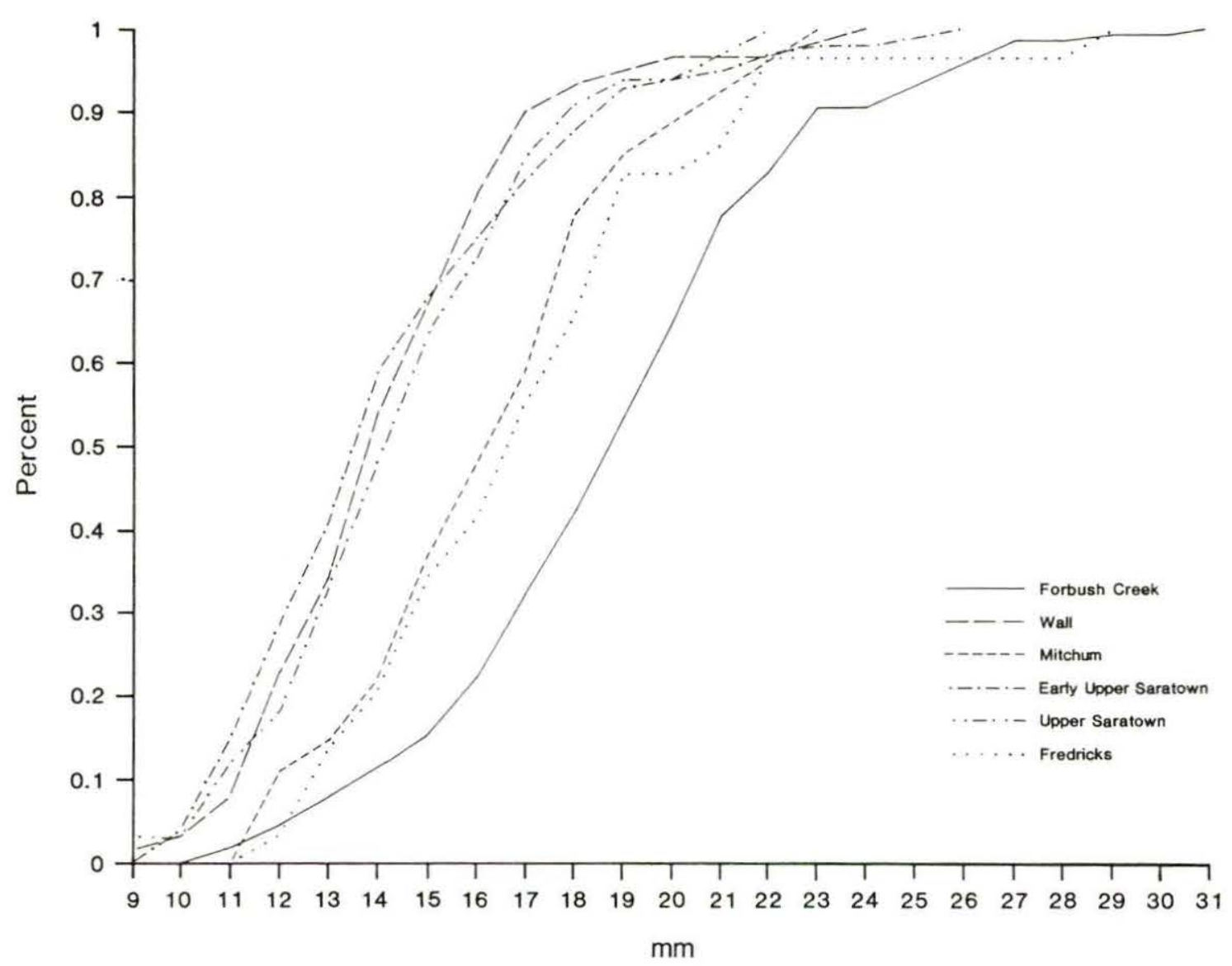

Figure 9.16

Cumulative frequency distribution of projectile point width.

negate the possibility for using individual triangular projectile points as chronological indicators.

Since lithic tool manufacture and use is a sequential subtractive process and changes in the shape and size of a tool can result in morphological changes as well, certain lithic tools may be less sensitive to relatively short term change. This would seem to be particularly true in the case of small triangular points.

Table 9.7 shows the distribution of triangular projectile points by blade form for the six sites. With the exception of Forbush Creek, points with straight blades and straight or incurvate bases account for the majority of point forms. Forbush Creek has the greatest number of projectile point forms of all the sites. The Wall site, however, contained more straight-sided, straight-based projectile points and very few incurvate-sided, incurvate-based projectile points. Although these projectile points are small, it is possible to see evidence of reworking and resharpening. Many of the incurvate-sided, incurvate base forms appear to be resharpened or reworked straight-sided, incurvate-base projectile points, indicating possibly a trend toward more curation and maintenance in the later sites (e.g., Fredricks and Mitchum). An increase in the curation of projectile points may have occurred as the bow and arrow continued to be used for certain types of hunting while overall chipped-stone tool production began to decline due to replacement by European items.

Finally, the third research question addressed patterns of lithic raw material utilization and what these patterns might reveal about mobility. This question was approached from two levels. The first level involved the identification of the range of raw material types used at the Wall, Mitchum, and Fredricks sites. The second level required using geological information from the surrounding areas to identify possible sources of specific raw materials. This task was accomplished by using a geological map of Orange County to identify potential areas for certain raw material outcrops and then making field inspections to collect samples from these areas for comparison with the archaeological specimens. It was beyond the scope of this project to determine definite aboriginal use of certain sources; only potential acquisition areas were identified. A source of felsic tuff, identical to archaeological specimens from the Wall and Fredricks sites, was located less than a river-mile from these sites. Outcrops of rhyolite, argillite, 


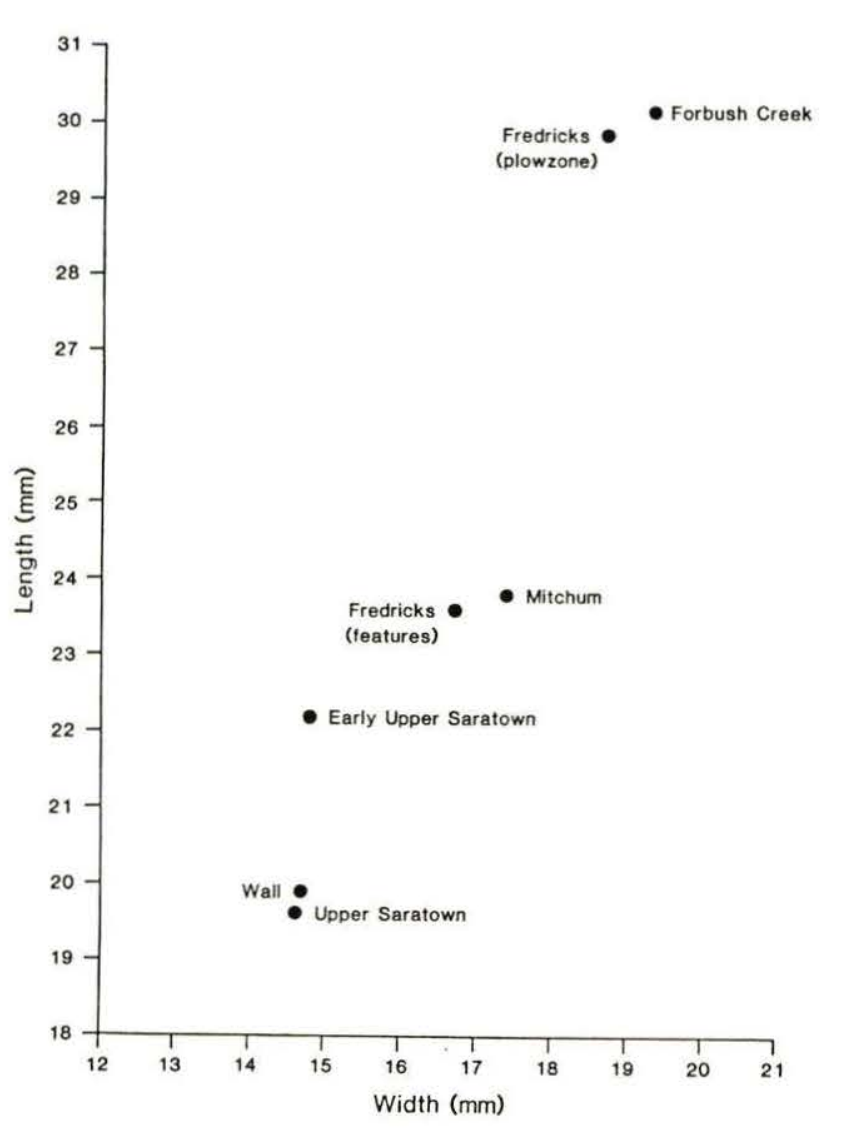

Figure 9.17

Bivariate plot of mean projectile point length versus mean width. and various vitric tuffs were located within three miles of the sites.

Fourteen raw material types were identified at the three sites. A summary of the raw material distribution is presented in Table 9.8 . The majority of the raw materials utilized at all three sites are locally available materials (Table 9.9). Identification of the raw material type, because of the small size of the artifact or degree of weathering, was not possible for approximately $2-3 \%$ of the flakes and chippedstone tools from all three sites. Raw material types that could not be identified during the analysis but might be identified later were classified as "indeterminate." Between $2-4 \%$ of the flakes from all three sites were classified as indeterminate. However, an interesting pattern is revealed by the distribution of chipped-stone tools classified as indeterminate. At the Wall site, only $2 \%$ of the chipped-stone tools were indeterminate; however, this increased to $8 \%$ at the Fredricks site and $10 \%$ at the Mitchum site. This pattern could result from either the movement of groups of people, or tools constructed at special-use sites being brought back to the habitation areas.

The majority of debitage and tools from the Wall and the Fredricks sites were made from locally available materials. But, at the Mitchum site, although $96 \%$ of the tools is of locally available materials, only $84 \%$ of debitage is local. of the $16 \%$ that is nonlocal, $2 \%$ is Coastal Plain Chert, $8 \%$ Ridge-and-

Table 9.7

Distribution of triangular projectile point forms by site.

\begin{tabular}{|c|c|c|c|c|c|c|c|c|c|c|c|c|}
\hline \multirow{2}{*}{$\begin{array}{l}\text { Projectile } \\
\text { Point Form }\end{array}$} & \multicolumn{2}{|c|}{$\begin{array}{c}\text { Forbush } \\
\text { Creek }\end{array}$} & \multicolumn{2}{|c|}{ Wa11 } & \multicolumn{2}{|c|}{$\begin{array}{l}\text { Early Upper } \\
\text { Saratown }\end{array}$} & \multicolumn{2}{|c|}{ Mitchum } & \multicolumn{2}{|c|}{$\begin{array}{l}\text { Upper } \\
\text { Saratown }\end{array}$} & \multicolumn{2}{|c|}{ Fredricks } \\
\hline & $\mathrm{n}$ & $\%$ & $\mathrm{n}$ & $\%$ & $\mathrm{n}$ & $\%$ & $\mathrm{n}$ & $\%$ & $\mathrm{n}$ & $\%$ & $\mathrm{n}$ & $\%$ \\
\hline $\begin{array}{c}\text { Straight Blade, } \\
\text { Straight Base }\end{array}$ & 14 & 7.61 & 78 & 69.03 & 10 & 23.26 & 18 & 52.94 & 26 & 38.81 & 44 & 48.89 \\
\hline $\begin{array}{l}\text { Straight Blade, } \\
\text { Incurvate Base }\end{array}$ & 28 & 15.22 & 26 & 23.00 & 8 & 18.60 & 5 & 14.71 & 22 & 32.84 & 17 & 18.89 \\
\hline $\begin{array}{c}\text { Incurvate Blade, } \\
\text { Straight Base }\end{array}$ & 8 & 4.35 & 1 & 0.88 & 0 & - & 4 & 11.76 & 2 & 2.99 & 2 & 2.22 \\
\hline $\begin{array}{c}\text { Incurvate Blade, } \\
\text { Incurvate Base }\end{array}$ & 35 & 19.02 & 5 & 4.42 & 3 & 6.98 & 6 & 17.65 & 1 & 1.49 & 14 & 15.56 \\
\hline $\begin{array}{c}\text { Excurvate Blade, } \\
\text { Incurvate Base }\end{array}$ & 21 & 11.41 & 3 & 2.65 & 4 & 9.30 & 0 & - & 2 & 2.99 & 4 & 4.44 \\
\hline $\begin{array}{c}\text { Excurvate Blade, } \\
\text { Straight Base }\end{array}$ & 12 & 6.52 & 0 & - & 2 & 4.65 & 0 & - & 3 & 4.49 & 0 & - \\
\hline Unidentified & 66 & 35.87 & 0 & - & 16 & 37.21 & 1 & 2.94 & 11 & 16.42 & 9 & 10.00 \\
\hline Total & 184 & 100.00 & 113 & 100.00 & 43 & 100.00 & 34 & 100.00 & 67 & 100.00 & 90 & 100.00 \\
\hline
\end{tabular}


Table 9.8

Percent of raw material types represented by debitage from the Wall, Fredricks, and Mitchum sites.

\begin{tabular}{|c|c|c|c|}
\hline Raw Material Type & $\underset{z}{\text { Wall }}$ & $\begin{array}{c}\text { Fredricks } \\
\qquad z\end{array}$ & $\begin{array}{c}\text { Mitchum } \\
z\end{array}$ \\
\hline Chert (Loca1) & 0.7 & 0.7 & 4.8 \\
\hline Chert (Non-Local) & 0.4 & 0.7 & 15.6 \\
\hline Quartzite & 0.2 & 0.6 & - \\
\hline Vein Quartz & 34.2 & 14.5 & 22.1 \\
\hline Crystal Quartz & 3.0 & 1.2 & 1.2 \\
\hline Rhyolite & 5.8 & 7.4 & 14.1 \\
\hline Argillite & 0.4 & 0.7 & 0.6 \\
\hline Metavolcanic (Other) & 1.8 & 6.0 & 0.6 \\
\hline Welded Tuf $\mathrm{f}$ & 4.5 & 7.2 & 2.6 \\
\hline Vitric Tuff & 40.0 & 34.6 & 31.0 \\
\hline Felsic Tuff & 2.5 & 7.1 & - \\
\hline Tuff (Other) & 0.5 & 0.7 & - \\
\hline Basalt & 1.6 & 9.3 & 0.6 \\
\hline Silicate & 1.5 & 4.1 & 1.6 \\
\hline Unidentified & 1.1 & 3.2 & 1.6 \\
\hline Indeterminate & 1.7 & 2.1 & 4.2 \\
\hline
\end{tabular}

Table 9.9

Percent of flakes and chipped-stone tools of local raw materials.

\begin{tabular}{lcc}
\hline & Flakes & $\begin{array}{c}\text { Chipped-Stone } \\
\text { Tools }\end{array}$ \\
\hline Wite & $99 \%$ & $98 \%$ \\
Fredricks & $99 \%$ & $97 \%$ \\
Mitchum & $84 \%$ & $96 \%$ \\
\hline
\end{tabular}

Valley Chert, and $6 \%$ unidentified chert. On visual inspection, the Ridge-and-Valley Chert appears to be very similar to the Chapultepec Formation of the Ridge-and-Valley province in Virginia. Since $10 \%$ of the materials from Mitchum is indeterminate, it is possible that further investigation will prove that these materials are also not local. Most of the Ridge-and-Valley flakes are $2 \mathrm{~cm}$ or smaller in size and may represent resharpening of tools manufactured elsewhere.

\section{SUMMARY AND CONCLUSIONS}

In summary, this study provides a preliminary analysis of lithic artifacts from a series of late prehistoric through historic sites on the North Carolina Piedmont. The analysis has revealed the following similar patterns for these sites: 1) use of predominantly local raw materials; 2) use of small triangular projectile points; 3) a tool kit dominated by small flake tools; 4) tools made on flakes as opposed to bifacial preforms; 5) very few formalized tools constructed for a long use-life; and 6) relatively few ground stone tools.

The results of the lithic analysis present a view consistent with Carnes' (this volume) conclusions concerning the impact of European trade goods on aboriginal society. Based on her analysis of historic artifacts from the Fredricks site, Carnes believes the acceptance of European trade goods did not result in a sudden abandonment of native skills or goods. While the introduction of European metal tools and weapons certainly had an impact on the Indian cultures of the North Carolina Piedmont, many of these items appear to have been integrated into the aboriginal social and political systems rather than replacing elements of the existing technology. The introduction of these alien goods certainly did not drastically alter the production and use of aboriginal stone tools and weapons. 


\title{
Faunal Remains from the Wall and Fredricks Sites
}

\author{
by
}

\author{
Mary Ann Holm
}

\section{RESEARCH QUESTIONS}

Analysis of the faunal remains from the Wall and Fredricks sites provides information important to interpreting cultural changes among the Piedmont Indians during the Protohistoric and Historic periods. Prior to the faunal analysis a series of research questions, based on information from the ethnohistorical record and from previous archaeological work, was formulated. Although several of these questions later proved to be unsuitable for the particular faunal assemblages found at the Wall and Fredricks sites, they did provide some insights that allowed this researcher to move beyond simple identification and toward an interpretation of faunal exploitation in the context of culture change.

The patterns of exploitation of faunal resources reported for several prehistoric North Carolina and Virginia sites (e.g., Waselkov 1977; Barber and Williams 1978; Runquist 1979; Egloff, Barber, and Reed 1980; Coleman 1982) are similar to the pattern reported by Smith (1974) for Middle Mississippi sites in the Mississippi Valley. In addition to showing a concentration on many of the same species as Smith's groups, the North Carolina and Virginia assemblages reflect a similar pattern of selective, seasonally oriented exploitation. Smith (1974:288) hypothesizes that

this cycle of selective, seasonal exploitation of certain animal species groups by Middle Mississippi populations was a procurement strategy that concentrated on those sections of the biotic community that would provide a maximum meat yield for a minimum of expended energy.

For the analysis of the faunal remains from the two Eno River sites, Smith's pattern provides two general research questions:

1. How did the overall pattern of faunal exploitation differ between the two sites?

2. Can the subsistence strategies exhibited at the two sites be explained in terms of maximization of meat yield and minimization of energy expenditure?
In order to answer the general research questions, more specific questions were formulated:

1. What was the relative importance of the various species of animals utilized by the occupants of the two sites?

2. Was faunal exploitation a seasonal activity at the two sites? If so, during what seasons was each species hunted?

3. What strategies were employed for procuring the exploited species?

4. How selective were the inhabitants of the sites in their exploitation of animal populations?

Other questions formulated prior to the analysis were:

1. Can patterns of butchering of the major species be identified?

2. Is there evidence of hunting species primarily for their hides?

3. How was faunal exploitation related to plant procurement and exploitation?

4. Was the pattern of faunal exploitation altered by the introduction of European technology?

5. Did introductions by Europeans of new plants and animals affect the existing pattern of faunal exploitation?

These questions formed the initial base from which methods were developed to describe and compare the assemblages recovered from the two sites. As the questions indicate, in addition to identifying the patterns of faunal exploitation of the inhabitants of the sites, a major goal of this research was to examine the possible effects of European contact on the use of faunal resources.

It was acknowledged, however, that differences between the Wall and Fredricks site assemblages could not be attributed automatically to European-induced changes in aboriginal 
subsistence. For example, differences could have resulted from the fact that the faunal remains from the two sites were retrieved from dissimilar contexts. Over $95 \%$ of the bones from the Wall site were found in a large midden associated with the palisade lines on the periphery of the village, and the remainder from the fill of a single burial pit. Nearly $88 \%$ of the bones from the Fredricks site, on the other hand, was obtained from burial fill and the rest from feature fill. All except one of the burial pits from the Fredricks site contained sizeable quantities of bone fragments in the zones of fill above the human skeletal remains. These deposits seem not to be the result of overlying midden having slumped into the pits, since the plowzone in the area around the burial pits contained relatively few artifacts. Although the differing contexts of the bones (sheet midden versus pit fill) are significant, the bones from the fill in the tops of the burial pits at the Fredricks site, and the bones from the midden at the Wall site can all be considered to represent the disposal of food refuse.

In addition to reflecting different methods of refuse disposal, the different contexts also may not have provided equal conditions for the preservation of bone. Whereas the midden at the Wall site probably represents the activities of many people over a period of several years, the remains from the Fredricks site, especially the remains from the burial pits, probably represent much briefer activities of fewer people. Thus, differences in the assemblages from the two sites may reflect differences in seasons of activity or differences in the behavior of large versus small segments of the representative communities.

Also, because the remains from the Fredricks site were primarily from burial fill, they may represent ceremonial activities, which could have been quite different from every-day subsistence practices. Finally, some of the differences between the two assemblages may relate to the fact that the sample from the Wall site $(n=30,257)$ is much larger than that from the Fredricks site $(n=16,393)$.

In spite of these problems, however, it should be recognized that the assemblages from these two sites offer an excellent opportunity to compare pre-contact and post-contact patterns of exploitation of animal resources in a setting in which variables of the natural environment can, for the most part, be held constant. Further, both sites were exposed to similar factors affecting the preservation of archaeological remains and they were excavated and recorded utilizing the same field techniques. Finally, the remains from the two sites were processed, sampled, and analyzed in an identical manner.

Given the rapidity with which European diseases and social manipulations succeeded in

disrupting and ultimately destroying aboriginal culture in piedmont North Carolina, it seemed likely that the faunal remains from the Fredricks site would show at least some evidence of a change in patterns of faunal exploitation from prehistoric to historic times. It was also expected that differences in the remains would reflect increased participation in the deerskin trade, rather than major changes in subsistence patterns, since ethnohistoric accounts (Lefler 1967:182-184; Swanton 1946:256-257) suggest considerable continuity between prehistoric and historic subsistence practices in North Carolina and Virginia. Prehistoric Dan River subsistence was based primarily on corn and bean agriculture and deer hunting, with other plants and animals utilized to a lesser extent.

The seasonal round emphasized deer hunting and food storage in winter, small game capture in spring, fishing and wild and domestic plant food harvesting throughout the summer, and nut gathering and turkey hunting in the fall and early winter (Waselkov 1977:230).

Swanton (1946:256-257) provides an outline of the historic Southeastern subsistence cycle.

Corn, beans, pumpkins, and a few other vegetables were raised, and the fields where these grew usually determined the sites of the towns. This was because they required labor and protection and because most of the crop was stored for later consumption. Dried meat was also stored there, but it was never possible to tell where game animals were to be found, while the location of the field was definite. This, of course meant that the people were generally in or near their villages in summer... Between planting and harvest, they did, however, often get time for a shorter hunt. After harvest they would remain in town until well toward winter to enjoy the produce of their fields and thus place it beyond the reach of human or animal predation. As the harvest was seldom sufficient to last - nor was it expected to last - until another crop came in, the Indians were obliged to seek natural food supplies elsewhere and, since such supplies were not usually concentrated, this meant that the people themselves scattered about in camps where they remained until planting time...

Swanton (1946:257) also mentions that fish were included in the diet during the summer.

In his account of the diet of the Siouan groups of North Carolina, Lawson named as staples many of the species found in the prehistoric sites of the same area (Lefler 1967: 182-184; Wilson 1983).

Whereas neither accounts give the kind
Swanton's nor Lawson's of information needed to 
quantify relative dependence upon any particular resource, both indicate that the historic subsistence pattern was similar to the prehistoric pattern.

In both the prehistoric and historic patterns, hunting for food was an important activity. It seems likely that if the inhabitants of the Fredricks site did participate in the deerskin trade, their participation involved (at least initially) only an expansion of the hunting activities which were already of major importance in their adaptive strategy. With increased participation in the deerskin trade over time it is expected that qualitative (rather than simply quantitative) differences would develop between the hunting activities prior to and after contact. Rather than merely hunting more often or killing a greater number of animals, it is possible that the Indians began to range further from their villages, exploit portions of the environment that previously had been rarely utilized, or hunt species that had not been hunted frequently in the past.

We know that during the period at least from 1650-1676, in which they occupied their island in the Roanoke River, the Occaneechis played an important role in the deerskin trade. It is not known, however, whether this participation increased after they moved to the site on the Eno River around 1680. If the Occaneechis maintained their strong participation in the deerskin trade after their move south (and the abundance of trade goods at the Fredricks site indicates that this is likely), the faunal remains from the Fredricks site might be expected to differ from those of the protohistoric Wall site by exhibiting some or all of the following characteristics:

1. more opportunistic hunting patterns--e.g., hunting should be less seasonally oriented and there should be more evidence of hunting at all times of the year.

2. less balance between maximization of meat yield and minimization of energy expenditure.

3. evidence of exploitation of portions of the environment that previously had not been heavily utilized.

4. changes in procurement strategies--e.g., Waselkov (1977) suggests that, the method of hunting deer may have evolved from stalking to community drives.

5. possibly less specialization and more variability in the faunal assemblage.

6. increased evidence of hunting for fur and hides rather than for meat, such as increased evidence that animals were butchered in the field with only portions of the carcasses being returned to the site.

7. possible increases in the numbers of tools and features associated with hide-working (such as smudge pits).

The first four expectations would reflect qualitative changes in hunting patterns that might have had the effect of increasing, at least temporarily, the quantity of animals (and thereby skins) obtained. The fifth expectation might have arisen if the Fredricks site inhabitants had begun to hunt any available fur-bearing animals, including those species that had not been desirable prior to the onset of European trade. The sixth expectation would reflect a marked increase in the number of animals killed beyond those required to fulfill the needs (subsistence and raw material) of the site inhabitants. The final expectation would manifest an increase in the number of tools and features associated with hide-working that might occur with an increase in hide procurement for trade.

Although this list of preliminary expectations is far from exhaustive, it provides a basis on which to compare the two faunal assemblages beyond merely comparing the frequencies of identified species from each site. As work with the assemblages has progressed, the initial list has been reevaluated, further questions added, and others eliminated. Some of these adjustments to the original list of research questions arose when new information was gleaned from the ethnohistorical record. More frequently, the original questions had to be modified because of limitations imposed by the faunal assemblages themselves.

\section{ETHNOHISTORIC REFERENCES TO THE USE OF FAUNAL RESOURCES}

Among the many ethnohistoric accounts for the Piedmont area of North Carolina and Virginia are those of Lederer, Needham and Arthur, Fallam, Bland, Wood (Alvord and Bidgood 1912), and Lawson (Lefler 1967). With the exception of Lawson's account, however, none of these documents provides detailed information about hunting, fishing, and other subsistence activities of the historic North Carolina Indians. In A New Voyage to Carolina, John Lawson described his 1701 exploration of the region from Charleston, South Carolina, through the North Carolina Piedmont, to New Bern, North Carolina. In addition to presenting the scenes and events of his trip, Lawson also wrote a chapter detailing the "Vegetables", "Beasts", "Insects", "Birds", and "Fish" of North Carolina. Lawson's account thus provides a wealth of information on the use of faunal resources by North Carolina Indians.

During his winter journey, in addition to making direct contact with the Occaneechis in 
their town on the Eno River (Lefler 1967:61), Lawson encountered a number of other groups including the Eno, Keyauwee, Saponi, and Tutelo. Although he gives considerable attention to the ways in which the Piedmont (and also the coastal) Indians utilized faunal resources, he provides only scanty information, about the ways in which the animals were procured (hunted, trapped, etc.).

\section{Mammals}

According to Lawson, deer was the most important mammalian resource to the North Carolina Indians. He mentioned "barbaku'd" and roasted venison; venison broth thickened with acorn meal; and "a Dish, in great Fashion amongst the Indians, which was Two young Fawns, taken out of the Doe's Bellies, and boil'd in the same slimy Bags Nature had plac'd them in" (Lefler 1967:51, 58). Parts of the deer were utilized in a variety of ways in addition to food. For example, deer hides were used for clothing, shoes, and as covers for drums, and were also an important commodity for trade with the Europeans. "The Bone of a Deer's Foot" was used for scraping the hair off of hides, and deer brains (after being baked and then soaked in water) were used in tanning hides (Lefler $1967: 217)$. Lawson also mentioned the use of the "Head of a Buck" as a decoy with which to hunt other deer (Lefler 1967:29).

Swanton (1946:249) lists a number of ways in which Southeastern Indians used various parts of the deer in addition to those mentioned by Lawson. Horns were boiled for glue and made into projectile points, ornaments, and needles; hooves were made into rattles; and sinews and skins were used to make fishnets and bowstrings. Ribs were made into bracelets, and tibiae into flutes. Tools constructed from deer bones that have been recovered from archaeological sites include metatarsal beamers, ulna awls, and antler flakers (Waselkov 1977; Runquist 1979).

In addition to describing the technique of stalking deer, Lawson mentioned that

when these Savages go a hunting, they commonly go out in great Numbers, and oftentimes a great many Days Journey from home, beginning at the coming of Winter.... Thus they go and fire the Woods for many Miles, and drive the Deer and other Game into small Necks of Land and Isthmuses, where they kill and destroy what they please (Lefler 1967:215-216).

Other techniques used by North Carolina and Virginia Indians for hunting deer were stalking them without the use of a decoy, and driving them to water without the use of fire (Waselkov 1977:108).

While visiting Occaneechi Town, Lawson was served "good fat Bear," and the next day, in Adshusheer, he feasted upon "hot Bread, and
Bears-oil". The Indians considered the paws to be the most edible part of the bear, whereas the head was always thrown away (Lefler 1967:122). In addition to being eaten, bear's oil was used for frying fish, and was mixed with "a certain red Powder" and daubed on the body and used for greasing the hair (Lefler 1967:121, 174). Lawson also mentioned that the "Oil of the Bear is very Sovereign for Strains, Aches, and old Pains" and that bear's fur was used for making muffs and facing caps (Lefler 1967:122-123). The only method of capturing bear mentioned by Lawson involved killing the animals that were flushed during the fire drives used for hunting deer (Lefler 1967:17).

Opossum was used for food by the Indians, but the fur of this animal was "not esteemed nor used" except when it was spun to make baskets, mats, and girdles (Lefler 1967:125-126, 195). Raccoon meat was served to Lawson on several occasions during his voyage, and raccoon skins and furs were used by the Indians for clothing and blankets (Lefler 1967:23, 126, 200). A1though skunks (or polecats) were used for food, Lawson stated that their skins were not used in any way (Lefler 1967:124).

Rabbits (or hares), and squirrels were roasted without being gutted, and their skins were used for clothing and blankets. Although Lawson stated that rabbits were caught during fire drives, he did not provide a description of the ways in which opossums, raccoons, skunks, or squirrels were hunted (Lefler 1967:182, 200).

Beavers were prized for their thick fur, and their skins were used in making shoes, mittens, and other clothes (Lefler 1967:125, 200). Beaver meat was also eaten, and its tail was considered a delicacy (Lefler 1967:66, 125). Lawson encountered a Saponi Indian who maintained traps for capturing beaver (Lefler 1967: 54 ).

Lawson listed a variety of rodents and insectivores that were found around the houses and fields of the Indians (Lefler 1967:120, 130-131). These animals may have been used for food, although Lawson did not mention such a practice.

European-introduced animals present in North Carolina and utilized by the Indians encountered by Lawson during his voyage consisted of horses and pigs. Although cattle were present, Lawson does not indicate that they were used by the Indians for food. According to Lawson, the only use made of the horse by the Indians was for carrying deer back to their villages (Lefler 1967:44). Although Lawson alluded to hog stealing by the Indians, he did not indicate that hogs were raised by them (Lefler 1967:64). He did mention, however, that the "Paspitank" Indians kept cattle at one time, although he was not sure if they were still raising these animals at the time of his travels. 
All of the mammals identified from the 1983-1984 faunal assemblages from the Wall and Fredricks sites (with the exception of the shrew and vole) were described by Lawson. Mammals mentioned by Lawson that were not identified in these archaeological assemblages are buffalo, panther, wild cat, "tyger", otter, minx (mink), $\mathrm{elk}$, and lion.

\section{Birds}

Lawson listed over 110 birds that could be found in North Carolina at the time of his journey (Lefler 1967:140-141). Of these, the turkey and the passenger pigeon were the most important to the Indians as sources of food. Turkey bones were also made into many different kinds of tools (e.g., awls and beamers) and ornaments (e.g., beads). Turkey feathers were used by Southeastern Indians in making feather mantles and fans, and in feathering arrows. Arrow points were also manufactured from turkey spurs (Swanton 1946:251). Turkey meat was offered as food to Lawson so often that it eventually "began to be loathsome" (Lefler 1967:34).

Although the passenger pigeon is now extinct, Lawson's description provides a vivid picture of this bird and the way it was hunted and used by the Indians.

Pigeons...were so numerous in these parts that you might see many Millions in a flock... You may find several Indian Towns, of not above 17 Houses, that have more than 100 Gallons of Pigeons 0il, or Fat; they using it with Pulse, or Bread, as we do Butter.... The Indians take a Light, and go amongst them in the Night, and bring away some thousands, killing them with long Poles, as they roost in the Trees. At this time of the Year, the Flocks, as they pass by, obstruct the Light of the Day (Lefler 1967:50-51).

Another bird identified in the faunal assemblages from the Wall and Fredricks sites is the bobwhite quail. This bird was probably an important source of food and it also provided feathers which could have been used for clothing and decoration. Other birds identified from the faunal assemblages include sparrows, killdeer, bluejay, great horned owl, woodpecker, and lesser scaup. Of these only the lesser scaup could be considered, with any certainty, to have been used for food. Lesser scaup is also the only bird identified in the faunal assemblage that was not mentioned by Lawson.

It is important to note that Lawson stated that "all small game, such as Turkeys, Ducks, and small Vermine, they [the Indians] commonly kill with Bow and Arrow, thinking it not worth throwing Powder and Shot after them" (Lefler 1967:216).

\section{Reptiles}

The box turtle was probably the most important reptile utilized by the Indians that Lawson encountered. Box turtle meat was eaten, and the shell was made into rattles, cups, and dippers (Lefler 1967:138). Other turtles represented in the faunal assemblages from the Wall and Fredricks sites were snapping turtle, painted turtle, musk turtle, and mud turtle. None of these others was mentioned specifically by Lawson, but all (with the exception of the musk turtle that was probably not eaten because of its offensive smell) probably were utilized in the same manner as the box turtle.

Vertebrae from a variety of poisonous and nonpoisonous snakes were identified in the two faunal assemblages. Lawson mentioned that "a11 Indians will not eat them [snakes], tho' some do," that the skin of the king snake was used to make girdles and sashes, and that rattlesnake teeth were used in an instrument for scarifying (Lefler 1967:137, 182, 223). He also noted that the coastal Indians avoided killing snakes "because their Opinion is, that some of the Serpents Kindred would kill some of the Savages Relations, that should destroy him" (Lefler 1967:219).

\section{Amphibians}

Amphibians identified in the archaeological assemblages were the spadefoot toad, and indeterminate frogs and toads. Although Lawson noted the presence of frogs in North Carolina and listed them among the "Insects," he did not mention whether they were used by the Indians for food or for any other purpose.

\section{Fish}

Lawson listed 20 types of fresh-water fish in North Carolina (Lefler 1967:156). Of these, two (catfish and suckers) were identified in the faunal assemblages from the Wall and Fredricks sites. The other two species identified archaeologically (gar and sunfish) were not mentioned by Lawson.

Fishing with hooks, weirs, and with bow and arrow (on the coast) were all described by Lawson (Lefler 1967:218).

\section{Summary}

In addition to descriptions of the ways in which individual species of animals were procured and utilized by the Indians, Lawson provided some additional information useful for interpreting the two faunal assemblages. He mentioned that the Indians "boil and roast their Meat extraordinary much, and eat abundance of Broth" (Lefler 1967:231). He also stated that "A11 the Indians hereabouts carefully preserve the Bones of the Flesh they eat, and burn them, as being of the Opinion, that if they omitted that custom, the game would leave their Country, 
and they should not be able to maintain themselves by their Hunting" (Lefler 1967:58). Both of these statements provide information that is helpful in evaluating how accurately the faunal assemblages from the Wall and Fredricks sites reflect the original assemblages of bone produced at these sites and in interpreting any patterns observed in the surviving archaeological assemblages.

Nearly every species identified in the faunal assemblages from the Wall and Fredricks sites was mentioned by Lawson. Although Lawson's descriptions of the ways in which the Indians utilized these animals are not consistently detailed, they do provide information that cannot be obtained from the archaeological record alone.

\section{EXCAVATION AND RECOVERY TECHNIQUES AFFECTING FAUNAL REMAINS}

At the Wall site, after removal of the plowzone, the midden was excavated (with shovels in the first square excavated and with trowels in each subsequent square) in two levels. These levels correspond with a slight change in color between the upper and lower midden soil. The soil from each level in each $10 \times 10-f t$ square was kept separate and waterscreened through a sluice box equipped with a sequence of 1/2-inch, $1 / 4-i n c h$, and $1 / 16$-inch screens.

Excavation of burials and other features was performed with small hand-tools such as trowels, dental probes, and brushes. Each natural zone within a feature was removed separately, and all fill from each zone was waterscreened as a unit through the sequence of graduated screens. Special care was taken with the animal bones to ensure that, although dried thoroughly before being placed in plastic storage bags, they did not become cracked and brittle from excessive exposure to sunlight. Ten-liter samples of soil from each zone in each feature was processed by flotation. The bones retrieved through this procedure were subsequently screened in the laboratory through 1/2-inch, 1/4-inch, and 1/16-inch screens to permit comparison of these bones with the faunal remains recovered through field waterscreening.

\section{SAMPLING AND ANALYTIC PROCEDURES}

Only those bones and bone fragments recovered from undisturbed contexts were included in the material analyzed from the Wall and Fredricks sites. In other words, bone from the plowzone was excluded. The vast majority of the analyzed faunal remains from the Wall site was from four $10 \times 10-\mathrm{ft}$ units of undisturbed sheet midden. Although three burial pits were excavated at this site in 1983, the fill from only one of those pits contained more than a few poorly preserved bone fragments. Therefore, the remains from the fill of only one burial pit and four squares of midden make up the sample analyzed from this site. The faunal assemblage from the Fredricks site was recovered from the fill of fourteen pits. Nine of these were burial pits, one was a fire pit, one a storage pit, and three pits of indeterminate function. As yet, no sheet midden has been found at the Fredricks site.

Identical analytical procedures were used on the assemblages from both sites. All of the bone recovered in the $1 / 2-i$ nch and $1 / 4-i$ nch mesh screens was analyzed. There were numerous tiny, unidentifiable fragments of bone retrieved by the 1/16-inch screen. Because it would have been a time-consuming and (probably) pointless task to separate all of these minute fragments from the fine gravel that was also recovered in this size screen, only those bones and bone fragments which appeared to be identifiable were pulled from the $1 / 16$-inch washings. The bones and bone fragments from each excavated unit (10x10-ft square of midden, or feature) and from each level or zone within each excavation unit were kept separate during analysis. Also, bones from different sized screens were not combined during analysis.

The basic procedures followed in identifying and analyzing the faunal remains from the two sites closely follow those outlined by Smith (1976): 1) each bone fragment was initially sorted into one of three groups--unidentifiable, identifiable only to class, or identifiable as to skeletal element; and 2) each of these fragments (whether it was identifiable or not) was examined for evidence of modification such as burning or cutting.

For those bones that could be identified beyond the level of class, the side of the body (when applicable) and portion of the bone (proximal, distal, or shaft) was noted. After that, a taxonomic identification was made for each of the identifiable bones and bone fragments. Several of the variables that affected whether a fragment could be identified beyond family or order were: "(1) the specific skeletal element in question (i.e., rib versus mandible), (2) the amount of diagnostic surface present, (3) the ability of the person identifying the specimen, (4) the size of the comparative collection being employed, and (5) the degree of morphological similarity of species within the taxonomic group" (Smith 1976:281). To help minimize problems introduced by variables (3) and (4), a group of 205 bones and bone fragments was sent for identification to Elizabeth Reitz at the Zooarchaeological Laboratory, University of Georgia. This sample consisted of bones that appeared to be identifiable but for which the type collection at the Research Laboratories lacked comparative specimens.

In addition to determining the total number of fragments in each taxonomic category, all of 
the fragments in each category were weighed.

When possible, the age and/or sex of the animal represented by a particular fragment was assessed. In most cases, these characteristics could be determined only for the remains of white-tailed deer. For the deer, age was estimated by noting whether or not the epiphyses of the long bones were closed, and by using Severinghaus's (1949) criteria of tooth development and wear. Sex of the deer was determined by using the pelvic girdle criteria set forth by Edwards et al. (1982). Attempts to determine age and/or sex of several other species, such as rabbits, squirrels, and raccoons, were less successful than for deer. This problem resulted, in large part, from characteristics of the faunal assemblages themselves. Many of the bones, or portions of bones, that display the characteristics used to distinguish between animals of different ages or sexes simply were not present in the remains being studied.

Information obtained from the procedures discussed above constitute primary data or "direct quantification of identified material" (Wing 1979:119). Several factors can influence how accurately these primary data reflect the original faunal sample. All bones, for example, do not stand an equal chance of being represented in an archaeological assemblage. The survival of bone after it has been discarded is affected, primarily, by two factors: its physical condition at the time of disposal, and the nature of the environment in which it was placed. Whether a bone was burned, boiled, or roasted affects its chemical and physical properties, which, in turn, influences preservation (Chaplin 1971:15). Also, the basic structure of the bone must be considered. Teeth and phalanges are stronger than ribs and vertebrae, and, thus, are less likely to be destroyed (Payne 1972:68).

The manner in which a particular bone was discarded further affects its survival. If the bone were buried in a trash pit, for example, the rate of disintegration would depend on factors such as the "acidity or alkalinity, degree of aeration, movement of water, bacterial population, as well as the structure and seasonal properties of the soil" (Chaplin 1971:16). If it remained on the surface of the ground, it would be more accessible to scavengers, and more likely to be damaged by weather, and stepped on and crushed.

Excavation techniques also affect the number and kinds of bones eventually available for analysis. The portion of the site excavated, sieving techniques utilized, and steps taken to protect the fragile bone after excavation affect the sample.

For these and other reasons, one can assume that any collection of archaeological bone will represent only a portion of the faunal remains originally associated with the site. Thus, the primary data obtained probably will not provide enough information for reliable interpretations of what the assemblage represents in terms of past behavior. For this reason, secondary data, "which involve interpretation, extrapolation, or estimations based on primary data" (Wing 1979: 118) are neccessary. Examples of secondary data include calculations of minimum numbers of individuals, and estimations of usable meat weight.

Chaplin (1971) lists three of the most commonly named methods for quantifying the species represented by a collection of animal bones: 1) the fragments method, 2) the weight method, and 3) the minimum number method. Whereas there are advantages to each method, Chaplin and most others (e.g., White 1953; Daly 1969; Smith 1976; Styles 1981; and Klein and Cruz-Uribe 1984) prefer the minimum numbers method.

With the fragments method one counts the total number of identifiable bones and fragments of each species and determines the ratio of different bones or different species. The number of identified specimens (bones or bone fragments) per species is sometimes abbreviated as NISP (Payne 1975; Grayson 1979; Klein and Cruz-Uribe 1984). The NISP is little more than a list of bones of different animals present in an assemblage. The number of bones of a particular species represented in an assemblage does not necessarily indicate what percent of the diet of the original inhabitants was made up of the meat from that animal. For example, some species of animals have more bones than others. Also, although hunters may bring back the entire carcass of a smaller animal, they are liable to return with only the more useful parts of a larger one. Thus, only the broadest questions about subsistence can be answered using NISP.

In another approach, used to arrive more directly at conclusions about the relative dietary importance of each species, the analyst weighs the bone from each species and then multiplies that weight by a factor to determine the amount of meat represented by each type of animal. In using this method, however, every scrap of bone must be utilized in order to arrive at an unbiased approximation of amount of meat (Daly 1969:149). Because much of the bone analyzed usually is fragmented, it is nearly impossible to place each scrap into its appropriate species category. Further, it is impossible to account for all of the bone missing from the site or not retrieved during excavation. Also, the weight of the bone is affected by whether or not it was burned or charred and by the thoroughness with which it was cleaned and dried after excavation. Another objection to the weight method is the fact that it begins with the assumption that there is a fairly constant relationship between the weight of an animal and the weight of its bones. Although there is a correlation between these two factors, the relationship is variable (Smith 
1975:100). To counteract this bias it would be necessary to apply a different live weight value for each age and sex category for each species analyzed. Because it is not always possible to identify the species to which a fragment belongs, let alone the age or sex of the animal, the weight method is only appropriate for use with relatively few completely identified fragments.

The minimum numbers of individuals (MNI) method avoids many of the problems that plague the other two methods. Using the simplest form of this procedure, the minimum number of animals of each species is determined by counting the maximum number of any particular bone. When possible, the age, sex, and size of the animal is taken into account to increase the accuracy of this method. This analytical procedure is superior to the other procedures for a number of reasons.

The minimum number of animals that the bones could have come from is an indisputable fact. It is, moreover, a direct measure of a number of animals involved and is an abstraction of the true number of animals involved only within fixed limits. It also involves no assumptions about differential preservation of bone which can not be checked by examination of the specimens or by a site inspection. It is therefore using verifiable facts throughout (Chaplin 1971:70).

Grayson (1973:70) notes that the minimum numbers method "provides us with units which are necessarily independent of one another, and which may therefore be validly used in further statistical manipulation."

In spite of its advantages, the minimum numbers method also has several shortcomings. First, there is more than one way to derive the minimum number figure from an assemblage. Variation in the way in which faunal material from a site is grouped, for example, affects the results of analysis. If the material is separated into clusters according to the stratum and excavation unit in which it is found, it will yield the largest estimation of MNI. If the excavation unit is ignored, the minimum number decreases, and if neither excavation unit nor stratigraphy is used in grouping the material, the number will be even smaller (Grayson 1973: 433). The comparability of the data produced by the minimum numbers method is still suspect unless the analyst explicitly states how he arrived at his figures.

Three methods were used to quantify the faunal remains from the Wall and Fredricks sites. The NISP method was used because it was calculated automatically as the bone fragments were identified. Also, the weight of the bone identified for each taxonomic category was calculated. Comparison of the relative abundance of each species, as revealed by the number of identified fragments and by the weight of these fragments, provided information useful not only in determining the possible importance of these animals to the original inhabitants, but also information about the conditions (such as fragmentation or preservation) that affected how much of the assemblage could be identified and to what taxonomic level. The weights of the identified bones were not converted to meat weights because of the vast array of biases introduced by the use of the weight method.

The minimum numbers of individuals method was relied on most heavily in interpreting the two faunal assemblages. In comparing the assemblages from the Wall and Fredricks sites, MNI was calculated from each site as a whole, with neither the excavation unit nor site stratigraphy taken into consideration. Although it yielded the smallest number of individuals, this method was necessary because of the different contexts from which the two assemblages were recovered.

\section{RESULTS OF ANALYSIS: WALL SITE}

This analysis of the faunal remains from the 1983-1984 excavations at the Wall site concentrated on the bone from four $10 \times 10-f t$ squares of undisturbed midden located just inside the outermost palisade surrounding the village. Although several burials were excavated at this site in 1983, the fill of only one contained more than a few poorly preserved fragments of bone. The remains from the fill of this one burial were also included in the analysis. As previously mentioned, all fill from the midden and the one burial was waterscreened through a sequence of three sized screens. A total of 30,257 fragments was examined from the 1983-1984 excavations. This total consists of 6,040 fragments from the $1 / 2$-inch screen, 19,688 fragments from the $1 / 4-i n c h$ screen, and 4,529 fragments from the $1 / 16$-inch screen. Approximately $42 \%$ of the collection $(12,714$ fragments) could not be identified. The majority of these fragments seem to be pieces of long bones of large mammals (probably deer).

A complete account of the faunal remains recovered in the 1983-1984 excavations is provided in Table 10.1. Burial 1 had two zones of fill containing a total of 1,340 bone fragments. The only passenger pigeon remains represented in the 1983-1984 assemblage were recovered in the fill of this burial. As there were no other obvious qualitative differences between the bones recovered from the burial and those recovered from the midden, the assemblage will be treated in the following discussions as though it were retrieved from a single context.

The first excavations at the Wall site were carried out in 1938, 1940, and 1941 (Coe 1952, 1964). Analysis of the faunal remains from 
Table 10.1

Animal remalns from the Wall site.

\begin{tabular}{|c|c|c|c|c|c|c|}
\hline Species & Frag. & ₹ Frag. & Wt. $(g)$ & \% wt. & MNI & $\chi$ MNI \\
\hline$\frac{\text { Odocolleus }}{\text { tailed }} \frac{\text { virglnianus, }}{\text { Deer }}$, White- & 4731 & 15.64 & 13305.5 & 61.42 & 36 & 12.50 \\
\hline Didelphis marsupialis, Opossum & 23 & .08 & 12.55 & .06 & 1 & .35 \\
\hline$\frac{\text { Sciurus }}{\text { Squirolinensis }}$, Gray & 35 & .12 & 5.18 & .02 & 1 & .35 \\
\hline Sciurus sp. & 297 & .98 & 16.47 & .08 & 9 & 3.12 \\
\hline Procyon lotor, Raccoon & 105 & .35 & 51.85 & .24 & 4 & 1.39 \\
\hline$\frac{\text { Sigmodon hispidus, Hispid Cotton }}{\text { Rat }}$ & 24 & .08 & .90 & .00 & 2 & .69 \\
\hline$\frac{\text { Peromyscus }}{\text { Mouse }}$ leucopus, White-footed & 22 & .07 & .63 & .00 & 2 & .69 \\
\hline 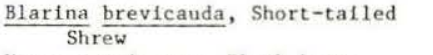 & 12 & .04 & 5.40 & .02 & 2 & .69 \\
\hline Ursus americanus, Black bear & 1 & .00 & 21.70 & .10 & 1 & .35 \\
\hline Sylvilagus sp., Cottontafl & 86 & .28 & 7.80 & .04 & 4 & 1.39 \\
\hline Castor canadensis, Beaver & 1 & .00 & 1.30 & .01 & 1 & .35 \\
\hline$\frac{\text { Microtus }}{\text { Vole }}$ pennsylvanicus, Meadow & 13 & .04 & .48 & .00 & 2 & .69 \\
\hline Glaucomys volans, Flying Squirrel & 1 & .00 & .11 & .00 & 1 & .35 \\
\hline Tamias striatus, Chipmunk & 1 & .00 & .10 & .00 & 1 & .35 \\
\hline Canidae, Wolf, Dog, Fox & 4 & .01 & .30 & .00 & 1 & .35 \\
\hline Ondatra z1bethecus, Muskrat & 1 & .00 & .10 & .00 & 1 & .35 \\
\hline Unidentified Mammal & 7652 & 25.29 & 4542.45 & 20.97 & - & - \\
\hline Meleagris gallapavo, Wild Turkey & 103 & .34 & 194.25 & .90 & 3 & 1.04 \\
\hline$\frac{\text { Ectopistes }}{\text { Passenger Pigeon }}$ & 2 & .00 & .10 & .00 & 1 & .35 \\
\hline Colinus virginianus, Bobwhite & 4 & .01 & $\cdot 30$ & .00 & 1 & .35 \\
\hline Cyanocitta cristata, Blue Jay & 4 & .01 & .27 & .00 & 1 & .35 \\
\hline Bubo virginianus, Great Horned Ow1 & 1 & .00 & 2.00 & .00 & 1 & .35 \\
\hline Unidentified B1rd & 514 & 1.70 & 126.07 & .58 & - & - \\
\hline Terrapene carolina, Box Turtle & 1000 & 3.30 & 687.24 & 3.17 & 5 & 1.74 \\
\hline$\frac{\text { Chelydra }}{\text { Turtile }} \frac{\text { serpentina, Snapping }}{1 e}$ & 8 & .03 & 8.50 & .04 & 1 & .35 \\
\hline Chrysemys picta, Painted Turtle & 6 & .02 & 13.20 & .06 & 1 & .35 \\
\hline$\frac{\text { Kinosternon }}{\text { Turtle subrubrum, Mud }}$ & 2 & .00 & 0.20 & .00 & 1 & .35 \\
\hline Unidentiffed Turt le & 1261 & 4.17 & 249.88 & 1.15 & - & - \\
\hline Crotalid sp., Poisonous Snake & 1 & .00 & .90 & .00 & 1 & .35 \\
\hline Unidentified Snake & 666 & 2.20 & 27.14 & .12 & - & - \\
\hline$\frac{\text { Scaphiopus }}{\text { Spadef holbrooki }}$ & 1 & .00 & .10 & .00 & 1 & .35 \\
\hline$\underline{\text { Rana }}$ catesbelana, Bullfrog & 19 & .06 & .20 & .00 & 1 & .35 \\
\hline Rana sp., Frog & 62 & .20 & 3.19 & .01 & 7 & 2.43 \\
\hline Bufo sp., Toad & 25 & .08 & 1.27 & .00 & 4 & 1.39 \\
\hline Unidentified Amphibian & - & - & - & - & - & - \\
\hline Ictalurus sp., Catfish & 194 & .64 & 2.95 & 0.01 & 187 & 64.93 \\
\hline Catostomus sp., Suckers & 10 & .03 & .55 & .00 & 1 & .35 \\
\hline Lepisosteus sp., Gar & 8 & .03 & .34 & .00 & 1 & .35 \\
\hline Lepomis, Sunfish & 3 & .01 & $\cdot 30$ & .00 & 1 & .35 \\
\hline Unidentified Fish & 641 & 2.12 & 10.25 & 0.05 & - & - \\
\hline Sub-Total (Identified to Class) & 17545 & 57.99 & 19302.02 & 89.10 & - & - \\
\hline Sub-Total (Unidentified) & 12712 & 42.01 & 2361.62 & 10.90 & - & - \\
\hline Total & 30257 & 100.00 & 21663.64 & 100.00 & 288 & 100.04 \\
\hline
\end{tabular}


these excavations was performed by Jeanette Runquist (1979). The majority of the remains that Runquist examined were recovered from a zone of undisturbed midden that was sifted through 1/4-inch mesh screen. A sample of the midden from each $10 \times 10-f t$ square was waterscreened, as was the fill from the few burials and features included in Runquist's sample. Her total assemblage consisted of 6,000 bones and bone fragments. Runquist's findings are occasionally included in this discussion of the results of analysis in order to provide the most complete description possible of the animals originally represented at the Wall site.

A total of $856 \mathrm{fish}$ bones representing 190 individuals ( $66.0 \%$ of the total number of individuals for the assemblage) was identified. The majority of these individuals were catfish. Other fish identified were sucker, sunfish, and gar.

Amphibians accounted for a minimum of 13 individuals $(4.5 \%$ of the total number of individuals), identified from 107 fragments. Reptiles accounted for $16.78 \%$ of the identified bone and $3.1 \%$ of the number of individuals identified. Remains of box turtle formed a significant portion of the assemblage, as this species was second only to white-tailed deer in percent of fragments identified to species. Snake bones accounted for $2.2 \%$ of the fragments recovered and less than $1 \%$ of the individuals.

With the exception of the wild turkey, birds do not seem to have been used frequently by the inhabitants of the Wall site. Three individuals (turkeys), representing $1.0 \%$ of the total number of individuals, were identified in the present analysis.

From a count of spurs, Runquist determined that three of the eight individuals in the 1938-1941 assemblage were males, whereas one of the three individuals in the 1983-1984 sample was male. In both cases, the proportions of males to females are somewhat higher than one might expect. In a study of over 6,000 turkeys harvested over a five-year period in Virginia, for example, on 1 y $18.9 \%$ of the turkeys captured were adult males (Gwynn 1964). The combined totals from the two Wall site samples indicate that four of the eleven individuals identified are male. This is a considerably higher percentage ( $36.4 \%$ versus $18.9 \%$ ) than Gwynn's (1964) studies indicate would occur in the same general area today.

Other than turkey, birds identified in the 1983-1984 assemblage from the Wall site consist of bobwhite quail, bluejay, great horned owl, and passenger pigeon. Passenger pigeon is represented by a single individual in the 19831984 assemblage. The bluejay, great horned ow 1 and bobwhite quail also are represented by only a single individual.

A total of 13,010 bones, representing a minimum of 69 mammals, was identified in the 1983-1984 assemblage. With the exception of the white-tailed deer $(M N I=36)$, squirrel $(M N I=10)$, raccoon (MNI=4), and rabbit $(M N I=4)$; none of the mammals in the assemblage accounted for more than two individuals $(0.7 \%$ of the total number of individuals).

White-tailed deer comprised 36 individuals (12.5\% of the total number of individuals), determined from 4,732 fragments. Because of the small number and fragmentary nature of the deer mandibles in this assemblage, it was not possible to determine the age distribution of all of the deer represented. Of the six mandibles that could be aged, using the method described by Severinghaus (1949), one was approximately 13-17 months old, one was approximately $2-1 / 2$ years old, one was approximately $5-1 / 2$ years old, and three (two lefts and one right) were approximately $7-1 / 2$ years old.

Additional information about the ages of deer hunted by the inhabitants of the Wall site was obtained by examining the epiphyses of the long bones. A minimum of six individuals in the population had open epiphyses (distal femur). This adds another five deer between the ages of $2-1 / 2$ and $4-1 / 2$ years (Lewall and Cowan 1963: 635). Using the criteria of pelvic suture closure (Edwards et al. 1982) it was determined that five individuals were less than one year old. Assuming that none of the long bones or pelves represented the same deer as the mandibles, it was possible to determine the ages of a maximum of 17 individuals. A more cautious approach assumes that a long bone, mandible, and/or pelvis falling in the same age category belonged to the same individual. Using this approach, a minimum of 15 individuals could be aged. Of these 15 individuals, $33.3 \%$ were less than $1-1 / 2$ years old, $46.7 \%$ were between $1-1 / 2$ and $5-1 / 2$ years old, and $20 \%$ were approximately $7-1 / 2$ years old. This sample is clearly too small to provide an accurate indication of the age distribution of the exploited population.

The sample studied by Runquist included 145 individuals $(46.0 \%$ of the total), 144 of which could be aged. Of these individuals, $17 \%$ were fawns, $63 \%$ were between $1-1 / 2$ and $7-1 / 2$ years old, and $20 \%$ were $7-1 / 2$ years old or older (Runquist 1979:229).

One method of determining the sex ratio of the deer represented by a faunal assemblage is through an examination of frontal bones for the presence of antlers, antler pedicles, or the denser bone that distinguishes males from females. This method was not useful for the 1983-1984 assemblage from the Wall site because very few deer skull fragments were recovered, and because the few antler fragments that were recovered were very small. However, it was possible to utilize a technique developed by Edwards et al. (1982) which uses characteristics of the pelvic girdle to distinguish male from female deer. For deer in which the sutures 
between the ilium, ischium, and pubis are fully ossified (deer one year old or older), the shape and position of the ilio-pectilineal eminence are different in males and females. Fourteen right and thirteen left innominate bones complete enough to display the ilio-pectilineal eminence were recovered in the 1983-1984 assemblage. Of these, five right and four left represented individuals below the age of one year and thus could not be used. On one left and one right innominate bone the characteristics of the ilio-pectilineal eminence were neither clearly male nor clearly female. Finally, however, it was possible to determine that five right and five left innominates represented males, and that three left and three right represented females.

An attempt was made to determine the ages of individuals of several species other than deer that were represented in the assemblage. Marks and Erickson (1966) developed criteria for determining ages of black bear based on skull morphology, canine cementum layers, tooth replacement and wear, epiphyseal suture closure, and baculum growth and maturation. As the only element identified as black bear in the Wall site assemblage was a single fragment of thoracic vertebra, it was not possible to determine the age of this individual. Although the age of raccoons can be determined using tooth wear criteria (Grau et al. 1970), this technique could not be applied successfully to the 1983-1984 faunal remains because no intact raccoon mandibles with enough teeth to permit aging were preserved in the assemblage. Age determination in fox and gray squirrels and in cottontail rabbits is based upon the degree of epiphyseal closure. The distal radius and ulna were utilized by Carson (1961) to develop age classes for squirrels. Of the 332 fragments identified as squirrel, only one was a distal radius and no distal ulnae were preserved. The epiphysis of the single distal radius was closed and thus indicated the presence of an individual at least 33 weeks old (Carson 1961:91). Hale's (1949) technique for aging cottontail rabbits is based on the degree of epiphyseal closure in the humerus. Four individuals from the present sample were represented by distal humeri, the epiphyses of which were all closed, indicating that these individuals were at least nine months old (Hale 1949:222).

No butchering marks were observed on any of the bones identified from the 1983-1984 Wall site assemblage. Guilday et al. (1962:64) indicate that it is possible to butcher an animal without leaving any marks on the bones, and that the probability that a bone will be cut in some way is greater if the person butchering the animal is unskilled, careless, or in a hurry. The absence of butchering marks on bones in the Wall site assemblage, thus, may indicate that the animals represented by the assemblage were dismembered by skillful and unhurried butchers. Although the majority of the bone from the Wall site was well preserved, the outer surface of most of the bones was somewhat eroded. It is possible, therefore, that if the original butchering did not leave deeply cut marks, those marks could have become worn away with the passage of time.

The only bone tools found in the 1983-1984 assemblage were one deer metatarsal beamer, one complete turkey tarsometatarsus awl and fragments of three more awls (Figure 10.1). Three small pieces of worked antler and one cut bird bone that might have been a bead were also found. In sum, analysis of the faunal remains from the 1983-1984 excavations at the Wall site identified a total of 288 individuals representing 32 species. The five most important species in terms of percent of MNI were catfish $(64.93 \%)$, deer $(12.50 \%)$, squirrel $(3.47 \%)$, frog $(2.78 \%)$, and box turtle $(1.74 \%)$.

\section{RESULTS OF ANALYSIS: FREDRICKS SITE}

The faunal remains from the Fredricks site were recovered from the fill of nine burials and five features. A total of 16,393 fragments from this site was examined. This total consists of 3,428 fragments from the 1/2-inch screen, 11,494 fragments from the 1/4-inch screen, and 1,469 fragments from the $1 / 16$-inch screen. A total of 142 individuals representing 35 species was identified.

A full listing of the faunal remains from the Fredricks site is provided in Table 10.2. A brief discussion of the results of analysis of the site as a whole is provided below. Following that, a more detailed treatment of the same remains is provided within a discussion of the features and burials from which the remains were recovered.

A total of 727 fragments from the assemblage were identified as fish. These fragments

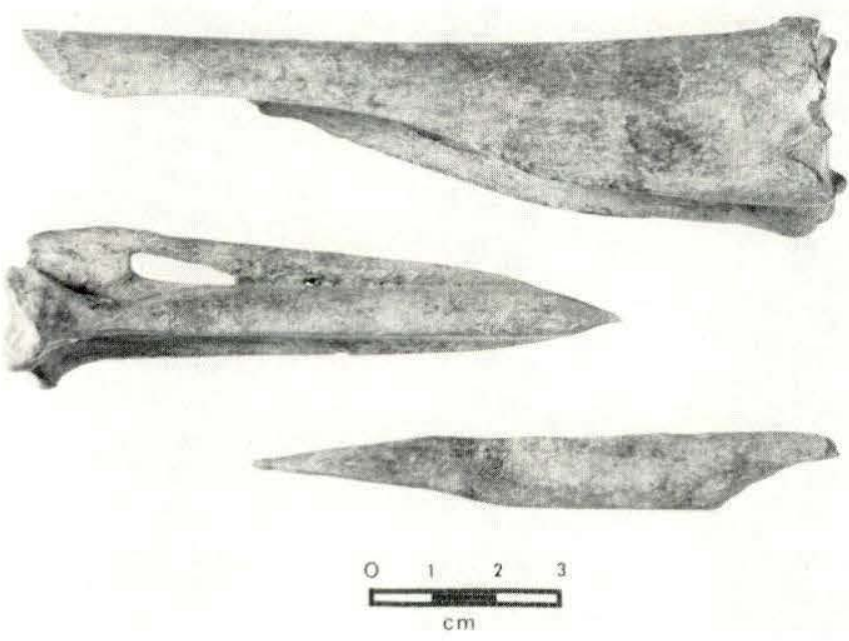

Figure 10.1

Bone beamer and awls from the Wall site. 
Table 10.2

Animal remalns from the Fredricks Site.

\begin{tabular}{|c|c|c|c|c|c|c|}
\hline Species & Frag. & \% Frag. & Wt. $(\mathrm{g})$ & \% wt. & MNI & $\%$ MNI \\
\hline$\frac{\text { Odocolleus }}{\text { talled }} \frac{\text { virgtnianus }}{\text { Deer }}$, White- & 1134 & 6.92 & 4227.34 & 44.30 & 9 & 6.34 \\
\hline Didelphis marsuplalis, Opossum & 1 & .01 & .30 & .00 & 1 & .70 \\
\hline$\frac{\text { Sciurus }}{\text { Squirrel }} \frac{\text { carolinensis, }}{\text { rray }}$ & 8 & .05 & 3.01 & .03 & 2 & 1.41 \\
\hline Sciurus niger, Fox Squirrel & 5 & .03 & 1.73 & .02 & 1 & .70 \\
\hline Sciurus sp. & 82 & .50 & 4.58 & .05 & 2 & 1.41 \\
\hline Procyon lotor, Raccoon & 22 & .13 & 11.04 & .12 & 1 & .70 \\
\hline Mephitis mephitis, Striped Skunk & 1 & .01 & .70 & .01 & 1 & .70 \\
\hline 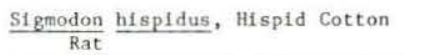 & 11 & .07 & .22 & .00 & 2 & 1.41 \\
\hline$\frac{\text { Peromyscus }}{\text { Mouse }}$ leucopus, White-footed & 29 & .18 & .29 & .00 & 2 & 1.41 \\
\hline$\frac{\text { Blarina brevicauda }}{\text { Shrew }}$, Short-tafled & 1 & .01 & .01 & .00 & 1 & .70 \\
\hline Ursus americanus, Black bear & 10 & .06 & 90.60 & .95 & 1 & .70 \\
\hline Equus caballus, Horse & 1 & .01 & 22.70 & .24 & 1 & .70 \\
\hline Sus scrofa, P1g & 1 & .01 & 24.50 & .26 & 1 & .70 \\
\hline Felis concolor, Mountain Lion & 1 & .01 & 2.50 & .03 & 1 & .70 \\
\hline Urocyon c1nereoargenteus, Gray Fox & 38 & .23 & 3.60 & .04 & 1 & .70 \\
\hline Canis $\underline{\text { fam1l1aris, }}$, Dog & 5 & .03 & .10 & .00 & 1 & .70 \\
\hline Unidentifted Mamnal & 3487 & 21.27 & 2332.57 & 24.45 & - & - \\
\hline Meleagris gallapavo, Wild Turkey & 148 & .90 & 221.81 & 2.32 & 4 & 2.82 \\
\hline$\frac{\text { Ectopistes }}{\text { Passenger Pigeon }}$ & 47 & .29 & 18.76 & .20 & 6 & 4.22 \\
\hline Charadrifdae, Plovers & 1 & .01 & .10 & .00 & 1 & .70 \\
\hline Fring1llidae, Sparrows & 7 & .04 & .15 & .00 & 2 & 1.41 \\
\hline Colinus virginfanus, Bobwhite & 3 & .02 & .11 & .00 & 1 & .70 \\
\hline$\frac{\text { Centurus }}{\text { Woodpecker }}$ carolinus, Red-bellied & 1 & .01 & .02 & .00 & 1 & .70 \\
\hline Anat 1dae, Duck & 2 & .01 & 1.70 & .02 & 1 & .70 \\
\hline Aytha affinis, Lesser Scaup & 7 & .04 & 2.50 & .03 & 1 & .70 \\
\hline Unidentified Bird & 374 & 2.28 & 72.66 & .76 & - & - \\
\hline Terrapene carolina, Box Turtle & 1065 & 6.50 & 1013.73 & 10.62 & 10 & 7.04 \\
\hline$\frac{\text { Chelydra }}{\text { Turt }} \frac{\text { serpentina, Snapping }}{1 \mathrm{e}}$ & 2 & .01 & 18.90 & .20 & 1 & .70 \\
\hline Chrysemys picta, Painted Turtle & 3 & .02 & 8.00 & .08 & 1 & .70 \\
\hline$\frac{\text { Sternothaerus oderatus, Musk }}{\text { Turt le }}$ & 3 & .02 & .60 & .01 & 1 & .70 \\
\hline$\frac{\text { Kinosternon }}{\text { Turt le }}$ subrubrum, Mud & 1000 & .04 & .63 & .01 & 3 & 2.11 \\
\hline Unidentified Turtle & 1090 & 6.65 & 244.38 & 2.56 & - & - \\
\hline Crotalid sp., Polsonous Snake & 2 & .01 & 1.56 & .02 & 1 & .70 \\
\hline Unidentified Snake & 226 & 1.38 & 10.05 & .10 & - & - \\
\hline$\frac{\text { Scaphiopus }}{\text { Spadefoot Toad }}$ & 31 & .19 & .63 & .01 & 3 & 2.11 \\
\hline Rana sp., Frog & 60 & .37 & 2.92 & .03 & 4 & 2.82 \\
\hline Bufo sp., Toad & 1 & .01 & .80 & .01 & 1 & .70 \\
\hline Unidentified Amphibian & - & - & - & - & - & - \\
\hline Ictalurus sp., Catfish & 72 & .44 & 1.80 & .02 & 69 & 48.59 \\
\hline Catostomus sp., Suckers & 57 & .35 & 1.36 & .01 & 1 & .70 \\
\hline Lepisosteus sp., Gar & 48 & .29 & 1.56 & .01 & 1 & .70 \\
\hline Lepomis sp., Sunfish & 7 & .04 & .58 & .01 & 1 & .70 \\
\hline Unidentified Fish & 543 & 3.31 & 12.11 & .13 & - & - \\
\hline Sub-Total (Identifled to Class) & 8643 & 52.76 & 8363.21 & 87.66 & - & - \\
\hline Sub-Total (Unidentified) & 7750 & 47.28 & 1178.30 & 12.35 & $\overline{2}$ & $99 . \overline{9}$ \\
\hline Total & 16393 & 100.04 & 9541.51 & 100.01 & 142 & 99.90 \\
\hline
\end{tabular}


represented a minimum of 72 individuals ( $50.7 \%$ of the total number of individuals). The vast majority of these were catfish, the most abundant species (in terms of MNI) in the assemblage. Other fish identified were sunfish, sucker, and gar.

Amphibians accounted for 8 individuals (5.6\% of the tota1), represented by 92 fragments. The only amphibians identified were spadefoot toad, frog, and unspecified toad.

Reptiles were represented by 17 individuals $(12.0 \%$ of the tota 1$)$ determined from 2,397 fragments. Most of the fragments identified as reptiles were small fragments of turtle carapace. Box turtle accounted for 10 of the individuals $(7.0 \%$ of the total) and was the second most abundant species in terms of MNI. A large number (228 fragments) of snake bones was recovered, but many of these were ribs or fragmented vertebrae that could not be identified as to species.

Turkey and passenger pigeon were the most abundant bird species identified. Passenger pigeon accounted for six individuals $(4.2 \%$ of the total), identified from 47 fragments. Turkey was represented by 148 fragments, accounting for four individuals $(2.8 \%$ of the total). Based on the presence of spurs, three of the four individuals were males. Other birds identified were bobwhite quail, red-bellied woodpecker, lesser scaup, and members of the Charadriidae (plover) Fringillidae (sparrow), and Anatidae (duck) families.

Approximately $56 \%$ of the identified bone fragments from the Fredricks site belonged to mammals. With the exception of the white-tailed deer $(M N I=9)$ and squirrel $(M N I=5)$, none of the mammalian species identified was represented by more than two individuals. The presence of European-introduced mammals in the assemblage is indicated by a single femur fragment of a pig and a single horse molar.

The presence of a minimum of nine deer (6.3\% of the total) was determined from 1,134 fragments. There were four deer mandibles in the assemblage that were complete enough to be aged using the technique based on tooth development and wear described by Severinghaus (1949). of these four, one was approximately $4-1 / 2$ years old, one $5-1 / 2$ years old, one $7-1 / 2$ years old, and one $8-1 / 2$ to $9-1 / 2$ years old. Through an examination of the epiphyses of the long bones of the deer, it was determined that two individuals had unfused distal femora and could thus be aged at between $2-1 / 2$ and $4-1 / 2$ years (Lewal1 and Cowan 1963:635). A sample of six individuals is too small to permit conclusions about possible exploitation strategies based on age for the Fredricks site. Of the deer that could be aged, however, $50.0 \%$ were between $2-1 / 2$ and $4-1 / 2$ years old, $16.7 \%$ were approximately $5-1 / 2$ years o1d, $16.7 \%$ were approximately $7-1 / 2$ years old and $16.7 \%$ were approximately $8-1 / 2$ to
$9-1 / 2$ years old.

There were no innominate bones preserved in the Fredricks site assemblage, upon which Edward's (1982) criteria for sex determination could be applied. Two of the deer frontal fragments recovered at this site were fairly delicate and did not possess antlers, and another frontal piece had an antler attached. These fragments indicate the presence of at least one male and possibly two females.

of the ten fragments identified as black bear, only one (a proximal metacarpal) could be utilized with the methods described by Marks and Erickson (1966) for determining age. This single bone indicated an individual between the ages of one and two years (Marks and Erickson 1966:404).

The technique proposed by Grau et al. (1970) for determining the age of raccoons could not be applied to the faunal assemblage from the Fredricks site. This technique is based on an analysis of wear on the lower teeth of the raccoon. No mandibles with adequately preserved dentition were recovered.

Although 95 bones and bone fragments were identified as squirrel, none of these was distal radii or distal ulnae. Because of the lack of these elements, it was not possible to use Carson's (1961) technique for determining age of gray and fox squirrels.

Cut marks were observed on a total of twenty of the deer bones in the Fredricks site assemblage. The neck portion of one scapula exhibited several transverse cut marks, as did the distal epiphyses of four humerii. The proximal epiphyses of one tibia and two radii al1 exhibited several cut marks. One pubis fragment exhibited what appears to be a cut made by an axe and two ilium fragments exhibited cut marks. Three rib fragments, one cervical vertebra, three lumbar vertebrae, and one astragalus also had cut marks. A cut mark on one of the rib fragments may have been inflicted with an axe. These fragments represent $1.8 \%$ of the deer bones recovered at the Fredricks site. Because this is such a small percentage, it is difficult to reconstruct the butchering process utilized by the original inhabitants. However, most of the cut marks are consistent with the skinning and butchering procedures reported for several prehistoric sites in the east (e.g., Guilday et a1. 1962).

Fragments of three bone knife handles and a highly polished, tapered splinter of bone that might have been a needle were the only examples of worked bone found at the Fredricks site. All four items had been manufactured from mammal bones but it was not possible to determine the species. 


\section{Feature Fil1}

There were three zones of fill in Burial 1 containing a total of 3,169 bone fragments, 504 of which could be identified to species. The majority of the bones $(89.2 \%)$ were retrieved from the top zone of fill, which was a dark brown organically rich soil. The mammals identified were white-tailed deer, opossum, gray squirre1, squirrel sp., and raccoon. Birds consisted of turkey, passenger pigeon, bobwhite quail, red-bellied woodpecker, and a single fragment belonging to the family Charadriidae (plovers). The reptiles and amphibians identified were frog, box turtle, and musk turtle. The four types of fish identified from this pit were catfish, sucker, sunfish, and gar.

There were only two zones of fill in Burial 2 , and the top zone, a dark brown humus, contained $84.5 \%$ of the bone fragments. The fill of this pit contained only 129 animal bone fragments, 30 of which were identified to species. Deer, squirrel, and raccoon were the only mammals identified, and the only birds identified were turkey and passenger pigeon. Box turtle was the only identifiable reptile, there were no amphibian remains, and there was only one fish bone (catfish).

The two zones of fill in Burial 3 contained 5,008 fragments of bone, 873 of which could be identified to species. Of the total number of animal bone fragments recovered from the site, $30.5 \%$ were recovered from the fill of this pit. Although a few unidentifiable fragments were located in the lower zone of fill, $99.4 \%$ were in the top zone of dark brown humus. Identified mammals consisted of black bear, white-tailed deer, gray squirrel, raccoon, skunk, and cotton rat. A single fragment was identified as domestic pig. The birds identified were turkey, passenger pigeon, and lesser scaup. Reptiles and amphibians were comprised of box turtle, snapping turtle, painted turtle, musk turtle, mud turtle, Crotalidae (poisonous snake), and frog. Fish identified were catfish, gar, and sucker.

Feature 1 had two zones of fill, the uppermost of which contained $95.6 \%$ of the 1,539 animal bone fragments. Of these, 257 fragments could be identified to species. The mammals represented were white-tailed deer, squirrel, raccoon, and cotton rat. The only birds represented were turkey and passenger pigeon. Remains of box turtle, mud turtle, poisonous snake, frog, catfish, sucker, and gar were also recovered.

Two major zones of fill were identified in Feature 2/Burial 4 and a total of 982 bone fragments (157 of which could be identified) was recovered. The first zone, a dark brown soil with charcoal fragments, contained $65.6 \%$ of the bone in this pit. The second zone, a mottled orange clay, contained $34.4 \%$ of the bone. White-tailed deer, raccoon, white-footed deer mouse, turkey, passenger pigeon, and box turtle were identified.

of the 2,375 bone fragments in the fill of Feature 3/Burial 5, 467 were identified. There were two major zones of fill. The uppermost zone (a brown loam with ash) contained $82.8 \%$ of the bone and a second zone (mottled orange clay) contained $17.2 \%$. The mammals represented in the fill were white-tailed deer, gray squirrel, fox squirrel, squirrel sp., raccoon, cotton rat, meadow vole, white-footed deer mouse, shorttailed shrew, mountain lion, and black bear. Turkey and passenger pigeon were the only birds present; whereas toad, frog, box turtle, mud turtle, and unidentifiable snake made up the reptiles and amphibians. Fish identified were catfish, suckers, and gar.

The five zones of fill in Feature 4/Burial 6 contained a total of 301 bone fragments. Only 23 of these fragments could be identified. In the other burial pits, the majority of the animal bone was located in an uppermost zone of dark organic soil. In Feature 4/Burial 6, however, $65.4 \%$, of the bone fragments were from two deeper zones of mottled orange clay, and $23.6 \%$ were from two zones of brown loam mottled with orange clay. Only $11.0 \%$ of the bone was retrieved from the uppermost zone of dark organic soil. All of the bone fragments which could be identified from this pit were white-tailed deer.

No animal bone fragments were found in the fill of Feature 5/Burial 7.

Six major zones of fill were distinguished in Feature 6/Burial 8. These zones contained a total of 683 bone fragments, 110 of which were identifiable to species. The first zone, a brown loam with numerous small pebbles, contained $39.8 \%$ of the bone fragments. The third zone, also a brown loam, contained $37.2 \%$, Zone 5 contained $10.5 \%$, and the rest $(12.5 \%)$ was contained in the bottom zone. Animals represented were white-tailed deer, squirrel, raccoon, white-footed deer mouse, passenger pigeon, box turtle, snapping turtle, and painted turtle.

In Feature 7/Burial 9, there were two primary zones of fill containing 217 fragments of animal bone. Only 15 of these fragments were identifiable, and all were white-tailed deer. The deepest zone of fill, a mottled orange clay, contained $65.9 \%$ of the bone, and the rest $(34.19 \%)$ was contained in the upper (brown loam soil) zones of fill.

Feature 9 has been interpreted as a fire pit associated with Structure 1, probably the remains of a sweat house. The bottom of this pit was lined with charred bark, and clusters of charred maize kernels were found lying within the charred remains of woven containers, probably baskets. Along with the maize kernels, one of these clusters contained the charred foot 
bones of a gray fox. The uppermost zone of fill in this pit (a dark yellowish-brown sandy ash) contained $26.1 \%$ of the bone fragments, the center zone (a combination of fill similar to that in Zone 1 mixed with orange clay) contained $6.7 \%$, and the deepest zone (charcoal, reddish clay, and ash), which contained the charred maize, accounted for $67.2 \%$ of the bone. All of the bone fragments in this third zone of fill were charred. In addition to the fox bones and a single horse molar, there was white-tailed deer, raccoon, bear, and dog.

Feature 10 was a trash-filled storage pit with two zones of fill. The uppermost zone was a dark brown loam, which contained $96.3 \%$ of the 722 animal bone fragments. Of these fragments, 134 could be identified as white-tailed deer, squirrel, turkey, and box turtle.

Feature 11 contained 13 identifiable bones (from a total of 94 fragments), all of which were identified as white-tailed deer. There was only one zone of fill in this feature.

Feature 12 had two zones of fill containing 282 bone fragments. The upper zone, a dark reddish-brown soil, contained $54.2 \%$ of the bone, and the lower, a brown sandy loam mottled with orange clay, contained 45.7\%. The 75 identifiable bones were comprised of white-tailed deer, squirrel, white-footed deer mouse, black bear, and box turtle.

There were two zones of fill in Feature 13. An uppermost shallow zone of mottled yellow clay, which contained almost no bone, intruded into a thicker zone of dark brown, highly organic soil, which contained $98.1 \%$ of the bone. of the 755 bone fragments, 209 were identifiable. Animals represented were white-tailed deer, fox squirrel, squirrel sp., raccoon, bear, turkey, passenger pigeon, sparrow, box turtle, frog, sunfish, and sucker.

The four burial pits most similar in terms of fill were Burial 1, Burial 2, Burial 3, and Feature 1. In all of these pits, the vast majority of the animal bone was recovered from the uppermost zone of fill, a dark, organically rich soil. The bone from these pits was well preserved and each pit contained most of the 31 species identified in the overall assemblage. The four pits were also very closely aligned in terms of spatial arrangement.

Feature 2/Burial 4 is somewhat similar to these four pits in that the majority of the bone fragments were recovered from an upper zone of dark organic fill. Only $65.6 \%$ of the bone from this pit was recovered from this zone, however, as opposed to the $84.5-99.4 \%$ for the same zone in the other aforementioned pits.

Feature 3/Burial 5 likewise could be grouped with the burial pits mentioned above. The majority of the bone was recovered from an upper zone of fill that consisted of a dark organic soil. Also, the species identified in Feature 3 /Burial 5 were almost identical to those identified in Feature 1.

Feature 7/Burial 9 and Feature 4/Burial 6 were very similar to one another and quite different from the other pits. In addition to being in adjacent positions, the two pits are similar in that the only identifiable remains recovered in either is white-tailed deer. The remainder of the bone fragments were too poorly preserved to identify. In both pits, approximately $65 \%$ of the bone was recovered in a deep zone of mottled orange clay. It is likely that the acidic nature of this clay is responsible for the poor preservation. Zones of brown loam or humus were identified in each of these pits, but unlike Burials $1-3$ and Feature 1, these zones contained very few animal bones.

Feature 6/Burial 8 was unique in that the faunal remains were recovered in zones of brown loamy soil separated from one another by zones of orange and brown mottled clay. No single zone contained the vast majority of bone. The preservation of the bone in this pit was not as good as in the other burial pits.

Finally, Feature 5/Burial 7 was unique in that it was the only burial pit from which no faunal remains were recovered. This pit was also more shallow (by $0.75 \mathrm{ft}$ ) than any of the other pits and lacked an upper zone of dark organic soil (which may have been plowed away).

\section{COMPARISON OF THE TWO ASSEMBLAGES}

Before a discussion of the use of faunal resources by the inhabitants of the two sites can be attempted, the state of preservation of the two faunal assemblages should be evaluated. As noted earlier, the contexts from which the bones were retrieved at the two sites were dissimilar. The majority of the bones from the Fredricks site were recovered from burial pit fill, whereas the majority of those from the Wall site were recovered from deposits of sheet midden. It has been suggested that "small fragments just would not survive" in a midden deposit (Runquist 1979:342) and that bones deposited in pits are less likely to be stepped on, exposed to scavengers, or damaged by weather than are bones which are not placed in pits (Chap1in 1971:16; Waselkov 1977:84).

At the Wall site, $19.96 \%$ of the bone was retrieved from 1/2-inch screen, $65.07 \%$ from $1 / 4-i n c h$ screen, and $14.97 \%$ from $1 / 16$-inch screen. At the Fredricks site, $20.91 \%$ was recovered from 1/2-inch screen, $70.12 \%$ from $1 / 4-$ inch screen, and $8.96 \%$ from 1/16-inch screen. Obviously, more small bone fragments were preserved in the midden deposits from the Wall site than in the pitfill at the Fredricks site. It should be noted that only those bones and bone fragments that appeared to be identi- 
fiable were pulled from the material recovered in the 1/16-inch screen. Thus, the percentage of small, identifiable fragments is actually higher in the Wall site assemblage than in the Fredricks site assemblage. At both sites, much of the bone recovered in the $1 / 2$-inch screen consisted of identifiable fragments of bones of larger animals and complete, or nearly complete, bones from medium-sized animals. Identifiable bone from the 1/16-inch screen belonged, for the most part, to smaller species, such as fish and amphibians. The vast majority of the bone recovered in the $1 / 4-i n c h$ screen, however, consisted of fragments of bone that were too small and/or too fragmented to be identified. The percentage of bone fragments that could not be identified was higher for the Fredricks site assemblage $(47.28 \%)$ than for the Wall site assemblage $(42.02 \%)$. It is likely that this is a result of the fact that the percentage of bone recovered in the $1 / 4-i n c h$ screen was also higher at the Fredricks site than at the Wall site.

Another way in which the condition of the bones from the two sites can be evaluated is by comparing the extent of fragmentation of the bones in the two assemblages. Extent of fragmentation can be determined from the number of fragments of deer bones present per individual identified (Runquist 1979:172). At the Wall site, a minimum of 36 individuals and 4,731 fragments were identified as white-tailed deer, which yields a ratio of 131.42 fragments per individual. For the Fredricks site, nine individuals and 1,128 fragments were identified as white-tailed deer, which yields a ratio of 125.33 fragments per individual. Thus it seems that, at least for the white-tailed deer, the bones in the Fredricks site assemblage are only slightly less fragmented than those in the Wall site assemblage. As noted earlier, the faunal remains from the Fredricks site may represent refuse cleaned from house floors, which would make the original contexts of the analyzed bone from both sites quite similar. Therefore, the slight difference in the ratios suggests to some extent that large bones deposited in pits may not be subjected to factors causing fragmentation as frequently as those deposited in sheet midden.

It should be noted that $30.7 \%$ of the bone from the Fredricks site was burned whereas only $8.9 \%$ of the bone from the Wall site was burned. This suggests the possibility that the deposits from which the Fredricks site assemblage were derived represent a limited range of activities such as cleaning house floors or hearths. A higher percentage of activities that did not produce burned bone may be represented by the Wa11 site assemblage. Table 10.3 shows the percentage of deer skeletal elements represented in the Wall and Fredricks site assemblages. With the exception of five elements (innominate, atlas, axis, cervical 3-7 vertebrae, sacrum, and patella) there is a higher percentage of every element represented at the Fredricks site than at the Wall site. This is one indication that the Fredricks site assemblage is better preserved than that from the Wall site. However, it could also be an indication that deer bones were treated differently by the inhabitants of the two sites. If, for example, the inhabitants of one of the sites frequently utilized deer bones as tools, it is possible that certain skeletal elements would not be discarded in the midden as food refuse. These tools would be curated, and thus would not be recovered in the midden in the same percentages as would be

Table 10.3

Expected and actual representation of deer skeletal elements.

\begin{tabular}{|c|c|c|c|}
\hline Element & $\begin{array}{l}\text { Expected } \\
\text { Freq./Ind. }\end{array}$ & $\begin{array}{l}\text { Wall } \\
\text { (36 Ind.) }\end{array}$ & $\begin{array}{r}\text { Fredricks } \\
(9 \text { Ind.) }\end{array}$ \\
\hline Occipital & 1 & 11.1 & 22.2 \\
\hline Frontal & 2 & 4.2 & 11.1 \\
\hline Hyoid, half & 2 & 1.4 & 11.1 \\
\hline Mandible, half & 2 & 56.9 & 61.1 \\
\hline Maxi11a & 2 & 23.6 & 33.3 \\
\hline At las & 1 & 11.1 & 0.0 \\
\hline AxIs & 1 & 38.9 & 33.3 \\
\hline Cervical 3-7 Vertebrae & 5 & 23.3 & 8.9 \\
\hline Thoracic Vertebrae & 13 & 8.1 & 17.1 \\
\hline Lumbar Vertebrae & 6 & 24.5 & 86.1 \\
\hline Sacrum & 1 & 16.7 & 11.1 \\
\hline Scapula & 2 & 77.8 & 88.9 \\
\hline Humerus, proximal & 2 & 20.8 & 44.4 \\
\hline Humerus, distal & 2 & 87.5 & 100.0 \\
\hline Radius, proximal & 2 & 47.2 & 61.1 \\
\hline Radius, distal & 2 & 15.3 & 55.6 \\
\hline Ulna & 2 & 47.2 & 66.7 \\
\hline Metacarpal, proximal & 2 & 13.9 & 94.4 \\
\hline Innominate & 2 & 95.8 & 83.3 \\
\hline Femur, proximal & 2 & 44.4 & 66.7 \\
\hline Femur, distal & 2 & 37.5 & 72.2 \\
\hline Tibia, proximal & 2 & 48.6 & 72.2 \\
\hline Tibia, distal & 2 & 4.2 & 66.7 \\
\hline Patella & 2 & 11.1 & 5.6 \\
\hline Metatarsal, proximal & 2 & 45.8 & 77.8 \\
\hline Metatarsal, distal & 4 & 33.3 & 72.2 \\
\hline Astragalus & 2 & 27.8 & 66.7 \\
\hline Calcaneur & 2 & 30.6 & 94.4 \\
\hline Proximal Phalanx & 8 & 17.4 & 54.2 \\
\hline Second Phalanx & 8 & 8.3 & 27.8 \\
\hline Distal Phalanx & 8 & 16.3 & 23.6 \\
\hline
\end{tabular}


expected if preservation were the only factor being considered.

For example, proximal metacarpals and metatarsals should survive better than lessresistant elements such as proximal humeri or frontals. Proximal metacarpals and metatarsals were made into beamers by some Piedmont groups, and several of these hide-working tools have been identified in the Wall site assemblage (one in the 1983-1984 assemblage and nine in the assemblage examined by Runquist). As yet, however, no tools of this kind have been recovered from the Fredricks site, whose original inhabitants had access to metal tools that may have made bone beamers obsolete. The percentages of proximal metacarpals (13.9\%) and metatarsals $(45.8 \%)$ recovered at the Wall site are not much higher than the percentages of other elements which could have been expected to be less wel1-preserved. At the Fredricks site, the percentages of proximal metacarpals ( $94.4 \%)$ and of metatarsals $(77.8 \%)$ is considerably higher than the percentages for many of the other elements. Thus, it is likely that the different representation of deer skeletal elements at the two sites is a result of differential patterns of use and/or discard of the bones by the inhabitants of the two sites in addition to the possible effects of differential preservation.

There is no evidence, therefore, that the bone from one site is appreciably better preserved than the bone from the other site. It follows also that there is little indication, in this case, that bones deposited in a pit will be better preserved than those discarded in an open midden. It is possible, however, that large bones deposited in pits will be slightly less fragmented than bones deposited in sheet midden.

Overall, the faunal assemblages from the Wall and Fredricks sites are very similar. Only seven species were identified at the Wall site that were not present in the Fredricks site assemblage. These were rabbit, beaver, chipmunk, muskrat, meadow vole, flying squirrel, and bluejay. With the exception of rabbit $(M N I=4)$ and meadow vole $(M N I=2)$, none of these species was represented by more than a single individual. Whereas meadow vole was represented by two individuals, it is very likely that these burrowing animals were intrusive in the deposit and were not utilized by the site's inhabitants. Rabbit is the only species from the Wall site assemblage that can be considered notable in its absence from the Fredricks site assemblage.

Ten species were identified from the Fredricks site that were not identified in the 1983-1984 assemblage from the Wall site. These were horse, pig, skunk, gray fox, mountain lion, red-bellied woodpecker, lesser scaup, musk turtle, sunfish, one individual belonging to the family Charadriidae (plover), and one individual belonging to the family Fringillidae (sparrow). As only one of these species, skunk, was present in the assemblage analyzed by Runquist, it is likely that none of these species was utilized to any great extent, if at a11, by the inhabitants of the Wall site. With the exception of the sparrow (MNI=2), these species were only represented by a single individual each in the Fredricks site assemblage. The presence of two European-introduced mammals, pig and horse, in the Fredricks site assemblage is important. However, pig was represented by only one femur fragment and horse by only one molar.

Thus, based on the presence or absence of individual species, the data suggest there were no major differences in the utilization of faunal resources by the inhabitants of the two sites. The two exceptions noted are the absence of rabbit and the presence of two European domesticates in the Fredricks site assemblage.

Although the gross inventories of species utilized by the inhabitants of the two sites are virtually the same, there seem to be differences in the ways and/or relative amounts in which these species were procured and/or utilized. In an attempt to determine which species were most important in the diet of the inhabitants of the sites, the amount of meat available from each was calculated, using estimations by Smith (1975a), White (1953), and Cleland (1966). These figures are presented in Table 10.4. It should be noted that the bones, skins, furs, and carapaces of these animals were often important to the Indians as materials for tools, clothing, utensils, and other material goods. Thus, a particular species would not always have been selected on the basis of its value as a source of food. The presence of only a single molar identified as horse indicates that this animal probably was not used for food by the inhabitants of the Fredricks site. Therefore, the amount of meat provided by this animal was not included in the calculations of available meat at this site.

The most important animals in the Wall site assemblage, listed in rank order of estimated meat yield, were deer, catfish, bear, raccoon, beaver, and turkey. At the Fredricks site the order was deer, bear, catfish, pig, mountain lion, turkey, and raccoon. Again, the assemblages appear to be quite similar.

In an attempt to gain a more detailed indication of the relative importance of the various species utilized, thirteen species or species groups were ranked according to a technique proposed by Smith (1975b:125-127). Using this approach, the species were ranked by their relative importance in terms of both the minimum number of individuals and projected meat yield. The results are shown in Figure 10.2.

At both sites, the species cluster into four groups. Deer and fish ranked very high on both scales and were evidently the most important faunal resources at the two sites. The second cluster consists of animals that 
Table 10.4

Estimated meat yteld in pounds.

\begin{tabular}{|c|c|c|c|c|c|}
\hline Spectes & $\begin{array}{l}\text { Estimated } \\
\text { Meat Yield/Ind. } \\
\text { (L.bs.) }\end{array}$ & \multicolumn{2}{|c|}{ Wa11 } & \multicolumn{2}{|c|}{$\begin{array}{l}\text { Fredricks } \\
\text { ibs. }\end{array}$} \\
\hline White-talled Deer & 85.0 & 3060.0 & 82.3 & 765.0 & 58.2 \\
\hline Opossun & 8.5 & 8.5 & 0.2 & 8.5 & 0.6 \\
\hline Gray Squirrel & 1.0 & 1.0 & 0.0 & 2.0 & 0.2 \\
\hline Fox Squitrel & 1.5 & - & - & 1.5 & 0.1 \\
\hline Squirrel sp. & 1.2 & 10.8 & 0.3 & 2.4 & 0.2 \\
\hline Raccoon & 15.0 & 60.0 & 1.6 & 15.0 & 1.1 \\
\hline Htspid Cotton Rat & 0.2 & 0.4 & 0.0 & 0.2 & 0.0 \\
\hline White-footed Deer Mouse & * & - & - & - & - \\
\hline Short-talled Shreu & * & - & - & - & - \\
\hline Meadow Vole & * & - & - & - & - \\
\hline Flying Squirrel & 0.1 & 0.1 & 0.0 & - & - \\
\hline Black Bear & 210.0 & 210.0 & 5.6 & 210.0 & 16.0 \\
\hline Rabblt & 2.0 & 8.0 & 0.2 & - & - \\
\hline Beaver & 31.5 & 31.5 & 0.8 & - & - \\
\hline$P 1 g$ & 75.0 & - & - & 75.0 & 5.7 \\
\hline Dog & 6.0 & - & - & 6.0 & 0.4 \\
\hline Mountain Lion & 60.0 & - & - & 60.0 & 4.6 \\
\hline Muskrat & 2.0 & 2.0 & 0.0 & - & - \\
\hline Gray Fox & 5.0 & - & - & 5.0 & 0.4 \\
\hline Chi pmunk & $*$ & - & - & - & - \\
\hline Total Mammal & - & 3392.3 & 91.0 & 1150.6 & 87.5 \\
\hline Turkey & 8.5 & 25.5 & 0.7 & 34.0 & 2.6 \\
\hline Passenger Plgeon & 0.7 & 0.7 & 0.0 & 4.2 & 0.3 \\
\hline Plover & * & - & - & - & - \\
\hline Sparrov & $*$ & - & - & - & - \\
\hline Bobwhite & 0.3 & 0.3 & 0.0 & 0.3 & 0.0 \\
\hline Red-bell1ed Woodpecker & * & - & - & - & - \\
\hline Blue Jay & 0.1 & 0.1 & 0.0 & - & - \\
\hline Lesser Scaup & 1.0 & - & - & 1.0 & 0.1 \\
\hline Great Horned Owl & 2.0 & 2.0 & 0.0 & - & - \\
\hline Unidentified Duck & 1.0 & - & - & 1.0 & 1.0 \\
\hline Total Bird & - & 28.6 & 0.7 & 40.5 & 3.1 \\
\hline Frog & $\cdot$ & - & - & - & - \\
\hline Tosd & • & - & - & - & - \\
\hline Spadef oot Toad & * & - & - & - & - \\
\hline Total Amphlblan & - & - & - & - & - \\
\hline Box Turtle & 0.3 & 1.5 & 0.0 & 3.0 & 0.2 \\
\hline Snapping Turtle & 10.0 & 10.0 & 0.3 & 10.0 & 0.8 \\
\hline Pafnted Turtle & 0.3 & 0.3 & 0.0 & 0.3 & 0.0 \\
\hline Musk Turtle & 0.3 & - & - & 0.3 & 0.0 \\
\hline Mud Turtle & 0.3 & 0.3 & 0.0 & 0.9 & 0.1 \\
\hline Snakes & 0.2 & 0.2 & 0.0 & 0.2 & 0.0 \\
\hline Total Reptile & - & 12.3 & 0.3 & 14.7 & 1.1 \\
\hline Catfish & 1.5 & 280.5 & 7.5 & 103.5 & 7.9 \\
\hline Sunf 1 sh & 1.0 & 1.0 & - & 1.0 & 0.1 \\
\hline Gar & 1.0 & 1.0 & 0.0 & 1.0 & 0.1 \\
\hline Suckers & 4.0 & 4.0 & 0.1 & 4.0 & 0.3 \\
\hline Total Fish & - & 286.5 & 7.6 & 109.5 & 8.4 \\
\hline Total & - & 3719.7 & 99.6 & 1315.3 & 100.1 \\
\hline
\end{tabular}

WALL SITE

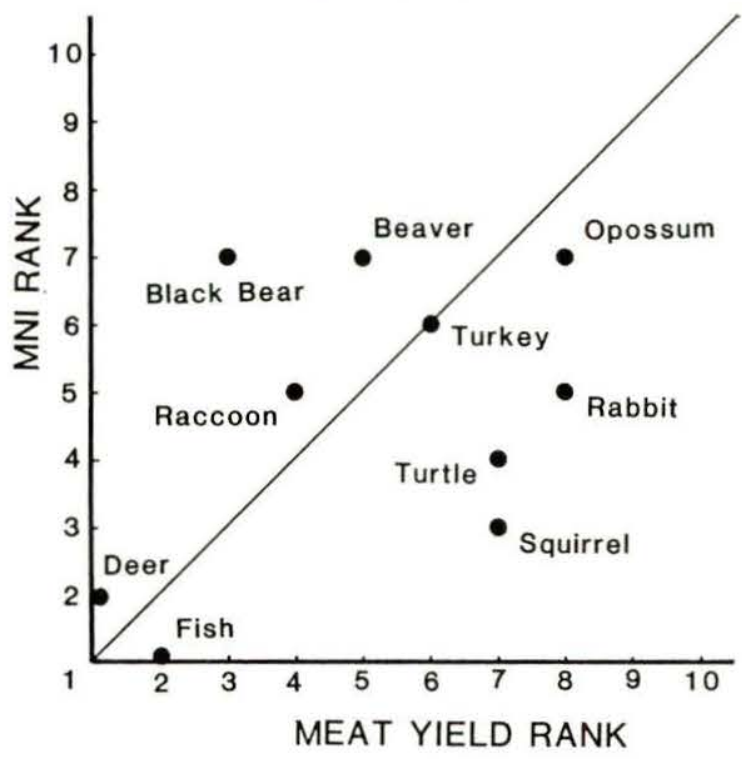

FREDRICKS SITE

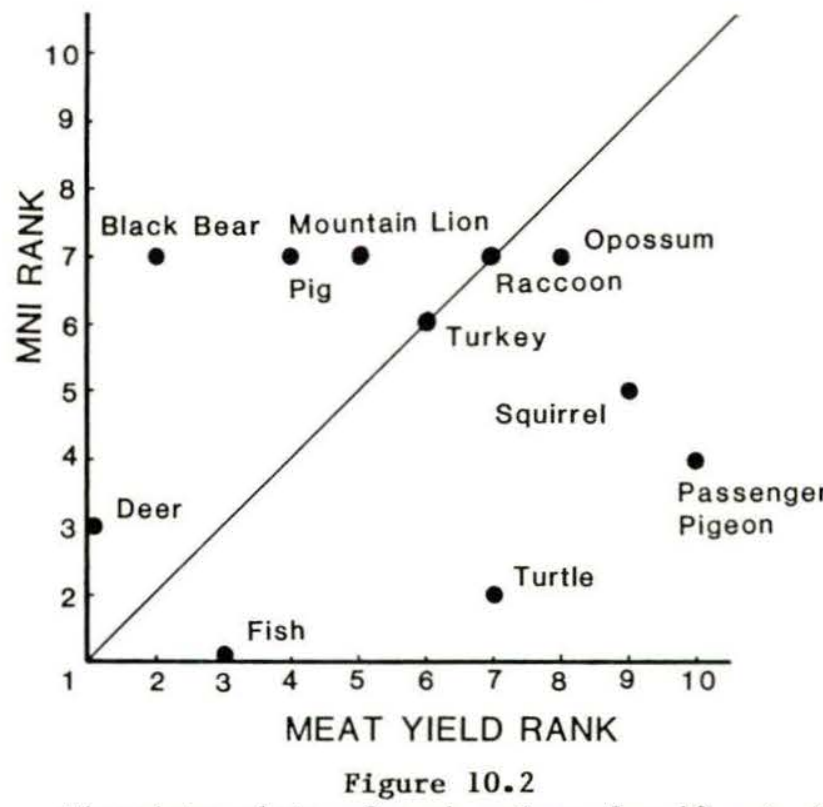

Bivariate plots of rank values for 12 animal species at the Wall and Fredricks sites.

ranked fairly high in terms of meat yield but were not frequently utilized. At the Wall site these animals were black bear, mountain lion, and beaver; at the Fredricks site, they were black bear and pig. Smith (1975b:126) notes that the low exploitation of bear and beaver at the Middle Mississippian sites whose faunal remains he analyzed may have been due to the fact that these species have low rates of reproduction, and thus were rarely encountered. It is interesting to note, however, that in his description of his visit to Occaneechi Town, Lawson (Lefler 1967:61) mentioned that the Indians brought him "good fat Bear" and that "Their Cabins were hung with a good sort of 
Tapestry, as fat Bear, and barbakued or dried Venison." It is also unlikely that pig would have been readily available to the inhabitants of the Fredricks site since this site was occupied early in the colonization process.

The third cluster consists of species that were utilized in high numbers but which yielded relatively small quantities of meat. At the Wall site, these species were rabbit, squirrel, and turtle. At the Fredricks site, they were turtle, squirrel, and passenger pigeon. At the Fredricks site, turtles were represented in higher numbers than were deer.

The fourth cluster of animals includes those species whose MNI and meat yield ranks were nearly equal. None of these species ranks highly in terms of either criterion of importance. At both sites these species were raccoon, turkey, and opossum.

At both sites, then, deer and catfish were the most important faunal resources. Turtle and squirrel were major secondary resources, as was rabbit at the Wall site and passenger pigeon at the Fredricks site. Raccoon, turkey, and opossum were utilized on a more limited basis at both sites. Bear and beaver at the Wall site, and pig and mountain lion at the Fredricks site provided large quantities of meat but were not as frequently encountered as were other species.

\section{HABITAT PREFERENCES AND SEASONALITY}

The species utilized by the inhabitants of the Wall and Fredricks sites can be divided into three groups based on their preferred habitats. Evidence for the seasons during which each species would have been procured is very limited.

Fish and all of the turtle species except box turtle are aquatic. Beaver are also dependent on an aquatic habitat. There is no archaeological evidence indicating at what seasons these species were collected. However, both turtles and fish are less readily available for exploitation during the winter. As only one beaver incisor was identified from the Wall site, it was not possible to determine the age of the individual or the season in which it was killed. The lesser scaup (identified in the Fredricks site assemblage) winters in North Carolina and occurs on lakes, rivers and ponds.

Shelford (1963:59-60) lists white-tailed deer, black bear, gray squirrel, fox squirrel, raccoon, opossum, striped skunk, and turkey among the species of the oak-hickory forest. Flying squirrel and mountain lion are also forest species. Of these animals, deer, gray squirrel, raccoon, and opossum also commonly utilize the forest edge. Other forest edge species identified in the assemblages are cottontail rabbit, gray fox, and bobwhite. With the exception of the passenger pigeon, which was present during the fall (Schorger 1955:268, 280), all of these forest and forest edge species were year-round residents of the North Carolina Piedmont. Thus, their presence in the assemblages provides little indication of the seasons during which they were exploited. The low representation of juvenile rabbits in the assemblages may indicate that this species was exploited primarily during the spring when the ratio of mobile juveniles to adults would have been lower than at other times of the year (Smith 1975b:100, 115-116). Turkey and passenger pigeons would have congregated in large flocks during the fall, in order to take advantage of the mast available at that time, and thus would have been more easily exploitable during those months.

The fact that no rabbits were identified in the faunal assemblage from the Fredricks site, and that passenger pigeon was represented by only one individual at the Wall site, makes it possible that the deposits from which the Fredricks site assemblage was derived are more representative of fall activities, whereas those deposits from which the Wall site assemblage was derived are more representative of spring activities.

Archaeologically, it is possible to determine the season during which deer were killed for those individuals represented by skulls having antlers attached (indicating May-February) or shed (indicating December-May). It is also possible to determine the season during which fawns (less that twenty months old) were killed based on stages of tooth eruption (Severinghaus 1949). At the Wall site it was only possible to determine the season during which two of the 36 individuals were killed. One individual was killed between May and February, as indicated by an antler attached to a frontal fragment, and another individual was killed during the spring or early summer, as indicated by the stage of dental eruption evident in one mandible. From the Fredricks site assemblage, it was possible to determine that one of nine individuals had been killed between May and February. The seasons during which the other individuals had been obtained could not be determined.

There are no clear indications that the inhabitants of one of the sites exploited specific portions of their environment to either a greater or lesser extent than the inhabitants of the other site. Likewise, there are no indications that there were major differences in the seasons during which the species were exploited. This apparent similarity, however, may simply be the result of a lack of evidence discernible in the archaeological record. 


\section{DIVERSITY}

One way in which it was possible to distinguish differences in the use of faunal resources by the inhabitants of the Wall and Fredricks sites was through the calculation of diversity. The formulas used and their results are shown in Table 10.5.

Using the Shannon-Weaver Index, species diversity was calculated as 1.46 for the Wall site assemblage and 2.19 for the Fredricks site assemblage. These numbers indicate that there is a greater diversity of species represented in the Fredricks site assemblage than in the Wall site assemblage. Using the same formula, Wing (1977) calculated diversity for assemblages from 43 other sites in the Southeast. The diversity indicated for the Wall and Fredricks site assemblages is lower than that indicated for all 43 of Wing's assemblages. The three sites that displayed diversity nearly as low as that of the Wall and Fredricks sites were sites at which the economy was based on specialized fishing (Wing 1977:87). As neither the techniques used in analyzing the faunal remains nor lists of species identified at each site were presented in Wing's discussion, it is difficult to evaluate whether or not a comparison of the Wall and Fredricks site assemblages with those reported by Wing is valid. However, at both the Wall and Fredricks sites, fish represented over $50 \%$ of the individuals identified and were the second most important resource, following deer, in terms of meat yield. At both sites, deer and fish were the most important resources. At the Fredricks site these species accounted for $56.68 \%$ of the MNI, whereas at the Wall site they accounted for $79.5 \%$ of the MNI. It is the dominance of these two resources that accounts for the fact that the two sites appear to be similar, in terms of diversity, to the specialized fishing sites described by Wing (1977).

Another method chosen for calculating

Table 10.5

Sunmary of spectes diversity measures.

\begin{tabular}{|c|c|c|c|c|}
\hline \multirow[b]{2}{*}{ Diversity Measure } & \multicolumn{4}{|c|}{ Site } \\
\hline & Fredricks & Wall & $31 \mathrm{Sk} 1$ & $315 k 1 a$ \\
\hline Shannon-Weaver Index ${ }^{1}$ & 2.19 & 1.46 & 2.54 & 2.29 \\
\hline Lieberson's Diversity Index ${ }^{2}$ & 0.73 & 0.55 & 0.88 & 0.88 \\
\hline Simpson's Index of D1versity ${ }^{3}$ & 0.73 & 0.54 & 0.85 & 0.87 \\
\hline
\end{tabular}

\footnotetext{
$1 H^{\prime}=\sum P_{1} \log _{e} P_{1}$,

where $P$ is the percentage of Individuals of 1 species Identified (Wing 1977:81).

$2 D_{w}=1-S=\left[\left(x_{1}\right)^{2}+\left(x_{2}\right)^{2}+\left(x_{3}\right)^{2} \ldots\right]$,

where $D_{w}$ stands for diversity within a population and is deternined by derfving the sum ( $\mathrm{S}$ ) of the squared percentages of each variable trait and subtracting that sum from one (Dickens 1980:40).

${ }^{3} D=1 \pm\left(P_{1}\right)^{2}$,

where $D$ is Simpson's Index of Diversity, $P_{1}$, is the proportion of individuals of species it in the assemblage (Styles 1981:45).
}

diversity is Lieberson's variation of Simpson's Index of Diversity. This method is described by Dickens $(1980: 40)$ as providing an "index that represents statistical probability of obtaining unlike characteristics in a population." The percentages of individuals of each species identified from the Wall and Fredricks sites were used with this formula. The resulting percentages were 0.55 for the Wall site and 0.73 for the Fredricks site. This indicates that there were only 55 chances out of 100 that any two randomly selected individuals identified from the Wall site assemblage will be different, whereas the chances of two individuals from the Fredricks site being different species are 73 out of 100 .

The final method is Simpson's Index of Diversity. Using this index, the lowest possible diversity would be 0 whereas maximum diversity for an assemblage is $1-1 / \mathrm{s}$, $s$ being the total number of species (Styles 1981:45). At the Wall site maximum diversity is 0.969 and actual diversity is 0.539. For the Fredricks site assemblage, maximum diversity is 0.966 and actual diversity is 0.726 . Thus, using Simpson's Index of Diversity, the Fredricks site assemblage exhibits more diversity than the Wall site assemblage. Also, the Wall site assemblage is only moderately diverse, whereas the Fredricks site assemblage exhibits fairly high diversity.

From the results of all four calculations, it is clear that the faunal assemblage from the Fredricks site exhibits more diversity than that from the Wall site. Increased diversity of faunal exploitation may have been a trend already developing in the Piedmont prior to European contact or it may represent a response to increased disruption of the social and natural environments following contact. To further investigate this problem, calculations were made of the diversity exhibited by assemblages from an Early Contact site and a Middle Contact site, both located in the North Carolina Piedmont on the upper Dan River. 31Skl dates ca. 1650-1675, and 31Skla dates ca. 1680-1690 (Wilson 1983:225). In age, 31Skl falls between the Wall and Fredricks site, whereas 31Skla may overlap slightly with the early portion of the occupation of the Fredricks site. The later of the two Dan River sites exhibited greater diversity than the earlier site when calculated using the first and third formulas, whereas the second formula yielded equal values for both sites. The results when each formula was used, however, indicate that the assemblages from 31Skl and 31Skla exhibited greater diversity than either the Wall or the Fredricks site. Thus there is no evidence to indicate that increased diversity in faunal exploitation was a general trend from protohistoric through historic times in the Piedmont. Likewise, there is no clear indication that the utilization of a greater diversity of species was necessarily a response to environmental disruption created by the presence of Europeans. 


\section{CONCLUSIONS}

Although the inhabitants of the Wall and Fredricks sites exploited a wide variety of species, both relied most heavily on deer and catfish. Turtle and squirrel were important secondary resources at the two sites, as were rabbit and raccoon at the Wall site and passenger pigeon at the Fredricks site. Turkey and opossum were supplementary resources at both sites, as was raccoon at the Fredricks site. Bear, at both sites, beaver at the Wall site and pig and mountain lion at the Fredricks site were only occasionally utilized. European domesticated animals, although present at the Fredricks site, had not become important as subsistence items.

The lack of data on the age and sex of most of the animals utilized made it impossible to determine with any certainty how selective the inhabitants of the two sites were in their exploitation of particular species. Nor was it possible to determine whether or not the patterns of exploitation can be explained in terms of maximization of meat yield and minimization of energy expenditure. Neither of the two most reliable methods for determining seasonality was very useful in interpreting the assemblages from the two sites. The presence of migratory fowl, passenger pigeon and lesser scaup, indicates some exploitation by the inhabitants of the Fredricks site of fall and winter species. The presence of juveniles of particular species (e.g., rabbit and squirrel) also provides evidence of seasonality. The fact that only adult rabbits were identified indicates that the inhabitants of the Wall site may have utilized this species in the spring.

It is possible that the reliance upon deer as a primary resource reflects an effort to minimize energy expenditure while maximizing meat yield. Deer congregate in relatively high densities during the fall and early winter in order to feed upon mast. They are thus easier to exploit at these times of year than at others (Smith 1975b:138). Ethnohistoric accounts and prehistoric evidence (Lefler 1967:215-216; Swanton 1946:256-257; Waselkov 1977:230) indicate that Southeastern Indians hunted deer primarily in the fall and winter. As it is not possible to determine the season during which the deer in the Wall and Fredricks site assemblages were killed, it is not possible to determine whether the inhabitants of the two sites utilized the same strategies as other Southeastern groups.

The knowledge of the age and/or sex of a few of the deer identified from the two sites, however, makes it possible to hypothesize about the methods used to hunt this species. At both sites, a nearly equal number of males and females was identified. Because such a low percentage of the total number of individuals could be sexed, though, these figures may not be an accurate reflection of the actual sex dis- tribution of the animals utilized. In both assemblages, the majority of the individuals were neither very young nor very old. This indicates that it is likely that drives or surrounds were the methods used in hunting the deer rather than stalking (Waselkov 1977:120).

Catfish was the second most important resource at both sites in terms of meat yield. The preferred water habitat of this species is small rivers with sluggish current (Smith 1975b:61), conditions which are met by the Eno River. Catfish are available in large numbers during the spring spawning season and also in the summer when the water level is low (Smith 1975b:60). The seasons during which the inhabitants of the Wall and Fredricks sites exploited this resource cannot be determined. However, Swanton (1946:257) proposes that many Southeastern Indian groups relied on fishing during the summer.

The secondary resources identified from the Wall and Fredricks sites differ from those reported for other sites which seem to represent minimized energy expenditure-maximized meat yield strategies. At the Middle Mississippi sites reported by Smith (1975:137-138) and the prehistoric Dan River sites reported by Waselkov (1977:101) raccoon and turkey were reported as important secondary resources. These species, like deer and catfish, exhibit high population densities during the fall and winter, when they were most likely to have been hunted. With the exception of passenger pigeon (at the Fredricks site), the species identified at both sites as important secondary resources do not congregate in easily exploitable groups at any time of the year. Squirrel, turtle, and rabbit may have been abundant near the sites and fairly easy to capture. That these species were such important resources to the inhabitants of the Wall and Fredricks sites suggests that the exploitative strategy used by these people was not entirely dominated by a concern for maximization.

Calculations of diversity indicated that the inhabitants of the Fredricks site used a greater diversity of species than the inhabitants of the Wall site. There is no indication, however, that this increased diversity through time was a general trend in the Piedmont. Nor is there any clear indication that it was necessarily a response to the disruption of the social and natural environments produced by Europeans.

From the data available thus far, contact (either direct or indirect) with Europeans seems to have had little effect on the basic pattern of faunal exploitation of the inhabitants of the Fredricks site. The presence of one horse molar and one fragment of pig bone indicates that animals introduced by Europeans probably were not important to the diet of these people. The increase in butchering marks found on deer bones from the Fredricks site, however, may be the 
result of different butchering practices following contact.

The many European artifacts found at the Fredricks site indicate considerable participation in the deerskin trade by the inhabitants of this site. There is, however, no direct evidence for this in the faunal assemblage. There is no indication that species were being hunted primarily for their hides rather than for meat, at least not in the near vicinity of the village. Nor is there evidence that portions of the environment were being exploited either more or less heavily than in the past. Even though good evidence for the exact strategies used to hunt deer is lacking, there is an indication that procurement strategies at the Fredricks site were not very different from those at the Wall site. Also, no increase in the number of tools or features associated with hide-working is evident at the Fredricks site. In fact, no hide-working tools have been found at the Fredricks site. There are three possible explanations for the discrepancy between the presence of a large number of European artifacts at the Fredricks site and a lack of evidence for participation in the deerskin trade in the faunal assemblage. The majority of the remains from the Fredricks site were recovered from burial pitfill and may reflect special ceremonial behavior that was not related to hunting activities associated with the deerskin trade. A second possibility is that activities associated with the deerskin trade, in general, were carried out at hunting camps away from the village. A third possibility is that in their role as trade "middlemen", the Occaneechis were not directly involved in the hunting activities associated with the deerskin trade.

Analysis of the ethnobotanical remains from the Wall and Fredricks sites (Gremillion 1984, this report) also shows surprisingly little evidence of differences in plant utilization between precontact and postcontact sites. With the exception of peach, no plant species introduced by Europeans were identified at the Fredricks site. Although acorn was not as plentiful at the Fredricks site as at the Wall site and hickory was more abundant at the former, corn, beans, and squash were important resources at both sites. The faunal remains from the Wall and Fredricks sites, when combined with this ethnobotanical evidence, support the contention that a basic late prehistoric subsistence pattern was maintained well into the Historic period of aboriginal occupation in the Carolina Piedmont. 


\section{Plant Remains from the Fredricks, Wall, and Mitchum Sites}

by

Kristen Johnson Gremillion

\section{INTRODUCTION}

The relationships between humans and their environment are conditioned to a large extent by the search for food and other material resources. And, since subsistence and other aspects of culture are mutually interdependent, the study of food acquisition is an important aspect of any examination of cultural stability and change. Decisions made by individuals and groups about which resources to exploit reflect and are affected by changes in social organization and communication with outside groups as well as by changes in the natural environment. For aboriginal Piedmont groups, the period between the first European entrance into North America and their ultimate loss of cultural identity was one in which many such changes took place.

The project which is the subject of this report seeks to describe and ultimately explain changes in aboriginal Piedmont culture during the Protohistoric and Historic periods using archaeological and ethnohistorical data. Unlike the ethnographer, the archaeologist does not have access to living informants, a situation which multiplies the problems normally encountered in describing a way of life and its historical development. The database for this study of the use of plant foods is primarily archaeological, supplemented only by the few available historical records. Nevertheless, it is felt that the analysis of plant remains has a great deal of potential for adding to our knowledge of European contact and culture change in the North Carolina Piedmont.

Despite the fact that archaeologists do not have the advantage of direct observation of food-getting practices, reconstruction of subsistence patterns can be accomplished with due attention to the transformations undergone by plant remains both before and after they enter the archaeological record. In addition, possible explanations can be offered for changes in plant procurement patterns. In this study, these explanations will be based upon a costbenefit model derived from economic and ecological theory.
METHODOLOGICAL ISSUES

Like archaeologists in general, paleoethnobotanists are faced with the problem of how to interpret human behavior on the basis of material remains and their context. The transfer of items from a systemic to an archaeological context (Schiffer 1976) introduces a number of factors which tend to mask relationships between the remains themselves and the behavior that produced them. For the most part, techniques developed for determining true relationships between plant remains and subsistence practices are still rudimentary. However, recognition of intervening biases is a first step towards guarding against incorrect interpretations and constructing ones that are relatively secure. Such questions of method are quite complex and invite detailed discussion. However, a brief look at some basic problems and their relevance to the current project is sufficient to establish a background for the interpretations that follow.

\section{Material and Physical Factors}

The material makeup of plant parts is one important factor influencing the probability of their preservation in an archaeological context. At open-air sites, only carbonized plant remains can be securely associated with a past occupation (Yarne 11 1982; Dimbleby 1979). Conditions that result in the preservation of durable materials (such as peach pit and thick nutshell) may tend to destroy more fragile materials (e.g., roots, leaves, and flowers) or simply render them unrecognizable to the analyst. Although items like acorn shell are amenable to carbonization, they tend to fragment easily both during and after deposition. Post-depositional disturbance in the form of pedoturbation thus affects the distribution of preserved plant remains in the soil by dispersing, breaking, and abrading them.

\section{Behavioral Factors}

Food Processing and Consumption. The activities of site occupants also affect the probability that certain kinds of plant parts will be recovered archaeologically. Foodprocessing practices that make use of fire, such as drying or parching, increase the likelihood 
of carbonization. Lawson (Lefler 1967:112, 182) has documented the fruit-drying techniques of Piedmont Indians. Corn and other grains may also have been dried or parched for storage and/or consumption. The fact that beans were probably boiled rather than parched may be responsible for their relatively sparse representation archaeologically in the East (Yarnell 1982). The practice of using food wastes such as corncobs and hickory nutshells for fuel may similarly result in their overrepresentation relative to other kinds of remains that may actually represent greater quantities of food.

Deposition. Plant remains may thus be deposited (that is, transferred to an archaeological context) either intentionally or unintentionally. Edible parts can be lost accidentally during consumption or processing, whereas waste products usually are intentionally deposited, often in a circumscribed area such as a village midden or trash pit. Frequently, therefore, food remains rather than food itself are preserved archaeologically (Dennell 1976). In the case of hickory nutshe11, the remains are more representative of some quantity of food consumed. However, fruit and weed seeds must be evaluated more carefully. These sometimes may have been carbonized fortuitously rather than having been lost during processing. Amounts of food represented by seeds must be determined with reference to quantity, fruit size, and number of seeds per fruit.

Determination of the relative amounts of food represented by archaeological plant remains thus depends to a great extent on an understanding of the contexts in which they occur. In trash-filled pits, for example, a fairly heterogeneous mixture of plant remains can be expected to occur. As garbage, these deposits are perhaps more representative of subsistence practices during a given time period than those that result from more specialized activities. A village midden, the type of context from which most of the Wall site sample was drawn, is also a heterogeneous mixture. However, it has been shown (Johnson 1983) that different parts of a midden can have lower or higher densities of plant remains than pit fill from the same site. In any case, the chief difference between midden and feature fill may often lie in relative density of plant remains rather than the kinds of remains represented.

Most of the Mitchum site plant remains samples were drawn from trash-filled pits, as were several each from Wall and Fredricks. Some upper burial fill from Wall and Fredricks seems to represent intentional deposition of food remains (see Ward, this report), possibly associated with special activities. Feature 9 at the Fredricks site is a deep stratified pit surrounded by a wall trench. Its formation seems to have been associated with a complex series of activities. Problems encountered in interpreting plant remains assemblages from this feature will be discussed in more detail below.
In recognition of the seasonal and behavioral parameters of deposit formation and their effect upon plant remains collections, results from different contexts are presented separately in the tables.

\section{Recovery}

The behavior of the archaeologist also has significant effects upon the composition of plant remains assemblages. The amount of a site that is excavated is such a factor. However, recovery methods are equally important. Flotation, or water separation, has been shown to be the most effective recovery method since it minimizes damage to fragile materials and employs a fine screen (in this case, a $0.71 \mathrm{~mm}$ mesh) that recovers even small seeds. Most of the manipulations of plant remains data in this study will, therefore, rely on flotation samples (both heavy and light fractions), which have the advantage of being documented as measured quantities of fill. Although one can process large (unmeasured) quantities of fill by waterscreening, it is necessary to use a highpressure water source that tends to damage fragile plant remains. Waterscreened material from the 1983 field season was examined for purposes of comparison with flotation samples and to supplement the flotation database. These results are presented separately, but included in site totals. Midden flotation samples from 1983 are also included in the tables, but are excluded from further manipulations since only light fractions were analyzed.

\section{Sample Analysis and Methods of Comparison}

Analysis. The standardized analysis procedure followed for all samples approximates that presented by Yarnell (1974). Each sample is weighed and then sifted through a series of U.S. Standard geological sieves ranging in mesh size from $6.35 \mathrm{~mm}$ to $0.21 \mathrm{~mm}$. Material greater than $2.00 \mathrm{~mm}$ in diameter is completely sorted. Material passing through the $2.00 \mathrm{~mm}$ screen is searched for seeds only, and for items not represented in the larger size category. Quantities of each component in the $1.41 \mathrm{~mm}$ to 0.71 $\mathrm{mm}$ size class are extrapolated on the basis of their representation in the $6.35 \mathrm{~mm}$ to $2.00 \mathrm{~mm}$ category (Table 11.1). It is assumed that components are represented equally in these two size classes. Even though this assumption is not always satisfied, the procedure provides a better estimate of actual quantities than quantification of only the largest size class. Material passing through the $0.71 \mathrm{~mm}$ screen is excluded from calculations, since it is composed mainly of fine soil particles.

Methods of Comparison. Several techniques will be used to compare plant remains data from different sites and contexts. Percentage of plant food remains by weight is especially useful for comparing remains of the same class (Table 11.2). However, since these classes differ in their durability and likelihood of 
Table 11.1
Summary of plant remains from the Wa11, Fredricks, and Mitchum sites (weights in grams).

\begin{tabular}{|c|c|c|c|c|c|c|c|c|c|c|c|c|c|c|c|c|}
\hline $\begin{array}{l}\text { Proveniencel } \\
\text { Sample Type }\end{array}$ & $\begin{array}{l}\text { Soil Volume } \\
\text { in Liters } \\
\text { (Flotation) }\end{array}$ & $\begin{array}{c}\text { Total } \\
\text { Plant } \\
\text { Remains }\end{array}$ & $\begin{array}{l}\text { Wood } \\
\text { Charcoal }\end{array}$ & $\begin{array}{l}\text { Unid, } \\
\text { Plant }\end{array}$ & $\begin{array}{l}\text { Rhizome/ } \\
\text { Tuber }\end{array}$ & $\begin{array}{l}\text { Plant Food } \\
\text { Remains }\end{array}$ & $\begin{array}{l}\text { Hickory } \\
\text { Nutshell }\end{array}$ & $\begin{array}{l}\text { Acorn } \\
\text { She11 }\end{array}$ & $\begin{array}{l}\text { Acorn } \\
\text { Meat }\end{array}$ & $\begin{array}{l}\text { Unid. } \\
\text { Nutmeat }\end{array}$ & $\begin{array}{r}\text { Walnut } \\
\text { She11 }\end{array}$ & $\begin{array}{l}\text { Peach } \\
\text { Pit }\end{array}$ & $\begin{array}{r}\text { Total } \\
\text { Corn }\end{array}$ & $\begin{array}{c}\text { Common } \\
\text { Bean }\end{array}$ & Seeds & $\begin{array}{l}\text { Cucurbit } \\
\text { Rind }\end{array}$ \\
\hline \multicolumn{17}{|l|}{ Wall Site } \\
\hline \multicolumn{17}{|l|}{ Flotation } \\
\hline Fea. Fill & 71 & 18.84 & 16.86 & 0.84 & - & 1.14 & 0.74 & 0.20 & 0.08 & - & - & - & 0.06 & - & 0.06 & - \\
\hline Bu. Fill & 15 & 4.95 & 4.21 & 0.39 & - & 0.35 & 0.21 & 0.04 & - & - & - & - & 0.08 & - & 0.02 & - \\
\hline Midden & 200 & 107.34 & 68.11 & 8.09 & - & 31.14 & 23.07 & 2.76 & 0.23 & 0.23 & 1.10 & - & 3.14 & 0.25 & 0.36 & - \\
\hline Midden ( $L F$ only) & 110 & 3.54 & 3.14 & 0.12 & - & 0.28 & $\mathrm{x}$ & $\mathrm{x}$ & - & - & - & - & 0.26 & - & 0.02 & - \\
\hline Total Flotation & 396 & 134.67 & 92.32 & 9.44 & - & 32.91 & 24.02 & 3.00 & 0.31 & 0.23 & 1.10 & - & 3.54 & 0.25 & 0.45 & - \\
\hline \multicolumn{17}{|l|}{ Waterscreen } \\
\hline Bu. Fill & & 85.33 & 73.88 & 4.02 & - & 7.43 & 5.18 & 0.45 & - & - & - & - & 1.75 & - & 0.05 & - \\
\hline Midden & & 23.37 & 16.16 & 1.28 & 0.05 & 5.88 & 3.82 & 0.33 & - & - & - & - & 1.64 & 0.05 & 0.04 & - \\
\hline Total Waterscreer & & 108.70 & 90.04 & 5.30 & 0.05 & 13.31 & 9.00 & 0.78 & - & - & - & - & 3.39 & 0.05 & 0.09 & - \\
\hline Total & & 243.37 & 182.36 & 14.74 & 0.05 & 46.22 & 33.02 & 3.78 & 0.31 & 0.23 & 1.10 & - & 6.93 & 0.30 & 0.55 & - \\
\hline \multicolumn{17}{|l|}{ Mitchum Site } \\
\hline \multicolumn{17}{|l|}{ Flotation } \\
\hline Fea. Fill & 180 & 97.62 & 91.25 & 2.61 & - & 3.76 & 2.55 & 0.26 & - & - & 0.04 & 0.31 & 0.37 & - & 0.23 & - \\
\hline Bu. Fill & 10 & 1.92 & 0.70 & 0.20 & - & 1.02 & 0.96 & 0.03 & - & - & - & - & 0.03 & - & - & - \\
\hline Total Flotation & 190 & 99.54 & 91.95 & 2.81 & - & 4.78 & 3.51 & 0.29 & - & - & 0.04 & 0.31 & 0.40 & - & 0.23 & - \\
\hline \multicolumn{17}{|l|}{ Waterscreen } \\
\hline Fea. Fill & & 190.87 & 177.22 & 4.33 & $\mathrm{x}$ & 9.32 & 6.76 & 0.34 & - & - & 0.30 & 1.08 & 0.61 & - & 0.22 & 0.01 \\
\hline Bu. Fill & & 2.34 & 1.82 & - & - & 0.52 & 0.34 & - & - & - & - & - & 0.18 & - & - & - \\
\hline Total Waterscreer & & 193.21 & 179.04 & 4.33 & $\mathrm{x}$ & 9.84 & 7.10 & 0.34 & - & - & 0.30 & 1.08 & 0.79 & - & 0.22 & 0.01 \\
\hline Total & & 292.75 & 270.99 & 7.14 & $\mathrm{x}$ & 14.62 & 10.61 & 0.63 & - & - & 0.34 & 1.39 & 1.19 & - & 0.45 & 0.01 \\
\hline \multicolumn{17}{|l|}{ Fredricks Site } \\
\hline \multicolumn{17}{|l|}{ Flotation } \\
\hline Bu. Fill & 477 & 95.67 & 70.18 & 4.40 & - & 21.09 & 14.49 & 0.14 & 0.05 & - & 0.63 & 0.86 & 4.27 & 0.10 & 0.53 & 0.02 \\
\hline Fea. $9 \mathrm{ZI}$ & 40 & 17.24 & 4.11 & 0.83 & - & 12.30 & 10.00 & $\mathrm{x}$ & - & - & 0.02 & 0.07 & 1.91 & 0.22 & 0.08 & - \\
\hline Fea. 9 ZII & 30 & 10.01 & 3.61 & 0.33 & - & 6.07 & 4.46 & - & - & - & - & 0.15 & 1.37 & 0.05 & 0.04 & - \\
\hline Fea. 9 ZIII & 70 & 113.89 & 51.89 & 2.97 & - & 59.03 & 15.81 & 0.29 & - & - & 0.01 & 2.00 & 40.31 & 0.17 & 0.44 & - \\
\hline Total Fea. 9 & 140 & 141.14 & 59.61 & 4.13 & - & 77.40 & 30.27 & 0.29 & - & - & 0.03 & 2.22 & 43.59 & 0.44 & 0.56 & - \\
\hline Struc. 1 & 10 & 0.69 & 0.30 & 0.01 & - & 0.38 & 0.37 & $\mathrm{x}$ & - & - & - & - & 0.01 & - & - & - \\
\hline Fea. $10-13$ & 95.5 & 52.38 & 45.06 & 1.10 & - & 6.22 & 5.75 & 0.05 & - & - & 0.04 & 0.14 & 0.17 & 0.04 & 0.03 & - \\
\hline & 722.5 & 289.88 & 175.15 & 9.64 & - & 105.09 & 50.88 & 0.48 & 0.05 & - & 0.70 & 3.22 & 48.04 & 0.58 & 1.12 & 0.02 \\
\hline \multicolumn{2}{|c|}{ Bu. Fill Waterscreen } & 184.01 & 158.23 & 4.24 & 0.20 & 21.34 & 11.19 & 0.04 & - & - & - & 5.81 & 3.84 & 0.05 & 0.39 & 0.02 \\
\hline Total & & 473.89 & 333.38 & 13.88 & 0.20 & 126.43 & 62.07 & 0.52 & 0.05 & - & 0.70 & 9.03 & 51.88 & 0.63 & 1.51 & 0.04 \\
\hline
\end{tabular}


Table 11.2

Percent of plant food remains from the Wall, Fredricks, and Mitchum sites.

\begin{tabular}{|c|c|c|c|c|c|c|c|c|c|c|c|c|}
\hline $\begin{array}{l}\text { Provenience/ } \\
\text { Sample Type }\end{array}$ & $\begin{array}{l}\text { Soil Vol. } \\
\text { in Liters } \\
\text { (Flot. only) }\end{array}$ & $\begin{array}{l}\text { Plant Food } \\
\text { Remains, } \\
\text { Grams }\end{array}$ & $\begin{array}{r}\text { Hickory } \\
\text { Nutshell }\end{array}$ & $\begin{array}{l}\text { Acorn } \\
\text { Shell }\end{array}$ & $\begin{array}{l}\text { Acorn } \\
\text { Meat }\end{array}$ & $\begin{array}{l}\text { Unid. } \\
\text { Nutmeat }\end{array}$ & $\begin{array}{l}\text { Walnut } \\
\text { Shell }\end{array}$ & $\begin{array}{l}\text { Peach } \\
\text { Pit }\end{array}$ & Corn & Bean & Seeds & $\begin{array}{c}\text { Cucurbit } \\
\text { Rind }\end{array}$ \\
\hline \multicolumn{13}{|l|}{ Wall Site } \\
\hline \multicolumn{13}{|l|}{ Flotation } \\
\hline Feature Fill & 71 & 1.14 & 64.9 & 17.5 & 7.0 & - & - & - & 5.3 & - & 5.3 & - \\
\hline Burial Fill & 15 & 0.35 & 60.0 & 11.4 & - & - & - & - & 22.9 & - & 5.7 & - \\
\hline Midden & 200 & 31.14 & 74.1 & 8.9 & 0.7 & 0.7 & 3.5 & - & 10.0 & 0.8 & 1.2 & - \\
\hline Midden(LF only) & 110 & 0.28 & $\mathrm{x}$ & $\mathrm{x}$ & - & - & - & - & 92.9 & - & 7.1 & - \\
\hline Total Flotation & 396 & 32.91 & 73.0 & 9.1 & 0.9 & 0.7 & 3.3 & - & 10.8 & 0.8 & 1.4 & - \\
\hline \multicolumn{13}{|l|}{ Waterscreen } \\
\hline Burial Fill & & 7.43 & 69.7 & 6.1 & - & - & - & - & 23.6 & - & 0.7 & - \\
\hline Midden & & 5.88 & 65.0 & 5.6 & - & - & - & - & 27.9 & 0.9 & 0.7 & - \\
\hline Total Waterscreen & & 13.31 & 67.6 & 5.9 & - & - & - & - & 25.5 & 0.8 & 0.3 & - \\
\hline Total Wall & & 46.22 & 71.4 & 8.2 & 0.7 & 0.5 & 2.4 & - & 15.0 & 0.8 & 1.1 & - \\
\hline \multicolumn{13}{|l|}{ Mitchum Site } \\
\hline \multicolumn{13}{|l|}{ Flotation } \\
\hline Feature Fill & 180 & 3.76 & 67.8 & 6.5 & - & - & 1.1 & 8.2 & 9.6 & - & 6.1 & - \\
\hline Burial Fill & 10 & 1.02 & 94.1 & 2.9 & - & - & - & - & 2.9 & - & - & - \\
\hline Total Flotation & 190 & 4.78 & 73.4 & 6.1 & - & - & 0.8 & 6.5 & 8.4 & - & 4.8 & - \\
\hline \multicolumn{13}{|l|}{ Waterscreen } \\
\hline Feature Fill & & 9.32 & 72.5 & 3.6 & - & - & 3.2 & 11.6 & 6.5 & - & 2.4 & 0.1 \\
\hline Burial Fill & & 0.52 & 65.4 & - & - & - & - & - & 34.6 & - & - & - \\
\hline Total Waterscreen & & 9.84 & 72.2 & 3.5 & - & - & 3.0 & 11.0 & 8.0 & - & 2.2 & 0.1 \\
\hline Total Mitchum & & 14.62 & 72.6 & 4.3 & - & - & 2.3 & 9.5 & 8.1 & - & 3.1 & 0.1 \\
\hline \multicolumn{13}{|l|}{ Fredricks Site } \\
\hline \multicolumn{13}{|l|}{ Flotation } \\
\hline Burial Fill & 477 & 21.09 & 68.7 & 0.7 & 0.2 & - & 3.0 & 4.1 & 20.2 & 0.5 & 2.5 & 0.1 \\
\hline Fea.9, Zone I & 40 & 12.30 & $81 \cdot 3$ & $\mathrm{x}$ & - & - & 0.2 & 0.6 & 15.5 & 1.8 & 0.7 & - \\
\hline Fea.9, Zone II & 30 & 6.07 & 73.5 & - & - & - & - & 2.5 & 22.6 & 0.8 & 0.7 & - \\
\hline Fea.9, Zone III & 70 & 59.03 & 26.8 & 0.5 & - & - & $\mathrm{x}$ & 3.4 & 68.3 & 0.3 & 0.7 & - \\
\hline Total Fea. 9 & 140 & 77.40 & 39.1 & 0.4 & - & - & $\mathrm{x}$ & 2.9 & $56 \cdot 3$ & 0.6 & 0.7 & - \\
\hline Structure 1 & 10 & 0.38 & 97.4 & $\mathrm{x}$ & - & - & - & - & 2.6 & - & - & - \\
\hline Fea. $10-13$ & 95.5 & 6.22 & 92.4 & 0.8 & - & - & 0.6 & 2.3 & 2.7 & 0.6 & 0.5 & - \\
\hline Total Flotation & 722.5 & 105.09 & 48.4 & 0.5 & $\mathrm{x}$ & - & 0.7 & 3.1 & 45.7 & 0.6 & 1.1 & $\mathrm{x}$ \\
\hline Burial Fill Watersc & ceen & $21 \cdot 34$ & 52.4 & 0.2 & - & - & - & 27.2 & $18 \cdot 0$ & - & 1.8 & 0.2 \\
\hline Total Fredricks & & 126.43 & 49.1 & 0.4 & $\mathrm{x}$ & - & 0.6 & 7.1 & 41.0 & 0.5 & 1.2 & $\mathrm{x}$ \\
\hline
\end{tabular}


preservation, between-class comparisons are sometimes misleading if based upon relative representation by weight. Ubiquity measures assess the frequency of occurrence of plant taxa without regard for their quantity (Table 11.3). Here, ubiquity is calculated by finding the percentage of flotation samples comprising both light and heavy fractions in which an item occurred (most of these samples represent 10 liters of fill; the few exceptions probably do not introduce significant bias).

A modified version of Simpson's diversity index (Peet 1974) will also be used to compare data. This index, designed for ecological community studies, measures both richness (numbers of taxa represented) and equitability (evenness of representation of taxa), and its value can range from zero to one. Diversity (D) is computed as

$$
1-\sum \mathrm{p}_{i}{ }^{2},
$$

where $p$ is the proportion of item $\underline{i}$ in the assemblage. Proportions of each plant food in a sample are determined using a ubiquity measure in which values sum to one and using equal numbers of samples from each site. It is assumed that these proportions reflect proportions in the diet to some extent, but the diversity of the plant food resource base for each community as a whole is of primary interest.

Seeds (except for common bean, corn, peach pit, and nut remains) are combined and their aggregate weight presented for each context. Numbers of seeds are presented in separate tables along with estimated corn cupule and kernel counts (Tables 11.4-11.5). In addition, actual counts are converted to number per gram of plant food remains, a procedure that takes into account differences between sample sizes. All comparative techniques are of course subject to the cautions presented above.

Most of the categories in the tables are self-explanatory. Non-botanical materials and uncarbonized plant parts are sorted completely for light fractions and weighed as an aggregate for heavy fractions, but only plant remains are reported in the tables. The "wood charcoal" category also includes stem charcoal and bark. "Residue" is the unsorted material less than $0.71 \mathrm{~mm}$. Corn remains are presented as aggregate weights of cupules and kernels but are itemized in the seed count tables.

\section{COMPARISON OF PLANT REMAINS ASSEMBLAGES}

Although plant remains from three sites are included in this study, comparison will focus on the Wall and Fredricks sites, which represent sequential occupations of the same approximate location. The Mitchum site occupation, temporally intermediate between the first two, is anomalous in certain respects. In fact, calculation of a similarity index (King 1982) based upon plant taxa (expressed as twice the number of shared taxa divided by the total number of taxa for both sites) shows greater similarity between Wall and Fredricks than between either of them and Mitchum. One reason for this distinctiveness is that some deposits at the Mitchum site display a seasonal profile different from those of the other two sites. This factor, as well as the location of Mitchum in a different river valley and the relatively small area of the site that was sampled, makes it difficult to assess change using all three sites. The Mitchum site plant remains should therefore probably not be considered representative of a transitional subsistence pattern in a developmental sequence from the Wall site occupation to that of the Fredricks site. Although the cultural connectedness of the Wall and Fredricks site populations is still in question, they shared a similar local environment and probably had a similar range of plant foods from which to choose. Comparison, therefore, will focus on the use of plant foods by these two populations.

\section{Nut Resources: Hickory, Acorn, and Walnut}

Nuts were important aboriginal foods throughout the East, beginning at least during Early Archaic times. Acorn was probably the most important plant food in the Southeast prehistorically until Mississippian times, although hickory seems to have dominated in some locations. Walnut is generally less abundantly represented than either hickory or acorn (Yarne11 and Black 1983).

A11 three nut types are represented at the three sites in question. Hickory is the most abundant nut type at all three sites based on percentage by weight (Table 11.6). However, acorn shell fragments more easily than thick nutshe 11 and is therefore probably greatly underrepresented. In addition, hickory nutshel1 represents smaller quantities of nutmeat than does acorn shell because of acorn's higher meat-to-shell ratio. Acorn shell may represent anywhere from five to 200 times as much nutmeat as an equivalent quantity of hickory shell (Lopinot 1983). Accordingly, Yarnell and Black (1983) have recommended multiplying acorn she11 quantities by 50 in order to arrive at a corrected acorn-to-hickory ratio. Using site totals, the acorn-to-hickory ratio (based on estimated food quantities) for the Wall site is 5.72 , in contrast to the Fredricks site value of only 0.42 . The Mitchum site value is 2.97 .

It is clear that acorn is much better represented at the Wall site than is hickory. By the time of the Fredricks site occupation, a shift to greater emphasis on hickory seems to have occurred. The drop in hickory nutshell as percentage of plant food remains from Wall to Fredricks may be a consequence of its highly variable representation in different contexts. 
Table 11.3

Ubiquity of plant remains, as percent of flotation samples, at the Wall, Fredricks, and Mitchum sites.

\begin{tabular}{|c|c|c|c|c|c|c|c|c|c|}
\hline Taxon & Features & $\begin{array}{r}\text { Wall } \\
\text { Burial }\end{array}$ & $\begin{array}{l}\text { Site } \\
\quad \text { Midden }\end{array}$ & Total & Burials & $\begin{array}{ll} & \text { Fredri } \\
\text { Fea. } & 10-13\end{array}$ & $\begin{array}{l}\text { ks Site } \\
\text { Fea.9/Str.1 }\end{array}$ & Total & $\begin{array}{l}\text { Mitchum Site } \\
\text { Burial/Features }\end{array}$ \\
\hline Hickory & 37.5 & 100.0 & 100.0 & 83.3 & 100.0 & 100.0 & 100.0 & 100.0 & 100.0 \\
\hline Acorn & 87.5 & 100.0 & 100.0 & 100.0 & 42.2 & 40.0 & 60.0 & 45.7 & 52.9 \\
\hline Walnut & - & - & 50.0 & 33.3 & 6.6 & 10.0 & 13.3 & 8.6 & 5.9 \\
\hline Peach & - & - & - & - & 28.9 & 30.0 & 60.0 & 35.7 & 23.5 \\
\hline Cucurbit & - & - & - & - & 4.4 & - & 6.7 & 4.2 & - \\
\hline Corn & 25.0 & 100.0 & 100.0 & 80.0 & 93.3 & 90.0 & 100.0 & 94.3 & 70.6 \\
\hline Grape & - & 50.0 & 65.0 & 46.7 & 8.9 & 10.0 & 53.3 & 18.6 & - \\
\hline Hawthorn & 12.5 & - & 5.0 & 6.7 & - & - & 13.3 & 2.9 & 5.9 \\
\hline Blueberry & - & - & - & - & 8.9 & - & - & 5.7 & - \\
\hline Bedstraw & - & - & 5.0 & 3.3 & 2.2 & - & 20.0 & 5.7 & 5.9 \\
\hline Maypops & - & - & 30.0 & 20.0 & 8.9 & - & 73.3 & 21.4 & 5.9 \\
\hline Sumac & - & - & - & - & 2.2 & - & 13.3 & 4.2 & - \\
\hline Persimmon & - & - & 5.0 & 3.3 & 13.3 & - & - & 8.6 & - \\
\hline Groundcherry & - & - & - & - & 2.2 & - & 13.3 & 4.2 & - \\
\hline Grass Family & - & - & - & - & 2.2 & - & 13.3 & 4.2 & 17.6 \\
\hline Spurge & - & - & - & - & 2.2 & - & - & 1.4 & - \\
\hline Bramble & - & - & - & - & - & - & 13.3 & 2.9 & 5.9 \\
\hline Poke & - & - & 5.0 & 3.3 & - & - & 20.0 & 4.2 & - \\
\hline Chenopod & - & - & - & - & - & - & 33.3 & 7.1 & - \\
\hline Elderberry & - & - & - & - & - & - & 6.7 & 1.4 & - \\
\hline Legume Family & 12.5 & - & 5.0 & 6.7 & - & - & 6.7 & 1.4 & - \\
\hline Morning glory & - & - & - & - & - & 10.0 & - & - & - \\
\hline Dogwood & - & - & 5.0 & 3.3 & - & - & - & - & - \\
\hline Pink Family & 12.5 & - & - & 3.3 & - & - & - & - & - \\
\hline Bearsfoot & 25.0 & - & - & 6.7 & - & - & - & - & - \\
\hline Maygrass & - & - & - & - & - & - & - & - & 35.3 \\
\hline Unid. A & - & - & - & - & - & - & - & - & 11.8 \\
\hline Little barley & - & - & - & - & - & - & - & - & 5.9 \\
\hline Barley/Fescue? & - & - & - & - & - & - & - & - & 11.8 \\
\hline \# of samples & 8 & 2 & 20 & 30 & 45 & 10 & 15 & 70 & 17 \\
\hline
\end{tabular}

Note: Based on flotation samples from which both light and heavy fractions were analyzed. 
Table 11.4

Seed counts from the Fredricks site.

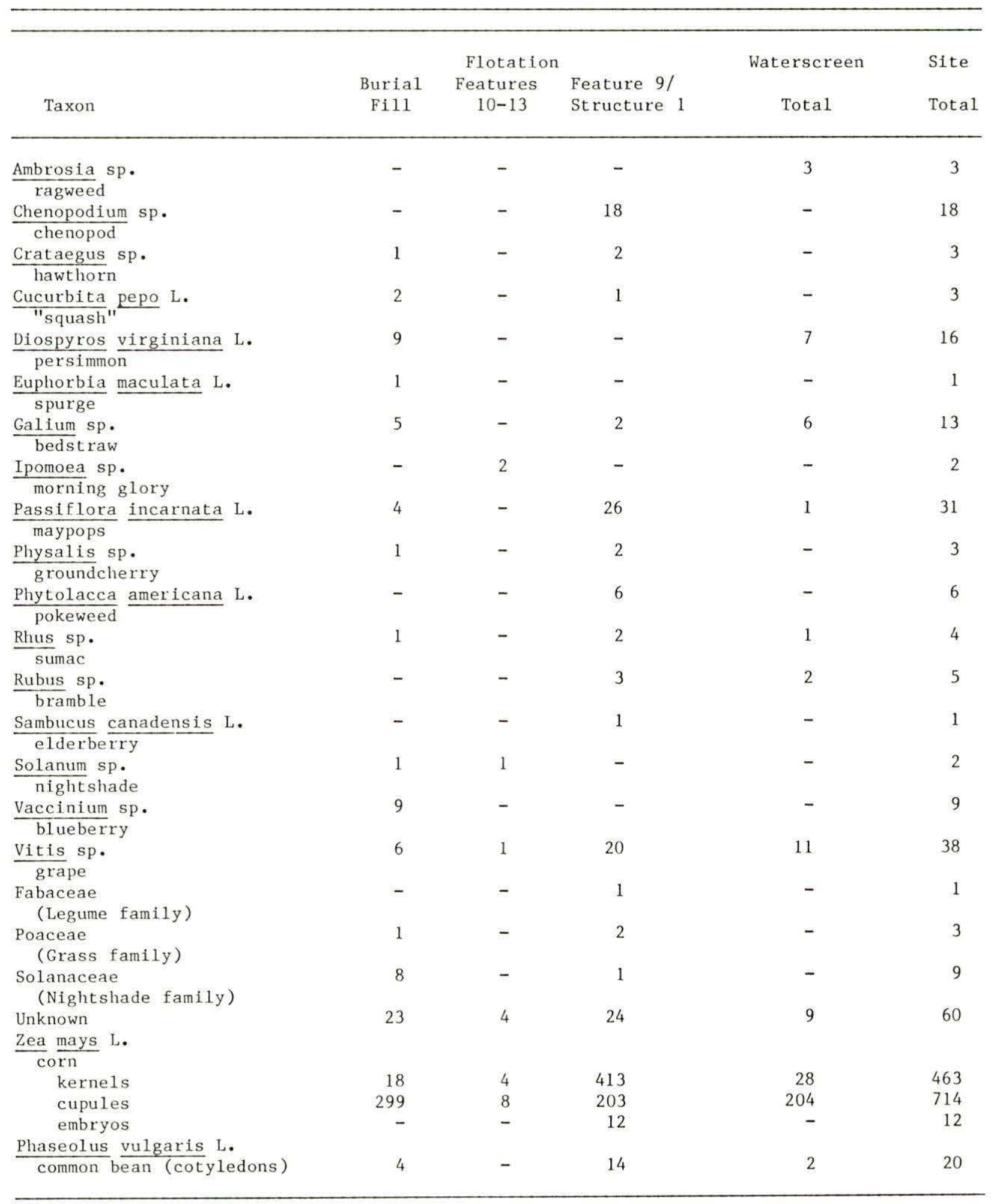


Table 11.5

Seed counts from the Wa11 and Mitchum sites.

\begin{tabular}{|c|c|c|c|c|c|c|c|c|c|c|}
\hline \multirow[b]{2}{*}{ Taxon } & \multirow[b]{2}{*}{ Midden } & \multirow{2}{*}{\multicolumn{2}{|c|}{$\begin{array}{cc} & \text { Wall } \\
\text { Midden } & \text { Burials } \\
\text { (LF only) } & \end{array}$}} & Site & \multirow[t]{2}{*}{ WS } & \multirow[b]{2}{*}{ Total } & \multirow{2}{*}{\multicolumn{2}{|c|}{$\begin{array}{l}\text { Mitchum } \\
\text { Flotation } \\
\text { Burial Features }\end{array}$}} & \multirow[t]{2}{*}{$\begin{array}{l}\text { Site } \\
\text { WS }\end{array}$} & \multirow[b]{2}{*}{ Total } \\
\hline & & & & Features & & & & & & \\
\hline$\frac{\text { Centrosema }}{\text { butterfiypea }} \frac{\text { virginiana }}{\text { Lea }}$ & - & - & - & - & - & - & - & - & 1 & 1 \\
\hline$\frac{\text { Chenopodium }}{\text { chenopod }}$ & - & 1 & - & - & - & 1 & - & - & - & - \\
\hline$\frac{\text { Cornus sp. }}{\text { dogwood }}$ & 1 & - & - & - & - & 1 & - & - & - & - \\
\hline$\frac{\text { Crataegus }}{\text { hawthorn }}$ sp. & 1 & - & - & 1 & 1 & 3 & - & 1 & 4 & 5 \\
\hline$\frac{\text { Diospyros }}{\text { persimmon }}$ virginiana L. & 1 & - & - & - & 2 & 3 & - & - & 4 & 4 \\
\hline$\frac{\text { Galium sp. }}{\text { bedstraw }}$ & 1 & - & - & - & 1 & 2 & - & 1 & - & 1 \\
\hline$\frac{\text { Hordeum }}{\text { little }} \frac{\text { pusillum }}{\text { barley }}$ L. & - & - & - & - & - & - & - & 13 & - & 13 \\
\hline $\begin{array}{l}\text { Iva annua } \frac{\text { var macrocarpa }}{\text { Jackson }} \\
\text { sumpweed }\end{array}$ & - & - & - & - & 1 & 1 & - & - & - & - \\
\hline$\frac{\text { Passiflora }}{\text { maypops }}$ incarnata $\mathrm{L}$. & 8 & - & 1 & - & 1 & 10 & - & 1 & 4 & 5 \\
\hline$\frac{\text { Phalaris }}{\text { maygrass }}$ caroliniana Walter & - & - & - & - & - & - & - & 440 & - & 440 \\
\hline$\frac{\text { Phytolacca }}{\text { poke }}$ americana $\mathrm{L}$. & - & - & - & 1 & - & 1 & - & - & - & - \\
\hline$\frac{\text { Polymnia }}{\text { bearsfoot }} \frac{\text { uvedalia }}{\mathrm{L}} \mathrm{L}$. & - & - & - & 2 & - & 2 & - & - & - & - \\
\hline$\frac{\text { Rubus }}{\text { bramble }}$ & - & - & - & - & - & - & - & 1 & - & 1 \\
\hline$\frac{\text { Vitis }}{\text { grape }}$ & 21 & - & 1 & - & 1 & 23 & - & - & 5 & 5 \\
\hline Unknown & 26 & 47 & - & 2 & 2 & 77 & - & 19 & 5 & 24 \\
\hline Unidentified Type A & - & - & - & - & - & - & - & 11 & - & 11 \\
\hline Barley/Fescue ? & - & - & - & $\overline{-}$ & - & $\bar{j}$ & - & 14 & - & 14 \\
\hline $\begin{array}{r}\text { Caryophyllaceae } \\
\text { (Pink Family) }\end{array}$ & - & - & - & 1 & - & 1 & - & - & - & - \\
\hline $\begin{array}{l}\text { Fabaceae } \\
\text { (Legume Family) }\end{array}$ & 1 & - & - & - & 1 & 2 & - & - & - & - \\
\hline $\begin{array}{l}\text { Poaceae } \\
\text { (Grass Family) }\end{array}$ & - & - & - & - & - & - & - & 30 & - & 30 \\
\hline $\begin{array}{l}\text { Solanaceae } \\
\text { (Nightshade Family) }\end{array}$ & - & - & - & 1 & - & 1 & - & - & - & - \\
\hline \multicolumn{11}{|l|}{$\frac{\text { Zea }}{\text { corn }} \frac{\text { mays }}{\mathrm{rn}} \mathrm{L}$. } \\
\hline cupules & 196 & 30 & 7 & 4 & 263 & 500 & 1 & 21 & 30 & 52 \\
\hline kernels & 26 & 1 & - & 1 & 21 & 49 & 1 & 6 & 11 & 18 \\
\hline \multicolumn{11}{|l|}{$\frac{\text { Phaseolus }}{\text { common bean }} \frac{\text { vulgaris }}{\text { Le. }}$} \\
\hline
\end{tabular}


In addition, hickory nutshel1 is more ubiquitous at both Fredricks $(100.0 \%)$ and Mitchum (100.0 $\%)$ than at Wall (83.3\%). Acorn percentage and ubiquity both show significant drops between the Wall and Fredricks sites (Table 11.6).

There is, therefore, good evidence for increasing emphasis on hickory nut collection during the Historic period with a concomitant decrease in the use of acorn, although the results from the 1985 excavations indicate that the differential representation of these two foods at Fredricks is not as great as was previously thought. Walnut is represented by small quantities at all three sites, but shows a similar decrease in both ubiquity and percentage. The Wall site population does seem to have exploited the range of available nut resources more evenly, whereas the Fredricks site population focused on hickory. Using the modified Simpson index discussed above, the Wall site flotation samples show a higher diversity for nut taxa $(D=.6272)$ than do an equal number of Fredricks site samples $(D=.5412)$. Since richness and sample size are held constant (both sites have three nut taxa), differences in equitability must be responsible for this contrast. This finding, in contrast to the more evenly distributed representation of nut types at the Wall site, supports the notion of a focus on exploitation of one nut type at Fredricks. This conclusion is based upon ubiquity; comparison of food represented could present a different picture. Differential use of hickory and acorn seem more pronounced at Wall than at Fredricks, based on the corrected ratio.

The Mitchum site assemblage is similar to that of the Wall site in this respect. At Mitchum, acorn and walnut are both better represented as percentage of nut remains than they are at Fredricks. However, ubiquity values for the three nut types at Mitchum are closer to those for Fredricks. It is difficult to decide how to interpret these data, but it does seem that acorn was used to a greater extent than hickory at the Mitchum site, judging by its corrected acorn to hickory ratio.

Table 11.6

Percent of nutshell from the Wall, Fredricks, and Mitchum sites.

\begin{tabular}{|c|c|c|c|c|}
\hline Context & $\begin{array}{l}\text { Nut she11 } \\
\text { (grams) }\end{array}$ & $\underset{z}{\text { Hickory }}$ & $\underset{z}{\text { Acorn }}$ & $\underset{z}{\text { Wal nut }}$ \\
\hline \multicolumn{5}{|l|}{ Wall Site } \\
\hline Flotation & 28.12 & 85.4 & 10.7 & 3.9 \\
\hline Waterscreen & 9.78 & 92.0 & 8.0 & 0.0 \\
\hline Total & 37.90 & 87.1 & 10.0 & 2.9 \\
\hline \multicolumn{5}{|l|}{ Mitchum Site } \\
\hline Flotat ion & 3.84 & 91.4 & 7.6 & 1.0 \\
\hline Waterscreen & 7.74 & 91.7 & 4.4 & 3.9 \\
\hline Total & 11.58 & 91.6 & 5.4 & 2.9 \\
\hline \multicolumn{5}{|l|}{ Fredricks site } \\
\hline Flotation & 51.69 & 97.7 & 0.9 & 1.4 \\
\hline Waterscreen & 11.23 & 99.6 & 0.4 & 0.0 \\
\hline Total & 62.92 & 98.1 & 0.8 & 1.1 \\
\hline
\end{tabular}

\section{Tropical Cultigens}

Corn. Corn remains, like hickory nutshe11, are well represented at all three sites. Ubiquity and percentage are both highest for the Fredricks site, based on site totals. However, corn percentage is highly variable between contexts at the Fredricks site, ranging from a low of $2.6 \%$ (Structure 1) to a high of $68.3 \%$ (Feature 9, Zone 3). The high proportion of corn in Feature 9 is largely responsible for the surprisingly high site total of $41.0 \%$.

Whether or not to accept the Fredricks site corn percentages as directly comparable to those from the other two sites depends to a large extent upon how Feature 9 differs from other features in the composition of its plant remains assemblage. Feature 9, especially Zone 3, contains a high number of seed taxa compared to upper and lower burial fill from selected burials, fill from Features 10, 11, 12, and 13 (all shallow pits), and Structure 1 (associated with Feature 9) (Table 11.7). Corn percentage is also unusually high, particularly for Zone 3 . In Zone $3 \mathrm{~b}$, which is defined as clusters of charred corn kernels and bark resting on the floor of the pit, corn comprises $95.8 \%$ of plant food remains. For this reason, results from this zone were not included in site totals. Zone $3 b$ possibly represents some specialized activity involving food processing and/or consumption, and, as such, it is not directly comparable with other contexts (such as midden and shallower pits) that probably represent trash deposits.

Although Feature 9 is a rich source of information, some care must be exercised in interpreting its plant remains. For comparison between sites, similar contexts should be compared in this case. Burial fill from Wall and Fredricks contains similar proportions of corn remains. Similarly, the number of kernels per gram of plant food remains is quite close based upon waterscreened material (no corn kernels were found in the Wall site burial flotation samples). Neither an increase nor a decrease in use of corn can be established between the Wall and Fredricks site occupations. It is certain that corn remained an important crop into historic times, and likely that it may have retained its former level of importance.

Although the overall percentage of corn at the Mitchum site is relatively low, the presence of two cob-filled pits at the site points to its importance there. Additionally, a study of the seed assemblage from several features at the site indicates that some of those features probably were formed during early- to midsummer. There is a fairly strong correlation between low corn percentage and summer seasonal profiles $(r=0.82$, Spearman rank order correlation coefficient, significant at the 0.05 level, two-tail), based on selected feature data from all three sites. Thus, the unexpectedly low representation of corn at Mitchum may be a 
Table 11.7

Comparison of plant remains from burials, features, and structures at the Fredricks site.

\begin{tabular}{|c|c|c|c|c|c|c|c|c|c|c|c|c|}
\hline & Upp & er Burial & Fill & Lower & Burial & Fil1 & \multicolumn{4}{|c|}{ Fea. 9} & \multirow{2}{*}{$\begin{array}{l}\text { Fea. } \\
10-13\end{array}$} & \multirow{2}{*}{$\begin{array}{r}\text { Str. } \\
1\end{array}$} \\
\hline & Zone I & Zone II & Zone III & $\mathrm{Bu} .4$ & $\mathrm{Bu} .5$ & $\mathrm{Bu} .8$ & Zone I & Zone II & Zone III & Zone IIIb & & \\
\hline $\begin{array}{c}\text { Liters of } \\
\text { Fill }\end{array}$ & 20 & 20 & 20 & 70 & 62 & 98 & 40 & 30 & 70 & 10 & 95.5 & 10 \\
\hline $\begin{array}{l}\text { Plant Food } \\
\text { Remains }(\mathrm{g})\end{array}$ & 1.40 & 2.94 & 1.85 & 1.25 & 1.37 & 4.43 & 12.30 & 6.07 & 59.03 & 8.15 & 6.22 & 0.38 \\
\hline Hickory $(\%)$ & 74.3 & 65.6 & 67.0 & 86.4 & 54.7 & 77.9 & 81.3 & 73.5 & 26.8 & 3.4 & 92.4 & 97.4 \\
\hline $\operatorname{Acorn}(\%)$ & $\mathrm{x}$ & - & 0.5 & - & 2.2 & 0.2 & $\mathrm{x}$ & - & 0.5 & - & 0.8 & $\mathrm{x}$ \\
\hline Peach Pit (\%) & 1.4 & 9.9 & 3.8 & - & - & 3.6 & 0.6 & 2.5 & 3.4 & - & 2.3 & - \\
\hline $\begin{array}{l}\text { Walnut (\%) } \\
\text { Corn: }\end{array}$ & $4 \cdot 3$ & - & - & - & - & 0.7 & 0.2 & - & $\mathrm{x}$ & - & 0.6 & - \\
\hline Cupules (\%) & 18.6 & 21.8 & 23.2 & 11.2 & 29.9 & 13.5 & 6.5 & 8.1 & 5.8 & 0.5 & 1.8 & - \\
\hline Kernels (\%) & $\mathrm{x}$ & 2.7 & $\mathrm{x}$ & $\mathrm{x}$ & 2.9 & 0.7 & 9.0 & 14.5 & 62.4 & 95.8 & 1.1 & 2.6 \\
\hline Common Bean (\%) & 0.7 & - & 0.5 & 1.6 & - & 1.4 & 1.8 & 0.8 & 0.3 & 0.2 & 1.0 & - \\
\hline Seeds $(\%)$ & $\mathrm{x}$ & $\mathrm{x}$ & 4.9 & $\mathrm{x}$ & 6.6 & 2.0 & 0.7 & 0.7 & 0.7 & $\mathrm{x}$ & 0.5 & - \\
\hline \multicolumn{13}{|l|}{ Seeds } \\
\hline "Squash" & - & - & 1 & - & - & - & - & - & 1 & - & - & - \\
\hline Unidentified & 1 & 2 & 5 & 2 & - & 3 & 3 & 1 & 19 & - & 4 & 1 \\
\hline Maypops & 1 & - & 1 & - & - & 1 & 4 & 2 & 20 & 1 & - & - \\
\hline Blueberry & 1 & - & - & 1 & 1 & 1 & - & - & - & - & - & - \\
\hline Grape & - & 1 & - & - & 1 & 1 & 1 & 1 & 18 & - & 1 & - \\
\hline Persimmon & - & - & 1 & - & 1 & 3 & - & - & - & - & - & - \\
\hline Sumac & - & - & - & - & - & - & - & - & 2 & - & - & - \\
\hline $\begin{array}{l}\text { Nightshade } \\
\text { family }\end{array}$ & - & - & - & - & - & - & 1 & - & - & - & - & - \\
\hline Grass Family & - & - & - & - & - & 1 & - & - & 2 & - & - & - \\
\hline Spurge & - & - & - & - & - & 1 & - & - & - & - & - & - \\
\hline Hawt horn & - & - & - & - & - & - & 1 & 1 & - & - & - & - \\
\hline Chenopod & - & - & - & - & - & - & - & - & 17 & - & - & 1 \\
\hline Elder & - & - & - & - & - & - & - & - & 1 & - & - & - \\
\hline Bramble & - & - & - & - & - & - & 2 & - & 1 & - & - & - \\
\hline Groundcherry & - & - & - & - & - & - & - & - & 2 & - & - & - \\
\hline Legume Family & - & - & - & - & - & - & - & - & 1 & - & - & - \\
\hline Bedstraw & - & - & - & - & - & - & 1 & - & 1 & - & - & - \\
\hline Poke & - & - & - & - & - & - & 1 & 2 & 3 & - & - & - \\
\hline Nightshade & - & - & - & - & - & - & - & - & - & - & 1 & - \\
\hline Morning Glory & - & - & - & - & - & - & - & - & - & - & 2 & - \\
\hline
\end{tabular}


reflection of a seasonally-specific strategy of harvesting grasses (primarily maygrass, a relatively reliable seasonal indicator) during the spring and summer when corn stores were low and other crops were not yet available. Corn was an important crop for Mitchum site people, but its relative importance on a year-round basis cannot yet be determined.

Beans. Common bean (Phaseolus vulgaris L.) is rare on archaeological sites in the East until after ca. A.D. 1200 (Yarnell 1983:5). It was found at both the Wall and Fredricks sites. Its representation at these two sites is quite similar in both ubiquity and percentage. The fact that beans may have been prepared by boiling rather than parching or roasting may account for its limited occurrence at both sites. Beyond this, little can be said about the importance of beans for the Wall and Fredricks site populations.

Cucurbits. Rind fragments of cucurbits (members of the gourd family, Cucurbitaceae) were found at Mitchum and Fredricks. They represent either bottle gourd (Lagenaria siceraria Standley) or "squash" (Cucurbita sp.). The latter taxon is also represented at the Fredricks site by three seeds of Cucurbita pepo L., probably a gourdy (as opposed to fleshy) variety, on the basis of seed size (R. Yarnell, personal communication 1984). It is likely that the fruits were used as containers, and perhaps for food as well.

Native Grains. Several different kinds of grasses are represented at the Fredricks and Mitchum sites. Most of these could be identified only to the family level (Poaceae) or to the category "barley/fescue", which includes grains similar to little barley except for their size. However, large numbers of maygrass (Phalaris caroliniana Walter) and smaller numbers of little barley (Hordeum pusillum L.) grains were found in Feature 6 at the Mitchum site. Chenopod (Chenopodium sp.) from Wall and Fredricks and sumpweed (Iva annua var. macrocarpa Jackson) from Wall will also be discussed as grain seeds (in some cases more properly called fruits; here the term "seeds" will be used to refer to both).

A single sumpweed seed was recovered from the fill of a Wall site burial. Its dimensions ( $6.7 \times 5.3 \mathrm{~mm}$ with corrections for carbonization) place it well within the range of the cultigen variety of Mississippian times (Yarnell 1978). This large-seeded variety, which was presumably the result of human-mediated selection, is known only archaeologically, and its decline as a crop is not yet well understood. Although the Wall site population may have grown sumpweed, its importance there cannot be assessed on the basis of a single specimen. It is important to note, however, that this native crop plant persisted so late in the Piedmont, and that it was dispersed so far to the east from its native range in the Mississippi drainage.
A small number of chenopod seeds was recovered from the Fredricks site, and a single specimen was found in a Wall site midden flotation sample. Like sumpweed, chenopod was an important crop plant in some parts of the East, and shows some evidence of morphological change under domestication (B. Smith 1985; Fritz 1984). However, chenopod is also a weedy taxon, and its presence in small numbers does not argue convincingly for its use here as food.

The Mitchum site shows the strongest evidence for utilization of native starchy seeds, mainly in the form of maygrass and little barley grains. Maygrass has been found archaeologically outside of its modern natural range, which is considered evidence of its role as a crop (Yarnel1 1983; Cowan 1978). Since it is an annual species that fruits in the early part of the year, maygrass has been classified as a "cool-season" grass (Bohrer 1975). In westcentral Illinois, little barley has also been classed as a crop plant because of its abundance in archaeological contexts (Asch and Asch 1983:687). The large numbers of maygrass grains found at Mitchum provide strong evidence of harvesting and perhaps also husbandry.

The deposits at Mitchum that contain maygrass have in general a relatively early seasonal profile based upon seed types. They also contain relatively small amounts of corn remains. Therefore, the presence of large quantities of maygrass may be evidence of a seasonal pattern of grass-collecting in the spring and early summer months, when most edible fruits as well as crops would have been in short supply. Although there is as yet no evidence for similar patterns at Wall or Fredricks, the possibility of their existence should not be ruled out until material from deposits with similar seasonal parameters can be examined.

\section{Fleshy Fruits}

Seeds of fleshy fruits are quite common at all three sites, with persimmon (Diospyros virginiana L.), grape (Vitis sp.) and maypops (Passiflora incarnata L.) being the most common taxa. A greater number of fruit types is represented at Fredricks than at the other two sites, and most are from Zone 3 of Feature 9, which contained a particularly rich assemblage of plant food remains. Most of the fleshy fruit types found at the Wall site were also found at Fredricks. In general, the fruit types recovered can be classified as weedy taxa adapted to early successional habitats, forest edges, and disturbed ground. Some of these plants, such as maypops, may have been tended or simply tolerated as garden volunteers because of their food value. The peach (Prunus persica L.) was certainly introduced as a crop, but its weediness contributed to its rapid spread to aboriginal groups.

Except for peach, which is present at Fredricks but absent from the Wall site, it is 
difficult to discern any major differences in the use of fleshy fruits. Persimmon, grape, and maypops all remained important, although grape shows a slight decline based on number per gram of plant food remains (Table 11.8). Hawthorn (Crataegus sp.) seems to have been relatively important at all three sites. The abundance of fruit types in Feature 9 at the Fredricks site may be a reflection of special activities such as fruit drying rather than of a trend toward use of a greater variety of fruit types. In any case, although fleshy fruits were not staple foods, they were commonly used.

Peach does, however, deserve some attention here as a species introduced by Europeans that was readily adopted by aboriginal groups. Sheldon (1978) has correctly pointed out the significance of peach remains as indicators of European contact. However, historical accounts (Lawson, in Lefler 1967; Salley 1911) of the presence of populations of peach trees in the seventeenth and early eighteenth centuries attests to the spread of this species somewhat independently of intentional propagation. The domesticated peach grows well with little or no tending (Hedrick 1972) and bears fruit within several years of germination. Therefore, peach remains are more properly considered signs of indirect rather than direct contact with Europeans.

At the Mitchum site, peach pits comprise $9.5 \%$ of plant food remains and occur in $23.5 \%$ of samples analyzed. Its representation at Fredricks is similar. Peach was certainly used during both occupations, although the density and durability of peach pit probably results in its over representation relative to other plant foods. The rapid diffusion of peach and its partial independence from human care may be responsible for its abundance at Mitchum despite the scanty evidence there of contact in the form of trade goods.

\section{Miscellaneous Seeds}

Several of the seed types present at these sites probably were not used as food. They may have become included in the deposits fortuitously because they grew on the site, or may have had non-food uses. Bedstraw (Galium sp.) seeds can be used to make a beverage, but it is more likely that the vegetative part of the plant was used as bedding. Morning glory (Ipomoea sp.), spurge (Euphorbia maculata L.), and bearsfoot (Polymnia uvedalia L.) were probably garden weeds. Dogwood (Cornus sp.) and the Pink family (Caryophyllaceae) may have been incidental inclusions. Unidentified Type $A$ is fairly distinctive, but has not yet been identified using standard reference works (Martin and Barkley 1961; Montgomery 1977; USDA 1974), and thus is classed as miscellaneous.

\section{Diversity}

A Simpson diversity index was computed for
Table 11.8

Distribution of seeds from the Wall, Mitchum, and Fredricks sites (number per gram of plant food remains).

\begin{tabular}{|c|c|c|c|}
\hline Seed Type & Wal1 & Mitchum & Fredricks \\
\hline Plant Food Remains ( $\mathrm{g}$ ) & 32.63 & 4.78 & 105.09 \\
\hline \multicolumn{4}{|l|}{ Tropical Cultigens } \\
\hline Corn kernels & 0.83 & 1.46 & 4.14 \\
\hline Corn cupules & 6.13 & 4.39 & 4.85 \\
\hline Bean cotyledons & 0.28 & - & 0.17 \\
\hline Cucurbit & - & - & 0.03 \\
\hline \multicolumn{4}{|l|}{ Native Grains } \\
\hline Legume family & 0.06 & - & $\mathrm{x}$ \\
\hline Maygrass & - & 92.05 & - \\
\hline Little barley & - & 2.72 & - \\
\hline Barley/fescue & - & 2.93 & - \\
\hline Grass family & - & 6.69 & 0.03 \\
\hline Chenopod & - & - & 0.17 \\
\hline Sumpweed & 0.03 & - & - \\
\hline \multicolumn{4}{|l|}{ Fleshy Fruits } \\
\hline Grape & 0.64 & - & 0.26 \\
\hline Maypops & 0.25 & 0.21 & 0.29 \\
\hline Hawthorn & 0.06 & 0.21 & 0.03 \\
\hline Persimmon & 0.03 & - & 0.08 \\
\hline Bramble & - & 0.21 & 0.03 \\
\hline Blueberry & - & - & 0.09 \\
\hline Sumac & - & - & 0.03 \\
\hline Nightshade & - & - & 0.02 \\
\hline Groundcherry & - & - & 0.03 \\
\hline Nightshade family & 0.03 & - & 0.09 \\
\hline E1derberry & - & - & $\mathrm{x}$ \\
\hline \multicolumn{4}{|l|}{ Greens } \\
\hline Poke & 0.03 & - & 0.06 \\
\hline \multicolumn{4}{|l|}{ Miscellaneous } \\
\hline Bedstraw & 0.03 & 0.21 & 0.07 \\
\hline Dogwood & 0.03 & - & - \\
\hline Pink family & 0.03 & - & - \\
\hline Bearsfoot & 0.06 & - & - \\
\hline Unid, type A & - & 2.30 & - \\
\hline Spurge & - & - & $\mathrm{x}$ \\
\hline Morning glory & - & - & 0.02 \\
\hline
\end{tabular}

Notes: Based on flotation samples from which both light and heavy fractions were analyzed; $\mathrm{x}=$ trace.

the Wall and Fredricks site assemblages as well as for nut types. Based on equal numbers of samples from each site, diversity values for the Wall and Fredricks sites are quite close (.8384 and .8812 , respectively). Both are also high, indicating relatively heterogeneous assemblages. The Fredricks site assemblage is somewhat more diverse than that of Wall; however, the difference is not as great as might be expected considering the fact that 23 taxa are represented at Fredricks, compared with only 12 from Wa11. This finding indicates that the relatively high Wall site index reflects greater equitability of representation of plant taxa in that assemblage. The Fredricks site assemblage, on the other hand, is richer.

One interpretation of these results is that 
more plant resources were used during the later occupation but that there was a concentration on exploitation of only a few of these. Although these findings must be considered highly tentative, diversity indices may prove to be useful tools for interpreting subsistence remains, with appropriate attention to potential sources of bias (cf. Holm, this report; Wilson 1983). Since ecologists working with observable organisms often disagree on the meaning and proper use of diversity indices (Peet 1974), archaeologists must exercise extra caution in using them.

Summary

Several differences in patterns of plant use between the Wall and Fredricks site occupations are apparent. Acorn and walnut were used more frequently at Wall, whereas hickory dominates at Fredricks. An earlier pattern of more equitable use of all three nut types gave way to one of concentration on hickory with retention of acorn and walnut as relatively minor resources. Corn remained the chief staple crop, and may have become even more important in the Historic period. Native grasses available in spring and summer were seasonally important at least to the Mitchum site population. The Wall site population either grew or acquired cultigen sumpweed, but there is no evidence of the use of indigenous grain crops at Fredricks. The peach was used and probably cultivated by the time of the Mitchum site occupation, but had not yet become available to the Wall site population. There may have been a trend at the Hillsborough locality toward use of more plant resources with intensive use of a small number of these, but this interpretation is still tentative.

\section{Additional Data from North Carolina and Tennessee}

Comparison of plant remains from the Wall and Fredricks sites is particularly informative about changing aboriginal subsistence in one part of the Eno River valley, and the Mitchum site data provide a glimpse of Historic period plant use in the adjacent Haw River valley. However, another Historic period Piedmont site has yielded paleoethnobotanical data that are useful for comparison with these results. This is the Upper Saratown site in the Dan River drainage of the northwestern North Carolina Piedmont, located about 50 miles west of the Mitchum site. This site was occupied between A.D. 1650 and 1700; therefore, its occupation overlaps with that of the Fredricks site. Information on plant remains from Upper Saratown is taken from Wilson $(1977,1983)$.

Since the Upper Saratown plant remains were recovered by waterscreening through $1 / 16-i n$ mesh, they will be compared with waterscreened material from Fredricks. The assemblages are similar in a number of ways. The hickory nutshell percentage for Upper Saratown ( $51.6 \%)$ is quite close to that for Fredricks (52.4\%). However, the corrected acorn-to-hickory ratio for Upper Saratown is nearly one (0.94), which suggests that the two nut types had similar importance there; the ratio for Fredricks based on waterscreened samples is only 0.17. Walnut and hazelnut are also better represented at Upper Saratown than at Fredricks.

Corn percentage is higher at Upper Saratown $(37.5 \%)$, but this may be a consequence of the greater variety of feature types represented there. Common bean and squash occur in small amounts at Upper Saratown, in addition to bottle gourd. Except for sunflower (represented by one seed), no native grain seeds were recovered from the site. However, watermelon, another European introduction, is represented in addition to peach. As at Fredricks, grape, persimmon, and maypops are the most abundant seed types. A Simpson diversity index of .8876 was obtained for Upper Saratown, which is quite close to that calculated for Fredricks (.8812).

Thus, the Upper Saratown population exploited the same types of resources that were used at Fredricks. Fleshy fruits, including peach, occur in similar proportions at both sites. The same is true for all non-indigenous crop plants except for corn. Both sites show evidence of considerable European contact. A number of factors including local environment, group size, trade relations with Europeans, and archaeological excavation procedures may account for the differences between the two assemblages. In any case, plant use seems to have been similar in both areas during the Historic period. Data on plant remains from earlier sites in the Dan River drainage would be useful for determining whether there were similar changes in plant use between the Protohistoric and Historic periods there as were noted at the Eno River sites.

Elsewhere in the East, changes in plant food use during the Contact period are in some respects different from those assessed for the North Carolina Piedmont. In the lower Little Tennessee River valley (Chapman and Shea 1981), corn percentage is generally higher than in the Piedmont for both the Late Mississippian Dallas phase (ca. A.D. 1300-1600) and the Historic Cherokee period (ca. A.D. 1700-1819), with corn percentage increasing sharply during historic times. Although acorn seems to have undergone a similar decline in importance, walnut and butternut were still used frequently in the Historic period. In eastern Tennessee, sunflower, chenopod, maygrass, and knotweed (Polygonum sp.), all indigenous grain seed sources, are as abundant, or more abundant, at Historic period sites as they are in Late Mississippian components. 


\section{A COST-BENEFIT APPROACH TO CHANGE AND CONTACT}

Having described changes in the archaeological record of plant use in the North Carolina Piedmont during the Protohistoric and Historic periods, the task of explaining those changes remains. Although the following suggestions should be considered informal hypotheses rather than conclusions, they can be used to guide further research. Additionally, this discussion is intended to integrate archaeological information on plant use with consideration of other aspects of aboriginal Piedmont culture during the Historic period, including relationships with Europeans.

Cost-benefit models are derived mainly from microeconomic theory. However, ecologists have incorporated some economic concepts into their own models of diet choice, territoriality, and group organization. Optimal foraging theory has integrated economic and ecological concepts in attempting to model optimal subsistence and survival strategies for both human groups and non-human animals. Examples of the use of cost-benefit models in interpreting archaeological subsistence data include the work of Earle (1980) and of Keene (1981), who also uses an optimal foraging approach.

Cost-benefit and optimization models include the assumption that humans, like other animals, tend to exploit resources in such a way that costs are minimized and benefits maximized. Subsistence practices vary in their efficiency in terms of time and energy costs, and it is assumed that increased efficiency results in increased fitness (Winterhalder 1981). Although these assumptions are not always justified, they will be used here for heuristic purposes. The proximate cause of changing frequencies of different behaviors within a population (i.e., decision-making by individuals or groups) will not be discussed here. However, since costbenefit relationships affect decision-making, it should be acknowledged that the selection of certain behaviors ultimately will be made at the expense of others.

\section{Currencies, Costs, and Benefits}

The currency (i.e., measure of efficiency) most commonly used in cost-benefit assessments is energy (Winterhalder 1981). Complex currencies such as nutrients are sometimes used in linear programming approaches (Keene 1981). Time factors should also be taken into account, which allows for assessment of acquisition rates (E. Smith 1979). Since the non-quantitative approach used here eliminates the complexities of mathematical modeling, time, energetic, and nutritional factors will all be considered where appropriate.

Cost components of resource acquisition include search costs, pursuit and handling costs, and opportunity costs. Search costs, which are incurred while locating prey, vary according to technological resources and distribution of prey in space. For agricultural products, for instance, search time would be essentially zero, in contrast to that for mobile fauna. Pursuit and handling costs consist of prey capture, transport, processing, consumption, and other related activities. All of these costs reflect time spent as well as energy expended on a given task.

Time spent on one activity is necessarily lost for other activities. Therefore, the cost of procurement of a given resource can be seen to include the value of activities in which resources could have been alternatively invested, or what has been called its opportunity cost (Winterhalder 1983:15). This opportunity cost model can be especially useful for comparing activities that result in different types of material gain (e.g., hunting for trade purposes versus hunting or other procurement for subsistence purposes).

Returns exist in the form of both energy and nutrients. It is assumed that decisions on what resources to use are made in an effort to maximize at least short-term returns, even though people lack complete and perfect information about their environment. Non-material benefits may also be relevant, but will not be considered in detail here.

\section{Specific Applications of a Cost-Benefit Approach}

Relationships between subsistence activities, environmental variability, group organization, and information flow in human groups are always complex. When Europeans arrived in the North Carolina Piedmont they brought with them new potential resources as well as epidemic disease and attendant changes in population (Dobyns 1984) and group organization. By the time of the Fredricks site occupation, there is evidence of ongoing trade between aboriginal and European groups. We know from historical sources that trade in deerskins was prevalent in this period, and certainly must have affected the costs and benefits of other activities. This evidence is persuasive, despite the fact that studies of faunal remains from the Wall and Fredricks sites are inconclusive regarding changes in the frequency or kind of deer hunting (see Holm, this report). With this information in mind, we can begin to assess the relationships between the European presence (particularly in the form of trade networks) and changes in plant food use as reflected in the archaeological record.

Trade and Opportunity Cost. At the Hillsborough locality, use of acorns declined in the Historic period, whereas hickory nuts remained an important resource. In the East as a whole, acorn use seems to have declined prehistorically after a peak during the Early Woodland period in Tennessee, reaching its lowest levels during the Historic period (Yarnell and Black 1983). Its decline may in part reflect an increasing 
importance of corn and other grain crops.

Some modern time and energy expenditure studies comparing nut processing techniques suggest that the costs of processing acorns for consumption may be higher than that for hickory nuts, depending on the techniques employed (Talalay, Keller, and Munson 1984; Petruso and Wickens 1984). Although acorns may actually yield more meat per unit time spent shelling, most types of acorns require leaching to render the meats palatable. Hickory nut meats, on the other hand, can be easily extracted by pulverizing whole nuts and boiling the mixture to extract oil and hickory "milk" (provided that appropriate containers are available), a practice documented for Historic period North Carolina Indians (Lawson, in Lefler 1967). Put into a cost-benefit framework, a prehistoric scenario might to as follows. If acorn processing costs were in fact relatively high, other resources with lower associated costs, higher benefits, and similar nutritional content (such as corn) could have outranked acorns.

This scenario is not, however, adequate for explaining why acorn use declined even further during the Historic period. Trade may have played a role in decreasing acorn's ranking. As trade items exchanged for deerskins became, in effect, a new resource for aboriginal groups, the opportunity cost of procuring acorns (as well as certain other plant foods) might have risen. Increased opportunity cost induced by the introduction of trade goods might have acted to lower the rank of acorns still further.

A similar argument can be advanced to explain decreasing use of walnut in the same area. Except for a few time periods at a few locations, walnut has not been a primary resource in the East based upon available archaeological evidence. Its distribution in the Carolinas is scattered (Radford et. al. 1968), resulting in higher pursuit costs (in the form of travel time) relative to other nut resources. With the advent of the deerskin trade, a shift to a focus on the highest-ranked nut resource (hickory) could have made more time available for trade-related activities, particularly during the fall, when the deer rut and the fruiting times of most nut-bearing species partly coincide.

In the case of corn, the change in cost-benefit ratio was probably less severe. As a crop, corn would have had negligible search costs, moderate handling costs (including garden maintenance), and probably high yield compared to other food crops. High energetic and nutritional gains would have compensated for any increased opportunity costs resulting from trade. The symbolic significance of corn and its prominence in ritual are non-material factors that perhaps acted to encourage its maintenance. Trade brought in ornaments, tools, and alcohol, none of which could have replaced a staple food such as corn except through further complication of trade networks.

If use of "cool-season" grasses did in fact become less common, trade could have similarly introduced scheduling conflicts and increased opportunity costs. However, it seems that maintenance of grass harvesting as a seasonal strategy would have had relatively low associated costs and high benefits during a period of relative resource scarcity. Another possibility is that information about using these resources was simply lost because of depopulation and reorganization of human groups. Discontinuities introduced into the path of information flow between generations could have led to loss of some traditional procurement techniques. This suggestion is highly speculative, but presents some possibilities for further research.

The adoption of peach is easily explained using a cost-benefit approach. All fleshy fruits represented at the three sites are somewhat weedy species that tend to be highly productive in disturbed habitats such as those around Indian settlements. Search costs would have been correspondingly low, and processing cost negligible. The peach was simply a newly available fruit with similar qualities. In addition, peach fruits had the large size and perhaps the increased palatability often associated with selection under domestication. Even as a crop, the maintenance costs of peach use would have been low because of its ability to grow well with relatively little care. Processing costs for storage would not have been any higher than those associated with native fruits.

Division of Labor and Group Size. Until this point, subsistence activities have been discussed as if they were participated in equally by all group members. It should at least be pointed out that sexual division of labor is a factor to be considered. For instance, if deer hunting and trade with Europeans were male activities, and plant procurement a female activity, there would not necessarily have been direct scheduling conflicts between trade-related activities and nut collection, grass collection, and corn harvesting and planting. However, if women were the processors of deerskins, their activities would have closely tracked those of the men, resulting in a similar overall effect of increased opportunity cost. Similarly, group size and population structure would have affected the efficiency ratios of different activities.

\section{RECONSTRUCTION OF PLANT USE PATTERNS AT THE FREDRICKS SITE}

Differences between pre-contact and postcontact plant use and the explanation of apparent subsistence changes have been the main focus of the ethnobotanical research discussed 
here. However, it is also important to establish as complete a reconstruction as possible of plant use during a given occupation. Since of the three sites discussed here, Fredricks has provided the largest body of ethnobotanical data, it offers the best opportunity for such a reconstruction. Using these data as we11 as information about contemporary vegetation and historical sources, the pattern of plant use and plant management at the Fredricks site in the Middle Contact period can be outlined. Two basic parameters of plant resource use will be used to orient this discussion, namely spatial and temporal variation in resource occurrence and human responses to them.

\section{Spatial Variation}

Even in a mature ecological system, such as the oak-hickory forest that was probably the dominant vegetation pattern in the pre-contact Piedmont, areas of contrasting vegetation inevitably occur. This is because environment is not simply a stable backdrop for human activity, but rather a dynamic phenomenon subject to historical processes that produce both spatial and temporal variability (Winterhalder 1980:136). If mature hardwood forest is taken as a matrix, areas that contrast in some way with these surroundings can be termed patches (Wiens 1976; White and Pickett 1985). Natural disturbance in the form of fire or fallen trees are likely to create patches of varying sizes. The presence of human populations produces additional agents of disturbance, sometimes intentional (e.g., firing of forested tracts to drive game or encourage browse for herbivores) and sometimes less so (patches of herbaceous or shrub vegetation in fallowed agricultural fields, at various stages of succession). Thus the surroundings of the village represented archaeologically by the Fredricks site should be viewed as a mosaic of vegetational patches in a forested matrix, including anthropogenic (human-generated) patches as well as ones differentiated on the basis of natural factors such as disturbance, slope, elevation, soil substrate, and hydrology. Patch distribution is variable in time as well as space, producing a mosaic of vegetational patches at different stages of development (Wiens 1976:82), each with its own assemblage of species.

A formal survey of existing vegetation in the immediate vicinity of the Fredricks site has not yet been undertaken. Although such a survey would be useful and may be done in the future, its usefulness would be limited because of two important factors. First, because of the shifting nature of patches, especially in the context of considerable human disturbance, the distribution of vegetational patches at the time of occupation would be impossible to reconstruct on the basis of modern distributions. Even during the site occupation (estimated at some 30 years) the dynamics of patch distribution would have become increasingly complex as agricultural fields were abandoned and new patches created through clearance. Second, the present-day composition of early successional habitats (i.e., areas recently disturbed and characterized in forested areas by sun-loving herbaceous species that grow and reproduce rapidly) has been drastically altered through introduction of 01d World weeds, which have in many cases out-competed indigenous species usually dominant in such situations. Nineteenth-century land survey records (Delcourt 1976) and charcoal analysis (Chapman et al. 1982) have also been used in the Eastern Woodlands to reconstruct past environments. Pollen analysis, if feasible, would also be a useful tool for this task. However, none of these methods have yet been used at the Fredricks site.

Therefore, only a speculative reconstruction of environmental patches near the Fredricks site can be offered, based on general knowledge of community types common in the Piedmont today (Moore 1973; Moore and Wood 1976; Shelford 1963). Ethnographic information is available from a different cultural and geographical area. Alcorn (1984) has done a careful analysis of anthropogenic vegetation zones and their management for the Teenek of northeast Mexico. Direct extrapolation from modern to prehistoric land use would of course be inappropriate since different cultures and vegetational environments are considered, as well as different time periods. No formal analogies are being drawn between observed and past behavior-to-artifact relationships. However, information about relationships between a farming society and its land can be used to generate ideas about what kinds of patches might have been used by the Fredricks site inhabitants. Although such a reconstruction is admittedly speculative, it is valuable nonetheless as a background for understanding subsistence behavior as it is revealed archaeologically.

One of Alcorn's (1984) important findings was that most vegetation zones used by the Teenek can be classed as anthropogenic, even local forest. This was probably true of the Fredricks site locale as we11, since hardwood forest was an important source of food (hickory, acorn, and walnut) and may thus have been managed to some extent (including the most drastic form of management, burning). However, hardwood forest may be considered the naturally occurring matrix vegetation within which anthropogenic patches would have occurred. Hardwood forest was probably the most common ecological community near the village. Except for nut harvests, though, hardwood forest with its thick canopy and sparse understory probably provided little in the way of edible herbaceous plants. Extensive management of hardwood forest was probably minimal, although selective removal of certain trees can provide additional light for nut-bearing species and other food plants. Although oak and hickory trees have too long a generation time to allow for extensive human manipulation through planting and harvesting, limited management of some kind may have been 
practiced. A close relationship involving some sort of management even seems likely, since acorns and hickory nuts were apparently staple foods, but there is no direct evidence of this.

Within the forest, large patches in various stages of succession may have been present as a consequence of burning. Firing of forest to encourage browse for game or to encircle deer has been documented for Southeastern groups (Hudson 1976; Swanton 1946). Lawson (in Lefler 1967:31, 215) notes extensive burning of forest during game drives. Nut-bearing trees might have been either damaged or temporarily removed from these areas, but the resulting herbaceous vegetation would have produced more edible seedand fruit-bearing species than closed-canopy forest. Also, trees growing in more open locations, such as forest edges, are more productive. Plant species that dominate in such disturbed areas typically are annuals that produce large numbers of propagules (Horn 1974; Odum 1976). Most of the fleshy fruits used by the Fredricks site inhabitants (including bramble, grape, and elderberry) grow well in such disturbed habitats. Lawson (1967:34) mentions "savannahs" near Congaree full of fruit-bearing bushes.

Closer to the village, anthropogenic patches were probably even more common. Perhaps most obvious would have been agricultural fields in which the dominant crop was corn, beans, cucurbits, and other crops. The quantities of corn apparently consumed by the site inhabitants, as well as historic references to "fields" (Lefler 1967:56), indicate that separate agricultural fields (as opposed to small mixed gardens) were probably located near the village. Along with crops, weeds would have been present in fields, some of which may have been useful and hence spared. Teenek often spare useful trees when clearing a field in which to plant maize and beans (Alcorn 1984:346). Sun-loving weeds of agricultural fields near the Fredricks site might have included some of the fleshy fruit species mentioned above, as well as less useful ones such as morning-glory (Ipomoea sp.), still a common cornfield weed today. We do not know how extensively cornfields were weeded. However, William Hilton's "A Relation of a Discovery" (1664), speaking of the Carolina coast near the mouth of the Cape Fear River, mentions the high productivity of cornfields, "although the Land be overgrown with weeds through their lazinesse" (Salley 1911:44). It may be that European ethnocentrism mistook sparing of useful weeds for sloppy husbandry.

Abandoned agricultural fields may constitute a distinct type of anthropogenic patch. Among the Teenek, these are used in various ways before being replanted; sometimes they are replanted as mixed gardens, and sometimes simply maintained as habitats for useful wild or weedy species, especially medicinals (Alcorn 1984: 367-370). Old fields were potential sources of fleshy fruits and medicinal plants. At
Fredricks, old field habitats may have been similar to those of other patches deforested through burning, though perhaps somewhat closer to the village.

Still closer to or within the village, patches of ground maintained in a more or less disturbed state were undoubtedly common. Among the Teenek, dooryard "gardens" constitute an anthropogenic zone near houses. Some of the plants managed in this zone are conscientiously tended and propagated; others are simply spared. Many are medicinals (Alcorn 1984:331). The existence of dooryard "gardens", or clusters of useful plants, is purely speculative for the Fredricks site. Lawson did not mention such "gardens" in Piedmont villages, but it is possible that an Englishman would not have recognized a confusion of apparent weeds as a garden (in contrast to cornfields, which were apparently similar enough to European agricultural fields to be noted as such).

Another anthropogenic patch is equally speculative for the Fredricks site, and would be analogous to the cafetal, or coffee orchard of the Teenek (Alcorn 1984:372). The cafetal is essentially a managed forest, planted sometimes with coffee, and/or a variety of other fruitbearing trees. The possible existence of such zones near the Fredricks site is of particular interest because of the importance of fruit-bearing trees like persimmon, hawthorn, and peach. Certainly some amount of management of fruit trees is likely, particularly in the case of peach, which was a domesticate when it was introduced to North America. Lawson's account does not mention stands or orchards of fruit trees. However, he makes the statement that peaches "are the only tame Fruit, or what is Foreign, that these People enjoy. .." (Lefler 1967:173). Piedmont Indians apparently had a long-standing relationship with native fruit trees before contact, and with the peach thereafter. Certainly the existence of maintained stands of fruit trees analogous to the Teenek cafetal is a possibility.

In sum, a speculative reconstruction of main anthropogenic vegetational zones and patches near the Fredricks site village, with economic plants possibly found in each, can be summarized as follows:

1. Hardwood forest: oak, hickory, walnut; also blueberry, hawthorn (on poorly-drained soils), and grape (in low woods and on stream banks).

2. Large non-forested patches: Lawson's "savannahs"? Patches in various stages of succession after burning. Sumac, bramble; possibly maypops, poke, and hawthorn.

3. Active agricultural fields: corn, common bean, cocurbits; weedy annuals (groundcherry, poke, maypops). 
4. 01d fields: groundcherry, poke, maypops, hawthorn?, bramble?

5. Dooryard "gardens"?: medicinals and herbs?

6. Fruit tree stands?: persimmon, peach, hawthorn?

\section{Temporal Variability}

In addition to spatial variability resulting from vegetational patchiness on the landscape, Fredricks site inhabitants had to cope with temporal variability. For purposes of scheduling subsistence activity, the more-orless predictable seasonality component of temporal variability was probably of utmost importance. Unfortunately, most archaeological deposits at the Fredricks site contain remains of plants that ripen in the late summer and early fall. The storage of some of these foods (e.g., corn and nuts) complicates any attempt to determine season of deposition for particular deposits. However, a general outline of the Fredricks site "seasonal round" for plant foods can be proposed on the basis of the general plant remains assemblage from the site, botanical evidence of fruiting seasons for the species involved (Radford et al. 1968) and historical information from Lawson's account (Lefler 1967).

Mid-Summer to Early Fall. This was undoubtedly the season during which most crop harvesting took place. Perhaps most importantly, the chief crop, corn, would be ripe in late summer to early fall, as would common bean and cucurbit. If more than one corn crop was sown in a given year, a summer harvest, perhaps of "green corn", might have taken place as well. Fleshy fruits also become ripe during this broad time period, among them grape (August to October), hawthorn (August to October), elderberry (July to August), maypops (July to October), peach (June to July), persimmon (September to October), and blueberry (June to october). Summer and early fall would thus have been busy times for harvesting crops (including tree crops such as the peach) as well as collecting fruits growing mainly in old fields and other disturbed areas. Lawson reports (Lefler 1967:217) that fruits were dried and pounded into cakes for winter storage. Processing and storage of corn was also a fall activity.

Early Fall to Early Winter. The most important nut resources for the Fredricks site population, acorn and hickory, would have been available for collection roughly from September to November. The best time for collecting acorns and hickory nuts may have overlapped somewhat with the time of crop harvesting. After collection, acorns probably were processed to make acorn oil and hickory nuts prepared for storage (Lefler 1967:51, 105).

Mid-Winter to Early Spring. This part of the year is something of a terra incognita for the paleoethnobotanist seeking archaeological evidence of seasonal plant use. Most plants are dormant in the winter, and except for late nut crops, few or no fresh plant foods would have been available. It is during this part of the year that foods stored in the fall would have been consumed. This also may have been a time for hunting forays (possibly to obtain deerskins for trade as well as meat for food), as was reported by Lawson (Lefler 1967:217).

Spring to Late Summer. Spring may well have been the leanest time of year for the Fredricks site people. Most plant species resume growth in the spring, and some flower during this time, but fruiting generally does not occur until later in the year. Fresh greens would, however, have been available. Stores of crops, nuts and fruits from the previous fall would be near-depleted by this time. Perhaps animal foods dominated the diet in the spring; in fact, Lawson (in Lefler 1967:217) notes the use of weirs to take herring coming upstream to spawn in March and April. Even farther inland, the Fredricks site people may have turned to the nearby Eno River in springtime for fish (catfish bones are abundant in Fredricks site deposits, as reported by Holm [1985]). Another important spring activity was planting corn and other crops, which, like the harvest, was probably accompanied by rituals. Perhaps trade with Europeans became more active in the spring.

\section{SUMMARY AND CONCLUSIONS}

An analysis of plant remains from three archaeological sites that span the Protohistoric to Middle Contact periods has produced some useful working hypotheses about the effects of European-Indian interaction on aboriginal subsistence practices. Differences in the amounts of various nut remains (e.g., more acorn and walnut relative to hickory at the later sites) may indicate a trend toward displacement of already low-ranked resources because of increased time and energy devoted to trade-related activities, principally deer hunting. In other words, the opportunity costs of exploiting certain low-ranked plant resources would have increased as trade-related activities increased. If corn remained important, as evidence so far suggests, its persistence in Piedmont subsistence may have been due to its role as a high-ranked staple not easily substituted by European domesticates or other traditional aboriginal foods. An apparent decline in "cool season" grasses is not easily explained in terms of changing costs and benefits, since these species would have continued to constitute a valuable resource during a part of the year when most other plant foods were unavailable. Scheduling conflicts resulting from trade activities or information loss about traditional plant procurement techniques are two possible explanations for such a decline. Adoption of 
the peach, a European introduction, was certainly related to low procurement and processing costs of this plant, as well as to its high energy returns and palatability.

Despite the differences noted between pre-contact and post-contact plant use, there is no evidence at the Fredricks site of adoption of European crops (except peach) or abandonment of native ones, and only moderate evidence of adjustments in the proportions of native plant foods contributing to subsistence. Perhaps the presumed position of the Occaneechi village on the Eno as a trading center made it atypical in this respect. For example, if individuals from other depopulated areas aggregated at this village, its pre-contact population level might have remained stable despite losses through disease. And if Fredricks site men acted as middlemen in the European trade network, they may not have traveled far afield to hunt specifically for trade. It is apparent that explanation of subsistence stability is as important for this project as the explanation or establishment of change. We may find that the apparent stability of subsistence as revealed archaeologically in fact reflects a considerable amount of behavioral change (Winterhalder 1980). Behavioral changes may have been necessary to maintain the traditional diet represented archaeologically in the face of considerable peturbation.
In future research, we should look beyond the Piedmont to other parts of the East for comparisons of subsistence change and possible correlations between contact and aspects of change in other areas. Archaeological data on changing plant use in the Historic period are still not abundant (except, for example, Chapman and Shea 1981; Cadde11 1981). As more data become available, however, it will be possible to profitably ask questions about variability in the effects of contact on subsistence. For example, were highly complex societies such as the Southeastern chiefdoms more resistant to change than simpler groups? Was change in subsistence more prevalent where trade with Europeans was most intense? Is depopulation really associated with "loss" of some traditions? Was subsistence change more rapid in areas where European settlers, as opposed to traders and travelers, were present as competitors for local resources? With regard to other effects of colonization, we might ask whether groups in heavily missionized areas such as Spanish Florida changed their subsistence practices more rapidly in response to pressures from European authorities. All of these questions are important ones, although they cannot yet be answered. However, studies focused on limited areas, such as the North Carolina Piedmont, will ultimately provide a basis for moving forward. 


\section{References Cited}

Abler, T.S. and Elisabeth Tooker

1978 Seneca. In Handbook of North American Indians Vol. 15, edited by Bruce G. Trigger. Smithsonian Institution, Washington, D.C.

Adair, James

1930 History of the American Indian. Watauga Press, Johnson City, Tennessee.

Alcorn, Janis B.

1984 Huastec Mayan Ethnobotany. University of Texas Press, Austin.

Alexander, Edward P. (editor)

1972 The Journal of John Fontaine. An Irish Huguenot Son in Spain and Virginia, 17101719. Colonial Williamsburg Foundation, Williamsburg, VA.

Allen, E. P. and W. F. Wilson

1968 Geology and Mineral Resouces of Orange County, North Carolina. Bulletin No. 81, North Carolina Department of Conservation and Development, Raleigh.

Alvord, Clarence W. and Lee Bidgood (editors)

1912 The First Explorations of the TransAllegheny Region by the Virginians, 16501674. Arthur H. Clark Co., Cleveland.

American Geological Institute

1962 Dictionary of Geological Terms. Doubleday, New York.

Anonymous

1715? "Map of Carolina. Showing the route of the Forces sent in the years 1711, 1712, and 1713, from South Carolina to the relief of North Carolina, and in 1715 of the Forces sent from North Carolina to the assistance of South Carolina, also showing the controverted Bounds between Virginia and Carolina. About 15 miles to 1 inch. [1715.] 29 in x 20 in." Public Record office, Colonial office Library, Carolina 4, London.

Arber, Edward and A.G. Bradley (editors)

1910 Travels and Works of Captain John Smith President of Virginia and Admiral of New England 1580-1631. 2 vols. John Grant, Edinburgh.

Asch, David and Nancy Asch

1983 Archaeobotany. In Excavations at the Smiling Dan Site: Delineation of Site Structure and Function During the Middle Woodland Period, edited by Barbara D. Stafford and Mark B. Sant, pp. 635-725. Center for American Archaeology, Contract Archaeology Program, Reports of Investigations No. 137.
Axte11, James

1981 The European and the Indian. Oxford University Press, Oxford.

Bahou, W.F.

1975 The Relationships of Particular Trace Elements in the Dickson Mounds Skeletal Population. Paleopathology Newsletter $11: 15-17$.

Baker, Steven G.

1975 The Working Draft of: The Historic Catawba Peoples: Exploratory Perspectives in Ethnohistory and Archaeology. Ms. on file, Department of History, University of South Carolina, Columbia.

Baker, Vernon

1974 Carillo's Statistical Study of English Wine Bottles: Some Comments and Further Considerations. Conference on Historic Sites Papers 1972, volume 7, part 4, edited by Stanley South.

Barber, Michael B. and Joseph A. William

1978 Faunal Analysis. In 44Cpl, the Onion Field Site, Dan River Focus. Unpublished BA thesis by J.A. Williams, Department of Anthropology, College of William and Mary, Williamsburg.

Barbour, Philip L.

1964 The Three Worlds of Captain John Smith. Houghton Mifflin, Boston.

Barbour, Philip L. (editor)

1969 The Jamestown Voyages Under the First Charter, 1606-1609. 2 vols. Cambridge University Press for the Hakluyt Society, Cambridge, England.

Barnwe11, Joseph W. (editor)

1908 The Tuscarora Expedition. Letters of Colonel John Barnwel1. South Carolina Historical and Genealogical Magazine 9:2854 .

Barte1, Brad

1982 A Historical Review of Ethnological and Archaeological Analyses of Mortuary Practice. Journal of Anthropological Archaeology $1: 32-58$.

Bass, William M.

1971 Human 0steology: A Laboratory and Field Manual of the Human Skeleton. Missouri Archaeological Society, Columbia.

Beals, Ralph

1962 Acculturation. In Anthropology Today, edited by Sol Tax, pp. 375-395. The University of Chicago Press, Chicago, IL. 
Beaudry, Mary C.

1981 Colonizing the Virginia Frontier: Fort Christanna and Governor Spotswood's Indian Policy. Ms. submitted for publication in The Comparative Archaeology of European Colonialism, edited by Stephen L. Dyson.

Bennyhoff, James A. and Robert Heizer

1958 Cross-Dating Great Basin Sites by California Shell Beads. University of California Archaeological Survey Reports 42: 60-92, Berkeley.

Bentha11, Joseph L.

1969 Archaeological Investigation of the Shannon Site, Montgomery County, Virginia. Virginia State Library Publications, No. 32. Virginia State Library, Richmond.

Beverly, Robert

1947 The History and Present State of Virginia. edited with an Introduction by Louis B. Wright. University of North Carolina Press, Chapel Hill.

Billings, Warren (editor)

1975 The 01d Dominion in the Seventeenth Century: A Documentary History of Virginia, 1606-1689. University of North Carolina Press, Chapel Hill.

Binford, Lewis R.

1959 Comments on the "Siouan Problem." Ethnohistory $6: 28-41$.

1962 A New Method of Calculating Dates from Kaolin Pipe Stem Samples. Southeastern Archaeological Conference Newsletter 9: 19-21.

1967 Smudge Pits and Hide Smoking: The Use of Analogy in Archaeological Reasoning. American Antiquity 32:1-12.

1971 Mortuary Practice: Their Study and Their Potential. In Approaches to the Social Dimensions of Mortuary Practices, edited by J.A. Brown. Memoirs of the Society for American Archaeology 25:58-67.

1972 An Archaeological Perspective. Seminar Press. New York.

1979 Nunamuit Ethnoharchaeology. Academic Press, New York.

Black, T.K.

1978 A New Method of Assessing the Sex of Fragmentary Skeletal Remains: Femur Shaft Circumference. American Journal of Physical Anthropology 48:227-231.

Blakely, R.L. and L.A. Beck

1981 Trace Elements, Nutritional Status, and Social Stratification at Etowah, Georgia. In Research Potential of Anthropological Museum Collections, edited by A. Cant- we11, J.B. Griffin, and N.A. Rothchild. Annals of the New York Academy of Science, Volume 376.

Blalock, Hubert M.

1960 Social Statistics. McGraw-Hill, New York.

Blatt, H., G. Middleton, and R. Murray

1972 Origin of Sedimentary Rock. PrenticeHal1, Inc., Englewood Cliffs, NJ.

Bohrer, Vorsila L.

1975 The Prehistoric and Historic Role of the Cool-Season Grasses in the Southwest. Economic Botany 29:199-207.

Bourne, Edward G. (editor)

1904 Narratives of the Career of Hernando De Soto in the Conquest of Florida . . . 2 vols. A.S. Barnes, New York.

Brain, Jeffrey P.

1979 Tunica Treasure. Papers of the Peabody Museum of Archaeology and Ethnology No. 71, Harvard University, Cambridge.

Brothwe11, D. R.

1981 Digging Up Bones. Cornell University Press, Ithica.

Brown, A. B.

1974 Bone Strontium as an Dietary Indicator in Human Skeletal Populations. Contributions to Geology 13(2):47-48.

Brown, Allen

1948 Shell Wampum Beads of North American Indians. Tennessee Archaeologist 4(3).

Brown, James A.

1971 Introduction. In Approaches to the Social Dimensions of Mortuary Practices, edited by J. A. Brown. Memoirs of the Society for American Archaeology 25:1-6.

Brock, R.A. (editor)

1885 The Official Letters of Alexander Spotswood, Lieutenant-Governor of the Colony of Virginia, 1710-1722. vol. 2. Collections of the Virginia Historical Society, n.s, vol. 2. The Society, Richmond.

Buikstra, Jane E.

1976 Hopewell in the Lower Illinois Valley. Northwestern University Archaeological Program, Scientific Papers, No. 2. Evanston, IL.

Buikstra, Jane E. and D.C. Cook

1980 Paleopathology: An American Account. Annual Review of Anthropology 9:433-470.

Bullen, A.K.

1972 Paleoepidemiology and Distribution of Prehistoric Treponemiasis (Syphilis) in Florida. Florida Anthropologist 25:133174. 
Bunze1, Ruth

1933 Ornament. In Encyclopedia of the Social Sciences, edited by Edwin R. A. Seligman, Vol. 11:496-497. The Macmillan Co., New York.

Bushne11, David I., Jr.

1920 Native Cemeteries and Forms of Burial East of the Mississippi River. Bureau of American Ethnology Bulletin 71, Washington.

1930 The Five Monacan Towns in Virginia, 1607. Smithsonian Miscellaneous Collections 82 (12). Smithsonian Institution, Washington.

Butler, James B.

1963 Rocks of the Carolina Slate Belt in Orange County, North Carolina. Southeastern Geology $4(3)$.

Cadde11, Gloria May

1981 Plant Remains from the Yarborough Site. Ms. in author's possession.

Caldwe11, Joseph and Catherine McCann

1941 The Irene Mound Site. The University of Georgia Press, Athens.

Carnes, Linda F.

1983 Appendix II: Identification of EuroAmerican artifacts. In Tomotley: An Eighteenth Century Cherokee Village, by William W. Baden. Report of Investigations No. 36, Department of Anthropology, University of Tennessee, Knoxville.

1985 Euroamerican Artifacts from the Fredricks, Wall, and Mitchum Sites. In The Historic Occaneechi: An Archaeological Investigation of Culture Change. Final Report of 1984 Investigations, edited by Roy S. Dickens, Jr., H. Trawick Ward, and R. P. Stephen Davis, Jr., pp. 340-415. Research Laboratories of Anthropology, University of North Carolina, Chapel Hi11.

Carrillo, Richard

1974 English Wine Bottles as Revealed by a Statistical Study: A Further Approach to Evolution and Horizon in Historical Archaeology. Conference on Historic Sites Papers 1972 vol. 7, part 4, edited by Stanley South.

Carson, James D.

1961 Epiphyseal Cartilage as an Age Indicator in Fox and Gray Squirrels. Journal of Wildlife Management 25:90-93.

Chaplin, Raymond E.

1971 The Study of Animal Bones from Archaeological Sites. William Claus and Sons, London.
Chapman, Jefferson

1978 The Bacon Farm Site and a Buried Site Reconnaissance. Report of Investigations No. 23, Department of Anthropology, University of Tennessee, Knoxville.

Chapman, Jefferson, Paul A. Delcourt, Patricia A. Cridlebaugh, Andrea B. Shea, and Hazel R.

Delcourt

1982 Man-Land Interaction: 10,000 Years of American Indian Impact on Native Ecosystems in the Lower Little Tennessee River Valley, Eastern Tennessee. Southeastern Archaeology 2:115-121.

Chapman, Jefferson and Andrea B. Shea

1981 The Archaeobotanical Record: Early Archaic Period to Contact in the Lower Little Tennessee River Valley. Tennessee Anthropologist $6: 61-84$.

Charles, Tommy

1983 Thoughts and Records from the Survey of Private Collections of Prehistoric Artifacts: A Second Report. Institute of Archaeology and Anthropology, University of South Carolina, Notebook 15:1-37.

Charlton, T.H.

1978 Teotihuacan, Tepeapulco, and Obisdian Exploitation. Science 200:1227-1236.

Clarke, David L.

1968 Analytical Archaeology. Methuen, London.

1977 Spatial Archaeology. Academic Press, New York.

Cleland, Charles E.

1966 The Prehistoric Animal Ecology of the Upper Great Lakes Region. Anthropological Papers, No. 29. Museum of Anthropology, University of Michigan, Ann Arbor.

Coe, Joffre L.

1937 Keyauwee, a Preliminary Statement. Bu1letin of the Archaeological Society of North Carolina 3(1), Chapel Hill.

1952 The Cultural Sequence of the Carolina Piedmont. In Archaeology of the Eastern United States, edited by James B. Griffin, pp. 301-311. University of Chicago Press, Chicago.

1964 The Formative Cultures of the Carolina Piedmont. Transactions of the American Philosophical Society n.s. 54(5), American Philosophical Society, Philadelphia.

1972 Field Report of Highway Salvage Archaeology at Site Yd ${ }^{\mathrm{V}} 1$, Yadkin County, North Carolina. Ms. on file at the Research Laboratories of Anthropology, University of North Carolina, Chapel Hill. 
Coe, Joffre L. and Ernest Lewis

1952 Dan River Series Statement. Prehistoric Pottery of the Eastern United States, edited by James B. Griffin, Museum of Anthropology, University of Michigan, Ann Arbor.

Coleman, Gary N., Douglas H. Ubelaker, Michae1 Trinkley, and Wayne E. Clark

1982 The Reedy Creek Site, 44Ha22 South Boston Virginia. Quarterly Bulletin 37(4):150188, Archaeological Society of Virginia.

Cotter, John and J. Paul Hudson

1957 New Discoveries at Jamestown. National Park Service, Washington.

Cowan, C. Wesley

1978 The Prehistoric Use and Distribution of Maygrass in Eastern North America: Cultural and Phytogeographical Implications. In The Nature and Status of Ethnobotany, edited by Richard I. Ford, pp. 263-288. Museum of Anthropology, University of Michigan, Anthropological Papers No. 67, Ann Arbor.

Cumming, William P.

1958 The Discoveries of John Lederer. University of Virginia Press, Charlottesville.

Daly, Patricia

1969 Approaches to Faunal Analysis in Archaeology. American Antiquity 34(2):146-158.

Davis, R.P. Stephen, Jr.

1983 Aboriginal Ceramics From the Eno and Haw River Valleys and Their Bearing Upon the Identification of John Lawson's Occaneechi Town. Paper presented at the Annual Meeting of the Society for Historical Archaeology, Williamsburg, VA.

DeBoer, Warren

1984 Subterranean Storage and the Organization of Surplus: The View from Eastern North America. Ms. on file at the Department of Anthropology, Queens College, New York.

de Bry, Theodore

1590 Thomas Hariot's Virginia. University Microfilms, Inc., Ann Arbor.

Delcourt, Hazel R.

1975 Reconstructing the Forest Primeval, West Feliciana Parish, Louisiana. Melanges No. 10, Louisiana State University, Museum of Geoscience, Baton Rouge.

Denne11, R.W.

1976 The Economic Importance of Plant Resources Represented on Archaeological Sites. Journal of Archaeological Science $3: 229-247$.
Depratter, Chester, Charles Hudson, and Marvin T. Smith

1983 The Route of Juan Pardo's Explorations in the Interior Southeast, 1566-1568. The Florida Historical Quarterly 62:125-158.

Dickens, Roy S., Jr.

1980 Ceramic Diversity as an Indicator of Cultural Dynamics in the Woodland Period. Tennessee Anthropologist 5:34-46.

1984 In Search of Occaneechi: Archaeology and History of the North Carolina Piedmont, Symposium Introduction. Paper presented at Annual Meeting of the Society for Historical Archaeology, Williamsburg.

1985 The Form, Function, and Formation of Garbage-filled Pits on Southeastern Aboriginal Sites: A Archaeobotanical Analysis. In Structure and Process in Southeastern Archaeology, edited by Roy S. Dickens, $\mathrm{Jr}$. and H. Trawick Ward. University of Alabama Press, University.

Dickens, Roy S., H. Trawick Ward, and R. P. Stephen Davis, Jr.

1984 The Historic Occaneechi: An Archaeological Investigation of Culture Change: Preliminary Report of 1984 Investigations. Research Laboratories of Anthropology, University of North Carolina, Chapel Hill.

1985 The Historic Occaneechi: An Archaeological Investigation of Culture Change. Final Report of 1984 Investigations. ReResearch Laboratories of Anthropology, University of North Carolina, Chapel Hill.

Dimbleby, Geoffrey

1978 Plants and Archaeology. Humanities Press, Atlantic Highlands, New Jersey.

Dobyns, Henry F.

1983 Their Numbers Become Thinned: Native American Population Dynamics in Eastern North America. University of Tennessee Press, Knoxville.

Driver, Harold E.

1969 Indians of North America. The University of Chicago Press, Chicago.

Droessler, J.

1981 Craniometry and Biological Distance--Biocultural Continuity and Change at the Late Woodland-Mississippian Interface. Center for American Archaeology, Evanston, IL.

Dumbre11, Roger

1983 Understanding Antique Wine Bottles. Antique Collectors' Club Ltd., Woodbridge, Suffolk, England. 
Earle, Timothy $\mathrm{K}$.

1980 A Model of Subsistence Change. In Mode1ing Changes in Prehistoric Subsistence Economies, edited by Timothy K. Earle and Andrew L. Christenson, pp. 1-30. Academic Press, New York.

Edwards J.K., R.L. Marchinton, and G.F. Smith

1982 Pelvic Girdle Criteria of White-tailed Deer. Journal of Wildlife Management 46: 544-547.

Egloff, Keith

1980 Crab Orchard Site: A Late Woodland Palisaded Village. Quarterly Bulletin 34: 130-148, Archaeological Society of Virginia.

Egloff, Keith, Michael B. Barber, and Celia Reed

1980 Leggett Site: a Dan River Agricultural/ Riverine Hamlet. Ms. on file, Virginia Research Center for Archaeology, Williamsburg.

Ewan, Joseph and Nesta Ewan (editors)

1970 John Banister and His Natural History of Virginia, 1678-1692. University of I11inois Press, Urbana.

Ewers, John C.

1957 Hair Pipes in Plains Indian Adornment: A Study in Indian and White Ingenuity. Bureau of American Ethnology Bulletin 164, Anthropological Paper 50, Smithsonian Insitution, Washington.

Faulkner, Charles H.

1977 The Winter House: An Early Southeast Tradition. Midcontinental Journal of Archaeology 2:141-159.

Flannery, Kent V.

1976 The Early Mesoamerican Village. Academic Press, New York.

Ford, Thomas

1979 A Descriptive Analysis of the Euro-American Artifacts from the Citico Site (40Mr7) 1978 Excavations. Unpublished report submitted to the Tennessee Valley Authority, Knoxville.

Fritz, Gayle J.

1984 Identification of Cultigen Amaranth and Chenopod from Rockshelter Sites in Northwest Arkansas. American Antiquity 49: 558-572.

Fundaburk, Emma L. and Mary D. F. Foreman

1957 Sun Circles and Human Hands: The Southeastern Indians ' Art and Industries. Paragon Press, Montgomery, AL.
Futer, A. A.

1969 The Strickler Site. In Susquehannock Miscellany, edited by John Witthoft and W. Fred Kinsey, III, pp. 136-147. The Pennsylvania Historical and Museum Commission, Harrisburg.

Gardner, Paul S.

1980 An Analysis of Dan River Ceramics from Virginia and North Carolina. M.A. thesis, Department of Anthropology, University of North Carolina, Chapel Hill.

Genoves, S. C.

1967 Proportionality of Long Bones and Their Relation to Stature Among Mesoamericans. American Journal of Physical Anthropology $26: 67-78$.

Gilbert, R.I.

1977 Applications of Trace Element Research to Problems in Archaeology. In Biocultural Adaptations in Prehistoric America, edited by R.L. Blakely, pp 85-100. University of Georgia Press, Athens.

Gleason, Henry A. and Arthur Cronquist

1964 The Natural Geography of Plants. Columbia University Press, New York.

Good, Mary Elizabeth

1972 Guebert Site: An Eighteenth Century Historic Kaskaskia Indian Village in Randolph County, Illinois. Memoir II of The Central States Archaeological Society, Inc.

Goody, J. R.

1962 Death, Property and the Ancestors. Stanford University Press, Stanford, CA.

Gould, Richard A.

1979 Exotic Stones and Battered Bones: Ethnoarchaeology in the Australian Desert. Archaeology $32(2): 28-37$.

Graham, Martha D.

1973 Dental Morphology, Attrition, and Pathology in Selected Skulls from Town Creek Indian Mound, Mount Gilead, North Caro1ina. Unpublished Master's thesis, Department of Anthropology, University of North Carolina, Chapel Hill.

Grau, G.A., G.C. Sanderson, and J.P. Rogers

1970 Age Determination of Raccoons. Journal of Wildlife Management 34:364-372.

Gravely, Richard P., Jr.

1969 The Madison Cemetery. Eastern States Archaeological Federation Bulletin 27:11.

Grayson, Donald K.

1973 On the Methodology of Faunal Analysis. American Antiquity 38(4):432-438. 
Grayson, Donald K.

1979 On the Quantification of Vertebrate Archaeofaunas. In Advances in Archaeological Method and Theory, vol. 2, edited by Michae1 B. Schiffer, pp. 200-238. Academic Press, New York.

Great Britain, Public Record Office, Colonial office

1682 Series 1, vol. 48. Microfilm copy in Virginia Colonial Records Project, Colonial Williamsburg Archives, Williamsburg.

1714 Series 5, vol. 1316. Transcript in the Library of Congress Manuscript Room, Washington.

Gremillion, Kristen

1984 Aboriginal Use of Plant Foods and European Contact in the North Carolina Piedmont. M.A. thesis, Department of Anthropology, University of North Carolina, Chape1 Hill.

Gremillion, Kristen J.

1985 Plant Foods at the Fredricks, Wall, and Mitchum Sites. In The Historic Occaneechi: An Archaeological Investigation of Culture Change. Final Report of 1984 Investigations, edited by Roy S. Dickens, Jr., H. Trawick Ward, and R. P. Stephen Davis, Jr., pp. 642-677. Research Laboratories of Anthropology, University of North Carolina, Chapel Hill.

Griffin, James B.

1945 An Interpretation of Siouan Archaeology in the Piedmont of North Carolina and Virginia. American Antiquity 10:321-330.

Grinnan, A.G.

1895- The Last Indians in Orange County, Vir-

1896 ginia. Virginia Magazine of History and Biography 3:189-191.

Gruber, J. W.

1971 Death in a Late Prehistoric Village in Pennsylvania. American Antiquity 36:6476 .

Guilday, John E., Paul W. Parmalee, and Donald P. Tanner

1962 Aboriginal Butchering Techniques at the Eschelman Site (36Lal2), Lancaster, Pennsylvania. Pennsylvania Archaeologist $32(2): 59-83$.

Gwynn, John V.

1964 Virginia Upland Came Investigations: Restoration of the Wild Turkey. Annual Report, Virginia Pittman-Robertson Project.

Hale, Horatio

1883 The Tutelo Tribe and Language. Proceedings of the American Philosophical Society $21: 114: 1-47$.
Hale, J.B.

1949 Aging Cottontail Rabbits by Bone Growth. Journal of Wildlife Management 13:216225 .

Hal1, Edward T.

1966 The Hidden Dimension. Doubleday and Company, Inc., Garden City.

Hamilton, T. M. (editor)

1960 Indian Trade Guns. Missouri Archaeologist 22. Columbia.

Hammett, Julia E.

1983 Preliminary Classification of North Carolina Shell Bead Artifacts: Some Indications and Implications. Ms. on file, Research Laboratories of Anthropology, University of North Carolina, Chapel Hill.

Harrington, Jean C.

1954 Dating Stem Fragments of 17 th and 18 th Century Clay Tobacco Pipes. Quarterly Bulletin 9(1):10-14, Archaeological Society of Virginia.

Harrington, Mark R.

1908a Catawba Potters and Their Work. American Anthropologist (n.s.) 10:399-407.

1908b Some Seneca Corn-Foods and Their Preparation. American Anthropologist (n.s.) 10: 575-590.

Hariot, Thomas

1972 A Briefe and True Report of the New Found Land of Virginia. The Complete 1590 Theodor De Bry Edition. Introduction by Paul Hulton. Dover Publications, New York.

Harris, Frances L. (editor)

1937 Lawson's History of North Carolina. Garrett and Massie, Richmond, Virginia.

Hatch, J.W. and R.A. Geide1

1983 Tracing Status and Diet in Prehistoric Tennessee. Archaeology 36:56-60.

Hazard, Samuel (editor)

1851 Minutes of the Provincial Council of Pennsylvania, From the Organization to the Termination of the Proprietary Government, vol. 2. Jo. Severns, Philade1phia.

Hedrick, U.P. (editor)

1972 Sturdevant's Edible Plants of the World. Dover Publications, New York.

Heye, George C. and George H. Pepper

1915 Exploration of a Munsee Cemetery near Montague, New Jersey. Contributions from The Museum of the American Indian, vol. 2, Heye Foundation, New York. 
Hodder, Ian

1978 The Spatial Organization of Culture. Duckworth and Co. Ltd., London.

1982 The Present Past: An Introduction to Anthropology for Archaeologists. B. T. Batsford, Ltd., London.

Holland, C.G.

1970 An Archaeological Survey of Southwest Virginia. Smithsonian Contributions to Anthropology 12, Washington.

1982 A Saponi Note. Quarterly Bulletin 37:42, Archaeological Society of Virginia.

Holm, Mary Ann

1985 Faunal Remains from the Wall and Fredricks Sites. In The Historic Occaneechi: An Archaeological Investigation of Culture Change, edited by R.S. Dickens, Jr., H.T. Ward, and R.P.S. Davis, Jr., pp.578641. Ms. on file at the Research Laboratories of Anthropology, University of North Carolina, Chapel Hill.

Holmes, William H.

1903 Aboriginal Pottery of the Eastern United States. Twentieth Annual Report of the Bureau of American Ethnology, 1898-99, pp. 1-237, Washington.

Horn, Henry S.

1974 The Ecology of Secondary Succession. Annual Review of Ecology and Systematics 5: 25-37.

1978 Optimal Tactics of Reproduction and LifeHistory. In Behavioral Ecology: An Evolutionary Approach, edited by J. R. Krebs and N. B. Davies, pp 411-429. Blackwell Scientific Publications, Oxford.

Hrdlicka, A.

1916 Physical Anthropology of the Lenape or Delawares, and the Eastern Indians in General. Bureau of American Ethnology Bulletin 32, Washington.

Hudson, Charles M.

1970 The Catawba Nation. University of GeorMonographs 18. University of Georgia Press, Athens.

1976 The Southeastern Indians. The University of Tennessee Press, Knoxville.

Hudson, Charles M., Marvin T. Smith, and

Chester B. DePratter

1984 The Hernando De Soto Expedition: From Apalachee to Chiaha. Southeastern Archaeology $3: 65-77$.
Hunter, W. A.

1959 The Historic Role of the Susquehannocks. In Susquehannock Miscellany, edited by John Whitthoft and W. Fred Kensey, III, pp. 8-18. The Pennsylvania Historical and Museum Commission, Harrisburg.

Huss-Ashmore, R., A.H. Goodman, and G.J. Armelagos

1982 Nutritional Inference from Paleopathology. Advances in Archaeological Method and Theory, Vol. 5, pp. 395-474. Academic Press, New York.

Jackes, M.K.

1983 Osteological Evidence for Smallpox: A Possible Case from Seventeenth Century Ontario. American Journal of Physical Anthropology 60:75-81.

Johnson, Kristen J.

1983 Formation of Archaeological Refuse Deposits at the Wall Site. Ms. in author's possession.

1984 A Preliminary Statement on Plant Remains from the Wall and Fredricks Sites. Paper presented at the Annual Meeting of the Society for Historical Archaeology, Williamsburg, VA.

Karklins, Karlis

1982 Glass Beads. History and Archaeology 59. National Historic Parks and Sites Branch, Parks Canada, Environment Canada, Ottawa.

Keene, Arthur S.

1981 Optimal Foraging in a Nonmarginal Environment: A Model of Prehistoric Subsistence Strategies in Michigan. In HunterGatherer Foraging Strategies, edited by Bruce Winterhalder and Eric Alden Smith, pp. 171-193. University of Chicago Press, Chicago.

Kent, Barry C.

1984 Susquehanna's Indians. The Pennsylvania Historical and Museum Commission, Anthropological Series, No. 6. Harrisburg.

Kenyon, W. A.

1982 The Grimsby Site: An Historic Neutral Cemetery. Royal Ontario Museum, Toronto, Canada.

Kidd, K. E. and M. A. Kidd

1970 A Classification System for Glass Trade Beads for the Use of the Field Archaeologists. Canadian Historic Sites: Occasional Papers in Archaeology and History $1: 45-89$.

1982 A Guide to the Artifacts of Colonial America. Alfred Knopf, New York. 
King, Chester D.

1982 The Evolution of Chumash Society: A Comparative Study of Artifacts Used in Social System Maintenance in the Santa Barbara Channel Region Before A.D. 1804. Unpublished Ph.D. dissertation, Department of Anthropology, University of California, Davis.

King, Frances B.

1982 Preliminary Analysis of Botanical Remains from Phillips Spring. In Holocene Adaptations Within the Lower Pomme de Terre River Valley, Missouri, Vol. 3, edited by Marvin Kay, pp. 701-727. U.S. Army Corps of Engineers, Kansas City District.

Klein, Richard G., and Kathryn Cruz-Uribe

1984 The Analysis of Animal Bones from Archaeological Sites. University of Chicago Press, Chicago.

Klippel, Walter

1971 Prehistory and Environmental Change along the Southern Border of the Prairie Peninsula during the Archaic Period. University Microfilms, Ann Arbor, MI.

Kramer, Carol

1979 An Archaeological View of a Contemporary Kurdish Village: Domestic Architecture, Household Size and Wealth. In Ethnoarchaeology: Implications of Ethnography for Archaeology, edited by Carol Kramer, pp. 139-163. Columbia University Press, New York.

Krogman, W.M.

1978 The Human Skeleton in Forensic Medicine. Charles C. Thomas, Springfield, IL.

Kupperman, Karen Ordahl

1980 Settling With the Indians. Rowman and Littlefield, Totowa, NJ.

Lambert, J. B., C. B. Szpunar and J. E. Buikstra

1979 Chemical Analysis of Excavated Human Bone from Middle and Late Woodland Sites. Archaeometry 21:115-129.

Larson, C. S.

1980 Human Skeletal and Dental Health Changes on the Prehistoric Georgia Coast. Excursions in Southeastern Geology: The Archaeology-Geology of the Georgia Coast 20:192-201.

Larson, Lewis H., Jr.

1971 Archaeological Implications of Social Stratification at the Etowah Site, Georgia. In Approaches to the Social Dimensions of Mortuary Practices, edited by J. A. Brown. Memoirs of the Society for American Archaeology 25:58-67.
Lawson, John

1967 A New Voyage to Carolina. edited with an Introduction and Notes by Hugh T. Lefler. University of North Carolina Press, Chapel Hill.

LeBlanc, Steven

1971 An Addition to Naro11's Suggested Floor Area and Settlement Population Relationship. American Antiquity 36:210-211.

Lederer, John

1958 The Discoveries of John Lederer. edited with Notes by William P. Cumming. University of Virginia Press, Charlottesville.

Lee, Richard B. and Irven DeVore

1976 Kalahari Hunter-Gatherers. Harvard University Press, Cambridge.

Lefler, Hugh T. (editor)

1967 A New Voyage of Carolina. University of North Carolina Press, Chapel Hill.

Lewall, E.F., and I.McT. Cowan

1963 Age Determination in Black-tail Deer by Degree of Ossification of the Long Bones. Canadian Journal of Zoology 41:629-636.

Lewis, C1ifford M. and Albert J. Loomie

1953 The Spanish Jesuit Mission in Virginia, 1570-1572. Published for the Virginia Historical Society by the University of North Carolina Press, Chapel Hill.

Lewis, Ernest

1951 The Sara Indians, 1540-1768: An EthnoArchaeological Study. M.A. thesis, Department of Sociology and Anthropology, University of North Carolina, Chapel Hill.

Lieberson, Stanley

1969 Measuring Population Diversity. American Sociological Review 34(6):850-862.

Lopinot, Neal H.

1983 Analysis of Flotation Sample Materials from the Late Archaic Horizon. Chapter VII in The 1982 Excavations at the Cahokia Interpretive Center Tract, St. Clair County, Illinois, by Richard W. Jefferies, pp. 77-108. Center for Archaeological Investigations, Southern Illinois University at Carbondale, Research Paper No. 37.

McCary, Ben C.

1957 John Smith's Map of Virginia. With a Brief Account of Its History. University of Virginia Press, Charlottesville. 
McCollough, Major C.R., Quentin R. Bass, II, William 0. Autry, and Duane R. Lenhardt

1980 Phase II Archaeological Investigations of Ten Specified Locales in the Falls Lake Reservoir Area, Falls Lake, North Caro1ina. Submitted to Wilmington District, U.S. Army Corps of Engineers by GAI Consultants, Inc., Monroeville, PA.

MacCord, Howard A., Sr.

1983 Indians of Piedmont Virginia: An Abbreviated Culture History. Paper presented at A Conference on Piedmont Archeology, Yorktown, VA.

McIlwaine, H.R. (editor)

1928 Executive Journals of the Council of Colonial Virginia. vol. 3. Virginia State Library, Richmond.

1930 Executive Journals of the Council of Colonial Virginia. vol. 4. Virginia State Library, Richmond.

McKern, T.W. and T.D. Stewart

1957 Skeletal Age Changes in Young American Males. Headquarters Quartermaster $\mathrm{Re}-$ search and Development, Technical Report EP-45, Natick, Massachusetts.

McManus, Jane M.

1985 An Analysis of the Lithic Artifact Assemblage from the Forbush Creek Site (31Yd1), Yadkin County, North Carolina. Honor's thesis, Department of Anthropology, University of North Carolina, Chape 1 Hill.

Marks, S.A., and A.W. Erickson

1966 Age Determination in the Black Bear. Journal of Wildlife Management $30(2): 389-$ 410.

Martin, Alexander C. and William D. Barkley

1961 Seed Identification Manual. University of California Press, Berkeley.

Merrel1, James $\mathrm{H}$.

1982a Natives in a New World: The Catawba Indians of Carolina, 1650-1800. Ph. D. dissertation, The John Hopkins University, Baltimore.

1982b "Our Bond of Peace": Patterns of Intercultural Trade in the Interior, 16501700. Paper presented at the Annual Meeting of the American Historical Association, Washington.

1984a "Their Very Bones Shall Fight": CatawbaIroquois Relations. Paper presented at the Forty-Fourth Conference on Early American History, "The 'Imperial' Iroquois," Williamsburg, VA.

1984b The Indians' New World: The Catawba Experience. William and Mary Quarterly 3rd ser., 41:537-565.
Meyers, J.T.

1970 Chert Resources of the Lower I11inois Valley. Reports of Investigations 18, Illinois State Museum, Springfield.

Miche1, Francis Louis

1916 Report of the Journey of Francis Louis Michel from Berne, Switzerland, to Virginia, October 2, 1701-December 1, 1702 . Translated and edited by William $\mathrm{J}$. Hinke. Virginia Magazine of History and Biography 24:1-43,113-141, 275-288.

Miller, Car1 F.

1957 Reevaluation of the Eastern Siouan Problem with Particular Emphasis on the Virginia Branches - The Occaneechi, the Saponi, and the Tutelo. Bureau of American Ethnology Bulletin 164, Anthropological Papers 52, Smithsonian Institution, Washington.

1962 Archaeology of the John H. Kerr Reservoir Basin, Roanoke River, Virginia-North Carolina. Bureau of American Ethnology Bulletin 182, Washington.

Molnar, S.

1971 Human Tooth Wear, Tooth Function and Cultural Variability. American Journal of Physical Anthropology 17:179-186.

Montgomery, F.H.

1977 Seeds and Fruits of Plants of Eastern Canada and Northeastern United States. University of Toronto Press, Toronto.

Mooney, James

1894 The Siouan Tribes of the East. Bureau of American Ethnology Bulletin 22, Washington.

Moore, Julie H.

1973 Preimpoundment Studies, Falls Project, A Survey of the Vascular Plants. Department of Environmental Sciences and Engineering, School of Public Health, University of North Carolina, Chapel Hill.

Moore, Julie H. and Emily W. Wood

1976 B. Everett Jordan Dam and Lake: Assessment of the Vegetation. Department of Environmental Sciences and Engineering, School of Public Health, University of North Carolina, Chapel Hill.

Morris, Percy C.

1975 A Field Guide to Shells of the Atlantic and Gulf Coasts and The West Indies. Houghton Mifflin Co., Boston.

Morrison, A.J.

1921 The Virginia Indian Trade to 1673. Wil1iam and Mary Quarter1y 2nd ser., 1:217236. 
Morton, Richard L. (editor)

1956 The Present State of Virginia from Whence is Inferred a Short View of Maryland and North Carolina, by Hugh Jones. The University of North Carolina Press, Chape1 Hill.

Mouer, L. Danie1

1983 A Review of the Archaeology and Ethnohistory of the Monacans. In Piedmont Archaeology: Recent Research and Results, edited by J. Mark Wittofski and Lyle E. Browning, pp. 21-39. Archaeological Society of Virginia, Special Publication No. 10, Richmond.

Murphy, T.

1959a The Changing Pattern of Dentine Exposure in Human Tooth Attrition. American Journal of Physical Anthropology, 17:167-178.

1959b Gradients of Dentine Exposure in Human Tooth Attrition. American Journal of Physical Anthropology 17:179-186.

Myers, Albert Cook (editor)

1970 William Penn's Own Account of the Lenni Lenape or Delaware Indians. The Middle Atlantic Press, Somerset.

Narol1, Raoul

1962 Floor Area and Settlement Population. American Antiquity 27:587-589.

Navey, Liane

1982 An Introduction to the Mortuary Practices of the Historic Sara. M.A. thesis, Department of Anthropology, University of North Carolina, Chapel Hill.

Neumann, Georg K.

1952 Archaeology and Race in the American Indian. In Archaeology of Eastern United States, edited by J.B. Griffin, pp.13-34. The University of Chicago Press, Chicago.

Neumann, George

1967 The History of Weapons of the American Revolution. Harper and Row Publishers, New York.

Newman, Robert

1977 An Analysis of the European Artifacts from Chota-Tanasee: An Eighteenth Century Overhil1 Cherokee Town. Unpublished M.A. thesis, Department of Anthropology, University of Tennessee, Knoxville.

Noel Hume, Ivor

1974 All the Best Rubbish. Harper and Row Publishers, New York.

Novick, L.A.

1978 Prehistoric Lithic Material Sources and Types in South Carolina: A Preliminary Statement. South Carolina Antiquities 10(1), Archeological Society of South Carolina, Columbia, SC.
Odum, Eugene P.

1976 The Strategy of Ecosystem Development. In Human Ecology: An Environmental Approach, edited by P. Richerson and J. McEvoy, III, pp. 81-95. Wadsworth, Belmont, CA.

Orchard, William C.

1929 Beads and Beadwork of the American Indians. Museum of the American Indian, Heye Foundation, New York.

Ortner, D.J. and W.G.J. Putschar

1981 Identification of Pathological Conditions in Human Skeletal Remains. Smithsonian Institution Press, Washington.

O'Shea, John M.

1984 Mortuary Variability, An Archaeological Investigation. Academic Press, Inc., Orlando, FL.

Payne, Sebastian

1972 On the Interpretation of Bone Samples from Archaeological Sites. In Papers in Economic Prehistory, edited by E. S. Higgs, pp. 65-81. Cambridge University Press, Cambridge.

1975 Partial Recovery and Sample Bias. In Archaeozoological Studies, edited by A. T. Clason, pp. 7-17. North Holland, Amsterdam.

Pearson, Philip Edward

1842? Memoir of the Catawbas, sent to Governor James Henry Hammond. Ms., typescript copy in the York County Public Library, Rock Hill, SC.

Peebles, Christopher S.

1971 Moundville and Surrounding Sites: Some Structural Considerations of Mortuary Practice II. In Approaches to the Social Dimensions of Mortuary Practices, edited by J. A. Brown. Memoirs of the Society for American Archaeology 25:68-91.

Peet, Robert K.

1974 The Measurement of Species Diversity. Annual Review of Ecology and Systematics $5: 285-307$.

Peterson, Harold

1956 Arms and Armor in Colonial America, 15261783. The Stackpole Company, Harrisburg.

Petherick, Gary L.

1985 Architecture and Features at the Fredricks, Wall, and Mitchum Sites. In The Historic Occaneechi: An Archaeological Investigation of Culture Change. Final Report of 1984 Investigations, edited by Roy S. Dickens, Jr., H. Trawick Ward, and R. P. Stephen Davis, Jr., pp. 53-178. Research Laboratories of Anthropology, University of North Carolina, Chapel Hil1. 
Petruso, Karl M. and Jere M. Wickens

1984 The Acorn in Aboriginal Subsistence in Eastern North America: A Report on Miscellaneous Experiments. In Experiments and Observations on Aboriginal Wild Plant Food Utilization in Eastern North America, edited by Patrick J. Munson, pp. 360378. Indiana Historical Society, Prehistory Research Series, Volume VI, No. 2, Indianapolis.

Pianka, E.

1978 Evolutionary Ecology. Harper and Row, New York.

Polhemus, Richard

1981 Aboriginal Burial Associations from the Plum Grove Site, 40Wg17. In Archaeological Investigations at the Plum Grove Site, Washington County, Tennessee, edited by Roy S. Dickens, Jr. Ms. on file, Laboratory of Archaeology, Department of Anthropology, Georgia State University, Atlanta.

Polhemus, Richard (editor)

1984 Toqua Report. Unpublished draft report submitted to the Tennessee Valley Authority by the Department of Anthropology, University of Tennessee, Knoxville.

Price, F. G. Hilton

1908 0ld Base Metal Spoons. B. T. Batsford, High Holborn, London, England.

Quinn, David B.

1977 North America From Earliest Discovery to First Settlements: The Norse Voyages to 1612. Harper and Row, New York.

Radford, Albert E., Harry F. Ahles, and

C. Ritchie Bell

1968 A Manual of the Vascular Flora of the Carolinas. University of North Carolina Press, Chapel Hill.

Rapoport, Amos

1969 House Form and Culture. Prentice-Ha11, Inc., Englewood Cliffs, NJ.

Raymond, Percy E.

1952 Latten Spoons of the Pilgrims. In Antique Metalware, edited by James Mitche1l. University Books, New York.

Rights, Douglas L.

1957 The American Indian in North Carolina. John F. Blair, Winston-Salem.

Rights, Douglas L. and William P. Cumming

1958 Essay on the Indians of Lederer's "Discoveries". In The Discoveries of John Lederer, edited by W. P. Cumming, pp. 111-126. University of Virginia Press, Charlottesville.
Rindos, David

1984 The Origins of Agriculture: An Evolutionary Perspective. Academic Press, New York.

Rothschild, Nan A.

1979 Mortuary Behavior and Social Organization at Indian Knoll and Dickson Mounds. American Antiquity 44:658-675.

Runquist, Jeannette

1979 Analysis of the Flora and Faunal Remains from Protohistoric North Carolina Cherokee Indian Sites. Ph.D. dissertation, Department of Zoology, North Carolina State University, Raleigh.

Sahlins, Marshall D.

1968 Tribesmen. Prentice-Hall, Inc., Englewood Cliffs, NJ.

Sainesbury, W.N. (editor)

1893 Calendar of State Papers, Colonial Series, America and the West Indies, 16691674. Printed for Her Majesty's Stationary office by Eyre and Spottiswoods, London.

Salley, Alexander S. (editor)

1911 Narratives of Early Carolina, 1650-1708. Charles Scribner's Sons, New York.

SAS Institute, Inc.

1982 SAS User's Guide: Statistics, 1982 Edition. SAS Institute, Inc., Cary, NC.

Saunders, William L. (editor)

1968 The Colonial Records of North Carolina. AMS Press, New York. Originally published 1886-1890, P.M. Hale, Raleigh, NC.

Saxe, A. A.

1971 Social Dimensions of Mortuary Practices in Mesolithic Populations from Wade Ha1fa, Sudan. In Approaches to the Social Dimensions of Mortuary Practices, edited by J. A. Brown. Memoirs of the Society for American Archaeology 25:39-56.

Schiffer Michael B.

1972 Archaeological Context and Systemic Context. American Antiquity 37:156-165.

1976 Behavioral Archeology. Academic Press, New York.

Schmor1, G.

1971 The Human Spine in Health and Disease (2nd American Edition). Grune and Stratton, New York.

Schoeninger, M.J.

1979 Diet and Status at Chalcatzingo: Some Empirical and Technical Aspects of Strontium Analysis. American Journal of Physical Anthropology 51:295-310. 
Schoeninger, M.J. and C.S. Peebles

1981 Effect of Mollusc Eating on Human Bone Strontium Levels. Journal of Archaeological Science 8:391-397.

Schorger, Arlie W.

1955 The Passenger Pigeon: Its Natural History and Extinction. University of Wisconsin Press, Madison.

Schroed1, Gerald F.

1980 Structure and Village Pattern at the Historic Overhill Cherokee Towns of Chota and Tanasee. Paper presented at the 37th Annual Southeastern Archaeological Conference, New Orleans.

1983 Eighteenth Century Overhill Cherokee Domestic Structures. Paper presented at the 48th Annual Meeting of the Southeastern Archaeological Conference, Columbia.

Seehan, B. W.

1980 Savagism and Civility: Indian and Englishmen in Colonial Virginia. Cambridge University Press, Cambridge.

Service, Elman R.

1964 Primitive Social Organization. Random House, New York.

Sever, L.E.

1974 Zinc and Human Development: A Review. Human Ecology 3:43-57.

Severinghaus, C.W.

1949 Tooth Development and Wear as Criteria of Age in White-tailed Deer. Journal of Wildlife Management 13(2):195-216.

Sheldon, Elisabeth Shepard

1978 Childersburg: Evidence of European Contact Demonstrated by Archaeological Plant Remains. Southeastern Archaeological Conference Special Publication No. 5, pp. 28-29.

Shelford, Victor E.

1963 The Ecology of North America. University of Illinois Press, Urbana, Illinois.

Siebert, Frank T., Jr.

1945 Linguistic Classification of Catawba. International Journal of American Linguistics 11:100-104, 211-218.

Simpkins, Daniel L.

1984 Some Spatial Configurations of Late Archaeological Components in the CarolinaVirginia Piedmont. Paper presented at the Annual Meeting of the Society for Historical Archaeology, Williamsburg.
Sizemore, Beverly A.

1984 "Gorges" and "Wampum": Shell Artifacts from the Wall and Fredricks Sites. Paper presented at the Annual Meeting of the Society for Historic Archaeology, Williamsburg.

Smith, Bruce D.

1974 Middle Mississippi Exploitation of Animal Populations: A Predictive Model. American Antiquity 39(2):274-291.

1975a Toward a More Accurate Estimation of Meat Yield of Animal Species at Archaeological Sites. In Archaeozoological Studies, edited by A. T. Clason, pp. 99-106. North Holland, Amsterdam.

1975b Middle Mississippi Exploitation of Animal Populations. Anthropological Papers 57. Museum of Anthropology, University of Michigan, Ann Arbor.

1976 "Twitching": A Minor Ailment Affecting Human Paleoecological Research. In Cultural Change and Continuity, edited by $\mathrm{C}$. E. Cleland, pp. 275-292. Academic Press, New York.

1985 The Role of Chenopodium as a Domesticate in Pre-Maize Garden Systems of the Eastern United States. Southeastern Archaeology $4(1): 51-72$.

Smith, Eric Alden

1979 Human Adaptation and Energetic Efficiency. Human Ecology 7:53-74.

Smith, Gerald P.

1965 An Archaeological Survey of the New Hope River Valley. M.A. thesis, Department of Sociology and Anthropology, University of North Carolina, Chapel Hill.

Sorohan, Bryan P.

1985 An Analysis of the Dental Pathology of Human Skeletal Remains from the Wall and Fredricks Sites, Hillsborough, N.C. Honor's thesis, Department of Anthropology, University of North Carolina, Chapel Hill.

South, Stanley

1959 A Study of the Prehistory of the Roanoke Rapids Basin. M.A. thesis, Department of Sociology and Anthropology, University of North Carolina, Chapel Hill.

1964 Analysis of the Buttons from Brunswick Town and Fort Fisher. The Florida Anthropologist 17:113-133.

1977 Method and Theory in Historical Archaeo1ogy. Academic Press, New York. 
Speck, Frank G.

1919 The Functions of Wampum Among the Eastern Algonkian. Memoirs of the American Anthropological Association 6(1):3-71.

1935 Siouan Tribes of the Carolinas as Known from Catawba, Tutelo, and Documentary Sources. American Anthropologist 37:201225.

Spencer, Herbert

1886 Part IV: Ceremonial Institutions. In The Principles of Sociology, Vol. II. D. Appleton and Co., New York.

Spicer, Edward H. (editor)

1961 Perspectives in American Indian Culture Change. The University of Chicago Press, Chicago.

Steinbock, R.T.

1976 Paleopathological Diagnosis and Interpretation: Bone Diseases in Ancient Human Populations. Charles C. Thomas, Springfield, IL.

Stone, Lyle

1974 Fort Michilimackinac 1715-1781. Publications of the Museum, Michigan State University, East Lansing.

Sturtevant, William C.

1958 Siouan Languages in the East. American Anthropologist 60:738-743.

1975 Two 1761 Wigwams at Niantic, Connecticut. American Antiquity 40:437-444.

Styles, Bonnie What ley

1981 Faunal Exploitation and Resource Selection: Early Late Woodland Subsistence in the Lower Illinois Valley. Northwestern University Archaeological Program, Evanston, I1.

Sumner, W. M.

1979 Estimating Population by Analogy: An Example. In Ethnoarchaeology, edited by Carol Kramer, pp. 164-174. Columbia University Press, New York.

Swanton, John R.

1946 The Indians of the Southeastern United States. Bureau of American Ethnology Bulletin 137, Washington.

Szpunar, C.B., J.B. Lambert, and J.E. Buikstra

1978 Analysis of Excavated Bone by Atomic Absorption. American Journal of Physical Anthropology 48:199-202.

Tainter, J.

1978 Mortuary Practices and the Study of Prehistoric Social Systems. In Advances in Archaeological Method and Theory, Vol. 1, edited by Michael B. Schiffer, pp. 105141. Academic Press, New York.
Talalay, Laurie, Donald R. Keller, and Patrick J. Munson

1984 Hickory Nuts, Walnuts, Butternuts, and Hazelnuts: Observations and Experiments Relevant to Their Aboriginal Exploitation in Eastern North America. In Experiments and Observations on Aboriginal Wild Plant Food Utilization in Eastern North America, edited by Patrick J. Munson, pp. 338359. Indiana Historical Society, Prehistory Research Series 6(2).

Tinling, Marion (editor)

1977 The Correspondence of The Three William Byrds of Westover, Virginia, 1684-1776 (2 vols.). University Press of Virginia, Charlottesville.

Todd, T. W. and D. W. Lyon

1925 Endocranial Suture Closure, Its Progress and Age Relationship Part I Adult Males of White Stock. American Journal of Physical Anthropology 7:325-384.

Trinkley, Michael, and S. Homes Hogue

1979 The Wachesaw Landing Site: The Last Gasp of the Coastal Waccamaw Indians. Southern Indian Studies $31: 3-20$.

Trotter, M. and G. C. Gleser

1952 Estimation of Stature from Long Bones of American Whites and Negroes. American Journal of Physical Anthropology 10:463514.

1958 A Re-evaluation of Estimation of Stature Based on Measurements of Stature taken During Life and of Long Bones After Death. American Journal of Physical Anthropology 16:79-123.

Turner, E. Randolph

1978 An Intertribal Deer Exploitation Buffer Zone for the Virginia Coastal Plain-Piedmont Regions. Quarterly Bulletin 32: 42-48, Archaeological Society of Virginia.

Turner, F.J. and J. Verhoogen

1960 Igneous and Metamorphic Petrology. McGraw-Hill, New York.

Tyler, Lyon Gardiner (editor)

1907 Descriptions of Virginia and Proceedings of the Colonie, by John Smith. In Narratives of Early Virginia. Charles Scribners' Sons, New York.

Ubelaker, Douglas L.

1974 Reconstruction of Demographic Profiles from 0ssuary Skeletal Samples: A Case Study from the Tidewater Potomac. Smithsonian Institution, Washington.

1978 Human Skeletal Remains. Aldine Publishing Company, Chicago. 
Ucko, Peter

1969 Ethnography and Archaeological Interpretation of Funerary Remains. World Archaeology $1: 262-280$.

U.S. Department of Agriculture

1974 Seeds of Woody Plants in the United States. Agriculture Handbook No. 450, Forest Service, Washington.

Varner, John G., and Jeanette J. Varner

(translators and editors)

1951 The Florida of the Inca. University of Texas Press, Austin.

Vaughan, Alden T.

1977 New England's Prospect by William Wood. University of Massachusetts Press, Amherst.

Voegelin, E. W.

1944 Mortuary Customs of the Shawnee and Other Eastern Tribes. Indiana Historical Society, Prehistoric Research Series 2(4).

Ward, H. Trawick

1980 The Spatial Analysis of the Plow Zone Artifact Distributions from Two Village Sites in North Carolina. Ph.D. dissertation, University of North Carolina, Chapel Hill.

1984a Intra-Site Patterns at the Warren Wilson Site. Paper presented at the Conference on Cherokee Prehistory, Warren Wilson College, Swannanoa, NC.

1984b The Spatial Dimension of Siouan Mortuary Ritual: Implications for Studies of Change. Paper presented at the Annual Meeting of the Society for Historic Archaeology, Williamsburg, VA.

1985 Social Implications of Storage and Disposal Patterns. In Structure and Process in Southeastern Archaeology, edited by Roy S. Dickens, Jr. and H. Trawick Ward, pp. 82-101. University of Alabama Press, University.

Waselkov, Gregory A.

1977 Prehistoric Dan River Hunting Strategies. M.A. thesis, Department of Anthropology, University of North Carolina, Chapel Hill.

Waselkov, Gregory, Brian M. Wood, and Joseph M. Herbert

1982 Colonization and Conquest: the $1980 \mathrm{Ar}^{-}$ chaeological Excavations at Fort Toulouse and Fort Jackson, Alabama. Auburn University, Montgomery.

Washburn, Wilcomb E.

1957 The Governor and the Rebel: A History of Bacon's Rebellion in Virginia. Univer- sity of North Carolina Press, Chape1 Hill.

Watson, Patty Jo

1976 In Pursuit of Prehistoric Subsistence: A Comparative Account of Some Contemporary Flotation Techniques. Midcontinental Journal of Archaeology 1:77-100.

Watts, Steve

1985 Native vs. Trade Items in the Eighteenth Century Material Culture of the Catawbas and Other Southeastern Native Peoples. Ms. on file, Schiele Museum, Gastonia.

Weisner, Polly

1974 A Functional Estimator of Population from Floor Area. American Antiquity 39:343350 .

White, P.S. and S.T.A. Pickett

1985 Natural Disturbance and Patch Dynamics: An Introduction. In The Ecology of Natural Disturbance and Patch Dynamics, edited by S. T. A. Pickett and P. S. White, pp. 3-13. Academic Press, New York.

White, T.E.

1953 A Method for Calculating the Dietary Percentage of Various Food Animals Utilized by Aboriginal Peoples. American Antiquity 18:396-398.

White, Wesley

1981 Historical Overview of the [Saponi] Tribe: Second Essay. Ms. in possession of author.

Whitthoft, John, W. Fred Kinsey, III, and

C. H. Holzinger

1959 A Susquehannock Cemetery: The Ibaugh Site. In Susquehannock Miscellany, edited by John Whitthoft and W. Fred Kinsey, III, pp. 99-119. The Pennsylvania Historical and Museum Commission, Harrisburg.

Wiens, J.

1976 Population Responses to Patchy Environments. Annual Review of Ecology and Systematics $7: 81-120$.

Williams, Roger

1643 A Key into the Language of America. George Dexter. London. (Reprinted for The Rhode Island and Providence Plantations Tercentary Committee, Inc., 1936, 5 th edition, Roger Williams Press, Providence).

Williams, S.C. (editor)

1930 Adair's History of the American Indians (Reprint of the 1775 London edition). The Watauga Press, Johnson City, Tennessee. 
Wilson, Homes Hogue

1983 The Use of Paleo-Nutrition in the Analysis of North Carolina Piedmont Aboriginal Populations. Manuscript on file, Research Laboratories of Anthropology, University of North Carolina, Chapel Hi11.

1984 European Trade Goods from the Fredricks Site (310r231). Paper presented at the Annual Meeting of the Society for Historical Archaeology, Williamsburg, VA.

Wilson, Homes $\mathrm{H}$.

1985 Human Skeletal Remains from the Wa1l and Fredricks Sites. In The Historic Occaneechi: An Archaeological Investigation of Culture Change. Final Report of 1984 Investigations, edited by Roy S. Dickens, Jr., H. Trawick Ward, and R. P. Stephen Davis, Jr., pp. 260-339. Research Laboratories of Anthropology, University of North Carolina, Chapel Hill.

Wilson, Jack H., Jr.

1976 Final Report: 1974 Excavations within the New Hope Reservoir. Ms. on file, Research Laboratories of Anthropology, University of North Carolina, Chape1 Hill.

1977 Feature Fill, Plant Utilization and Disposal Among the Historic Sara Indians. M.A. thesis, Department of Anthropology, University of North Carolina, Chape1 Hill.

1983 A Study of Late Prehistoric, Protohistoric, and Historic Indians of the Carolina and Virginia Piedmont: Structure, Process, and Ecology. Ph.D. dissertation, Department of Anthropology, University of North Carolina, Chapel Hill.

1985 Feature Zones and Feature Fill: More than Trash. In Structure and Process in Southeastern Archaeology, edited by Roy S. Dickens, Jr. and H. Trawick Ward, pp. 60-81. University of Alabama Press, University.

Wing, Elizabeth

1977 Subsistence Systems in the Southeast. The Florida Anthropologist $30(2): 81-87$.

1979 Paleonutrition: Method and Theory in Prehistoric Lifeways. Academic Press, New York.

Winkler, Helmut G.F.

1976 Petrogenesis of Metomorphic Rock. Springer-Verlag, New York.

Winterhalder, Bruce

1980 Environmental Analysis in Human Evolution and Adaptation Research. Human Ecology $8: 135-170$.
Winterhalder, Bruce

1981 Optimal Foraging Strategies and HunterGatherer Research in Anthropology: Theory and Models. In Hunter-Gatherer Foraging Strategies, edited by Bruce Winterhalder and Eric Alden Smith, pp. 13-35. University of Chicago Press, Chicago.

1983 The Analysis of Hunter-Gatherer Diet: Stalking an Optimal Foraging Model. Paper prepared in advance for WennerGrenn Symposium No. 94, Cedar Cove, FL.

Wright, J. Leitch, Jr.

1981 The Only Land They Knew: The Tragic Story of the American Indians in the 01d South. The Free Press, New York.

Wright, Louis B.

1947 The History and Present State of Virginia, by Robert Beverley. The University of North Carolina Press, Chapel Hill.

Wright, Louis B. (editor)

1966 The Prose Works of William Byrd of Westover: Narratives of a Colonial Virginian. Harvard University Press, Cambridge.

Yarne11, Richard A.

1974 Plant Food and Cultivation of the Salt Cavers. In Archaeology of the Mammoth Cave Area, edited by Patty Jo Watson, pp. 113-122. Academic Press, New York.

1978 Domestication of Sunflower and Sumpweed in Eastern North America. In The Nature and Status of Ethnobotany, edited by Richard I. Ford, pp. 289-299. Anthropological Papers 67, Museum of Anthropology, University of Michigan, Ann Arbor.

1982 Problems of Interpretation of Archaeological Plant Remains of the Eastern Woodlands. Southeastern Archaeology 1:1-7.

1983 Prehistoric Plant Foods and Husbandry in Eastern North America. Paper presented at the 48th Annual Meeting of the Society for American Archaeology, Pittsburgh.

Yarne11, Richard A. and M. Jean Black

1983 Temporal Trends Indicated by a Survey of Prehistoric Plant Food Remains from Southeastern North America. Revised version of a paper presented at the 40 th Annual Meeting of the Southeastern Archaeological Conference, Columbia, SC.

Yarne11, Ricard A. and M. Jean Black

1985 Temporal Trends Indicated by a Survey of Archaic and Woodland Plant Food Remains from Southeastern North America. Southeastern Archaeology 4:93-106. 


\author{
Roy S. Dickens, Jr. \\ H. Trawick Ward \\ R. P. Stephen Davis, Jr.
}

\begin{abstract}
INTRODUCTION
The archaeological excavations conducted at the Fredricks site during 1983-84 provided information sufficient to address general questions about the period of occupation, the overall configuration of the material-culture inventory, mortuary behavior, and subsistence activities; however, they did not provide a firm basis for addressing larger problems pertaining to overall settlement structure and composition. In order to address these latter research problems, a third season of fieldwork was undertaken at the Fredricks site, funded by a second grant from the National Geographic Society. The specific questions considered by this work included: 1) Is the existing cemetery the only one on the site, and was it the result of one episode of warfare; 2) What were the habitation structures like and how were they arranged in the settlement 3) Did more than one tribe reside in the village; and 4) What was the size and overall pattern of the settlement? Fieldwork undertaken to answer these questions consisted of excavating the remaining burials in the cemetery, isolating domestic structures in the northwestern and southeastern parts of the village, and uncovering a large portion of the village palisade.
\end{abstract}

\section{RESULTS}

The 1985 excavation at the Fredricks site was nearly twice as extensive as those undertaken during the two previous seasons, and was of sufficient size to permit an estimation of village size. Results of this excavation indicated that the site was substantially smaller than previously thought. At that point, it appeared that the community, as delimited by the palisade, covered approximately $10,000 \mathrm{ft}^{2}(.25$ acre). Over $60 \%$ of this area had, been excavated, and an additional $3,000 \mathrm{ft}^{2}$ had been excavated beyond the palisade in the vicinity of the cemetery and Structure 6 (see Figures 1.12-1.13). Despite this extensive work, the intrasite structure still was only generally understood because of the incompleteness of the architectural record. Plowing had reduced most house patterns to diffuse scatters of postholes, making their identification difficult.
Thirty features and burials were recorded during the 1985 field season (see Table 1.1). Twenty-five of these were excavated using procedures outlined in Chapter 1 ; the remainder were mapped and augered to determine depth. A preliminary description of archaeological features and structures is presented below.

\section{Burials}

Three burials were excavated during 1985 and comprise the remaining burials within the village cemetery. In all, this cemetery contained 12 burials and an additional probable burial (Feature 1).

Burial 10 (Feature 27) contained the remains of a sub-adult, 4-5 years of age at of death (Figure A.1). The burial was loosely ly flexed and placed on its left side. Skeletal remains were very poorly preserved. Artifacts accompanying this burial included two checkstamped ceramic pots, one small plain ceramic pot, one greenstone celt, one turtle carapace "cup", an iron hoe, lead shot, and nine brass bells. Numerous small white, red, and blue glass beads were scattered on the pit floor near the skull. Remnants of fiber matting across the pit bottom indicate that this individual was "wrapped" prior to interment.

Burial 11 (Feature 14) was a young adult of indeterminate sex, approximately 15-17 years old at death (Figure A.2). Bone preservation was generally poor. This burial was loosely flexed and lay on its right side. Several artifacts associated with Burial 11 occurred as clusters and appear to represent separate bundles or containers. The first cluster, located near the feet, contained several Cornaline de Allepo beads, vermillion and red lead, wire Cbracelets, and a snuff tin. An adjacent cluster contained a case knife, two Jews harps, and lead shot. A third cluster, located near the right knee, consisted of a concentration of Cornaline de Allepo beads. In addition to these artifact clusters, four pewter buckles were found near the skull and a large cord-marked ceramic bowl lay near the chest.

Burial 13 (Feature 26) was an adult male, 37-42 years old at death (Figure A.3). This burial was also loosely flexed and was placed on 


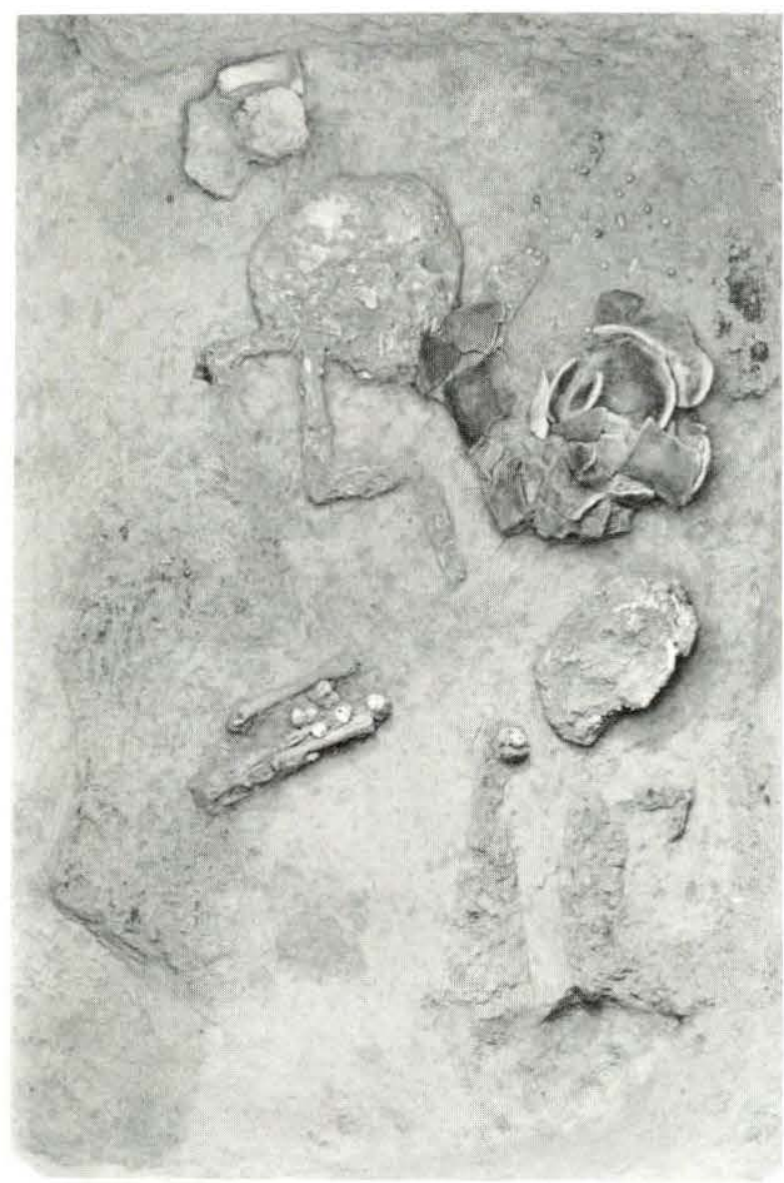

Figure A.1

Burial 10, excavated.

its left side. As with the two previous burials, skeletal remains were in generally poor condition. Artifacts accompanying Burial 13 included two bone-handled case knives, a pewter porringer, and a kaolin trade pipe.

\section{Storage Pits (Figures A.4-A.5)}

Six of the features excavated during 1985 (Features 17, 19, 28, 29, 30, and 41) are interpreted as storage pits. These pits varied from 2.5-3.5 ft in plan dimension and all but one exceeded two feet in depth. Two had straight sides; the remainder had "bell-shaped" profiles. Features 17 and 19 were located within Structure 9 at the southeastern end of the village. Features 30 and 40 were located just west of Structure 5. Feature 30 was the only pit identified at the Fredricks site that does not appear to be associated with the Historic period occupation. In addition to an absence of Euroamerican artifacts, the pottery from this feature was mostly net impressed, indicating a late prehistoric cultural association. Finally, Features 28 and 29 were both located outside the village palisade and just northeast of Structures 4 and 6 . The location of these pits and the paucity of Euroamerican artifacts within their fill indicate that they may date to an initial period of Occaneechi settlement pre-

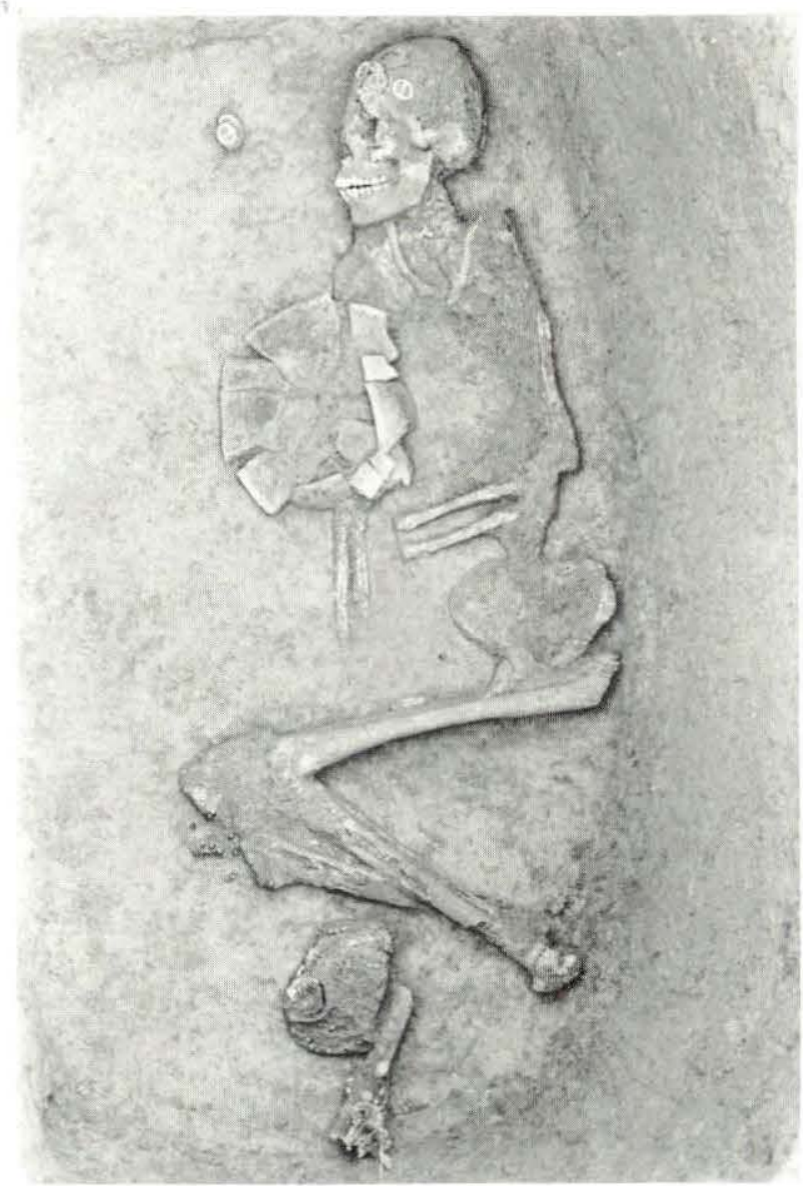

Figure A.2

Burial 11, excavated.

ceding the establishment of the palisaded village.

All of these features contained moderately rich assemblages of artifacts and subsistence remains, and provided substantial new information for studying domestic activities at the site.

\section{Pits (Figure A.6)}

In addition to storage pits, four other pit features (Features 18, 20, 23, and 33) were excavated. These also may have served as storage facilities; however, their shallow depth (ca. 1.0-1.5 ft) makes functional interpretation less certain. These pits tended to be straightsided to slightly "bell-shaped" in profile. Features 18 and 20 may be associated with Structure 9; Features 23 and 33 were located inside Structure 5. All of these features contained numerous artifacts. Feature 18 was particularly interesting in that it contained a heavy concentration of sherds and vessel sections from three large, simple-stamped and check-stamped jars.

\section{Shallow Basins}

Seven shallow basins (Features 16, 24, 25, 


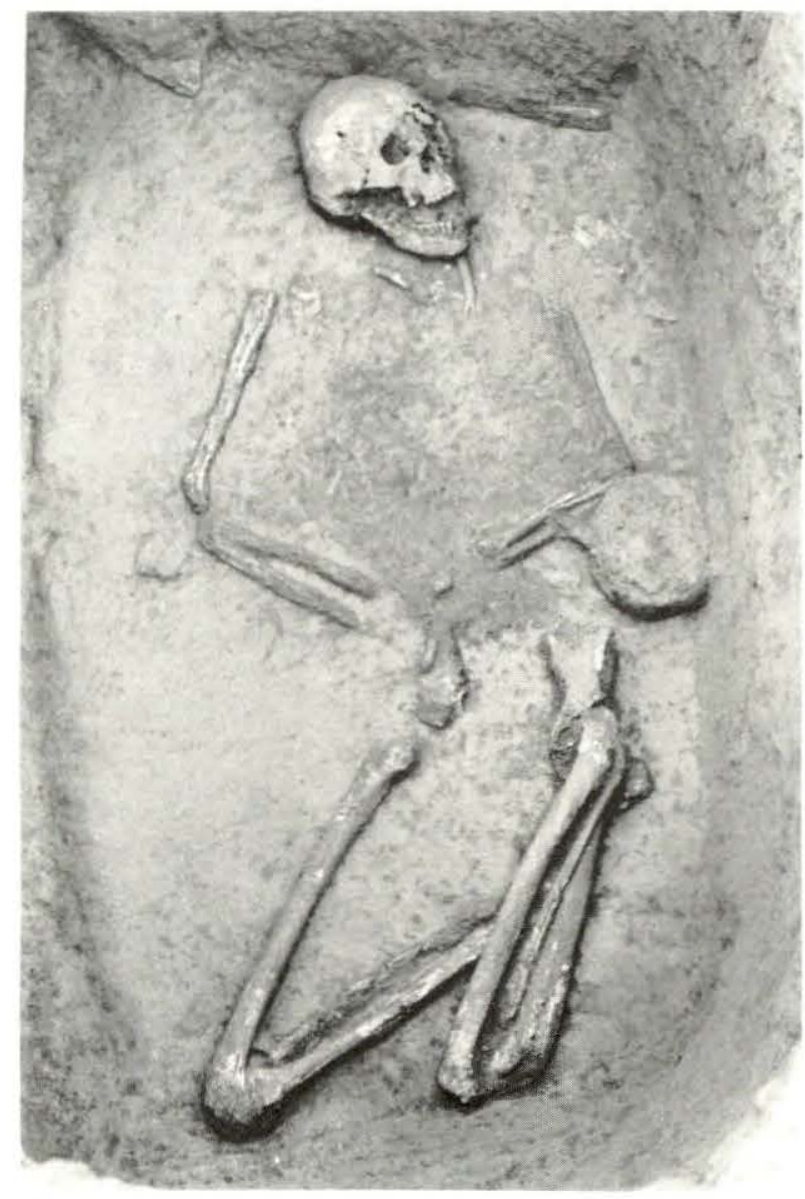

Figure A.3

Burial 13, excavated.

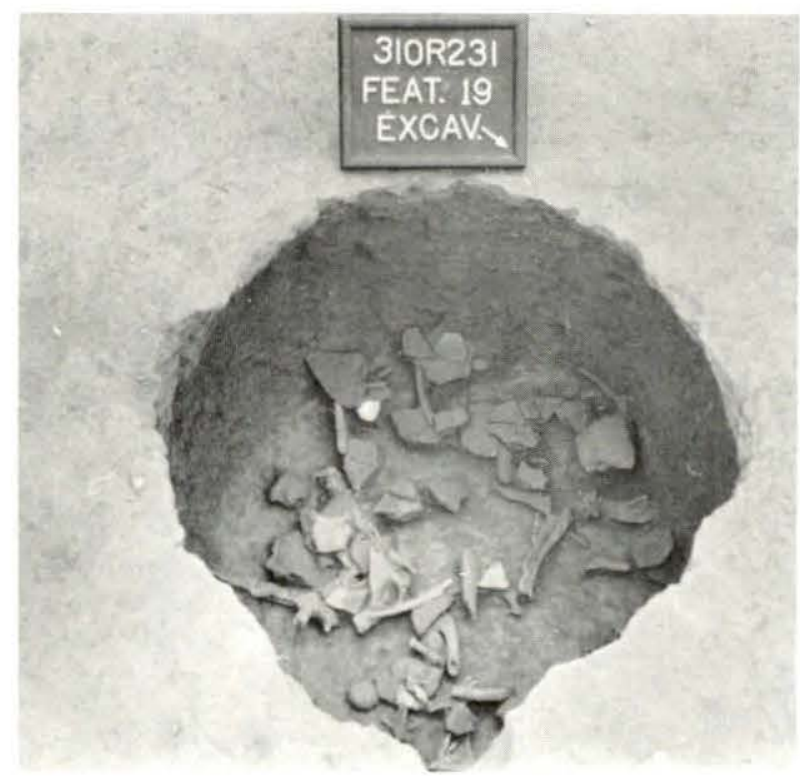

Figure A. 4

Feature 19, excavated.

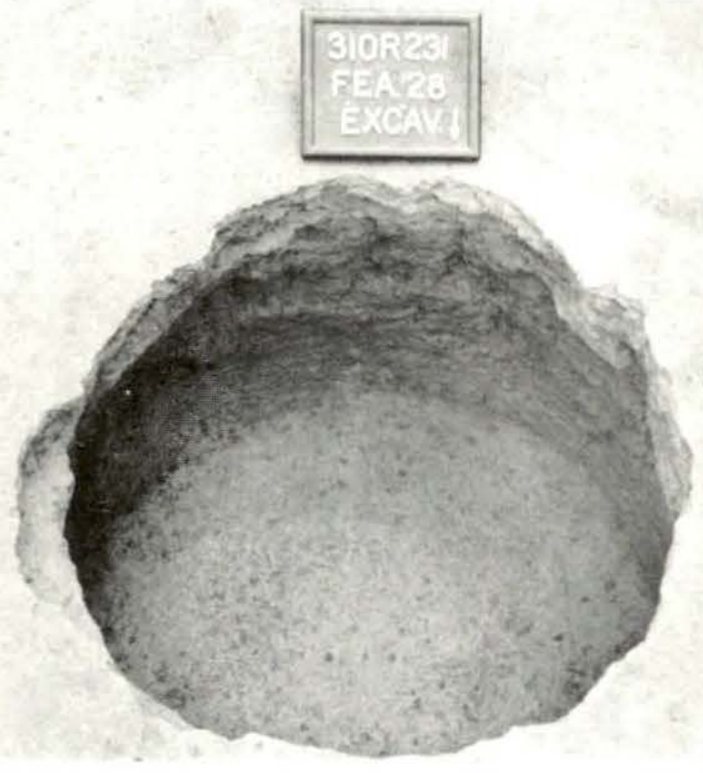

Figure A. 5

Feature 28, excavated.

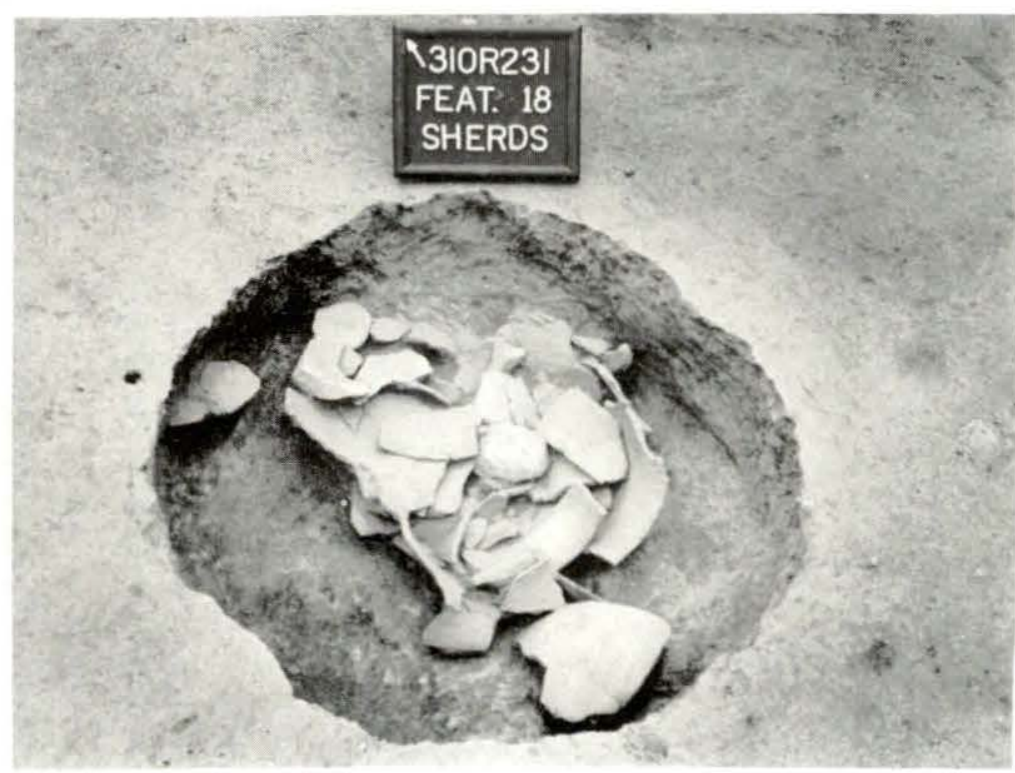

Figure A.6

Feature 18, excavated. 
$37,38,39$, and 40 ) were excavated. These features were mostly oval in plan, shallow, and lacked distinct pit walls. The probable use of these features is undetermined. Feature 16 was located within Structure 8; Feature 24 lay within Structure 6; and Feature 40 was located inside Structure 4. Associations for the other shallow basins are indeterminate. Most of these features contained only a small number of artifacts.

\section{Sha1low Depressions}

Features 21 and 22 were classified as shallow depressions and consisted of little more than dark stains at the top of subsoil. They were only 0.1-0.2 ft deep and probably represent the bottoms of either large postholes or small basins. Both were situated within Structure 8.

\section{Misce1laneous Features}

Other features excavated at the Fredricks site during 1985 include two charred corncobfilled pits (Features 35 and 36 ) in the vicinity of Structure 6 and a tree stump (Feature 15) located just northeast of the cemetery. Features that were mapped but not excavated include: a hearth stain (Feature 34) associated with Structure 5; one possible burial (Feature 31); two probable pits (Features 42 and 43); and one probable rodent disturbance (Feature 32 ).

\section{Structures (Figure A.7)}

Three structures were exposed by the 1983-84 excavations at the Fredricks site. Structure 1 was an oval wall-trench structure located in the center of the village, and is interpreted as a communal sweat lodge. Structures 2 and 3, located adjacent to the palisade and defined by concentrations of postholes and poorly defined wall-post alignments, represent domestic structures.

During 1985, six additional structures were exposed. These structures are briefly described below.

Structure 4 was a small, circular wal1trench structure located outside the palisade at the north end of the excavation. It was approximately five feet in diameter and contained Feature 40 , a small shallow basin. Its proximity to Structure 6 suggests that it probably was associated with this larger structure. Structure 4 may represent either a small sweat house or an above-ground storage facility.

Structure 5 was a well-defined wall-trench house located at the northwestern end of the village. It was oval-to-rectangular in form and measured approximately $16 \mathrm{ft} \times 19 \mathrm{ft}$. In addition to a centrally located hearth (Feature 34), two pits (Features 32 and 33 ) and a large sha1low basin (Feature 24) were also located inside this structure.

Structure 6 was a wall-trench house located at the northern end of the village, and was intruded by both the village palisade and Structure 5. As a consequence, it may represent one of the initial houses constructed at the site by the 0ccaneechis. It was roughly circular and measured about $17 \mathrm{ft}$ in diameter. No features were associated with this structure.

Structure 7 lay immediately east of Struc-

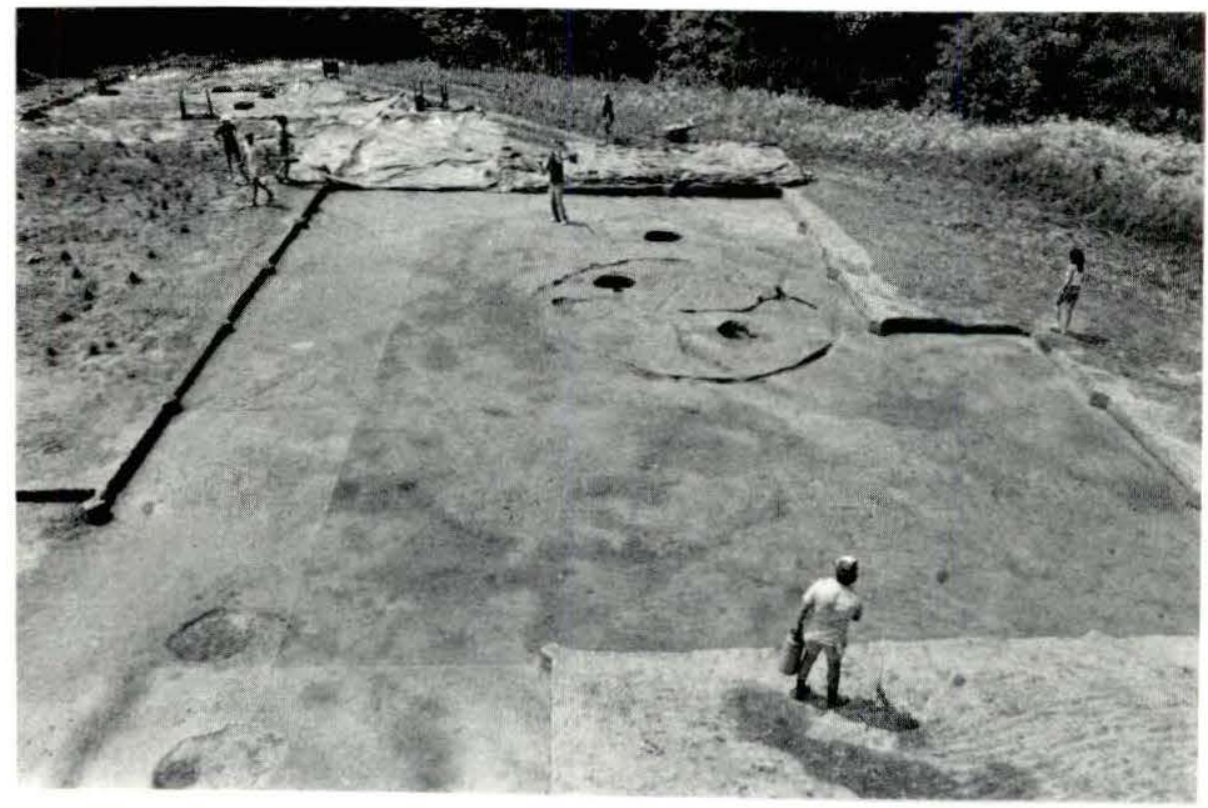

Figure A.7

Structure 5 (excavated) and Structure 6 (unexcavated in foreground) at top of subsoil. 
ture 5, and was defined by a circular alignment of postholes. This house was approximately 18 $\mathrm{ft}$ in diameter and was the most clearly defined non-wal1-trench structure identified at the Fredricks site. No features were associated with Structure 7 .

Structure 8 was situated between Structure 3 and the palisade at the eastern edge of the village, and was represented by a concentration of postholes measuring about $14 \mathrm{ft}$ in diameter. Features associated with this house included a pit (Feature 13) excavated in 1984, a shallow basin (Feature 16), and two shallow depressions (Features 21 and 22).

Structure 9, situated at the southeastern end of the village, was a poorly defined, rectangular house that measured about $11 \mathrm{ft} \times 17 \mathrm{ft}$. Several of the postholes that comprised this structure pattern probably were eradicated by plowing. Two storage pits (Features 17 and 19) were associated with Structure 9; in addition, two other pits (Features 18 and 20) located west of the structure also may be associated.

Artifacts (Figures A. 8-A.10)

A large collection of artifacts and subsistence remains were recovered from both plowzone and feature contexts. The 1985 excavation not only greatly expanded the size of the Fredricks site artifact collection, it also provided a substantial increase in the number of samples used to address questions about domestic activities at the site.

\section{CONCLUSIONS}

The 1985 fieldwork at the Fredricks site was undertaken to address a series of research questions about intra-settlement patterning and possible ethnic variability among the village population. Part of the fieldwork focused upon completing the investigation of the cemetery and exploring the potential for additional cemeteries. Auger testing during 1984 indicated that five to seven additional burial pits would be encountered at the north end of the cemetery (Dickens et a1. 1984:27). Five pits were exposed in this area; however, only three of them were burials. The remaining two pits, located about $10 \mathrm{ft}$ beyond the cemetery, were deep storage pits associated with Structure 6 .

In a11, 13 burial pits (including Feature 1) comprised this cemetery and contained the remains of three infants or neonates, four

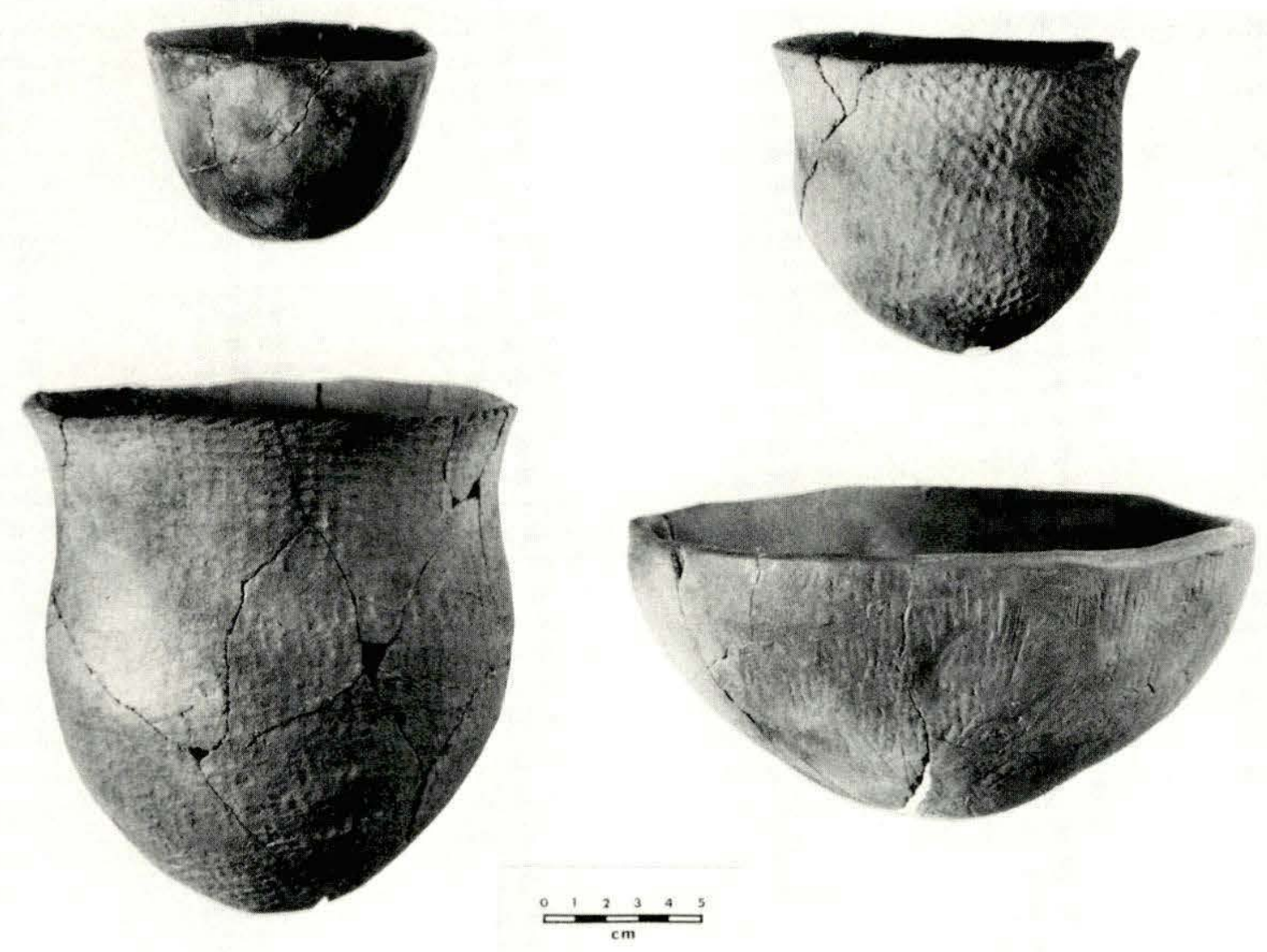

Figure A. 8

Clay pots recovered from Burial 10 (top row and bottom left) and Burial 11 (bottom right). 


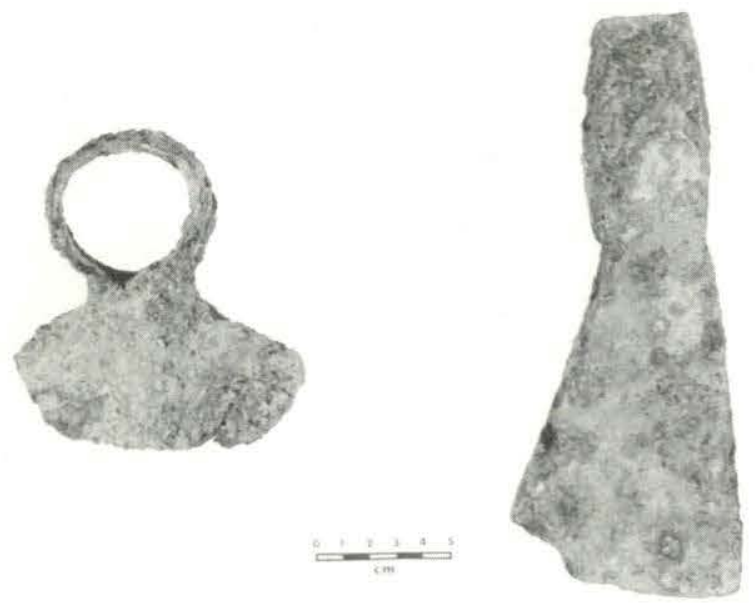

Figure A.9

Iron hoe and axe fragments.
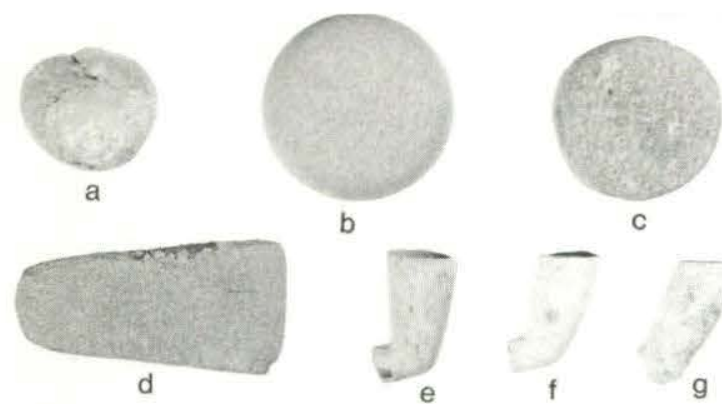

C
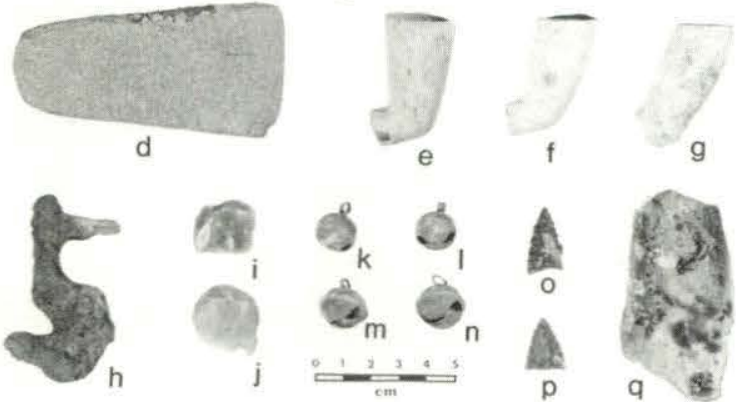

Figure A. 10

Selected small artifacts recovered during 1985 excavations: hammerstone (a); discoidal (b); stone disk (c); celt (d); clay pipes (e-g); gun lock (h); gunflints $(i-j)$; brass bells $(k-n)$; projectile points $(o-p)$; and bottle glass (q).

sub-adults, five adult males, one adult female, and one young adult of indeterminate sex. Although the spatial relationships among these burials and the kinds of associated artifacts indicated that all individuals were interred over a relatively short time period, it was difficult to determine if the cemetery was the product of a single event. The evidence of violent death observed on Burials 4 and 9 indicates that warfare may have been responsible for at least some of the deaths.

Archaeological excavations during 1985 also contributed significantly toward an understanding of village development, domestic architecture, and the overall village plan.
Evidence obtained in 1985 suggested that the site was initially settled by one or a few families. Remains of this initial occupation consisted of Structure 6 which was intersected by the palisade and Structure 5, Structure 4, and Features 28 and 29. Ceramics from both features were similar to those from features inside the palisade; however, a significantly smaller number and variety of Euroamerican artifacts were found. The fact that Structure 6 was intruded by the palisade suggested that it was no longer in use when the larger village was established.

In addition to identifying six more structures (including another possible sweat house), the expanded excavation in 1985 also permitted a much more secure interpretation of the two poorly defined domestic structures exposed in 1984. These new data indicated that houses were of wall-trench as well as single-post construction, were of variable shape ranging from sub-rectangular to cirqular, and provided approximately $175-250 \mathrm{ft}^{2}$ of interior floor space each. Hearths were centrally located, and some houses had interior, subterranean storage facilities.

Although much of the southwestern half of the village remained unexcavated at the end of the 1985 season, certain inferences could be drawn about the overall settlement plan. Apparently the Occaneechi village was small and compact, encompassing only about .25 acre. It was surrounded by a palisade constructed of small saplings and probably was comprised of 11-12 houses situated in a circle along the inside of the palisade. Assuming approximately five persons per household, this suggests that the community contained approximately 50-75 individuals. Interior to the houses was a common area, or plaza, which contained few architectural features. The central feature within this plaza, as well as within the larger village, was an oval sweat lodge (Structure 1). Its relatively large size (compared to Structure 4 ) and its location indicated that this was a communal facility. Because of the compact nature of the settlement, it was believed that a variety of domestic activities were conducted outside the palisade, probably between the village and the Eno River located about $50 \mathrm{ft}$ to the southwest.

Finally, the 1985 investigations sought to determine if other ethnic groups resided at the site along with the Occaneechis. Although direct ethnohistoric evidence is lacking, Lawson's accounts of Piedmont Indian societies indicate that many villages had become multi-ethnic communities by the early eighteenth century (Lefler 1967). This research question was approached in a preliminary way by an analysis of pottery from the 1983-84 excavations (see Chapter 8). This analysis concluded that most variability in the pottery assemblage could be best explained by multiple site occupations over time rather than by a single, multi-ethnic 
occupation. This question was addressed further with the ceramic data acquired in 1985; however, these data only added strength to the previous explanation and offered no evidence for the presence of other historic ceramic traditions at the site. At this time, it was believed that if members of other tribes were residing with the Occaneechis, then they probably occupied separate, nearby villages and thereby contributed little to the archaeological remains of the Fredricks site. 
Appendix B

\title{
Summary Report of 1986 Fredricks Site \\ Excavations
}

by

\author{
H. Trawick Ward
}

R. P. Stephen Davis, Jr.

\section{INTRODUCTION}

\begin{abstract}
Although the 1985 excavations did much to clarify the internal configuration of the Occaneechi village, additional fieldwork was proposed in 1986 to allow the total excavation of the habitation area within the palisade. Because the small village compound is unique in the Piedmont region, its complete excavation offered a rare opportunity to study the in situ remains of a spatially-bounded social unit larger than a household. And although approximately half the compound had been exposed by 1985, the intrasite patterns were still only generally understood because several structures were represented by diffuse posthole clusters. It was believed that the total excavation of the palisaded area would clarify the spatial definition and relationships of all the structures as well as expose all associated features. The data from the habitation area in conjunction with the cemetery data would permit fine-grained subsistence, social, and ritual reconstructions, and allow accurate estimates of population size. Sampling biases that plague most archaeological investigations would be reduced to a minimum. A detailed knowledge of the village spatial structure would also provide an excellent comparative background for assessing smaller scale excavations at other Contact period sites and facilitate the reconstruction of intrasite patterns from relatively small excavated samples. This phase of fieldwork was again supported by the National Geographic Society.
\end{abstract}

In addition to uncovering the remainder of the village compound, the extent of a slightly earlier occupation outside the palisade in the northern part of the site was also to be explored by auger tests in 1986. Unfortunately, extremely dry and compact soil conditions prohibited the implementation of this phase of the project. Because the palisade did not follow the regular oval outline projected in 1985, it was also necessary to excavate more squares than initially proposed to uncover all the area within the compound.

\section{RESULTS}

The 1986 excavation at the Fredricks site uncovered all the village area enclosed within the palisade except for a small section in the southwest corner where large trees prevented soil removal. Although still small, the village shape was more irregular than predicted after the 1985 field season (see Figure 1.13). The irregular outline resulted from the fact that the palisade "bowed-out" or expanded to the southwest, thus creating a D-shaped rather than oval configuration. Although much of the structural evidence continued to consist of vague posthole clusters, two additional wall-trench structures were defined and the pit features associated with the structures were extremely rich. Their depositional character and contents added significant new data that aided in clarifying general as well as specific behavioral patterns within the village.

Nineteen features and burials were excavated during the 1986 field season (see Table 1.1). Two additional features were mapped but not excavated and four domestic structures were recorded. A preliminary description of archaeological features and structures is presented below.

Burials (Figures B. 1-B.2)

Two burials were excavated in 1986 . Neither was associated with the cemetery, and both were contained in pits that were very different from those of the cemetery burials. Although human skeletal remains were absent, two other pit features were excavated that resembled burials in form and fill characteristics.

Burial 12 (Feature 50) was located in the southern part of the site, just outside the palisade in what appears to be a southern entrance to the village compound. It contained the remains of an infant about six months old. The body was placed in a shaft-and-chamber pit. Bone preservation was extremely poor, but it appears that the legs were flexed and the head pointed to the south-southwest. Brass bells, which preserved small fragments of cane matting, were found in the leg area. The presence of matting suggests that the body was wrapped prior to interment. A lead bale seal and several shell beads also were present in the leg area. 


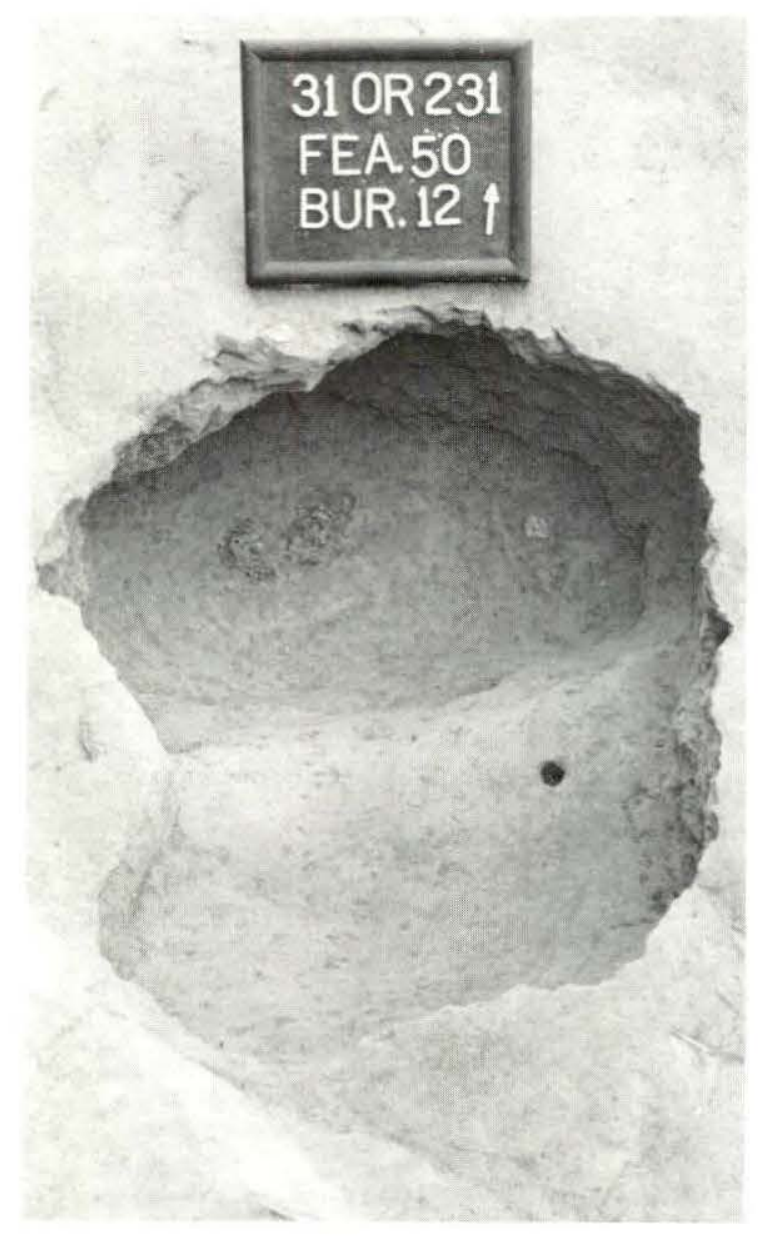

Figure B.1

Burial 12, excavated.

the southern part of the site within a cluster of pit features that formed a band paralleling the interior margin of the palisade. In this burial, the loosely flexed remains of a 12 year old subadult were placed in the side chamber of a shaft-and-chamber pit with the head oriented to the east. Shell beads were strung around the neck and the right wrist. European trade artifacts consisted of a brass buckle and several pewter buttons in the waist area, brass rings on the fingers of both hands, and numerous white glass beads in the area of the right hip.

\section{Probable Burial Pits}

Two features (Feature 31 and 49) possessed pit characteristics similar to other pits identified as human burials but contained no recognizable skeletal remains. Feature 31 was a rectangular, straight-sided pit ( $3.1 \mathrm{ft}$ long by $2.2 \mathrm{ft}$ wide by $2.0 \mathrm{ft}$ deep) that contained a clayey fill with few artifacts and a dark organic stain at the bottom of the pit suggestive of a decomposed burial. This feature was positioned along the inside wall of Structure 10. Feature 49, situated just north of Structure 13, was a small shaft-and-chamber

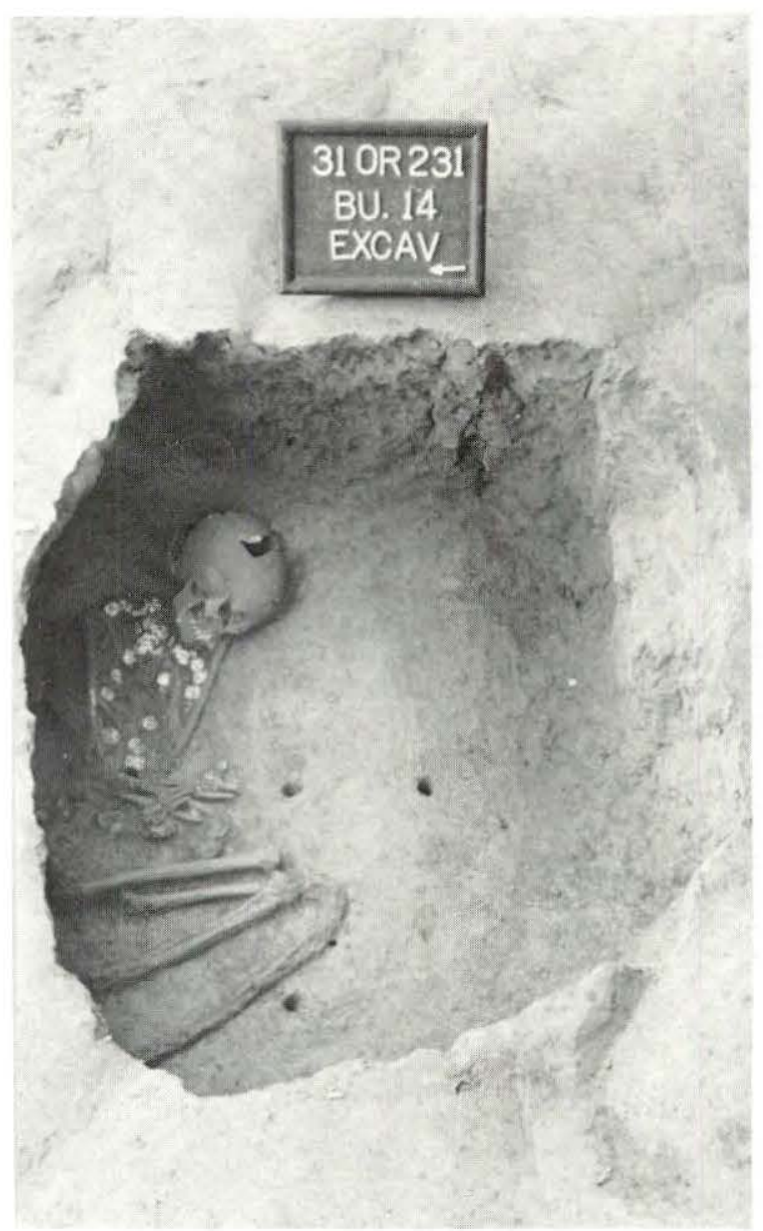

Figure B.2

Burial 14, excavated.

pit ( $2.5 \mathrm{ft}$ long by $1.5 \mathrm{ft}$ wide by $1.2 \mathrm{ft}$ deep). This feature also contained a clayey upper fill containing few artifacts, and had a dark organic zone on the chamber floor that yielded a single brass thimble.

\section{Storage Pits (Figures B.3-B.5)}

Five of the features excavated during 1986 (Features 44, 46, 51, 53, and 56) are interpreted as storage pits. These pits varied from 2.0-2.9 ft in plan dimension and ranged from 1.9 $\mathrm{ft}$ to $3.3 \mathrm{ft}$ in depth. All were deep cylindrical pits with either straight or slightly bowed sides. Unlike several of the storage pits excavated in previous seasons, none had distinctive "bel1-shaped" profiles. Fill within these pits was generally rich in artifacts. All of these features are located in the vicinity of domestic structures. Feature 44 was clustered with two other pits (Features 45 and 47) just east of Structure 13 while Feature 46 was situated just northwest of this structure. Feature 51 was located within Structure 12. Feature 53 was located be'ween Structures 12 and 13, and may be associated with an unidentified structure at that location. Finally, Feature 56 


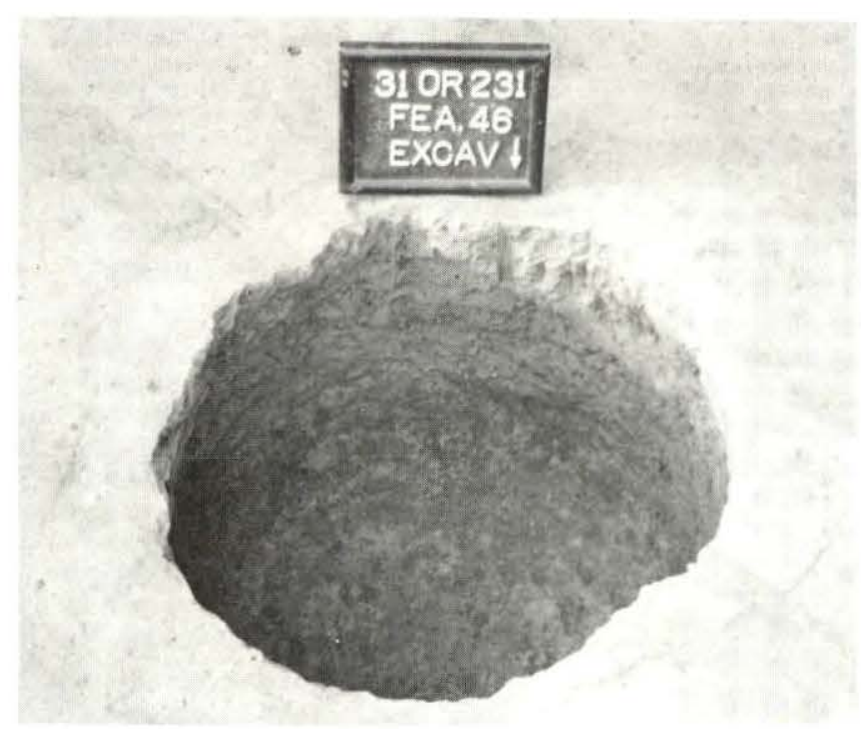

Figure B.3

Feature 46, excavated.

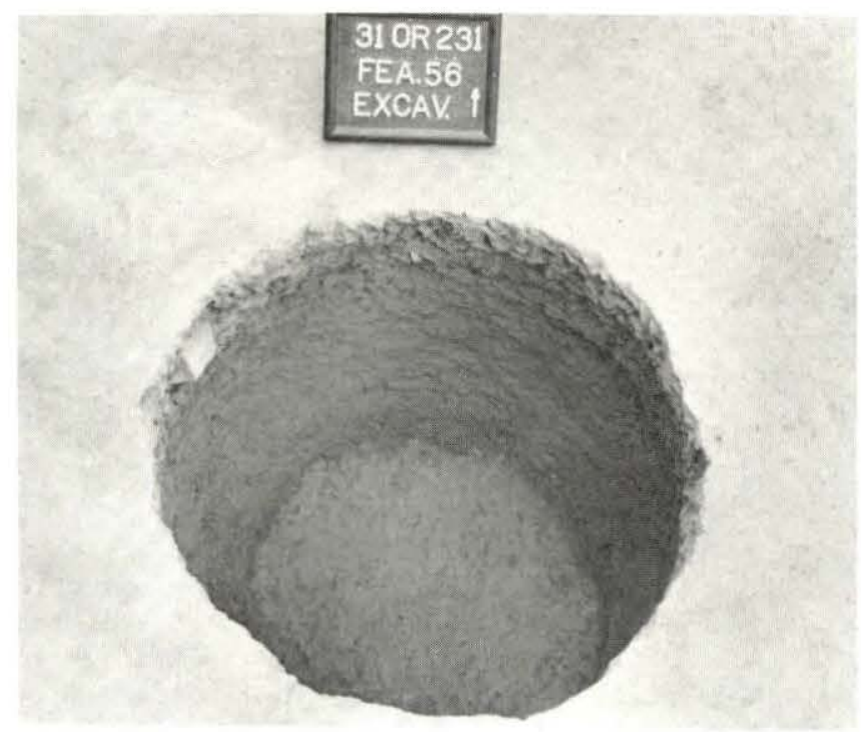

Figure B. 4

Feature 56, excavated.

was situated between the west wall of Structure 11 and the palisade, and apparently is associated with that structure.

\section{Pits}

In addition to storage pits, eight other pit features (Features 42, 45, 47, 55, 57, 58, 59, and 61) were excavated. An additional unexcavated feature (Feature 60) is probably also a pit. Several of these features (i.e., Features 42, 45, 47, 57, 59, and 61) may have served as storage pits; however, their shallow depth (ca. 1.3-2.1 ft) makes functional interpretation less certain. These pits were circular to oval in plan dimension $(2.2-3.7 \mathrm{ft}$ in diameter) and tended to be straight-sided to

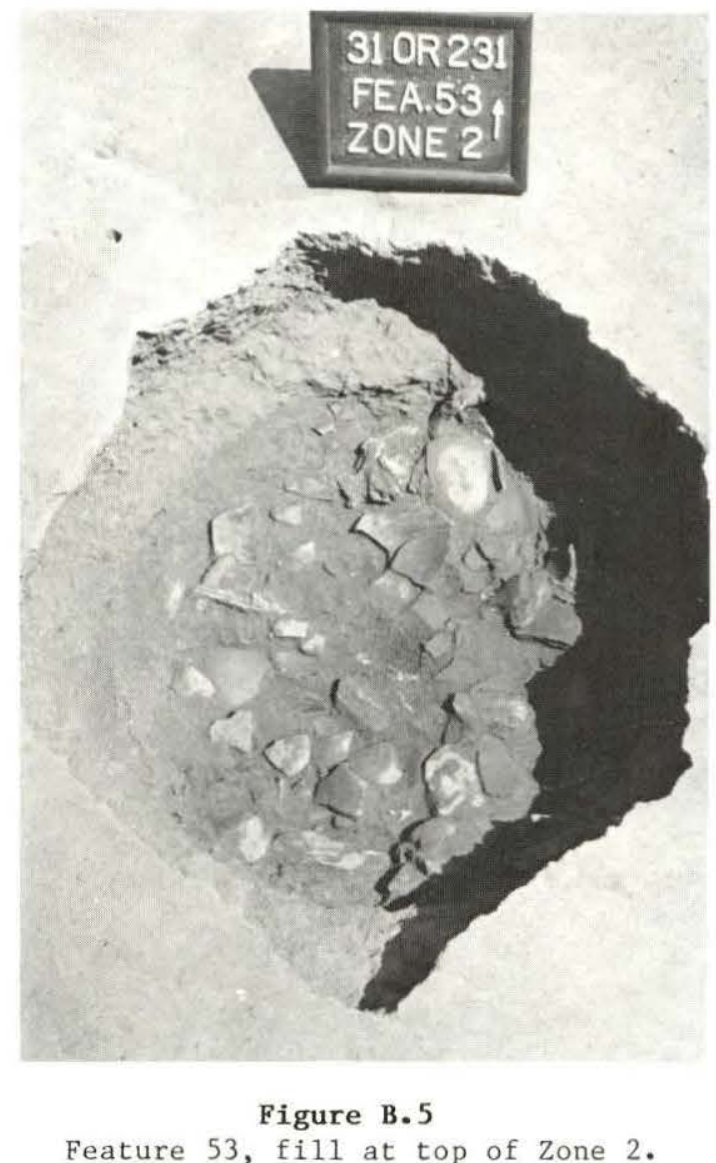

slightly "be11-shaped" in profile. While some of these features contained very few artifacts, most possessed fill characteristics similar to other storage pits. As with storage pits, these pit features all appeared to be associated with domestic structures. Feature 42 was situated inside the palisade between Structures 9 and 13, and may be associated with an unidentified structure at this location. Features 45 and 47 were clustered with Feature 44 just east of Structure 13 while Features 55 and 58 were situated along the southwest edge of this structure. Features 57 and 60 are probably associated with an unidentified domestic structure between Structures 12 and 13. Finally, Features 59 and 61 were associated with Structure 12 .

\section{Shallow Basin}

One shallow basin (Feature 52) was identified during 1986 but was not excavated. This feature was oval in plan dimension, approximately $2.0 \mathrm{ft}$ in diameter, and intruded the Structure 11 wall trench. Augering of the center indicated that it lacked any appreciable depth.

\section{Probable Hearth}

Feature 43, located between Structures 9 and 13, was a fire-reddened area at top of subsoil measuring approximately $1.5 \mathrm{ft} x 2.0 \mathrm{ft}$. 
Augering of the center indicated that it was less than $0.3 \mathrm{ft}$ deep.

\section{Irregular Trench}

Feature 48 was a long, irregular trench that ran in a north-south direction and intruded Structures 10 and 11 . It was about $2.5 \mathrm{ft}$ wide, approximately $0.3 \mathrm{ft}$ deep, and contained small amounts of pottery, rock, and historic artifacts (mostly beads). Thin traces of this feature were observed at the north end of the excavation in 1985 but were not excavated. This earlier evidence, coupled with the $35-\mathrm{ft}$ section excavated in 1986, indicate that the trench is at least $75 \mathrm{ft}$ long. Although this feature certainly post-dates both structures, it does not appear to be associated with later Euroamerican activity in the site vicinity. This conclusion is based on the fact that no Euroamerican artifacts were found that post-date the Indian occupation of the site. The exact nature and function of this feature is unknown.

\section{Structures}

Before 1985, nine structures had been exposed at the Fredricks site. Four (Structures $1,4,5$, and 6) were well-defined circular to oval wall-trench constructions. Two of these (Structures 1 and 4) probably represent nondomestic, ceremonial or special purpose building whereas the other two (Structures 5 and 6) probably served as houses. Except for Structure 7 , the remaining structures (Structures $2,3,8$, and 9) were represented by somewhat igue clusters of postholes and pit features, and probably represent the remains of houses. Structure 7 consisted of a well-defined circular alignment of wall posts. No features were associated with it.

During 1986, four additional structures (Structures 10,11, 12, and 13) were uncovered. Two were constructed using wall trenches, and two were represented by posthole clusters.

Structure 10 was located adjacent to Structure 5 and was defined by a segment of a wall trench and several wall posts. It was oval in shape and measured roughly $18 \mathrm{ft} x 15 \mathrm{ft}$. One pit feature (Feature 31) was located just inside the structure and another pit (Feature 30) was positioned immediately outside its northeast corner. The latter feature, however, appears to be associated with an earlier, prehistoric occupation of the site. The structure was intruded by a long shallow trench (Feature 48).

Structure 11 was adjacent to and south of Structure 10. Although a wall trench comprised most of its perimeter, individual wall posts were predominant along the southern and eastern walls. This structure was oval in outline and measured approximately $20 \mathrm{ft} \times 18 \mathrm{ft}$. It was intruded by Feature 52 and the long trench feature (Feature 48) that also intruded Structure
10. One pit (Feature 56) lay west of the structure near the palisade.

Structure 12 was an il1-defined wall-post structure located adjacent to and north of Structure 11. It was circular to slightly oval in outline and measured approximately $15 \mathrm{ft}$ in diameter. Three large pits (Features 51, 59, and 61) were dug around the periphery of the structure.

Structure 13 was the only structure defined along the southern perimeter of the site. Like Structure 12 , it was somewhat vague but appeared to be represented by a circular cluster of postholes adjacent to the palisade and near the southern entrance. Two pit features (Features 55 and 58) were located inside the circle of postholes whereas several pits formed a line north of the structure, separating it from the remainder of the village. Burial 12 was located immediately to the south in the palisade entrance. Structure 13 measured roughly $20 \mathrm{ft}$ in diameter.

\section{Artifacts (Figures B.6-B.9)}

A large collection of artifacts and subsistence remains were recovered from both plowzone and feature contexts during 1986, and add appreciably to existing artifact assemblages from the site. Specific artifact categories for which substantial new collections were obtained include: aboriginal lithic artifacts, aboriginal ceramic artifacts, Euroamerican artifacts, faunal remains, and ethnobotanical remains.

\section{CONCLUSIONS}

The primary objective of the 1986 fieldwork at the Fredricks site was to excavate the remainder of the village area encompassed by the palisade. These excavations were designed to provide a complete plan of the structures and facilities within the compound and to allow distributional studies of various artifact classes across the site. By studying the habitation area in conjunction with the cemetery complex located immediately outside the palisade, questions concerning intra-site settlement patterns, subsistence, mortuary behavior, and ethnicity can be addressed within the larger context of late seventeenth century culture change on the North Carolina Piedmont.

In general, the features excavated in 1986 were extremely rich in artifact content and displayed depositional characteristics that shed significant light on the questions mentioned above. The ceramic inventory was greatly increased, particularly in terms of the domestic vesse1 assemblage. There now appears to be greater diversity along both stylistic and functional lines than was indicated by earlier research. Numerous lithic artifacts also were 

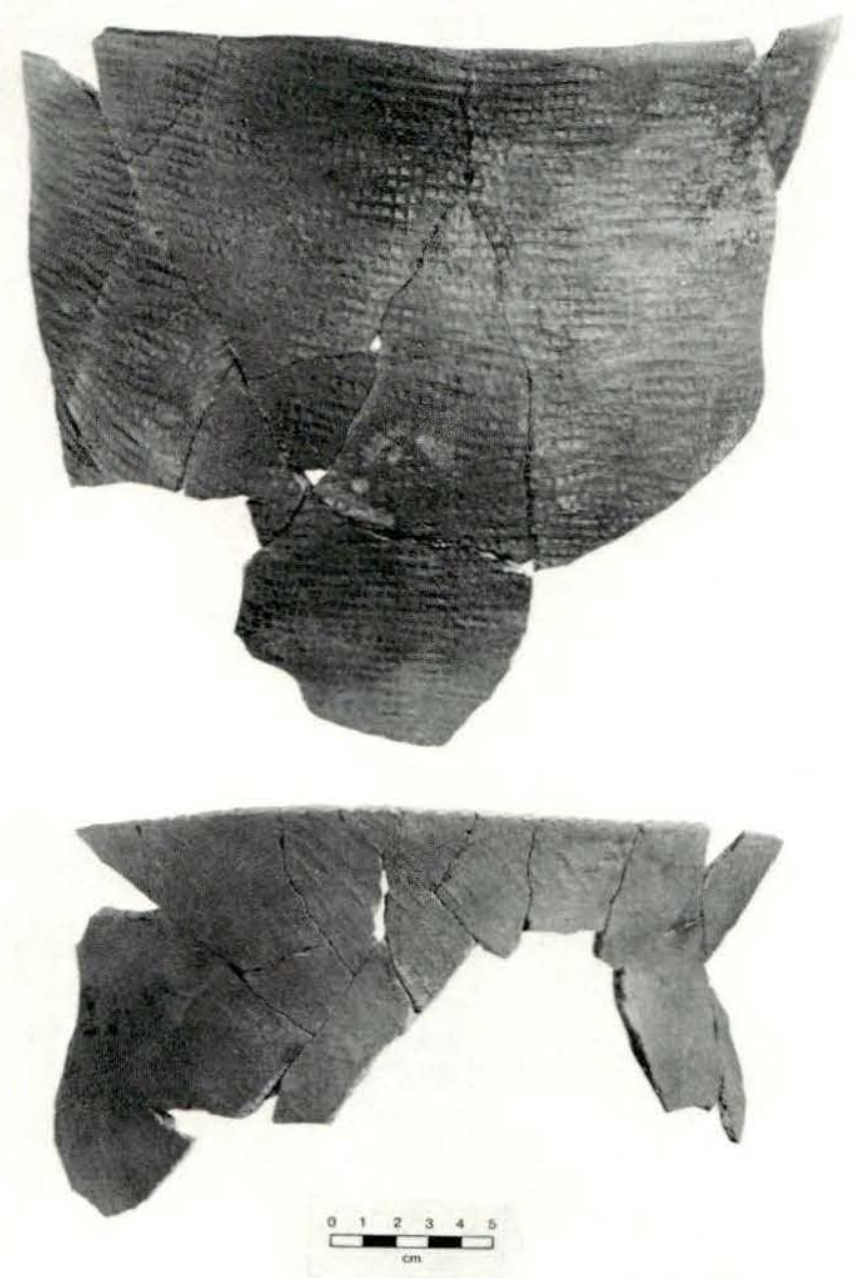

Figure B.6

Reconstructed check-stamped pots.
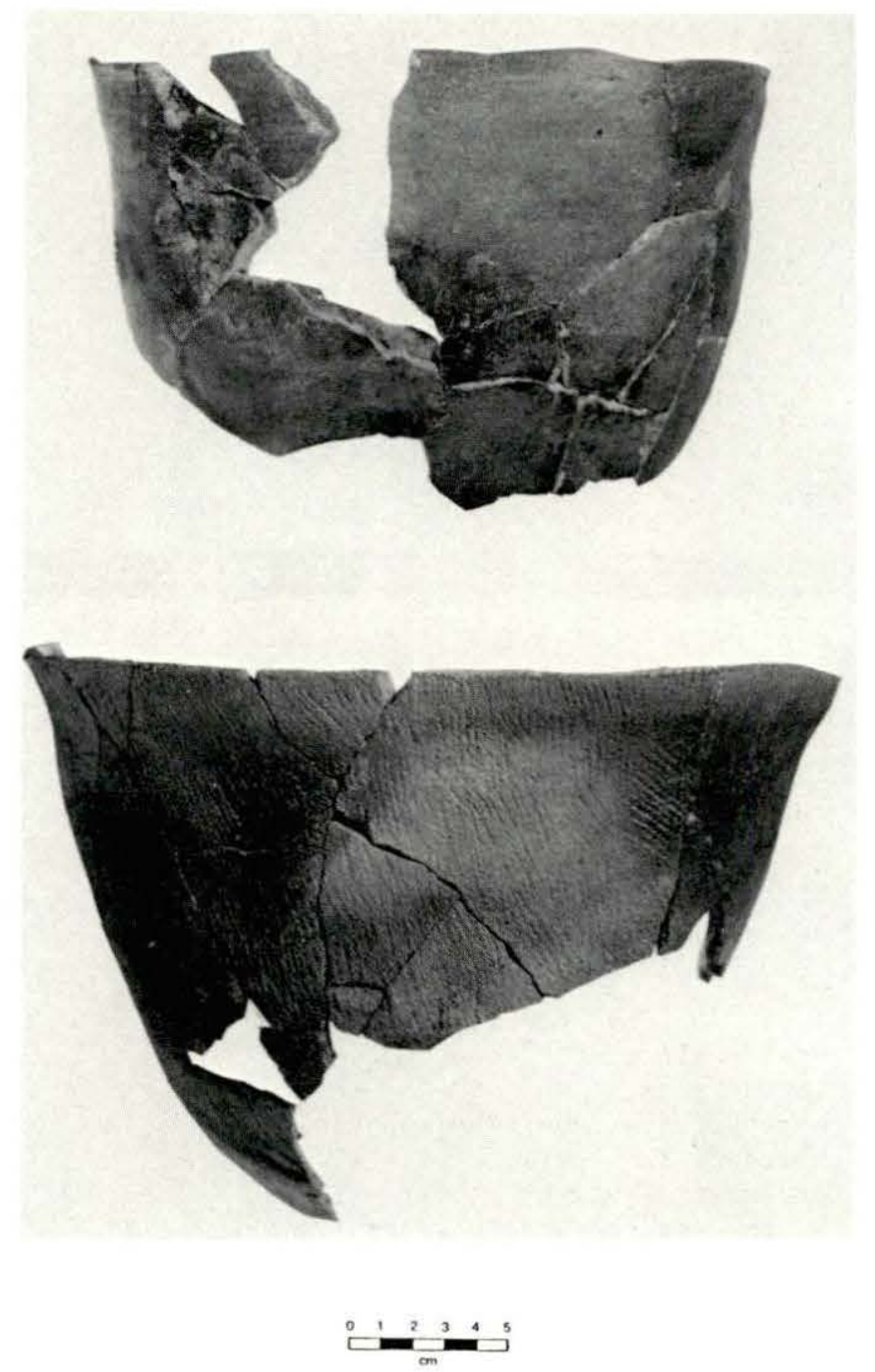

Figure B.7

Reconstructed plain and cord-marked pots.

contrast to the straight-sided, rectangular pits previously excavated. These shaft-and-chamber burials also lacked the distinct upper layer of refuse-laden soil that characterized most of the cemetery burials. The apparent differences in mortuary practices that are suggested by the burial data might be expected if different ethnic groups were living together in the village.

It was stated earlier that the cemetery burials and their attendant evidence of ritual death feasting might reflect northern influences (see Chapter 4). Certainly this pattern is not typical of the North Carolina Piedmont. On the other hand, the shaft-and-chamber pits that were found in 1986 are very similar to pit forms typically identified with Piedmont Siouan groups. The fact that they were 1ocated within the village rather than in a separate cemetery area also fits the Siouan mortuary pattern.

The size of the village compound, the number of houses contained within it, and the 

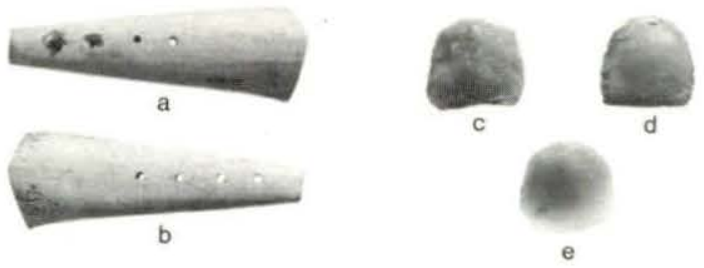

0<smiles>C[Si]1(C)[CH]CC1</smiles>
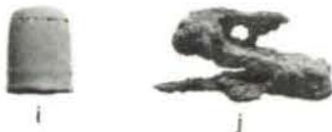

h

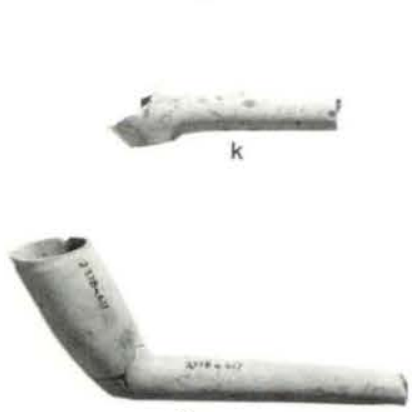

m

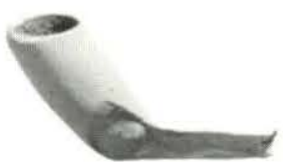

n

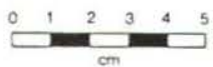

Figure B. 8

Small Euroamerican artifacts: bone knife handles $(\mathrm{a}-\mathrm{b})$; gunflints ( $\mathrm{c}-\mathrm{e})$; coiled wire $(\mathrm{f}-\mathrm{g})$; buckle fragment (h); thimble (i); gun part $(j)$; kaolin clay pipes $(k-n)$.

population estimates predicted at the end of the 1985 field season all seem to be accurate in light of the completed excavation (Figure B. 10). The palisade enclosed a little over a quarter of an acre on which at least 11 domestic structures were built. At any given time, probably no more than $50-75$ people inhabited the village compound and the occupation probably lasted no longer than five years. The size of the village and the population estimates support demographic models suggested by the ethnohistoric documents and contrast markedly with late prehistoric and early historic occupations on which there is adequate archaeological data for comparison.

Although the current phase of the Fredricks site research was completed in 1986, there are other avenues that should be explored through additional investigations. The question of the existence of other contemporary village compounds in the immediate vicinity has obvious and crucial significance not only in terms of clarifying the social and political standing of occaneechi Town but also in regards to larger questions of culture change and stability. Inter-regional comparisons are also necessary to draw into sharp focus a complete picture of Indian life on the Piedmont during this most critical era.
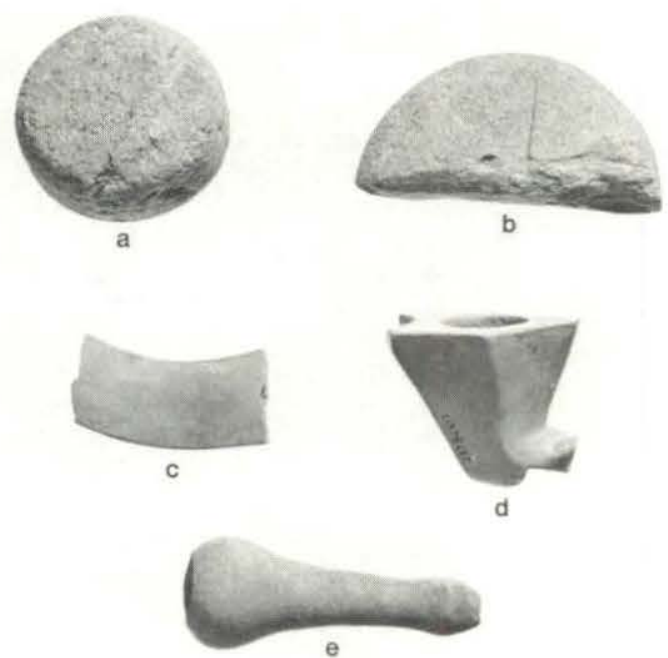

e
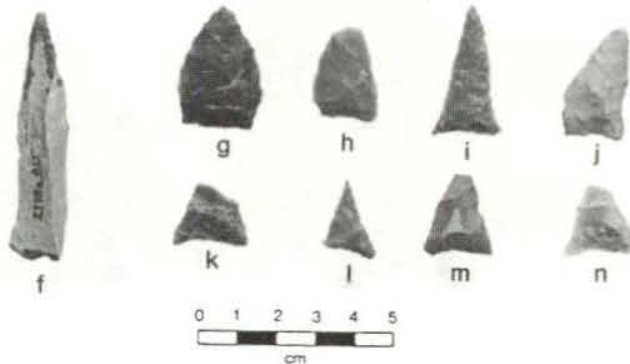

Figure B.9

Aboriginal artifacts: discoidals (a-b); clay pipes (c-e); perforator (f); projectile points $(g-n)$.

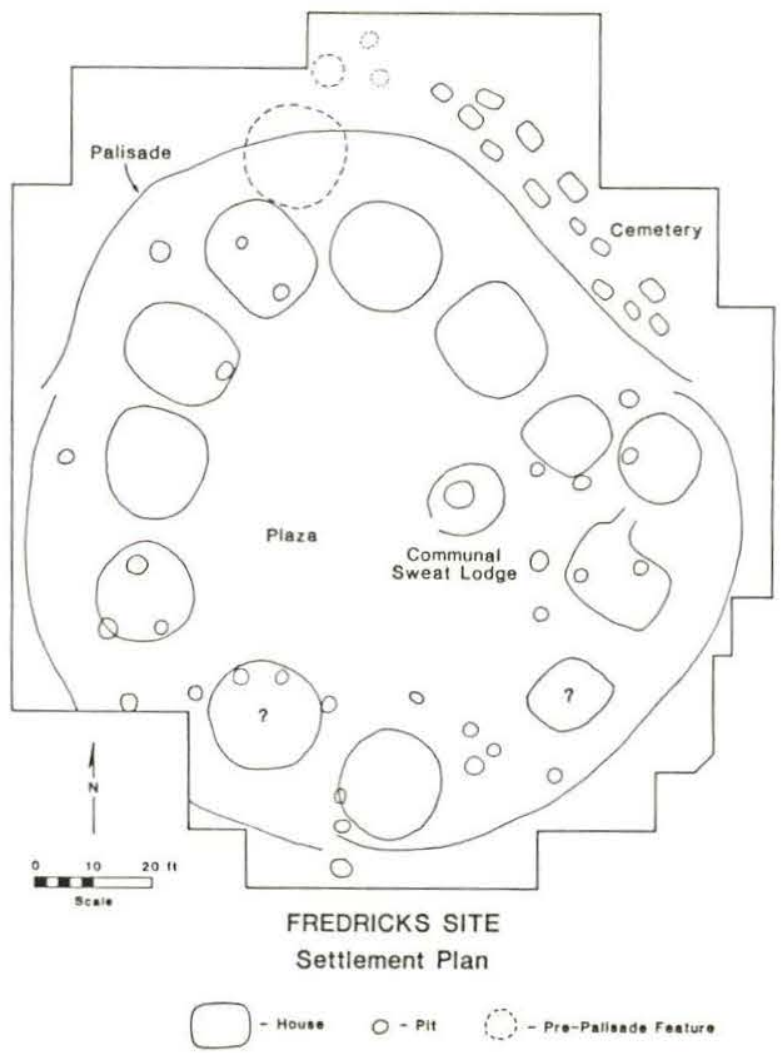

Figure B. 10

Settlement plan of the Occaneechi village. 


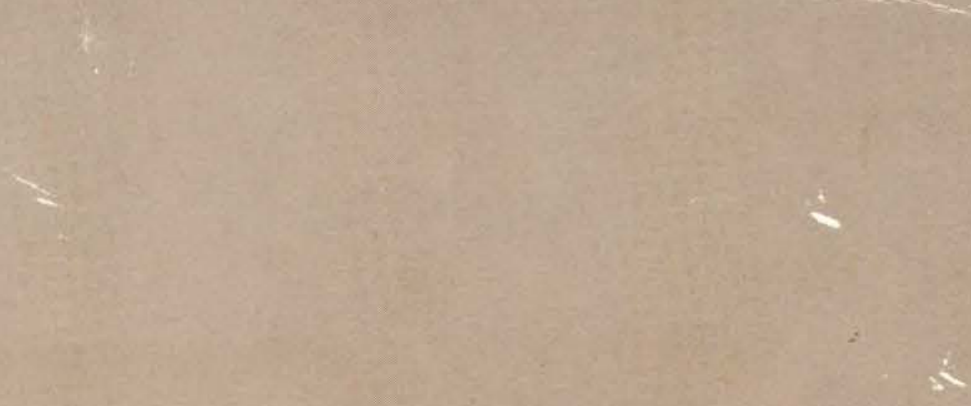

-...

,

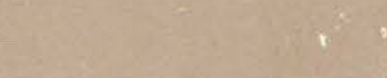

